\title{
Praceedings of the MASTER
} 1960 Idaha Conference on

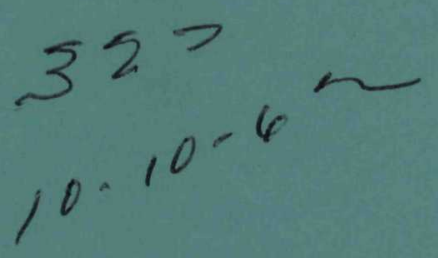

REACTOR
KINETICS

Sun Valley, Jdaha

Octaber 12-14, 1960
PHILLIPS

PETROLEUM COMPANY

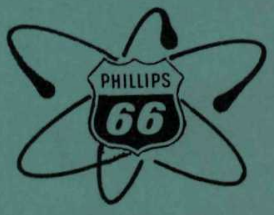

ATOMIC ENERGY DIVISION

\section{US ATOMIC ENERGY COMMISSION}


PRICE $\$ 4.50$

Available from the

Office of Technical Services

U.S. Department of Commerce

Washington 25, D. C.

\section{LEGAL NOTICE}

This report was prepared as an account of Government sponsored work. Neither the United States, nor the Commission, nor any person acting on behalf of the Commission:

A. Makes any warranty or representation, express or implied, with respect to the accuracy, completeness, or usefulness of the information contained in this report, or that the use of any information, apparatus, method, or process disclosed in this report may not infringe privately owned rights; or

B. Assumes any liabilities with respect to the use of, or for damages resulting from the use of any information, apparatus, method, or process disclosed in this report.

As used in the above, "person acting on behalf of the Commission" includes any employee or contractor of the Commission, or employee of such contractor, to the extent that such employee or contractor of the Commission, or employee of such contractor prepares, disseminates, or provides access to, any information pursuant to his employment or contract with the Commission, or his employment with such contractor. 


\section{DISCLAIMER}

This report was prepared as an account of work sponsored by an agency of the United States Government. Neither the United States Government nor any agency Thereof, nor any of their employees, makes any warranty, express or implied, or assumes any legal liability or responsibility for the accuracy, completeness, or usefulness of any information, apparatus, product, or process disclosed, or represents that its use would not infringe privately owned rights. Reference herein to any specific commercial product, process, or service by trade name, trademark, manufacturer, or otherwise does not necessarily constitute or imply its endorsement, recommendation, or favoring by the United States Government or any agency thereof. The views and opinions of authors expressed herein do not necessarily state or reflect those of the United States Government or any agency thereof. 


\section{DISCLAIMER}

Portions of this document may be illegible in electronic image products. Images are produced from the best available original document. 
IDO-16791

AEC Research and Development Report

Reactor Technology

TID-4500, Edition 17

Issued: July, 1962

\title{
Praceedings of the 1960 Idaha Conference an
}

\section{REACTOR KINETICS}

\author{
J. C. Haire \\ G. O. Bright \\ Editors
}

\begin{abstract}
The 1960 Idaho Conference on Reactor Kinetics, held October 12-14 at Sun Valley under the auspices of the Nuclear Safety Research and Development Branch, Division of Reactor Development, U. S. Atomic Energy Commission, was organized and conducted for the U. S. A.E.C. Idaho Operations Office by Phillips Petroleum Company, Atomic Energy Division.
\end{abstract}

General Chairman: G. O. Bright

\section{PHILLIPS \\ PETROLEUM \\ COMPANY}

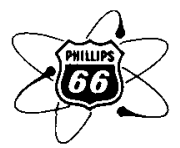

Atomic Energy Division

Contract AT $(101) \cdot 205$

Idaho Operations Office

U. S. ATOMIC ENERGY COMMISSION 


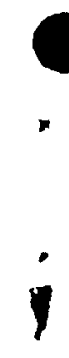

i 
The 1960 Idaho Conference on Reactor Kinetics was held at sun Valley, Idaho, October 12, 13, 14, 1960, under the auspices of the Nuclear Safety Research and Development Branch, Division of Reactor Development, U. S. Atomic Energy Commission. The organization of and arrangements for the conference were carried out by the Atomic Energy Division, Phillips Petroleum Company for the Idaho Operations Office, U. S. Atomic Energy Commission. The conference, the third on the subject organized by Phillips Petroleum Company, was unclassified.

The meeting consisted of six consecutive half-day sessions, each of which covered a particular part of the kinetics field. The emphasis in the program was to achieve a maximum of information exchange and to assess the work being done in terms of its relationship to the reactor safety program. Strictly technical detail was minimized to a degree consistent with keeping abreast of the rapidly developing field.

Attendance was by invitation with the purpose of restricting the size of the meeting so that a free interchange of information was possible, and also to ensure that a complete representation of the kinetics field was obtained. It was intended that every major group having an interest in reactor kinetics be represented.

The meeting was divided into six sessions organized by subject. These consisted of one introductory or general orientation session, four subject sessions, and a summary session having the purpose of reviewing the entire kinetics field and the accomplishments of the meeting.

Each of the sessions was conducted more as a discussion than as a series of progress reports from various projects. A typical session ran as follows:

A review paper on the state of the art including accomplishments, methods, amount of effort being applied and whatever else might be appropos to the subject was prepared and presented by a recognized expert in the field, and provided a stimulus to the discussion.

To supplement the review paper, presentations were made by a panel of about six experts representing as large a part of the field as possible. In addition to amplifying particular phases of work the panelists indicated the directions that their own effort would take in the future. 
Having scoped the field by means of the review and panel, the session then turned to open discussion from the floor. Questions to and from the reviewer, panelists, attendees and Chairman provided the impetus for a fruitful exchange of information, ideas and program plans. In some cases attendees with a particular a priori desire to participate came prepared with notes and slides to contribute to the subject under discussion.

The topics covered in the first session were very broad and the tone of the meeting, with emphasis placed on reactor safety and design considerations resulting from kinetics work, was firmly set. The subject sessions were devoted to power excursion programs, transfer function work, reactor instability and thermal-hydraulic problems, and analytical efforts in the field. The final review session pointed up the status of the reactor kinetics field as indicated by contributions during the conference. The accomplishments in the field and the areas needing emphasis were summarized and a number of constructive suggestions were made on program direction and information dissemination.

In order to obtain the latest developments in every area represented, the participants were not requested to supply the texts of their papers prior to the meeting. However, since previous such information sessions had indicated the desirability of having some record of the discussions, after the meeting, provision was made to record the entire conference proceedings, utilizing the sound amplification system set up for the speakers and supplementing this with microphones placed around the meeting room.

It was recognized immediately following the meeting that a permanent written record was desired by all who had attended and participated in the conference to ensure that the information exchanged would receive wider dissemination and to document a new point of debarkation for subsequent sessions devoted to reactor kinetics.

The proceedings were transcribed from the tape recordings and forwarded to the individual contributors for review and editing of their respective portions with the admonition to make only those changes required for reasons of clarity, factuality and policy, in an effort to preserve the conversational flavor of the proceedings. In preparing this document the editors have attempted to retain a "nontampering" attitude; however, some small portions of the transcript were deleted in editing for various reasons: some, at the suggestion of the participants; some, where discussion was non-tractable or completely irrelevant and some, where participants could not or did not supply copy of material placed on the blackboard and essential to the point of discussion. In addition, some deletion and rephrasing was considered necessary to provide continuity to those areas centered around blackboard discussions. Of those participants who provided review of their contributions, the last returned his copy to the editors in February, 1962. 
We would like to express our sincere appreciation to all participants for their cooperative efforts in bringing the proceedings into being. Other acknowledgements are included in Appendix I. Lists of conference attendees and organizational affiliation abbreviations are included in Appendixes II and III.

The titles shown for the panel presentations and review papers were selected by the editors who assume full responsibility for any failure to convey the main theme of the author's presentation. The editors also assume all responsibility for any errors and apologize for any mismatching of names to recorded statements or any misquoting which may have occurred in editing. It is hoped that the information exchange offered through this free expression of ideas far outweighs the consequence of any errors in the record.

J. C. Haire

G. O. Bright

Editors 
•... 
PROCEEDINGS OF THE 1960 IDAHO CONFERENCE ON REACTOR KINEIICS

Opening of the Conference . . . . . . . . . . . I W. E. Nyer

SESSION I - GENERAL CONFERENCE ORIENTATION . . . . . . . . . 3

Opening Remarks . . . . . . . . . . . . . . . 3

R. L. Doan (Chairman)

Problems Associated with Dynamic Behavior of Reactors . . 5 C. K. Beck

On the Application of Reactor Kinetic Data . . . . . 9 C. R. McCullough

GENERAI DISCUSSION . . . . . . . . . . . . 16

SESSION II - POWER EXCURSION PROGRAMS . . . . . . . • • • 35

Opening Remarks ................ 35

R. S. Stone (Chairman)

Review Paper

POWER EXCURSION STUDIES . • . • • • • . . • • • 37 F. Schroeder

PANEL PRESENTATIONS • • • . . . . . . . . . . 57

Presentation of Kewb Program . . . . . . . . . . 57 M. E. Remley

Presentation of Spert Program . . . . . . . . . 64 W. E. Nyer

Presentation of ORNL Program ........... 67 E. P. Epler

Presentation of Triga Program . . . . . . . . 71 R. H. Stahl

Presentation of Godiva Program ......... . 74 T. M. Wimett

Presentation of Treat Program . . . . . . . 78 J. F. Boland

GENERAI DISCUSSION . . . . . . . . . . . 86 
SESSION III - REACTOR TRANSFER FUNCTION DETERMINATION AND

APPLICATION . . . . . . . . . . . . . . . . . . . 105

Opening Remarks . . . . . . . . . . . . 105

W. C. Lipinski (Chairman)

Review Paper

REVIEW OF REACTOR TRANSFER FUNCTION FIELD . . . . • 107 J. A. Thie

PANEL PRESENTATIONS

A Statistical Stability Monitor . . . . . . . . 119 E. P. Gyftopoulos

Presentation of Brookhaven Transfer Function Program. • 125 H. J. C. Kouts

Presentation of Spert Oscillator Program . . . . . 133 A. A. Wasserman

Presentation of Westinghouse Reactor

Transfer Function Program . . . . . . . . . 145 J. N. Grace

Determination of $\ell^{*} / \beta$ by Pulsed Neutron Method . . . 151 E. R. Silver

Presentation of Nonlinear Stability Problems . . . . 157 F. Storrer

GENERAL DISCUSSIONS . . . . . . . . . . . 161

HRT Transfer Function Information . . . . . . . 161 P. R. Kasten

Discussion (Continued). . . . . . . . . . 170

SESSION IV - REACTOR INSTABILITY AND THE THERMAL-

HYDRAULIC PROBLEM . . . . . . . . . . . . . . . . . . . . 189 T. M. Synder (Chairman)

Review Paper

THERMAL HYDRODYNAMIC INSTABILITY . . . . . . . 189 N. Zuber 
TABLE OF CONTEIVIS (CONTINUED)

PANEL PRESENTATIONS . . . . . . . . . . . . . . 209

Transient Boiling Experiments . . . . . . . 209

V. E. Schrock

Instability Problems . . . . . . . . . . 230 E. S. Beckjord

Experimental Hydraulic Programs at ANL . . . . . . 234 R. P. Anderson

Presentation of Bettis Programs ......... 236 J. N. Grace

A Model for Hydraulic Instability . . . . . . . 242 J. A. Fleck

Discussion of Two Types of Reactor Instabilities . . 246 J. Chernick

Presentation of Ramo-Wooldridge Program . . . . . 251 D. B. Langmuir

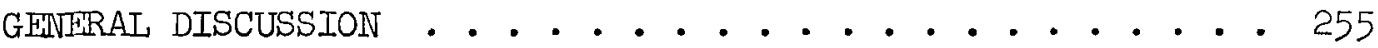

SESSION V - ANALYTICAI METHODS IN REACTOR KINETICS . . . . . . 261 J. Chernick (Chairman)

Review Paper

ANALYTICAL METHODS IN REACTOR KINETICS . . . . . . 263 A. F. Henry

PANEL PRESENTATIONS . • . . . . . . . . . . . 283

Review of Some Prompt Critical Accidents . . . . . 283 W. R. Stratton

Dependence of Reactor Burst Behavior on the Specific

Shutdown Law . . . . . . . . . . . 289 S. G. Forbes

Developments in Reactor Safety at ORNL . . . . . . 293 W. K. Ergen

Analytical Models for Burst Transients . . . . . . 297 S. O. Johnson 
Discussion of Extraction of Reactivity From Power Traces

and Equilibrium Bubble Size Analysis . . . . . . . 302 E. R. Cohen

Fast Reactor Kinetics . . . . . . . . . 306 H. H. Hummel

GENERAL DISCUSSION • . . . . . . . . . . . . 309 SESSION VI - GENERAL REVIEW . . . . . . . . . . . . . 319 W. E. Nyer (Chairman)

Summarizing Remarks . . . . . . . . . . . 319 C. K. Beck

Summarizing Remarks . . . . . . . . . . . 322 I. D. P. King

Summarizing Remarks . . . . . . . . . . . 325 E. P. Blizard

Summarizing Remarks . . . . . . . . . . 329 T. J. Thompson

Summarizing Remarks .............. 342 C. R. McCullough

GENERAI DISCUSSION . . . . . . . . . . . . 347

Appendix I - Acknowledgements . . . . . . . . . . 359

Appendix II - List of Attendees . . . . . . . . . . . 361

Appendix III - Organization Affiliation Abbreviations. . . . . 363 


\title{
PROCEEDINTGS \\ ON THE \\ 1960 IDAHO CONFERENCE ON REACTOR KINETICS
}

\author{
Opening of the Conference \\ by \\ W. E. Nyer (PPCo)
}

Nyer: Good moming, and welcome to the 1960 Idaho Conference on Reactor Kinetics. The organization and arrangements for this meeting were carried out by the Atomic Energy Division of Phillips Petroleum Company, for the Atomic Energy Commission and the Idaho Operations Office. Glenn Bright was the General Chairman for this conference and handled practically all of the arrangements; George Brockett has handled the sound system for us.

The objective of this meeting was to provide for free exchange of information and full discussion of the problems relevant to reactor kinetics and reactor safety so that, firstly, work in the field can be carried out with a background of sound understanding of the state of the art and the overall problems, and secondly, to assist in the diffusion of the information developed in the various programs to the level of everyday practice. To attain these objectives in the meeting, we considered a number of things. First of all, we thought of the people we would like to have at the meeting and there are a number of attributes that we would like attendees to have. For one thing, we wanted workers in the various programs in the field. We also wanted people who are cognizant of the needs of the reactor industry; people who can exert an influence on the dissemination of information, and people who exert an influence on the formation of opinions--opinions on what constitutes good safety and what does not constitute good safety. Now some of the people I have mentioned so far have, in a sense, a vested interest in the proceedings. We believe that for balance, we should also have people who do not have the vested interest of providing the information or having any real need for it in their own work. So, we also have included some people who do not take part in these various programs but, we believe, have a recognized expertness in the reactor field and can provide us with a good critique on the work that is being carried out.

Another factor we considered was, of course, the subject matter. We chose topics for discussion which we hoped would illuminate the principal problems and possible solutions. The particular topies were not intended to be either exclusive or all-inclusive. We felt that if we provided a few convenient bins into which people could throw various points for discussion, that this was all that was needed for the particular group that we had in mind getting together here.

The final point we considered and one which we believe has some importance, is the mechanics of these sessions--how we are going to run them. ( $I$ will cover that a little more in detail in just a moment.) We wished to provide an atmosphere that is conducive to a good exchange of information. To accomplish this, Sun Valley was chosen after a 
consideration of several points. We did not want the area to have so many distractions that people would have difficulty in staying at the meeting. I think you will agree that Sun Valley does not have that problem--there are no specialty restaurants to run off to, or any plays that must be seen that might interfere with the real business of people carrying out discussions of our areas of interest and work. For another thing, we wanted to keep the group and the meeting area small enough so that we could talk as a group with a high degree of informality and maximize the information-exchange. Now there have been some objections expressed to us about the remoteness of this location. If this turns out to have been an overriding disadvantage, we would like to hear about it in the future. In fact, if there are any comments at all on things that might improve the objective, or attaining the objective, of exchanging information, we would like your comments.

Now there is just a little bit of administrative trivia that I think we should mention at this point. I think just a word on the scheme of the session would help. We have provided, as you all know, a review paper at the beginning of each session and the function of the review paper is to try to discuss the state of the art. Now, clearly, no reviewer knows what the state of the art is at any given instant. For this reason, we have included a panel whose function is to bring up work which a reviewer can't reasonably expect to be familiar with. Another function of the reviewer, hopefully, is to introduce controversial subjects and in general try to promote discussion. We expect that the panelists will comment on any deliberate, or inadvertent, controversial points that the reviewer makes. Since we purposely also want to provide everybody with a soapbox, we hope that after we go through these first two portions of the meeting that people will feel free to make speeches from the floor. It will be quite easy to accommodate any slides that anyone wants to show at that time. I think that the only problem that the Chairmen will have will be to make sure that no one takes an inordinate amount of time for his soapbox rendition, but we do wish to encourage as much of an exchange of ideas as possible. I think that we have only one admonition in general, and that is, we really did not want to devote too much time to hardware. There are various things meant by hardware, there is mathematical hardware as well as the physical hardware. For the purpose of this meeting, we might also put containment in the hardware category. However, it does impinge on the kinetics problem and there may be reason to bring it in, but we are not primarily interested in it. Clearly, control systems are a real part of the safety problem and the kinetics problem; they all go together, so we do want to hear about that, but not about specific designs. This is all that's necessary to say about that point.

Well, for the first session this morning we have a number of people who, I think, have various of these attributes or characteristics that I mentioned earlier. Certainly you will all agree that they are in position to formulate opinions and to influence the dissemination of the information. These people are also cognizant of the needs of the field. To start this session, I would like to introduce Dr. Doan, Manager of the Atomic Energy Division of Phillips Petroleum Company. Dr. Doan has a long background you all know about in the atomic energy field and also has a long period of service on the ACRS; Dr. Doan. 


\author{
SESSION I \\ GENERAL CONFERENCE ORIENTATION \\ Chairman: R. L. Doan (PPCO) \\ Speakers: C. K. Beck (AEC) \\ C. R. McCullough (Mon) \\ Opening Remarks \\ by \\ R. L. Doan (PPCo)
}

Doan: I think I might ask the first two speakers to join me up here so I won't be so lonesome, so Cliff, if you and Rogers will, get up here and stand by. I guess the objective of this first session is to set the stage for the real discussion that will come later. Warren Nyer talks about wanting to encourage discussion on controversial subjects, so I guess he thought the proper way to open the session would be to have some controversial people, so that would possibly start it off. At any rate, both the HEB and the ACRS have the job of looking at the safety aspects of reactors which involve in a good many ways the kinds of things that are the subjects of the discussion this morning.

The basic problems of reactor kinetics and the objectives of experimental investigations in the reactor kinetics field, as I am sure you would all admit, can be stated a good bit more simply and easily than the answers can be found in the way of experimental data and interpretation of the data. Under a given configuration and composition of fuel, moderator, nuclear poisons and a given set of initial conditions, the problem is to determine the power response of the reactor, and the consequences of this response, to a given rate of reactivity addition continued over a specified length of time. The major objective of reactor kinetics research is to improve our understanding of reactor behavior in order to facilitate the design of safer and cheaper reactors. I think we would all be agreed that, in time, this certainly will result. Whatever can be built into a reactor that will improve its inherent safety characteristics represents that much less to be provided in the way of expensive instrumental controls and containment. Whatever factual information can be obtained and disseminated on the inherent limitations of a given type of reactor to the energy that can be generated by power excursion will in time serve to raise the confidence level of everyone, including government regulatory groups, in the built-in natural controls that already exist in reactors.

I recall, not too many years ago, we talked about certain limitations on delta $\mathrm{k}$ in swimming-pool types of research reactors. As the work on kinetics has gone forward we certainly have changed our viewpoints and modified them and revised them in the light of the data that have been obtained. Because it is presently impossible to consider any nuclear reactor to be self-stabilizing within safe limits under all conceivable reactivity additions, it is necessary to exercise judgment on the amount 
and kind of control instrumentation required to supplement the inherent control mechanism and keep the reactor within safe limits during both normal and abnormal operations. Such inherent factors as delayed neutron fraction, neutron lifetime, Doppler broadening and void coefficients, all play an important role in stabilizing the reactor against extreme power transients but they are not enough in themselves to effect all the necessary controls. The first two factors affect the time scale of the transient response, but only indirectly its terminal point. Within certain limits the last two factors can influence both the time scale and the terminal point, that is, the maximum power resulting from a given delta $k$. The extent to which natural shutdown mechanisms can be depended upon to safeguard the reactor, either in conjunction with, or in the absence of, control instrumentation is one important area of understanding which, it is hoped, will be increasingly illuminated by reactor kinetics studies. More detailed and quantitative knowledge of shutdown mechanisms and the range over which they are effective will almost certainly lead to simpler, cheaper, and more reliable reactor control systems. Reactor stability against changes in reactivity, which may have occurred during operation, due to a developing combination of circumstances peculiar to the particular power level or operational procedure being employed, is something that every designer tries to achieve but can never be quite certain that he has succeeded. One only needs to recall the severe oscillations that can be initiated in the reactor under certain circumstances by a large, delayed, negative temperature coefficient, a phenomenon relatively unknown a few years ago, to speculate on the possibility that there may be other sources of instability which will be revealed by more extensive, theoretical and experimental investigations in reactor kinetics. Slow oscillations due to xenon instability transients have been observed in at least some large reactors and have been controlled by proper programming of the control rods. The most famous power transient in reactor history caught the technical personnel flat-footed for lack of information to explain it. In this case the transient was a negative one and put the first Hanford production reactor out of commission for lack of neutrons until Fermi found the answer. It is unlikely that there will ever be any more surprises in the reactor behavior field comparable to the first one, although one must admit that there was no very well developed understanding of the oscillations at the time they were first observed. However, it is becoming increasingly apparent that a more detailed knowledge of reactor kinetics in all types of reactors is essential to a proper evaluation of reactor safety. Getting the necessary experimental background in this area is quite costly and for this reason it is essential to give careful consideration to selecting the kind of experimental program that will produce the most useful results.

I have with me on the platform today two gentlemen who spend a goodly portion of their time looking at various aspects of reactor safety. Many of these aspects, as I have stated previously, fall in the area of reactor kinetics. Dr. Beck, as you know, has been for sometime the Head of the Hazards Evaluation Branch in the Division of Licensing and Regulation and has recently been made Assistant Director of that division; and Dr. McCullough is a long-time member of the Advisory Committee on Reactor Safeguards and for a good many years its chairman. I am sure they will agree with me that there is no more single important field in the area of reactor safety than that of reactor kinetics. I am now going to call 
on these gentlemen to make whatever remarks they consider appropriate in this area, to state their own interests and the interests of the groups with which they are associated in the reactor kinetics field. I'll first call on Dr. Beck.

\section{Problems Associated with Dynamic Behavior of Reactors by C. K. Beck (AEC)}

Beck: Mr. Chairman, Gentlemen. In a gathering of this kind, which is devoted to the serious business of complex detailed technical problems, there appears to be some feeling of necessity that some extraneous material should come into the program, some deference to protocol, some courtesy to the patron sponsor, or what have you, that is necessary. And since the AEC is in one way or another involved in this business, there was a recognition, I presume, on the part of the program committee, that some representation from that body ought to be included on the program, even though not very much contribution might be expected therefrom. I was definitely given to understand that this part of the program was non-technical, it was sort of general, and more or less introductory in nature, and might very well have been omitted entirely without serious detriment to the conference. But whatever the reasons, I am here to make a few remarks that may or may not be helpful, and had intended that these be very brief. The chairman has informed me that there is a gap in the program and that my remarks ought to be exterded somewhat, if possible. However, while saying a few relatively unimportant things in a short period might be acceptable, saying an equally unimportant few things in a longer period would not be permissible, I should think.

In any case, I want to mention a few items which arise from the viewpoint we have in the Reactor Hazards Evaluation staff with respect to this problem of kinetic and dynamic behavior of reactors. The basic responsibility of our staff is not to design reactors so that appropriate measures of safety will be provided, but to analyze, and evaluate, and determine whether or not the reactor someone else has designed and proposes to build is provided with an appropriate measure of safety.

With respect to dynamic and transient phenomena, a reactor may be considered to have an appropriate measure of safety when either of two conditions are fulfilled:

First, if the potential combination and interaction of its assorted characteristics do not result in spontaneous instability; that is, oscillatory or erratic variations in behavior, mechanical, hydraulic, thermal, or nuclear, of such magnitude that components would be damaged, or would not result in rapid excursions of performance parameters; that is, flow rates or neutron flux, to ranges which would damage components in consequence of such impressed perturbations as are likely to occur, reactivity insertions or other.

The second condition: an appropriate measure of safety would be satisfied when, in any reactor, any inherent tendency toward spontaneous instability or excursion of performance parameters to unsafe values by likely perturbations, would be counter-balanced or could be contravened 
by sufficiently dependable safeguards, shutdown fuses, safety valves, or control rods. Stated differently, a reactor should not be likely to be unstable or to undergo excessive transients under conditions likely to be encountered, but should either of these possibilities exist, then appropriate and dependable safeguards against such possibilities should be available.

It is not possible to define quantitatively the dividing line between "an appropriate measure of safety" in these two criteria, and "an inappropriate measure," or to specify quantitatively the nuclear, thermal, hydraulic, and mechanical features or characteristics which must be included. There are so many different combinations that would be acceptable that it would be impossible to tabulate all of these and premature to specify some as being preferable. That is, in this area of dynamic and transient characteristics, I believe that our present position would have to be that we do not know enough about what the various factors are and their relative contributions to stability and resistance to excursions, to specify that this particular, or that particular one, or this particular combination, must be present in any reactor system, or to even attempt a tabulation of permissible ones, so that any proposal which might be submitted would have to conform with either this list, or that one. We are still in an experimental area of rapid development, of expanding understanding, and though some have suggested that in certain types of reactors we may be already at the position where there can be standardization and specification of required characteristics, I believe this is not true. For example, in power reactors, I think we are just at the beginning, even though some power reactors are somewhat similar to others. Even in those types which are more prevalent, it is my opinion that we have an undue measure of carryover from the earlier days of experimental restrictions and control and have not moved far enough into a broader consideration of what might really be the minimum necessary requirements and restrictions; the control or design features which must without fail be included.

Therefore, the judgment which must be arrived at in considering the appropriateness of the measures of safety provided, in any reactor project, must remain, for the time being, a subjective one. However, in arriving at these evaluation judgments, which to a considerable degree must remain subjective, at least in my opinion this is so, a number of questions are usually asked. At the risk of belaboring the obvious to some extent, I would like to mention a few of these questions which are customarily asked when one examines the dynamic aspects of reactors.

The first question would be, "What factors are present which would contribute to instability? What are the magnitudes and time factors of the various reactivity parameters in the fuel, the moderator, the coolant, and the physical structure? Temperature coefficents? Doppler effects in the fuel and in poisons likely to be present? Void coefficient? Heat transfer parameters? Circulation and flow rate? Poison buildup and burnout? Possible effects of structural deformations?" That is, are there combinations of these parameters that might lead to instability in the range of operations considered? 
The second question, "What are the response characteristics of the reactor; particularly the thermal and nuclear responses to impressed perturbation? Should there be a sudden reactivity insertion, what is the time response of the reactor in power level and in the secondary effects, such as changes in temperature and neutron flux patterns and the amount and distribution of generated (or burned out) poisons? How much perturbation can be impressed without exceeding counter-balancing effects?"

Third, "What are the non-uniformities over the core, in flux, temperature, poison distribution, and so forth? How do these vary during operation of the reactor? During core burnout and during shifting control rod patterns?" This was, for example, a particularly troublesome question when the initial operation of the Dresden reactor was being considered because of the very large size of its core and the possibility that there might be non-uniformities or feedback effects between various portions of this core which were acting somewhat independently of each other.

Another question, "What are the margins between normal values of thermal flux and burnout flux? That is, the average values of this margin and lesser margins in the hot-spot areas. What are the magnitudes and time factors in transient phenomena and how do these relate to burnout?" Farther along this line, "What are the microscopic hydraulic and thermal phenomena involved in the burnout process, and how can these be more reliably predicted?" There is a particularly great need for an experimental investigation of this matter, particularly in narrow channels peculiar to reactor cores. The phenomenon of boiling disease is clearly recognized, but microscopically the phenomenon is not clear, at least, I don't understand it. The factors which influence it, particularly in the narrow channels encountered in reactor structures, are apparently very complex and subject to substantial influences from components which, in ordinary situations, might be expected to have relatively little effect.

Another question, "What are the magnitudes and time factors required in safeguard devices used to control or prevent instability or excursive behavior? Can mechanical devices move fast enough to have any effect? How large reactivitywise must they be, if they are to prevent excursions or to control sudden onset of instability? Can instruments detect incipient departures from normality and invoke corrective measures in time to prevent trouble?"

Another question along this line, "What are likely to be the consequences of divergent oscillations, or nuclear runaway, should they occur? What energies are released, and other consequences? What are the natural termination processes of such abnormal out-of-bounds operation? Are such out-of-bounds phenomena self-limiting at once; that is, essentially stable, or are they self-limited by delayed action parameters, or self-limiting only by destruction and dispersal?" An evaluation of the likelihood of out-of-bounds behavior and the consequences of such, should it occur, are the two key criteria to the final judgment that must be made in safety approval.

It is frequently impossible to obtain satisfactory resolution of these problems in advance of reactor construction. Except in some wellexplored types, there is substantial uncertainty as to the detailed 
dynamic behavior to be expected. Even in well-proven types, such as boiling water reactors, a change in fuel geometry or materials, or a substantial change in core size or geometrical arrangement, for example, to include a superheater or some other additional component, may lead to uncertainty in the prediction of performance characteristics, and the feedback between various parts of the system. This latter consideration then brings into focus another series of questions of importance to the evaluation and regulatory processes; namely, "To what extent and by what means, can successful operation at low power level and one set of thermal and hydraulic conditions be extrapolated to predictions of the performance to be expected at higher power levels and other conditions?"

The development of techniques for analysis of forced oscillations, forced oscillation response, excursions due to small reactivity perturbations, and random-noise fluctuations in power level, has been of great assistance in stability predictions for reactors, largely boiling and pressurized-water types for which it has been most explored. A great deal of confidence has been obtained, for example, from the experiments on the EBWR, and the experimental verification in that reactor of these techniques and its applicability in those cases. Whether or not, and under what conditions, these techniques can be extended to other reactors will similarly serve a useful purpose when such has been explored.

It is obvious from this recital of questions we have to ask, and the number of them on which only partial answers can be obtained, how important it is to have under way a vigorous program of research in these areas. I am convinced, myself, without any minimization of the importance of the theoretical explorations, that the heart of this problem at our present stage must be an experimental one. Borax, the early Spert reactors, EBR-I, and EBWR have provided us with a good solid beginning for the development of conceptual understanding, and there are ample data for analysis and limited extrapolation for prediction of performance in other situations. But, as in most other situations, an abundance of experimental observations is the only way of identifying the valid extrapolations and explanations and verifying the foundations for more generalized understanding. The new flexible spert reactor and Borax $V$ are overdue extensions of the earlier experiments, and there are others that should be considered. There is a particular need for experimental investigation of other types of reactors and other combinations of conditions beyond those which have previously been explored.

Pending such new programs, however, there appears in the meantime to be before you kinetics experts, a considerable amount of overdue housecleaning on collection, organization, and interpretation of what has already been done. No less than three times in the last year, some member of the Spert organization has expressed to me his indignation at seeing Borax or Spert data quoted out of context in some hazards report, in situations where these data did not apply and invalid conclusions had been drawn. In two cases, it so happened, my staff had already recognized the fallacy of the arguments which had been submitted, and in one we had not. The point is, however, there is a huge need, a vacuum, in this area which is only fragmentarily filled by the work done thus far. It is very important that this work be organized and presented to the nuclear community so that all parties will understand the correct interpretations 
and its area of validity. I think this is urgently needed, not for development of standards or as a point of departure for specifications of requirements that must be included in reactors, but for maximum assistance to the designers and to the evaluative staffs who must reach some more or less subjective judgments on the proposal submitted. It is my full expectation that the discussions at this meeting will contribute in substantial part to a beginning on this point of explanation, interpretation, and indication of limitations, and areas of validity; that you experts in this field will fill in, during this meeting, some of the gaps in my own knowledge in this specialized field.

For this, as well as for the pleasant opportunity to re-establish contacts with old friends and to make some new ones, I am very happy to be here.

Doan: Thank you, Dr. Beck. I am sure a number of you have some comments you would like to make on this, or some questions, perhaps to clarify some of the things $\mathrm{Dr}$. Beck has said. After the next speaker, this will be encouraged and permitted, because only to the extent that there is full and free discussion of these things, can this meeting be considered to be a success. But before calling for these comments or any questions, I'll call on our next speaker, Dr. Rogers McCullough, to say a few remarks from the viewpoint of the Advisory Committee on Reactor Safeguards. Dr. MeCullough.

\section{On the Application of Reactor Kinetic Data by$$
\text { C. R. McCullough (Mon) }
$$

McCullough: Mr. Chairman, Friends. There are two angles to following a speaker like Dr. Cliff Beck; one is that he has pretty well covered most of the things that I had thought about saying. However, I have an advantage, perhaps, of picking up a few points and emphasizing them; but I must say that he has covered, quite thoroughly, most of the points which I am very much interested in.

I can't remember whether this is the second or third of these kinetic conferences--I seem to remember it's being the third--maybe the first one was quite informal--and I've always enjoyed coming. I'm very appreciative of being invited to this one. They are vigorous, effective exchanges of ideas.

Now, in my feeling, the kinetic part of reactors is one of the exceedingly important parts. From the safety point of view, over and over again, we look at two things--we look at the excursions, and we look at the loss of coolant. I have the uncomfortable feeling that there has been a trend to overemphasizing the examination of the loss of coolant accident, and sort of passing by the excursion. Partly, I believe, because we don't understand as much about the excursion phenomena as we ought to, and we are put to sleep with the assurances that, "Oh, we have inherent stability," and "We have adequate shutdown mechanisms" and so forth. This, perhaps, can be developed more thoroughly a little bit 
later in my own remarks and perhaps much more thoroughly in the conference which is going to follow. Now, I am sure you recognize that a reactor that hasn't started,--sitting quiescent,--is certainly an entirely safe reactor. It has neither the excursion problems nor the shutdown problems. Almost equally safe is a reactor which is running at steady power, which is another way of saying that the times we are apt to get into trouble is when we are either trying to start up or shut down these machines. Now, it happens that there are some reactors which will cook along with very little variation as long as they have been brought to steady power, and I think it is worth thinking--in trying to advance the art of reactors-that there may be real utility for a so-called "base-load" reactor; namely, one that is carefully brought to power and then left there, and only shut down when some failure occurs or you no longer need the base-load. This is a reactor which cooks along steadily at a given power and is not maneuverable. I think this ought to be given further thought as development of the utility of nuclear energy. Now, if you recall the very first pile, as they piled up the material in the West stands they were watching carefully for the first indication that the reactor was critical, and, if I remember correctly, instead of the word critical, in some other languages it's called "the reactor becomes exponential", and this impresses you, I think, with the idea that perhaps for the first time in history, we are now dealing with an exponential phenomenon. If you'll think back when you first began to have some concept of quantities and numbers that you knew how to add, one block, plus another block is equal to two blocks; then as you became a little bit more sophisticated you learned how to multiply, that when you took two blocks this time, and two blocks this time, and two blocks this time, you had six blocks. But many of us are just beginning to really appreciate what exponential means, and that's the characteristic of the nuclear business. These machines go up exponentially in power.

Now, what I want to say can be briefly broken down into three parts. I want to talk a little bit of how I feel about the necessity for more understanding of the fundamental characteristics, the theoretical characteristics, and the detailed characteristics of kinetics, shutdown mechanisms, and so forth. Secondly, I want to talk a little bit more-I'll try to add a little to what Dr. Beck has already said, about experiments. And the third subject I'd like to talk a little bit about is how you may use the kinetic properties of reactors.

Now, if you look at a reactor and its kinetics, I think it is important to understand just how the increase in power, which is another way of saying the increase in the rate of fission, which is another way of saying an increase in the amount of the neutron flux which is effective in fission--just what are the characteristics of this flux which influence the kinetics of a reactor. And I submit to you that I do not believe we understand this as thoroughly as we ought to. Now, I am probably not up to date, and hope to be corrected in the subsequent discussion if I am not, but I've seen many curves; I've seen considerable discussion of the properties of the excursions of the Spert reactors, but I am impressed with the fact that the curves are empirical fitting of the data. There is still, the last I knew, an unknown about the real characteristics of the shutdown mechanism. Now I'm not at all clear as to what this uncertainty is due to, but I think it's something that ought to tickle our 
imaginations, ought to keep on spurring us to further thinking as to what is involved here. You realize, of course, that there are neutrons which are generated in the heart of the core, and if you are talking thermal reactors, they very promptly get moderated and moved down to the thermal energy, and then they, of course, are absorbed in the fuel and cause fissions. Just to be arbitrary, I am calling those "local neutrons." There are some other neutrons that wander around the core, get out into the so-called reflector, which is a misnomer as we all know, and finally get back in and cause fissions. Arbitrarily, let's call these "errant neutrons." And, by the way, I'm stealing some other people's ideas when I use this concept, but you'Il pardon this--we practically all steal ideas all over and re-present them. There are other effects that happen during an excursion. I think we haven't looked thoroughly enough at the secondary and tertiary effects of the excursion--or perhaps, not only the first differential, but the second and third differential. We must look at the gamma rays and where they are generated. I have not seen enough discussion, in my opinion, of the prompt beta effect. I ran into a comment, not too long ago, to the effect that there are considerable soft gamma rays generated in the fission process. I haven't had time to look up the literature to find out whether this is in fact so, but let me point out that if this is true that there is a large fraction of energy--and by large, I mean a few per cent shall we say--which occurs at the time of fission in the form of soft gamma rays, or perhaps hard $\mathrm{X}$-rays. Then the thickness of the cladding makes a big difference as to how much energy is deposited in the moderator, and this is worth thinking about further. If it is a fact that there is this soft fraction--this might be a mechanism of heating the moderator and the shutdown effect.

Now, by the same token, in understanding the theory of what goes on, I think we should be aware of the trap of excessive formulation, on excessive formalism from the use of mathematics. Now, certainly you can't do any appreciable amount of theorizing and justifying one theory, or one part of a theory with another, without mathematical expressions, but you can surely get trapped by it. You'll be so concerned with manipulating the formula that you'll forget what you are talking about. And I think it is important to remember and avoid this trap. Now, Dr. Beck made the very strong point that theory is fine, but practice and experiment and verification of theory is essential. I most heartily second this. Obviously we like to see reactors built which have inherent stability characteristics, and we've done very well in certain reactors in designing it into them. Not too long ago I had the privilege of observing a naval reactor, and that reactor was in a submarine. We went back in the engine room and we had them maneuver the machines from zero power to full power. This can be done without moving a single control rod. This is all because of the temperature coefficient of the reactor. Now, actually they do manipulate the control rods a little bit because they have certain limits of temperature that they wish to stay within, so the operator trims the rods a little bit to be sure he doesn't get near the boundary, but it was not necessary at all, from full. power to zero power, to move these control rods, which is a very good example of inherent stability. Other stability effects--the Doppler effect, perhaps radiolytic gas and the time constants of these various effects--need to be verified experimentally, as Dr. Beck said. 
Another point which is troublesome to those of us who have to norry about safety is when you are depending on control mechanisms... and we always have to depend on control mechanisms--even if you have inherent stability, there comes a time where you have to do something to bring the thing really down to zero power and low temperature. How reliable are these devices? And when you start thinking about this you'd better start right back at the beginning and ask yourself how reliable is the ion chamber, or the fission chamber, or whatever other primary sensor you are using. Now this is hardware, I know, but it''s not, I submit, out of the subject matter of this conference because ycu must think what you are measuring and why you are measuring that particular quantity. If you are measuring neutron flux you must be sure that the neutron flux is the thing that's really important at that particular time. Look, if you're worrying about burnout of fuel elements, it's not the neutron flux that you're concerned with, it's about the heat flux and about the thing that's there to take the heat away. If we're talking of water reactors, the thing that's important to avoid burnout, is not primarily the neutron flux, although certainly this is important, it's to know that there's no steam blanket there which, of course, makes for poor heat transfer. Now, how you handle this problem, I don't know. You can't have thermocouples over every square centimeter of surface in the reactor core, and this is what is inferred by the comments I've just been making. But, we have to have some way of knowing what the temperature is. Now there are other parameters which you need to know; namely, the flow characteristics. And frankly, I think that some of our instrumentation has been elaborated because--well--we know how to measure these things and this is very intriguing instrumentation, very impressive looking, but I'm not sure it's really at the heart of what you're trying to do. And incidentally, if you're trying to advance the art, there are some economic reasons why you want to make the instrumentation just as simple as you possibly can. But the primary thing is, let's keep focused on what we're trying to measure, what we're trying to detect, and how we're trying to counteract it.

Now, in connection with these actual checkouts of the theory and the measurements and verification of the characteristics of the reactors, we need this data in order to write criteria or standards for safety, for acceptability of the reactor. I keep trying to say that safety is really only another word for reliability, and certainly anyone that has a reactor wants it to be reliable, and the reliable reactor, in my book, is a safe reactor. Cliff has referred to the difficulty of writing standards, that the matter is too complex for us to write them down now, and I agree heartily. At the same time I think we need to think about what we mean by criteria, and the truth is that we're using criteria every time we design a reactor. And the truth is that most reactor designers use the same criteria, in general terms. Criteria does not have to be a nice tabulation of accurate numbers; to get criteria, you start out with a philosophy. The first point that you make, and cliff made this point, for your criteria is that the reactor you design must be stable. Now, if it should have a characteristic such that it drifts away from the point where you want to have it, then the rate at which it drifts must be correctable. Well, there's a point before that. You must know that it's drifting and then secondly, you must be able to correct it before it's gotten to the point where it's damaged its components. So, I submit to you that we are in a position to take and write down the criteria that 
we're actually using. And if we do this intelligently, we will not fence ourselves in with numbers. Now, I'd like to put this point across, that when you start talking about these, call them qualitative concepts if you like, criteria which we are really and truthfully using, it's awfully easy to fall into the trap of expressing them by a nice simple number. We do this type of thing in all of our activities in life. We try to simplify the thing, and so thereby define it accurately, and when we do, and when this thing that we're using as a measure is not really expressible as what we are really trying to say, we immediately put ourselves in the hole. Now, for example, we really don't care how fast we withdraw control rods, what we really mean is how fast we're putting reactivity in. And that has no meaning unless we define the characteristics of a particular reactor that we're working with. We have a sort of a well-understood criteria that reactors must not have positive temperature coefficients. Well, we immediately start thinking of exceptions. Again, we don't object, really, to positive temperature coefficients at room temperature unless this coefficient is of such a character that when we try to get the reactor up to its design power that this will let the thing get out of hand. We recognize that we've got a lot more time to work with this thing as we start a reactor up. We would not like a positive temperature coefficient which is of a large magnitude when a reactor is in its operating range. so to say that it must not have a positive coefficient is misstating the problem. We've oversimplified it.

Now, the other point that I'd like to comment on is that I see in the literature more and more of the thing that Dr. Mark Mills, who I consider was one of the very able workers in this field, was emphasizing over and over again, and that is that you don't need to make elaborate experiments to settle some of these points. He was a great believer in small-scale, bench-type experiments which try to settle a particular issue, and I see evidence of more and more of this going on, and I think it's to be encouraged and to be increased. This is not to say that some of the larger scale experiments are not necessary--they are--they are essential. But there is plenty of room for well-designed experiments on the small scale.

Now on this matter of criteria and standards, I think it's important that we get busy and work on them. We're in the fortunate position today that in the nuclear business we're not forced to come up with some criteria or standards today, because there's been an accident. In other endeavors, the history has been that there were no standards, there were no criteria, until an accident occurred, then there was an investigation and the conclusion was that if there had only been a standard, if there had only been a rule, then this accident wouldn't have occurred. Immediately there is pressure to write this. Now we're not in the position today, so we have time to think it through and write the right kind of a standard. But let's don't let time get away from us. I think we ought to start on this-start with the general, make it more and more specific as we understand what we are talking about. Specify what we mean and not fall into the trap of picking a number just because it's an easy way of expressing it.

Now, a third point that I mentioned was the utilization of the kinetic properties or the dynamic properties of reactors. In my mind there are seven unique properties of fission which sets it apart from other means 
of producing energy, of releasing energy. And the seventh one is the extremely dynamic character of the fission process. I don't know of any other device which can be moved from low power to high power and back again as rapidly as the fission reactor. Now, you are all familiar with the time constants that we are concerned with here, the delayed neutron fraction, and the neutron lifetime. Now what useful purpose can we put this property to? I must confess that I don't have any good ideas. I only know of one possible use which is yet to be proven out, and that is medical. There's some work that Dr. Lee Farr of Brookhaven has done and which has the hypothesis that if you saturate a tissue, a diseased tissue, malignant tissue, with boron and then give it a flash of neutrons above a certain threshold; (his hypothesis is that below the threshold there will be some malignant cells left) above the threshold you'll kill them all, and he says it's very important to kill them all. Now, one way of doing this is to have a flashing reactor which saturates the particular tissue involved. To me this is an exciting possibility and I'm most anxious to see it proven out and something done about it. You can recognize right away there's no safety problem here because there's no buildup--no significant safety problem--because there's no buildup of fission products. Now what other uses can this property be put to? I can vaguely dream of using it for certain testing of materials; I can vaguely use it for certain research projects; but now I'm stumped, and the reason I'm bringing it out here is because there should be focuses on it. If you really want fission, nuclear energy, to move ahead fast, find things that it can do that you can't do any other way. This is what we want to focus on, and this dynamic property has not been exploited. I want to see some more original thinking on this point.

Now, in conclusion, I've made the point--I hope I've made it--that we need more theoretical work; we need more thinking about the real mechanism of what we're talking about; we need experimental verification on a small scale and on a big scale. Now, we have a problem, and Cliff talked about this one; we need to get this information out more promptly. We need to get it out in a form that can be understood by those of us who are not so well informed, or well grounded in the theory as you people are who are working in the field. Coming out here, I was reading a Quarterly Report of Spert. It was in our hands, available for me to read maybe two or three weeks ago, but the work was finished in December of last year, and some of the experiments that they were talking about doing I am very much interested in, and I don't know what's happened in the meanwhile, I hope to find out. Who is going to do this? I know that most fellows hate to write reports, and this is a failing of most theoretical and experimental people. I used to hate to write reports myself, and still do actually to be honest about it, but I've learned because I've lived a few more years than you people have that gee, what you know is maybe interesting and valuable to you, but if the rest of the world doesn't know it, it's not much good, and so we need to get this information distilled. Now how do you do it? I'd like suggestions of how we get this information written out in forms which are usable by all the people that are concerned and getting it out promptly. Thank you. 
Doan: Thank you, Dr. McCullough. Now I'm sure that we don't, any of us, want to work too hard here at this meeting. We've been sitting quietly for an hour or more and I'm also sure that some of you have some comments you would like to make on this; among other things, Spert would probably like to answer this last question (laughter), so since Warren Nyer told us there was some coffee waiting back there, I will hereby declare a coffee break of ten minutes, after which we will convene again and see what comments there are on these papers. 
SESSION I

\section{GENERAI DISCUSSION}

Doan: The Atomic Energy Commission has the responsibility for directing the reactor program for this country and also for setting up adequate regulations. There are, as you well know, some problems. At the top we have the Atomic Energy Commissioners, chosen for their abilities in various aspects of endeavor important to directing such a large program. These Commissioners are under a number of pressures and have occasion to make regulations, and often find themselves in the position of wanting very badly to say some things in some very specific terms that involve technical aspects. So, it occasionally becomes necessary to say to the Commissioners, "But, gentlemen, you can't quite do that--we'll try to tell you why, but for these technical reasons you just can't do what you'd like to do." The business of setting site criteria and criteria for the safety of reactors is one of these. It's a little difficult for these people, in general, to understand why, if the Advisory Committee on Reactor Safeguards can arrive at some judgments and conclusions with regard to the safety of the reactors, why they can't say in simple language that can be put in the form of regulations just what their criteria are. One step further down the line are the members of the Advisory Committee on Reactor Safeguards who are one step less removed from day-to-day problems, but I am sure, a good many times in connection with trying to get across the basic aspects of the relationship between various parameters in reactor kinetics and reactor safety, a lot of you would still like to say to the members of the ACRS, "Gentlemen, you haven't quite got the idea." Now, I'm accustomed to this. I get it all the time in my own shop, and so one of the purposes of this first session this morning, at which these gentlemen have made some remarks, is to enable you to say, either by questions or comments and in whatever delicate language you'd like to say it, something similar with respect to the remarks that have been made.

So, without getting into detailed discussions of a type that should be reserved for the specific sessions that have been set up here for later, anything pertinent to the opening session, which has been defined by Mr. Nyer to be a nontechnical one, but which has gotten slightly into the edges of technical aspects, this will be the proper place to make such comments, and we will accept these comments in the way of longer remarks. The mike is here on the podium for anybody that would like to make these remarks, or if you would like to make some short ones or ask some questions from the floor. Now, McCullough and I don't rate very high on our detailed technical understanding of this, but I would have you know the ACRS is not without its experts, even in this field, so that any questions that get too hard to us, we'll call on our member sitting on the floor out there, Bill Ergen, and he'll answer those questions. But, at any rate, I will now accept any comments or questions. If you'll raise your hand, we will acknowledge the speaker. Dr. Ergen.

Ergen: I want to make one remark with respect to $\mathrm{Dr}$. McCullough's statement on the naval reactors; namely, that you do not have to move the 
control rod at all as you go from zero power to full power and similar maneuvers. Of course, one way of accomplishing this in reactors is to overdesign the reactors so that you get nowhere near its limits and Admiral Rickover is very emphatic every time he gets an opportunity to say so, that the naval reactors, indeed, are overdesigned and consequently are very safe. This is one way of doing it in a field in which you don't have any competition, as Admiral Rickover of the Naval Reactors Branch has. However, I would feel that if you do want to make things as efficiently as possible, we cannot always go that far. And besides, it is not always obvious when it's a boiling water reactor that a reactor which is very much overdesigned is really the safest one, because, assuming that you have to extract a given amount of power, if you have (in a boiling water reactor, for instance) more steam if the power density is low, and the greater amount of stored energy is not exactly conducive to safety. So, my point is, that though it is always easy to be satisfied with very overdesigned reactors, it behooves us (those working in the field of reactor safety and also the ACRS) to look ahead and see how far we can go in the direction of relaxing overdesign practices, and go to, say, higher power density in boiling water reactors, so we would be prepared to pass intelligent judgment at the time an applicant actually proposes this, rather than holding him up, which may not even be the safest way of settling the question.

Doan: Are there any other comments?

Henry: I would like to comment on the word "overdesign". The Nautilus was a very conservative design by today's standards; at the time lack of knowledge justified such conservatism. Our improving ability to predict power distribution and thermal behavior is reflected in newer cores.

Perhaps I can, in this context, bring out a question I wanted to ask. It seems to me that some of the criteria of a safe design ought themselves to be examined; they have frequently been set merely because they were easy to meet or are "extra safe". "Extra safe" requirements may then be made a criterion of safe design and thus create unexpected difficulties in meeting this "criterion" in a more advanced design.

There is today a procedure being discussed which may again run counter to one of these "criteria" of safe design. I refer to the possibility of starting up a reactor without an external source. I expect that the immediate reaction of practically everyone in this room to such a suggestion might be categorically that it should never be done, and I suggest that, because in a couple of years people may be wanting to do this, we start examining our consciences on the matter and see whether there really is an actual need always to see a signal reading on an instrument during a reactor startup or whether this criterion is something that we have imposed merely because it was always easy and "extra safe".

Doan: I suppose we' $d$ all agree that if we had enough time to trade for $\overline{\text { lack }}$ of source this would be fine. Then, it gets into the question, 
"What are the compelling reasons for starting up without a source." I saw another hand over here some place.

Lipinski: I'd like to tell you about a blind startup. A series of experiments were performed in the AIPR reactor, but the criteria there was not to exceed the 10-sec period, and we never did have an opportunity to complete these experiments. But, from all indications, we would have had of the order of a l-sec period, and leveling off at power--on blind startup.

Nyer: This was for what withdrawal rate?

Lipinski: This would be for the order of $10^{-4} \Delta \mathrm{k}$ per second, which was the built-in reactivity rate on the drives.

McCullough: Well, the fact that you would level off, is that a characteristic of your reactor?

Lipinski: Yes, it was a boiling reactor.

McCullough: But it's characteristic of the reactor, so you couldn't use this as a generalization?

Lipinski: No. For this particular type.

Grace: What about the natural source level.

Lipinski: Well, there was an artificial strong source. To have carried these experiments further would have required source reduction.

Henry: I'm talking of a sourceless startup--blind only because there are too few neutrons for the instruments to detect.

Lipinski: Well, the experiments can be exterided to go to reduced source levels. But the reactor in this particular case does have inherent shutdown.

Henry: Yes, well, as Dr. Doan indicated, you slow down when you do this.

Ergen: Why can't you put the source in the reactor itself, so that it's an integral part of the reactor, rather than putting it in from the outside? 
Henry: For one thing, the lifetime of the reactor makes it difficult. The source either dies out or must be very high intensity and hence expensive to begin with.

Doan: Well, I think everybody understands that what we are really talking about is just the initial startup; after one's been running at power, then you don't have a sourceless startup any more; you have your inherent

sources. Any other comments?

Chernick: I must say that one would like to see very good and sufficient reasons for removing something which obviously, under assumed conditions, can be a very dangerous way of operating a reactor; namely, one with a detector with no signal. There are apparently, though, cases where we are going to operate reactors blind in terms of another very important variable and that is the temperature inside of our fuel elements. And again, this is a problem of the extreme difficulty of measuring temperatures in high pressure reactors--particularly important, for example, in oxide fuel elements, . . . . and it is clear that we are going to be operating reactors without knowing now close to burnout some parts of some fuel elements are going to be, and so on. Whether this is a good idea or not, I'm afraid that because of the extreme difficulty, the designers are being forced into this position. And again, only after experience on the operating reactor after a long period of time will you know that it is a safe way to run the reactor or not.

Doan: One time, in the course of looking at a reactor that had been started up--I'll not say what reactor--I inquired as to why the particular method involving some additional control instrumentation was employed, and the answer I got, "That's because the ACRS required it." Now, just to stimulate the discussion, are there any of you who have a tendency to build things into reactors, safetywise, because the ACRS seems to think it's necessary, and you really don't think it, and it would be easier to put it in than to argue with HEB or ACRS? If there are, now is your time to really talk about it.

Stone: This problem does come up, Dick--every reactor that is built. Since we are pushing the limits in the small utility reactor field, and we are also pushing the limits, material-wise, in the high-temperature, gas-cooled reactors, we don't have standards to work with and we don't have large backgrounds of experience on which to base our designs. So, that we do have some uncertainties in what we consider to be adequate, and perhaps in what the Hazards Evaluation Branch or ACRS might consider to be sufficient protection. I feel that this is a situation that is transient. I think it's something that we will live with, and want to live with, because we feel at this time that if one tried to establish hard and fast standards, the standards would be outmoded and could not be discarded rapidly enough to keep up with the state of the art. However, I would add this. That, after Dr. Beck's fine talk, I think that if that type of talk could be distributed as a very informal guide to people in our particular position where we are trying to get to 
commercial nuclear power now, it would aid us tremendously in coming in, either to the Hazards Evaluation Branch or the ACRS, with something that's reasonable and which has a much closer chance of being acceptable to all parties. Certainly, I would advocate from the floor that this talk be distributed.

Doan: I think this is an excellent suggestion and I am sure Dr. Beck will be glad to do this. If one may extrapolate these remarks a little bit, I believe it's the feeling of a good many of us that this is really the way, in the present state of the art, to handle ideas on criteria, standards, and so on--that through the delivery of technical papers by various people who are both skilled in the art and who have the job of arriving at decisions; that if we get our thoughts lined out, as Dr. Beck and $\mathrm{Dr}$. McCullough have here--this gives the basis of the thinking upon which the judgments are arrived at. Now, if you put these things in the form of documents that go into the federal register, for example, then, of course, they get a certain amount of congealing when they get in there. But, if they are put in the form of technical papers where there's some give and take in the discussion, where those of you who are designing reactors will get some notion of the thinking without being tied to it rigorously, it seems to me this is the best way to handle this problem at the moment; although one has to realize that since a lot of pressures are involved, there is a good reason for trying to do the other. It's a very difficult thing to do. Let me put my other question in a slightly different way just to see how the thinking is on that. I don't really think I need to ask this question here, but in some places it might be an appropriate question to ask. And that is, do any of you consider that if the Safeguard Committee or HEB has decided that something is safe, that you do not need to have your own reservations and your own standards which may go beyond those which are required by the regulatory or the recommending bodies? I'm sure that no one here really is in that category. In the course of putting specifications into licenses--construction and operating licenses--granted to the licensee, it seems to me there is always the possibility that if subsequently there is an incident, some manager or some lawyer for the managers will say, "Well, now look, this was agreed by the experts to be safe and we had an incident, so I guess they'II have to explain it." Am I just imagining something here, or could this happen? Cliff, do you have any comments on this?

Beck: I am not quite sure just how to answer the question, I am not sure quite what the question is--but my comment would be this. There have been two different feelings floating around within the AEC, which are now getting the first real consideration. One of these feelings is that the AEC, itself, bears a heavy burden of responsibility for the full safety of licensed reactors and, therefore, that appropriate steps must be taken to insure that the AEC has full control and knowledge, and acquiescence in all proposed actions or changes that an applicant or licensee might contemplate. Another viewpoint that has been held by a large number of people, and this is one that has not been fully agreed to--or, as I say, it hasn't been discussed, it's right now being considered fully for the first time, and that is that the AEC is responsible for safety only up to a certain point. This view is one that I concur in, and believe that 
it eventually will be the one adopted. This can be described as follows: to such an extent the major possibilities of hazard have been evaluated and safeguarded against, and assurance has been gained that responsible people have taken appropriate actions in anticipation of difficulties and have provided against all foreseeable accidents in all reasonable ways, and have provided a competent operating staff with procedures developed which require thoughtful and deliberate consideration of changes and proposals, the AEC has then discharged its responsibility. A major portion of responsibilities beyond this then rest with the applicant, himself. This I think is inescapable. The only way the former viewpoint could be implemented would be for the AEC to actually run the facilities, itself, or to have a full-time resident staff of inspectors there who would approve or acquiesce in every change or operation contemplated. This would be very impractical and is not contemplated.

In a sense, the $A E C$, from a regulatory standpoint, is going through growing pains, finding its true position, just as technical information is becoming clarified in many areas, I believe we are now on the threshold of moving toward a more clearly defined position of what our role is and what the complementary and equally important responsibility of the operators is in this matter.

To answer your specific question, "If an accident should happen", it is not just the responsibility of the reviewing bodies. Clearly, if they have overlooked a major point or if they were negligent or careless in their reviews, then certainly they ought to be called into account. But, despite the review by outside groups, a heavy responsibility rests on the operators to anticipate the difficulties that might arise; to use competent people and carefully prepared procedures, to minimize hazard even though things go wrong. Thus, there is a dual responsibility; it is not that of either party, alone. I don't know if that answers your question.

Doan: Well, I guess that since the question wasn't quite understood, this is a good answer. Let me try to make this a little more clear. And I'd like to call on $\mathrm{Dr}$. McCullough for it. You know, and I know, and everybody here knows, that the AEC cannot assume full responsibility for the safety of your reactor. Those of you who operate these reactors must assume this responsibility. A while ago I asked if any of you had put in some additional control gadgets at the insistence of HEB or ACRS, and I think that, on occasion, this might happen. Now, what I didn't say for the rest of the statement is, that suppose that some one thinks that we need an additional safety device which may actually have the effect of making the reactor not more safe, but less safe. Then, it seems to me that you have the responsibility for standing up and arguing on a technical basis for this thing and not accepting something which someone thinks may make the reactor more safe but which you really think makes it less safe, because this gets right to the question of who has the responsibility for safety. As the regulations go in the direction of making more and more requirements for more and more reporting and coming back for changes, it is easy to get the impression that Mecca is the one in Washington that assures the safety of the reactor--be that the ACRS or the HEB, and of course, neither Dr. Beck nor any of the ACRS members 
would subscribe to this. I just want to make clear that the philosophy in which you operate your reactors, in our case, we ourselves--our technical people--are responsible whether it's the Safeguard Committee and we have a continuing Safeguard Committee with a full-time chairman at the MTR and ETR who looks at all the experiments and all the safety aspects of the reactor. Nothing goes in the reactor that hasn't been carefully scrutinized by this group and approved by them. You have your own ways of doing this, and regardless of what has been approved officially, we have our own criteria over and above that which has to be satisfied, and I am sure you do, too. I just wanted to be sure that nobody is under any misapprehensions as to where this responsibility really lies. Dr. McCullough, I am sure you'd like to make some remarks on this.

McCullough: I would. Thank you. Well, again, repeating, I think it's very clear indeed, that the primary responsibility for the safety of a reactor resides in the operator of the reactor. There's nobody that knows better than he as to how close he is to the margin of trouble. I would say, the next step down of responsibility is the designer of the reactor-the fellow that puts in the devices that he thinks are necessary, and this is reinforcing what Dick says. Now, all the ACRS or all the HEB or all the other people can do, in my own opinion--I'm speaking of my own opinion now, not trying to represent any policy or group opinion; I'm talking of what I think--all we can do is to make sure, in our opinion that the designe $\bar{r}$ and the operator have really faced up to the problems that are important and are doing an adequate job. Now, there have been times in the past, and I think there will continue to be times, when we will be able to actually assist the designer and the operator in their understanding. But, this is relatively rare, and the only reason why we are able to do this, really, is because we see all kinds of reactors and we see some points which were important in reactor $A$, that the designer of reactor $B$ or the operator of reactor $B$ just wasn't quite aware of. Now, having said all this, I think I want to get back to point this up in some of the remarks that Henry and stone made here. But, to introduce these remarks I think you ought to realize that every operator and every designer has a built-in bias--the designer is trying to build a reactor as cheaply as possible--I mean he's in business--this is reasonable and proper, and this is a bias. The operator again wants to run it as economically as possible if this is a power reactor, he wants to get as much power out of it as he can. This gives him a built-in bias; he doesn't want to shut down to fix this, that, and the other thing; he doesn't want to shut down and examine some wiggles on the chart that, in his opinion, don't mean anything anyway. Furthermore it's worth saying that history has shown--experience has shown--that, no matter how careful you are in your theoretical analysis and your procurement of materials, you do not know all of the things which can go wrong. We have lived with steels for many, many years and we still find surprises in the way these steels behave under certain conditions. To give you a very simple example, stainless steel was used but it wasn't recognized for quite a while that there was a stress-corrosion problem with chlorides. This came upon us all of a sudden, and boy, we had lot's of trouble. This illustrates the kind of thing that sneaks up on you, so although you as a designer think you've got a good firm basis to stand on, when people don't agree with you and make you take additional precautions, you must recognize that in the 
beginning of a technology you have to live with this kind of conservatism within limits. Now, the other point. Al Henry said, "We'll live with the criterion of two stuck rods", and he recognized this was a trap he fell into because it was easy. I hope we recognize this kind of trap. On the other hand, if it's cheap and easy to do some additional thing that everyone agrees helps the safety of the machine, if we can do this without setting a precedent we ought to do it. Now notice I hedged on this--perhaps I should go further--in my opinion you ought to do it whether it sets a precedent or not--it's up to people to stop this matter of setting precedents where they don't mean anything. Back to this question of whether you add instruments, or gadgets, simply because ACRS or HEB says so, or--now, these are words that make me mad--to be real frank with you--because we try very hard not to tell you to do something that has no good reason. I do recognize that it's sometimes difficult to argue people into the point of view that it is nonsensical to put it on, and if it's because we're ignorant--because we don't understand the problem well enough--well--until you educate us that's something I'm afraid everybody has to live with. But we do honestly try not to be arbitrary and require things that have no good reason. I think it's up to you people that design reactors and are trying to introduce sensible approaches to the control of them to stand upon your hind legs and argue, when you really think you're right. Let's have a meeting of minds, and if we have to say, "Well, on the basis of experience we think maybe you're right, but we'll have to learn more"--okay, that's reasonable. But just to arbitrarily insist on a thing, this is not tolerable.

Stone: Rogers, the first point I'd make is that in my estimation, the operator is not the best possible and, in fact, may be the worst possible person to evaluate the hazards associated with the operation of a reactor. He's too close to it; he's too busy trying to meet his production schedule or keep his plant on the line at 80 per cent efficiency. This is also true in design, we're too close to the reactor design and I want to come back to this a little later. At the moment, it is a very valuable service that we receive in using both the Hazards Evaluation Branch and the ACRS as a sounding board; in other words, we sum up our ideas and present a case. Unfortunately, we do not have the depth of experience and have no way of obtaining the depth of experience of looking at a large number of different reactor designs and establishing that which is reasonable at a given time in our technology. So that we must, to a large extent, put up a straw man in the form of a case; here is what we would like to do, and in many cases the experience that is available either in the HEB or the ACRS comes along and knocks this straw man down. This is necessary. Any single reactor manufacturer does not now have the necessary experience to cover all of the ground, so where it would be very convenient for us to fight and it might knock a few dollars off the price of a reactor and give us time to fight this given thing, many times we feel that we should go along with the present evaluation of the state of the art which can be better made in a collective group such as the ACRS or the Hazards Evaluation Branch. Speaking about cheapness, or reducing the price of a reactor, I think I speak not only for my own company, but for every company that's involved in the reactor manufacturing business today; that is, we are not primarily motivated by producing a cheap reactor now, and everytime we build a given divice, whether it be a small research reactor or a power 
reactor, we put our professional reputation on the line--not for this year, not for this reactor, but for that in perhaps ten years or twenty years downstream. If our only interest were in making a dollar now, we wouldn't be building reactors.

McCullough: I would comment here that I think there's a real difference of opinion here when you say that the worst person to evaluate the safety is the operator. I don't buy this; this ought to be discussed at a later time.

Doan: Ep, you have the floor.

Epler: I'll be very glad to debate this point. I'm sure that you, Dick, have had a lot of experience on the reactors, and you've had a lot of experience designing reactors. And before we all get through, we're going to have a lot of experience with most everything. It is true that the operator is preoccupied in trying to prevent somebody getting out of line, a small accidental spill, or something, and he's right. But the real man who understands the reactor and the components that can get into it is the man who designed it, and who is designing the next one, and who sees the mistakes in his earlier designs; he's trying to prevent them in his later ones. Now, he sees a lot of reactors. I think, therefore, that I would argue that it cannot be the review body at some distance that finds a reactor's mistake because, after all, they'd only have a small amount of time to spend on this, but it must be the man who sits on top of the reactor and not necessarily operating it, who finds its mistakes. You mentioned committees at Phillips, and I'm sure that other organizations have the same sort of setup--committees established to find errors which have been overlooked by the designer, and I think that makes clear a point-ra firm point that perhaps the trouble was that the designer was on his first job. If he had been on as many jobs as the committee had been on, he would not need this reminder. So, I would make a plea to put the responsibility not solely on the operator but on a team of operators and designers, but put the responsibility as much as possible on the designer.

Doan: Someone else here had his hand up.

Stahl: It's my experience that you ought to distinguish, perhaps, between two things: the ultimate safety of the reactor and the things that are likely to go wrong. I would say that the operator or the designer will have a better idea of the thing which is likely to happen--the spill that's likely to happen or the rod that's likely to go. But, perhaps the committee will be able to take an independent view of--the remote view--and examine better the ultimate limitations of a particular design of a particular group of reactors, and perhaps have a better opinion on this particular subject. 
Doan: I'm sure that those here with me today would agree that the greatest service which $\mathrm{HEB}$ and ACRS render to the reactor industry is not in the sageness of their wisdom, but in the careful examination of the safety aspects which their discussions and comments encourage on the part of those that are designing and operating the reactors. That, of course, is where the safety must ultimately lie. The latitude that can be given under legal regulations to make changes in a reactor after it has been approved, of course, that is something that has to be decided and some limitations put on. The question is, how much? In other words, we have the problem; we review a reactor under certain design parameters of fuel, flow, controls, power, etc. Clearly, in order to have the required flexibility to make reasonable changes without coming back for more tools, there needs to be a certain amount of latitude allowed the licensee. But, equally clearly, it would be hazardous to allow a very substantial amount of latitude because, while everybody here by stipulation has good judgment, there are some people that we might want to question. Take the reactor which has been designed with certain accepted parameters, someone decides to perform some sort of a noble experiment-he gets curious as to how it would go if he were to double the power, or how it would go if he would cut down on the water flow by a factor of 50 to 75 per cent, or something of that sort--there have to be certain limits to this. But again, basically, the person or the group that has to assume responsibility is the operator. But, of course, under the law the Commission and its various bodies have also to accept their own responsibilities. Are there any other comments? Thoma.

Snyder: What we've been talking about is responsibility, and I think the thing we should keep in mind is that responsibility is something that can be multiplied but can't really be divided. First of all, I think every operator has to feel completely responsible for the safety of his plant and dependent on no one else. I think every designer has to feel completely responsible for the plant he designs--as built, at least--and as changes are made with his consultation. And I think in the same way that although the various review committees cannot feel quite the absolute degree of responsibility for one of these plants, as the operator or designer, they feel, certainly, responsible that every good practice that they know of has been adhered to. I obviously don't agree that the operator is a poor judge of the safety of his plant, nor do I agree that the designer is a poor judge of this.

deBoisblanc: I have a viewpoint which sort of rests intermediate between that expressed by Epler and that just expressed by Thoma. We actually are talking about a reactor as if it were something which was designed with an exquisite attention to detail and that everything about the reactor is known by the designer, and is written down by the designer into some sort of a manual so that the person who gets this machine can assimilate this, say, as a philosophy and undertstand completely this philosophy on which the design was based. Iet us presume that is the case. You then have the situation where the reactor goes along and operates for a long time and grows old on the job. It is in this area that the "operator" can have an accident. I think part of the confusion here is a semantic confusion of what we mean by "operator"--I think Dr. Doan means Phillips Petroleum 
Company operating a whole plant with responsibility for using the reactor, running the reactor, and conducting the experimental program, and to some extent designing reactors or changes in reactors. So, an operator in that broad sense becomes one who is responsible for all phases of the reactor, keeping it as it was if a change is not necessary, keeping it safe if a design change is necessary, and operating it within the frame work of the design philosophy, if that design philosophy has been substantiated by adequate tests. This is the reason for all the things like the shakedown programs, and the testing of the drop times of rods, and the shutdown features of the various instruments; if these have all been found to perform as designed and the margins of protections are validated by the test program, then it is the operator and his supporting staff's responsibility to see that it adheres to the philosophy of the designer. To do this, he only needs to understand the philosophy of the design, and this sort of information is communicable. If the reactor undergoes a series of changes, whether it be a change in power level or a basic reorientation of the design parameters, such as enlarging the core, or lengthening the core, or changing to plutonium; each such change is essentially a reactor design problem. The staff that accomplished that is a reactor design staff--it must be just as sophisticated, just as understanding as the original design staff. In that sense, if that staff also operates the reactor, then again, you see that the largest measure of responsibility is resting with the "operator". Now, very few installations in the future will be able to be both an operator of the reactor, a designer of the reactor, and a modifier of the reactor, because there will be power companies using reactors; there will be people who have to have an economically small staff, a minimum staff shall we say, to just do the jobs that must be done. And I think that that's the sort of concept of operators that Dick Stone was mentioning. If you deal with a minimal staff, then you cannot guarantee that that minimal staff, without supplementing wi.th certain types of talent, can indeed even remember the design philosophy. He may never know the design philosophy. He may be a seaman in the Nautilus, operating the valves that allow the reactor to go to full power and shut down with just turning a water valve. He may not know how close the reactor is to its design margin--to its operating limits. He may not care, and he may get extremely lax. Therefore, in that sense, you can't really expect that the reactor operator is the man that is the best one to decide whether changing something, say, like replacing a stainless steel valve with one which is made of carbon steel, is safe.

Snyder: But you do expect that the U. S. Navy, as the operator, is able to take that responsibility.

deBoisblanc: In that sense. That's where the conflict came. It was the fact that "operator" in the broad sense is the best one; "operator" in the limited sense is perhaps the worst one (to take that responsibility).

Doan: Other comments? 
Silver: I want to make something clear that may only be important for the research reactor operator. If you're talking about power reactors, where the limits are in the technology and materials, I think the encyclopedic viewpoint of the committee probably makes it the best qualified to judge the problems and limitations and make the decisions. But, if you're talking about research reactors where you don't usually have these limitations as the main factors, but the kind of experiments that you can do, the kind of instruments which go with it as a tool, there you have to be very sure that the operator is the one who decides, because otherwise you limit him very severely as to what he can do with this research tool and certainly, he cannot come to committees with every experiment he wants to do or every small modification he can make. So in these two types of reactors the chief responsibility naturally falls in these two directions.

Doan: Dick?

Stone: I want to thank Deslonde (deBoisblanc) for clearing up the difference between the day-to-day operation of the reactor and the operating agency. Now, there's one other thing I'd like to add to this, and I wish that Marvin Mann were here because he could make the point much better than I could. And that is this question of changes in the day-to-day-operations characteristics of a reactor, and of the operating agency itself. Initially, I can remember years ago at KAPL when we first started the critical assembly program, the only one that was allowed to manipulate the controls was the physicist-in-charge. This has slowly been downgraded as time goes on, and finally now they have people that are high school graduates, perhaps. On power reactors, I'm sure that we'll suffer this same downgrade. As a reactor grows old in its work, I expect here too the possibility of downgrading the day-to-day-operations crew of the reactor will come more and more into play. So that turning, even on a research reactor level, the responsibility of this reactor toward the individual or those individuals who are responsible for its daily operation, I think can lead to a major problem in our infant industry. I am sure that within the operating agency there must be a review function, and this review function must carry representation from responsible technical people within that organization. Now, having to judge whether an experiment is small or large I think must be settled within the operating agency. I'm sure that we do this and I'm sure that most other operating agencies do the same thing. But to hang a guy that's responsible for twisting the valves, or replacing the chambers, for the operational philosophy or operational safety of that particular installation, I believe is wrong.

\section{Doan: Comment?}

Grace: I'm Nelson Grace from Bettis. I agree with Dick (Stone) that most outfits do need some guidance with the theory of safety, but I agree also with the other statements that have been made that it's an extremely dangerous situation to imply that safety does not rest at home but rests with some committee abroad. I'd like to describe the situation we have 
at Bettis that I think works out very well. At Bettis the scientist-incharge of each critical facility is responsible for the safety of his facility. We operate a number of critical facilities, and they are all quite flexible in their operation; the core configuration in some cases changes every day, and they are doing different types of experiments. Now, whenever they plan a new experiment or run into a situation where a new question of safety arises, they discuss their proposed operations with a committee, a local committee made up of the scientists-in-charge of the other critical facilities and get their opinion. This committee does assume responsibility for their assigned task--that is, to review and advise to the best of their knowledge and judgment--but they certainly don't assume any responsibility for failure or mismanagement in the performance of the experiments. Beyond that committee there is still a higher committee, the Bettis Advisory Safeguards Committee, of which I happen to be a member. When the local steering committee feels that they have a proposal that they are not sure of, they have it reviewed by the higher committee, which is somewhat more remote from the operation of the cores and which has perhaps a slightly different viewpoint. But in any case, while these committees assume some responsibility, I'd say that about the primary responsibility there is no doubt--it must rest with the scientist-in-charge of the experiment. The operators do assume responsibility to operate within whatever scope has been defined for them by their supervisors. That responsibility and scope of freedom is quite limited so far as the guy who pulls the control rod is concerned. When it comes to changing the core configuration that night for the next day's tests, then the responsibility is at a higher level, etc. These responsibilities are well defined at Bettis. But, it bothers me to see that there is an implied ambiguity here as to who is responsible for these situations. That's a very dangerous situation.

Doan: Well, I think you brought out a good point there. When I mentioned that our MTR-ETR Safeguards Committee has a kind of working-level control over our operations of these reactors, that's the way we find it necessary to conduct these operations, because the people--the superintendents of operations and their supervisors simply don't have the time to give the kind of engineering and technical attention to the great variety of problems that these others do. However, in our other reactors we operate much the same way that you have described now; the responsibility for the safety of our operations in the Spert Project, for example, in the various Spert Reactors, resides with the Spert supervisors, and not with any Safeguard Committee. The same is true for our operations of the ETRC, the RMF and the ARMF; that also does not come under the jurisdiction of the Safeguard Committee. So, we operate both ways, but the objective is always the same; to place the responsibility in the most reliable hands for the operations we have in mind. Now, are there any other comments?

Boland: Back to your original point of use of "hardware" that some of us may put in because we feel that somebody's going to say, "Well, John Jones used that ten years ago, we've got to put it in our reactor". I would like to see the reviewing agencies feel some responsibility for suggesting that such useless hardware be taken out per se. To my knowledge this has not been done very much in the past, at least, in any of the projects I've 
been associated with. So, I would like to suggest that Mr. Beck's group and possibly the ACRS comment on these things when they see them and can see no need for them. If they're excess baggage, let's get rid of them.

Doan: Well, I'm sure the ACRS and the HEB both get a little borderline in the questions that come up. Basically, however, I think that both would agree that it is not a function of either of these groups to help design the reactor; it is their function to help arrive at some conclusions on the reactor as designed. But actually, and from a realistic viewpoint, one does get into these kinds of questions from time to time. I don't know if either one of you gentlement would like to say anything about this. If not, are there any other comments--we're getting pretty limited here, and I'm sure some of you are getting near your lunch time. In the back?

Jarrett: I think we 've exhausted this subject because there seems to be a general agreement. Therefore, I would like to change the direction. I'm not very pleased with the glib use of the words "likelihood", "probability", and "reliability". It seems to me there are three alternatives. I think this is a reasonable place to explore them. One is the possibility that there is a general agreement on what these terms mean. And I, only as an individual, can suggest that this is very unlikely because of the tremendous effort that's gone into this in the electronics industry which, after expenditure of many dollars, has not resulted in any clear cut definitions in these areas. The second possibility is to request that the people who use these words define what they mean by them. I heard Dr. Beck use "likelihood", and I heard Dr. McCullough use "reliability", and I'm not sure what they mean. I know what I think they mean, but I'm not sure that they mean the same thing. Now there's a third possibility, and it's the third possibility I want to avoid, and that is regulatory agencies defining these terms in such a narrow context as to not allow the flexibility to enable a longer-range prediction of safety of the reactor systems. I feel that there has to be more attention given in the nuclear industry to take positive steps on the first of these three possibilities; namely, general agreement on what the concept means.

\section{Doan: Cliff?}

Beck: This comment leads to a suggestion I wanted to make. I'd like to have the benefit of some discussion and comments from this group on a controversial issue on which pressure is building up from a variety of sources on both sides. I refer to the development of what is called "criteria" or "standards". If one takes almost any of the major components or systems of a reactor it is possible to write down the performance objective which this system should accomplish. Take, for example, the control system. The control system of a reactor should be such that it places at the disposal of the operator, under any conditions, the ability to close down the reactor. Once one has defined this performance goal for the system, and begins to move to the implementation of that goal by specifying what the characteristics of the system must be, or the components which must be included in that system, or how many, it becomes an 
exceedingly difficult problem. One might specify that the reactivity invested in the control system must always exceed by a certain amount the total reactivity ever anticipated in the core of the reactor, or that no single rod in the system should have more than a certain amount of reactivity invested in it and no automatic control rod should have more than a certain smaller reactivity value, and so on. It might be feasible, and generally could be, to specify these values for a given reactor, but it is impossible to specify values which would be applicable to all reactors. As another example, one might define as a performance criterion that the potential combinations and interactions of the assorted characteristics of any reactor should not result in spontaneous instability of the reactor. If one proceeds to elaborate that performance goal or general criterion into more specific things, it becomes exceedingly difficult.

On one reactor we do have an imposed requirement that the amount of reactivity added from room temperature by the integrated total positive temperature coefficient, from room temperature up to the point at which it changes over to a negative coefficient, could never be more than the value of beta. It is not clear that this would be a good general requirement. For some reactors this would not be a necessary requirement for safety.

It is not difficult to specify performance goals or axiomatic requirements such as these outlined above for each reactor system, but I would be hard-pressed to proceed to quantitative or even qualitative specifications that might be required to implement these. Earlier today I stated that each reactor should have "an appropriate measure of safety", and in response to the question raised by the Atomics International representative, I indicated that I did not know how to define "appropriate measure of safety". And it's true, I don't. What I'd like to ask this group is: To what extent can specific criteria or standards for reactors be written? Would they have any value at all, and how far can they be carried? As I indicated earlier, there is a great deal of pressure from various sources for extensive detailed standards for the reactor design to be written. Generally, those who are farther removed from the problems are more vocal in this respect. We have recommendations from responsible groups and individuals that we ought to be trying to do this in a very vigorous way. I myself find it not only exceedingly difficult; but have doubts as to the wisdom of doing this at this stage in reactor technology. What I'd like to ask is, what advantages would this have--how far might it be carried? Let us restrict it just now to the general subject area of this meeting, reactor kinetics and dynamics, for example.

Doan: Would you like to make a comment?

Chernick: I'd like to comment that I agree with Dr. Beck; in fact, I'd say it a little more strongly. I think there's a very great danger of leaving ourselves in the hands of semanticists and philosophers in an area like that; anything along this direction could easily do a lot more harm than good. I'd like to go a little further and say I think that the interplay between the reactor designers and the Safeguard Committee, ACRS, has been the thing that has really improved our knowledge of reactor 
safety. It was in the hands of our committee members that some of the questions first got asked which are now basic to studies of reactor safety, such things as loss of pump, or lowering coolant temperatures, and so on, all of which could have serious consequences. Everybody recognizes these things as necessary things to look at during reactor design, and all of this came about through one or another meeting between designers and the ACRS. Perhaps it's best to go to specific cases rather than talking in general terms. I know, for example, many years ago, when the Brookhaven reactor was first designed, and the first committee was set up, some of the questions were then immediately asked. As an example, Teller raised the question of what happens to a reactor in case of an earthquake. Well, I think we were able to answer his question for the area of Long Island, but I still think it's a pertinent question for a reactor that's going to be built in California, for example. At the request of the committee, we put in additional backup controls on the reactor. Now, frankly, this goes to another comment that was raised about getting rid of outdated safety devices. We have never used this backup control and it's a little bit of a nuisance. We had boron shot which were tested to see if they could work. It was a little nuisance vacuuming them back out of the reactors. Now, if we have never had to use it, does that mean we should get rid of it--this backup control--at this time? I don't think that we'd be willing to recommend getting rid of these devices when they are not damaging nor in any way obstructing the use of the reactor. There are many other questions like this that were asked by the AEC which led to experiments which could be done at laboratories which helped determine what happens under various conditions. Thinking specifically when we converted our fuel elements to enriched uranium (and here we were interested in increasing the flux), we were asked about the possibility of using steel elements because of their higher melting temperature, and so forth. Well, we didn't want to go to steel because of its poisoning effect and, in fact, we could shelve it for equivalent poisoning in aluminum. It was the question of flux and not the safety of the reactors as the primary goal of this conversion-that aluminum was preferable to steel. Nevertheless, we thought it was advisable to melt the aluminum in an experiment--let it melt on the graphite and see if it permeated through the graphite cracks and crevasses, and so on. In an oxidizing atmosphere, it turned out that it did not. I think these little points all help and go into the subject matter of what is known about reactor safety. So, I think this type of interplay-and I'm sure we're just talking about a narrow area here and I'm sure many other groups have had similar experiences--has been the thing that has been very helpful, and possibly the reason why we have such a good safety record so far in operating the reactors that have been built. So, I do think, to get back to the original point, that this is a healthy way of improving our knowledge of reactor safety and we ought to leave the philosophy to the philosophers.

Doan: Perce has been trying to make a comment here.

King: As a non-expert listener I'd like to make the following comments on the discussion. It seems to me that the discussion has been concerned with responsibility and I think this affects the AEC members--Rogers and 
Cliff, here--to some extent. How far should one write down rules and regulations without automatically taking over more and more responsibility? I think this is one of the big problems. The more detailed regulations on criteria and operations, and so forth, you people try to lay down, the less responsibility qualified operators will feel they have. So, this is a sort of philosophical question.

Doan: Yes, I'm sure it is. Dr. McCullough, you've been making some notes here. I'm sure you have some comments on this.

Mccullough: Well, I'd like to turn to Mr. Jarrett's comments and couple these with the comment that Cliff just finished making. Cliff made the point, if I state it correctly, that it's comparatively easy to write down generalizations of what you're trying to accomplish, but it is very, very difficult to go from those generalizations into more specific expression of these things, and I agree, heartily, that it is extremely difficult. In my own experience, I've tried to do this repeatedly in a number of places, and I have had a great deal of difficulty doing it. Now, Jarrett made the point that the words "probability", "likelihood", and "reliability," are loosely used. I agree with him; they are loosely used. And I think there is a real danger they will be defined by regulatory agencies and people in the regulatory agencies who do not understand what they are talking about. Now, I will offer my own point of view here to try to illustrate how important it is to do what Mr. Jarrett suggested and, also, how difficult it is. As far as I, personally, am concerned, the word "probability" can anly be expressed in terms of a statistical concept, and when you don't have any experience you can't have statistics, period. And I think we should be a little careful how we use the word. I probably am guilty of sloppy use of it, myself. Now, when you talk about "reliability"; this, in a sense, in my own view, does have some statistical implications but it has some other implications as well. Now, in one review that occurred some years ago, we were talking about the reliability of different components of the control system, and one of my good friends said, "Well, we're depending on gravity and I think you'Il agree that that's reliable." And he's right; this is one thing we refuse to question. But now when you start moving over into other things, like how reliable is a piece of steel 3 inches in diameter, this is not an easy question to answer. And I think we need to think and define what we mean by the order of reliability, so that when we say that the reactor must have a control system capable of shutting it down, for any insertion of reactivity which is possible, again you're in trouble with the word "possible". You could say "probable": then you're in trouble again, but you have to find the right word to say it, and then you say, for example, "In this particular reactor this means so and so." Now, this really says what you mean, but if you leave out the first part and only pick up the second, then you're in real trouble because the numbers you picked are not applicable to anything but that specific reactor, or an exact replica of it. Well, to come back. Cliff makes a point that you can write general things; it's ve:y, very difficult--almost impossible--to reduce 
this to more definite things. But, gentlemen, I recognize the difficulty but I think you've got to begin to learn how to do it. And, incidentally, if you sit down and try to do it, you really wake up to the fact that you have been a little sloppy in your thinking before. Now, the other question is who's going to do it? And, I don't know the answer to that.

Doan: Well, now there's a "likelihood" that some of you are getting tired and the "probability" that you are getting hungry, and despite your great general "reliability", the "possibility" that, if I don't call this session to a close pretty soon, some of you are going to walk out anyhow. There is a thing or two, however, we have to do before we draw this session to a close. Dr. Beck has a few comments to make on another proposition.

Beck: That was the one I had in mind.

Doan: Oh, you have--you've already raised that. Well then, in that case, we can call this session to a close, but before we do that, and so you won't get away too fast, I think Warren Nyer has some comments in the direction of bringing you back to the real purpose of this meeting after the straying afar that we have done in the discussion.

Nyer: Well, after we finished hearing from the panelists this morning, I somewhat doubted the wisdom of encouraging everybody to speak freely. But I do feel it necessary to reply a little bit on one point. As far as the delay in the spert reports are concerned--we can only plead guilty to that, and hope that by the next time we have a meeting, that I won't have to do that. On the other hand, it's worth noting that that particular frying pan is big enough to hold almost everybody here. However, I do think things are getting off to a good start, because I find a number of things that $I$ can disagree with in the statements made this morning. First of all, I would like to deny that there was any lack of need for comments by this morning's speakers; there was no attempt to have filler material. I feel that the framework of the discussion we had this morning is something that we have an interest in and an involvement in, just as any wage-earner has an involvement in income taxes. And the point of similarity doesn't end there--in just having a passing interest in it. I think I would also disagree with the statement--the reference--that the rest of us were experts and leaving this group out up here. I don't think that this is a necessary, or even correct, distinction to make. I also believe that there were a number of technical points that were raised that we don't need to go into any more in this session because they will be covered quite a bit later on. For example, I have reference to the discussion about the statistical problems in startup without sources. I believe that the Ios Alamos people are going to make some comments on that and we have some information to give you from spert. I would like, now, to come back to the business of the meeting. The Chairman this afternoon will be Dick stone, and the meeting will come to order at 2 o'clock. Now, I'd like to mention one other thing about the meetings in general. One of our problems, and one of the things that we have always heard a lot about in addition to hearing about it this morning, 
is that we have been asked time and again to make sure that we do whatever we can to get the information developed in our program into the hands of everybody. I believe that a meeting such as this is an attempt to fulfill that function and fulfill that request. We also have had comments on the past meetings that people wished that we had had a record of the meeting. Now we have tried to avoid having any degree of formalization which would restrict the exchange of information. For that reason, no formal papers were requested. We also have had some comments that people did not want their remarks published. I do believe that it would be valuable if we could have a desciptive report on the meeting that would be available to everybody. Now, we plan on taping the proceedings of each session. If anyone does not want this done when his particular comments are made, please let us know and we will turn off the tape recorder at that time. Maybe we should turn it off all the time--but if anyone wants to edit his own contribution to the proceedings, we will try to comply with this. We don't want anything in the proceedings that people do not feel is appropriate; on the other hand, we do want to be able to have some kind of descriptive report on the meeting available afterwards. So, if any of you have any comments on this plan, please pass them on to us later and we'll do what we can to satisfy everybody, and still meet our objectives of supplying information to other people and to maximizing the exchange of information here. Do you have anything else to say, Dick?

Doan: No, this is it.

Nyer: Then let's go to Iunch. 
POWER EXCURSION PROGRAMS

\author{
Chairman: R. S. Stone (GA) \\ Review Paper: F. Schroeder (PPCo) \\ Panelists: J. F. Boland (ANL) \\ E. P. Epler (ORNL) \\ W. E. Nyer (PPCO) \\ M. E. Remley (AI) \\ R. H. Stahl (GA) \\ T. F. Wimett (IASL)
}

Opening Remarks

by

R. S. Stone (GA)

Stone: This afternoon, Frank Schroeder will present a one-hour long review paper on power excursion programs throughout the country; at the end of that talk, I should like to take a break for coffee, and after this break I should like to introduce the panel, and to have each of the panelists spend perhaps 10 or 15 minutes on his particular program. After each of the panelists has had his say, I think we should have discussion from the floor, so I'll ask that we restrict discussion until after the panel has presented its program.

I should like to introduce Frank Schroeder of the Phillips Petroleum Company. 
- 


\title{
POWER EXCURSION STUDIES
}

\author{
Review Paper \\ by \\ F. Schroeder (PPCo)
}

Schroeder: This afternoon we are talking about power excursion studies, and I think it is a very appropriate phase of reactor kinetics work to start off our technical sessions with, since it is usually the problem which comes first to mind when one first starts looking at reactor safety. It may not turn out to be the most important aspect of reactor safety but it is usually the first one. I was about to say we have a panel of experts on the platform this afternoon, but since they are now out in front, I will just assure you that they are here. I know also that many of the experts in some of the programs that I am going to be referring to this afternoon are out in the audience, and based on this morning's experience I think we ought to be able to provoke a few controversies before the afternoon is over.

I want to attempt, in the next hour, to indicate in rather broad outline some of the things that I think we know about power excursion tests after what has been several years now of rather active experimental work in the field. I want also to indicate some of the gaps in our knowledge. The treatment will be rather cursory, but what I have intended here is to lay a background for the panelists and to provide a sort of checklist, if you will, for things which we would like to talk about in the panel and floor discussions. I want to apologize in advance, if I misrepresent anybody's work; I'm sure you won't hesitate to set me straight after I get throigh. Since I have depended for the most part on published work--as Rogers McCullough pointed out this morning, there is some time-lag there--and there are probably more recent results which some of you will want to mention.

Most of what we will be discussing this afternoon will be limited to discussions of the inherent self-limiting behavior of the reactor itself, with no credit taken for the performance of external control systems. Although there will be, I'm sure, some discussion which will get into that field, but for most of what I will say I'm taking no credit for external controls. The main reason for making the point is that I think we always have to keep the distinction well in mind, since the results we are talking about will be quite different, depending upon whether we are willing to include the effects of external controls.

First, what do we reasonably hope to gain from investigating the inherent response of a reactor system to a very large reactivity perturbation? Well, we find out in a gross way what happens to a given system under such conditions. This type of information itself may be quite useful in hazards evaluations, and such tests may provide demonstrations of the inherent safety of a given system, or at least may establish what the safe limits of operation are. But, all of us hope to obtain more than this from power excursion tests. We want to know why the system behaves as it does and we want our level of understanding 
to be sufficient to lead the way to intelligent design changes. Such design changes, if really based on a firm understanding, should achieve the objectives which $\mathrm{Dr}$. Doan referred to this morning of improving not only the safety of reactor systems, but their performance, and probably their economy through reduction in the cost of overdesign of control systems and containment vessels, and through more flexibility in site selection. An additional benefit which can also accrue from the intersive test programs carried out for just a few reactor types is that we may find--and I think we have already reached this point--that by virtue of experience in these intensive studies, we can propose some reasonable and safe programs of rather limited kinetic testing which can be applied to many new reactors as part of their initial startups.

Power excursion tests have now been performed on several reactor types, as most of you know; we've had such varied systems as fast, bare metal reactors, graphite-moderated, zirconium hydride-moderated, and water-moderated reactors. There now exists a general level of understanding of power excursion behavior which can be applied to all the systems. I want to make it clear, that when I say level of understanding, I don't mean that we really understand all the details of what's going on, but that we have correlative models which are quite good in predicting behavior, and which can be used to extrapolate behavior for certain design changes. This sort of an understanding is the result of analytical work, stimulated and verified by the experimental programs, and of additional experimental information about the nature and interaction of the actual mechanisms of self-shutdown.

I'd like to first point out some of the characteristics which all of the different systems which have been tested have in common. First, consider the self-limiting step-transient test, in which we investigate the response of the reactor to sudden, essentially instantaneous injections of reactivity. After the initial exponential power rise, some feedback effect will ultimately cause a reduction in the reactivity of the system and this will lead to a power maximum and subsequent shutdown. In general, we know that this decrease in reactivity introduced by the reactor itself will be some function of the nuclear energy release. We have found by experience in the various programs that the functional relation which couples reactivity changes with energy release may be expressed in a not-very-complicated form and still, with some adjustments of parameters, successfully describe all of the cores tested. Much of our ability to describe the general kinetic behavior of reactors is based on an examination of the properties of the reactor kinetics equations for certain forms of this coupling equation which relates the reactivity to the energy release. In order to fully utilize the understanding we have as a result of the tests, and, in particular, if we wish to extrapolate beyond our present experience, we must also have some knowledge of the details of the actual shutdown mechanisms involved; a correlative model is not going to solve all of our problems. It is at this point that our present level of understanding is least adequate.

In the analysis of excursion tests, we are concerned, of course, with the energy release as a function of the reactivity insertion and, perhaps more practically, with the pressures and temperatures developed. The extent to which we can measure the appropriate pressures and 
temperatures varies somewhat from reactor to reactor; but the power behavior is always obtained and by integration, we can always compute the energy release. In Slide 1, we have shown, in arbitrary units, the general behavior of the power and energy release at the time of the power maximum as functions of the initial reciprocal period, alpha. The plot is against the initial reciprocal period rather than the reactivity, since the period is directly observable while the magnitude of reactivity step is not. The energy at the time of the power peak has been selected since we are interested in deducing information about the feedback mechanisms from these data and the point at which the power rise is halted provides a convenient time reference. The general form of the curves shown here is similar for all the systems which have been investigated thus far. If we are willing to assume that the lumped parameter kinetic equations are an adequate description of the systems we are concerned with, we can also calculate, from the experimental powerversus-time history, the time behavior of the reactivity of the system. Also shown on this slide is the quantity which we call the compensated reactivity. It is the difference between the initial reactivity injection and the reactivity of the system at the instant of the power maximum and represents the reactivity compensated by the reactor itself in order to stop the power rise. Most of the general statements we make about power excursion tests in broad terms are based on these three curves. There is also some important information contained in the shape of the power burst, not all of which is reflected very sensitively in the reactivity and energy data.

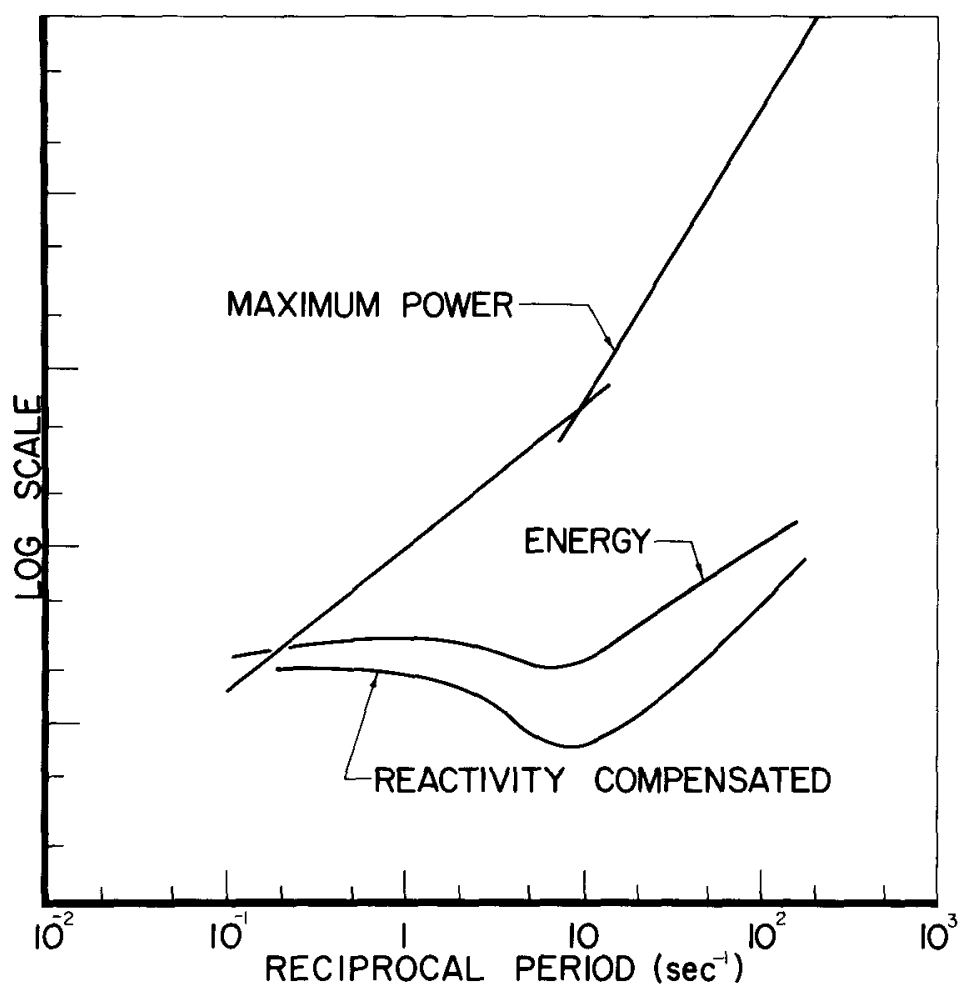

Slide 1. General Characteristics of Power Bursts 
Let us consider for a moment the general features of this compensated reactivity curve. Here, I think, one of the major points is that the reactivity change which is required to halt a power rise for transients up to, and slightly beyond, the prompt critical region may be appreciably less than that originally injected. This fact is clearly of importance to hazards analyses and is nothing more, of course, than another way of stating the well-known fact that a fast-acting safety system need only control a small amount of reactivity in order to check a power excursion in the region around prompt critical, even though one must ultimately remove all the injected reactivity in order to return to steady-state equilibrium. In Slide 2 , the curve marked $\Delta k(0)$ is the original reactivity injection given by the in-hour relation plotted again as a function of reciprocal period. For very long periods, one would expect to have to compensate most of this reactivity in order to stop the power rise. The curve marked $\Delta \mathrm{k}_{\mathrm{p}}(\mathrm{o})$ is simply the prompt excess reactivity, which should be the lower limit for the required compensation and will be the required curve for very short periods where we can ignore the contribution of the delayed neutrons. For intermediate periods, the curve for the required compensation must make a transition from the long-period asymptote to the short-period asymptote and the curve might be expected to exhibit a maximum and a minimum in this transition region, as shown in the slide.

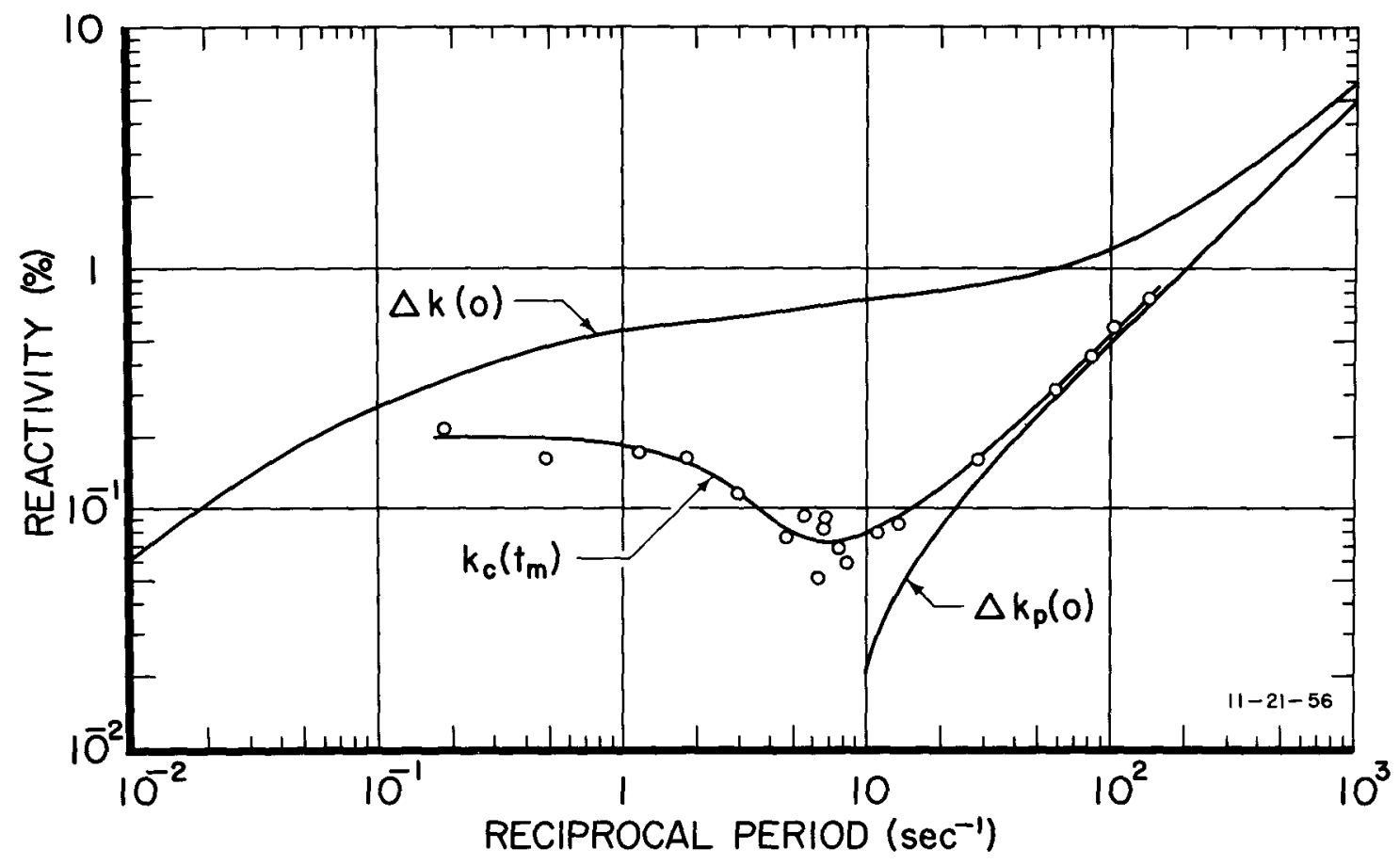

Slide 2. Reactivity Versus Reciprocal Period - Spert

Now I don't want to get into details which will probably be discussed in the session on analytical methods, but I would like to point out a few statements which follow from some rather simple analytical work. If one assumes an approximate analytical form for the power burst shape (and here we pick one which can be varied to include most of the 
experimentally observed shapes), and plug this expression into the kinetics equations and solve for the reactivity at the time of the power maximum; and if one performs this calculation for various shapes of the power burst and for various values for the nuclear parameters in the kinetic equations, the following conclusions are reached: first, the shape of the compensated reactivity curve plotted as a function of reciprocal period is dependent on the delayed neutron properties, on the prompt neutron lifetime, and on the shape of the power burst; but this last dependence is not very sensitive.

The analysis predicts further that the maximum and minimum in the transition region as shown for the particular case on slide 2 will appear for many reactor systems, but will not be present if the prompt neutron lifetime happens to be very long, or if the reactivity coupling produces either extremely broad or extremely narrow power bursts, but for most of the systems we would expect a maximum and a minimum in the curve.

Now, to what extent can we use observed compensated reactivity data to obtain an insight into the shutdown processes themselves? One might, at the outset, postulate the appropriate mechanisms for reactivity compensation, measure the reactivity coefficients in steady-state experiments, and describe from first principles the flow of energy in the system. From these data, the compensated reactivity can be calculated and compared with that obtained from the observed power burst. To the extent that agreement is obtained, one could feel that he had successfully described the situation. Such a check is really not definitive, however, in the absence of substantiative information as to the actual details of the mechanisms and the energy partition, but, of course, the same can be said of many of our physical models; they are true only insofar as they accurately predict the behavior of the systems to which they are applied. Such an approach has been tried in all the programs with rather different degrees of success. One difficulty that crops up in such a first-principles approach, is that some of the processes for the transfer and conversion of energy into reactivity effects are extremely complicated to calculate under transient conditions and in some cases the appropriate descriptions are not even available.

In the absence of good analytical descriptions for some of the complicated physical processes involved in shutdown, one can take an alternative approach and examine the properties of the kinetics equations for postulated forms of the equation which describe the reactivity feedback, without specifying the underlying physical processes which might give rise to the assumed form. This approach sheds little light on the mechanisms themselves, but can be quite useful in correlating behavior and in obtaining a general understanding of the way in which reactor transient behavior is affected by various forms of the coupling equation. The adjustment of the parameters in the rather simple forms postulated for the coupling, produces quite a variety of burst shapes which include any that would reasonably be expected. By comparing the calculated power burst shapes with those obtained experimentally, one may be able to determine which type of coupling equation is most appropriate to use for a given reactor system. Once a suitable coupling equation has been selected, the dependence of maximum power and energy release upon the 
various nuclear properties and reactivity coefficients may be obtained, and the very form of the required coupling equation may suggest some of the mechanisms involved. Most importantly, if the appropriate coupling equation can be determined for a given class of reactors by the performance of relatively few kinetic experiments, the performance of many members of the class can be predicted and the effect of design changes can be evaluated.

Now, I will spend a few minutes comparing some of the results of the various power excursion programs on the basis of the framework I have just described. First, consider the unpressurized, plate-type, water-moderated systems as represented by the Borax I and spert I programs. The Borax I work tested an aluminum-plate core in an open tank and investigated the period region between $100 \mathrm{msec}$ and $13 \mathrm{msec}$ at ambient water temperatures and down to $5 \mathrm{msec}$ period for tests initiated from boiling. Burst behavior was explored as a function of temperature for a selected number of periods. Spert I has now tested four aluminum cores and two stainless steel cores at various temperatures up to boiling and the period range has been extended to include from $10 \mathrm{sec}$ to $5 \mathrm{msec}$.

The various Spert I and Borax I cores differed rather widely in some of their nuclear properties. In particular, the average void coefficient for the various cores differed by a factor of 10 , and in fact, one core had a small positive coefficient for small voids in the center of the core. The prompt neutron lifetime varied by a factor of about 5 . For the Spert I core which was most similar in properties to the Borax I core, the onset of fuel melting is predicted to occur at periods of about $3.5 \mathrm{msec}$, whereas the well-known Borax I destructive test was run with an initial period of $2.6 \mathrm{msec}$. Extrapolation of the Spert data indicates agreement with the $135 \mathrm{Mw}-\mathrm{sec}$ nuclear energy release which was estimated for the Borax destructive test. In general, the behavior of all of the Spert I and Borax I cores is quite similar and I will discuss them as a group.

For this type of reactor, the principal mechanism for reactivity compensation is the decrease in effective moderator density arising variously from the thermal expansion of the water, thermal expansion of the fuel plates, and from the formation of steam voids. One would hope to be able to measure the appropriate density or void coefficients of reactivity for the various cores, and to be able to account for the experimentally observed reactivity compensagtions. As I mentioned before, for Spert and Borax cores this is quite difficult because of our lack of knowledge of the details of such things as transient boiling and also because in many cases, we don't know some of the pertinent effective heat capacities, local importance functions, etc.

For example, for the tests having initial asymptotic periods of less than about $50 \mathrm{msec}$, the measured fuel plate surface temperatures exceed the boiling point of water before peak power is reached, and boiling would be expected to contribute to the shutdown process. However, if one calculates the reactivity change at the instant of peak power from the power burst shape and uses experimental values for reactivity coefficients, it turns out that the steam volume required is at least an order of magnitude less than that which is predicted on the 
basis of steady-state heat transfer. A number of possible explanations exist, such as suppression of the boiling point by transient pressures, steam blanketing of the fuel plates, and transient superheat requirements, etc. As a first step, however, one could hope that a relatively simple picture of the process might provide a means for predicting the gross features of behavior. A particularly simple model for the boiling effect has been tried at Spert to permit making reactivity calculations. It has been called the "Conduction Boiling" model.

This treatment argues that the high heat-transfer rates which are observed in subcooled nucleate boiling are a result of bubble agitation of the boundary layer, with only a small fraction of the energy being transported by the bubbles themselves. It is assumed that under transient heating conditions, this "micro-convection" will not develop instantaneously, and the transient heat transfer rates may be treated as characteristic of conduction to the stagnant water rather than of boiling heat transfer. With this simple assumption, the temperature distribution in the moderator may then be calculated by a simple thermal diffusion equation. If it is also assumed that a fixed fraction of the transferred heat actually goes into steam formation, then the steam void growth and the compensated reactivity, due to steam, can be calculated. This fraction is used as an adjustable parameter in this model and is selected to fit the data at one point.

The effects of thermal expansion of the fuel and water, and a small contribution for direct gamma-ray and neutron heating of the moderator, have also been calculated and the total reactivity effect compared with experimental data from the Spert-I and Borax-I cores. Slide 3, illustrates

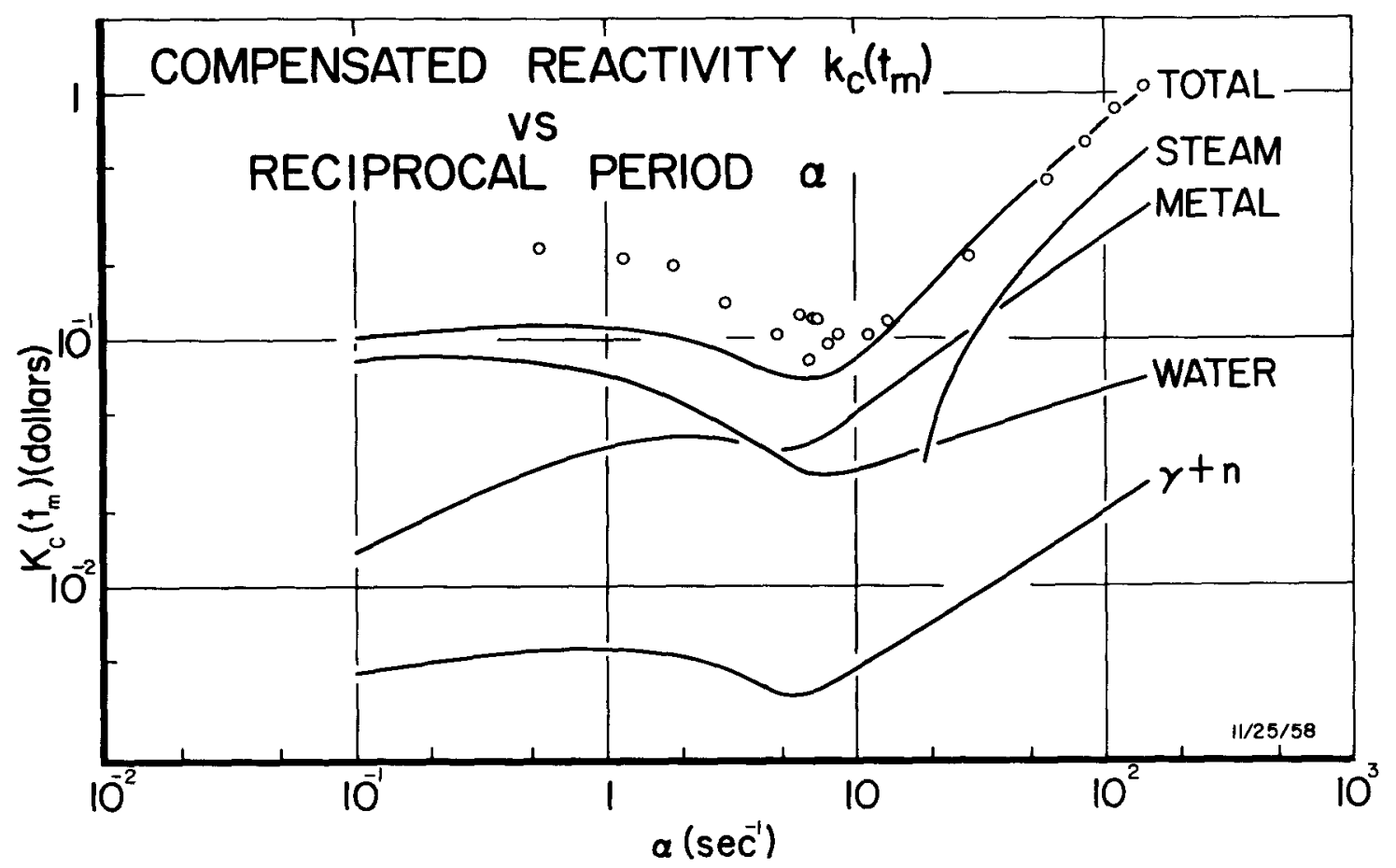

Slide 3. Compensated Reactivity at the Time of the Power Peak vs Reciprocal Period - Spert 
such a comparison for one of the Spert cores. The solid lines are predicted values and the points represent the experimental values. Comparing the calcilated total compensated reactivity curve with the measured values over the entire range of experimental data available, it is seen that the agreement is generally very good. There is one region in the neighborhood of alpha equal one where the calculated value falls somewhat short of accounting for the entire reactivity change. In this region, radiolytic gas is expected to contribute to the reactivity compensation but it has not been included in these calculations due to the lack of an adequate description of the gas evolution under transient conditions.

For alpha from 5 to 20 , the known effects account for the entire shutdown within limits of calculational errors. For alpha greater than 20 the inclusion of the steam shutdown term gives a good fit if the steam contribution is adjusted for alpha equal 100.

We would like to have a better picture of the actual physical processes going on under these transient conditions and hopefully develop some physical basis for the magnitude of the adjustable parameter in the model. To this end, Spert $I$ is presently engaged in a program of experiments in which pressure, volume, and temperature relationships are being investigated for sections of typical fuel plates which are exponentially heated in a closed water-filled capsule by using the reactor as a driving source. An attempt is also being made to photographically record the transient boiling process in the capsule.

One additional bit of information is available in connection with the relationship between boiling and non-boiling shutdown, as a result of some recent tests on Spert III. The particular tests were concerned with the effect of raising the system pressure for some ambient temperature step-transient tests. Here it was found that by increasing the pressure to 2500 psi, boiling could be eliminated as a shutdown mechanism for periods as short as $11 \mathrm{msec}$. Interesting enough, the peak power was only slightly increased and the energy to the time of power peak was increased only a factor of about 2, compared to the data for the atmospheric pressure tests in which boiling occurred. The interesting point is that when one takes proper account of the nonlinearities of water expansion which become important for the higher temperatures reached in these tests, the thermal expansion effects alone appear to be large enough to account for the shutdown without contribution from steam.

Now let us investigate the coupling equation appropriate for the plate-type reactors. One of the most successful approaches for the Spert data has been to assume a generalized nonlinear form for the coupling as indicated in the equation $k_{c}(t)=b[E(t-\tau)]^{n}$. The compensated reactivity is assumed to be proportional to the $n^{\text {th }}$ power of the energy produced by the reactor from the start of the transient to a time $(t-\tau)$, where $\tau$ is the delay time between the energy release 
and its manifestation as a reactivity effect. The exponent $n$ is taken as a positive constant and the constant of proportionality is called the shutdown coefficient $b$. Such a nonlinear dependence of reactivity upon energy release is easily rationalized since, for one thing, the thermal expansion of water is known to be nonlinear. The use of a fixed time delay is simply a convenient means of treating the time constants for heat transfer. This equation has been solved for superprompt-critical bursts for both zero-delay time and delay times much greater than the initial asymptotic period. It is found that by appropriate choice of the constant $n$, either the zero-delay or longdelay solutions will provide an adequate fit to the experimental power burst up to the time of the peak.

In Slide 4, the zero-delay burst shape is compared with the experimental data for a $9.5 \mathrm{msec}$ transient. The solid lines are the analytical results for different values of the parameter $n$. The case of $n=1$ here corresponds to the simple linear energy model for shutdown which predicts many of the general features of power excursions but, as you can see, does not represent the short period power burst shape very well for the Spert reactors. The choice of $n=2$ provides a good fit up to the peak. If we try to match the experimental shape immediately after the peak, however, we must go to the long-delay form of the model. Slide 5 shows the same sort of comparison for the long-delay model. Here, if $n=1.5$, the model gives good agreement for the whole burst.

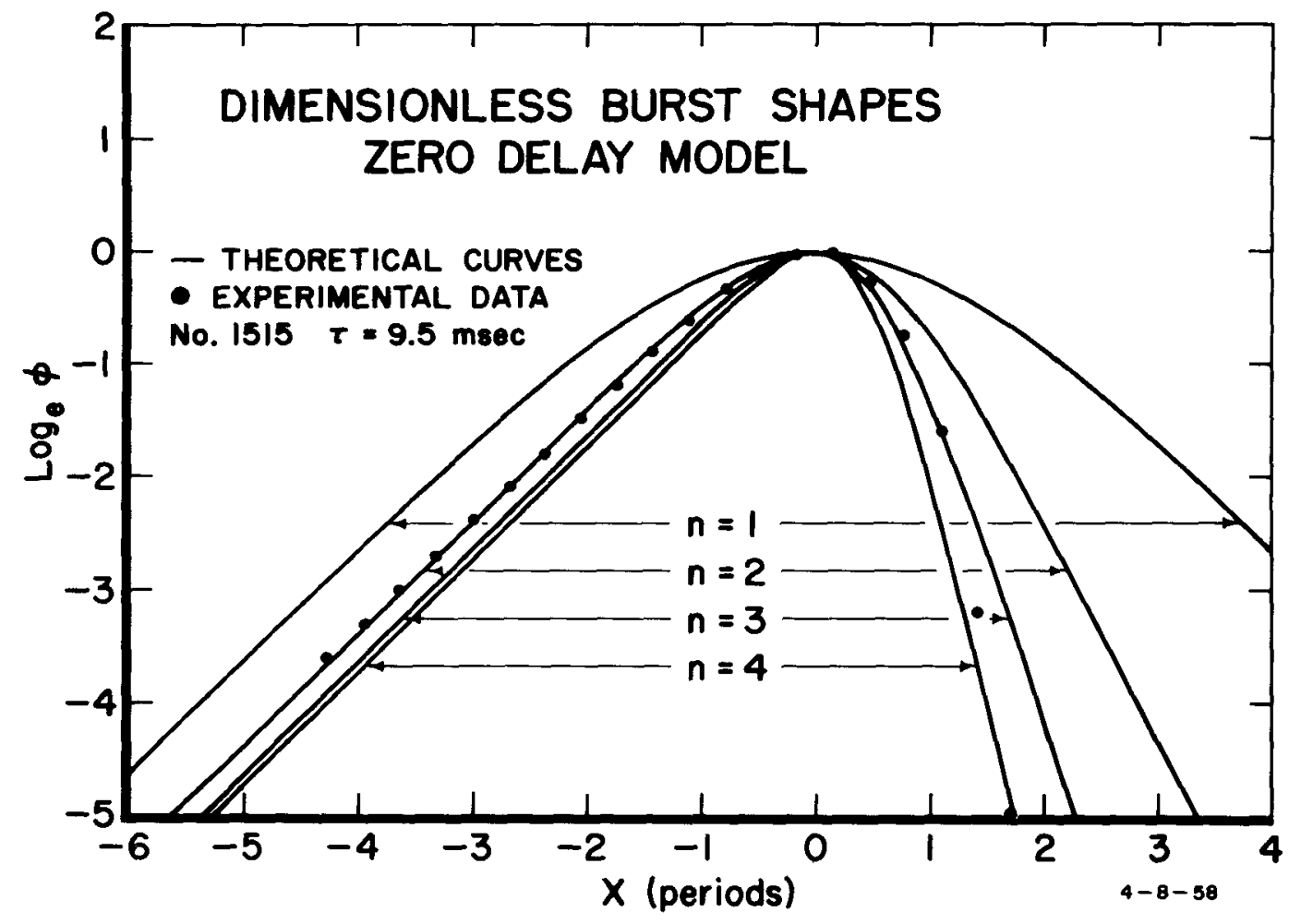

Slide 4. Comparison of Zero-Delay Model Burst Shapes and Spert Experimental Data 


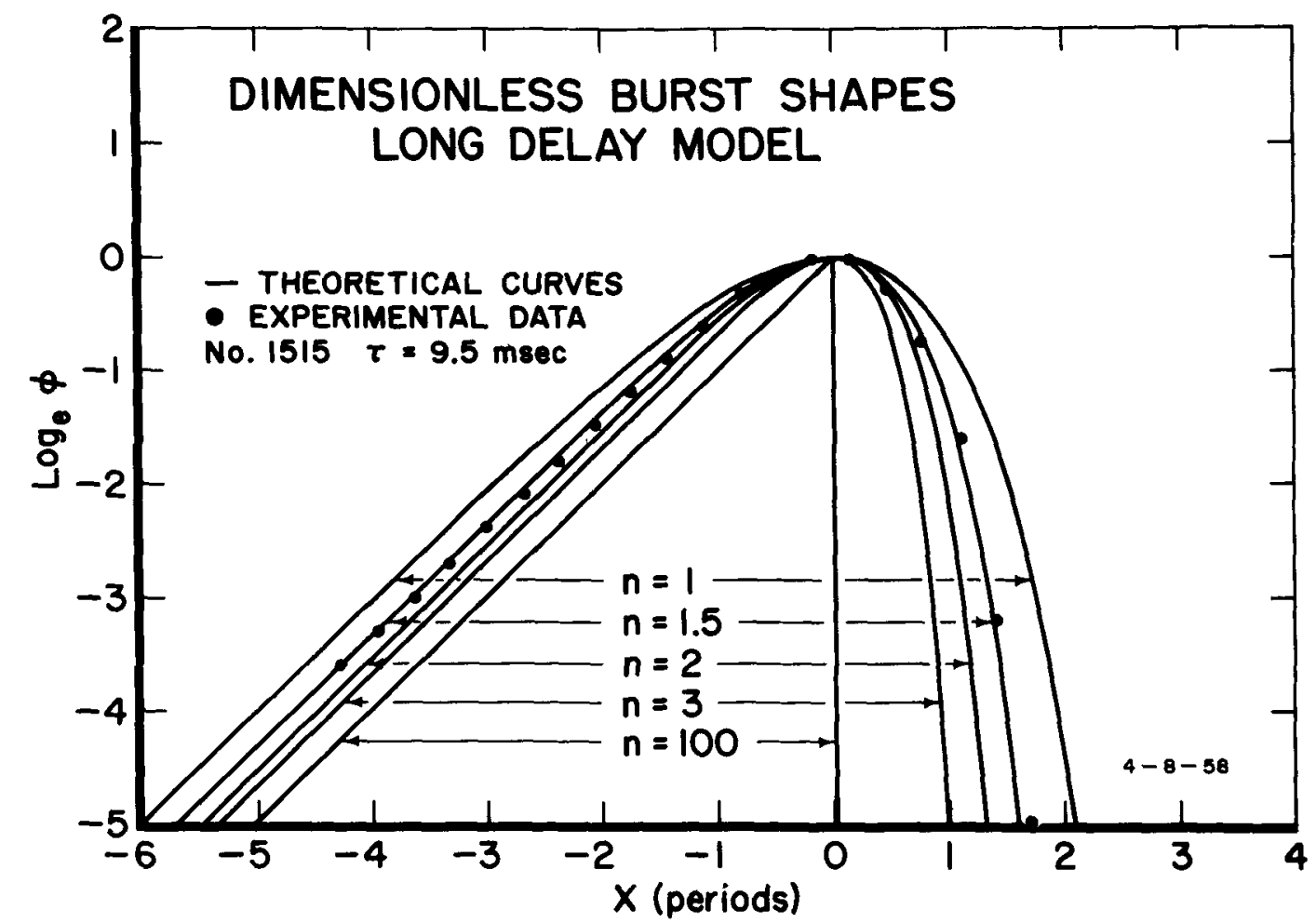

Slide 5. Comparison of Long-Delay Model Burst Shapes and Spert Experimental Data

A result of particular interest which arises from the coupling equation treatment is that it does allow the prediction of the dependence of peak power, energy release, etc., on the parameters $b$ and $n$. The analytical results for peak power for the zero-delay and long-delay cases are given in the equations

$$
\begin{aligned}
& \phi\left(t_{m}\right)=\frac{\frac{n+1}{n}}{b^{1 / n}}\left(\frac{n}{n+I}\right) \quad \text { Zero Delay } \\
& \frac{n+1}{n} \\
& \phi\left(t_{m}\right)=\frac{\alpha}{b^{1 / n}}\left(e^{\alpha \tau-1 / n}\right) \quad \text { Long Delay }
\end{aligned}
$$

In both cases, the peak power is seen to be proportional to the reciprocal of the $n^{\text {th }}$ root of the shutdown coefficient $b$. Since $n$ is found experimentally to be of the order of 2 for plate-type reactors, this implies that the dependence on the shutdown coefficient $b$ is weaker than the linear dependence predicted from the simple linear energy model. The coefficient $b$ is proportional to the ratio of the density coefficient of reactivity to the prompt neutron lifetime. Therefore, for the plate-type water-moderated reactors it would be expected that the peak power for short-period bursts would vary approximately as the reciprocal of the square root of the void coefficient over the lifetime. Investigation of six Spert cores for which the shutdown coefficient $b$ varies by a factor of 50 , has substantiated this 
prediction. The weak dependence of $\mathrm{b}$ is implicit in any reactor which displays a shutdown effect that increases more rapidly than the energy released, and is not, therefore, unique to the particular analytical form used here.

Now let us consider the step-transient behavior of aqueous homogeneous systems such as the water-boiler reactor. The Kewb project has investigated the behavior of a prototype $50 \mathrm{kw}$ water-boiler research reactor with a spherical vessel. The period range investigated has been from 30 sec to 2 msec, which, incidentally, is the shortest period to which any thermal reactor has been intentionally subjected, at last report. The initial temperature was varied over the full range of operating values from $10^{\circ} \mathrm{C}$ to $90^{\circ} \mathrm{C}$, and was found to have little effect on the kinetic behavior. Similarly, initial pressure, initial power and the fraction of the sphere initially filled by the fuel solution were found to be relatively unimportant. The most significant of these parameters appears to be the initial core volume--the transient pressures generated when the core is initially filled with fuel solution are two to four times those observed in the under-full cores. The highest transient pressures observed were of the order of 600 psi for 2-msec period tests.

In this type of system, the principal shutdown mechanisms are thought to be the thermal expansion of the solution and the formation of radiolytic gas. Steam formation does not play a significant role in the shutdown of this reactor. Slide 6 shows the experimental compensated reactivity data for the Kewb system. Note here that the abscissa is the period rather than $\alpha$. Using the measured energy release, the heat capacity and the measured temperature coefficient of reactivity, the reactivity compensated due to temperature alone has been computed and is displayed as the curve labelied "temperature". The remaining unaccounted-for reactivity has been labelled

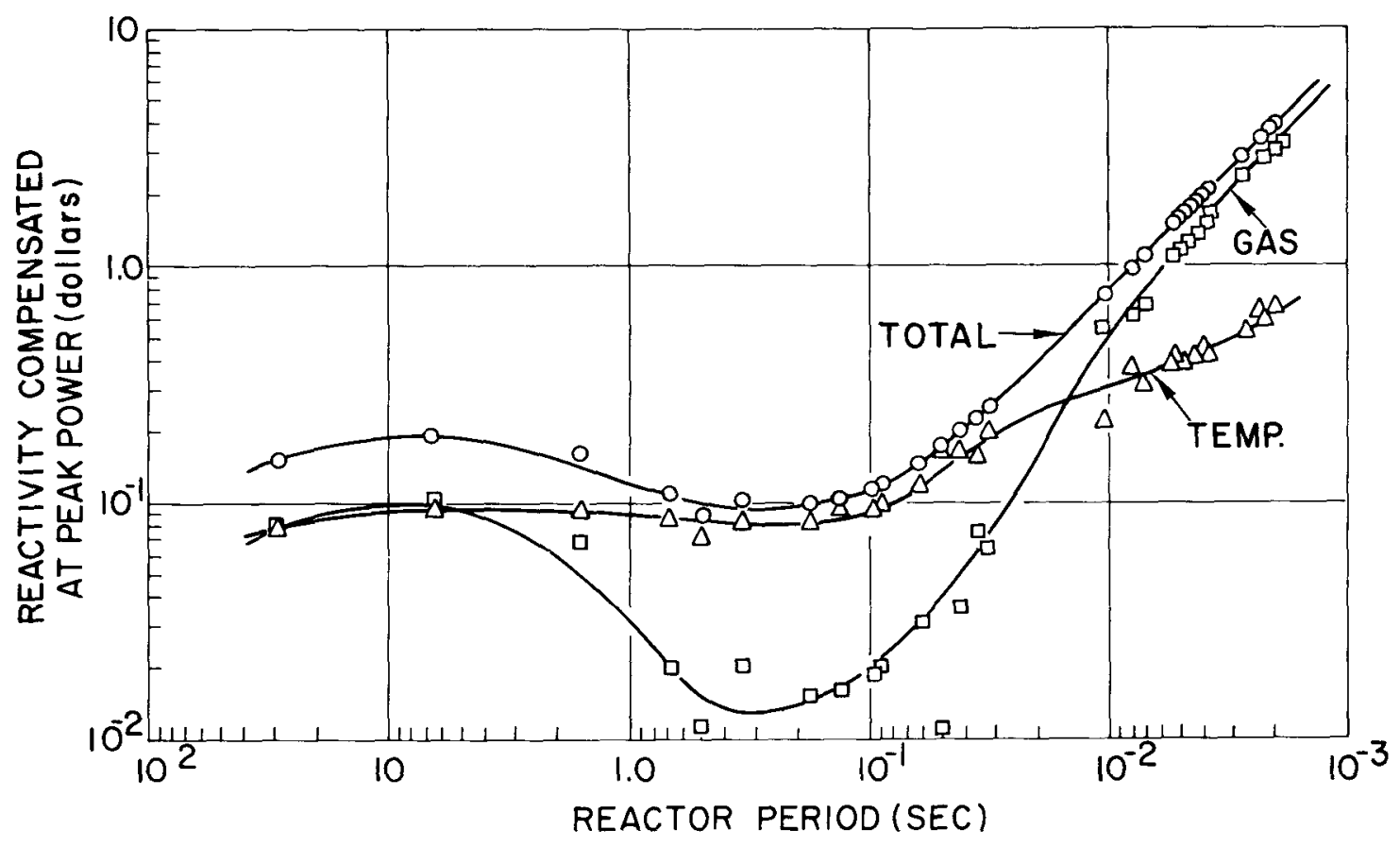

Slide 6. Compensated Reactivity at Time of Power Peak Vs Reactor Period - Kewb 
"gas". An examination of these data has led to the following conclusions. For periods of the order of $10 \mathrm{sec}$, void formation must supply about half of the required compensation, but for the period region from 1 sec to $20 \mathrm{msec}$, temperature effects dominate the picture. For very short periods, the gas formation must take control and must become increasingly more important as the period decreases. So here again as with the plate-type systems, the importance of various mechanisms changes with the period range.

A coupling model has been postulated at Kewb which assumes that the driving function for void formation is the product of the instantaneous power and the energy, the thought here being that void formation is influenced by a time-dependent "nucleation rate" (which might be proportional to the instantaneous power density), and by the time-dependent dissolved gas concentration which is assumed to be proportional to the instantaneous energy. The last assumption is based on the hypothesis that the growth of an individual void will depend on the amount of gas in solution in a small localized region, rather than on the average gas concentration over the reactor core. The coupling equations used were

$$
\begin{aligned}
k_{c}(t) & =A T(t)+B V(t) \\
\dot{T} & =C \dot{E}(t)-\alpha T(t) \\
\dot{V} & =v E(t) \dot{E}(t)-\sigma V(t)
\end{aligned}
$$

The compensated reactivity is given by the first equation where $A$ is the temperature coefficient and $B$ is the void volume coefficient. In the

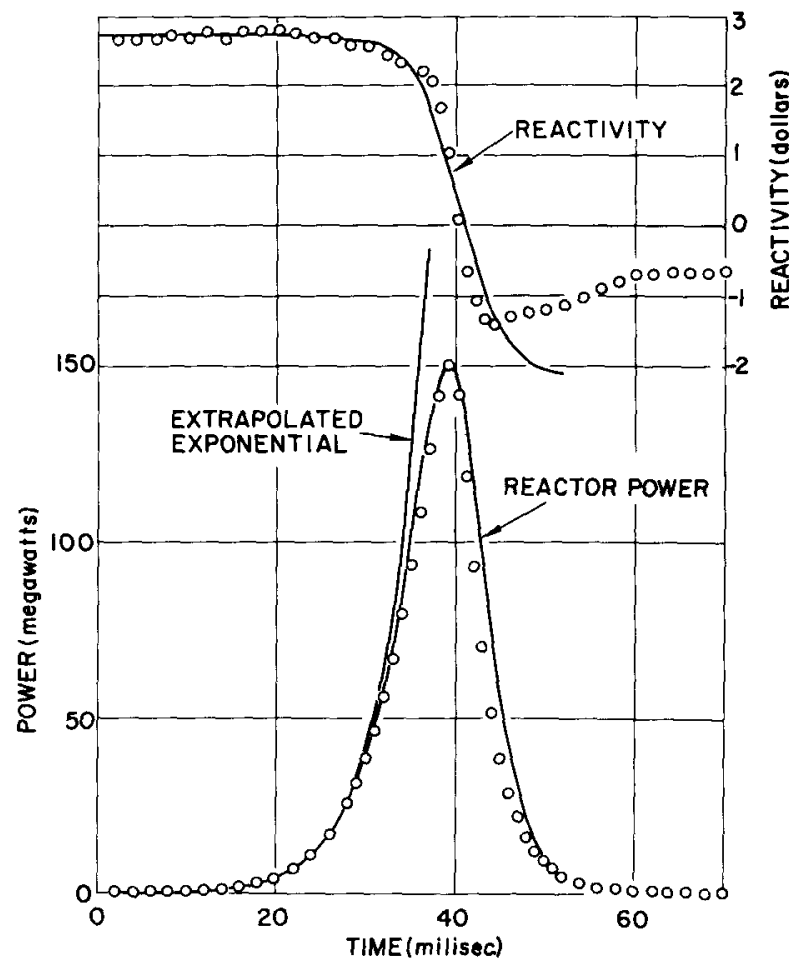

Slide 7. Comparison of Coupling Model Power and Reactivity with Kewb Experimental Data differential equation for temperature, $C$ is the reciprocal heat capacity, $\alpha$ is the time constant for heat removal and $E$ is the energy release.

As indicated in the second term of the void equation, bubbles are permitted to escape the system, and $\sigma$ is the reciprocal of the estimated bubble residence time. The void production term is proportional to the product of energy and power, with $\nu$ an adjustable parameter for the model. The parameter $\nu$ has been determined for each of a large number of transient experiments by adjusting its value until the calculated and observed peak powers agree. Slide 7 shows the typical kind of fit obtained for power burst shape for short-period test -- in this case a $4.5 \mathrm{msec}$ test. The plotted points are from the experimental power trace and the curve is that predicted by the model. The computed burst shape fits pretty well, except for the 
trailing edge of the burst, much as was the case for the Spert zero-delay model. This model has been successful in predicting peak power as a function of $\alpha$ over a range from 100 to 500; that is, periods of $10 \mathrm{msec}$ or less, with no additional adjustment of parameters.

For longer periods, however, the model was not as successful. In the intermediate period range where temperature effects account for most of the shutdown, the model over-estimates the gas effect. For periods around $10 \mathrm{sec}$, where the assumed bubble residence time of $10 \mathrm{sec}$ becomes important, the model under-estimates the effect of gas and produces too broad a peak, no matter what the value chosen for the residence time.

No provision is made in the model for the effect of transient pressure on the gas voids. Since these pressures are of the order of $100 \mathrm{psi}$ at the time of the power peak for short-period Kewb transients, the success of the model has suggested to the Kewb people that the voids may be in the form of very small bubbles with very high internal pressures.

As in the case of the steam formation in the plate-type systems, Kewb has had good success in describing short-period excursions by a simple model which ignores the microscopic details of bubble formation. And, in both cases, we have an adjustable constant which has not been derived from basic principles. It should be noted that the void formation portion of the Kewb model, being the product of power and energy, is quite similar to the general form of coupling equation which was used successfully for short-period Spert bursts, since up until the power peak, the product of energy and power is approximately an energy-squared dependence.

Now I would like, even more briefly, to mention three other systems which might be called solid homogeneous systems. Consider the Triga reactor first. In Triga, we have combination fuel-moderator elements of low enrichment uranium-zirconium hydride in the form of right circular cylinders clad with aluminum. Additional moderation is provided by the water-coolant and a graphite reflector.

The reactor possesses an interesting large negative coefficient of reactivity which is attributed to the bound hydrogen in the zirconium hydride and, because of the intimate mixture of fuel and moderator, the coefficient is quite prompt. Step-transient tests have been performed with Triga for periods as short as $3.2 \mathrm{msec}$. For super-prompt-critical bursts, it has been found that the simple model in which reactivity compensation is proportional to energy release describes the data quite well. In addition, the direct approach of calculating the coupling equation by a space-independent treatment of the thermal properties of the system has been successful. The thermal model includes the various heat capacities, thermal time constants and the temperature coefficient as determined from quasi-equilibrium experiments. The calculated behavior agrees with experiment within the expected limits of error.

A similar situation exists for the Treat reactor which is designed for use as a driving source for meltdown studies. The Treat fuel consists of uranium oxide particles distributed in graphite. Here, again, the measured temperature coefficient has been used to predict the burst behavior quite well on the basis of a simple energy shutdown. 
Lastly, the reactor which was probably the first for which power excursion studies were carried out--Godiva is a fast reactor composed of a highly enriched uranium metal sphere, which can be made superprompt critical by rapid assembly. The linear energy coupling model, which I have referred to several times today, was first developed to describe Godiva and it was found that until the period gets very short, the model fits the data, as would be expected since the shutdown mechanism is the thermal expansion of the metal itself, which is essentially prompt. However, for periods of the order of $20 \mathrm{\mu sec}$, the bursts become larger than predicted by the linear model, since the time scale of the burst is now short compared to the thermal expansion time constants of the sphere. For very large reactivity insertions, the energy release in the burst is about proportional to the cube of the reciprocal period, rather than the linear dependence given by the simple model. I believe the Los Alamos people have had good success in fitting the very shortperiod data using digital codes to include the spatial and inertial effects.

Now, I would like to compare the step-transient data from these varied systems in the following way. Suppose we consider an experimentally determined quantity called the effective dynamic energy coefficient of reactivity, call it epsilon. This is the ratio of the reactivity compensated by the system, to the nuclear energy release, evaluated at the time of the power peak. Now, since any shutdown mechanism will have some characteristic time constant we would expect that if we plot epsilon as a function of reciprocal period that, for a single mechanism system, there would be a region at low alpha where epsilon would be relatively constant, and could be determined by non-transient measurements, but for short enough periods, the effective value would begin to decrease. If, however, another more effective mechanism begins to act only at short periods, the value of the energy coefficient may level out or even rise, but ultimately for short enough periods, the curve should drop again. Suppose we now look at the coefficients as a function of reciprocal period for various systems as shown in Slide 8. The data for the various plate-type cores of spert and Borax are contained within the cross-hatched area. In general, for these plate cores, the effective energy coefficient decreases as the reciprocal period increases, and then levels out or slightly rises as we reach periods where boiling is important. That is, as the time scale of the burst is shortened, the effectiveness of the thermal effects, which depend on heat transfer rates, decreases; but in the shorter-period region we pass the threshold for the more effective mechanism of steam void formation. The various Spert cores are much alike and over the range of period available there is really very little variation in the coefficient and we have a rule of thumb that for this type of system, over the range of period available, a coefficient of $6 \pm 56 / \mathrm{Mw}-\mathrm{sec}$ will describe all the cores for all practicable periods. Data are also shown here for Spert III, the solid curve is for atmospheric pressure and the dashed portion is for tests at 2500 psi. Note that the suppression of boiling by pressurization pushes the curve downward in the short-period region, as we would expect, since the burst shape for the pressurized tests is not sufficiently different to affect the compensated reactivity, but the energy release is increased. 


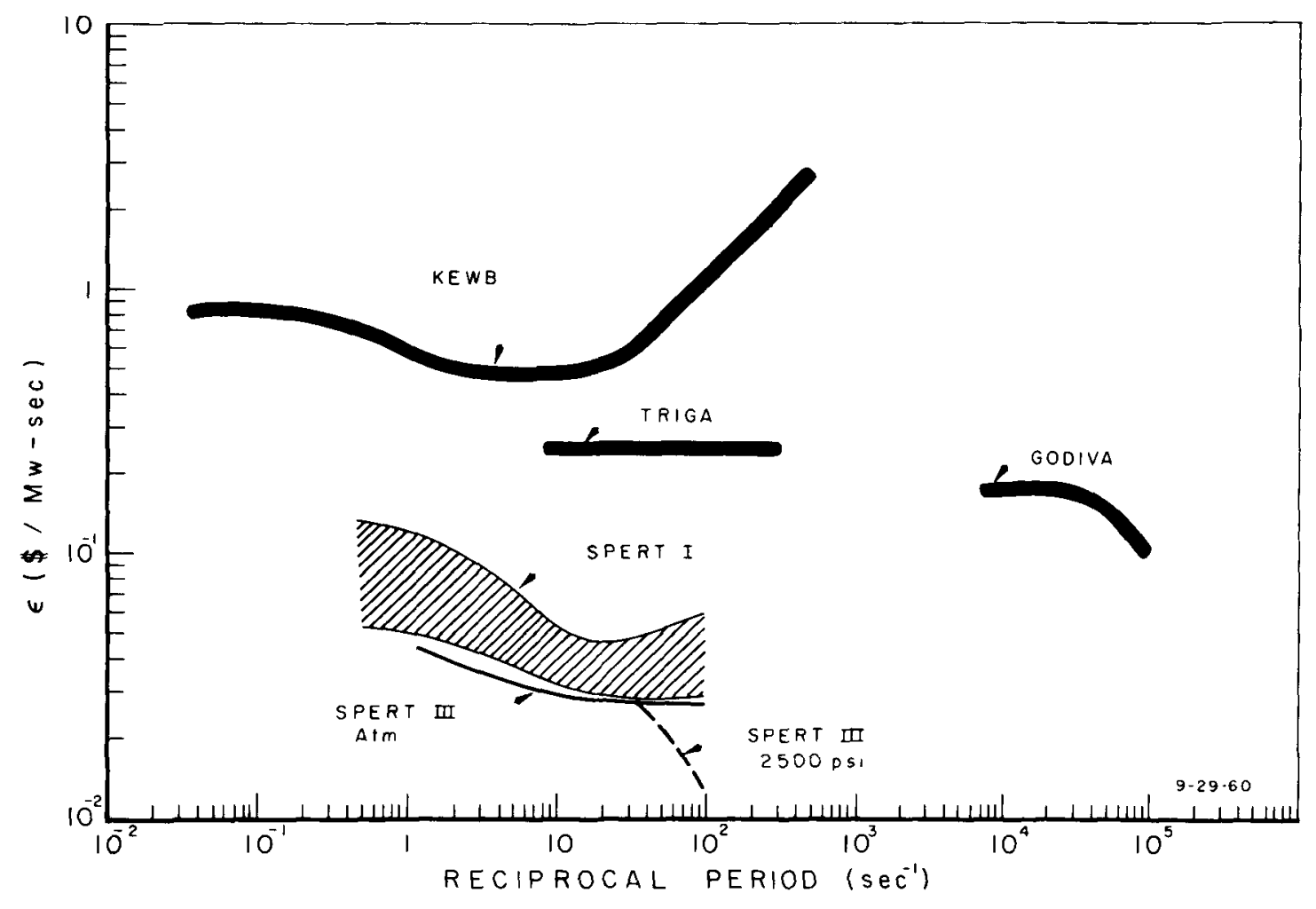

Slide 8. Effective Dynamic Energy Coefficient of Reactivity vs Reciprocal Period

For Kewb, the more prompt thermal effects present in the homogeneous system result in a larger coefficient, about $50 \% / \mathrm{Mw}-\mathrm{sec}$ in the prompt critical range. The onset of the increasingly effective void formation at short periods causes an increase in the coefficient to the order of 2.5\$/Mw-sec for $2-\mathrm{msec}$ period tests.

For Triga, the shutdown mechanism is quite prompt, the linear energy model seems to fit satisfactorily over the whole range of super-promptcritical tests, and thus the dynamic energy coefficient is constant. The experimental value is about $25 \phi / \mathrm{Mw}-\mathrm{sec}$.

Similarly, as long as Godiva is in the region of applicability of the simple model the coefficient i's constant at about $17 \phi / \mathrm{MW}-\mathrm{sec}$, but as the period gets very short, the effective energy coefficient of reactivity drops to about $10 \notin / \mathrm{Mw}_{\mathrm{w}}-\mathrm{sec}$ for $11-\mu \mathrm{sec}$ transients.

One must avoid the temptation to make sweeping conclusions about relative safety from a comparison of effective energy coefficients, however, since it is clear that the amount of energy release for a given period is not the whole story when different reactor types are compared. For example, while the attainment of energy releases which are sufficient to cause fuel assembly meltdown is probably the most important limit for solid-fueled reactors, the transient pressure developed may provide the limit for a homogeneous system such as Kewb. 


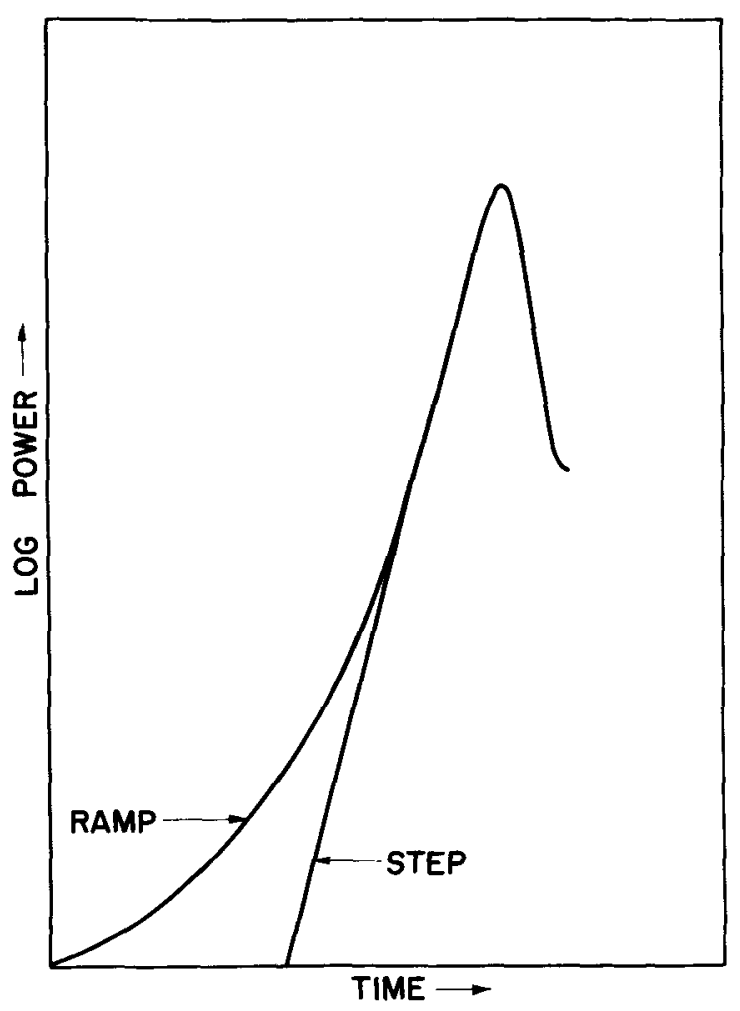

Slide 9. Comparison of Spert Power Burst Shapes Following Step and Ramp Reactivity Insertions
In the time remaining, I want to touch on some additional types of tests which have been performed in the field of power excursions. First, recall that all the experiments I have discussed so far have involved sudden, step-wise insertions of reactivity. From the point of view of those who must design or evaluate the safety of control rod drive systems, a very important consideration is that of determining the maximum reactivity insertion rates which should be permitted for a given reactor, and to what extent the initial power level influences this maximum rate. In order to provide answers to this type of question, many experiments have been performed in which the control rods are simply pulled continuously so as to produce an essentially constant rate of reactivity addition--in other words, the ramp test. Here, the most interesting point is that experimentally we find--and this is true for all systems which have been investigated-that an experimental equivalence can be found between the ramp-rate and the step-insertion tests. This equivalence can best be described by reference to Slide 9 where we

have plotted power as a function of time. In the ramp test, the reactor period decreases continuously until the rate of reactivity loss resulting from the energy release is equal to the rate of insertion. At this point, the reactor period has its minimum value. Experimentally, we find that the remainder of the initial power burst is essentially the same as that of a step-transient for which the initial period is equal to the minimum period for the ramp-test. Thus, if a ramp test is characterized by its minimum period, it is essentially equivalent to a step test of the same period. This same result can be obtained from some approximate analytical treatments, provided that certain initial conditions are satisfied.

This analytical work has also led to an approximate relationship between the ramp rate and the minimum period produced--and hence between the ramp rate and the period of equivalent step-burst. The exact nature of the definition of equivalence is open to some discussion in the analytical treatment but the experimental work indicates the validity of this sort of treatment for most cases of practical importance. The approximate relation says that the equivalent ramp rate varies as the square of the stepinjection and inversely with the prompt neutron lifetime. There is also a very weak dependence on the initial power level, which is also substantiated by experiment. Thus, if we determine the maximum permissible step 
injection for a system, we can also estimate the maximum permissible ramp rate. To say it another way--from this equivalence we can apply the general understanding we have obtained of step-type power excursion tests to the problem of reactor startup and prescribe limitations on the allowable rod withdrawal rates, provided only that the initial power falls within rather some wide limits. Furthermore, because of the square-law dependence between steps and ramps, design changes which slightly increase the permissible step (such as an increased void coefficient of reactivity) should significantly increase the allowable control rod withdrawal rates. In fact, in some it might be possible to extend the permissible rates so far that it becomes mechanically difficult to achieve these rates in practice. For example, the computed permissible ramp rate for the spert I A-core is about $12 \$ / \mathrm{sec}$. The Spert I reactor contains a transient rod especially designed to provide rapid reactivity injection for step-type experiments. The maximum rate of reactivity addition provided by this fast rod is about $25 \$ / \mathrm{sec}$, which is only a factor of 2 greater than the permissible ramp rate for this reactor. A 50 per cent increase in the permissible step would result in a factor of 2 increase in the permissible ramp and the reactor would be inherently safe against reactivity additions even from this very fast rod.

The statements we have made so far about the ramp-rate tests can be applied to startup accidents in reactor systems as we usually operate them--that is, with a sufficiently high initial neutron level that the power responds immediately to the reactivity increase. Suppose, however, we have a reactor core in which the inherent neutron source level is very, very low and that we have no artificial source present. This brings us to the next topic which I wanted to place on the agenda for later discussion and which was already introduced this morning-reactor kinetics for systems in which the initial power level is so low that we must deal with a statistical problem. If we begin pulling rods in such a reactor, there may be a substantial delay in establishing a sufficient number of long fission chains to insure that the fission rate behaves in accordance with the kinetic equations. Before the reactor is really operating as a well-behaved chain reacting system, it might be dangerously supercritical. From the point of view of using our ramp-to-step equivalence to evaluate the safety of the reactor itself, we would like to answer the following question: For a given rod withdrawal rate, what initial neutron level must be present in order to insure that the previously discussed experimental and analytical treatments of ramp transients can be assumed to apply? Or we could ask, for a given initial neutron level and ramp rate, what is the probability that the reactivity compensating effects can begin to act? This question is also pertinent for reactors in which we depend on safety circuits for shutdown since we usually require some minimum power level for the proper operatiol of the sensing circuits. This problem is complicated analytically--I am sure we will hear more about this point from the theoreticians. Some rather crude estimates of the region of interest for this problem have been obtained by analysis of some power excursion experiments. A series of step-transient tests had been performed in Spert I with a small, relatively cold stainless-steel core 


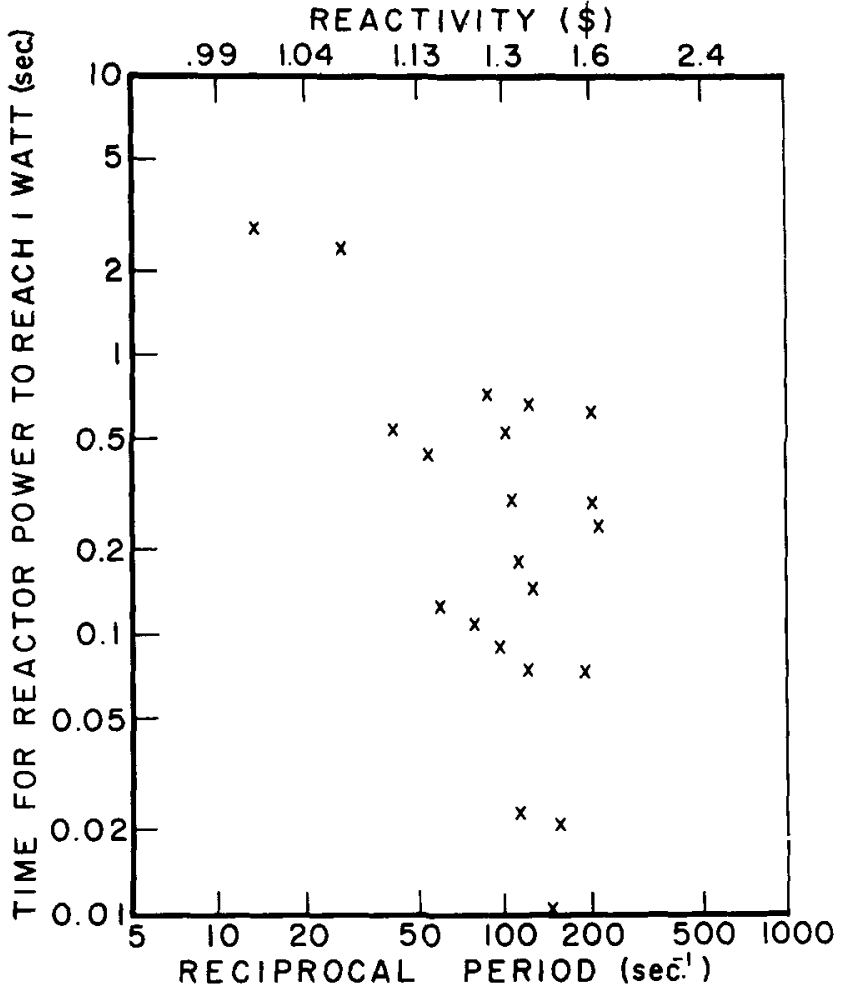

Slide 10. Spert Delay Times VS Reactivity Insertion and Reciprocal Period (the BSR-II core) with no artificial source present. For each test, the initial power levels were below 10 microwatts. From the power data obtained, it was possible to calculate the elapsed time from the step-injection of reactivity to the attainment of a power level of approximately one watt. These data are shown in SIide 10, plotted as a function of both the reactivity insertion and the reciprocal period. There is quite a bit of scatter, as would be expected, since we are investigating a statistical process. This sort of behavior with even longer delays had been noted long ago for the Godiva reactor but I think the significant point here is that we now have, for the first time, a demonstration that a thermal reactor system, of not-unusual design, can sit at prompt critical for the order of one second before the power reaches easily detectable levels. I think it is clear that the statistical problem of a sourceless startup is clearly not a trivial one for many reactors.

A large number of reactor startups were performed with this core at Spert with no source present and without incident, but the critical position was well-known. I think we must conclude that a good deal more experimental and theoretical work must be done before we are in a position to prescribe quantitative limits on the minimum source levels for safe startup of essentially unknown systems.

The third miscellaneous topic I want to mention concerns the benefits to be derived from reactor safety systems. The approach $I$ have used for most of this paper has been to consider the inherent behavior of the reactor itself without taking any credit for the mechanical safety system which might be provided. What differences are there in the consequences of a large reactivity addition if we add safety systems to the picture? I would like to postulate that the basic question here is: To what extent does a good, fast-acting safety device provide more protection to the reactor and, in particular, to the general public than is already present in the inherent properties of the reactor? Unfortunately, any safety system we can postulate has an inherent fixed time delay between the decision to scram and the actual accomplishment; whereas, the inherent shutdown properties of the reactor will be at least more closely geared to the time scale of the burst. Thus, there might well be a region of 
short-period power excursions where the mechanical shutdown initiated by scram circuits will be too slow and one must depend upon the inherent properties of the reactor anyway. We are not always sure, without some careful consideration, that the addition of reactor safety devices, be they conventional rod scram circuits or fuses, will necessarily result in a significant improvement in safety for the extreme accident condition. Of course, we always need some automatic scram feature in order to completely shut down the reactor, and prevent the possibility of successive self-limiting bursts. But since much of the expense of good safety systems is, at least sometimes, in the achievement of very fast action in order to protect against short periods, I think we owe it to the industry to at least make sure we are really buying additional protection in this region.

Now, I would like to briefly summarize our present position regarding reactor excursion studies. Our understanding of plate-type, watermoderated systems of the low power research type seems to be in good shape. While we don't know as much as we would like to about the details of the radiolytic gas formation and transient boiling phenomena, we do have coupling models which correlate behavior and predict the effects of design changes, and the present programs of in-pile and out-of-pile work have great promise of filling some of these gaps.

I would like to comment that we at Spert agree whole-heartedly with Rogers McCullough's statements this morning that we need more activity in the direction of small-scale tests designed to answer specific questions about mechanisms--both in-pile and out-of-pile tests. Additional tests investigating the influence of the prompt neutron lifetime, and of the temperature, pressure and flow parameters are presently underway.

The tests already performed have demonstrated the ability of platetype water-moderated systems to withstand, with only minor core damage, periods as short as $5 \mathrm{msec}$. Extrapolation of the results to shorter periods is, we feel, on fairly firm ground, but a test program in which the consequences of core meltdown or violent disassembly are investigated would be of considerable interest. There seems to be no reason to intensively test additional plate-type cores (except destructively) but different types of water-moderated cores need to be investigated as well as other moderators. The problems associated with the kinetics of reactors operating in the high power range have not yet been experimentally investigated to any large extent, but such investigations are planned.

The Kewb work has demonstrated the ability of small aqueous-homogeneous research reactors to withstand the transient pressures produced by excursions for periods as short as $2 \mathrm{msec}$. Again in this case, the general features of behavior are understood, but some of the details of radiolytic gas formation remain to be ironed out. The influence of core geometry is thought to be predictable and is presently being checked by tests of a cylindrical core.

From the data already obtained in the various power excursion programs and from the analytical work based on and stimulated by the data, we now have a greatly increased level of understanding of reactor kinetics 
under accident conditions. We can now approach the problem of predicting the response of quite a variety of reactor systems on a much firmer basis; even without knowing very much about details. We are also in a better position to know the crucial experiments whicn should be performed on new and quite different systems to enable us to extrapolate and apply our present knowledge.

One specific achievement of the power excursion work has been to remove prompt critical as an upper limit for safe reactivity injection. We now have convinced almost everyone that we should not assume that $\$ 1$ is the reactivity insertion corresponding to the brink of disaster. The performance of hundreds of self-limiting power excursion tests in the prompt critical region, in which behavior has been safe and predictable has, indeed, been responsible in part for some of the interest in building reactors for use as pulsed sources, and Treat and Triga are examples which are already in use. The fact that we can now, with a considerable degree of confidence, predict the self-limiting behavior of many systems leads to the conclusion, which I touched on in the beginning, that we should now begin to consider performing limited kinetics test programs on many systems at the time of initial startup. Such tests might provide the best means of checking hazards calculations, of determining the appropriate form of the coupling equation and the actual values of dynamic reactivity coefficients and would provide perhaps the only realistic check on the performance of safety systems. I would hope to hear some additional discussion of this subject, since I feel that the only way to accomplish such programs is for such groups as this to be convinced that the gains to be made from such testing far outweigh the hazards involved. I think there are many-at this conference--who feel that power excursion tests are inherently too hazardous to perform even on reactors whose hazards reports assure us of the safety of even more serious maximum credible accidents.

My time has run out, and I'm sure you are all painfully aware that I have dealt in generalities and left out a good many things which might have been discussed or amplified. I now leave it to the panel, in particular, and to all of you, in general, to fill in the fine structure. 
Stone: I will ask each of the panelists to talk for 10 or 15 mimutes. I would like now to call on Marlin Remley of Atomics International to talk on the Kewb program.

\author{
Presentation of Kewb Program \\ by \\ M. E. Remley (AI)
}

Remley: As Frank Schroeder has already covered--we are doing experiments on the aqueous homogeneous reactor which, at first glance, one would think is a simple system. We don't have the problems of heat transfer from the fuel to the cladding and from the cladding to the coolant. We actually have the fission process taking place in a solution so that we get a prompt temperature effect, and, in addition, there is a second energy-dependent mechanism which controls the dynamic behavior; this is the production of radiolytic gases. As Frank indicated, the first studies in this program were made with a spherical core, and we have recently changed over to a cylindrical core to investigate the effect of geometry of the core, and what I would like to do is review briefly some of our experimental results, compare the results from the two cores, and then ask the question--what do these mean, and how much do we understand about the system.

Warren indicated this morning the conference was not going to emphasize hardware, but I thought it might be pertinent to take a moment to look at a couple of slides which show our two pieces of hardware. Slide 1 shows what

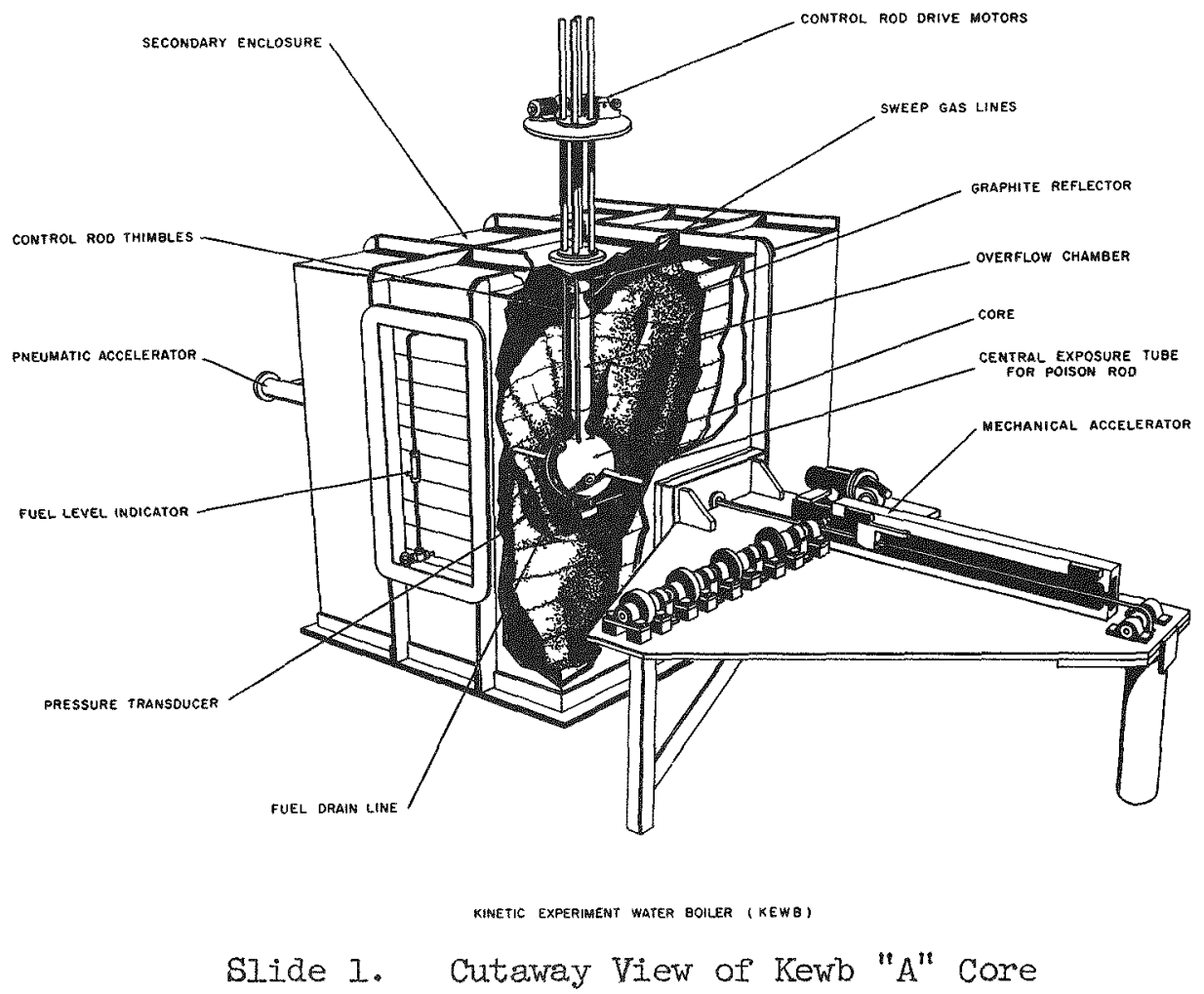


we call the Kewb "A" core, which is a spherical core, 12 inches in diameter, with uranyl sulfate solution, reflected by graphite. It has a conventional control system and a rod-accelerating mechanism for initiating transients. The significant feature here is the 12-in. diameter sphere that contains about 11-1/2 liters of uranyl sulfate solution in what we call the underfuII case and about 13-1/2 liters in the full case. In this latter, the fuel solution level is at the $2-1 / 2$ orifice at the top of the sphere. Slide 2 shows the installation of the cylindrical core, known as the " $B$ " core, which is a vessel also about 12 inches in diameter and about 36 inches high; it contains about 18 liters of uranyl sulfate solution, which occupies about the bottom third of the volume of the vessel, so that there is a void of some 35 liters above the fuel solution. It again is graphite reflected. This was installed and put into operation in March of this year.

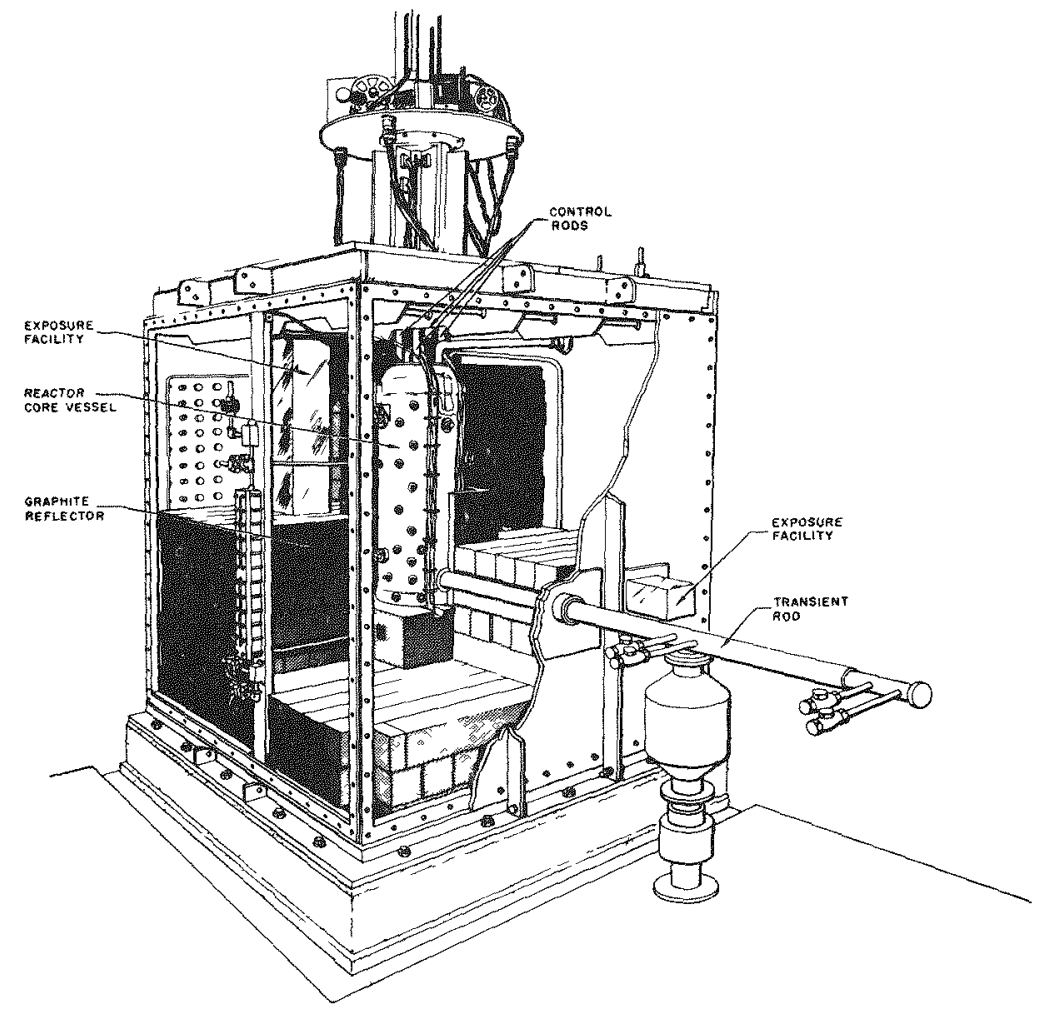

Slide 2. Cutaway View of Kewb "B" Core

Some of the experimental results are shown in Slide 3--this one gives the peak reactor power as a function of period. This gives the results from the " $A$ " core, which have already been discussed by Schroeder, along with recent results with the " $B$ " core. You will note that the cylindrical core does not show any significant deviation from the spherical core in the peak power characteristic. This peak power vs reactor curve--note that the period decreases going from left to right--has the characteristic shape that Schroeder pointed out. A significant addition is the extension of the experiments down to a much shorter period. The "A" core results stop, as Frank indicated, at about 2 milliseconds. We have now carried this down to 0.9 millisecond in the cylindrical core. Slide 4 shows the energy release as a function of reactor period. This again gives 


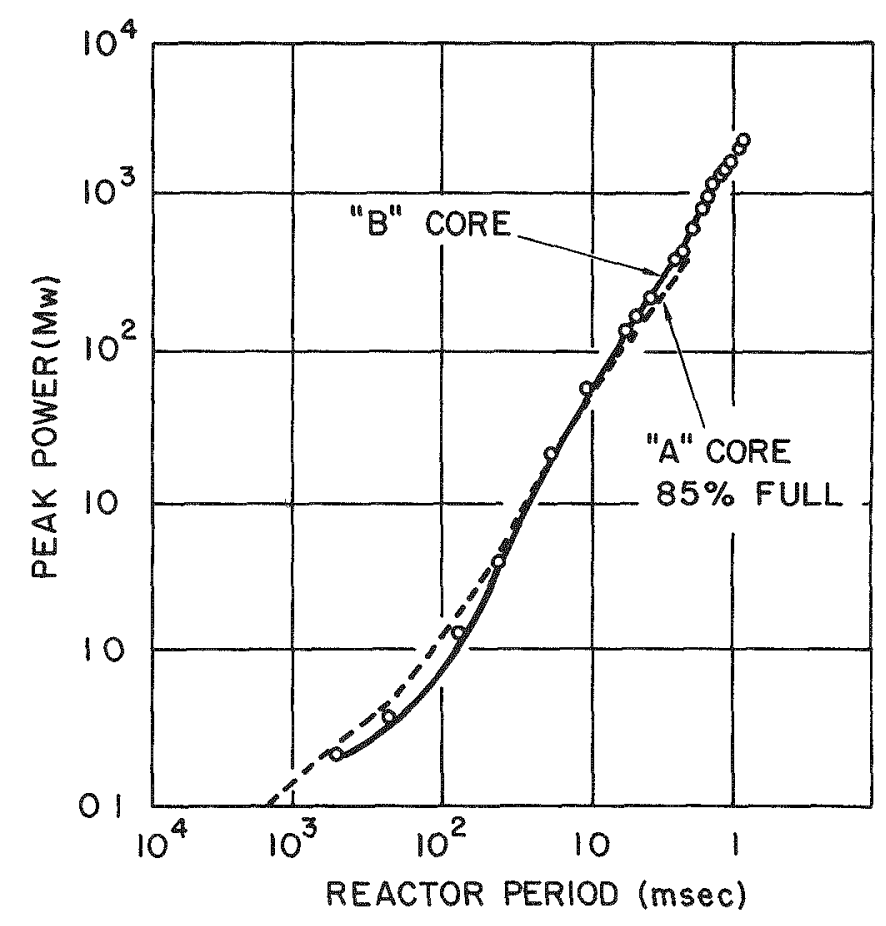

Slide 3. Maximum Reactor Power vs Reactor Period - Kewb

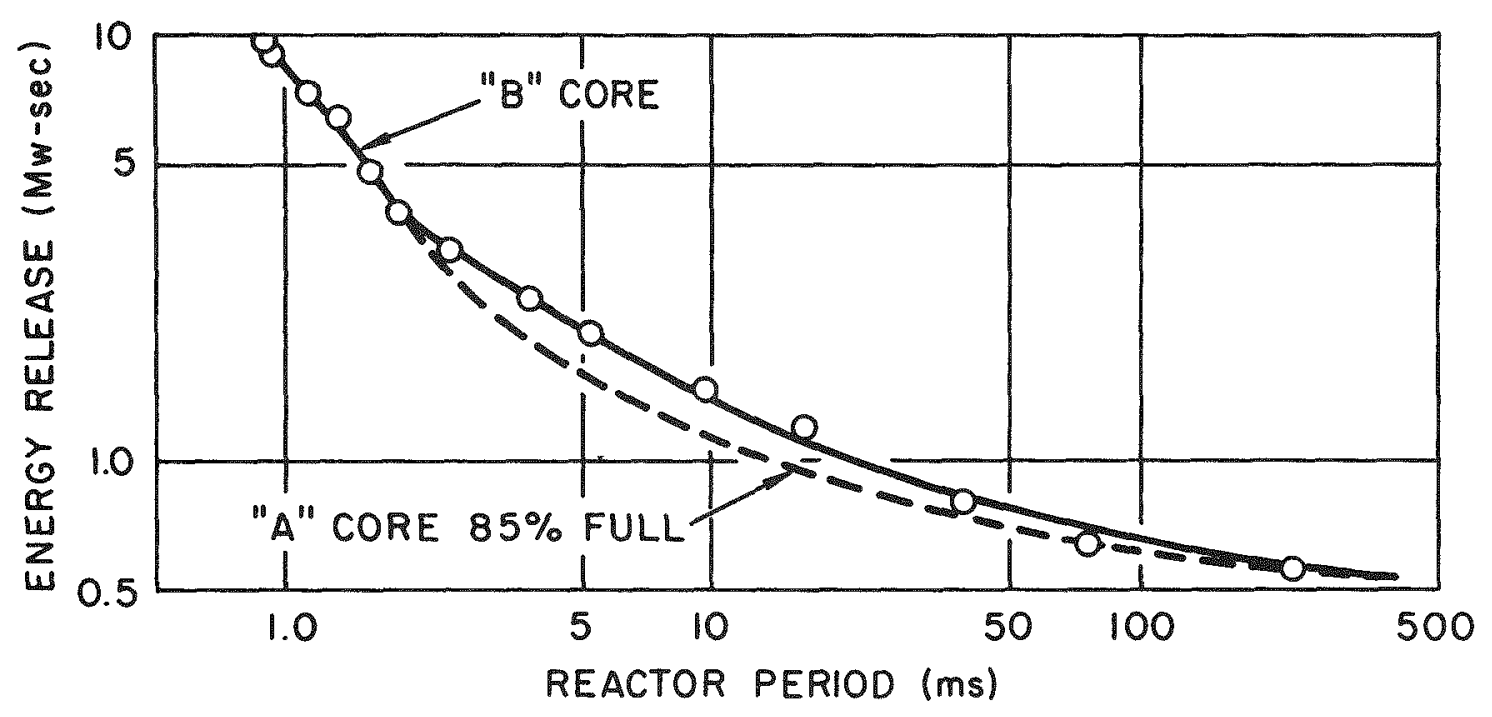

Slide 4. Energy to Time of Power Peak vs Reactor Period - Kewb 
the comparison of the " $A$ " core results with the recent " $B$ " core results down to the 0.9 millisecond period. Again, there is not mich effect from the difference in geometries. Slide 5 shows a comparison of the peak pressures in the two cores as functions of the reactor period. As I indicated, there are two reactivity feedback mechanisms which control the transients. One of these is the prompt temperature coefficient which gives a thermal expansion of the solution; the other one is the production of radiolytic gases, which displace the solution. Concident with the rapid production of the radiolytic gas and displacement of the liquid solution is the buildup of pressure in the core vessel. Two transient pressures are actually observed-one we call the expansion pressure and one we call an impact pressure. The expansion pressure is one that is measured with a transducer located at the bottom of the reactor vessel. In the "A" core the impact pressure is detected with a transducer located at the top of the sphere above the level of the fuel solution, and hence no indication is received until the fuel solution is expelled from the core. Thus the impact pressure is the result of the fuel solution striking the face of the transducer. In the " $B$ " core the impact pressure is detected by a transducer mounted on a deflection dome inside the vessel about 12 inches above the fuel solution surface. Both the pressures in the " $B$ " core are noted to be somewhat less than those in the "A" core for a given reactor period because of the larger free-surface area of the fuel solution.

In Slide 6 some results with ramp inputs of reactivity are shown, and I have included this to illustrate a point that schroeder has already

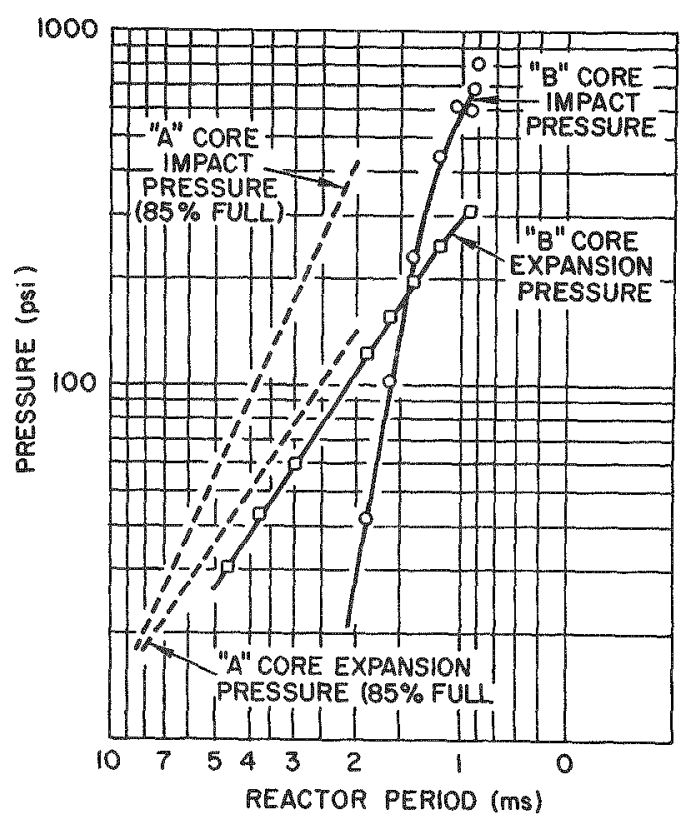

Slide 5. Comparison of Kewb Pressure vs Reactor

Period Data

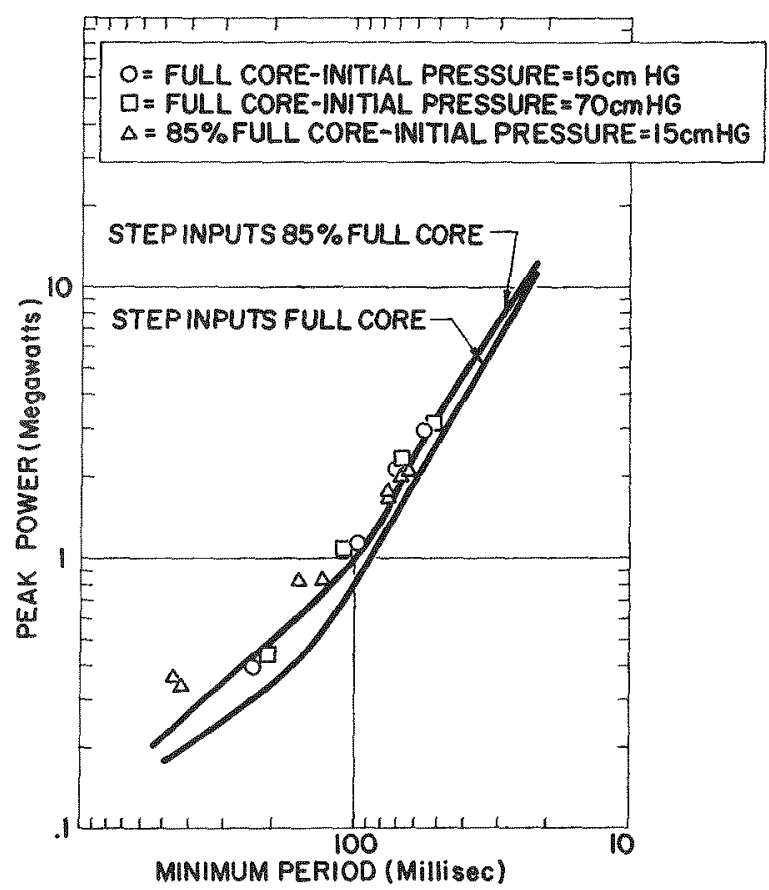

Siide 6. Maximum Power vs Minimum Period Data for Ramp Reactivity Insertions in Kewb Compared to step Inputs 
made: that the dynamic behavior with a ramp input is characterized by the minimum reactor period reached during the ramp. That is, a given ramp rate of reactivity input results in a specific minimum reactor period, and the transient reactor behavior is the same as that one would get from a step reactivity input giving a period equal to the minimum period during the ramp, as shown by the comparison of the ramp and step inputs on the slide.

Now, so much for these experimental results; let us now ask what these mean. As has been indicated, these have given a demonstration of the inherent shutdown capability. We have a coupling model that Frank has mentioned which apparently describes the behavior of the " $A$ "core system rather well, but we might ask the question--do we really understand what is taking place? We know there are two feedback mechanisms, the temperature coefficient due to the heating of the fuel solution by the fission process and the production of the radiolytic gas. As Schroeder gave, we have determined the reactivity compensated at peak power and knowing the temperature coefficient of reactivity have subtracted reactivity that is compensated at the peak by temperature. The rest of the compensated reactivity is then ascribed to the production of gas with the consequent increase in volume of fuel solution. The problem was then to relate this compensated reactivity to a proper description of the void formation by the radiolytic gas. Attempts to do this appeared to work fairly well until we observed what appeared to be an anomaly in the burst shape at short reactor periods with the "A" core. This anomaly became much more predominant when we carried the cylindrical core transients down to periods of less than a millisecond. If one plots the width at half-heighth of the transient power pulse as a function of reactor period, measured in reactor periods, one finds the width goes through a maximum at a reactor period of about 150 to 200 milliseconds--this is about prompt critical--with the width at half-height equal to about five periods. It then decreases with decreasing reactor period down to reactor periods of about 5 to 7 msec, at which the width at half-height is about two reactor periods. The width then starts to increase again, reaching a value of about $3-1 / 2$ periods at a reactor period of about a millisecond, indicating that there is something at these shorter periods preventing the very sharp cut-off of the power burst on the trailing edge. Normaliy the predominant shutdown mechanism at short periods is the gas formation and the major portion of it is produced after sufficient reactivity has been compensated to stop the power rise; i.e., after the transient power peak. This results in a very large negative reactivity swing and a very sharp cut-off in reactor power, until we get to the shorter periods below about $5 \mathrm{msec}$. Looking at this more closely we have ascribed this behavior to the reflector effects that Dr. McCullough mentioned this morning.

In SIide 7, the top curve is the theoretical inhour curve with the six-group delayed neutrons and with a prompt neutron lifetime in the reactor of $60-1 / 2$ microseconds. The experimental points represent the actual results obtained during transients with the " $A$ " core with both the full and underfull sphere, indicating that we do have a contributor to the inhour relation, in addition to the six groups of delayed neutrons. 


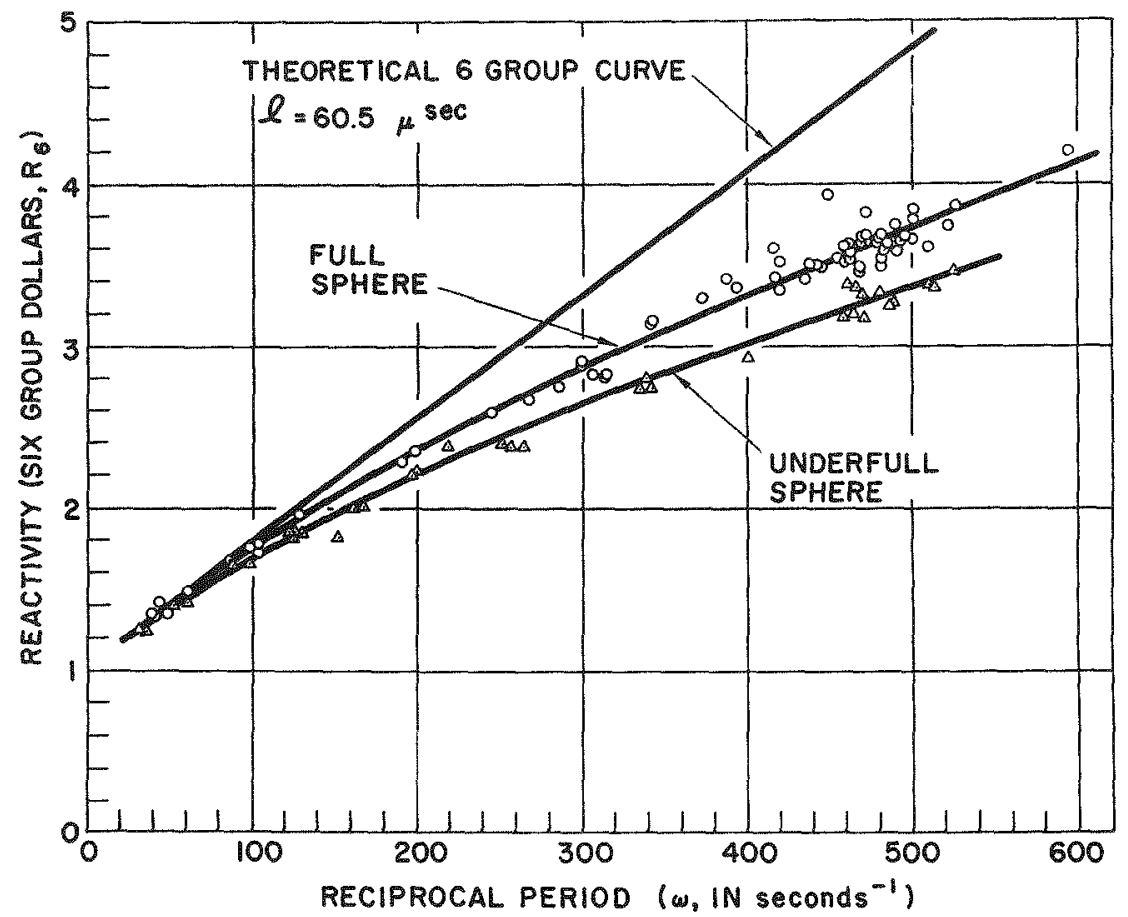

\section{SI1de 7. Kewb Inhour Curves}

The reactivity input for the transients is taken from control rod calibrations made with very small reactivity perturbations where period vs reactivity is determined almost solely by the delayed neutrons. These results show that the effect of the additional contributor appears to increase as the reactor period is shortened. You notice that these results are for reactor reciprocal periods of up to about 500 reciprocal seconds (reactor periods down to about 2 msec). Results with the " $B$ " core show the effect to become even larger when we get down to the l-msec region, so that in determining the amount of reactivity compensated at peak power, we are faced with the question of how much reactivity is attributable to what we have called a "seventh delay group". We have been able to ascribe to this seventh delay group the characteristics of a mean lifetime of about 2 msec and a fraction of about 2.3 per cent. Using these values in the kinetics equations we can get a fit to the experimentally observed inhour relationship. Now, one of the probrems that remains is using this to obtain the reactivity compensated at peak power and then determine how much of the reactivity compensation is due to gas formation.

So we have a demonstration of the dynamic behavior and the inherent shutdown characteristics of the aqueous homogeneous reactor. We have a model, a coupling model, which appears to describe the system behavior in the longer period regions. We have uncovered another factor which plays an important role in the very short period behavior. This is the "seventh delay group", which is in addition to the temperature coefficient of reactivity and the gas production. Now a further question that we need to answer is how much gas void is actually produced in the system and the 
rate at which the void is produced. This we cannot measure directly in the integral reactor experiment so we are now doing a capsule experiment in which an initially void-free capsule filled with fuel solution is located adjacent to the reactor core and is pulsed with the neutrons from the reactor pulse. The volume change in the capsule is measured as a function of time during the pulse, and this will be related to the energy deposition in the capsule. This result will then be used to determine the time-dependent void produced in the core, which can be related to the reactivity compensation in the core during the pulse, and to the energy coefficient of reactivity which Frank mentioned earlier. Also, we should get information to lead to better understanding of the actual mechanism of void formation in the fuel solution.

I think our results point up that in a system we originally thought was a simple system the integral reactor experiment alone is not going to give us a complete basic understanding of what is taking place in the system. We must turn to something more than just the integral reactor experiment, such as the capsule experiment we are now doing, in order to really understand the kinetic behavior and shutdown characteristics of the reactor.

Stone: Thank you, very much. 
SESSION II

PANEI PRESENTATTONS

Stone: I would like to now call on Warren Nyer, who will talk on the Spert program.

\author{
Presentation of Spert Program \\ by \\ W. E. Nyer (PPCO)
}

Nyer: I only have a few comments to add, since a fair amount of time has already been spent on spert results. I thought I would mention one thing about the effects of pressure on excursion behavior in order to go just a little bit beyond what Frank Schroeder mentioned. This is to bring out some of the background considerations. For some time, I think most of us have felt that it was important to have boiling take place to assist in the self-limiting of bursts and there was some concern as to what would happen if, at elevated pressures, the boiling was completely suppressed. I think that the one thing that came out of these experiments is that we need not have any great fear of this, at least as far as we were able to determine from the limited number of preliminary measurements made so far. There was no great increase in the energy released--we are talking about increases of the order of a factor of 2 .

There is a rationale for this behavior that I think should be discussed briefly. The shutdown effects in Spert take place by a variety of mechanisms. If one of them--in particular the boiling--is suppressed, we don't do anything about slowing down the energy release. We still have the energy going somewhere and it must go into one of the other shutdowns--in this case, perhaps just direct water-heating. Thus, there is some compensation that takes place when one mechanism is lost and apparently because of this, the loss of boiling as a shutdown mechanism is not really a serious thing.

One other point ought to be mentioned in the matter of bringing you up to date on our recent results. Some experiments have been conducted in Spert III on the effect of flow on self-limitation. Again, there is some concern that with relatively high flow rates, say, of the order of 18 feet a second through the core, some of the heated water or some of the voids that are formed during an excursion will be washed out of the core with the result that the effective shutdown coefficient is diminished. Therefore, we would expect a larger energy release. Well, the first effect that we saw in this case was that there was not much of a change in the peak powers in the bursts. There was indeed an increase in the total energy release, which means that there had to be a change in the burst shape in order for both of those things to be true. Since the burst shape did change, this doesn't introduce any surprising behavior. However, one other thing that did occur was that the peak temperatures were less in the case of fuIl flow than they were without flow even though the energy release had gone up. So the inference one makes from this is that we have improved the transfer of energy from the fuel plates to the 
water, and the net effect is to increase safety rather than to reduce it. I think you should be cautioned that this is still somewhat early data and we might have some question about the meaning of the temperature measurements.

One other general thing not related specifically to Spert work could be mentioned briefly. I feel we have somewhat of an obligation to try to provide people with rules of thumb that can be useful in formulating criteria safety. Now there is a danger mentioned by Rogers this morning that perhaps if such things are too simple and too easy to memorize, they will in themselves be restrictive rather than a help. One example of what I think we should strive for in this direction can be pointed out by coming back to the discussion of the sourceless startup. As has been mentioned earlier, the theoretical problem here is really very difficult. I don't think we have mentioned that there is also an experimental problem that is a little bit difficult. The experimental problem is to figure out a really meaningful experiment that can be done relatively easily that will give a lot of information on the statistical problem. In spite of these difficulties, I think we can say something useful. If we have a very low source rate, or very low rate at which neutrons appear in the system, we can establish this kind of a condition: during the time that it takes for a neutron to appear in order to start the chain, the reactivity addition rates should not have been so high that we have added more reactivity than the system can stand. Now this really is only a necessary condition and not a sufficient condition. That is, if the source is higher than given by this rule, we haven't guaranteed that we have a safe system because we have't taken into account the probability problem. On the other hand, if the source is less than this rate, clearly the system is unsafe, so that we have a means of specifying as a limiting form either the rate of reactivity or the source level. I think that the general thought I would like to leave everybody with is that examples like this are useful and we should try to express our results in such simple formulas because they are easy to apply. I should like some comment from $\mathrm{Dr}$. McCullough on this because of his remarks this morning. (To McCullough) Do you agree that this is the sort of thing we should strive for, or are you indeed afraid as mentioned earlier this morning that this might be a misleading thing? Maybe we will get a comment from you later. I would like to add one other thing brought up in Marlin's discussion. We are in agreement that to really understand the mechanisms that are responsible for shutdown, we have to go to something other than the integral experiments and we, too, for some time, have had some capsule experiments under way which some of our people here can discuss later if there is interest in that. I would like to direct a question to Marlin on this: I understand that there may be some work with organics--is this correct? If anything can be said about it, I would be very interested in hearing it, and I am sure everybody else would too.

Remley: We have started a program on the void formation in organics-transient void formation. This is a program that has just gotten started. It will be a capsule program in which we look at the formation of voids in capsules of the type we are using now with Kewb fuel solutions. In this case it will be organics and heated fuel elements, that is, pieces 
of fuel elements that will be heated with Kewb as a driver. This program is currently in its initial stages and we don't have any results on it. I have high hopes for it because as is indicated, I feel that it is this type of experiment that is actually somewhat analogous to what Dr. McCullough mentioned this morning--what Mark Mils had referred to as a capsule, modular or cellular experiment that can be much more economical than an integral experiment and may give us a real insight into the basic mechanisms and a basic understanding of what is taking place so that we can develop a model, approaching first principles, if you will, that will lead to real understanding and really improved design changes. 
Stone: I would like now to call on E. P. Epler of Oak Ridge National Laboratory.

$$
\begin{gathered}
\text { Presentation of ORNL Program } \\
\text { by } \\
\text { E. P. Epler (ORNL) }
\end{gathered}
$$

Epler: I should like to apologize for injecting a discordant note into this otherwise smooth program. I'm not sure that I belong in this part of the program because what I plan to talk about, although not necessarily hardware, is the consequence of the application of hardware. I have one slide for you to be looking at, and I will describe the electromechanical safety applications to the BSR-II core in Spert. Now, just a few things about the core and about the safety system. The core is a 15-in. stainless steel cube. The mean neutron lifetime is about $25 \mu \mathrm{sec}$. The safety system that was applied to this was one in use for many years with very little alteration. The principal alteration was the application of a spring which worked over a stroke of approximately a couple of inches in order to have initial acceleration which amounted to about 6-G. I would like to reiterate that otherwise the system is the system in use for many years and the response was not made better for this test.

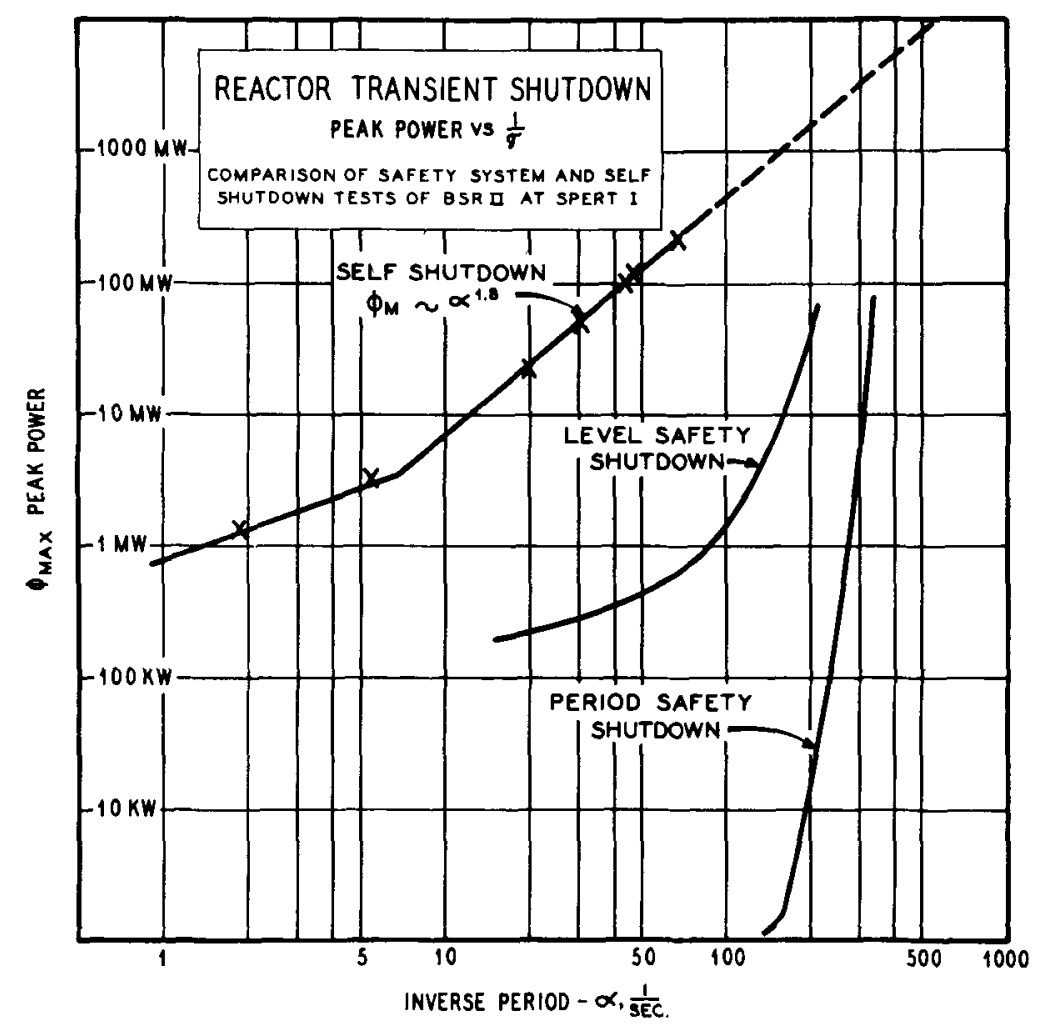

Slide 1. Maximum Reactor Power vs Reciprocal Period BSR-II Core 
Now the first curve is the usual self-shutdown curve which proceeded to--I think that last point was about a $13-\mathrm{msec}$ period on the self-shutdown series of excursions at which point the core starts to show some evidence of wrinkling. As we had some doubt as to whether we could replace the core, the tests had to be discontinued at that time. The next test series then, was conducted with the electromechanical safeties set so that the scram would occur at slightly above $100 \mathrm{kw}$, this being established as the arbitrary power rating of the core for this test. Now this is about the same level at which temperature becomes significant in the self-shutdown effect. So the next series was run with the electromechanical safeties and the tests were continued to a period of about $5 \mathrm{msec}$ at which time we again arrived at a peak power of $100 \mathrm{Mw}$ and were forced to discontinue the tests again.

Now at this point, we connected up the period scram. Now we were particularly interested in the effect of the period protection because there is some question as to what the response of the period meter is at extremely short periods. Our calculations showed that we should expect the period meter to respond so as to give us an advantage of about four decades over the level safeties on periods shorter than 10 msec. We proceeded with the test and again approached the limiting condition of $100 \mathrm{Mw}$ and at that time had attained a period of $3 \mathrm{msec}$.

Now the question has been raised as to whether it pays to put in these expensive shutdown devices when obviously, if you extrapolate these curves, you will see that the self-shutdown effect affords protection for shorter periods than can be handled by the safeties. In evaluating the relative merits of the self-shutdown effect and the period safety trip on the basis of reactor period, it is readily apparent that the self-shutdown will protect against the shorter period. The period safety, however, will prevent the development of short periods unless the accident mechanism produces a ramp substantially shorter than that produced by the burst mechanism used in this experiment. I would like to say, first, that the first two curves were conducted with a conventional source in place. This source was the kind and strength that is usually used in order to have an appreciable count rate at the time you begin to pull rods in starting up a reactor. Now for the burst mechanism that we used to produce the reactor excursions for these first curves and for all of them, as a matter of fact, we took an ordinary doubleended safety rod and just turned it upside down and, at the same time we pulled out poison, we threw in fuel. We accelerated it in with a net acceleration of about $6-G^{\prime} s$ and we got the reactor subcritical so we could get a good start before this thing hit. Now I would like to call that a sort of a modular accident. I think it's a rather severe accident. There are accidents, I think in nature that could approach this, like for example, a fuel element falling into the center of the core and being driven in by full water flow would, I think, approximate this accident. Now the interesting thing is that in this last series of tests we were forced to remove the source; otherwise, we could get no data. We were able to get data on the level safety protection because it occurred at $100 \mathrm{kw}$, that is, the scram point was $100 \mathrm{kw}$. Since the scram point was approximately four decades below this in the case of the period meter protection, we were unable to get the burst rod fully inserted and get all the reactivity in, in the allotted time which was about 75 msec. 
Therefore, it was necessary to remove the source and sit around and wait for a time, like up to a second or three-quarters of a second, at which time the burst occurred. Now this is a step insertion of reactivity.

Now, nature doesn't ordinarily provide such steps so I would like to compare the step or ramp in reactivity that nature provides with the kind that we put in with our safety system. I think some work should be done on that and as I said, I would like to speak in rather general terms, and I think that it is indicated that those who are responsible for reactor safety should ask themselves, does any critical accident to this core develop any more rapidly than the method for taking away the reactivity that I can attain with my safety system providing only that I have a source, and providing that the delay, if delay enters into the response of the safety system, is reasonable. Now in this test we had a delay which we had intended to be around $8 \mathrm{msec}$. So we have results here just a little bit better than we had intended. We should have had, for these very short periods, a rise of, perhaps, another e-factor or so.

Now it has been said that any electromechanical safety system incorporates a delay. This delay is the time required for the safety rods to take out the reactivity which, first, is only the amount in excess of beta. The accident must put in first beta then this same excess so the safety system is required to take out a smaller amount. Secondly, there are $\mathbb{N}$ safety rods taking out this reactivity wherein, in this simulated accident, one rod is putting it in, and then again there is a considerable difference in the time allotted for putting in the accident and for taking it out. If you use the period meter, and this trips at low levels you do have a considerable amount of time for the excursion to proceed, over, maybe, up to 10 decades during which time the safety rods are being inserted. This compares to a rise of six decades at most from source level to period trip level during which time the accident must develop. Now, I think in answer to Frank Schroeder's question as to whether this is economical--I would like to ask, first, is it economical to put in a safety system which is admittedly expensive and which should have the capability of this performance, but which does not attain it for reasons which have not been examined.

Stone: I think there might be just a little confusion on the concluding statement, so why don't we entertain a question at this point. Shall we? Frank, would you like to question this statement?

Schroeder: I think I know what Epler is driving at but I'm not sure I'm the proper one to ask the question. As I interpret your remarks, you are concerned with the fact that many expensive safety systems do not perform as this one performs. Is that correct?

Epler: Yes, I would say that if you were to have this performance, the delay must be kept in bounds, that is, the time for magnet decay preceeding release of the rods. Now, as you can see, that if you equate the time for the accident to grow with the time for the safety system to take out this excess reactivity, the only remaining delay must be the magnet 
decay or it is substantially the principal delay. Now, if you have used as a criterion for your systems that the delay is $200 \mathrm{msec}$ in order that you can use a contact-making ammeter then you will have no success with a 3-msec period. If you have used as your criterion a delay associated with some equipment which you can buy off the shelf, which may be 50 msec, you will not cope with a 3-msec period. You have spent money but you have not been able to handle the 3 -msec period. Now, furthermore, I will point out that you cannot expect to experience--a 3-msec period--with any reasonable accident mechanism if you have a source. Reactivity is added but it cannot develop a short period.

McCullough: If you have a source of how much?

Epler: Some work should be done in examining the maximum rates at which various accidents can develop. This will establish the importance of source strength and the level at which period trip will occur. It has not occurred to me until recently how important this has turned out to be. 
Stone: I would now like to go in the direction of the solid homogeneous reactors, and the first transition case in its deyelopment would be the Triga reactor which is a solid homogeneous fuel and some water moderation. Ralph Stahl of General Atomics.

\section{Presentation of Triga Program \\ by \\ R. H. Stah] (GA)}

Stahl: I will take just a very few minutes to talk about some data recently obtained with the Triga Mark F reactor which, in general, confirms many of the things that Schroeder talked about earlier. This is a second Triga reactor using the same fuel elements in the same array; in other words, it is the quasi-homogeneous or semi-inhomogeneous reactor, as the case may be, a reactor using uranium-zirconium hydride fuel elements of an inch and a half in diameter, and sitting in a tank of water. The same fuel arrangement is used for both reactors, the Triga Mark-I reactor has a graphite reflector and the new Triga Mark $F$ reactor has a water reflector. The motivation in doing these

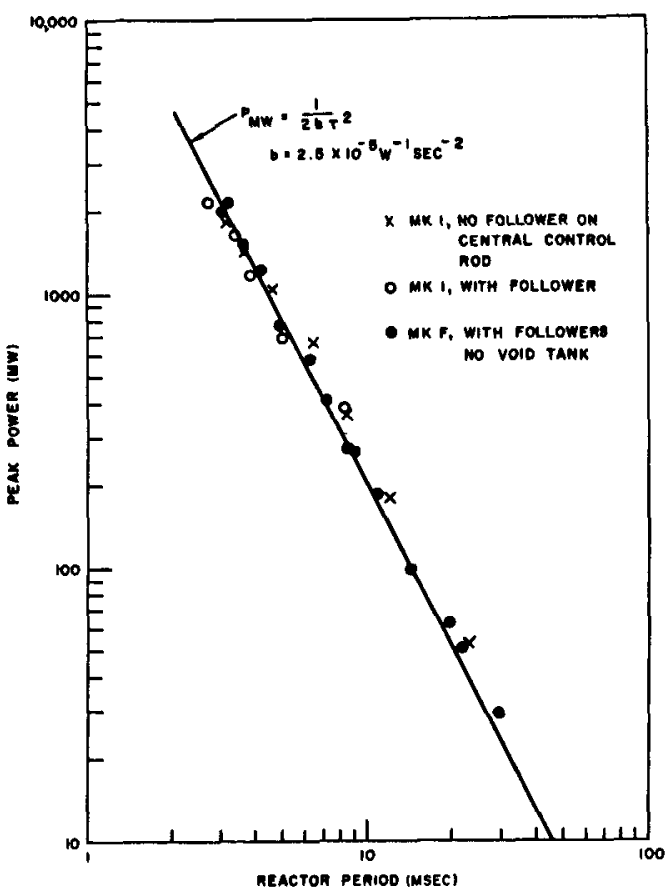

Slide 1. Peak Power vs Period During Triga Transients experiments was that we sold a reactor of this type as a pulsing reactor for two laboratories in Washington and we wanted to make sure that they performed as expected. Slide 1 summarizes the period--peak power relationship for these various reactors--the Mark-I reactor has a graphite reflector, Mark $F$ is the waterreflected reactor. The followers mentioned in the slide are followers on the central control rod which we use to flatten the flux and decrease the flux gradient at the center. It is seen that both reactors, for miraculous reasons, behave the same way. Furthermore, this thing is, as I surely indicated, a simple device; it's a Fuchs model reactor; it has a shutdown coefficient proportional to the energy release and also-as a later slide will show--puts out symmetrical pulses. The shutdown coefficient is shown in the 
slide. It depends on the temperature coefficient which, of course, is the mechanism of shutdown, and the lifetime and the effective heat capacity of the core. The water-reflected reactor has a shorter lifetime and a larger effective heat capacity and critical mass so that the shutdown coefficients of the two reactors turn out to be identical. Slide 2 is essentially the same data. We are interested in fuel temperature for this reactor and we step up to $500^{\circ} \mathrm{C}$, which is the upper limit for this particular fuel mixture for reasons of a phase transition which occurs at about $530^{\circ} \mathrm{C}$.

Slide 3 shows a comparison of a pulse width and measured period for various size pulses and shows that the shape of the pulse is constant--in other words, the shutdown mechanism stays constant. The period-pulse width relationship is just about what you would expect from a Fuchs model--the relationship is a wee bit narrower. So that, in agreement with Schroeder's review, we have a reactor which has a simple shutdown mechant.sm. The fuel temperature coefficient is, of course, something which you can measure on equilibrium experiments, also. We've done it on quite a few other experiments having to do with dosage available from such a reactor, which is probably not of interest to this group, and additional metallurgical experiments having to do with the effects of large numbers of transients on fuel elements. We had earlier run into fuel element ratcheting after hundreds of pulses and by making modifications on the cladding--a clad

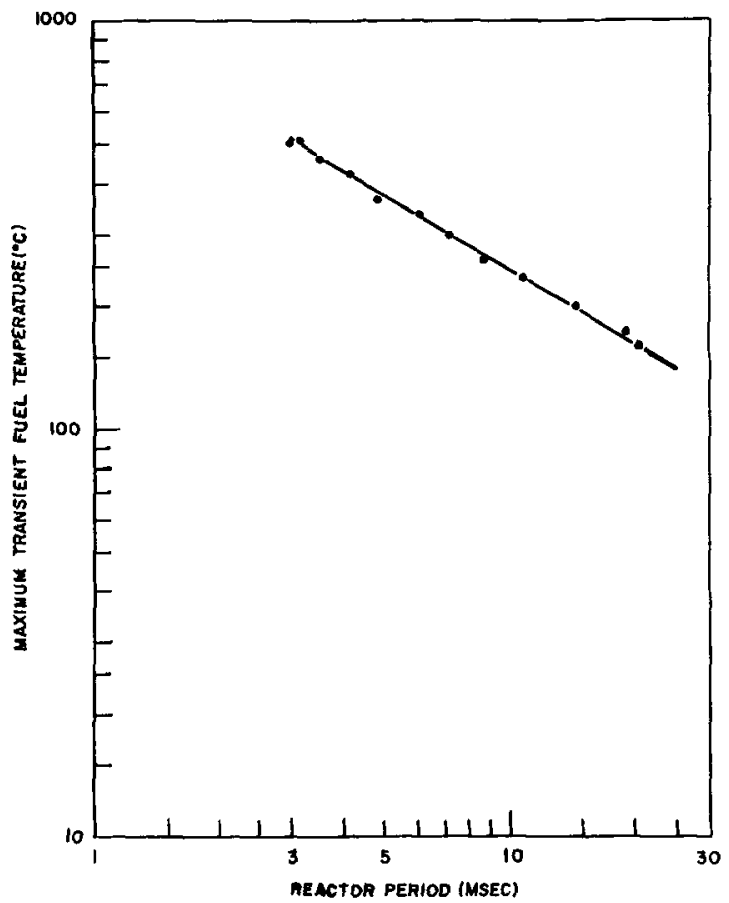

Slide 2. Maximum Central Fuel Temperature During One-Second Transients--Triga Mark $F$

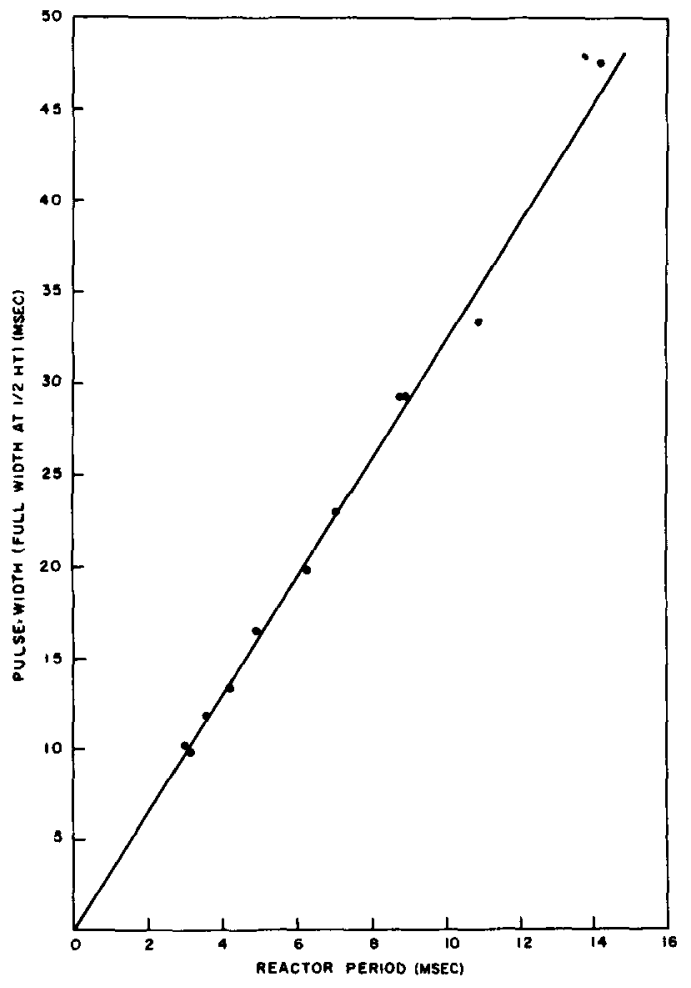

Slide 3. Experimental Period Pulse Width Relationship-Triga Mark F 
with aluminum with a non-metallurgical bond--we seem to have improved the behavior of the fuel elements to the extent that after the latest series of about 500 transients, there are no visible changes. It has been our experience that if you increase the fuel temperature above the phase transition region--above $530^{\circ} \mathrm{C}-$-that a certain amount of bending of the fuel element takes place, although this does not seem to alter the shutdown characteristics of the reactor. If you people have questions on the metallurgical experiments or the dosage measurements, you can ask them in a later question period. 


\title{
SESSTON II
}

\section{PANEL PRESENTATIONS}

Stone: Thank you, very much, Ralph. Now proceeding further in the direction of homogeneous reactors, I should like to call on Tom Wimett of Los Alamos.

\author{
Presentation of Godiva Program \\ by \\ T. F. Wimett (LASL)
}

Wimett: I propose to discuss a few results of recent measurements on Godiva II which might be of interest to this group. Godiva II, as you probably know, is an improved version of the old spherical godiva. It is cylindrical in shape, has a hollow cylindrical center cavity which accommodates the safety block instead of having a large parting plane as the old Godiva had. We now have two models of Godiva II, as a matter of fact. The first model is nickel-clad and the second one has cadmium plating over the nickel. An interesting feature of the nickel-clad Godiva, as also of Godiva I, is that we observed additional delayed neutron groups very much as Mr. Remley pointed out, except that in our case they were from room return. The neutrons scatter back from the room--in fact, one could represent the room return by two delayed neutron groups, each having less than a percentage abundance. This is in a particular room, of course, about 50 feet, I believe, by 30 , something like that--15 or 20 feet high. The interesting thing is that one group appeared to be in the 50 microsecond region and was therefore, fairly important in its affect on the shutdown mechanism. In bursts around prompt critical its effect was to increase the peak power by a factor of 2 .

I think one experiment that may be of interest to people here--I know that warren Nyer asked us a couple of years ago if we would perform this experiment--is a sub-prompt-critical excursion in which the reactivity was increased stepwise to something under prompt critical. Slide 1 shows three oscillograms of typical bursts obtained in Godiva II. I would like to call your attention to the bottom one, for which the reactor period was about a quarter of a second, which for Godiva is extremely long. You will see that the power level rises on the quarter-second period and levels off at about a half megawatt, and notice the time scale here--2 seconds-we scrammed it by a delayed type scram at the end of 2 seconds, so you see that this is an interesting mode of operation and I think it's probably of interest to the reactor hazards people. We have done this over quite a region and it always levels off at about half a megawatt, as one can predict on the basis of the ordinary kinetics equations. The temperature rise produced by this burst, for example, is about $150^{\circ} \mathrm{C}$ in Godiva. So we can't operate Godiva as a power reactor at this level for too long.

I would like to just mention briefly that the shutdown coefficient, the epsilon, for example, which Mr. Schroeder mentioned, is reduced by about a factor of 2 in Godiva II, because of a gap that exists between 


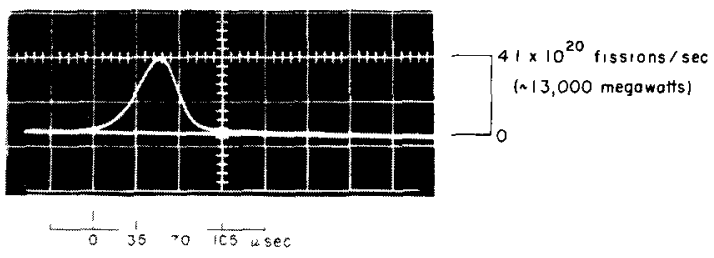

Oscillogram A

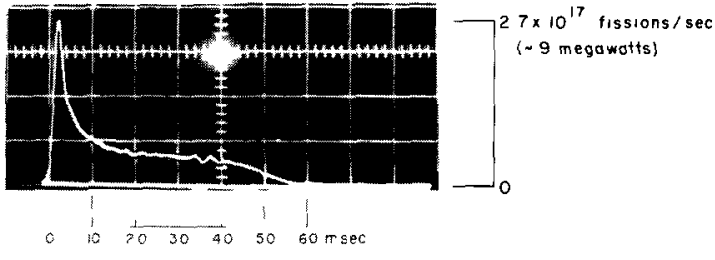

Oscillogram B

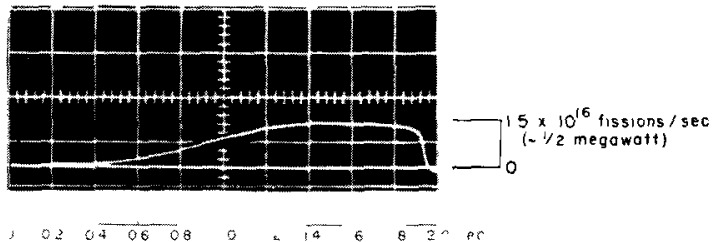

Oscillogram C
Slide 1.

Typical Godiva II

Power Bursts

the safety block at the center and the wall of that cavity. Thus, a plot of $\mathrm{Mr}$. Schroeder's epsilon for Godiva II would be more of a flat curve than he showed for Godiva I. If you recall the left-hand side was raised-that part would be lowered so that that curve would be more or less flat; and inertia, or the time for a thermal expansion to take place has been reduced because of the subdividing of material. In other words, the time for pressure waves to travel through any piece of metal in the system is now reduced so that we feel that we can go to higher yield bursts with Godiva II. We haven't done it because we've limited ourselves arbitrarily to about $100^{\circ} \mathrm{C}$ temperature rises. I think it would be very interesting to take the device to destruction, but there is a lot of material, $57 \mathrm{~kg}$ of enriched uranium 235. I don't know if we will be able to do this or not.

The other experiment that I think would be most important here has to do with startup with no source. Generally we operate Godiva this way, but only because we know what the final reactivity is in most cases. As you know, for the last burst in Godiva I, we didn't. Slide 2 shows a burst-wait-time distribution which we obtained under this condition. As you know, to get at this problem of the hazard of startup with no source-it's essentially a problem of the statistical distribution of the startup of chains; having to do with distribution between the initiation of persistent chains and finite chains, and as it shows up as a fluctuation in time at which a given power level is reached, this turns out to be convenient to measure in the super-prompt critical region of Godiva II a and that's what we did here. We simply measured this wait time, from the time of assembly to about $5 \phi$ over prompt critical, until the time of 


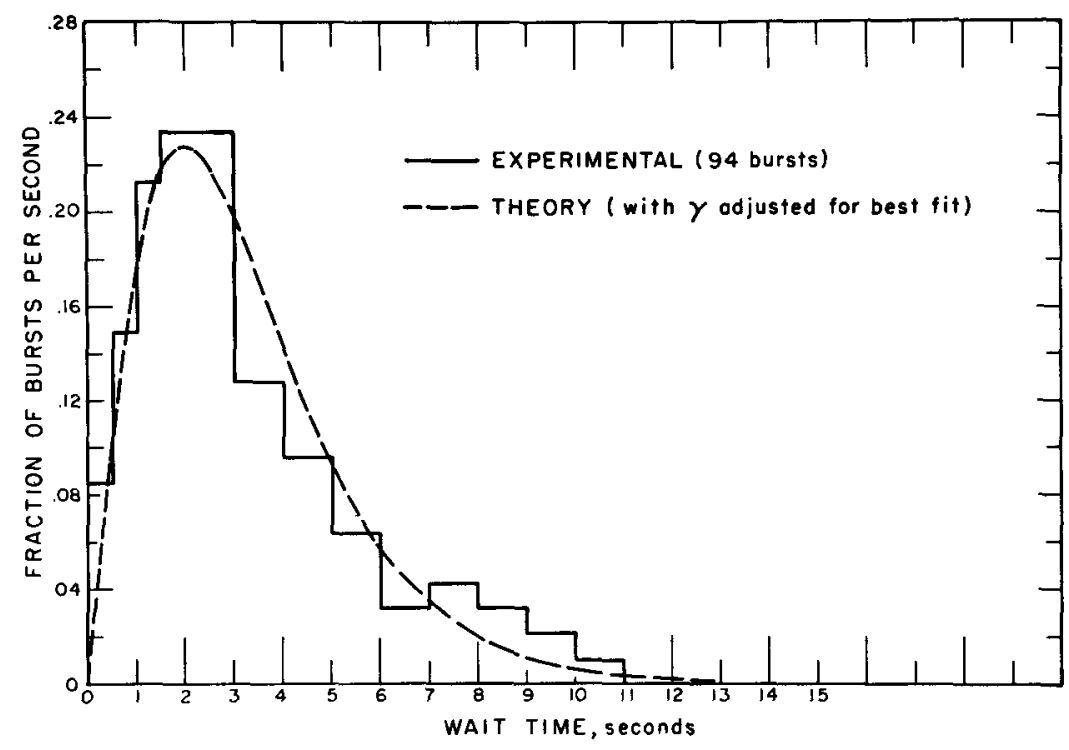

Slide 2. Godiva II Burst-Wait-Time Distribution

occurrence of the burst and on the basis of measurements of 94 bursts, we obtained this function. The ordinate is the fraction of bursts per second or the distribution of bursts on a per second basis, and the interesting thing is that it tends to start toward the origin. For example, the first measurement, from zero to one-half second, included four bursts, as I remember. In two of the four bursts the delay was under a half second. The data definitely shows a peak around 2 seconds. The average wait time is about 3 seconds. The dotted curve was calculated on the basis of Gordon Hansen's theory, which I believe, he presented to this group a couple of years ago, in which he considers the fluctuation of the initiation of persistent chains and also the additional fluctuation in the growth of the chains to a prescribed power level. It turns out if one considers only the fluctuation of the initiation of chains, this would be just a negative exponential--it would be maximum at the origin. The fluctuation in the growth tends to broaden it and reduce its amplitude at the origin, oddly enough. I think that's about enough to say about this.

We also ran another series of measurements of fluctuations and wait time in the sub-prompt critical region where the period was about 1.76 seconds. For these measurements the initiation was again stepwise and the time was measured to the time when the power level reached a given value, somewhat like an experiment mentioned earlier. For that case, the fiducial power level was about $3 \times 1011$ fissions per second. The measured mean square deviation, known as

$$
\left(\overline{\mathrm{T}^{2}}-\overline{\mathrm{T}}^{2}\right)
$$


was 21 seconds. In other words, data from 22 runs spread from about 44 seconds. The source in this case is spontaneous fission--about 90 neutrons a second, so you can see that is quite a fluctuation. The mean square deviation is twice that predicted on the basis of source initiation alone. So we feel that there are many more measurements that can be made of these time fluctuations and we have in mind an experiment to perform the same sort of experiment with ramp reactivity changes which, I think, would be more appropriate to this field. 


\title{
SESSION II
}

\section{PAINEL PRESENTATIONS}

Stone: In conclusion of the panel discussion, I would like to call on Jim Boland from the Argonne National Laboratory to speak on the Treat Reactor Program.

\author{
Presentation of Treat Program \\ by \\ J. F. Boland (ANL)
}

Boland: Since Treat is probably the only reactor which has been designed specifically to study the meltdown phenomena of reactor fuel elements, I think the data from the experiments as well as reactor operating data will be of interest to this group. Therefore, I would like to briefly review three aspects of the Treat program which I hope will be of interest to you. First is reactor operation and characteristics; second is the Treat program on fast reactor fuel elements being conducted by the Fast Reactor Safety Section of our Reactor Engineering Division; thirdly, the metal-water reaction program being conducted by our Chemical Engineering Division. Robert Liimatainen and Richard Ivins of this division will present a paper(I) at the 1960 ANS winter meeting on the results of their experiments, so I will not cover them in detail here; just indicate the nature of the experiments and a few interesting results.

First, coming to the reactor, the reactor has operated on transients which have reached peak powers of $3000 \mathrm{Mw}$ with integrated energy releases up to $900 \mathrm{Mw}-\mathrm{sec}$. This is one or two orders of magnitude above anything reported on the other transient reactors described previously. The shutdown mechanism, as observed, agrees well with the calculated model of moderator heating. It should be very prompt and we have no indication that it is appreciably delayed. Our shortest period to date has been about $45 \mathrm{msec}$. The results of the initial physics measurements in Treat and the supporting theoretical studies will be published shortly in two reports: ANL-6173, entitled Reactor Physics Measurements in Treat; and ANI-6174, entitled The Kinetics of Treat. Therefore, I will not go into the characteristics of the reactor which have been seported at other meetings and which will be available very shortly in report form.

Recently we have conducted transients in which we attempted to hold the reactor power at a constant value for several seconds, one to twentyfive seconds, after the negative temperature coefficient would have shut it down had we allowed the transient to proceed in the normal manner. This was done by withdrawing a control rod, to introduce positive reactivity. Slide 1 shows the shape of the so-called flat-top or constant power transient. If we miss on the initiating reactivity, compared to the ramp rate we have selected, our power will peak and then come back down and level off at some value. The transient shown was terminated by a scram after 6 seconds and it could have extended considerably longer if we had wanted a greater energy release. Notice, that this transient reached a peak power of around $68 \mathrm{Mw}$; integrated power, $113 \mathrm{Mw}-\mathrm{sec}$. A normal temperature- 


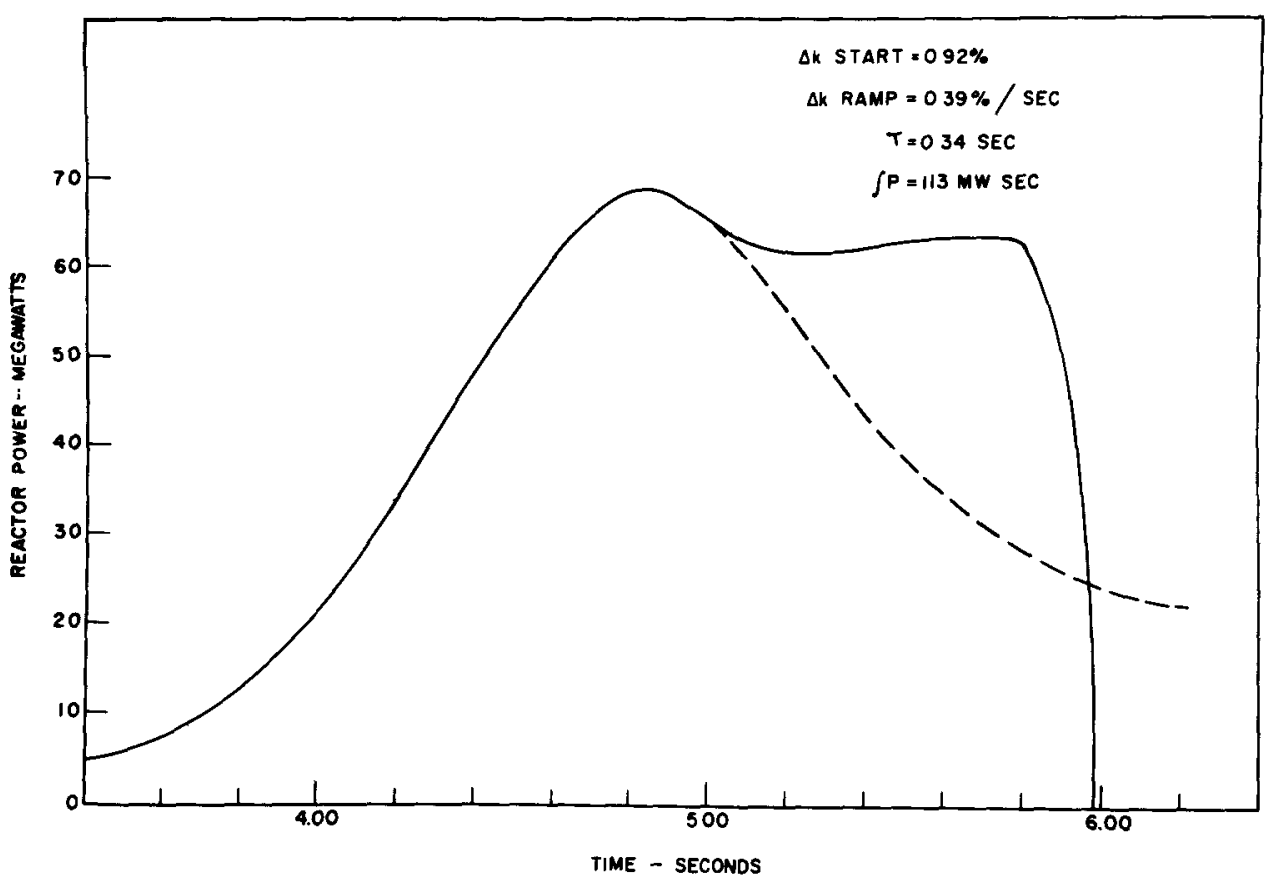

Slide 1. Treat Power Burst Shape

limited transient started with a $\Delta \mathrm{k}$ addition of 0.92 per cent would have had the shape indicated by the dashed line. The constant power type transient is desirable for some of our tests since this enables us to produce energy at a constant rate over most of the transient compared to the constantly changing rate which results from the normal peaked type transient. The slides previously shown by Frank Schroeder in nearly every case indicated a feedback of so many dollars per megawatt second which was usually flat over a range for all of the reactors covered. In our case we find that the shutdown coefficient, or whatever you want to call it, changes as a function of integrated power. This is due to the large change in the heat capacity of the graphite as a function of temperature. However, our shutdown coefficient as a function of temperature is essentially constant.

Turning to the fast reactor fuel element test program, experiments up to this time have been limited to single EBR-II type or Fermi-type pins in an inert gas atmosphere. Preliminary results on the EBR-II experiments were present in an article in the June 1960, Nucleonics (2); therefore, I will not repeat these but will try to cover some of the recent results in which I think you will be interested. Slide 2 shows several EBR-II type fuel elements after being subjected to transients in the Treat reactor. The first three, starting from the left, were natural uranium, EBR-II type pins, bonded with sodium in the normal manner, with stainless steel cladding. The first pin reached a temperature of about $900^{\circ} \mathrm{C}$. This was indicated by thermocouples on the cladding and is not uranium temperature. Note that there's some distortion, the helical spacer wires have come loose in several spots. Next, we have one that reached a temperature of 


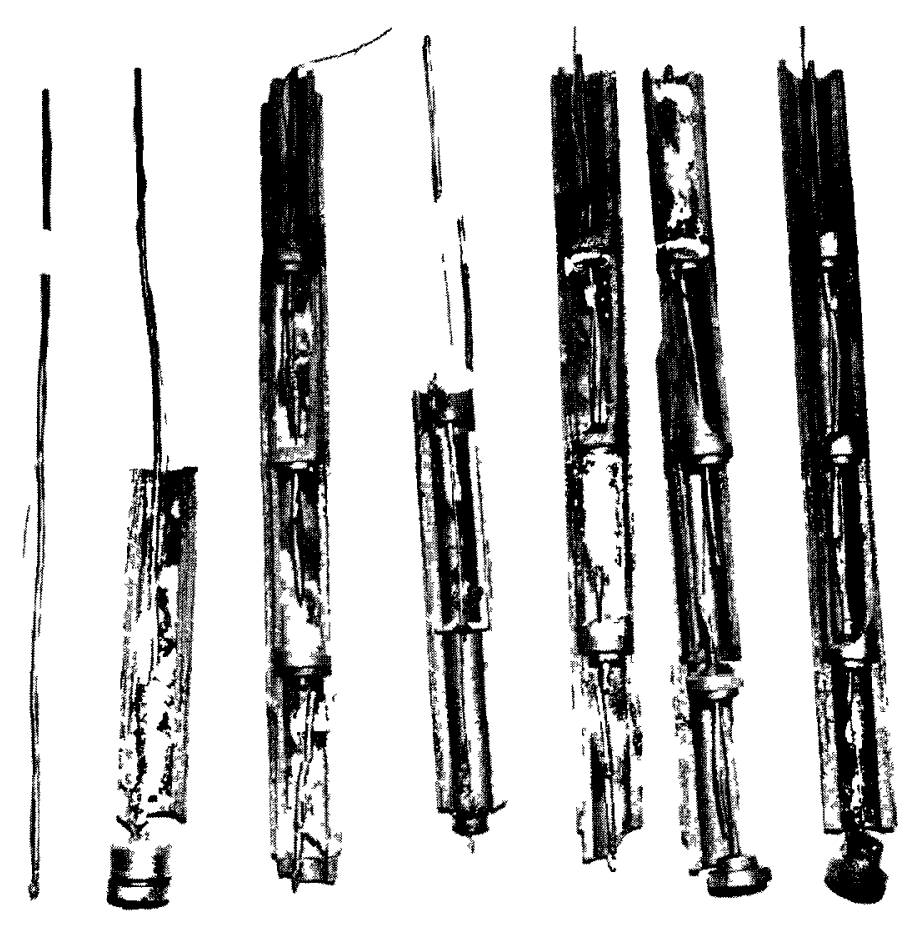

Slide 2. EBR-II Fuel Elements

about $1000^{\circ} \mathrm{C}$. Notice that there was not too much deformation in the pin. We have a penetration at the bottom through which the uranium was forced out by the sodium vapor pressure inside the cladding. It deposited on the cold wall of the crucible where it stuck and solidified. Generally, on this type of failure, we find that all of the uranium has left the pin. This is not 100 per cent true but is the general result in the temperature range of about 1000 to $1050^{\circ} \mathrm{C}$. The third pin is the same type of pin subjected to about the same type of transient, but for some reason it deformed more; notice that there are a number of areas of penetration. We have found that on tests of pins without the helical spacer wires the failure usually resembles the second pin. The uranium comes out near the bottom and failure does not occur at quite as low a temperature as it does with the helical spacers. We believe the helical spacers tend to twist the element and bring the stainless steel and uranium into more intimate contact forming eutectic which causes failure all along the line. The fourth pin and the seventh pin were natural uranium EBR-II type fuel elements which contained no bonding sodium. You will notice in the case of sample No. 4 a large portion of the cladding material has been dissolved and, where catchers were provided, this molten uranium collected in the manner you would expect if you punched a hole in a can containing a viscous fluid and allowed the fluid to run out under the forces of gravity alone. On the seventh specimen, apparently, there was a failure in the lower area early in the transient and, therefore, we did not have as much eutectic formed. I might add that the failure of Fermi-type pins looks quite similar to this; that is, a failure occurs and the material just runs out since there is no bond sodium to drive it out under pressure Pins 5 and 6 were the normal natural enriched EBR-II type pins with sodium 
bonding present but with a vent hole provided at the top of the pin to see if we could let the sodium vapors escape and determine what would happen under this condition. Apparently, in sample 5 this hole was plugged and most of the molten uranium came out of the center of the element. In the case of sample 6, apparently the hole stayed open and the sodium vapor and also a lot of the uranium came out the top. Notice that this pin came apart, this possibly happened during disassembly of the test capsule. The cladding temperature ran from around $900^{\circ} \mathrm{C}$ to $1100^{\circ} \mathrm{C}$ on these various samples. Reactor integrated powers of the order of 300 to $500 \mathrm{Mw}-\mathrm{sec}$ are required to heat the samples to these temperatures. We have a design integrated power of $1000 \mathrm{Mw}-\mathrm{sec}$, which is more than twice that required to cause extensive failure of the natural uranium samples.

I would like to say a few words about the future program on fast reactor fuel elements. We will run single pins in stagnant sodium, single pins in flowing sodium, clusters of pins in an inert gas, clusters of pins in stagnant and flowing sodium, repeats of these tests with pre-irradiated pins, and will eventually test oxide elements for fast reactors. I don't know just when we will test oxide elements. This will depend on the fuel development program. We are planning on taking photographs of the failure phenomena. We are having some trouble designing a transparent container which will contain the molten sodium and uranium. The first attempt was unsuccessful. However, I hope that in the next few months we are successful and I think that there is a very good chance that the new design will be successful.

Thirdly, coming to the metal-water reaction program, we have tested 46 samples. Included in this program were samples of unclad uranium alloy pins, unclad uranium wires, Zircaloy-2 clad aluminum-uranium oxide cermet pins, zirconium-clad uranium pins, unclad aluminum-uranium oxide cermet pins, and aluminum, stainless steel and Zircaloy-clad oxide pins. In the case of the oxide pins, we are merely using the oxide as a heat source for melting the cladding material. We have run a few tests on pre-irradiated Zircaloy-clad uranium-zirconium alloy plates; however, we have not reached the melting temperature of the plates as yet, so we have no significant results to report. The experimental procedure on these metalwater reaction experiments is to place a sample in a high pressure autoclave either with the sample in the water or suspended above the water so it will melt and fall into the water. We obtain records of reactor power, sample temperature and autoclave pressure during the transient operation. The autoclave is then opened and the gas collected for hydrogen gas analysis. From the basic reaction equations the amount of metal-water reaction can be determined from the amount of hydrogen gas evolved. Slide 3 shows the shape of a uranium sample before and after irradiation. The transient energy release on this experiment was $115 \mathrm{Mw}-\mathrm{sec}$ and we obtained 3.2 per cent metal-water reaction. Slide 4 shows the flux, the sample temperature, and the autoclave pressure records obtained from the oscillograph. The pressure indication was caused by radiation sensitivity of the transducer. Notice that one thermocouple came off quite early at fairly low temperature, the other one stayed with the sample and indicated a maximum temperature of $1550^{\circ} \mathrm{C}$. Slide 5 shows a Zircaloy-clad uranium pin before and after a transient. There was a 15-mil diameter hole drilled in the end of the Zircaloy cladding to provide a means for the molten uranium to escape prior to the time that the Zircaloy cladding melted. Notice 

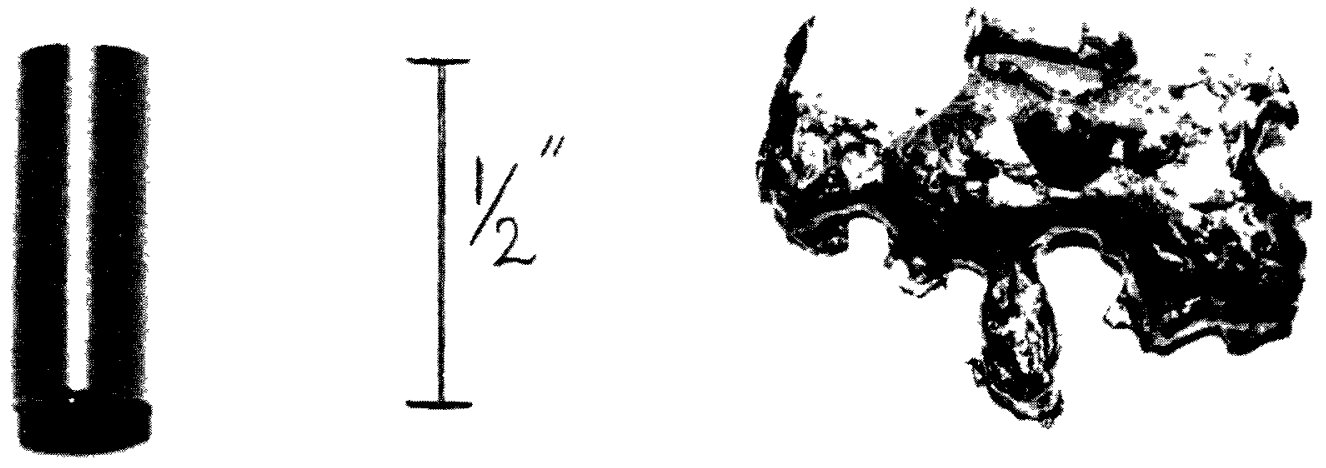

Slide 3. Uranium Sample Before and After

Irradiation in Treat

TREAT TRANSIENT CEN-8

METAL-WATER TEST

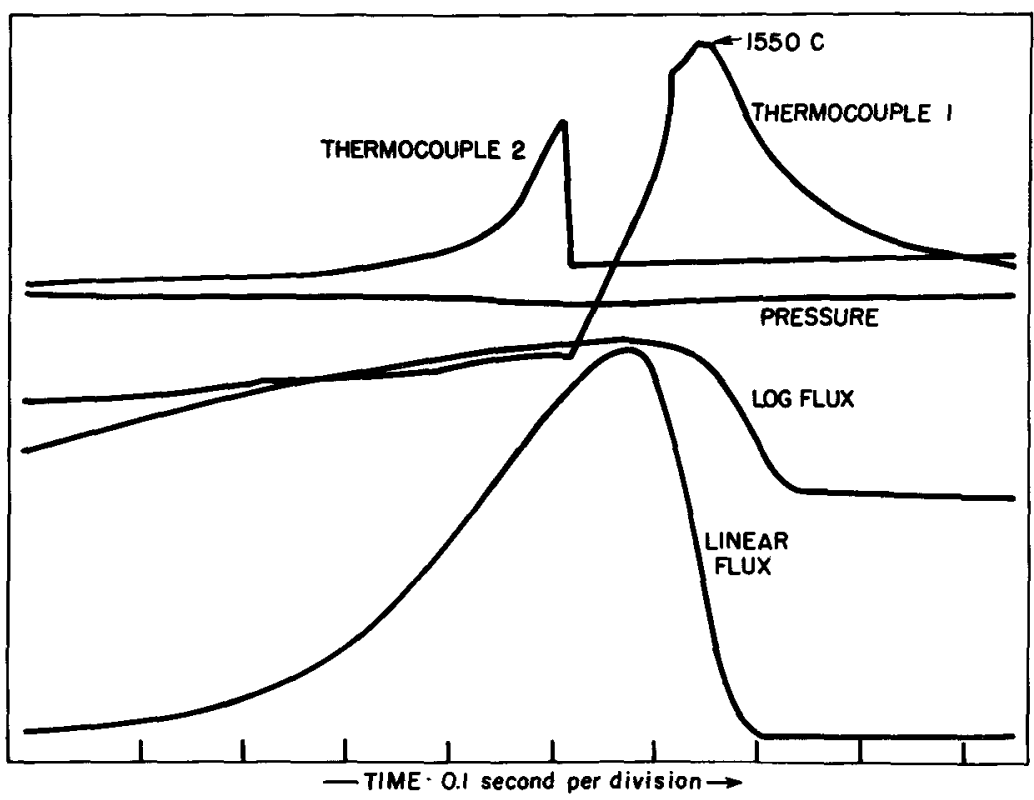

Slide 4. General Behavior of Sample Parameters During Test - Treat 


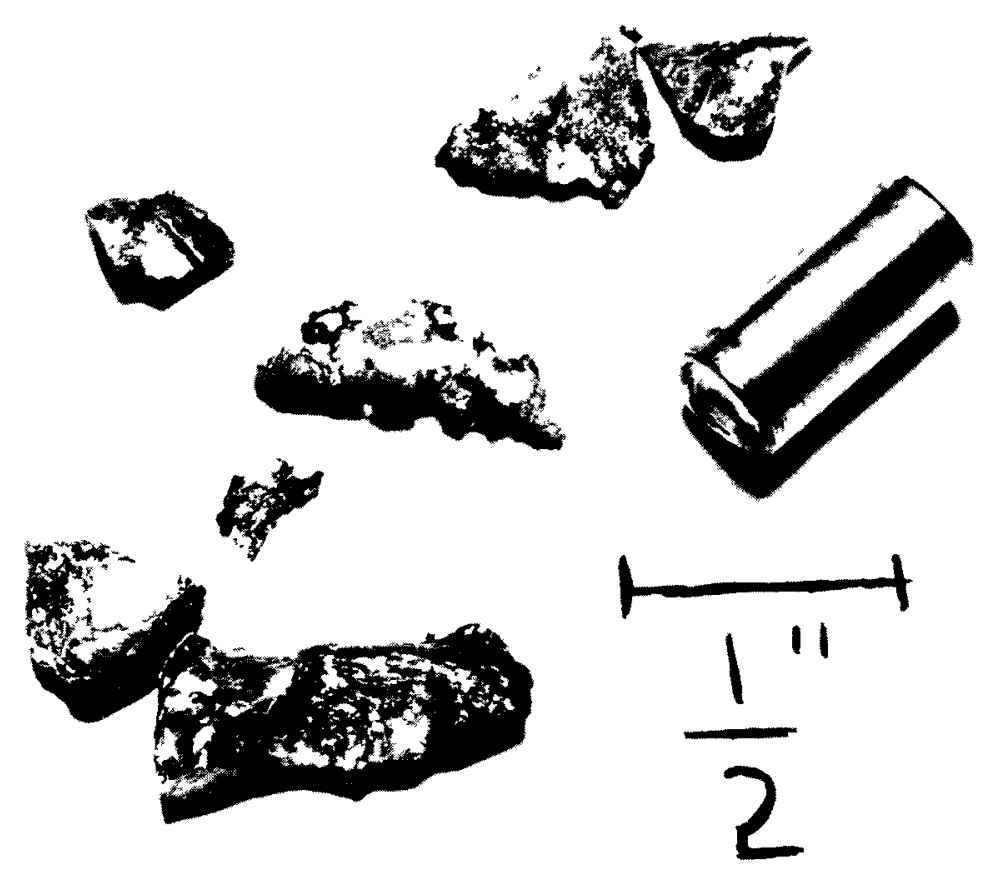

Slide 5. Zr-Clad Uranium Pin Before and After Irradiation in Treat

that the Zircaloy eventually all melts and conglomerates with the uranium forming several large pieces. Slide 6 shows the fines that were collected after this transient, the dark pieces are the uranium, the light particles are pieces of the crucible which contained the water and pin. Slide 7 shows an aluminum-clad oxide core pin after a $490 \mathrm{Mw}-\mathrm{sec}$ transient. The purpose of the experiment was to measure the aluminum-water reaction. An interesting sidelight was the fact that the oxide, which had been in pellet form inside the cladding, came out through the hole in the form of fine particles. We did not expect this and $I$ don't know whether anyone has an explanation for this or not. There was only an 0.8 per cent reaction of the aluminum with the water. This powdering of the oxide should provide food for thought for people using oxide fuel elements in their reactors. What would happen if a major transient melted a hole in the fuel element cladding? Would the oxide come out and end up in a pile at the bottom of the core which might be considerably super-critical and cause a second transient more violent than the first? Slide 8 shows a cross section of an oxide core pin clad with stainless steel. This sample received about $440 \mathrm{Mw}-\mathrm{sec}$ of reactor energy release. The cladding was not melted, the center of the oxide pellet was definitely melted as you can tell by the change in crystalline structure. However, an annulus between the center and the cladding was not melted but was cracked considerably. A fuel element might look like this if it were subjected to a transient which did not rupture the cladding but caused melting of the oxide at the center of the element. This also might indicate the condition of fuel elements which are routinely operated with the oxide in the molten state at the center of the fuel elements. 


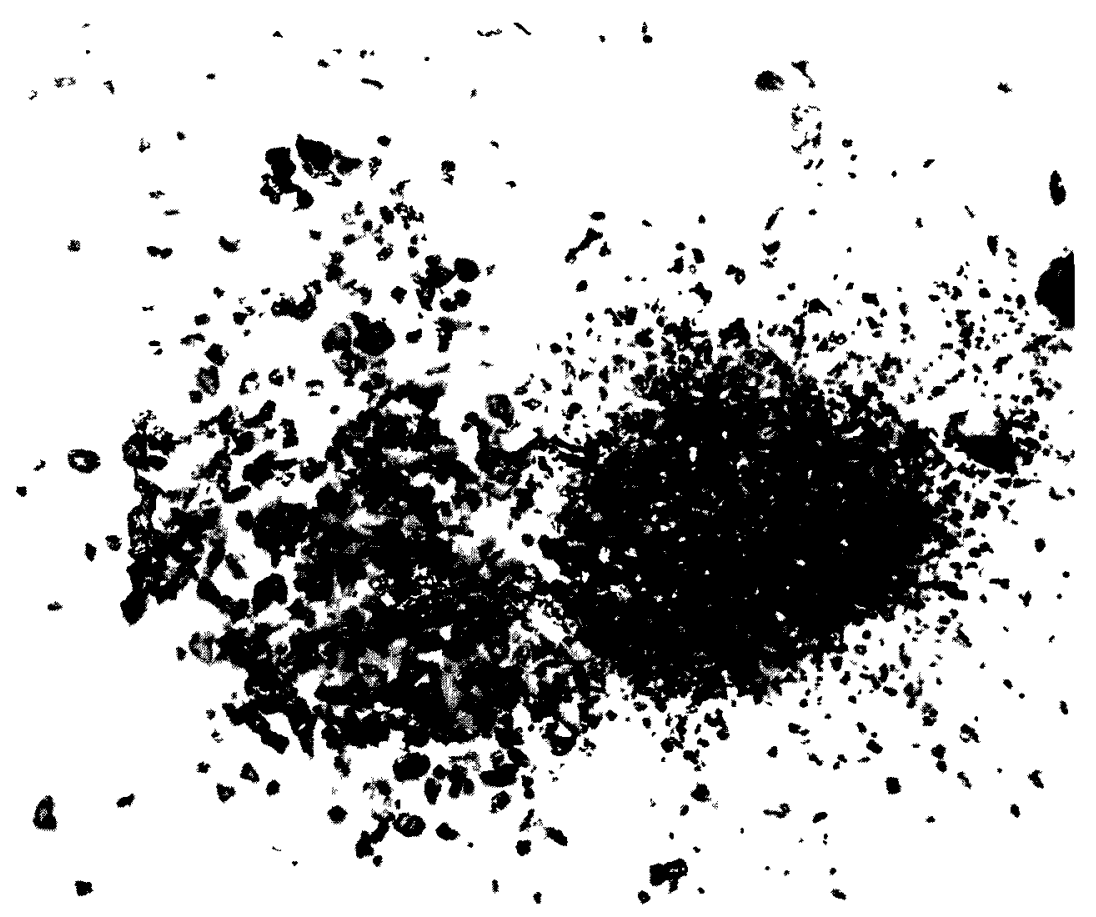

Slide 6. Sample Fines Collected Following Transient

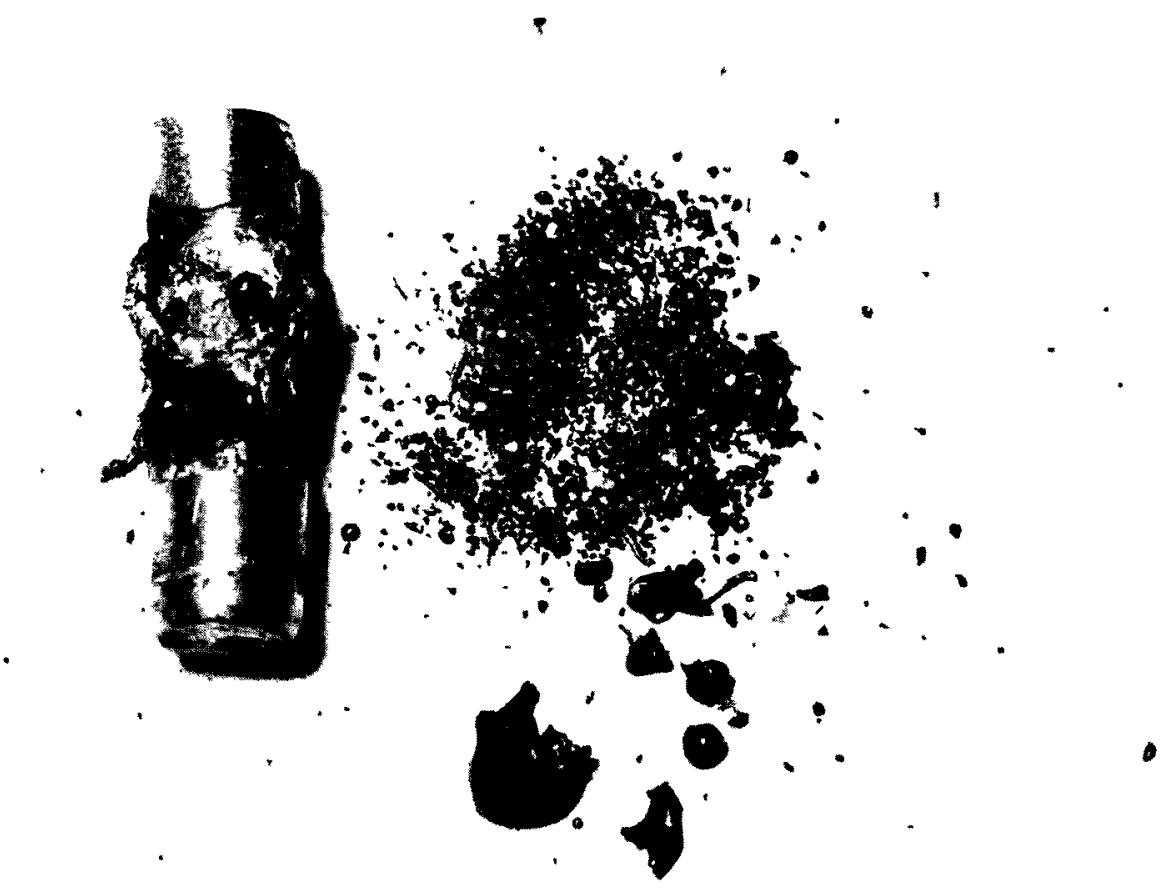

Slide 7. Al-Clad Oxide Fuel Pin Following Irradiation in Treat 


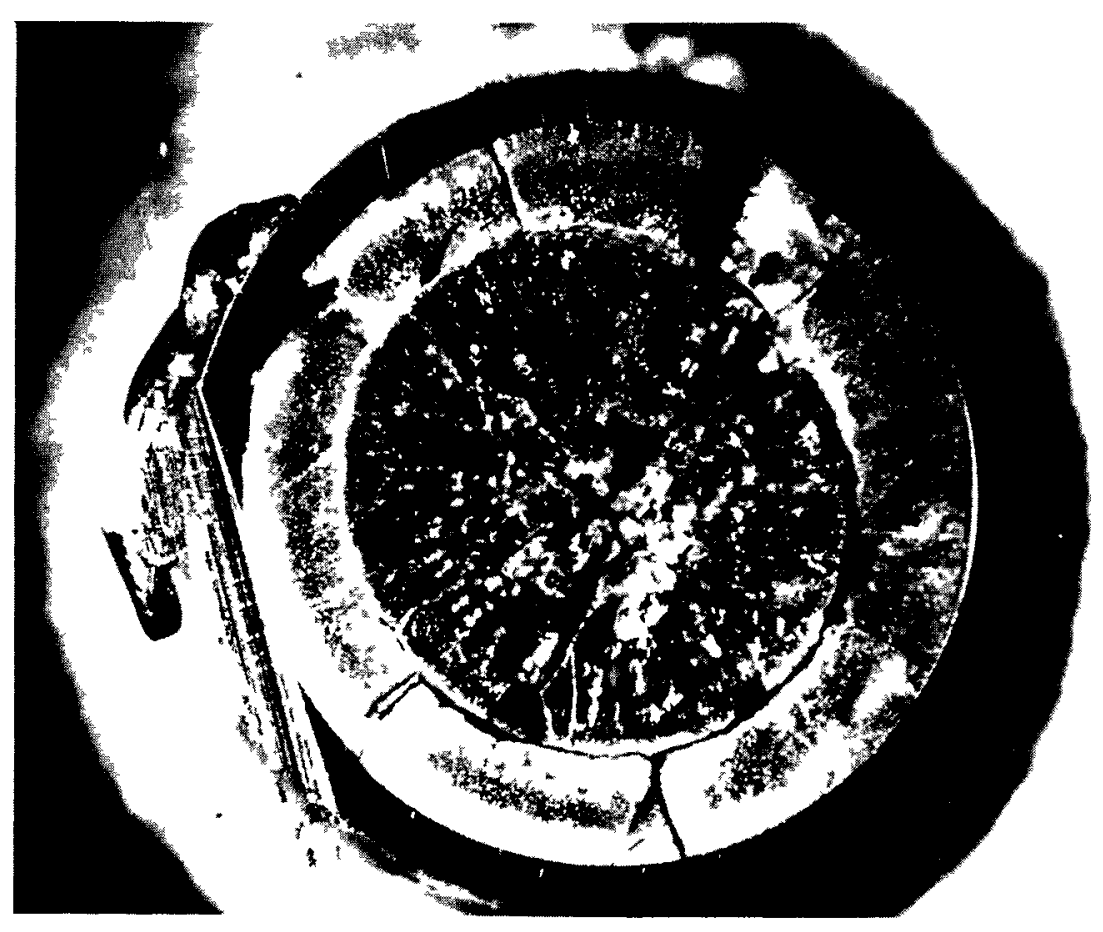

Slide 8. Cross Section of a SS-Clad Oxide Fuel Pin Following Irradiation in Treat

The future metal-water reaction program in Treat includes more experiments of the types I have briefly described but with higher energy inputs. Experiments will also be designed specifically to obtain information to be used in correlating in-pile data with data obtained in laboratory experiments where condenser discharge methods are used to melt wire samples.

\section{REF'ERENCES}

1. R. C. Liimatainen and R. O. Ivins, "Experimental Study of MetalWater Reactions Initiated by Muclear Reactor Transients, "Trans. Amer. Nuclear Soc., 3, p. 430 (December, 1960).

2. E. S. Sowa, "First TREAT Results - Meltdown Tests of EBR-II Fuel," Nucleonics 18, \#6, p. 122 (June, 1960). 
Stone: Thank you very much, Jim. During the questions from the floor I'd ask each one of you to stand and identify yourself so we'll know who we're talking to.

Grace: The statistical problem of reactor startup has been mentioned several times. I think it should be pointed out that significant progress is being made in being able to analyze this problem. The first analytical model has already been mentioned, that is Gordon Hansen's model. It was de reloped in connection with the Godiva experiments, and this model is quite relevant to super prompt critical transients; that is, above prompt criticality. The model has been improved since he no longer assumes that the persistent chains all develop in the same manner.

There is another model, developed by Don Harris at Bettis last winter, which includes the effect of delayed neutrons and therefore is applicable to transients below prompt criticality, which is really of more interest in our particular case. His method of the analysis suffers from the fact that calculations using his method involre the moments of the probability distribution of reactor fluctuations, which enter into the calculation of the probability of safety of the transients. It is very difficult to calculate as many moments as you need to describe the probability distribution, unless you assume a probabllity distribution, such as the normal distribution or the gamma distribution. Then, only the first and second moments are sufficient to completely define the distribution. Now he's been trying analytically with simple models to justify the use of some standard distribution and is running into difficulty in including the delayed neutron effects in this particular model. But we feel that by pursuing this approach we can justify the use of one of these standard distributions and have an analytical model that will work.

There is still another method, developed just recently by Henry Hurwitz, which is based on the assumption that a prompt chain of fissions occurs instantaneously; that is, there is a fission, then there is a fis sion chain that occurs instantaneously, and then you calculate the probability that a precursor was formed during that chain. His method looks good for transients close to delayed critical; that is, it cannot apply to transients at prompt criticality and above since it assumes that the prompt change occurs instantaneously.

Somewhere in all this I think a method will come out which can be used. We have a useful method now based on Harris' model, but we have to assume a conservative probability distribution because of our lack of knowledge of the actual distribution.

One final point: of course, a very important parameter in the evaluation of low-level startup is the natural source level of the core. Harris has also done a lot of research on what the different contributions to the natural source levels are. He has just recently published a report 
on this, (WAPD-TM-220, "Calculation of the Background Neutron Source in New, Uranium-Fueled Reactors," by D. R. Harris (March 1960) and he's also going to discuss this at the San Francisco ANS meeting ( $D$. R. Harris, "Natural Neutron Sources in New Uranium-Fueled Reactors", Trans. Am. Nuclear Soc., 3, 2, p.534 (December 1960)). He covers spontaneous fission, alpha-n reactions in different materials and cosmic rays.

I think the one thing we do need is many more experiments of the type that were described, as done in Godiva and Spert. As Warren knows full well, we've been pushing for more experiments of this type in the delayed neutron range; that is, below prompt criticality.

Stone: Thoma?

Snyder: In the talks about these experiments, today, one of the things that was not mentioned was the onset of the formation of shock waves. Perhaps rush experiments have been avoided thus far; however, we should think of the importance of such experiments in terms of our provisions for safety. We have usually three barriers between the fission products and the outside world; the fuel cladding, the pressure vessel and the containment structure around the reactor. Shock waves can have quite a bearing on the integrity of these barriers so that it would be worth learning more about the gray area between silow transients which certainly don't produce shocks and rapid ones which surely do.

Stone: Would someone on the panel like to comment on that? Warren?

Nyer: I think we agree with you entirely, Thoma. Our program just hasn't come to that point yet. We have been considering, recently, doing experiments of this sort. This is what Frank (Schroeder) meant when he was talking about getting into the region of destructive experiments. We, too, recognize that this is a gap in what we have done.

Chermick: I wonder if Thoma is aware of the work at Stanford on the sized-down models of reactors with the use of known explosives.

Snyder: Well, I'm not sure that I feel that an explosion test is altogether adequate for this because the time constants are not so easily controlled.

Stone: Yes? If the Chair may, I would like to comment on the fact that most of the discussions have been based on the first burst description of transients in test reactors. Since the thing that enters into a hazard evaluation is not peak power but total energy release, and since such a large fraction of total energy release occurs not in the prompt burst but in the following decay to some equilibrium power level or perhaps in some type of divergent oscillation, I am somewhat concerned that the larger portion of our work in reactor transient programs is not devoted to studies in this area of the time spectrum. Would someone in the panel like to comment on this aspect of our work?

Remley: I think that this is very definitely an area that needs some further exploration, because in the case of an excursion one gets an augmentation of the fission products by the excursion, and one of the things 
that one is concerned about is the containment of these fission products. So that one needs to look at what happens after this first burst, and we have done some looking at this, particularly in the case of ramp transients. In the Kewb, we have put the reactivity into the system and then allowed the reactor to continue to run for a period of several minutes up to 30 minutes; what happens is that one gets the first burst characterized by the minimum period of the machine, then the reactivity is held both by the temperature and the void and, in addition, the void is augmented in this case by actual steam formation, because one gets a boiling in the reactor core so that we then go into a regime of boiling control, and the system sits there and bolls at about $5 \mathrm{kw}$ with about $4 \%$ in reactivity held in the combination of principally the steam voids and the radiolytic gas production at some $10 \%$ of the design power of the machine; that is, this particular machine is a research reactor designed for 50-kw operation. Hence, the augmentation to the fission product inventory is a minor one resulting from the relatively small energy release in the first burst.

Stone: Yes, Sir?

Silver: I would Ilke to make a couple of comments based on the experience we have had in Spert BSR-II tests. The first is a point that has been touched on before, but I think there are some things that should be emphasized. The first is simple enough--the no-source startup, as pointed

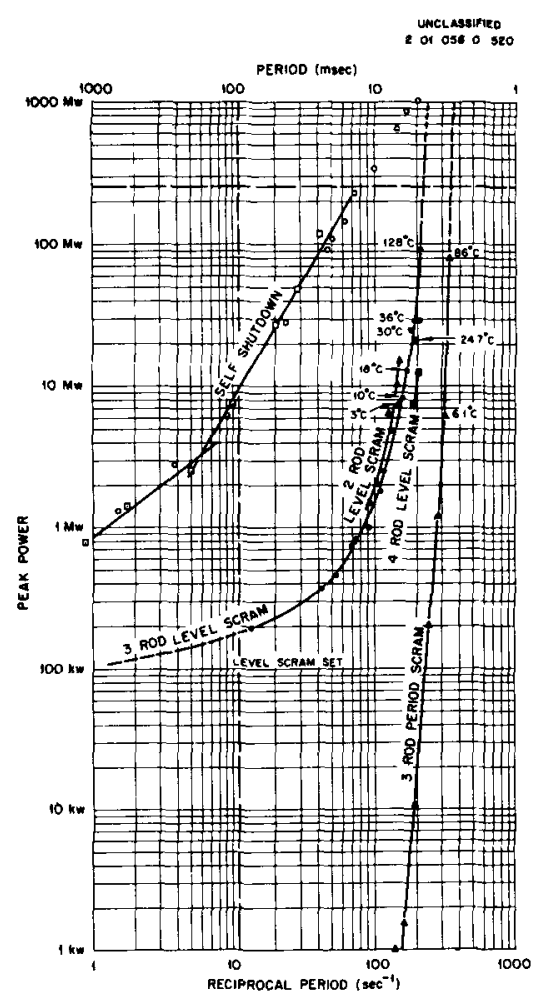

Slide 1. BSR-II Spert Tests - Peak Power Versus Inverse Period. Zero Initial Power out, gives you very long times (of the order of a second) between the transient insertions of reactivity and the occurrence of transients-so one really has to look for relatively slow methods of reactivity insertions in $100 \mathrm{king}$ at the concelvable accidents: collapse of experimental void, and things of this sort that are fairly slow. The other point is illustrated in the slide Mr. Epler showed, Slide 1. It concerns the ultimate effectivity limits of the control system that was used. The rods were arranged $I$ in. preinserted in a 15-in. high core in order to increase the initial effectiveness of them; the total rod worth is of the order of $12 \%$ reactivity worth and, yet, if you look at the extrapolation of the shutdown--control-shutdown curves, you will see it indicates that they wi,I meet and become equal to self-shutdown curves at about a power level in the range of one thousand to two or three thousand megawatts peak power. Now, that 
is just about the threshold for a catastrophe you might call it--oh, meltdown, possibly, and fission product release. Now I think that this indicates that this control system, which is as good as any I am aware of, will not protect you against a transient which can real1y cause trouble other than just damage to the reactor itself, and I don't know that any reactor system now can rely simply on a control system for protection against such a catastrophe. I think this points up the need for looking at fuses and other very fast means of helping if such a very large transient is at all conceivable. I know there have been some fuse tests done at Spert but they were only carried down to $10 \mathrm{msec}$, I believe, which is certainly a range in which ordinary control systems are quite effective and one really ought to look for very fast means of putting small amounts of negative reactivity in--in the range of 2 msec on down for plate-type swimming-pool reactors where I think this is a real consideration.

Epler: I would like to comment on that, please, Dick. What your comment implies, of course, is that you propose to run the reactor without a source because, as the data show, you cannot get this 3-msec period with this system unless you remove the source. Now, this raises the question--If people really want to protect their reactor, should they not concentrate on a way of putting in a source and making sure it's there? This, I think, is an issue that could well be discussed. Now it seems to me that keeping the source in the reactor is a fairly easy thing to do.

McCullough: I'm not at all clear that it's possible that we can get a practical reactor--and I'm talking of power and test reactors, not merely experimental ones--on a 2 -msec period even without a source. Now I think the question is very pertinent, "Is it possible to get a millisecond period?"

Stone: I'd like someone to tell me what reactor period you obtain, say at the MTR, if you drop the central fuel element in. Warren?

Nyer: I think, Deslonde, maybe you would be the one to answer that question.

deBoisblanc: The worth of the central fuel assembly is about $3 \%$. Somebody else can convert that into currency.

McCullough: How fast does it go in?

Stone: Stu?

Forbes: We did some dropped fuel-assembly tests in the Spert "P" core made up of APPR assemblies in which a peripheral assembly was suspended by magnets and then dropped into position with some guides so it would go in straight; increasing the rod position to limit the total $\mathrm{k}$ addition, hoping that we would be able to drop it the full two feet approximately of fuel length. We chickened out because the period was getting 
down around $5 \mathrm{msec}$, we had not yet made a full rod drop at $5 \mathrm{msec}$, somebody can refine these numbers for me but it's in that region. There was some indication at this point that the ramp nature of the insertion was perhaps beginning to show up, in that the burst was perhaps beginning to turn before the rod assembly seated but also, as you know, these stainless-steel plates go to pieces pretty fast at $5 \mathrm{msec}$, from warping and so on, so we quit. If that had been a central assembly I think you could safely say we would get into severe warping and possibly get down to where it might melt, but it's not certain.

\section{Stone: Silver?}

Silver: We measured the reactivity worth of the central fuel element in BSR-I aluminum swimming-pool reactor with the pulse method and got $4 \%$ which is the order of about $\$ 5$ of reactivity worth there. And, again, even in the power reactor I guess they get a new fuel charge, cold fuel when they haven't had it up to power. You have lots of time to put the reactivity in. I think it's at least a conceivable accident, although probably not a likely one.

Stone: Aren't those the questions that must be asked? What do you think a single piece of hardware is worth? How fast can you move it? How much is the source at the time that you move it? And what is your control system worth?

Forbes: Well, I think certainly on the "P"-core test one would say that you can drop it fast enough to get into trouble. I think it fell at about $90 \%$ of free-fa.11 velocity.

Remley: I was wondering what the ramp rate was? Do you know, Stu?

Forbes: No, I don't remember.

Silver: Was that source-free, Stu?

Forbes: No, it wasn't source-free. Well, it had the natural source and the core had been run a lot. I don't know--the initial power, I guess, was probably around a few watts from the normal procedure.

Chernick: I must say that in a power reactor it's very hard to conceive of an accident like this--even if you have the wrong core in there--you've got a source, normal shutdown of rods, normal withdrawal rates, it would be pretty hard to get an accident of this type.

Stone: On the normal control system, I believe that anyone would be inclined to agree with you--it's under those strange circumstances where you're handling a large piece of hardware by hand. Warren?

Nyer: Well, the problem in the case of the Spert reactor is that a single fuel assembly contains so much reactivity, and I think that what Jack

(Chernick) was saying is that in power reactors no group of fuel that is 
handled at any one time contains the amount of reactivity that we are talking about in these experiments. But I think the point is still correct, that the question is not an academic one where reactors like Spert are concerned.

\section{Stone: Deslonde?}

deBoisblanc: There is one interesting type of accident--if you have this situation in the MTR you wouldn't have any controls to drop, if you have already taken the top plug off. This accident can occur when all the shutdown $k$ that you have available inserted in the system to begin with, but it is an unlikely accident.

Ep has once talked about such an accident whereby in some types of cores you have an adjacent drive which picks up a fuel element and then it drops the fuel element. This, I think, is a very credible accident. It should be analyzed because, although the ETR uses a guide tube, in the MIR there is no guide tube. The fuel element is right adjacent to the rods and this is kept in bounds by inspection, by dropping the rods and things like that. But that is a very possible accident in MTR-like reactors. Of course, you remember that in the MTR the fuel elements are held down by a grid under ordinary circumstances. It will be very hard for them to break the grid and pull the fuel element out, but there may be designs contemplated which would have these control rods adjacent to fuel elements without the containment of the fuel elements by a grid and, in this case, it can be a very serious problem.

\section{Stone: Rogers?}

McCullough: I'd like to turn to another subject. I'm very much interested in some of these test experiments in $K E W B$, and I noticed that the pressure got up as high as 800 psi, if I remember the graph correctly. I wonder if in those tests you had made any tests by inserting some kind of a capsule in the middle of the core and measured the pressure rise during the excursion in that immediate capsule. The reason I'm asking this is because, conceivably, there can be a certain amount of pressure wave absorption in the core as a whole, whereas the center capsule might not have this effect and this--I would come back to the questions that have been raised about shock waves. The point was made that we are depending upon the integrity of the primary system. Now, if shock waves or focusing waves of any sort can develop, then you have a right to question the integrity of the primary system. However, if the pressure rise in the primary system is of the order of a factor of e below several milliseconds, shall we say, then, that this is not a serious problem because, if anything, the properties of the metal will respond to this and you have pressure relief devices on these things of one sort or another. So what is important is whether we have a true shock wave or whether we have any focusing effects-now this, I think, is an important thing to consider.

Remley: In answer to your direct question of, "have we measured the pressures in a capsule inside the core", we have not. I think some comment is appropriate on these pressures. There are two pressures that I indicated, one which we call expansion pressure and one which we call an 
impact pressure. The expansion pressure is measured with a pressure transducer located at the bottom of the core and one finds that the pressure measured with this one rises at a rate consistent with the increase in reactor power and with gas formation, so it essentially results from a push downward on the core vessel. The pressure at the top, which is the impact pressure, results from the response of the transducer that is located, in the case of the sphere, right near the top of the sphere between the Iiquid level and the orifice. The gas is formed in the core solution, then it expels the liquid upward and you get a slapping against this transducer, and this results in a rather sharp pressure pulse, and the same thing is true in the cylindrical core. There is no indication of pressure during the transient on this particular transducer until after peak power; that is, there is no expulsion of the liquid from the spherical core before shutdown. In addition to this first impact pressure pulse, there is a reflected wave that travels back down and one sees a secondary pulse on the bottom transducer. But when the spherical core is filled with fuel solution, and the top transducer is inundated, both transducers are then underneath the fuel solution and one gets an inertial pressure rise at the top of the core which is very similar to the one at the bottom of the core; in other words, the pressure is transmitted throughout the sphere in accord with Pascal's Law. Also there is a slightly higher pressure inside the spherical core than in the cylindrical core because of the fact that we are actually accelerating the fuel solution out through an orifice; whereas in the case of the cyllndrical core there is a free surface area 12 in. in diameter that permits the gas to escape more readily. I do agree that it is a very important matter to look at this for the possibility of buildup of shock wave because one gets a decided decrease in the velocity of sound in the fuel solution, as the gas is formed and as the density decreases with the consequent increase of compressibility of the fuel solution, because of the formation of void.

Stone: Jack?

Chernick: I would like to raise a question which is perhaps close to what you are talking about, and that is, are there any plans to carry any of these tests, specifically the Spert tests, to destruction. Now this happened, of course, in Borax I; but people are apparently loath to destroy large and expensive assemblies, and obviously this is a rather important thing, not only for the Spert tests but also for many of these pulsed reactors that we've been talking about. It would be very interesting to see how far they could be taken safely; for example, the solid-state physicists are getting quite interested in the pulsed system in Godiva for fast neutron damage and another factor of 5 probably would be quite important, but whether it reaches a factor of 5 or not is uncertain. I wonder if any members of the panel would like to comment on this.

Stone: Warren?

Nyer: I think, Jack, to answer your request for information--we have gone a little bit further than just saying we think we should look into this, we have actually made some attempts. Stu (Forbes) wrote a report 
on what we would like to do in the way of destructive tests and what we would hope to get out of such tests; we had in mind actually starting some, perhaps next year. I don't know yet whether we will get to them at all. I think one thing that maybe your mind should be set at ease on is that we are not reluctant to destroy the equipment anymore. The first year or two of operation we were reluctant because we only had one system in operation and, clearly, we could gain more from the tests we were doing than if we put the system out of commission. Now that argument doesn't hold anymore and we feel that we can go into these tests.

Stone: Jim?

Boland: I might say a word in regard to this. In the Treat programs we hope to find out something about what might happen in destructive tests of a whole reactor by testing a single pin, a sub-assembly or something of this nature so that the expense and the time involved are not so great. I think that if we know how much heat flux it takes to do a certain amount of damage to an element, then we might be able to predict what would happen in the actual reactor. Possibly one could determine if there is a possibility of a second pulse due to the redistribution of the materials after the first pulse if it did not produce enough energy to destroy the integrity of the cladding and things of this nature. The primary pirpose of the fast-reactor program in Treat is to obtain information which can be used to predict what could happen during an excursion in a reactor like EBR-II. One of these elements does melt down, and by testing them in stagnant sodium and flowing sodium we hope to get some fundamental ideas as to where this fuel is going to go, how it's going to redistribute, and things of this nature, and thereby predict, maybe with some certainty, what could happen in the event of a major meltdown of one of the fast reactors which is sodium cooled. I don't know of any program at the present time for similar tests on thermal reactor elements--there may be.

Stahl: Triga has no plans to go into destruction, we do hope to go to larger pulses without going to damage to fuel elements perhaps going to I-msec pulses, but we do hope to avoid destruction for the time being.

McCullough: Mr. Chairman, I would like to respond to a question that Warren Nyer asked me before the discussion. That is--whether these ruleof-thumb approaches to various phenomena is a worrisome matter? The answer is clearly, yes,--they are worrisome--but, at the same time, I think they are extremely worthwhile; in other words, I think we should derive the rule-of-thumb parameters and apply them to reactor systems with a clear understanding that these are tentative and that each fellow who comes up with a system using this rule of thumb and tries to apply it in his reactor, should clearly understand that this is not a rule, this is not a standard, but it is a tentative thing. In trying to use the rule of thumb we will begin to arrive at what Iimitations have to be set on such a rule.

Stone: I would like to add perhaps one thing to it, Rogers, and that is the important thing about these rules of thumb is to be real careful about extrapolating on the range of applicability. When we are up to $4 \%$ or so, 
we can tell what is happening here, we see Godiva in the change of coefficient, I think along in here, Triga, where we start to take thydrogen from a bound state to a free state. Here, again, we could well expect to see a change in the shutdown mechanism--I think this is an area where a high degree of caution is required. Any other questions? Bill?

Ergen: In this experiment where the $\mathrm{UO}_{2}$ ended up outside, is it possible that the $\mathrm{UO}_{2}$ vaporized? This is a question which frequently comes up-what is the vapor pressure of $\mathrm{UO}_{2}$ or where does $\mathrm{UO}_{2}$ boil?

Boland: I cannot answer this question for sure, although if you look at the energy input you would not expect vaporization of the $\mathrm{UO}_{2}$ and $\mathrm{I}$ believe that if you did have vaporization you would probably be able to tell it by post-mortem examination of the residue. I am not sure whether this has been done or not. Routinely, it would be done on this type of experiment, but I cannot answer your question at this time. Perhaps by December Bob Liimatainen will be able to shed more light on the results of this type of experiment.

Ergen: May I make one more remark?

Stone: Certainly.

Ergen: Warren Nyer mentioned that, if the steam formation is suppressed by higher pressure, then the energy has to go some place else but it is my recollection--from the steam tables--that a given amount of energy, one calorie, for instance, is much more efficient in decreasing the average density if it goes into steam formation, than if you just expand the water. And, I think it is more than one order of magnitude and I was wondering whether this would show up in the lack of effect.

Nyer: I don't think there is any disagreement with what I said. The point I was trying to make was we then put more energy into another mechanism than it would ordinarily have had and, in this case, the large amount of energy that was being generated still puts enough into the shutdown mechanism just due to the water heating to keep the overshoot from being very, very large. However, there was an increase in energy release as you would expect.

\section{Ergen: There was some?}

Nyer: Yes.

\section{Stone: Tom?}

Wimett: I would like to make a few comments on several of these questions. First, on the calculations of fluctuations relative to no-source startup. Gordon Hansen has just completed a paper which will be published this December, I think, in Nuclear Science and Engineering, in which he treats the case--that I mentioned, of sub-prompt critical type excursions with one delayed neutron group. Apparently it is a fairly difficult theoretical problem. The title of his paper is, "Assembly of Fissionable Material in the Presence of a Weak Neutron Source", and on the question of extending 
the yield of Godiva-like systems, I would like to mention that NDA and Oak Ridge have worked out a proposal for extending Godiva II to obtain yields almost a factor of ten greater and it is very much like Godiva II. Also, Bill Stratton and I are trying to stir interest in Ios Alamos to do some experimental work along this line.

Stone: Stu, you had a question.

Forbes: I just wanted to throw in a comment to Bill Ergen's comment. As the water temperature approaches the critical point, this distinction between Iiquid and vapor, of course, loses its meaning and these temperatures are beginning to get to the point where the nonlinear expansion of water is quite important so that, although at lower temperatures your statements seem quite correct, it begins to be less correct as you get to these higher plate temperatures. That is, the difference in energy and the difference in volume changes per unit energy gets to be essentially zero at the critical point.

Ergen: Well, even at a 1000 psi, there's still a lot of difference between them.

Forbes: Well, 2500 is pretty close to critical pressure--3200--so you're up to 250 some degrees centigrade, I believe, in these tests, Frank? And here the water expansion is switched over from square law approximately to ex-type thing--it's really going up.

Stone: Wa.It?

Lipinski: I'd like to go back to the suggestion earlier on conceivable accidents with regard to the insertion of reactivity. There have been a lot of proposals that require acceleration of rods in reverse opposition to gravity and it's quite concelvable that, in a reactor containing this design, a rack and pinion could fail during startup operation near criticality and have a control rod fall out of the reactor, giving you a fairly fast, large increase in reactivity. You've got to come up with a 2-g force that will equal the performance of a natural drop.

Head: I've come to the conclusion from Mr. Epler's statements that this reactor does not require a scram system. With the burst turned around inherently and a run in of a.ll rods at normal speed, the reactor could be safely shut down.

Stone: Al?

Henry: In certain classes of reactors, fast insertion of the control rods is a.11 that is needed to protect the core from most accidents. In some cases, the emergency safety system may be merely a fast insertion.

Nyer: One thing can be added to this discussion--one thing we're trying to do in these studies with the relative effectiveness of the trips that Epler mentioned was to really define and allow us to define what we're asking the control system to do. And, in this case, I think it's clear we are not asking the control system to protect the reactor against a 
catastrophe, but the control system is being asked to protect the reactor against core damage. That's just what it did, and this action was very effective in the region where core damage would take place and, if we had allowed inherent shutdown mechanisms to be the only operative ones, then we would have rippled the core and done all sorts of damaging things like that.

Grace: You're agreeing that you didn't have to mun the rods in fast, then.

Nyer: I would agree in principle that you do not have to do it in all cases.

Johnson: I would like to comment on one of the statements Frank Schroeder made at the end of his talk. In regard to proposing transient testing in new cores when we start them up. In view of his previous remarks, I interpreted this to mean--running experiments, determine parameters for some type of correlation, so that you could then determine perhaps the ultimate safety of the core. I think most of us would be rather hesitant in performing such tests as they do in Spert. We certainly don't want to get into the millisecond range; in fact, we'd have considerable difficulty in doing it without really altering the control systems in the reactors. On the other hand, the correlations proposed so far do not fit in the, oh, one- or two-second period range were we could conceivably measure these things; so I'm a little bit dubious about Frank's proposals.

Stone: Frank, would you like to answer that?

Schroeder: It seems to me he's left out the region where it would be conceivable to mun the tests; namely, that region right around prompt critical where we have fair success in relative correlations and where, if the experiments were approached intelligently and were well planned, I believe they could be carried out without undue risks to the systems.

Johnson: Of course the risks to the system--to your system--are somewhat different than to ours. I don't think you care very much whether you ripple the plates. We're in the course of determining this for the core which is to be operational. If we ripple the plates even very slightly, they would be essentially worthless or would not be able to operate as designed.

Schroeder: I'd like to point out that in the case of the tests performed with the BSR-II core, which have been mentioned here several times today, we were in such a situation. We very cautiously approached the point at which we began to get limited core damage and we did not go past it in these tests.

Stone: Frank, I think we're talking about two different things, really. One case we're talking about reactor cores that cost in the order of $10^{5}$ dollars, and in the other case we're talking about reactor cores which cost in excess of $10^{6}$ dollars, and they're not replaceable.

Henry: I think that another pertinent factor is that we want also to control excursions which will lead only to slight damage--not even come 
close to a catastrophe. Generally, protection of this kind mast be designed into the thermal and hydraulic character of the core beforehand; it cannot be added after the fact by changes in, say, the scram system. Thus, you can't wait till the core is built in order to test and adjust the protection system empirically. It could be perfectly disastrous if you found you were not, as a result of these tests, adequately protected.

I also agree with Stan Johnson's point that you can't risk damage to the core in testing its degree of self-protection.

Stone: I think that postulation not only applies to the naval reactor program, but to portions of the power reactor program; namely, we cannot in any way in initial testing compromise the integrity of that core. We're asking years of operation on one core and if, in the initial operation, we damage the core, we are defeating our purpose. Stu?

Forbes: Aren't you, though, in the rather incongruous position of having assured the HEB and ACRS that this reactor, under no conceivable circumstance, can get into trouble for, say, periods of $\mathrm{x}$ milliseconds--but for heaven's sake let's don't try it.

Stone: Yes; however, this assurance is given for a range of accidents up to a maximum credible accident which may well damage a core. Yes, Jim.

Boland: I'd like to ask a question of Frank Schroeder. Maybe we're getting inta something that'Il come up in the next session, but you people have run both excursion-type tests and oscillator-type tests at Spert. Is there any plan to present a correlation of the results? Can you predict what will happen on excursion from your oscillator results and, if so, is there any reason to propose excursion-type tests?

Schroeder: I'd like to let Mr. Wasserman answer that one.

Wasserman: The analysis of the high-power tests which were done earlier has not been carried far enough so that question can be adequately answered. Furthermore, it's not yet sure that the tests that were done were extensive enough to answer the question. So, I must defer for the moment.

Nyer: Dick?

Stone: Yes, Warren?

Nyer: I think there's just a little bit of a misunderstanding here. One of the objectives that we think we should have in the Spert program is to try to develop field tests that people can use with complete safety on any reactor--to find out what the actual dynamic characterics of a given reactor are. We don't want to specify these tests at the moment, they may simply be oscillator tests, for example. If this is to be the case, we've got to establish at some time that the oscillator tests are adequate to do this. So we have to make a connection between the excursion tests and the oscillator tests. This has not yet been done, it is something that is in the process of being done. Another thing occurred to me when we were talking about the excursion tests, and that is the image that the word "excursion" brings to mind for most people. I think we ought to remind everybody that 
every time a reactor is started up you do an excursion test. The thing is that, in this case, you don't try to find out what the reactor itself can do, you manipulate the rods and let that determine the history of the power rise. I think we have always made the condition that, if these tests are to be undertaken, they should be undertaken without any possibility of damage to the reactor or to increasing any hazard to the public. Based on our experience, and this is the only guide that we have at present, we believe these tests could be carried out with safety--with safety to the reactor and with safety to the public--and still get additional information that is not available in any other way. And this is all we were trying to propose here.

Wright: I'd like to mention briefly some results Arthur Morse of the SII Research Laboratory has obtained on the growth of steam voids under exponential power excursions. These are out-of-pile experiments done in a 6-in. section of a simulated Spert-IA moderator-coolant channel at atmospheric pressure. It is a somewhat unusual setup in that the boiling section is in a feedback loop which measures the resulting steam void volume and controls the power through an analog computer programmed with the reactor kinetics equation. It's also a little odd in that the channel expands outward so that the metal expansion and the water expansion with temperature compensate each other pretty well so that essentially what you have is a onechannel reactor which doesn't have a temperature coefficient, but it boils. Slide 1 shows the results from one of the power excursion tests. Thermocouples $1,2,3$, and 4 are $1,2,3$, and 4 in., respectively, from the top of the 6-in. channel. The temperature gradient shown is a spurious effect caused by a small inverse flow from a cold-water void-quenching system.

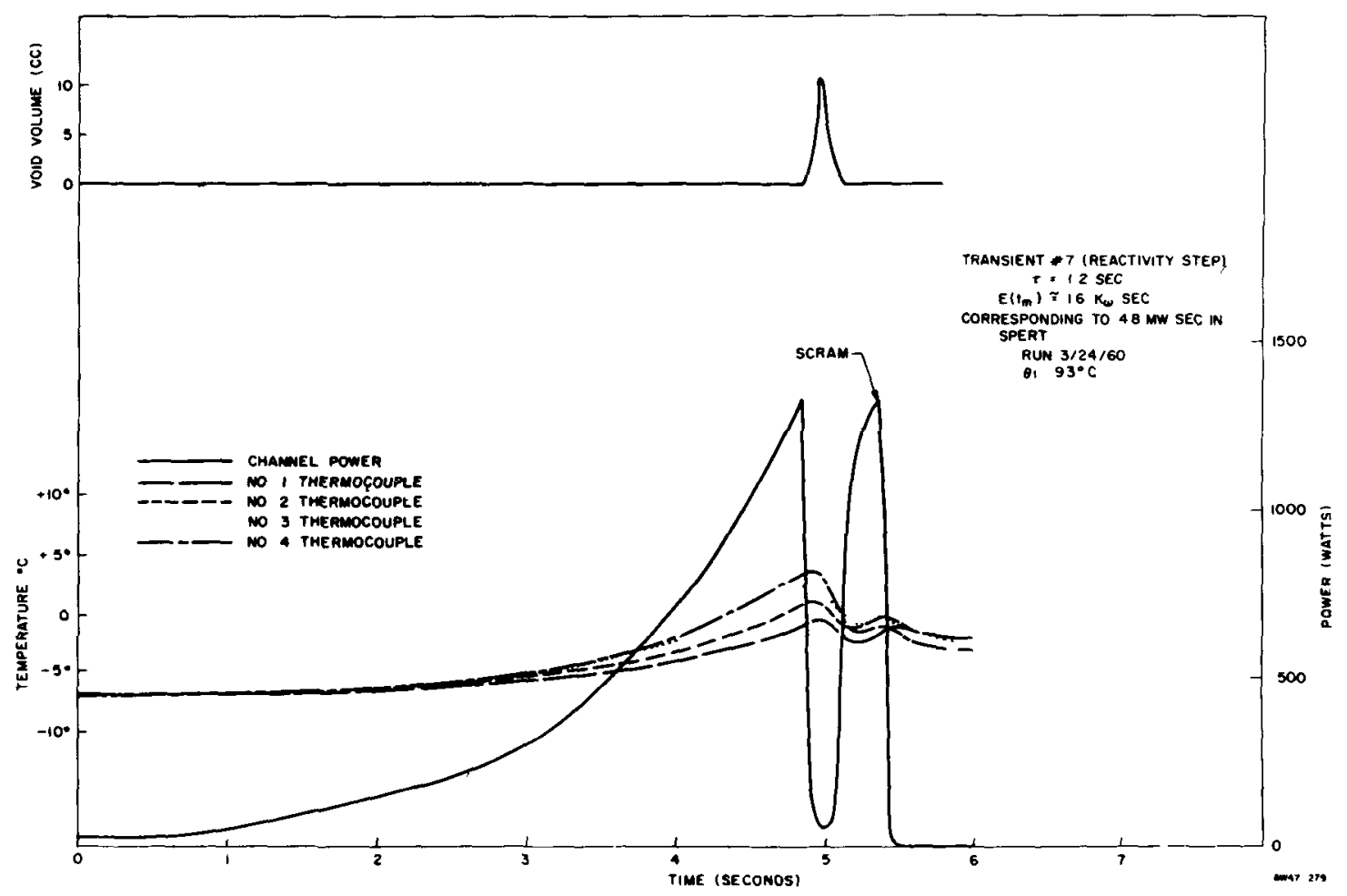

Slide 1. Typical Slow Simulator Transient Showing Void, Power, and Surface Temperature at 4 Positions on the Channel 
Return to power follows void collapse in this system because of its negligible temperature coefficient of reactivity. The sawtooth sort of curve there is the power and the top curve is the measured void volume. You see that the sawtooth power curve is quite different from any sort of reactor. The channel essentially empties in about $50 \mathrm{msec}$. I should say that this particular transient has about a one-second period. There are some temperature measurements down here that I guess I won't go into. An interesting effect that has been observed thus far--when these measurements were pushed down to a period of $150 \mathrm{msec}-$-we're trying to push further--is that the peak pressure that is observed is essentially independent of the period. With $30^{\circ}$ sub-cooling, about $4 \mathrm{psig}$ is observed on the pressure transducer. This is in direct contrast to what's been observed in Spert-IA tests where the pressure in the end-box goes about as the cube of the inverse period, $\alpha$. The observed behavior here is essentially that the whole channel just empties rapidly upon reaching a threshold superheat. The peak pressure correlation here is consistent with the picture that this threshold behavior is happening in individual channels, but in the reactor you are averaging over many channels. A similar averaging over many channels takes place in the reactor to round off the reactivity compensation and, hence, the power burst shape. The plate "superheat" seems to be, over this range, roughly independent of $\alpha$. The picture is--we are building up to the same water temperature independent of $\alpha$, putting the same amount of superheat energy in, and then the thing fires off with rapid voiding of the channel. Slide 2 shows the energy vs $\alpha$. The encircled crosses are these out-ofpile measurements with shutdown by boiling only. The other two sets of points are Spert I data in which the circles are for ambient temperature, while the squares at the bottom are for boiling. You see the behavior here in the region for $\alpha$ less than ten is essentially constant energy release. Slide 3 shows peak power. We are interested in what we will

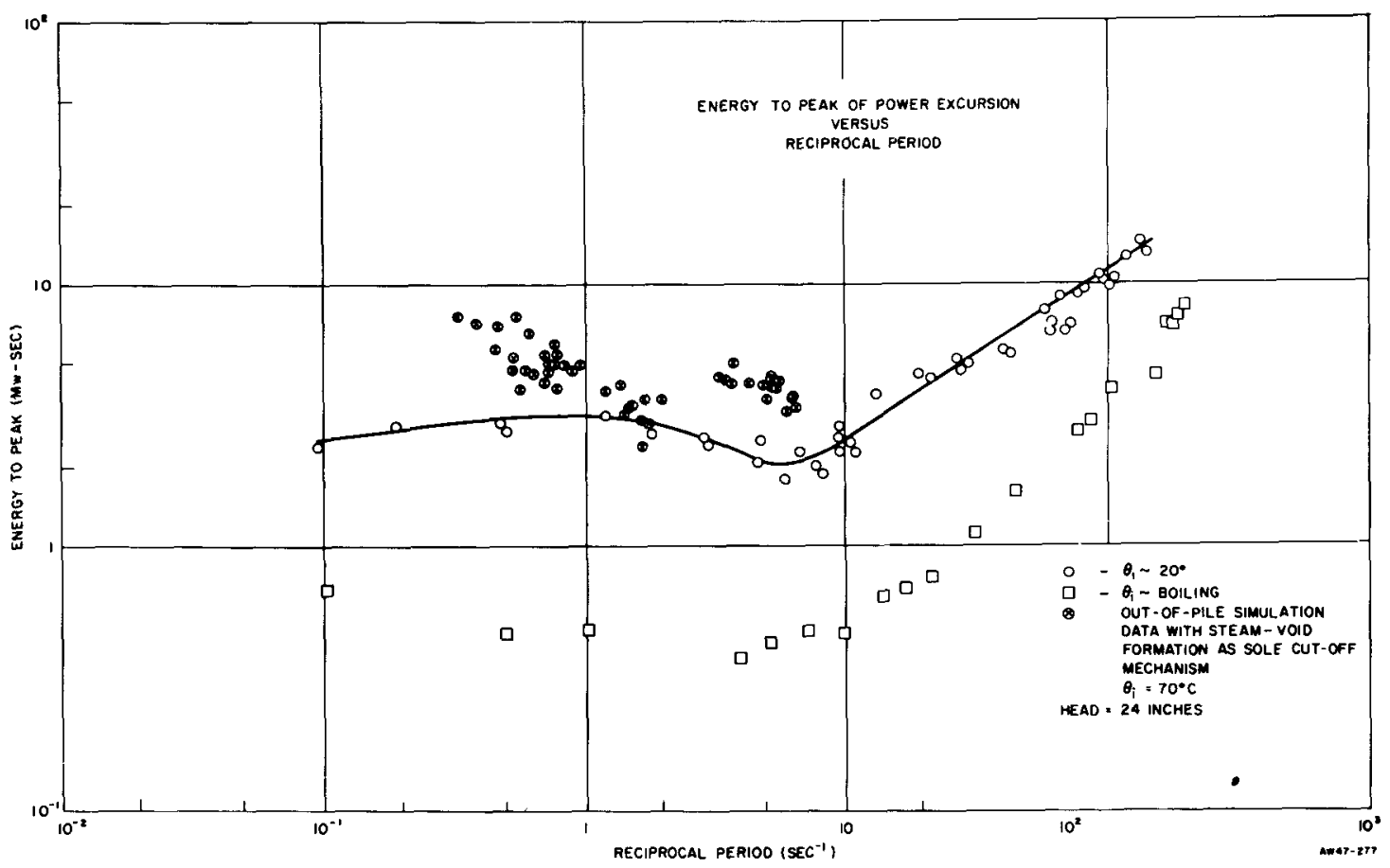

Slide 2. Energy Release in Transients in the Simulator and in Spert IA. Simulator energy release is normalized to the center of a hot channel in Spert IA. 


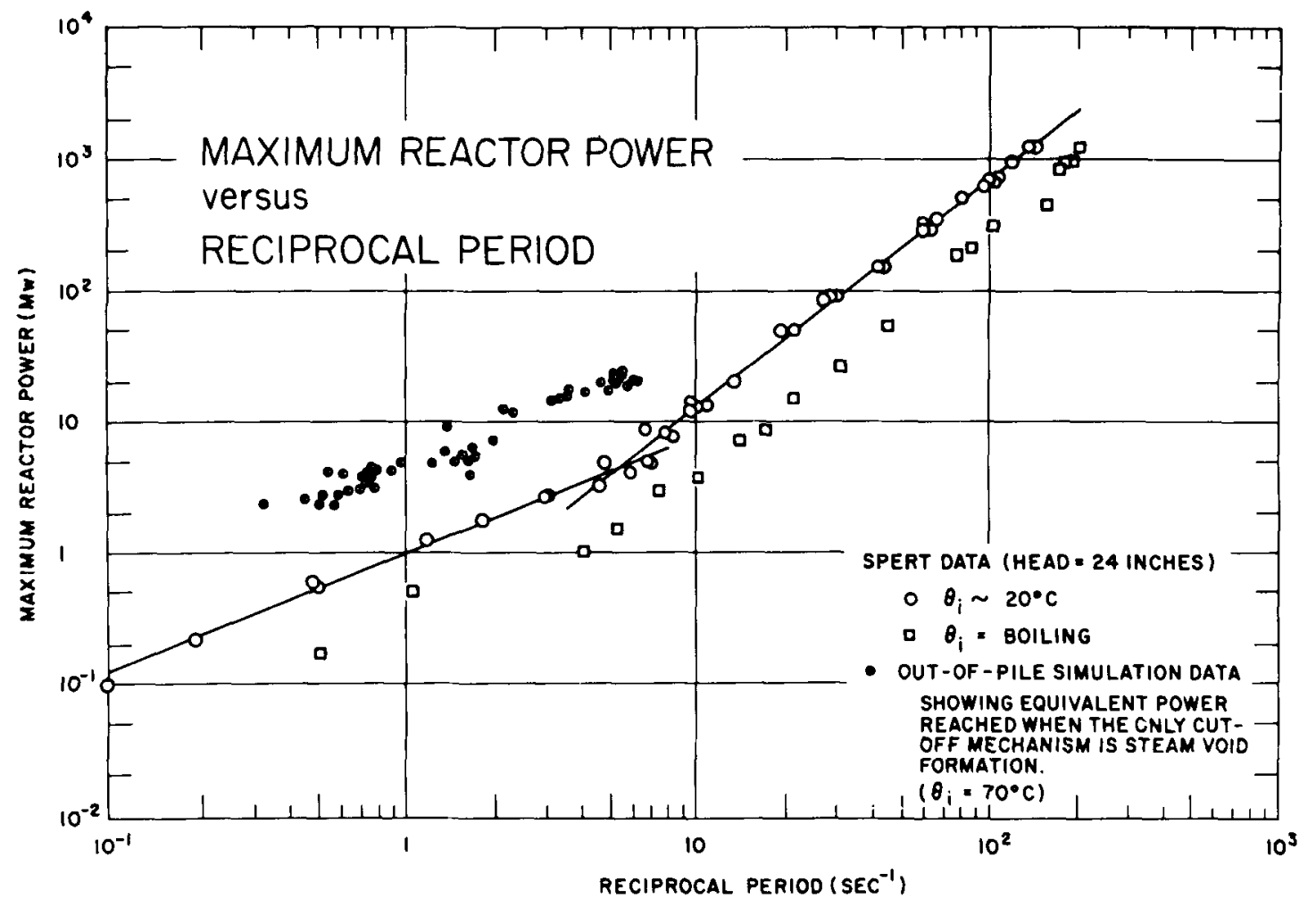

Slide 3. Peak Power in Transients in the Simulator and in Spert IA. Simulator peak power is normalized to the center of

a hot channel in Spert IA.

find out, of course, when we go on to the faster transients where boiling is the important shutdown mechanism in the reactor.

Stone: Yes, Sir?

Grace: I wanted to ask a question in connection with the proposed use of additional delayed neutron groups to represent a spatial effect--the neutrons slowing down in the reflector, room effects, and so forth. Has any attempt been made to analyze the problem directly with some modal analysis?

Wimett: I'm not sure I know just what you mean by modal analysis.

Grace: Well, rather than treat total reactor power as if space and time were separable, one should examine the waves of power as they propagate to the reflector and back.

Wimett: I think you are talking about a power reactor, aren't you?

Stone: Certainly in a core as small as Kewb or Godiva the propagation time of the higher order modes is so fast that you could not resolve these characteristic times of these frequencies. 
Cohen: We have done some preliminary calculations using sort of "bucket" models. We just had one bucket of core neutrons and a bucket of reflector neutrons; a two-group, two-region model. This is still quite preliminary but it seems, at the moment, not to be in disagreement with experiments.

\section{Stone: Al?}

Henry: There is another possible explanation of this particular series of experiments and I think it would be very interesting to try to decide what is actually happening. Is it in fact a nonseparable phenomena for which different spatial modes must be considered? Or is it a separable situation in which there is a single predominant flux shape throughout the core and reflector having a time behavior represented by a sum of exponentials

$$
\sum_{i} a_{i} e^{\omega_{i} t}
$$

the $\omega_{i}$ being roots of an inhour equation? (Actually, the spatial shapes for each $a_{i}$ are slightly different for a reflected core; but the difference is usually ignored.) Now it happens that the prompt neutron lifetime, which appears in the inhour formula, is not actually a constant but depends on the instantaneous flux shape throughout the core. Moreover, the flux shape associated with a very fast period tends to be dipped in the reflector and, as a result, leads to a shorter prompt neutron lifetime. Thus, if a series of exponential rises for a range of periods is analyzed by the inhour formula with a prompt neutron lifetime taken as constant, it will appear necessary to include another, very fast decaying, delayed neutron group. Actually, a more acceptable explanation is that the prompt neutron lifetime is period dependent, and failure to account for this makes it appear that another group is needed. Thus, the extra "delayed neutron group" could be due to a period-dependent prompt neutron lifetime or it could be due to the time behavior of some higher order spatial mode (in which case the use of the inhour formula has little justification). It would be most interesting, as I say, to try to decide this question.

Incidentally, the calibration of control rods also depends on the instantaneous flux shape. Consequently, calibrations made statically may not give the correct reactivity associated with a fast transient.

Cohen: We recognize this rod-calibration problem. This is another reason for quoting reactor transients, not in terms of reactivity, but in terms of initial stable period. There may be some indication of this flux change. A peaking of the flux in the core, if you're on a single, stable, very fast period--and you're right--it is equivalent to having the prompt lifetime change in this initial period. This is what I'm saying, the two-bucket model does not agree.

Henry: The two-bucket model assumes that there is not separability of space and time, and this is very possibly the case. It seems to me it 
would be very interesting to measure the time behavior in the core and in the reflector, simultaneously. That would decide between whether one should go ahead and try to analyze in terms of two-bucket models or in terms of a single mode.

Cohen: We may be able to get a little bit of information of this sort from the capsule experiments. We have a bellows capsule with which we measure the flux by measuring the void volume. The expansion in the capsule will measure the power level.

Wasserman: I would just like to ask you, if there were no difference in period of two counters, one in the center of the core and one in the reflector, what did you say this would imply?

Henry: It seems to me this implies there is a single spatial mode, that there is separability in space and time and, therefore, that the inhour formula, if used with a period-dependent prompt neutron lifetime, is quite appropriate.

Stone: You might see this in Godiva; you might see it in Kewb where we apparently have a varying inhour relationship.

Henry: Kewb might be better because the reflector is very near the core; it's graphite, and you would expect this phenomena to be accentuated.

Nyer: Spert II might be better, A1, because of the heavy-water reflector.

Henry: Yes.

Stone: Jim?

Boland: I would like to comment a little bit on this. During oscillator tests, both at EBR-I and at Treat, we observed a phase shift of the power signal with respect to the reactivity that was dependent on detector location. Treat was the more dramatic example of this, possibly because of its large size, long lifetime, big reflector, graphite, etc. At five cycles per second, I think we measured an extraneous phase shift of something like five or six degrees with the detector in the reflector compared to that measured with the detector in the core. We have also measured the period of the reactor using a detector in the core and a detector in the reflector and have not been able to distinguish between the period measured either place. Of course, our periods have been long compared to what some of the other people are talking about.

Stone: I don't believe you can see this effect in Treat; if you make the corrections for the heat capacity you find a constant shutdown coefficient, this then infers that you have a constant prompt generation time. The same is true in Triga; we can see variations in Godiva and can already see variations in other cores.

Henry: The fact of having a period changes the flux shape. $\mathrm{D}_{2} \mathrm{O}$, as Warren said, would be the best medium in which to observe such an effect; there the absorption is practically nothing until you get up to fast 
periods (Power $\sim e^{\omega t}$ ), In which case, the "effective absorption" (mathematically represented by (the true absorption cross section plus $\infty$ )/(neutron velocity)] would become significant and would in effect lead to a core with much different reflector properties.

Snyder: Is anyone familiar with an analysis that Dick Feynman made in about 1944, where he found that, if you use a modal analysis, the reflector could be considered as giving a fine structure to each of the core modes, and you could mu into situations where all of the--these different modes have about the same period. In effect, this would mean you get a slowly varying shape. I don't know whether this would help or not, but it is an easy way to do it.

Stone: Eugene Wigner also made an analysis on propagation times in the reactor on just this oscillator response question.

Cohen: Well, of course, there is another effect of the reflector if you make this modal analysis, it slightly changes all the delayed neutron theories. That is, if you make a modal effect, the second mode has slightly different delayed neutron eigenvalues, if you can identify the prompt neutrons, the overall eigenvalues, and then the other six delayed neutron eigenvalues. Those are also slightly different and that further smears out the inhour equations.

Stone: I would suggest that we continue this discussion under more appropriate conditions at Trail Creek Inn.

Nyer: I think there is one point to be discussed before you leave. Glenn Bright said there's a little confusion on the part of some people as to what is going to happen tonight, and what is going to happen is that there will be buses at $6: 15$ on this side of the Challenger Inn which will take us to Trail Creek Inn. The party will be inside--you aren't going to be asked to stand outside in the rain or snow.

Stone: I would like to take this opportunity to thank Frank Schroeder and also the panelists for a very pleasant and informative afternoon. 
- 
SESSION III

REACTOR TRANSFER FUNCTION

DETERMINATION AND APPLICATION

Chaiman: W. C. Lipinski (ANL)

Review Paper: J. A. Thie (McL-R)

Panelists: J. N. Grace (W-Bettis)

E. P. Gyftopoulos (MIT)

H. J. C. Kouts (BNL)

E. R. Silver (ORNL)

F. Storer (APDA)

A. A. Wasserman (PPCo)

\author{
Opening Remarks \\ by \\ W. C. Lipinski (ANL)
}

Lipinski: Yesterday we heard some excellent papers and discussions on the transient behavior of reactors through multi-decades of operation which is essentially nonlinear. This morning's session is going to be devoted to reactor transfer function determination and application, which will necessarily be limited to small signal analysis, and unless some of the panelists or attendees have information to offer in terms of models including the nonlinear aspects, we will probably hear everything devoted to the small signal analysis. The May conference covered transfer function measurements and reactor stability analysis, and today's session following 5 months later should be rather limited in terms of presentation of new material due to the short lapse of time in between these sessions.

The transfer function measurements are primarily of importance in terms of the evaluation of power operation of reactors. The transient cases cover the startup operation, whereas the transfer functions are very beneficial in determining the stability of a power plant and its maximum capabilities. $\mathrm{Mr}$. Joe Thie will present the review paper and bring us up to date on reactor transfer functions. Mr. Thie. 
0 
REVIEW OF REACTOR TRANSFER FUNCTION FIELD

by

J. A. Thie (McL-R)

Thie: The purpose of this paper is a rather general and broad coverage of the reactor transfer function field, and is not intended at all to present data and details of theories and the like. I think this is well covered in the literature. To the extent that certain recent developments are not covered, I'll mention a few, and I hope the panelists and the discussions from the floor will bring out interesting recent details. Most of the ideas that we'll cover in this paper I think will be applicable to many reactor types. You'll probably find the boiling reactor being used as an example because of the previous experience of the speaker, but I think if you're good at abstracting methods, you'll find that the examples we cite will be quite generally applicable; and insofar as applications of transfer functions go, we don't intend to cover all of these. If any important ones are left out after this paper and the review panelists are through, I hope the discussion from the floor rounds out the field.

We'll divide this paper into three categories: the first, touching on experimental methods involving transfer functions and related more general tests; second, the successes and/or failures of these experiments (unfortunately, I may talk mostly about successes because people do not report their failures); and finally, a category which might be called unsolved problems. This last category might be of interest just to see where we stand today and what we might do or what we should be looking at.

To start the ball rolling, let's look at the following list of incentives for running transfer function tests:

Oscillatory Tendencies in Reactors

Feedbacks to power (internal)

Flow

Startup

Xenon

Feedbacks from plant

Of course, the academic incentive is just to get dynamic information about your reactor. I think we all know that. But, as Lipinski mentioned, one does use transfer function tests, especially on power reactors which have oscillatory tendencies, in order to experimentally and on the spot, actually see where they stand in regard to these oscillatory tendencies. A few of these are mentioned here. The first, internal feedbacks to power, which covers a host of potential oscillatory tendencies like the one in EBR-I and like the one you see in many boiling reactors. The second, a flow oscillatory tendency I would like to distinguish from the first. One presumably might have a flow instability while running at constant power. In this case, you would have the reactor acting just as an instrument 
which measures the flow instability by virtue of--say, the voids changing or something, rather than the reactor being an inherent part of it. Of course, sometimes you can't separate the first and the second. The third one is a novel oscillatory tendency that was observed in EBWR, in starting up at low powers and low pressures. Very interesting oscillations would develop. They are interesting in the sense that they were anharmonic; that is, they were not sinusoidal, yet they were quite small in amplitude. The xenon-oscillation tendency is well known, of course; and the final category, feedbacks from the power plant. An example which occurs to mind on that occurred once on Borax-III. We were operating a feed-water pump, somewhat outside of its designed range, and my recollection is that the pressure-flow characteristic of the pump in the range we were operating was unfavorable and a feedback resulted, giving a 30-second oscillation to the entire power plant system, the reactor plus condensate-return pump. These, then, are some of the incentives to look at transfer functions because, presumably, one might hope they would help one detect tendencies well before they give one any trouble.

It isn't necessarily true that transfer functions are a panacea that will inevitably detect any tendency that might develop. In particular, one might envision oscillatory tendencies which have a sharp threshold in some nonlinear phenomena, and below that there is no indication whatsoever. An example of this type of oscillation in electronics would be your neon oscillator; that is, a condenser, resistor, neon tube, and battery combination. I don't think transfer function tests would indicate that your neon circuit is going to oscillate, yet, if you raise the battery voltage enough, it will. So there are other problems in detecting these oscl1latory tendencies, and we might look at what one might do to cover those which are not predictable by transfer functions, if there are any of that nature. here.)

The following is a table without data. (I promised not to show data

Excitation Form

Oscillation

Step

$f(t)$
Excitation Mechanism

Control Rod, Dynamic Variable, Self

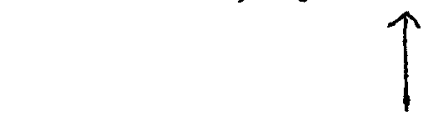

Test Type

The reason will be apparent. I intend to divide dynamic tests into this type of organization: the form of your excitation (the time function that your excitation mechanism is giving) versus what the excitation mechanism is itself. Where the abscissa and ordinate intersect defines the type of dynamic test you are running. This is not necessarily a complete organization of all tests, but it covers most of them of interest.

In the first column of the first row, where the control rod is being oscillated, is the transfer function in its narrowest sense. One oscillates a control rod sinusoidally and measures the reactor power and the reactivity. 
The quotient, of course, is the usual transfer function.

However, one should think more generally when he talks about transfer functions, and possibly we should encourage transfer function measurements in which something else is being excited, some dynamic variable other than reactivity. This has been done and, in particular, I think Borax-V will do some of this. They have an oscillatory flow valve that will be oscillated as the exciter.

Self-oscillation as an excitation mechanism (the last column of the first row) is of some interest, since it's about the most hazardous way you can mun a transfer function. You pick a system which is already in the unstable state, and it is exciting itself, and if you measure all the dynamic variables, such as pressure, temperature, flow, etc.--and power, of course--then by taking quotients of the various amplitudes and noting the phases, you are essentially evaluating the transfer function at the resonant frequency. This has been done for the Borax-II quite extensively, mainly because that reactor was quite free to take into the unstable state at liberty, and those days may be gone, but nevertheless, a lot of information was obtained by munning the reactor in an unstable state.

The next row, the excitation form being a step (the step control rod test, of course) was talked about in great detail yesterday. However, there is a different philosophy, I think, in this session as opposed to yesterday's. The analysis of the step tests, especially taking place over many decades, tends to be along the lines of time analysis, whereas the analysis of step tests which are just within one decade can be done in frequency space; that is, the Laplace transform solution of the problem represents the convenient way of doing it. So step tests where the control rod is moved a little bit to raise the power maybe 10 per cent -these have been done quite extensively, and for obtalning transter functions. The step you put in, of course, contains all the frequencies, so in principle you get the entire transfer function by just dividing out the Fourier transforms of your step and your resulting power. EBWR and Borax-IV have done this, and along the same lines a rather interesting step test was done, I think, on PWR, not to measure so much the power-to-reactivity transfer function but rather to excite a potential xenon instability. The reactor presumably of its own accord would not go into xenon instability, but the control rods (I heard this about 3 years ago; it's a little vague in my mind, maybe) were tilted and then brought back to a level point. This step excited the xenon flux tilt effect, and one was able to get information that way. Step tests are easier, of course, than oscillatory tests. However, they lack in precision and you may have to do a hundred steps to get the same precision as for a hundred oscillations.

Dynamic variables can be stepped. This is the most convenient thing to do in a power plant, and power plants are doing this. They drop a load, or they turn off the flow, turn on the flow to a higher value, etc.

This type of testing I think should be encouraged, and also the analysis of it can proceed along the lines of transfer function analysis. Or, it can be done in time space, depending on the philosophy of the person doing the experiment. 
Finally, in the last column of the second row, one is doing steps which are somewhat accidental; that is, the excitation mechanism being the system itself. This would be an accidental experiment, in my opinion, and typically is not of much value. There are examples where reactors have spontaneously excited themselves but, typically, these are not used for scientific measurements.

In the final row the excitation form is anything else, $f(t)$, not previously covered. If the mechanism is a control rod, we might cite in this area a novel experiment which took place on ZPR-III, a fast critical: the control rod was moved at random, and this is a function of time which lends itself, of course, to a transfer function analysis. I think succeeding speakers will talk about this in more detail for other systems. Another thing that has been done where a function was generated by the control rod to get dynamic information, was by the duPont group at Savannah River. They used a trapezoidal rod movement, something that could be done in almost any reactor. The information one gets is restricted to the low frequency range, but I think they were able to actually measure two time constants, a slow one and a fast one, in their system by means of an appropriately chosen function (some trapezoidal function) of the control rod. These experiments, of course, are more complicated than oscillatory ones, but sometimes, being more easily done, one accepts the complication and puts it into the analysis.

Where the dynamic variable is some arbitrary function, an example that occurs to mind here is a very simple experiment on EBWR. The operator moved the feed-water or condensate-return pump in a trapezoidal function, and all the system variables were measured and transfer functions were measured from one to the other. There, again, is something that was quite easily done on a power plant and lended itself to obtaining transfer functions in regard to quantities other than just reactivity and power.

And, finally, in the last category where you have a self-excitation in an arbitrary way, the most popular example here, I think, is the use of noise analysis. You do not necessarily obtain a transfer function when you do this, but I don't think you really have to. You obtain a frequency spectrum of the reactor, which is an end in itself.

We have now looked into the various categories of dynamic experiments and many, in fact almost all, of them lend themselves to analysis in frequency space; that is, transfer functions. The data, of course, that you get out of these is in proportion to how ambitious you are instrumenting the experiment. I think we are doing better now than we were in the original dynamic experiments on early Boraxes. One thing I'd like to mention in particular: the voids in a boiling reactor are a very crucial variable which are very elusive to measure as a function of time. You might infer them from the reactivity as the function of time, but that is not a direct measurement. So there has been some work at Argonne which hasn't been reported yet on the measuring of voids as a function of time and space in a dynamic system. The results obtained so far have been for a steady-state void distribution, but an attempt will be made to do a dynamic one later. The method is quite elementary and ingenious. You might guess this from its inventor, which happens to be Sam Untermeyer, who is noted for novel ideas. You just measure the cadmium ratio, and 
a. little look at the diffusion equations will convince you that the cadmium ratio (the thermal-to-fast flux ratio, virtually) is a good measure of the amount of void in the local area. So the EBWR was cadmium-ratio mapped, and this was converted to a void map, and agreed quite well with steady-state predictions. One might assume then or hope that this can be done dynamically, and there are plans to put in a pair of cadmium-ratio chambers and dynamically measure the local voids as a function of time. I think experiments of this type are barking up the right tree in pinning down unknowns in certain reactor systems. You have to measure more than just reactivity and the power to really make stringent tests of the type of theories that are being proposed to explain dynamic performance.

Now let's pass into the category of how successful have experimental attempts been. Of course, any transfer function test at all has its academic value in giving one an experiment which one can take, compare with theory, and, whether or not there are any oscillatory tendencies, some conclusions can be drawn as to how good one's dynamic understanding is. However, another goal of transfer function tests is to see how one can predict instabilities. Along these lines, there have been some successful attempts where the transfer function measurements were made at a low power, compared with theory, and then accurate predictions of transfer functions made at higher powers, and then these verified. I'm referring, in particular, to EBWR tests by deShong and Lipinsky. However, to be sure that you can always predict your instabilities, you must have a mechanism which resonates at low power and the resonance grows as you increase in power. It is not obvious that all oscillatory tendencies you meet will fall in this category, but so far this apparently is the case.

Where do we stand on a priori transfer function calculations? This probably requires honest answers from the people who are making them, and I don't intend to give any answers myself. What I think has to be done is a person should make predictions on these just like he predicts critical masses before the reactor is built and you make wagers on how close you are, and so on. I don't think this is being done yet on transfer functions, simply because the calculations probably are not as precise as the critical mass calculations.

The following equations show the analogy actually between transfer function tests and the approach to critical tests insofar as either might be a predictor of a critical system.

$$
\begin{aligned}
\text { Transfer Function } & =\frac{G_{O}}{1-G_{O} H} \\
\text { Multiplication } & =\frac{1}{1-k_{0 O} P}
\end{aligned}
$$

In one case the system is critical with respect to oscillations. The transfer function is the zero power transfer function over one minus the feedback times the zero power transfer function. The second term in the denominator approaches unity here, and the entire expression goes to infinity at the unstable point, which is well known. By the 
same line of reasoning, of course, the multiplication goes to infinity when your non-leakage probability here, $P$, gets to be $l / k_{\infty}$. So, it is well known that in multiplication tests one plots the reciprocal of multiplication against some parameter such as radius, and is able to accurately predict the criticality point. And he is even more successful if he gets fancy and compares various multiplications with theory along the way. By the same line of reasoning, one can do this with transfer functions. You might, for example, do something as empirical as plotting the reciprocal of your transfer function against some feedback parameter which controls $\mathrm{H}$, such as the reactor power itself, and have an empirical plot pointing to an unstable point. Hopefully, though, you might have a theory which will enable you to refine this and more accurately predict.

This empirical approach can really be extended over a large number of variations. You can plot the reciprocal of your transfer function; you can plot its half-width; or, if you are doing a rod-step experiment, and you get a decaying sine wave, you might plot the rate of decay, i.e., reciprocal of the decay time of any oscillatory transient you get. All of these things are proportional approaches to instability and can be empirically used as measures of how close you are.

The simplest of all is one which may have escaped us, and there will be a paper by Akcasu at the next ANS meeting on this (1); this is where you regard just the reactor power fluctuations as your measure of the approach to instability. And you plot, let's say, the reciprocal of the rms value of the power fluctuations against the reactor power. There is reason to believe that if, for some reason or other, all of these other methods, including rod oscillator tests, somehow fail to predict instability, your last ditch may be at a simple one, just the rms value. I think the details of that will come out in the next ANS meeting.

Noise tests have been used in experiments, and I think there have been some successes there. I believe some of the panelists, maybe for discussion, will report these. By means of the following diagram, let's look at the philosophy of what these noise tests hope to do.

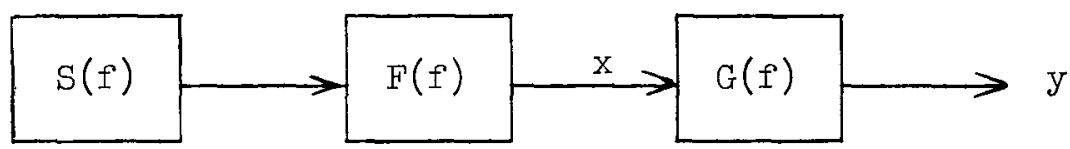

Schematically one might envision a reactor transfer function or any transfer function whatsoever between two variables, " $x$ " and " $y$ " in a system. These might be reactivity and power, but if you're ambitious they might be any two variables you have to play with. For self-excitation by noise, you have some source of noise which you may or may not know about, and this source is presumably filtered, perhaps, by some function before it really is converted to the exciting variable that is of interest. Suppose the output function, "y", is the reactor power. The Fourier spectrum of its fluctuation is not, of course, the transfer function. It is rather the transfer function times the filter characteristic, and if the noise source is not white, it even reflects the shape of that. However, as I pointed out a minute ago, you need not require that this experiment give 
you a transfer function. It is sufficient to require that it give you the power spectrum of your reactor. This in itself is a useful piece of information which actually supplements any direct measure of $G(f)$, because if you know $G(f)$, and if you have additional information about the power spectrum, you are in some position to talk about what is going on in the self-excitation mechanism.

Another category in which there have been, I think, some successful applications of experiment has been in the analyses done by the spert and the Ramo-Wooldridge groups in regard to the Spert oscillatory tendencies. The approach there is quite interesting because it shows that one can use out-of-pile transfer function measurements to predict in-pile instabilities, with rather few theoretical uncertainties. The philosophy is to measure out-of-pile, the non-nuclear aspect of your reactor. In the case of Spert, they measure transfer functions of a mocked-up Spert fuel element which is electrically heated; i.e., transfer functions between the power and the void as a function of height along the assembly, and so on. I think these will be reported in more detail later, but I would like to mention that this may be a powerful tool, since this non-nuclear aspect of the transfer function almost encompasses all aspects of the " $\mathrm{H}$ " in the transfer function formula presented above. This experimentally determines a lot of unknowns, and what remains is to just couple this with the zero power kinetics equations and a reactivity coefficient, which hopefully you know something about, and you can, indeed, come up with a reliable transfer function with a minimum of uncertainties.

There is another point that this experiment showed which is of some significance and $I$ would like to make it here. ( $I$ think it will be made again in the Reactor Instability and Thermal-Hydraulic Problem Session.) The experiment of running a transfer function between an oscillatory electrical power and voids, pointed out the difference, experimentally, between the two types of oscillations postulated for boiling systems: whether they are primarily hydraulic-flow oscillations, with power being constant, or whether they are primarily void oscillations, with flow being constant. And the results of these Ramo tests show that you can generate a resonance with a hydraulic transfer function which is not at all resonant. That is, it is actually the nuclear feedback that gave them the resonance and not the out-of-pile measurements per se.

We might pass on into the category of unsolved problems, if there are any; and I think I will mention a few, but $I$ hope in the discussions and from the floor, and so on, we either solve these here if there are solutions I am not aware of, or we add to the list, which is more likely. The following is a listing of just the general categories which might include a host of specialized topics.

\section{Problems Involving Transfer Functions}

Versatility in dynamic variables

Excitation of little understood oscillatory tendencies

Statistical aspects of transfer functions 
The first one is versatility in dynamic variables. We have been measuring transfer functions the easy way for many years between reactivity and power, but now that instrumentation is reaching its peak in regard to incore instruments, we have the possibilities of measuring the transfer function in great detail. In particular, I would envision a description of a reactor where you have $\mathrm{N}^{2}$ transfer functions, as you have $\mathrm{N}$ variables, and you have the transfer function between any two of these. Some of those, of course, are redundant, but there is really an $\mathbb{N}^{2}$ set of transfer functions if you measure enough variables. Possibly one needs more precision in some of the transfer function tests, too. If you take this analogy with critical mass predictions, if you are approaching optimum design, you have to be closer on your prediction on unstable points, or whatever is limiting you; and experiments may require more precision if one is to achieve his goal.

The second category is excitation of little-understood tendencies. I mentioned earlier some oscillatory tendencies that may not be completely understood. Let me cite two of them right here that occur to mind. Let's say that in a very large boiling system, possibly larger than Dresden, for example, there is some tendency to have a spatial void oscillation, analogous to spatial xenon oscillations. Well, how could you get information about this in advance? Possibly by doing an excitation of the system before it comes to that point, in the same way that the PWR group informed themselves of their xenon oscillation. The system is subcritical with respect to the oscillation, but it can be excited, and the flux-tilting and void-tilting effects can be measured during this excitation.

Another possibility of little-understood oscillatory tendencies is looking to see whether or not control rods can be used to obtain experimental information about oscillatory tendencies which do not have resonances preceding them. For example, the EBWR startup instabilities probably are not predictable by transfer functions, but one does not know this. One only speculates. And I think even in the case of the hydraulic instability--possibly the Ramo-Wooldridge people will shed some light on this--whether in out-of-pile transfer function tests one can, by measuring electrical power to void transfer functions, state anything about the threshold of hydraulic instability before it is reached.

The third category is one which may be relatively new to reactors, but it's certainly old in regard to mathematics and just the general theory of dynamical systems. This is the statistical aspect of transfer functions. We have been maybe a little naive in assuming that the transfer function is always a real smooth, well-defined concept having no complications, and let's talk about what complications might cloud the issue. And, in particular, we found on boiling systems these complications, and this is why I mention them. I would guess that possibly other reactor systems by analogy might run into a need of statistical analysis of transfer functions.

Let me get more specific on this point. Let's look at the following equations and postulate a resonant system. 


$$
\begin{aligned}
\left(\frac{1}{2 \pi f_{0}}\right)^{2} \ddot{y}+\frac{R}{2 \pi f_{0}} \dot{y}+y=A(t) & \frac{A(f)}{1-\left(\frac{f}{f_{0}}\right)^{2}+j R\left(\frac{f}{f_{0}}\right)}
\end{aligned}
$$

This is quite general, actually. This is the usual second order differential equation of a system which is potentially oscillatory, and there is some resistance, $R$, which if positive keeps the system in the stable condition, but should $R$ go to zero, then it could be oscillatory. $A(t)$ is a driving force. To be concrete, let's suppose that $\mathrm{y}$ is the reactor power, and $R$ is some collection of miscellaneous constants controlling the stability, and $A(t)$ is the reactivity noise in the bubbles or the boiling boundary or something. This equation is an approximation to describing the system near its oscillatory peak in the transfer function. Next is just a solution of that equation by Laplace transforms, and we see that indeed this is a resonance. The resonant frequency, $f_{O}$ and $R$ appear in the denominator, and in particular, if $f$ equals $f_{0}$, the transfer function becomes quite large, especially if $R$ approaches zero.

Now, where can the complications arise? Well, first of all $A(t)$ need not be a smooth sinusoidal control rod motion; it might be, in the case of self-excitation, a random function, and possibly a random t'unction with a constant Laplace transform, which for simplicity would help simplify things. But let's suppose also that $R$ is not constant, but also random. Now this may be more interesting: can you solve the equation this way, first of all? Mathematically, of course, you can't, but physicists do these things, anyway. And you find there's a certain limit where this solution might be approximately true, and let's assume that $R$ is slowly varying, compared to the time it takes to go through a resonant cycle. Then, roughly speaking, you have a transfer function which is slowly varying as $R$ fluctuates around at random.

We have reason to believe that this may be a model which would have some applicability in boiling systems. Let's refer back to the block diagram illustration for noise tests. Keeping in mind what we have just talked about, the system here is being excited by some noise. Let's suppose that $G(f)$ is constant in time, as one always assumes. Then what will be the time function that you get if $G(f)$ is highly resonant and being excited, let's say, by a white noise? This time function, regardless of what system, that is, reactor or electronic, tends to be a series of wave-trains. Actually, I noticed some on this conference's program manual (see cover of Program): those real little wiggles are exactly what you see on reactors. It's wave-trains that sort of come and go, and they slowly rise and they slowly decay. And, in particular, you can show that the average length of the wave-train is related to the halfwidth of the resonance in a very simple fashion. The sharper the halfwidth, the longer the average length of the wave-train. Roughly speaking, one is the reciprocal of the other. This, incidentally, is a very good visual indicator of an approach to instability. You just, with your naked eye, inspect the average length of wave-trains and take the 
reciprocal mentally and you have approximately the half-width of your transfer function; this is the most simple transfer function device on the market, the eye and the human brain. However, let's suppose it's more complicated than this. We have found on boiling systems this can be the case. In addition to observing wave-trains which come and go very slowly, as one might expect theoretically from a system where white noise excites a very sharp resonance, let's suppose that $G(f)$ is not constant, but instantaneously, maybe once every three or four seconds, it becomes infinite. Then superimposed on this phenomena you have another phenomena where, when the $G(f)$ has become momentarily unstable, you get exponentially rising sets of oscillations, which (if you have the guts not to scram) will damp out and go back into small wave-train patterns. The reason this model has been postulated is that we have observed systems which give almost in the same reactor condition both types of behavior; large divergent oscillations as well as small wave-trains.

So, I think there are some interesting theoretical problems, and experimental problems as well, in systems which have statistical fluctua tions, not only in excitation mechanisms, but possibly also in controlling constants. When you approach an unstable point, slight changes in almost anything can shift you momentarily into instability. So it's not at all unreasonable to postulate the possibility of these things. This is a potential area for exploration.

In particular, one might speculate, "What can a transfer function test do in instances where you might expect or look for non-constant transfer functions?" Well, typically, the transfer function measurement extends over all time. One averages one's signal over a long length of trace, and so on. However, there might be more information at one's disposal, and I think the next equation might show an example of what might be possible should the transfer function have a dispersion.

The Dispersion of a Transfer Function

$$
\frac{\Delta G}{G_{\infty}}=\frac{I}{G_{\infty}}\left(\sum_{I}^{N} \frac{\left(G_{i}-G_{\infty}\right)^{2}}{N}\right)^{1 / 2}
$$

The term $G_{\infty}$, let's say, is the transfer function measured over an infinitely long trace. Then this is just a standard dispersion formula if you measure the transfer function over a finite length of time. Suppose $G_{i}$ is the finite transfer function. For example, it might be measured over as few as three or four cycles in the resonant range. Then $G_{i}$ will not equal $G_{\infty}$, of course, but the average value of $G_{i}$ is $G_{\infty}$. The dispersion, then, in the transfer function is given by the standard formula. Now this dispersion might result from two things. First of all, you have a noise which is exciting your system but, secondly, even if you do not have a noise, you may have a fluctuating transfer function itself. Then if this is the case, the dispersion actually measures the fluctuating resistance, or whatever is controlling it. So the only point is, there may be some additional information one can obtain from more flexible transfer function tests measured over finite lengths of time; that is, looking into the statistical analysis of the experiments. 
Since the ball game is about to start, I didn't want to talk too long. I hope the succeeding panelists bring out various points which will round out the picture.

\section{REFFEREIVES}

1. A. Z. Akcasu, "Mean Square Instability in Boiling Reactors," Trans. Amer. Nuclear Soc., 3, pp 475, (1960). 
0 
SESSION III

PANEL PRESENTATIONS

Lipinski: Perhaps instead of taking a break now, it might be best to pass on to a few of the panelists, and then take a break a little later when the ball game gets interesting. Our first panelist will be Mr. Gyftopoulos from MIT.

\section{A Statistical Stability Monitor \\ by \\ E. P. Gyftopoulos (MIT)}

Gyftopoulos: I would like to talk about a statistical stability monitor. The title I am using is for "commercial purposes" because I know that Dr. Kouts is going to describe something similar.

The monitor is based on the method of using externally applied statistical signals to get information about the transfer function of a system. Currently, an MIT graduate student is working on the method at Los Alamos, and I think today, if things run on schedule, he will be trying it on one of the Rover reactors.

Basically, the method consists of the following: Suppose that we have a system which is characterized by a system function $h(t)$ and that we wish to determine this function experimentally. Then, as we heard from the previous speaker (Mr. Thie), there are several methods, such as oscillation tests or correlation of inherent statistical fluctuations, which we can use to derive the desired information, either in the frequency or in the time domain.

Another method, relatively new in the nuclear reactor field, is to excite the system under investigation by an externally applied random input $R(t)$, measure the corresponding output $O(t)$ and crosscorrelate the input with the output. It turns out--and I will not go into the details--that the crosscorrelation of the input and output is equal to the convolution integral of the system function and the autocorrelation function of the input:

$$
\phi_{\mathrm{RO}}(\tau)=\int_{0}^{\mathrm{T}} \mathrm{h}(\mathrm{t}) \phi_{\mathrm{RR}}(\tau-\mathrm{t}) \mathrm{dt}
$$

where

$$
\begin{aligned}
\phi_{\mathrm{RO}}(\tau)= & \begin{array}{l}
\text { the crosscorrelation of input and output over a } \\
\text { period } T
\end{array} \\
\phi_{\mathrm{RR}}(\tau)= & \text { the autocorrelation of the input over a period } T \\
T & =\text { the settling time of the system function. }
\end{aligned}
$$


If the random input is chosen appropriately, and in particular if it is chosen so that the autocorrelation function has an impulse-like shape (delta function), then the convolution integral reduces effectively to the value of the system function at time $\tau$ :

$$
\phi_{\mathrm{RO}}(\tau)=\mathrm{h}(\tau)
$$

In other words the crosscorrelation function is equal to the system function.

A random function with an impulse-like autocorrelation function can be created by several methods. One way is to use a binary chain which resumes values plus or minus one unit with equal probability and may (but need not necessarily) change sign at event points spaced at $\Delta t$ seconds apart. If the correlation time were infinite, this binary chain would have a triangular autocorrelation function of half-width $\Delta t$. However, since in any practical case the correlation time is finite, in general the autocorrelation function is more or less as shown in Figure 1. In order to avoid the side lobes of the autocorrelation function, which are sources of unpredictable errors, one can use special idealized binary chains. Such chains have been developed and, among other requirements, they fulfill the condition that they are periodic of period $T=N \Delta$ t where $N=251$, 1019, etc. The autocorrelation function of an idealized chain is shown in Figure 2.
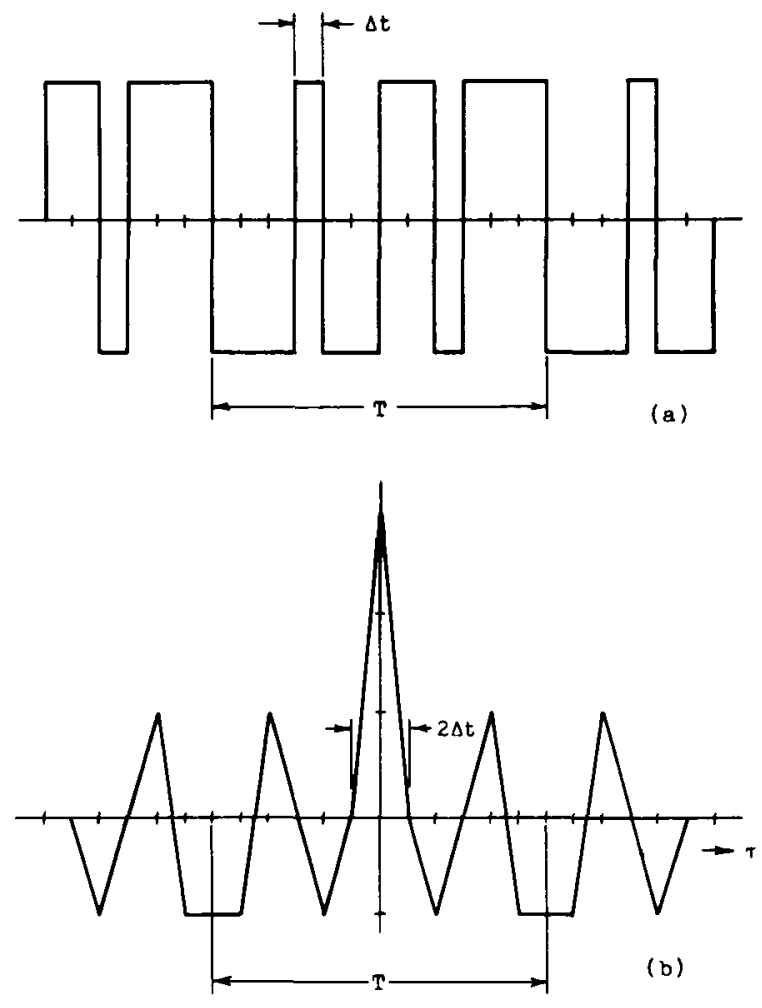

Figure 1. Arbitrary random binary signal (a) periodic over $T$ and autocorrelation function (b) of the same. The autocorrelation side lobes are introduced by the finite correlation time $\mathrm{T}$. 


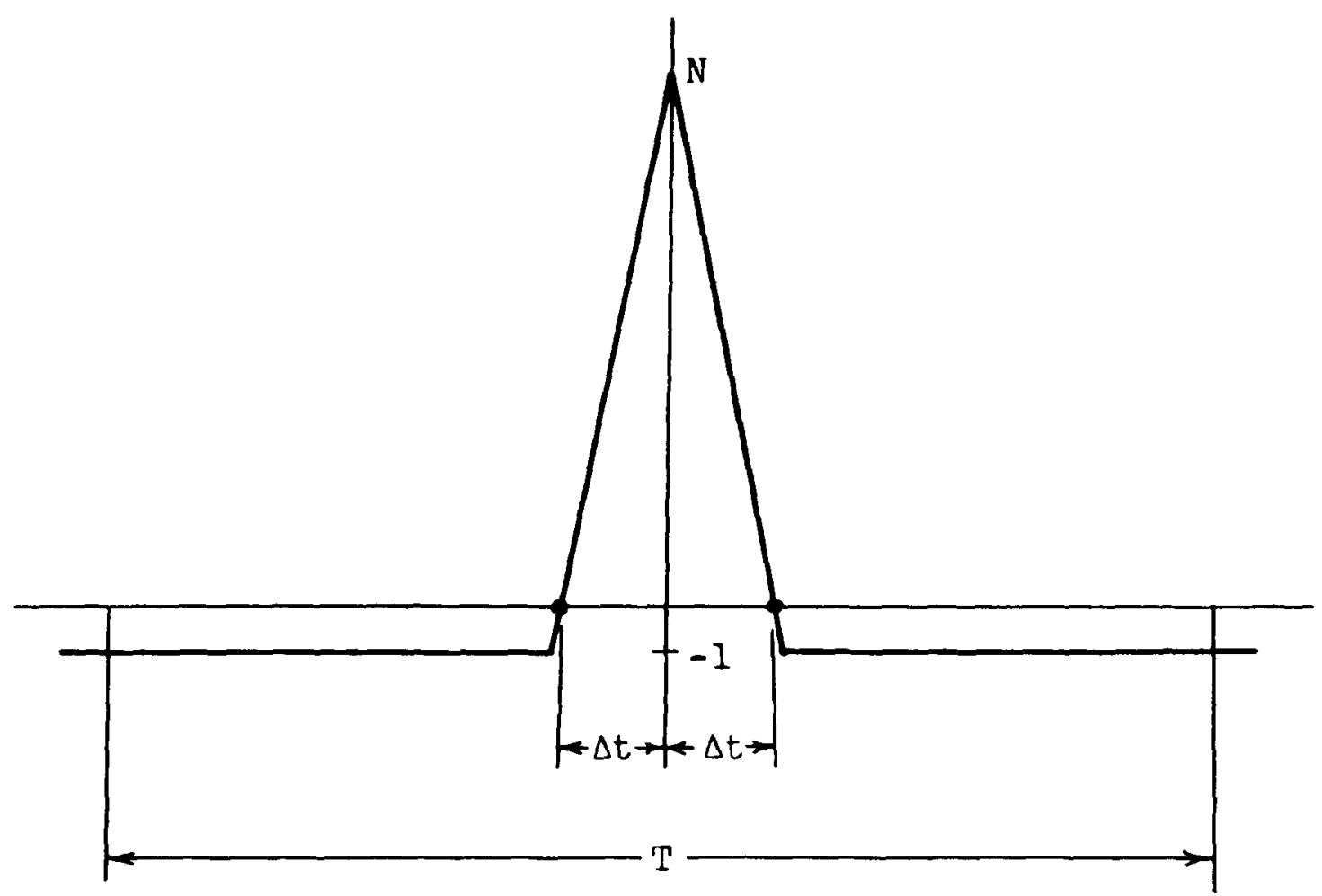

Figure 2. Autocorrelation function of an "idealized" random binary chain, periodic over $T(T=N \Delta t$, $N=251$ or 1019 , etc.)

The constant value of the autocorrelation beyond the spike is relatively small and can be easily accounted for in the experimental results. In particular:

$$
\phi_{\mathrm{RO}}(\tau) \sim \mathrm{h}(\tau)-\frac{1}{\mathrm{~T}} \int_{0}^{\mathrm{T}} \mathrm{h}(\mathrm{t}) \mathrm{dt}
$$

The meaning of Eqs. (I) or (3) can be graphically interpreted as shown in Figure 3. Suppose that the system function is somewhat as in Figure 3a. If the autocorrelation function of the input is as in Figure 3b, the convolution integral (l) implies effectively that the system function is sampled at the time $\tau$, determined by the delay time of the cross-or autocorrelation. Notice that the correction term in Eq. (3) can'be determined by using a delay time $\tau=-\Delta t$.

Well, this is the essence of the crosscorrelation method for the derivation of the system function. The latter, of course, can be easily transformed into the frequency domain to yield the transfer function. Now it is a simple matter to use this method and build a stability monitor. More specifically, assume that a system is excited by an idealized binary chain as shown in Figure 4. The input is delayed, say by an amount $\tau_{j}$, and multiplied by the output. The product is fed intc an integrator which integrates over a period $T$. The output of the integrator is approximately the value of the system function $h\left(\tau_{j}\right)$ at the time $\tau_{j}$. By introducing a series of delays, of different values, and a series of multipliers and integrators in parallel, we can have a series of points of the system 


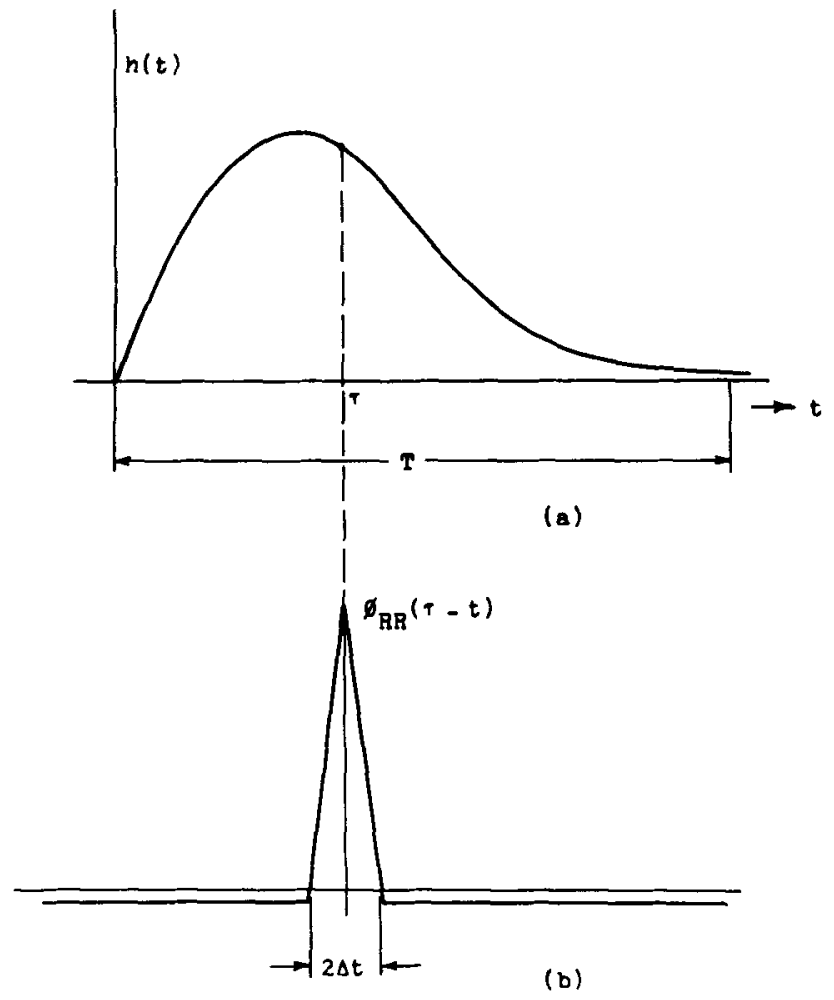

Figure 3. Typical system function $h(t)$ (a) and autocorrelation $\phi_{\mathrm{RR}}(\tau-t)$ of an "idealized" random binary chain $(b)$. The convolution of $h(t)$ and $\phi_{R R}(t)$ is equivalent to sampling $h(t)$ by the window function $\phi_{R R}(\tau-t)$. This sampling yields $\sim \mathrm{h}(\tau)$.

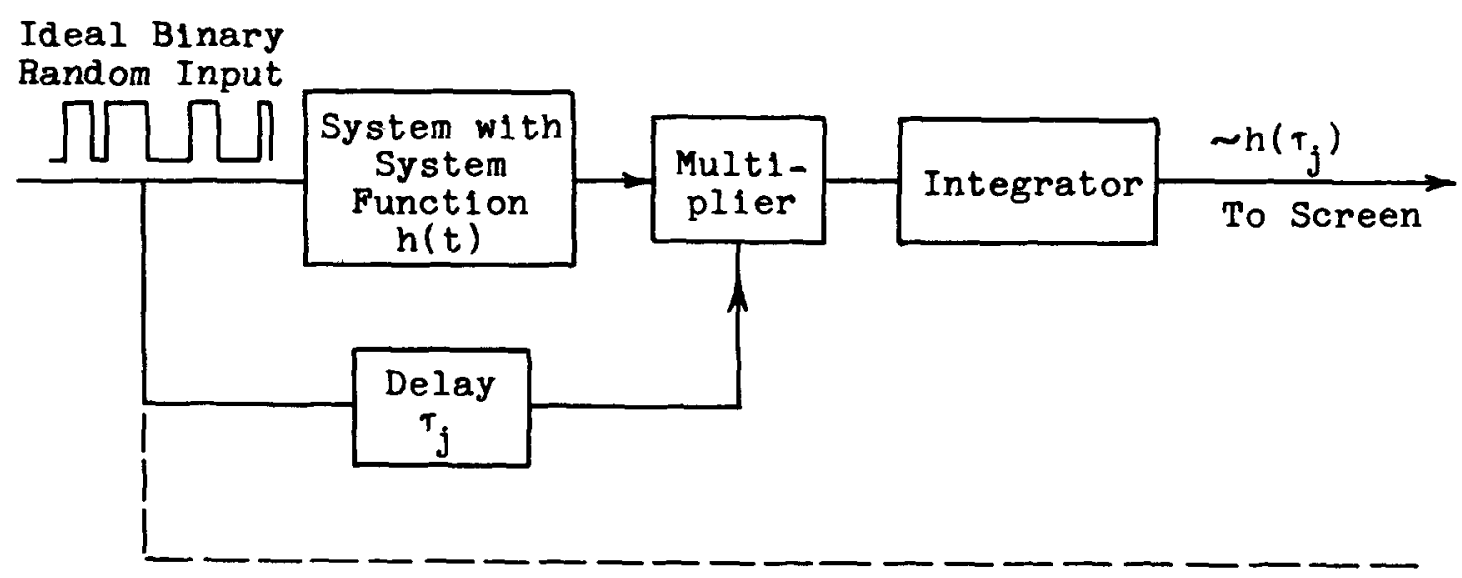

Figure 4. Schematic of Stability monitor or of experimental setup for the measurement of the system function by the crosscorrelation method. 
function. These points can be displayed on a screen to provide a continuous visual record of the system function. From the behavior of the system function we can easily infer whether the system is approaching instability or not as it was pointed out by Dr. Thie.

Equipment of the type suggested by Figure 4 has been built by my student, Mr. Balcomb, at Los Alamos. This equipment has been tested on Godiva followed by a second-order filter and the results were very satisfactory. The equipment was also tried on several simulated reactor systems with equally satisfactory results. Today or in a couple of days the same equipment is scheduled for use to study the dynamic characteristics of KIWI-A3 at the Nevada test site. I hope to report to you the experimental results in the near future.

The crosscorrelation method has several advantages over other methods used for the measurement of the system function of nuclear reactor systems.

First, one can get the entire information about the system function $h(t)$ in a very short time, as opposed to the case of oscillation tests. This is due to the fact that when the crosscorrelation method is used all the frequencies of the spectral density of the reactor system are excited at once while in the case of oscillation tests each frequency must be excited separately.

Second, the crosscorrelation method is not as limited as other methods by the presence of internal noise. The reason why this is true is because the random input has different statistical properties than the inherent noise and therefore during the crosscorrelation process the noise part of the output, due to internal noise, crosscorrelates out. This property is somewhat limited by the finite time of correlation; however, the experimental results so far show that the limitation is not very serious.

Third, since the crosscorrelation method is not limited by internal noise, one can introduce a very small amplitude signal and perform the experiment safely and without consideration of nonlinearities. Of course, the smallness of the input is a function of the correlation time (if you like, it is a function of the error that can be tolerated). The indications are that in a really noisy system the input may have to be as large as the inherent noise. However, this can be traded with correlation time.

A fourth minor advantage is that the experimental equipment necessary for the use of the crosscorrelation method, whether used for transfer function measurement or as a stability monitor, can be accommodated in a small package, say, $10^{\prime \prime} \times 10^{\prime \prime} \times 20^{\prime \prime}$. Los Alamos is planning to build such a device and have it used for reactors which are available for testing only for a short period of time.

Now, there was another comment that I wanted to make, which I forgot-no, I guess that's all. 
Unidentified: Isn't this the same system essentially that Goodwin developed for testing a cat-cracker several years ago?

Gyftopoulos: I am not aware of this particular development, but I wouldn't be surprised because this development came out of some work that Professor Lee did at MIT for the statistical study of communication systems. He made use of correlation techniques for cases where the signals involved are characterized by statistical properties rather than analytical functions. The method is also well known in the field of automatic control.

The comment that I wanted to make earlier is that on account of mechanical limitations of the equipment and economic considerations, it may not be easy to evaluate a system by this method if the bandwidth of the system is very large. Now, by "large" here, one means about 50 cycles per second. However, in case you are interested in the impulse response of a system as a whole, like a nuclear reactor system, the bandwidth is usually not that large. It may be of the order of 10 cycles per second, and then the method is applicable with no need for elaborate electronic instrumentation.

Unidentified: It has one disadvantage for a power reactor application, which is that you have to have a disturbance mechanism operating in the reactor continually, and this may not be too convenient in large power stations.

Gyftopoulos: Yes, but if--and I'm not certain yet how much reactivity it would require--if the noise doesn't bother you and if the signal you have to put in is not very large, it seems to me that it wouldn't be very difficult to put a small control rod in the reactor, which you oscillate.

Unidentified: Or you could oscillate the flow or something selse.

Gyftopoulos: You could do it this way and you introduce the kind of versatility that Dr. Thie was talking about. Of course, you would get a different kind of transfer function--but this serves the purpose just as well.

Unidentified: If it's noise in the system, it doesn't matter much in which variable you put it on.

Gyftopoulos: Exactly, but you would have a different kind of transfer function. 
SESSION III

PANEL PRESENTATIONS

Lipinski: Our next panelist is Mr. Kouts from Brookhaven.

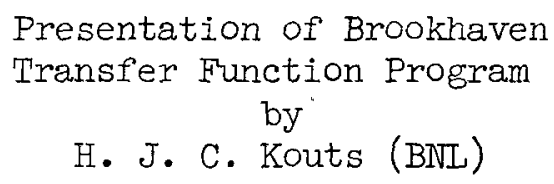

Kouts: Gyftopoulos and Brookhaven have had graduate students running neck and neck for awhile now on the problem which he's just introduced. As Joe Thie mentioned, this system has received attention for some time, and it is beginning to yield results in several places. Now, the graduate student at Brookhaven who has been concerned with transfer function analysis is Mr. Rajagopal, from Rensselaex Polytechnic Institute, and he has been studying exclusively this problem in the frequency domain-that is, use of random inputs and statistical analysis techniques for the finding of transfer functions. Mr. Rajagopal's research consists of a study done on one of our small reactors at Brookhaven, a neutron source reactor, which is just a little more than a zero power system. We have applied these results so far entirely to zero power systems. There is quite a bit of distance to go yet before we get into systems which have feedback processes.

I'll start off by presenting some of the earlier work in the sequence, and this is just the application of the usual methods of noise analysis to finding transfer functions. This work consisted of ion chamber measurements of the noise at various points within a reactor, Fourier decomposition, of the noise, and finding the transfer function from this. However, it differs a little from the usual technique in that in between there is sandwiched a process of finding the autocorrelation function of output, which is not one of the correlation functions Gyftopoulos has given. The problem one encounters is that in a system like this you can't get the autocorrelation function of input, because the input is the statistical process itself which cannot be separated from the output. Moreover, the noise that one measures is actually a combination of reactor noise and instrument noise, and since we are dealing with an autocorrelation function, the instrument noise just can't be gotten rid of. It is selfcorrelated with essentially flat frequency content.

Slide 1 shows a picture of some of the noise measurements. These are the fluctuations measured by the ion chambers, with the ion chambers at two different points; one where the ion chamber is more sensitive to the reactor noise, one where it is less sensitive to the reactor noise. When the chamber is well below the bottom of the reactor, the instrument noise is very evident, giving the trace an entirely different character from that where the chamber is in the core. One calculates from these outputs the autocorrelation function by means of a system which I'll describe after a bit, does a frequency spectrum decomposition of this autocorrelation function, and essentially finds the amplitude of the 


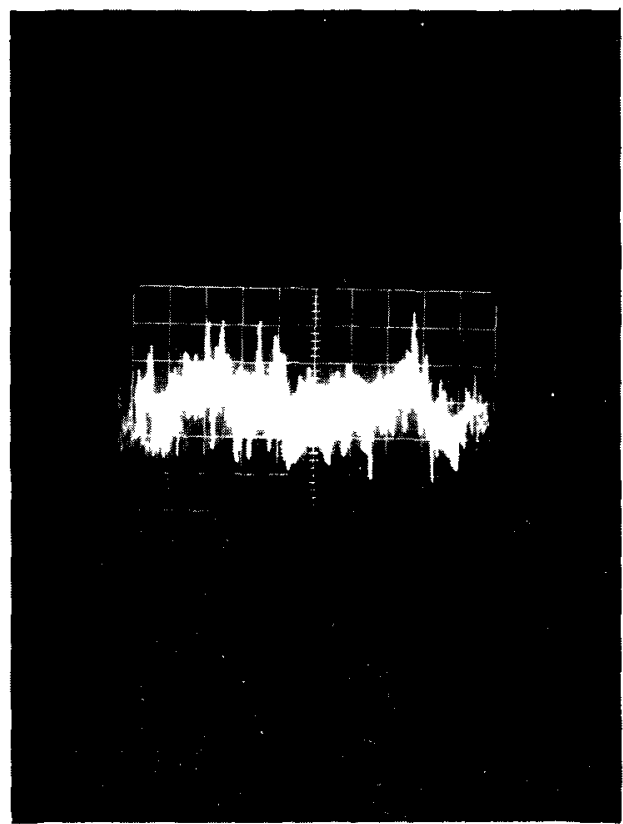

(a) Chamber in the Center of Core of Reactor

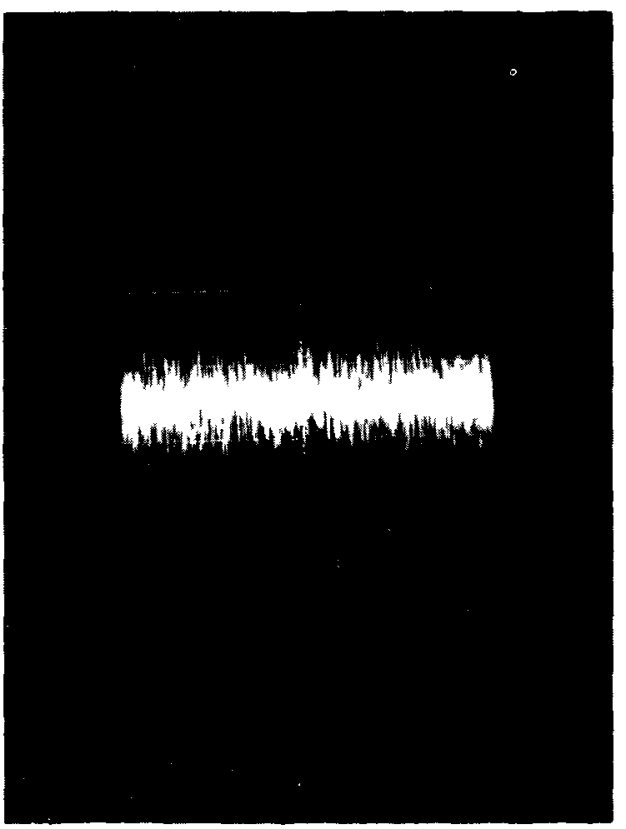

(b) Chamber at the Base of Reactor

\section{Slide 1. Typical signals of reactor fluctuations \\ (Horiz. time scale 50 millisec per large div.)}

transfer function as the square root of this.

Slide 2 shows the autocorrelation function which is obtained. It has a standard form. The transfer function as derived is shown in slide 3 . Now, you will see that there are measurements here at three power levels, and these correspond actually to three positions of the detector. In places where the instrument noise dominates, it adds a constant to the autocorrelation function, which prevents an accurate separation of the reactor response. But when attempts are made to make the detectors particularly sensitive to the reactor properties and not to the noise, then the reactor transfer function shines through with its usual form. Now this reactor is well known--it's a zero power system and has no feedback phenomena. The transfer function is also well known, and here it is measured and also calculated with the Keepin-Wimett delayed neutron data and the lifetime we have measured in the system by pulsed neutron methods. So you see that noise analysis does give this very nicely.

The crosscorrelation techniques we have been developing differ a little bit from these just discussed by Gyftopoulos. Random signals are fed in not as square wave inputs, but they are actually randomly generated by a radioactive source, the signals being integrated and then differentiated, and the result is, as will be seen later in Slide 5, a rather nondescript function which wanders around. Again, the content is white, at least as white as one can get with a finite time of introduction of the signal, and one can expect the analysis Gyftopoulos has discussed to apply to a system like this as well. 


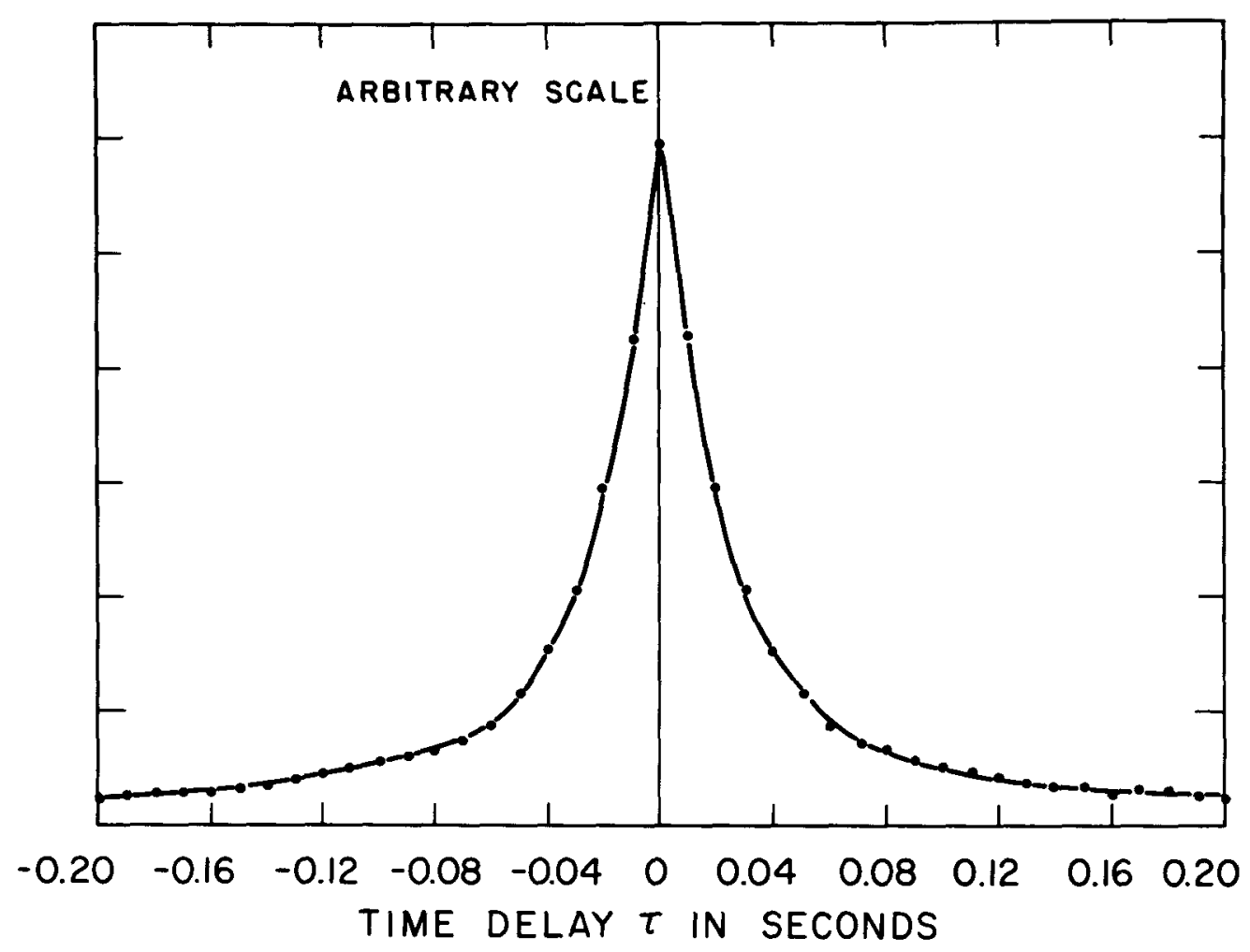

Slide 2. Autocorrelation function of reactor fluctuations at 0.08 watt

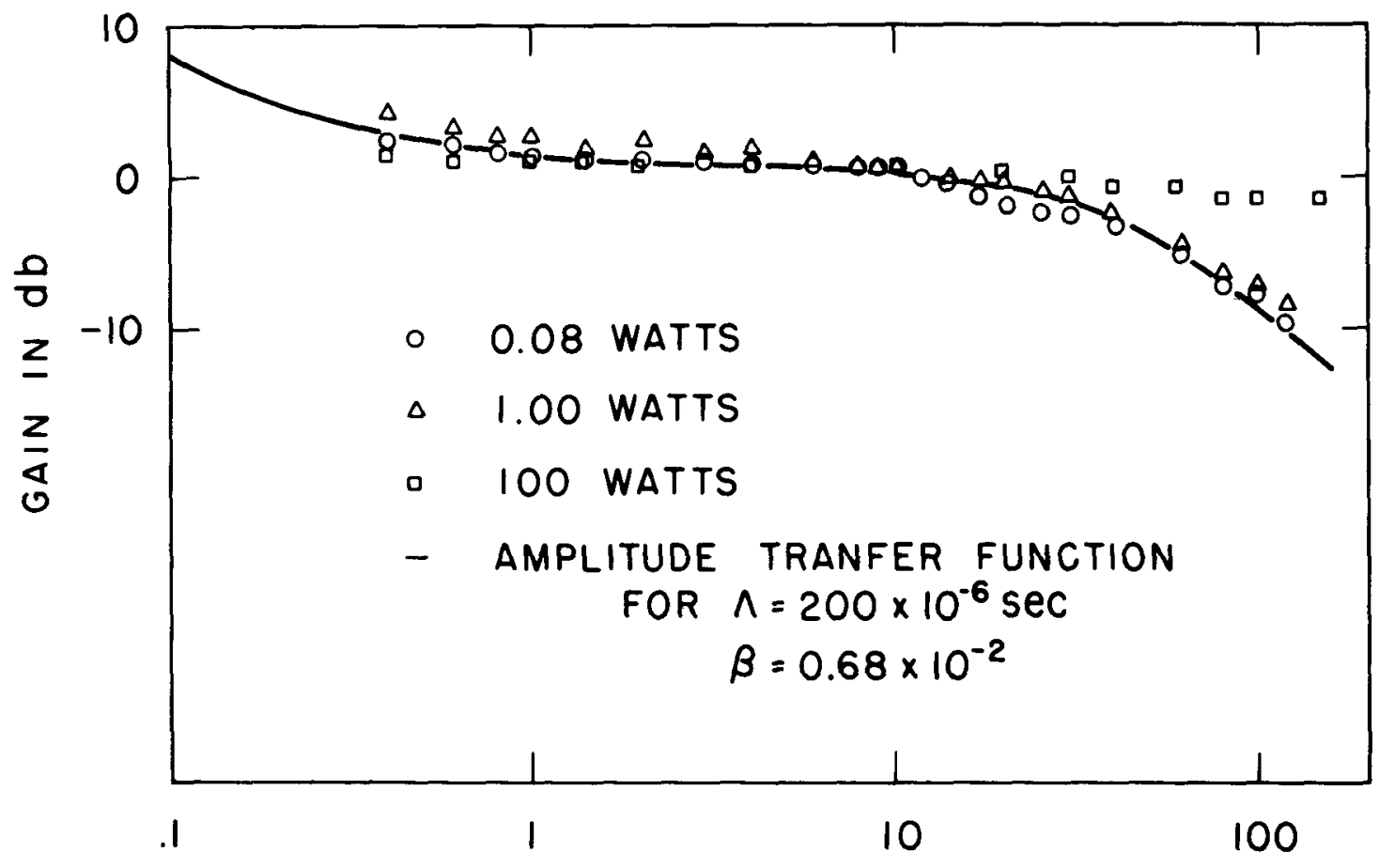

FREQUENCY IN RADIANS/second

Slide 3. Square root of spectra of reactor fluctuations 


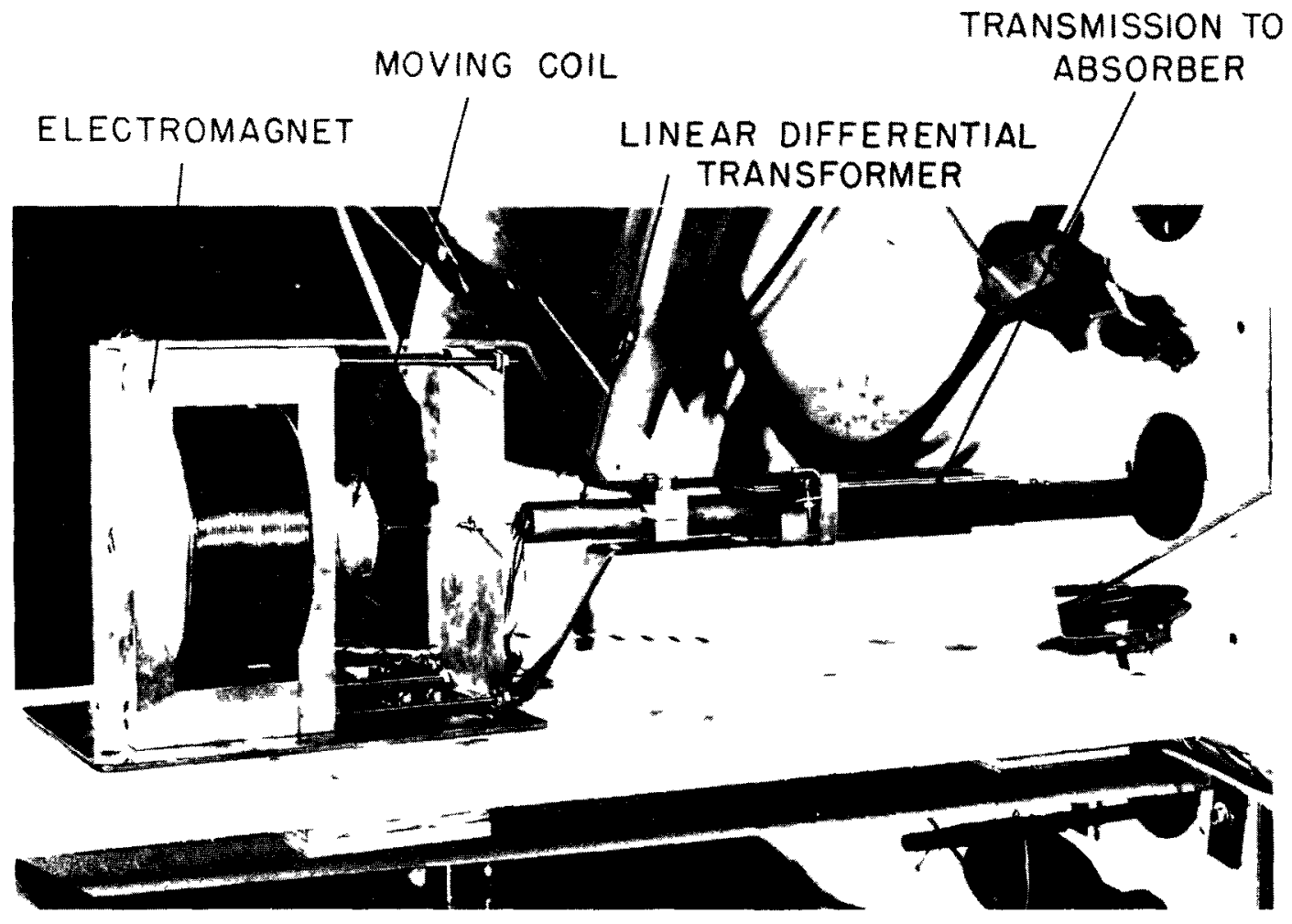

Slide 4. Reactivity input system - drive assembly

The apparatus which is used to create the input is shown in Slide 4. Inside the reactor, which one gets to through a hole in the shield, is a cadmium sleeve which oscillates inside another cadmium sleeve. In the process of oscillating, it adds or subtracts reactivity. The oscillation takes place through attachment to the end of a long, low-mass wire to a low-mass coil inside an electromagnet. The coil is also attached to a spring. This gives a restoring force, the electromagnet pulling one way, with a linear response function, and the spring pushing the other way. Now this apparatus can actually put out signals which are flat to about 100 cycles a second, and it has been tested to 100 cycles a second, so that one is able to investigate the response up to quite high values of the frequency. In this case, the measurements are certainly reliable up to about 30 cycles a second, and they'll be presented within that region. The output of the random signal source is applied to the electromagnet, and a high feedback system causes the position to be quite accurately proportional to the signal input.

Some of the results are shown in Slide 5. The input function here is of the character I roughly described, and the response function of the reactor again is measured with an ion chamber whose response is flat over the frequency spectrum we're feeding in. This is not a very stringent requirement. The output signal and the input signal are recorded on a tape recorder system shown in slide 6.

Now this is a magnetic tape system. We have two channels of input and two channels of output--altogether, four channels on a single tape. The reason for this is that in order to get the autocorrelation function 


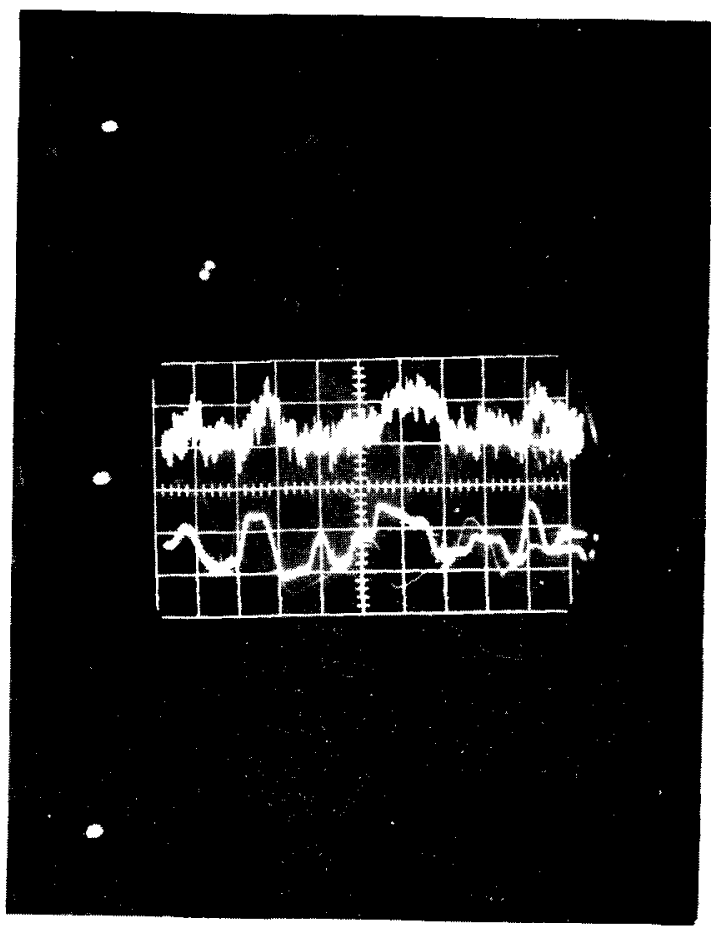

RESPONSE INPUT

Slide 5. Typical random reactivity input and neutron level response signals (Horiz. time scale 50 millisec per large div.)

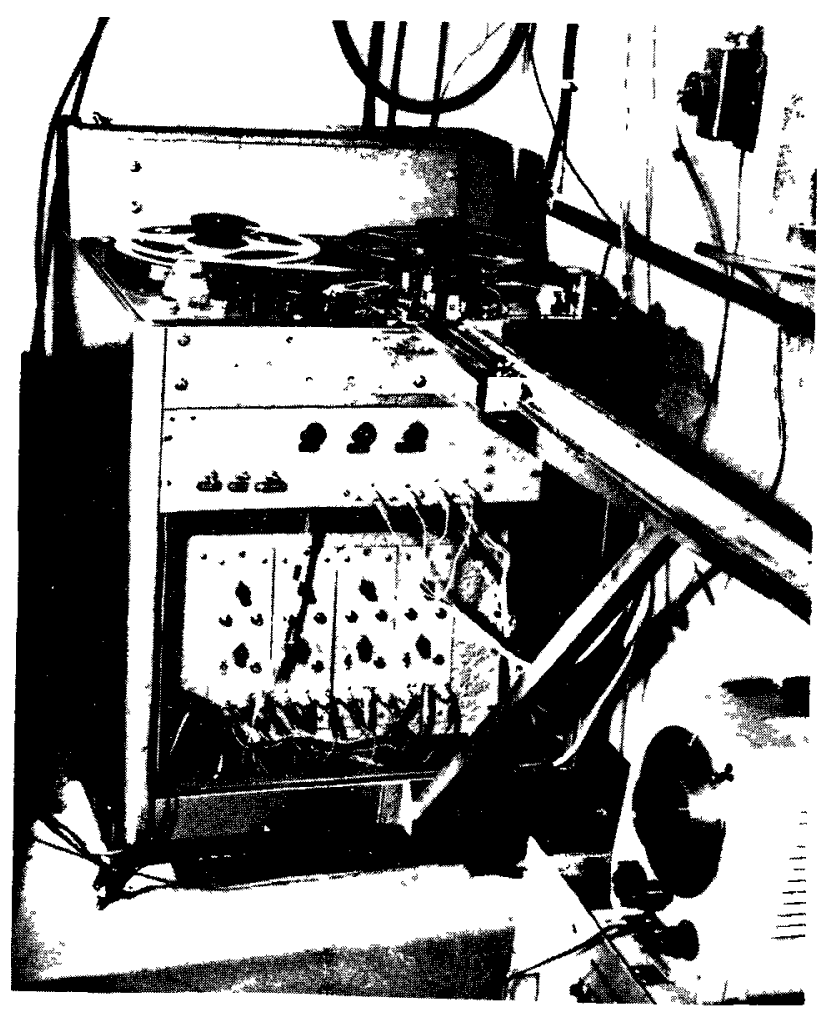

Slide 6. Tape recorder and time delay system 
and the crosscorrelation function, one has to sort of slide the signals across each other in time and integrate, so that you have to have available at one time also the full response of the system at every other time. The history is therefore poured onto the tape recorder and is taken off the tape recorder for analysis. It's recorded as an FM signal. This FM signal is, after suitable transformation back to a DC signal, fed to an analog computer through two reading heads spaced a proper distance apart, and the analog computer does the integration for us. It calculates simultaneously the autocorrelation function and crosscorrelation function. Slide 7 shows results.

The signals are fed in through filters, as they have to be because there is a lot of high frequency noise in the instrumentation, and the effect of the filter is clearly to spread out the signals. The autocorrelation function of input actually is a delta function, as Dr. Gyftopoulos said, if we have measured for a long enough period of time. Since the measurements here took five minutes each, one should expect a much narrower function than this one on the slide, except for the effects of the filter. Now, the filter characteristics are well known. We used two filters, which are variable band-pass filters of known characteristics, and of course their effect on these functions can be factored out by the Fourier convolution theorem.

The next two slides, Slides 8 and 9, show the results of Fourier decompositions of these. Dr. Gyftopoulos wrote down an expression here upon which this frequency analysis depends, which is that the inputoutput correlation function is equal to an integral from minus infinity

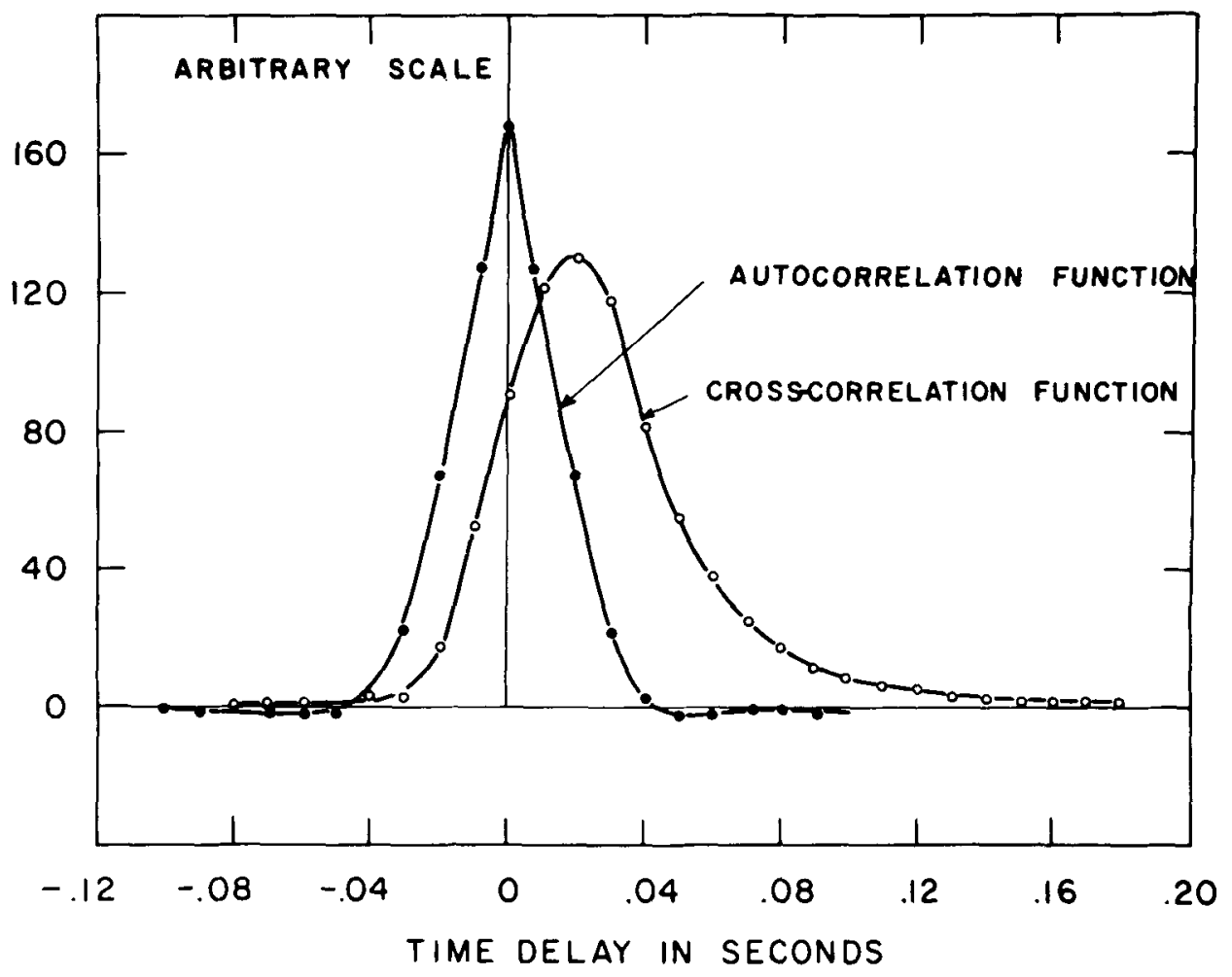

Slide 7. Typical correlation functions for random input 


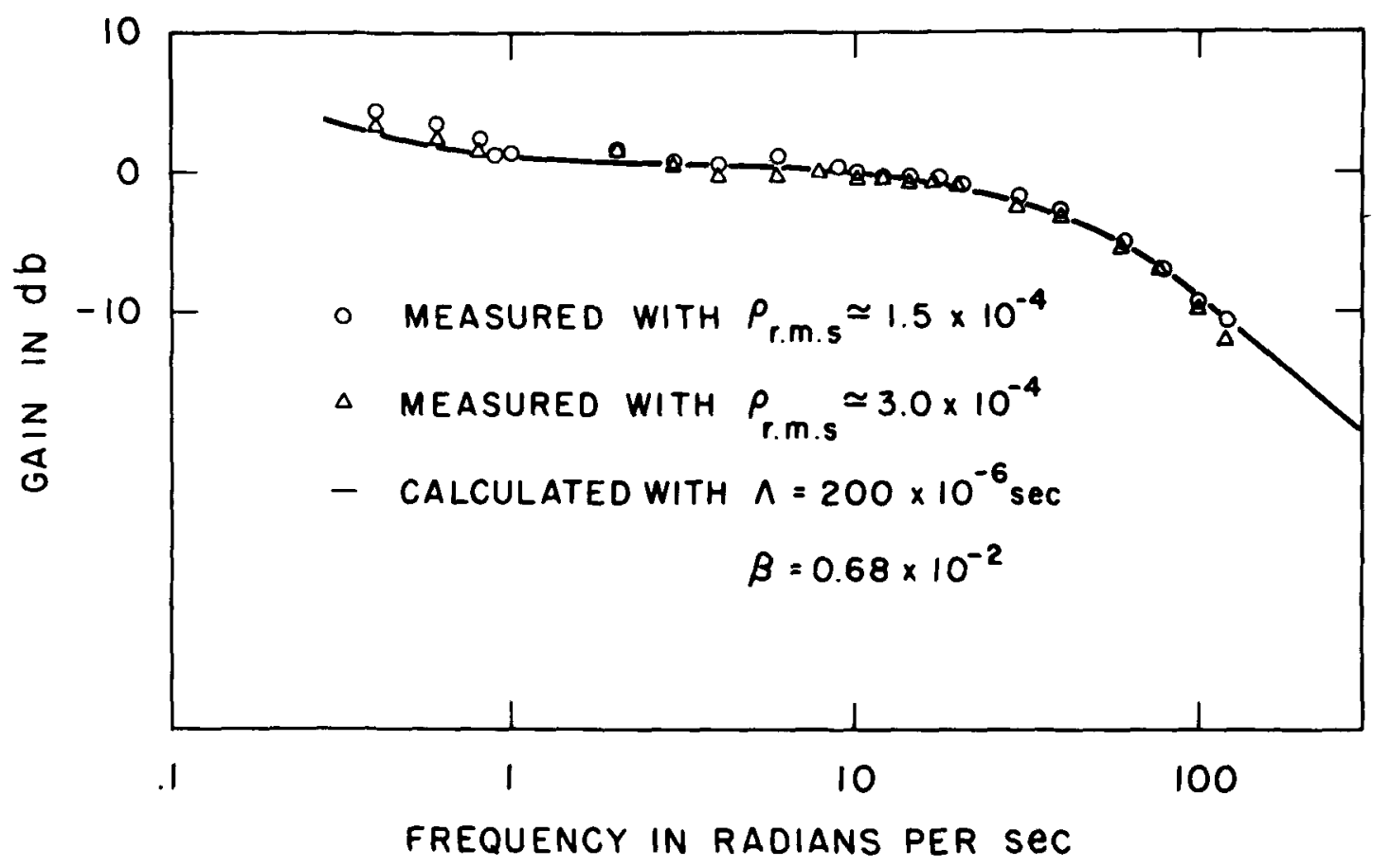

Slide 8. Amplitude transfer function of reactor

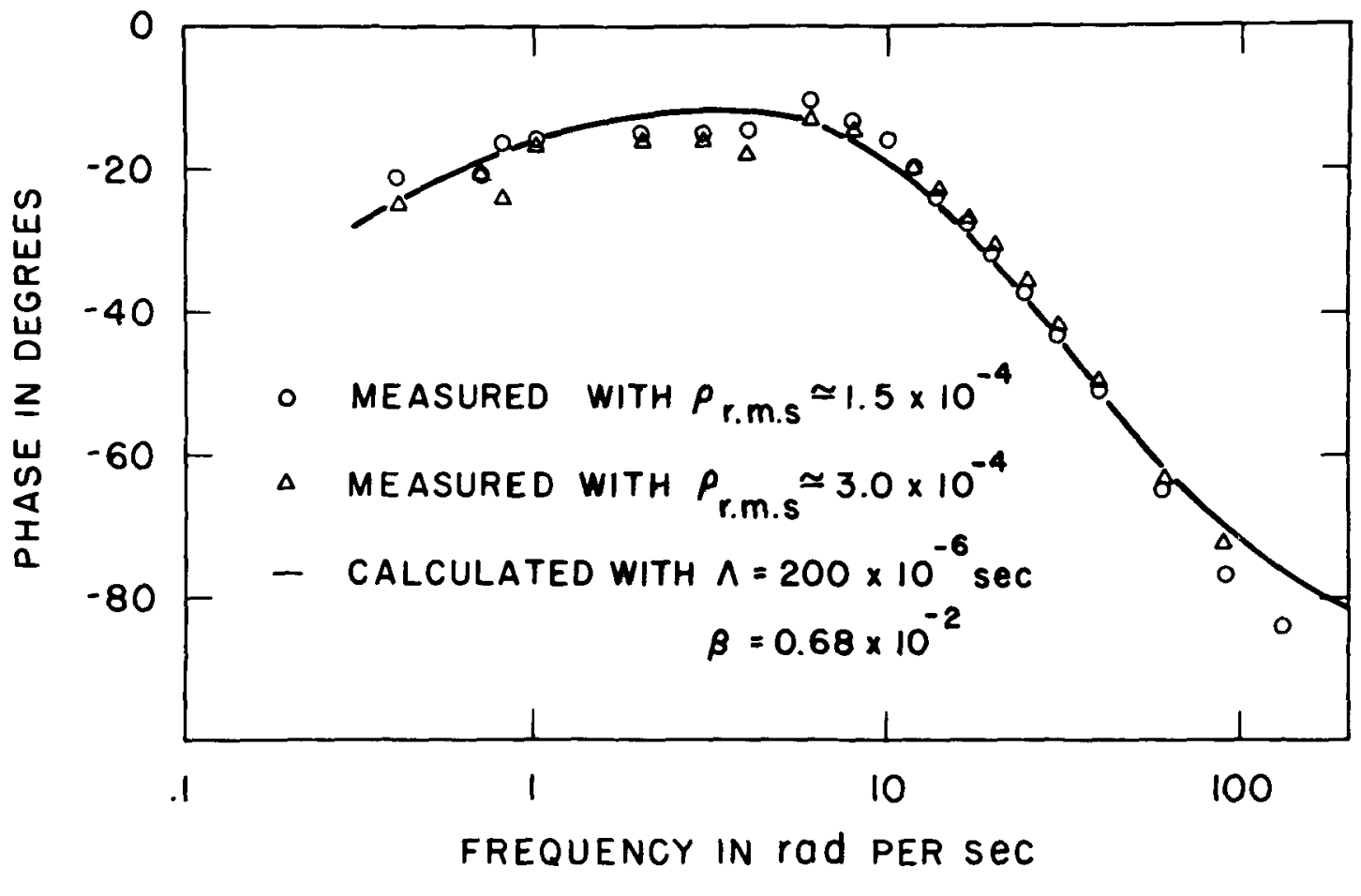

Slide 9. Phase transfer function of reactor 
to plus infinity of the impulse response function, times the input correlation function. If we do a Fourier transform of this, through the Fourier convolution theorem, get simply that the frequency spectrum of the input-output correlation function is the product of the Fourier transforms of these, and the input-input of omega. Or, the transfer function itself, with all information, not only on amplitude but on phase as well, is contained completely in the crosscorrelation function of input and output.

Slide 8 shows the real part of the Fourier decomposition of the input-output crosscorrelation function, compared with the theoretical expression. Slide 9 shows the phase, calculated the same way. So that here, by knowing the input, which one can't get normally by ordinary noise analysis, one can extract the additional information needed; that is, that of the phase of the system.

Unidentified: I wondered about the oscillograph records you had of the response in the middle of the reactor. Is it possible that the picture was turned around then because the flux response seemed to be leading the disturbance? In the picture which showed the disturbance . . . .

Kouts: That first picture I showed? . . . Oh, I'm sorry--it could be, I didn't take the pictures.

Lipinski: We'd like to postpone the questions until after the panel so that we can get into our discussion.

Kouts: Let's assume that it's turned around. (Laughter) 
SESSION III

PANEL PRESENTATIONS

Lipinski: The next panelist is Mr. Wasserman, from Phillips.

Question: Would you like to take a break first?

Lipinski: Why don't we go on with another panelist? Maybe the bases will be loaded by then.

Presentation of $\frac{\text { Spert Oscillator Program }}{\mathrm{by}}$

A. A. Wasserman (PPCo)

Wasserman: What I have to say today is in the way of being a status report on an oscillator program that has been going on at Spert for some time, trying to look at a number of different things. Much of the material about this program has been reported at the Chicago Transfer Function Conference (1) in May, and there is only a little bit new to say. But I'll take a moment--a long moment--to review very briefly for those who may not have been at Chicago, some of the basic things that have been done, and then wind up with what new there is to say about the program. I should mention that this work is being done at Spert as a regular part of the Spert program, in addition to being, for me, a doctoral thesis subject under the supervision of Professors Gyftopoulos and Thompson of MIT.

The purposes of the measurements which have been made at Spert are the following: first, to determine $\ell / \beta$, a long-honored oscillator objective, and to compare this with other measurements of $\ell / \beta$ which have been made by various other means in one particular reactor at Spert which I'll very briefly describe in a moment. These other measurements include poison perturbation tests, the regular step-transient measurements at short periods, and some pile noise measurements.

The second objective, about which I will speak more at the end of this talk, is to determine experimentally the effect of reactivity amplitude on the measured transfer function at low power, for comparison with theoretical results which have been derived in the literature in which the input-amplitude-dependent transfer function has been called a "describing function".

The third objective has been to predict the upper limit of stable reactor power for comparison with experimental results in this reactor, and to determine to what extent it is possible to use the transfer function measurements to predict measured reactor transient beharior (which was a question raised by Jim Boland yesterday, and to which we aren't yet able to give a satisfactory answer). It is recognized that success in the objective of predicting stable reactor power doesn't imply, 
necessarily, success in being able to predict transient behavior. A reactor may yield to linearized treatment for estimation of the limit of absolutely stable power from results of reactor transfer function measuremeasurements at lower powers, but this is no guarantee that the course of dynamic behavior at powers even slightly above this limit could be predicted with accuracy, due to the impact of the nonlinearities which occur only when the power amplitudes become quite large.

The fourth and final objective is simply to gain some experience with pile oscillators, and if they show promise, to do some pile oscillator experiments later, perhaps, in Sperts II, III, and IV where the conditions, the control of dynamic variables, is much better in many respects than it is in spert $I$, and from which a more definitive answer might be obtainable on the question of the possibility of correlating transfer function predictions with transient experimental results.

I'd like next to review very, very quickly, via a few slides, some of the basic information about the reactor and the oscillation experiment. Slide 1 shows the Spert I reactor system which many of you are familiar with, and I won't go into any more detail than to say simply that it's a light-water, heterogeneous, plate-type core. In the case of the oscillator experiments, the APPR core was used, containing stainless steel$\mathrm{UO}_{2}$ fuel elements.

Slide 2 shows the basic core arrangement used for the oscillator tests. The oscillator was located in a corner position, in the upper left, in the reflector at the edge of the core. For low-power measure-

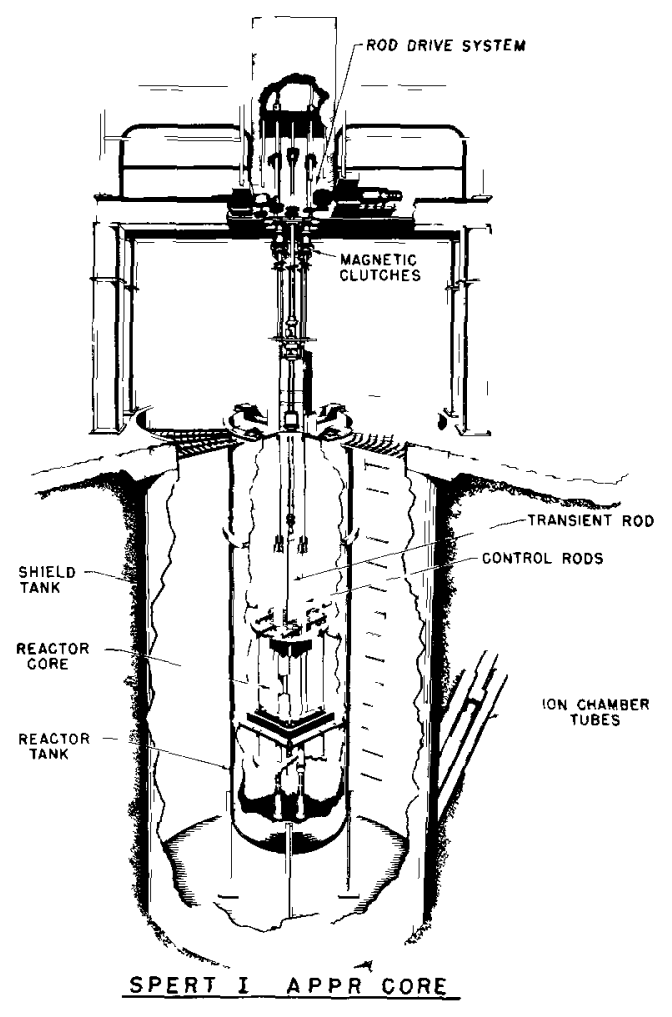

Slide 1. Cutaway view Spert I reactor 


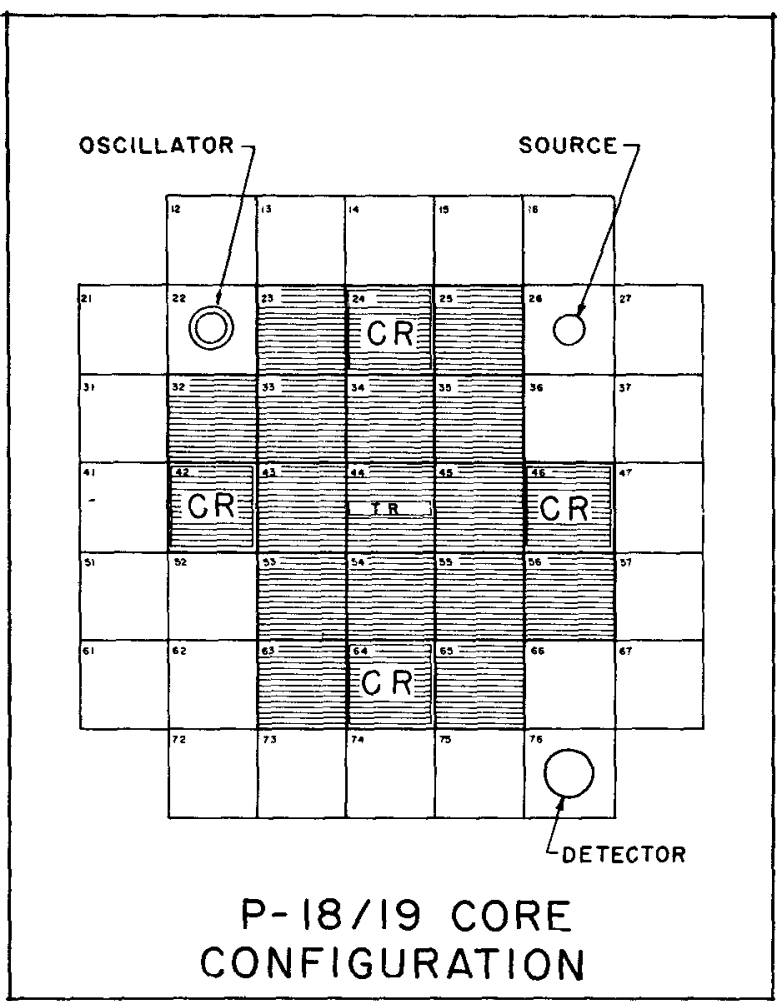

Slide 2. Core configuration for Spert oscillator tests

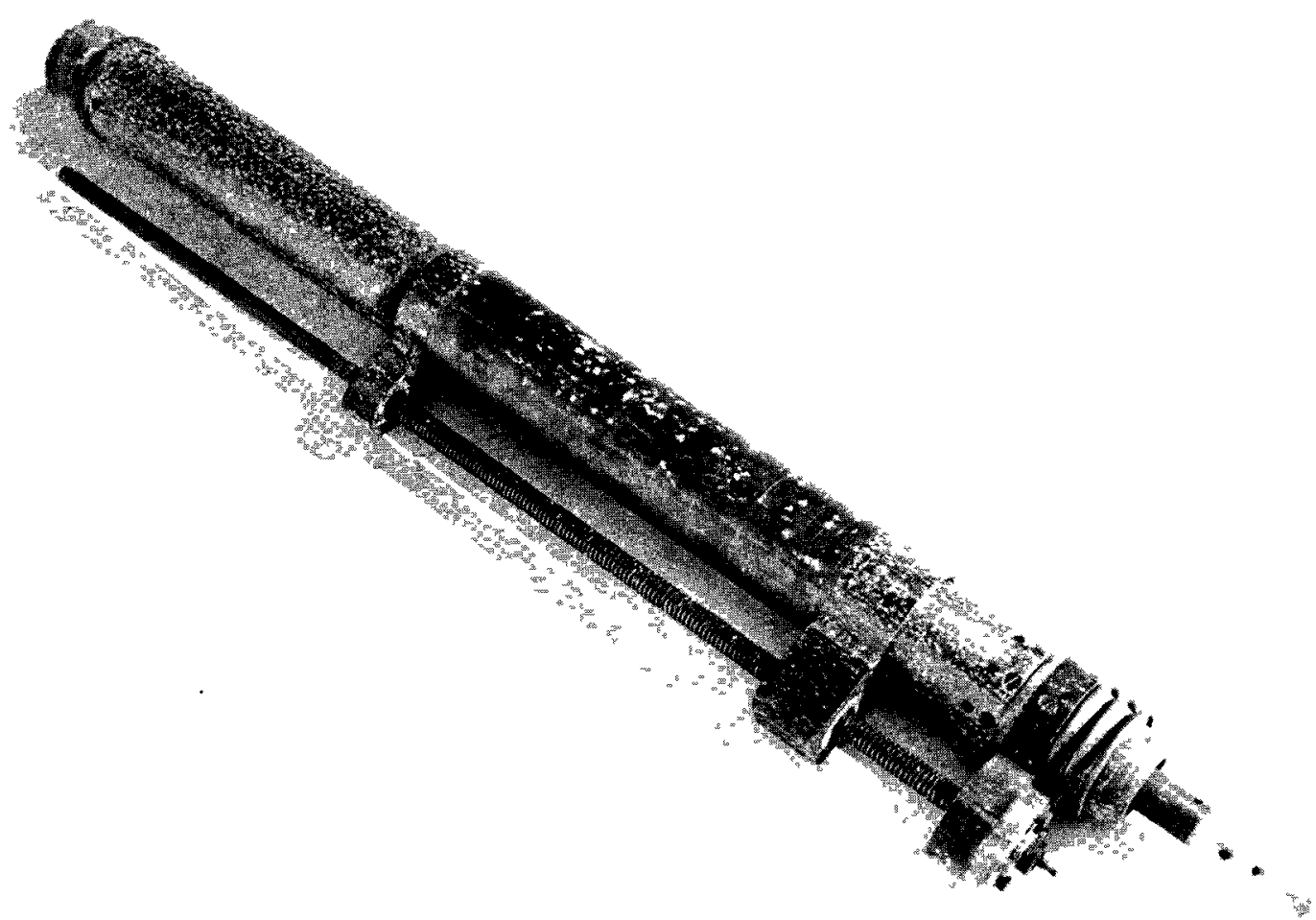

Slide 3. Spert oscillator mechanism 
ments a neutron detector was placed at the edge of the core on the opposite side of the reactor. For higher power tests the detector was moved away, outside the reactor tank. A neutron source was located near the edge of the reactor.

Slide 3 shows a photograph of the oscillator apparatus which was used. This was a rotary type of apparatus in which an essentially rectangular sheet of cadmium was embedded in the wall of a 2-1/2 inch aluminum cylinder, so that it made up approximately a half-cylinder of the rotor. As-this was rotated round and round, it perturbed the leakage in the reflector near the oscillator mechanism. The oscillator mechanism had one unique feature which permitted a variation in the amplitude of the reactivity, and that was this curtain and lead-screw mechanism. The curtain was another cylinder of aluminum, containing a full cylinder of cadmium embedded in it. The curtain surrounded the housing cylinder, inside of which the rotor was located, and by movement of this curtain cylinder up and down, variations in the worth of the oscillator rod pak-to-peak, amplitude-wise, could be achieved. The range of variation from the no-curtain position to the curtain in its most effective position was from about 2 to 3 cents half peak-to-peak in about $15 \phi$ or $16 \phi$ half peak-to-peak.

Slide 4 shows a typical reactivity wave shape achieved with this oscillator mechanism. The experimental points shown were determined by period measurements following small step changes in angular orientation of the oscillator rotor. The curve, the smooth curve, is a least-squares fit to the data. The equation of that curve is shown, and it indicates

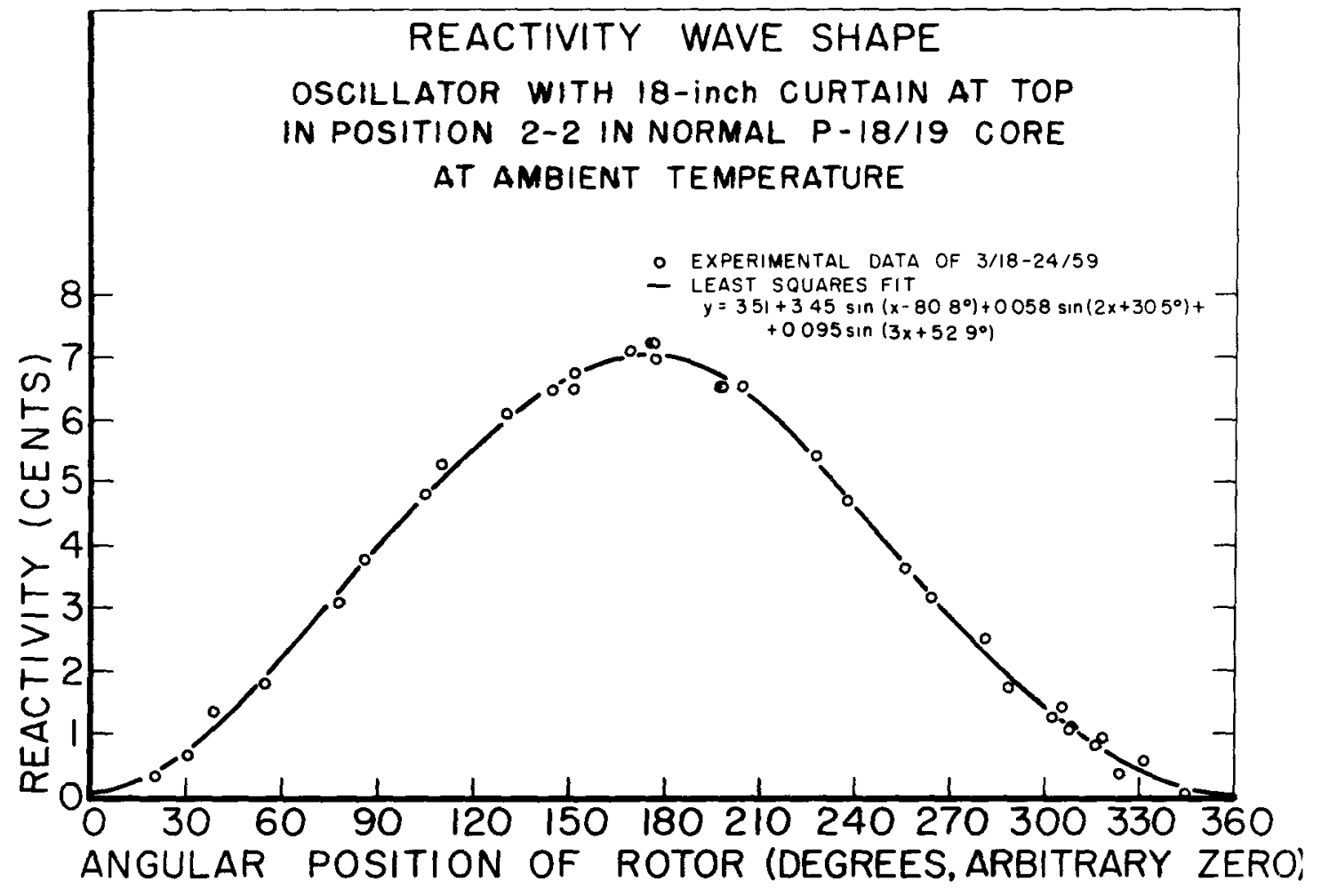

Slide 4. Reactivity wave shape of Spert oscillator 
there was about a 2 per cent second harmonic and about a 3 per cent third harmonic content to the sine wave, so it was a pretty good "pure" sine wave.

Slide 5 gives an indication of the effectiveness of the curtain in modifying the worth of the oscillator. The plot is of the peak-to-peak amplitude versus the displacement of the curtain. We used two different length curtains. The slide gives some indication of how one could get a continuous variation of reactivity amplitude, if desired.

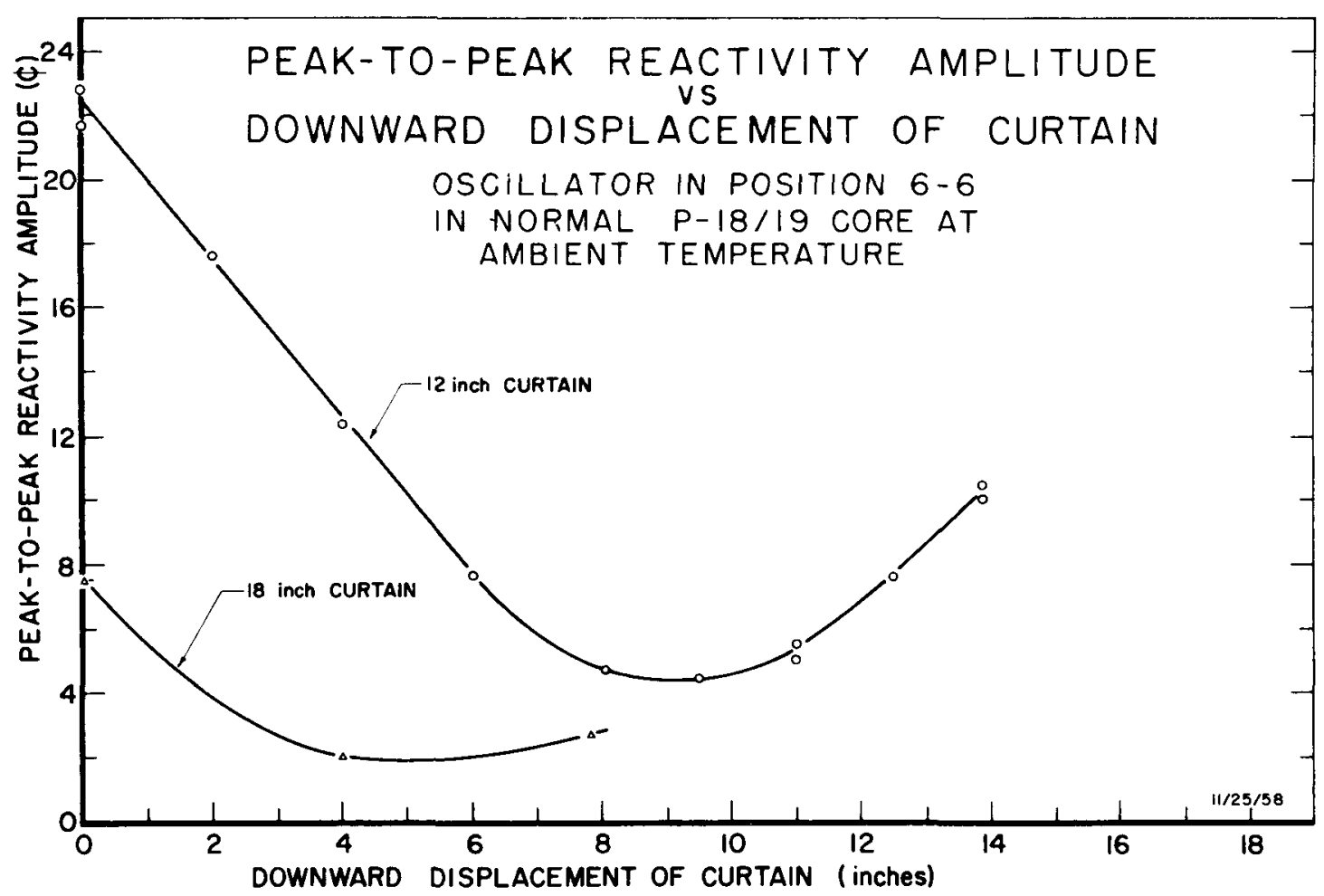

Slide 5. Curtain effect on Spert oscillator

The Table shows the range of variables used in the tests. I should have mentioned that there were two series of tests conducted. A first one included some low-power, small-amplitude studies; some high-power, small-amplitude studies; and some limited, large-amplitude, low-power studies, which were extended in the second series of tests which were conducted recently. The frequency spread was from 0.01 cycle per second to just short of 20 cycles per second in the earlier tests; and, in the later large-amplitude tests, this was extended down to about 0.002 cycle per second, to exaggerate the differences between the small-amplitude transfer function and the modification which the increase in reactivity amplitude brings about.

As you see, the highest power at which useful data could be obtained in this system was about 600 kilowatts. The system was a basically uncooled system with no forced circulation, and heat removal was effected by inserting cooling coils into the reactor tank and also flowing water in and out continuously, letting it flow out over a weir. The low-power 


\section{TABLE OF OSCILLATOR TESTS SPERT I P-18/19 CORE}

\begin{tabular}{|c||c|c|}
\hline \multirow{2}{*}{ MEAN } \\
POWER & \multicolumn{2}{|c|}{ PEAK-TO-PEAK } \\
\hline \multirow{4}{*}{ REACTIVITY } & AMPLITUDE \\
\hline \multirow{4}{*}{ LOW } & $\begin{array}{c}\text { LOW } \\
50-1000 \text { WATTS } \\
7-13 \mathrm{CENTS} \\
10-50^{\circ} \mathrm{C}\end{array}$ & $\begin{array}{c}\text { HIGH } \\
50-1000 \text { WATTS } \\
22-30 \mathrm{CENTS} \\
10-20^{\circ} \mathrm{C}\end{array}$ \\
HIGH & $\begin{array}{c}50-600 \mathrm{~kW} \\
7-13 \mathrm{CENTS} \\
50-100^{\circ} \mathrm{C}\end{array}$ \\
\hline
\end{tabular}

tests were conducted from about 50 to 100 watts. Some of the later ones were conducted at slightly lower powers, but not too much so. The amplitudes of what I call the small-amplitude tests were, as you see, from about 7 to $13 \phi$ peak-to-peak. That's about 3 to $7 \phi$ half peak-to-peak. The large-amplitude tests went up to $30 \phi$ peak-to-peak, as I mentioned.

I might just repeat one additional comment I made at Chicago, because it was of interest to some people there. In the earlier tests, two instrumentation systems were used. One was a direct recording on an oscillograph of the power signal plus a signal providing a phase reference with respect to the reactivity amplitude. Another system used was a wave analyzer of the nulling type which was originally used at KAPL and then adapted and used very successfully by Jim Boland on EBR-I. When I got into the high-power tests, I ran into so much boiling noise that the wave analyzer was rendered unusable by virtue of ringing of a band-pass filter in the system. Just to give you an illustration of the kind of noise that I faced, and perhaps also to point up the significance of the papers by Professor Gyftopoulos and Dr. Kouts on how useful it is to have a system that can ignore noise of this sort, Slide 6 shows a typical power trace on an oscillographic record. The top curve there is the phase reference derived from a sine pot following the oscillator rod round and round, and the bottom one is the signal from the ion chamber. The DC component is bucked out and this is the amplified AC portion. You can see that there's quite a bit of garbage to fight. This is not as bad as some cases, but is as bad as I felt could be meaningfully processed by hand. Any higher power or temperature of the bulk 


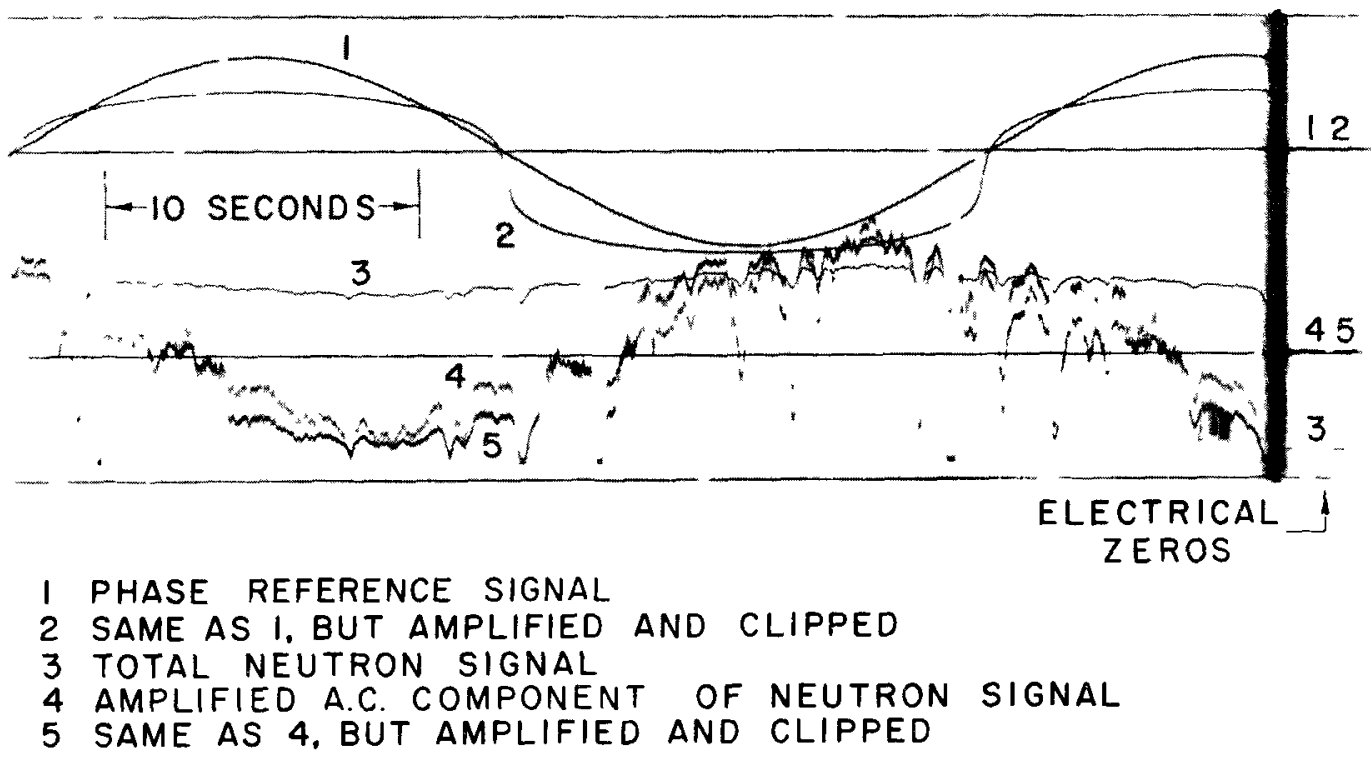

\author{
PILE OSCILLATOR RAW DATA FOR SPERT - I \\ $P-18 / 19$ REACTOR \\ $500 \mathrm{kw}, 75^{\circ} \mathrm{C}$ BULK WATER TEMPERATURE \\ $0.03 \mathrm{cPS}, 13$ \& PEAK-TO-PEAK REACTIVITY AMPLITUDE \\ Slide 6. Data record from Spert oscillator test
}

water in the reactor tank would have increased the noise to a sufficient extent that, without some kind of a correlation process, it would just have been hopeless.

Well, that's all I want to say about the earlier tests, except to remark that this earlier high-power data was given preliminary processing, and a feedback function was extracted which showed the same essential shape as the EBWR results, so I felt that there was information in these high-power results, even though the power levels reached were not nearly as high as one might have wished to get a very accurate extraction of the feedback function. But one other problem came up which was disturbing and which had to be cleared up before further work on the high-power data was advisable. This was the fact that the low-power, small amplitude results showed a discrepancy in the amplitude of the transfer function which was monotonically frequency dependent and which amounted to about 5 per cent deviation from the theoretical transfer function over the frequency range of about 0.015 cycle per second to 20 cycles per second, with most of this variation occurring below half a cycle a second. And because this well-behaved discrepancy was not then understood, I felt that it was important to try to pin it down before pushing the analysis of the high-power work, only then to discover that there was something basically wrong with the data. So the second series of tests were conducted both to extend the large amplitude measurements which had not been completed in the first series, and also to try to improve the system and to see if this amplitude discrepancy could be eliminated. 
I'd like to take a diversion here, and before going on to say what the results of the more recent experiments were, to speak a little bit about some analytical work which has been done as a parallel effort in connection with the large-amplitude, low-power measurements. A number of papers have appeared with respect to the so-called describing function of a reactor as a control system, concerned with the possibility of applying essentially linear techniques for dealing with the nonlinearities which occur, both in the neutron-kinetics equations themselves when the power amplitudes get large, and also in the feedback processes. And if you'll permit me, I'd like to read a few paragraphs of some notes that I've written up on this subject--nothing original, just sort of a correlation of background information, extracted in large part from the last two chapters in Referencel2).

The results of small-amplitude, pile-oscillator studies for which the reactor-kinetics equations are linearized can be used with some success to predict the limiting power for absolute reactor stability, and the EBWR and others are notable examples of this. The results are inapplicable, however, for predicting, first, the amplitude and frequency of the natural oscillations which occur when the power is raised to or above this limit; and, second, the course of reactor transients in which the peak power is significantly higher than the initial power. As Joe Thie remarked, if you make a step transient in which you only have of the order of a decade variation in power, you can hope to Fourier invert and to determine the transfer function, but not if you're considering very large reactor transients. And the reason, of course, is that the inherent nonlinearities in both the neutron kinetics and the reactivity feedback equations determine the behavior.

There are two general methods which exist for dealing with such cases. One is the topological or phase-plane approach, and this method gives general results only for a system which can be approximated by a secondorder, ordinary differential equation of a proper type; that is, one which has no terms involving explicit functions of the independent variable (in our case, time), and also a system which is subject to a zero external forcing function. In other words, this method only applies to studying natural oscillations for a rather simple model of a nonlinear system. To the extent that a reactor system can be described approximately by such an equation, the method permits the determination of the transient response to specified initial conditions, the stable and unstable equilibrium states of the system, and the existence and nature of "limit cycles" which describe sustained oscillations of the nonlinear system.

In many cases, when a reactor system is not adequately described by an autonomous second-order differential equation, the second, so-called describing function, method may be applicable. This method applies to systems of any order, tending usually to be more accurate the higher the order of the system. The method assumes that there is only one significant nonlinearity in the system, that the nonlinearity has no time-varying characteristics, that the input to the system is sinusoidal, and that the only significant frequency component of the output which contributes to the input is that having the input frequency. This last assumption requires that the system have a low-pass frequency response characteristic for all relevant operating conditions; that is, no very sharp resonances. 
Like the phase-plane method, the describing function method can be used to determine the conditions for stability and the conditions for, and general features of, any self-.sustained oscillations or limit cycles. In addition, the method permits evaluation of system performance for sinusoidal input functions. However, it does not, in general, provide more than an indication of the general characteristics of the transient response of the system.

The describing function, $D$, is defined as the ratio of the amplitude of the fundamental component of the output to the amplitude of the input. In general, for nonlinear systems, D is the function of the frequency, $\omega$, and the input amplitude, I. For the limit of small input amplitudes the equations describing the system behavior may be linearized, and $\mathrm{D}$ becomes independent of the input amplitude. In this limit, the describing function is equal to the transier function of the linearized system.

To analyze a nonlinear system by the describing function method, one first determines how $D$ varies with frequency and with input amplitude, and this may involve Fourier analysis to determine the fundamental components in the output. The results may be presented as plots of the gain and phase of $D$ versus frequency, with the input amplitude as parameter. These plots consist of families of curves representing different values of the input parameter. The curve for I (the input parameter) being zero, is identical with the transfer function of the linearized system.

Using such plots, the absolute and relative stability of the system may be analyzed as a function of the input signal level, using the stability analysis techniques which are available for dealing with purely linear systems. The approximate amplitude and frequency of any sustained oscillations may be determined but not their detailed wave form.

Some efforts to apply the describing function method to reactor problems have been made. In most cases, only the nonlinearity of the neutron kinetics equations have been considered. $(3,12)$ In others, the nonlinearity in the reactivity feedback equations has also been considered. $(4,9,10)$

With respect to the activities of the Spert program, it would be of interest to predict analytically (for comparison with observed results) the amplitude and frequency of sustained power oscillations at megawatt power levels by using the describing function of the neutron kinetics part of the system, coupled with some linear reactivity feedback model. The feedback model could be a simple, approximate analytical model derived from physical analysis, or it could be an experimentallydetermined, graphic representation, such as that obtained in the RamoWooldridge out-of-pile studies. (13) As an aside, it would also be of interest to couple the linearized neutron kinetics with an experimentallydetermined describing function for the feedback. The feedback describing function could be measured by a straightforward extension to large power variations of the techniques which the Ramo-Wooldridge people have already employed in their out-of-pile studies. 
Concerning the analytical derivation of the describing function of the neutron kinetics of a reactor, several authors have used a number of approximation techniques to obtain the desired results analytically. The results obtained in some of these analyses $(8,11)$ appear to be incorrect due to improper treatment of the mean value of the power during the oscillation and due to premature neglect of higher order terms. In particular, these analyses leal to a describing function magnitude which exceeds the transfer function magnitude at a given frequency, whereas the reverse is true for other analyses $(6,10)$ in which higher order terms seem to have been carried along properly.

The results of the large-amplitude, low-power reactivity oscillator tests conducted at Spert are important, therefore, in attempting to provide an experimental basis for checking the ranges of validity of these various analyses before efforts are made to apply the describing function method to the problem of predicting the sustained oscillations which have been observed in spert reactors at high-power levels.

Well, this has been kind of a long harangue in trying to give the motivation for these studies. Regarding the analytical determination of the describing function of the neutron kinetics, another fellow (Dick Wagner) and I have developed a code for calculating the describing function using an analytical formulation of Akcasu of Argonne (14), in which the form for the describing function is given as a power series in the reactivity amplitude. If the reactivity amplitude is designated as $\mu$, we would write that the describing function is given by the transfer function $G$, times a correction factor, which is a series

$$
\left(1+\mu^{2} C_{2}+\mu^{4} C_{4}+\cdots \cdot\right) \text {. }
$$

$\mathrm{C}_{2}$ is a function of the transfer function at the fundamental frequency $\mathrm{G}_{1}$ and of the transfer function at the second harmonic frequency $G_{2} \cdot C_{4}$ is a function of $G_{1}, G_{2}$, and $G_{3}$ (the transfer function at the third harmonic), and so on. As you go up the scale, the C's become more and more complicated expressions. They are, of course, complex quantities.

Our code permits calculation of the describing function through the $\mu^{4}$ term; over a frequency range from 0.001 cycle per second to 100 cycles per second, in steps of 20 frequency points per decade; for any number of values of the reactivity amplitude $\mu$; and capable of accomodating various values of neutron lifetime and up to 25 groups of delayed neutrons, so that heavy water reactors and beryllium-moderated reactors can also be accommodated. The calculation has been carried out and tabular data is currently available for the APPR stainless steel core in the spert I reactor. I plan to get some of this material done up in graphs which I hope to be able to make available for general interest.

In addition, to test how severe the cutoff at the $\mu^{4}$ term actually is, I have hand-calculated the $\mu^{6}$ term for a frequency of 0.002 cycle per second and for a half peak-to-peak reactivity amplitude of $0.156 \$$. The result shows that there is only about a half-per cent error due to leaving off the $\mu^{6}$ term at this low frequency, and the error will be less for higher frequencies. 
The results also show that above a reactivity amplitude of about $0.20 \$$ half peak-to-peak, probably very many additional terms would be necessary to get correct results, because the result of truncating at the $\mu 4$ term gives describing function magnitudes which are greater than the transfer function magnitude, which does not seem to be reasonable.

Well, so much for the parallel calculational effort. Getting back to the recent measurements, I'm sorry to say that I still have the same frequency-dependent amplitude discrepancy between the experimental results and the theory. For the small-amplitude case, this is a discrepancy between experiment and the theoretical reactor transfer function; for the large-amplitude work, it's a discrepancy between experiment and the calculated describing function values.

I'm still trying to determine whether the cause of the discrepancy rests with some part of the experimental apparatus, or if there is something further missing in the theory. In talking with Jim Boland here at the meeting, I have obtained one new lead. This is concerned with the effects of radiation on the insulators of the chambers that we used in the experiment. We used an Argonne-built, plate-type chamber, similar to a type that Jim has used successfully for much of his work. In one instance, he found that there was an apparent power-dependent leakage in one of the insulators, and I propose to see if I can't prove whether or not this could be the cause of this discrepancy.

The results, as they stand, show agreement with the describing function at about 0.01 cycle per second, and the experimental results are monotonically too low at higher frequencies and too high at lower frequencies. This is the pattern of the results. Well, that's about all I have to say. I'm sorry this is such a sketchy status report, but that's where things stand at the moment.

Lipinski: I assume there's coffee in the back, so this would be an appropriate time for everybody to take a break. 


\section{RFFERENCES}

1. A. A. Wasserman, "High and Low Power Spert I Transfer Function Measurements, "Proceedings of the Conference on Transfer Function Measurement and Reactor Stability Analysis, ANL-6205, p. 156, (May 2, 1960).

2. J. G. Truxal, "Automatic Feedback Control System Synthesis," Chapters 10 and 11, McGraw Hill, New York (1955).

3. V. H. Runsey, "Time Variation of Pile Power Due to Periodic Modulation of Effective Multiplication Constant," CRP-333 (PD-217) (June 19, 1957).

4. R. Siegel, and H. Hurwitz, Jr., "Effect of Temperature Coefficients on Reactor Stability and Reactor Transfer Function," KAPL-1138, (March I, 1955). (Appendix E, "Nonlinear Effects," by M. Nelkin).

5. I. G. Barrett, "Effect of Reactivity Modulation Amplitude on Measurement of the Reactor Transfer Function, "KAPL-M-LGB-10, (June 29, 1956).

6. Z. Akcasu, "General Solution of the Reactor Kinetic Equations without Feedback," Nuc. Sci. and Eng., 3, (4) pp. 456-457 (April, 1958).

7. H. B. Smets, "Low and High Power Nuclear Reactor Kinetics," Sc.D. Thesis in Nuclear Engineering, MIT (June, 1958).

8. V. Raievski, "Study of Neutron Transport by the Modulation Method," CEA-1095 (April 22, 1959). (D.Sc. Thesis in Physics, University of Paris).

9. H. A. Sandmeier, "Kinetics and Stability of Fast Reactors with Special Consideration of Nonlinearities," ANL-6014 (June, 1959). (D. Nat. Sci. Thesis in Physics, Swiss Federal Institute of Technology in Zurich).

10. H. A. Sandmeier, "Nonlinear Treatment of Large Perturbation in Power Reactor Stability," ucc. Sci. and Eng., 6, (2) pp. 85-92 (August, 1959).

11. H. B. Smets, "Describing Function of Nuclear Reactors", IRE Transactions on Nuclear Science NS-6 (4) pp. 8-12 (December, 1959).

12. C. N. Shen and D. C. Dougherty, "Some Nonlinear Aspects of Zero Power Kinetics of a Pile Oscillator," Trans. Amer. Nuclear Soc., 3, p. 111 (June, 1960).

13. R. W. Wright and S. M. Zivi, "Out-of-Pile Power-Void Transfer Function Measurements and Prediction of Spert I-A Boiling Oscillations," Trans. Amer. Nuclear Soc., 3, p. 1.14 (June, 1960).

14. Z. Akcasu, Private Communication (August, 1960). 
Lipinski: The next panelist on our program this morning is Mr. Grace from Bettis.

\author{
Presentation of Westinghouse \\ Reactor Transfer Function Program* \\ by \\ J. N. Grace (W-Bettis)
}

Grace: This morning $I$ wish to review and up-date the boiling reactor work which I described at the Argonne meeting on transfer functions last spring. After that, I want to mention some recent work by Steve Margolis which applies frequency response techniques to the spatial stability problem.

It was about two years ago that we began to develop a method of predicting instability of the reactivity feedback loop in a reactor under boiling conditions. Our object was to be able to predict stability from basic parameters--that is, the core dimensions, static heat transfer coefficients, coolant flow rates, etc.-without depending on experimental results from the actual core. We went about this in a straightforward manner and came up with two different models which were quite similar except that one contained more detail. One was a two-section model of the core; that is, the core was divided axially into two sections, and the coolant transport time delay connected the lower region to the upper region. This model was developed by Margolis and Kaplan(1).

The other approach, developed by Neil Curlee and John Reihing, involved a digital computer program for extracting the feedback transfer function from transient response. They used what was basically the ART transient heat transfer code for the IBM-704 computer(2), modified by adding a Fourier transform operation such that one could calculate the frequency response of the feedback transfer function by Fourier-transforming the calculated transient (identified as ART-03). This method employed a 30section model of the core. It was sometime later that we had an opportunity to test these methods of analysis in a power reactor. We made an attempt to predict at what pressure the reactor would oscillate due to reactivity feedback. Hydrodynamic instability often occurs in the same neighborhood of operating conditions as reactivity instability. Therefore, special care was given in the design of the experiment to avoid hydrodynamic instability so that we would get a test of reactivity instability.

The results indicated that the two-section model came within about 1 cycle per second of predicting the frequency of oscillation of $5.8 \mathrm{cps}$, and the 30-section model which was based on the ART-03 code was off in the other direction by about $1 / 2 \mathrm{cps}$. Now, both of these were singlechannel models; that is, we did not take into account parallel channels

\footnotetext{
*This paper was.issued as WAPD-OP-ll-Part I.
} 
or the fact that some regions were boiling sooner than others. Nevertheless, they came rather close to predicting at what conditions this system could oscillate.

In the experiment done last winter--Stan Johnson participated in the running of that experiment, and he is here in case anyone wants to ask him about any of the details--the specified power and flow were set up in the plant. Then the pressure was reduced in successive steps, and the frequency response was measured at each pressure. Now, as soon as the pressure was reduced enough to get some bolling in the core a resonance appeared, and each succeeding frequency response showed an increasing resonant peak which approached a frequency in the neighborhood of 6 cycles per second. Eventually, the last pressure reduction, which was just a couple of psi, resulted in sustained oscillation of power, through the reactivity feedback loop. In fact, these oscillations were allowed to persist for five or six hours--there was actually a shift change of operating personnel while the reactor was sitting there oscillating. The amplitude was limited, but the limiting envelope of the oscillations was a slowly varying function of time, varying between about $10 \%$ and $60 \%$ of the existing average level.

After the experiment had been done, a more detalled analysis was carried out by Ira Kohlberg using a multi-channel model with constants calculated for the core at its depleted conditions. We used the multichannel model to let some regions boil sooner than others. At the time when the experiment was performed, the core was actually almost fu, Iy depleted--near the end of its life--and the fuel distribution, of course, was nonuniform. A three-dimensional depletion calculation had been done using the synthesis technique developed by John Meyer. The results were used to determine density and temperature coefficient distributions as well as flux distributions for the depleted core.

The details of this analys is are quite intricate; Kohlberg did a very thorough and meticulous study. He took into account the recent power history to get exactly what the xenon situation was. From the motion of the control rods, corrected for xenon, he calculated how much reactivity was actually tied up in voids when each transfer function was being measured.

The one slide I have on this subject, Slide 1 , indicates the trend in the calculated frequency response curves, the solid lines, as pressure is reduced leading to increased reactivity tied up in voids. You will note that they follow a trend that one would expect. As the pressure is reduced, the low frequency gain is reduced by virtue of a stronger feedback, which is aegenerative at zero frequency. At the same time, because the void reactivity and the feedback gain are increased, the resonance increases in amplitude at a frequency where sufficient phase lag exists to make the loop essentially regenerative. We also have plotted on this slide two experimentally determined transfer functions for different void reactivity values.

The results amounted to this: that the errors in the calculations using the multi-channel model were not much different from the errors using the simplified one-channel models. The frequency of oscillation 


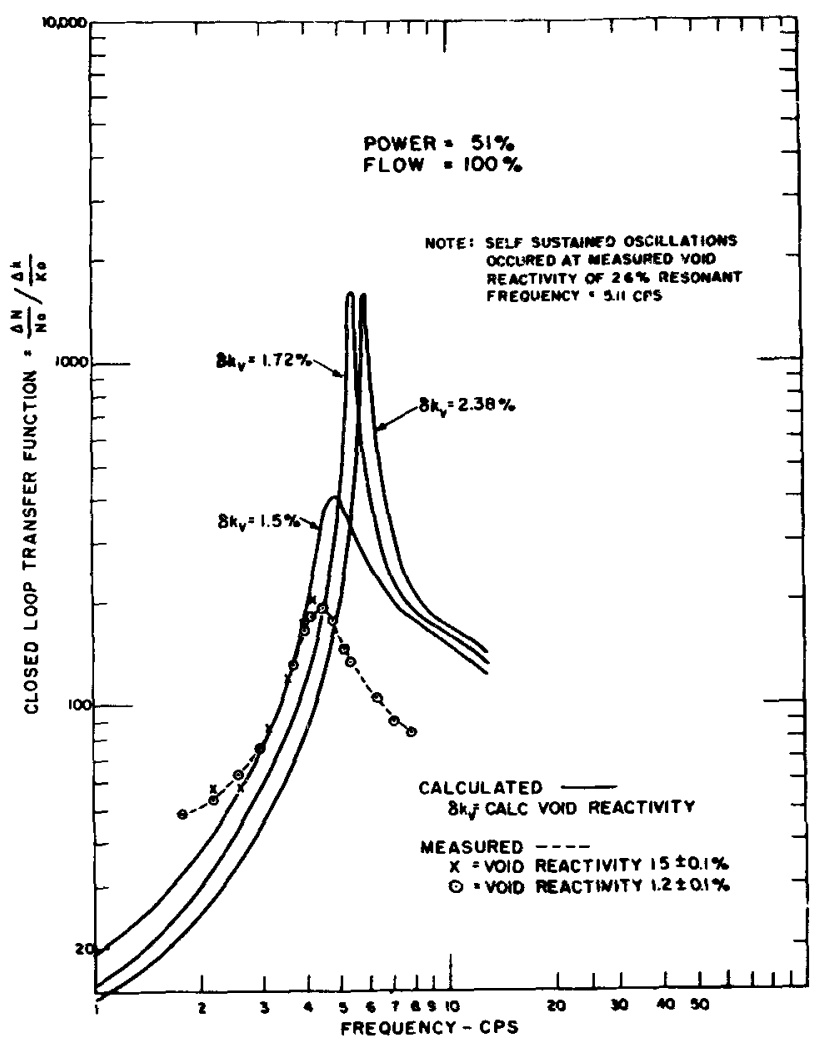

Slide I.

Comparison of Measured

and Calculated Closed

Loop Transfer Functions

was off by about one cycle per second. This more detailed analysis predicted 6.8 cycles per second, while the actual frequency of oscillation was about 5.8. The loop gain calculated at the conditions of oscillation was almost $10 \%$ less than what was really needed for instability. Reflecting this in terms of void reactivity, it amounted to about 1\%. The error in the calculated pressure for instability was actually within the pressure bounds of the core; that is, the calculated pressure was less than the inlet pressure and greater than the outlet pressure. Pressure affects the void reactivity very strongly in this system.

The question naturally comes up: How do you know you didn't have hydrodynamic oscillations? Well, we had seen hydrodynamic oscillations in a previous experiment at $\mathbb{N R F}$, using an earlier core. These are characterized by periodic fluctuations in the pressure measurements within the channel--it was a special hot channel subassembly that produced the oscillations. In this particular experiment no such fluctuations were observed in the pressure indications, in the several instrumented locations in the core. Again, for the details of what was done in this latest analysis, I would refer you to the report soon to be issued.

Since this is our first attempt, and our first experiment, in predicting void reactivity oscillations, I guess it would be unwise to make too great a claim at this time; but at least we can say we are optimistic about using design parameters to predict reactivity feedback oscillations under boiling conditions. 
The next thing--I guess I'd better just touch on--is the extension of frequency response methods to the spatial stability problem. My description will have to be brief, but perhaps I can at least give the gist of what has been done recently and then refer you to reports (pending) for the details. The method, developed by Steve Margolis, is a combination of modal analysis and a Laplace transformation of the xenon transfer function. What he gets is a determinant of transfer functions. Then, by setting the determinant equal to zero, he obtains what really can be called the characteristic equation of the system. Any of the various modal expansions can be used and any number of modes can be included. However, if only one overtone mode is used, the determinant has but one transfer function in it, located at the 1-1 position, and is much simpler to calculate and to interpret.

The method amounts to evaluating the determinant for values of $\mathrm{s}$, the Laplace variable, along the imaginary axis to determine whether or not the characteristic equation has any roots in the right-half plane. It can be extended to actually locate the roots by, in effect, moving the axis over which the variable is allowed to move. In other words, if we move the imaginary axis over a certain distance, we can actually enclose any contour that we want and "home-in" on the location of the zeros, and Margolis has done this.

By including the higher overtone modes one can approximate as closely as may be desired the true natural mode of oscillation. Since this method involves an expansion in static modes, in some cases it is possible that the higher modes may play a part by their interaction with the fundamental modes. This method of analysis allows additional overtone modes to be used in describing the characteristic equation of the system for the xenon oscillation.

When on,ly one overtone mode is adequate, it is very simple to deter mine the effect of a change in the temperature coefficient of reactivity or a change in the core coupling. Such changes simply translate the Nyquist plot along the real axis to the right or to the left, improved coupling moving it to the left, making it more stable, as does a stronger negative temperature coefficient.

I have one slide from Steve's report which shows this Nyquist plot for different levels of thermal flux, Slide 2. You can see that as the thermal flux is increased from 0.5 times $10^{14}$ to 1 times $10^{14}$ the plot encircles the origin, indicating a change from stability to instability. of course, for still higher flux levels it is more unstable. These plots were based on one overtone mode. He has also done this for two, three, and four overtone modes, and has found very little change in results, indicating that, at least in this particular example which was a simulated model of the PWR core, one overtone mode is adequate. In addition, he has done more detailed calculations in the vicinity of the instability and has determined that the unstable flux is somewhere between 0.8 and 0.83 times $10^{14}$.

This method uses modes calculated by means of a digital program called MULE which has just been debugged--in fact Steve's problem is the first test problem using it. The MULE program was developed by Dick Ewen, and he will present a paper on it at the San Francisco meeting (3). 


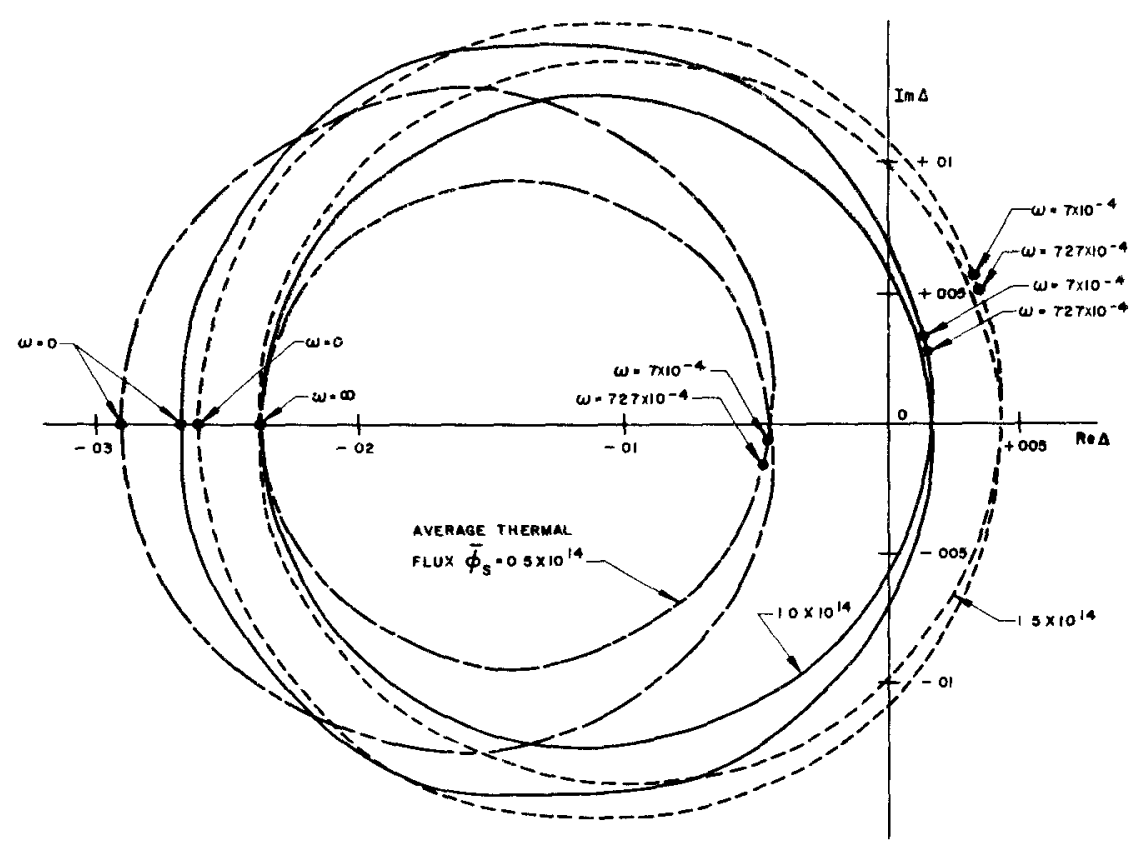

Slide 2. Nyquist Plots for Slab Reactor as a Function of Thermal Flux

In summary, Margolis' method, which also has been coded for digital solution, uses modes derived from the MULE code of Ewen and then goes through the transfer function determination to ascertain stability. When only one mode is used, one can observe the relative importance of temperature coefficient and coupling by simply translating the Nyquist plot, as I said before, and this provides a useful tool for evaluation or optimization of core design.

For a more adequate description of the frequency response method of spatial stability analysis and the MULE program for calculating modal expansions, I refer you to papers by Margolis 4 ) and Ewen(3) to be given at the AIS meeting in San Francisco and to the pending WAPD reports. 


\section{REFERENCES}

1. S. G. Margolis and S. Kaplan, "Transfer Functions for Boiling Reactor Stability Calculations," Trans. Am. Nuclear Soc., 3, p. 182 (June 1960).

2. J. E. Meyer, R. B. Smith, H. G. Gelbard, D. E. George, W. D. Peterson, "ART--A Program for the Treatment of Reactor Thermal Transients on the IBM-704," WAPD-TM-156 (November 1959).

3. R. L. Ewen, "MULE - A Code for the Calculation of Two Types of Overtone Modes," Trans. Am. Nuclear Soc., 3, p. 397 (December 1960).

4. S. G. Margolis, "A Nyquist Criterion for Spatial Xenon Stability," Trans. Am. Nuclear Soc., 3, p. 397 (December 1960). 
Lipinski: Our next panelist will be Mr. Silver from Oak Ridge.

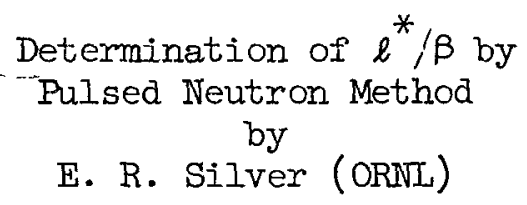

Silver: I'll keep my remarks very short since it appears to me, in any case, that they're only somewhat marginally related to the main topic of today's discussion. I propose to describe briefly some measurements of $\ell / \beta$, the neutron generation time, that we have carried out at oak Ridge using the pulsed-neutron method.

Our sources of pulsing have been two. We have used in some experiments a 300 kilovolt accelerator pulsed in the conventional way with a deuterium beam on a tritium target--which we have actually tilted up on end, and pointed down at the swimming pool reactor with the flight tube of the accelerated deuterons some 25 feet long, a target down next to the BSR reactor, and a fission chamber also adjacent to the core as the detector.

The other method that we have used is a little more unusual, and I'll just mention it very briefly, therefore. We used two reactors, since we happen to have two in the swimming pool--one as a source and the other as the experimental reactor. Before I talk about that, let me review very quickly, although I'm sure most of you are familiar with it, the equations pertaining to the measurements.

$$
\begin{gathered}
\rho=\frac{\ell^{*}}{T k_{\text {eff }}}+\sum_{i} \frac{\beta_{i} \gamma_{i}}{1+\lambda_{i} T} \\
\frac{\rho}{\beta \gamma}=\frac{\ell^{*}}{\beta \gamma} \frac{1}{T k_{e f i}}+\sum_{i} \frac{\left(\beta_{i} \gamma_{i} / \beta \gamma\right)}{1+\lambda_{i} T}
\end{gathered}
$$

where

$$
\sum_{i} \beta_{i} \gamma_{i}=\beta \gamma
$$


Equation (1) is just the standard asymptotic inhour equation, which in equation (2) has merely been modified to the usual dollar scheme by dividing by beta times gamma, the effective delayed neutron fraction.

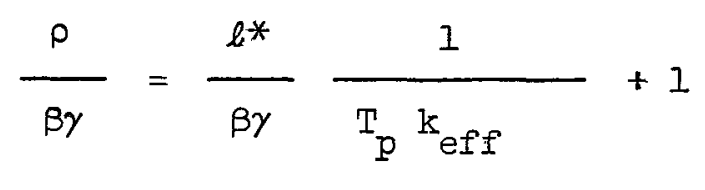

$$
[\rho(\$)-1]=\frac{\ell^{*}}{\beta_{\text {eff }}} \lambda_{p}
$$

where

$$
\frac{\rho}{\beta \gamma}=\rho(\$), \quad T_{p}=\frac{1}{\lambda_{p}}, \quad \beta \gamma=\beta_{\text {eff }} .
$$

For prompt neutrons only, in other words, at times so short after the burst that delays do not enter, we get equation (3), which can then be rewritten as shown in equation (4) where $\lambda_{p}$ is now the decay of the prompt neutrons with no delays, and the parameter to be measured is

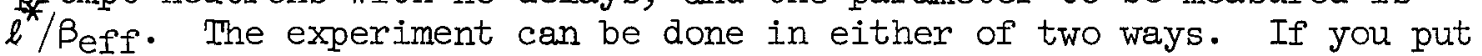
the reactor into a known configuration of reactivity, $\rho$, and you measure the decay constant, $\lambda_{p}$, then $l^{*} / \beta$ is determined directly. The experiment can also be turned around, of course, and we have done this: Once $b^{*} / \beta$ is known, you can measure unknown reactivity configurations, and thereby get control rod measurements and I'll mention this briefly.

The simplest and least problematic interpretation of the results is when $\rho$ is zero. If you can pulse a critical reactor, then $\rho$ is known quite accurately to be zero, and you get the simple equation that the $\ell^{*} / \beta$ is the reciprocal of the decay constant $\lambda_{p}$. However, obviously your pulsing represents a long-range, linear, flat source on a critical reactor and you thus have a linearly rising background in time which makes it difficult to get data, and the natural background, even when you first start it is quite high, so it poses real experimental problems. However, we have done this, because we were apparently running into difficulties with inhour calibrations of rods accurate enough so as not to affect the results. 
Slide 1 is a diagram of the apparatus that I've mentioned--the two-reactor experiments. Our standard BSR-I had a $D_{2} \mathrm{O}$ aluminum tank next to it; at right angles thereto--this is looking down at the top of the reactors-an air-filled tube, a mechanical chopper, a rotor some four inches in diameter, filled with a boron-10 powder, in order to be as black as possible--and three slits designed to give a total transmission of about 10\% of each half cycle, and then the BSR-II reactor--although other reactors have also been measured--in this case the BSR-II reactor, which is the experimental assembly. In the slide we show a lithium detector, although most of the work was done with a fission chamber because the gamma background is rather high, and you get much better signal-to-noise ratios with a fission chamber.

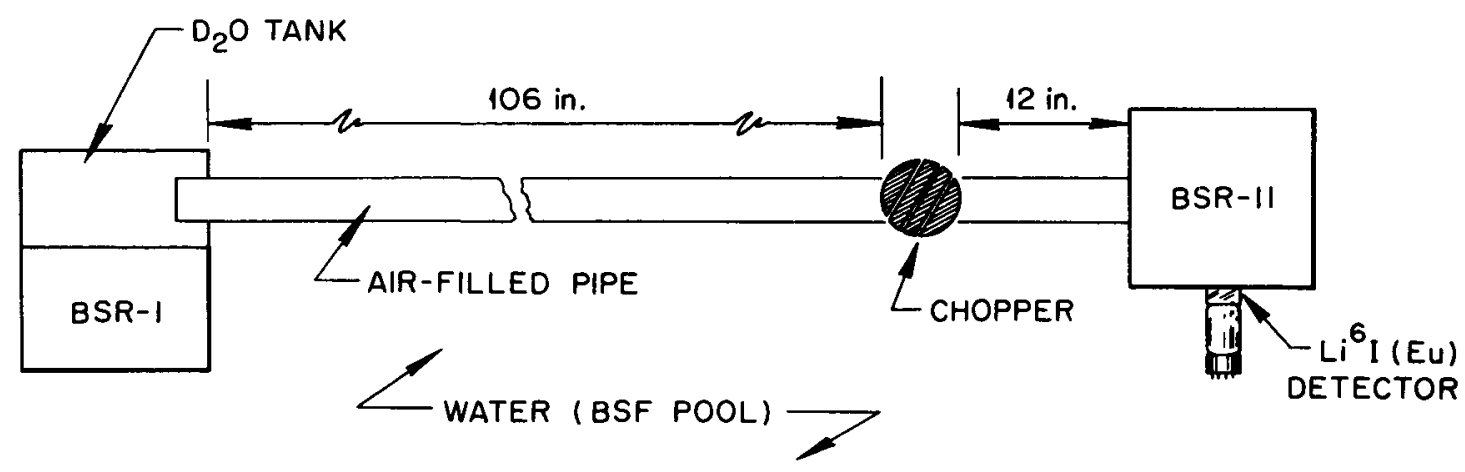

Slide 1. Reactor Beam Pulsing Experimental Arrangement

When you are pulsing a reactor which is close to critical, and it is, at best, a dollar or so subcritical for these experiments, in order to get good value of $\ell^{*} / \beta$, your background is quite high. Some typical curves obtained with the chopper are shown in Slide 2. In the upper one you will note that the minimum in the count rate, which is essentially the background, is a factor of about six or so lower than the peak. You would start measuring about where the fundamental mode (the asymptotic mode) has become established. (The measurement interval is shown in the slide). However, when you get close to critical, as we did in the lower configuration, even your total ratio of peak-to-background is only a factor a little better than two--and you are measuring a decay only something like a factor of one-and-a-half to one to your background, subtraction becomes rather crucial. However, we have coded a method due to Cornell which does the subtraction and extracts the exponential quite nicely.

Slide 3 shows what we get from these curves, the top one being at a reactivity of half a dollar subcritical, the next one, one dollar, and the lowest a little over one-and-a-half dollars subcritical, and 

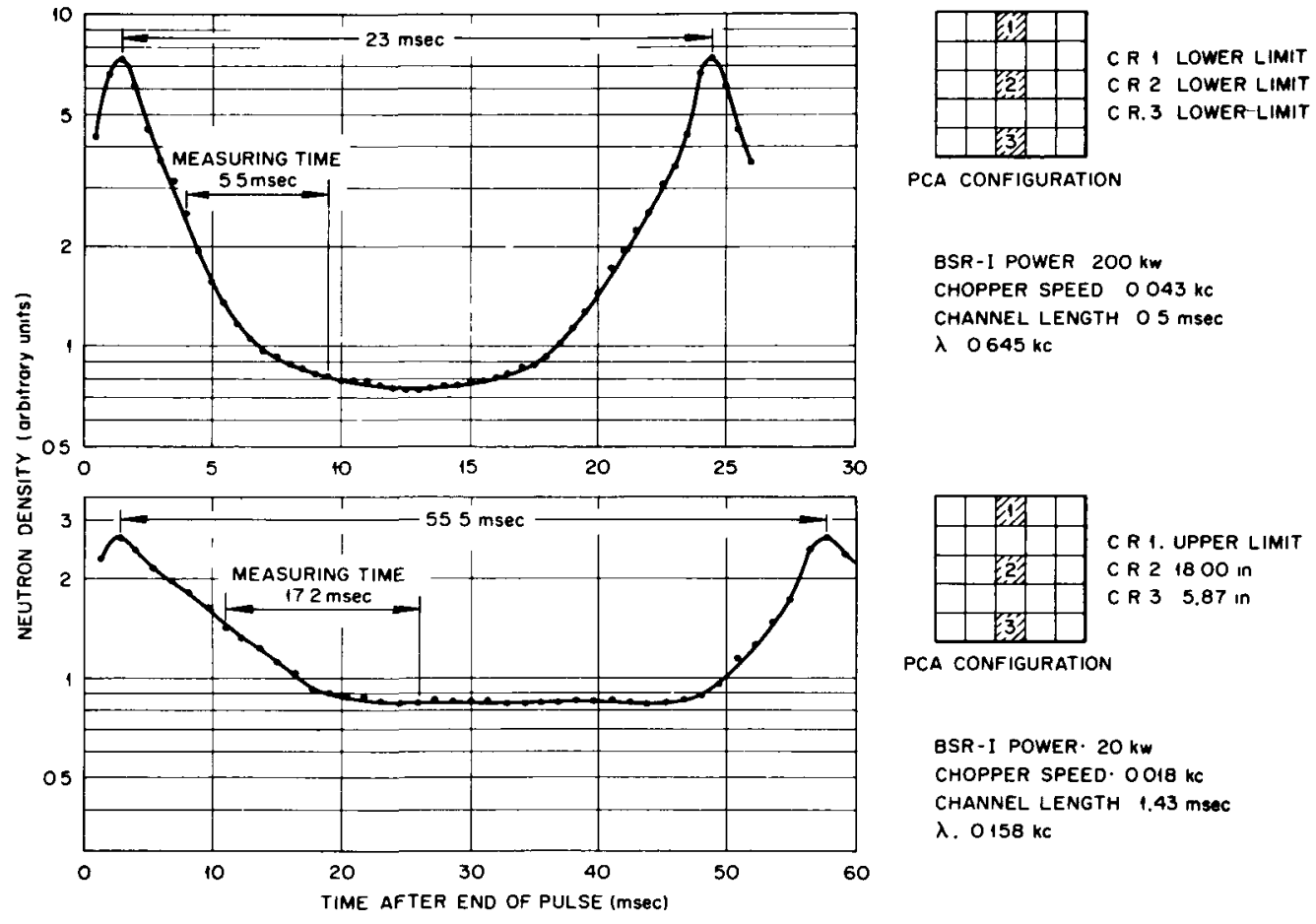

BSR - I POWER $200 \mathrm{kw}$

CHOPPER SPEED $0043 \mathrm{kC}$

CHANNEL LENGTH O $5 \mathrm{msec}$

$\lambda O 645 \mathrm{kc}$

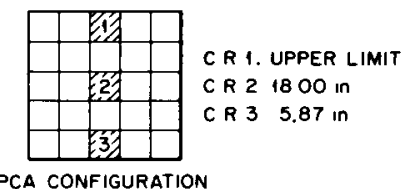

BSR-I POWER $20 \mathrm{~kW}$

CHOPPER SPEED $0018 \mathrm{kc}$

CHANNEL LENGTH $1.43 \mathrm{msec}$

$\lambda$. $0158 \mathrm{kc}$

Slide 2. Thermal Neutron Pulse Shape Due to Reactor Beam Pulse, for Two Different Reactivities of the Experimental Assembly

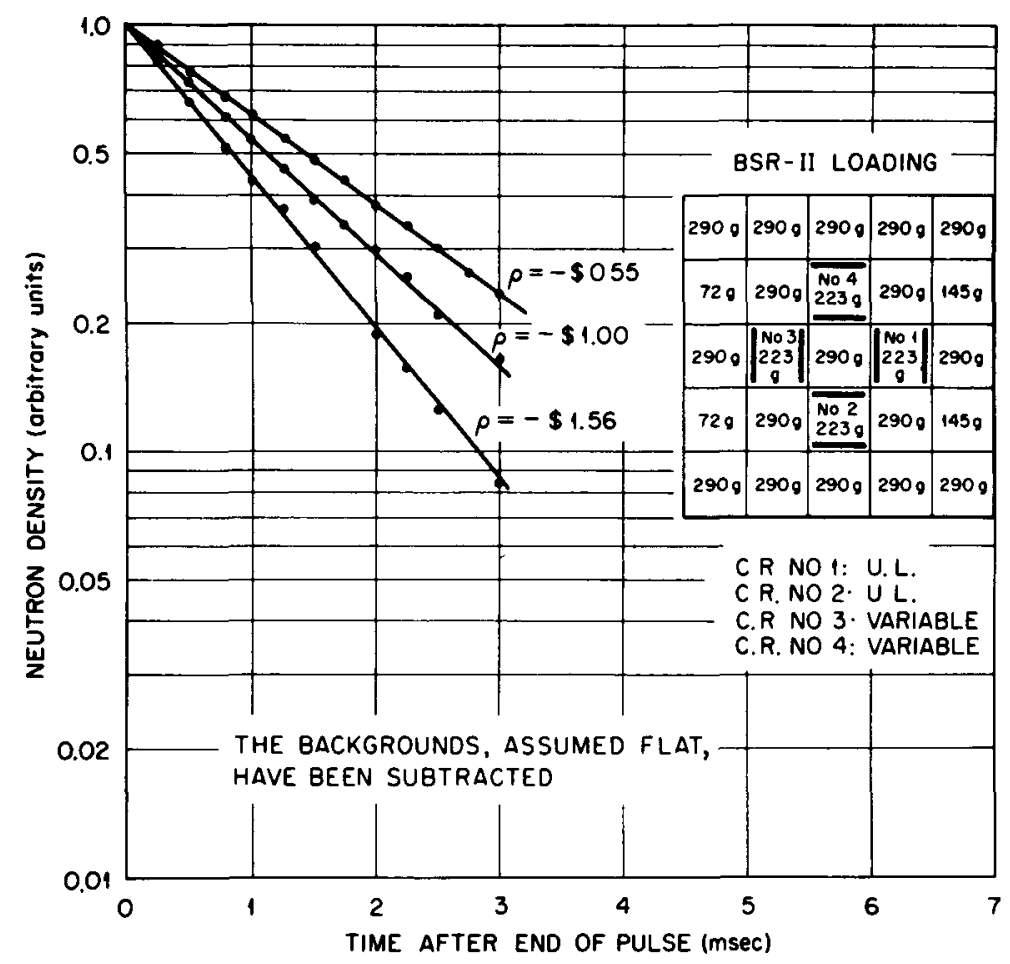

Slide 3. Calculated Results from Pulsed Neutron Measurements 
you note that after the background has been subtracted we get some pretty neat looking single exponential decays. These particular points, again were taken on the BSR-II reactor. You do this over a range of reactivities in order to see whether there's any effect of your control rod calibrations. Shown in the table are the results obtained with an aluminum reactor, where the decay constants are varying over a factor of two, one starting at about $40 \notin$ subcritical, and extending to oneand-a-half dollars. Notice that $\ell^{*} / \beta$ in this range varies only by about 3\%. Apparently our inhour calibration methods, in this case at least, are reasonably good, that there is no large-scale change in the measured $\ell^{*} / \beta$ as you change the reactivity over this range of one-half dollar to one-and-a-half dollars.

TABIE

Decay Constant and $\ell^{*} / \gamma \beta$ Values for Aluminum Pool-Type Reactor at Various Reactivity Values

Reactivity

Measured By

Inhour Method

(dollars)

$-0.40 \pm 0.02$

$-0.52 \pm 0.02$

$-0.80 \pm 0.03$

$-1.00 \pm 0.04$

$-1.48 \pm 0.06$
Decay Constant
$(\mathrm{kc})$

$0.121 \pm 0.004$

$0.135 \pm 0.004$

$0.158 \pm 0.005$

$0.177 \pm 0.005$

$0.221 \pm 0.007$

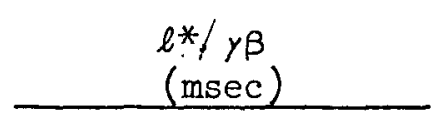

$11.57 \pm 0.58$

$11.26 \pm 0.56$

$11.39 \pm 0.57$

$11.29 \pm 0.56$

$11.22 \pm 0.56$

AVERAGE $11.3 \pm 0.6$

However, because there is some problem with the inhour measurements of rod worth since you always have to move other rods to compensate, and so flux perturbations occur, we have actually done most of our work recently using the accelerator which has a good enough on-off ratio of the pulse so that we can work at critical itself. We have measured the BSR-I in this fashion; we've measured $\ell^{*} / \beta$ with a clean core, and obtained about $9 \mathrm{msec}$, in a somewhat poisoned or dirty core, about $8.9 \mathrm{msec}$. 
The measurement we made on the BSR-II, I want to bring out briefly because, it's been discussed otherwise, or at least touched on by Art Wasserman. In the measurements that he described on the BSR-II the values of $\ell^{*} / \beta$ all cluster about 2.9 to $3.0 \mathrm{msec}$. We measured $3.2 \mathrm{msec}$ using the chopper and the reactor; we never measured at critical because with the chopper the background was a little too high to permit you to get a good data at critical itself, and we now have some reason to doubt the calibration of the control rods that we used for putting the known amount of negative reactivity in. Our value is 3.2 , plus or minus .2 msec, which is not in bad disagreement with the values of about $2.9 \mathrm{msec}$ that were obtained by the other method at spert. I hope that sometime in the future when we get the BSR-II back into the pool we will pulse it with the accelerator at critical and refine that value somewhat more. 
SESSION III

PANEL PRESENTATIONS

Lipinski: Our last panelist will be Mr. Storrer from APDA.

\author{
Presentation of Nonlinear Stability Problems \\ by \\ F. Storrer (APDA)
}

Storrer: I'd like to talk about nonlinear stability problems. Actually this is unfinished work; we started doing some work on this about two months ago, but we haven't been able to complete it yet due to other work. The transfer function method and the osclilator test are used only to determine stability for small perturbations. We have tried to extend this method of transfer function to study the problem of nonlinear instability.

I'll first review briefly the problem of stability for small perturbations. You can write that the transfer function $G$ at some power is $G_{0}(s) /\left[1-P X(s) G_{0}(s)\right]$. By the way, we limit ourselves to the case of linear feedback--we only consider the nonlinearity in the kinetics equation for large perturbations. If we call $Q(s)$ the inverse of the zero power transfer function, we can write this expression for $G$ by dividing the numerator and denominator by $G_{0}, 1 /[Q(s)-P X(s)]$. The usual Nyquist criterion is based on looking at the poles of the denomina tor and looking whether you have any roots, $s$, with zero or positive real parts.

So much for the stability for small perturbations. Can we extend this method now to large perturbations? First, let me say that, as far as I know, the only general criterion which has been developed for large perturbations is the one which was developed by Dr. Ergen and Dr. Welton, and some other people who have improved on it since, taking into account delayed neutrons and so on, and this is essentially that the real part of $X(1 \omega)$ is negative. Here, I define $X(0)$ to be negative when you have a negative power coefficient: Usually you define it differently, but since I'm going to talk about positive coefficients also, I would rather have $X$ positive for a positive coefficient and negative for a negative coefficient. Now, this is an unconditional stability criterion. If a reactor satisfies this condition, you can do anything to it. It will always be stable--even if it had no delayed neutrons.

Unfortunately, I don't think that any heterogeneous reactor satisfies this condition. So, we would like to have some more realistic stability criteria that we could use, and we are thinking in particular of the possibility of operating reactors with a prompt positive component of the total (negative) power coefficient--it's not that we want to do it, (laughter in audience), but we think that future fast reactors may possibly have some prompt positive components. This may be Doppler for one thing, and in some larger reactors we may have a positive sodium 
coefficient. We11, Fermi has all negative coefficients, I want (laughter in audience) to reassure you. We have been extremely conservative, but I don't think we could say a priori that we should not accept reactors in the future whose power coefficient has a prompt positive component. So, we have tried to develop some stability criteria.

This is the principle of the method. (I'II repeat that this work has not been completed, so if you ask me too many questions I may be embarrassed. But I think it's useful to present something even when it's not completed, so that people can criticize it and tear it to pieces if they want to.) Usually, the transfer function is for small perturbations around a steady state; one has small reactivity oscillations, and one looks for the resulting small power oscillations. Well, the idea--let's say that I'm not in steady state, but I have programed my rods so that the power would go up at a certain rate. Let's say that power here is a function of time--I want the power to go up exponentially. For this I insert so many cents of reactivity and when some feedback comes in, I insert some more reactivity to compensate for the feedback, and I try to stay on a constant period. Is there any danger that at some stage you'lil depart from this curve, from this programmed curve? Well, here's how we studied this problem. It's a very similar technique to the one for small departures from equilibrium. Now, we are going to talk about small departures from a programed power change and a programmed reactivity change. If you know the feedback--this $X(s)$ that describes the feedback--you can calculate how much reactivity you have to insert to stay on this constant period.

Let's assume that superimposed on this programed $R(t)$ which would give us this power variation, we have a little wiggle, aest, and then we study the departure of power from the programmed value that would correspond to $R(t)$, so the power would go at $P_{o} e^{p t}$-for an exponential this would be my progranmed value--times $\left(1+b^{s t}\right)$. I can define the transfer function as the ratio of $b / a$, and $I$ can again $100 k$ for the roots in the denominator, and if, for some value, the real part of the root is positive, then I may depart from my programed power variation. Well, when you do this, you substitute this into the reactor kinetics equation--the steady state or the programed parts cancel out--and then you also keep only the small perturbations from this programmed variation. And then you find the following transfer functions. For $b / a$, you find $1 /\left[Q(p+s)-R_{e f f}-P X(p+s)\right]$. (Maybe I should have kept my expression here that the power goes at $P_{0} e^{p t}$, so $p$ is the programed reciprocal period, and $s$ describes my little wiggle here.) So, we can look at the roots of this denominator. We have not looked into what happens if you have roots which are complex, but we are pretty sure about our results for real positive roots. By the way, this expression here is exactly the same as we had for small perturbations, except that we have $R_{\text {eff }}$ now. As I say, we have not arrived at definite consequences when you have roots which are complex and whose real part is positive, but we arrived at some definite conclusions, I think, about real positive roots. And I think those are the most dangerous. The other kind of instability which is oscillatory in nature can usually be checked; I mean, the period of the oscillation is usually relatively long, because it involves the time constant of the feedback. So if you start up your reactor--and of 
course you know that it is stable for small perturbations--so you start it up, and if you go on too short a period, you may get some kind of oscillation like this. Well, you'a know then that you went up in power too steeply, so you can reduce your rate of insertion. The dangerous thing would be if you go up in power at a reasonable period, and a.1 of a sudden you are at much shorter period.

The criterion for this is that--you'li have such a situation if you have a root--now instead of $(p+s)$ I can write just $p--i f$ I have a root of this equation for a positive real $p$, a small $p$, with a.lso positive $X$, then you'd run into this situation. If you plot the results of this thing, you will find that if you have a prompt positive coefficient (because it's only if you have a prompt positive coefficient that this would become positive), you can find a plot where you have power in abcissa and $R_{\text {eff }}$ in ordinate, and at any one power you know the maximum value of $R_{\text {eff }}$.

We11, I think I'Il stop here and I would, of course, welcome any questions, even though I may not be able to answer all of them. This will be presented at the San Francisco meeting of the ANS $(1)$. I hope it's going to be in better shape by then.

\section{REFFERENCES}

1. F. Storrer, "Stability Criteria in Iinear and Nonlinear Reactor Dynamics", Trans. Am. Nuclear Soc., 3, p. 432 (December 1960). 


\section{GENERAL DISCUSSIONS}

Lipinski: Mr. Kasten from Oak Ridge had a few comments he wanted to make. Kasten: I have some information on the transfer function of the Homogeneous Reactor Test to present. I have some slides.

Lipinski: How long will it take for that stack of slides?

Kasten: I'm going to mun through them very quickly. As you know, the Homogeneous Reactor Test had some difficulty with fuel instability.

Lipinski: Would you like to come up here and use the microphone?

$$
\begin{gathered}
\text { HRT Transfer Function Information } \\
\text { by } \\
\text { P. R. Kasten }
\end{gathered}
$$

Kasten: The Homogeneous Reactor Test developed some holes in the core wall because of fuel deposition. This deposition led to local hot spots, and melting of the metal at two positions. Deposition of fuel was associated with chemical instability of the fuel. The reactor-noise-analysis presented here was performed to see if the shape of the transfer function was dependent upon factors which lead to fuel instabilities.

During normal operation of the HRT, power oscillations occurred which were $I$ to $2 \%$ of the average power; after some fuel had deposited at various portions in the system, there was a loss in nuclear temperature and the power oscillations were larger, (about twice as large as normal). In this study we investigated the magnitude of the transfer function by means of autocorrelation analysis. Prior to presenting the results, I'd like to give a brief analysis review. Slide I shows conventional analysis and noise analysis procedures. We normally take the conventional kinetics

\section{CONVENTIONAL ANALYSIS}

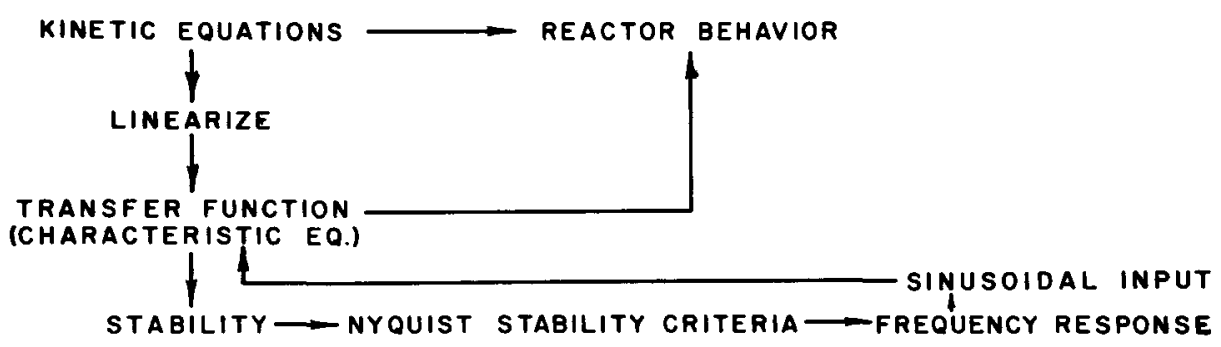

NOISE ANALYSIS

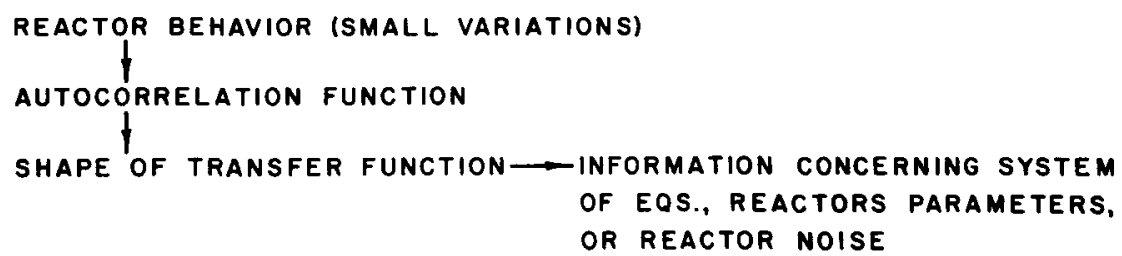

Slide 1. Methods Used to Analyze Reactor Behavior 
equations and use these to interpret reactor behavior. Under many circumstances the equations are linearized, which permits one to obtain the transfer function, which is essentially the inverse of the characteristic equation. We can analyze the transfer function by means of Nyquist's stability criteria to see if the system is stable. Frequency response information is associated with a particular interpretation of the Nyquist stability criteria; we can use the frequency response along with a sinusoidal input signal to bring us back to our transfer function. The above information is indicated in the first part of Slide 1 . In noise analysis we essentially start with the reactor behavior and obtain the autocorrelation function (in this particular instance); we use this function to get the shape of the transfer function, and from the shape we can get information concerning the system of equations, or, if we know the system of equations, we can determine parameter values, or, if we know both, we can get information on the reactor noise. This is indicated in the bottom half of Slide 1 .

Slide 2 gives the definition of the autocorrelation function which we used. The value of $X$ in Slide 2 is the power, and so the autocorrelation function correlates the power at a given time against itself at a future time. Slide 3 shows the value of the integral of interest, using the Fourier integral representation. As shown, the integral of interest is equal to the inverse Fourier transform of $X(v)$ times $X(-v)$ where $X$ is the Fourier transform of the power. Also, the Fourier transform of the power is equal to the transfer function times $\Delta \mathrm{k}(v)$, because we have

$$
\phi(\tau) \equiv \frac{\overline{x(t) x(t+\tau)}}{\overline{x(t)}^{2}}=\frac{\lim _{T \rightarrow \infty} \frac{1}{2 T} \int_{-T}^{T} x(t) x(t+\tau) d t}{\lim _{T \rightarrow \infty} \frac{1}{2 T} \int_{-T}^{T} \overline{x(t)}^{2} d t}
$$$$
=\frac{\int_{-\infty}^{\infty} x(t) x(t+\tau) d t}{\int_{-\infty}^{\infty} \overline{x(t)^{2}} d t}
$$

Slide 2. Definition of Autocorrelation Function 


\section{CONVOLUTION THEOREM}

$$
\begin{aligned}
& \int_{-\infty}^{\infty} x(t) x(\tau+t) d t=\int_{-\infty}^{\infty} d t x(t) \frac{1}{2 \pi} \int^{\infty} x(\nu) e^{-i \nu(\tau+t)} d \nu \\
& =\frac{1}{2 \pi} \int_{-\infty}^{\infty} x(\nu) e^{-i \nu \tau} d \nu \int_{-\infty}^{\infty} x(t) e^{i(-\nu) t} d t \\
& =q^{-1}\{x(\nu) x(-\nu)\}
\end{aligned}
$$

Slide 3. Relation Between Autocorrelation Integral and Fourier Transform of Reactor Power

a linear system of equations with constant coefficients. This is indicated in Slide 4. Slide 5 shows what information the autocorrelation function can give. Using the information in Slides 3 and 4, we see

$$
\begin{aligned}
& D\left(\frac{d}{d t}\right) x(t)=f(t) \\
& W(s)=\frac{\bar{x}(s)}{\bar{f}(s)}=\frac{1}{D(s)} \\
& X(\nu)=W(\nu) \overline{\delta k}_{e}(\nu)
\end{aligned}
$$

Slide 4. Relation Between $X(v)$, Transfer Function, and Transform of the Driving Function $\left(8 \mathrm{k}_{\mathrm{e}}(v)\right)$ 


$$
\begin{aligned}
& \mathscr{F}\{\phi(\tau)\}=k|W(\nu)|^{2}\left|\delta k_{e}(\nu)\right|^{2} \\
& \int_{0}^{\infty} \phi(\tau) \cos \nu \dagger d t a|W(\nu)|^{2} \\
& \text { IF }\left|\overline{\delta k}_{e}(\nu)\right|^{2} \text { is coNSTANT }
\end{aligned}
$$

Slide 5. Relation Between Autocorrelation Function and Transfer Function

that the Fourier transform of the autocorrelation function is proportional to the absolute value of the square of the transfer function; thus, with $\delta \mathrm{ke}(v)$ independent of frequency, we obtain the shape of the transfer function--no information on the phase angle. Slide 6 shows a typical HRT trace under normal operations. You see that the power variation is relatively small, $I$ to $2 \%$, and the predominant frequency in this trace was about 10 cycles per minute. Slide 7 shows the variation in power level as a function of probability of occurrence. Note that the histogram is very close to Gaussian in shape, and so encourages

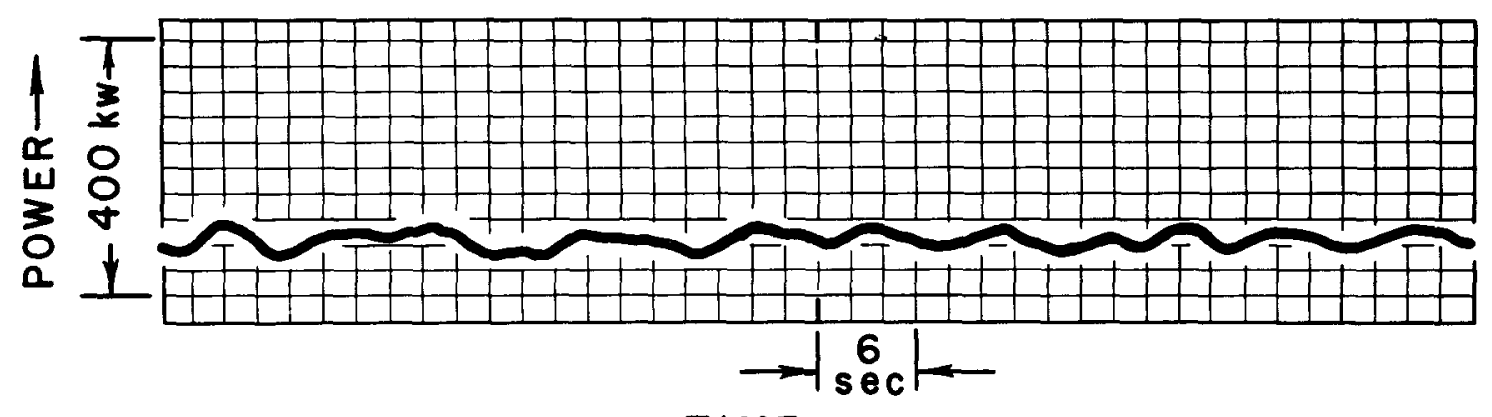

TIME $\longrightarrow$

\author{
AVERAGE POWER LEVEL $=2.26 \mathrm{MW}$ \\ CHART SPEED $=2.5 \mathrm{~mm} \mathrm{sec-1}$
}

SANBORN-RECORDER TRACE OF REACTOR POWER

Slide 6. Typical HRT Power Trace Under Normal Conditions 


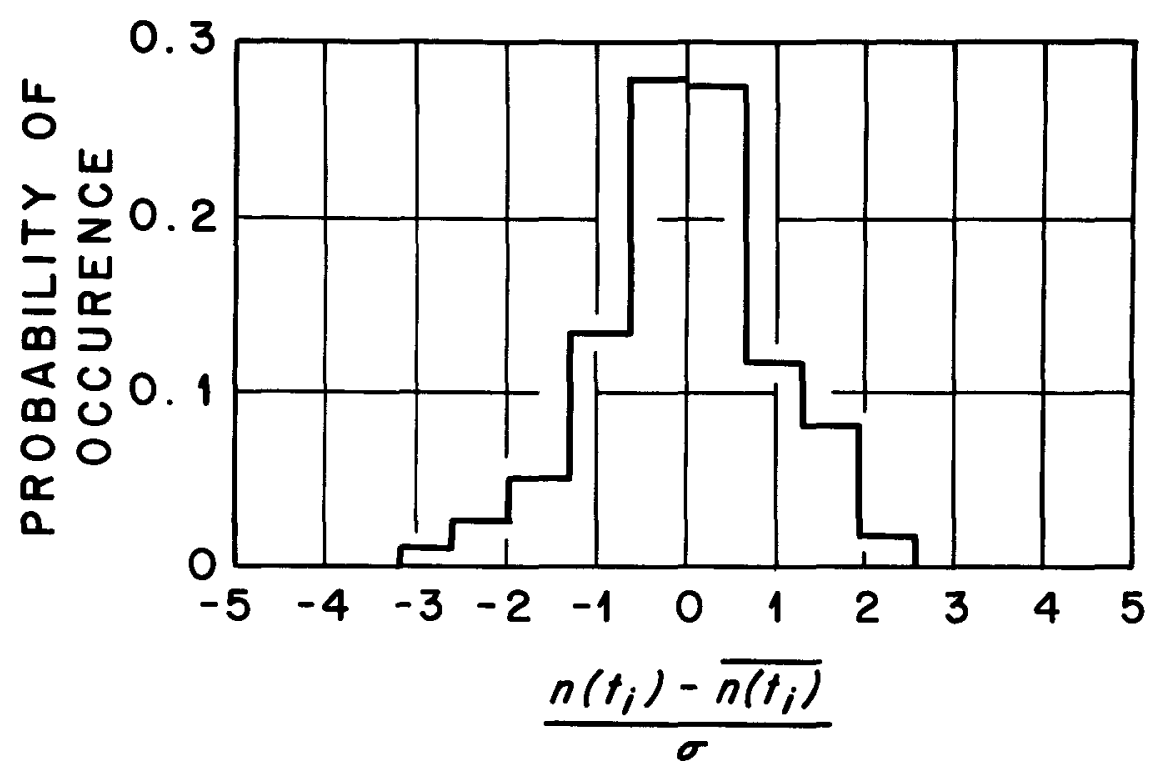

Slide 7. Histogram of HRT Power Deviations

gutocorrelation analysis. Slide 8 gives some results of the autocorrelation function for different operating conditions. The curve (I), curve (2), and curve (3), are all associated with stable reactor operation. You notice the shapes of the curves are very similar. SIide 9 shows the square root of the power spectrum, which is proportional to the transfer function, as a function of frequency. You see we get a

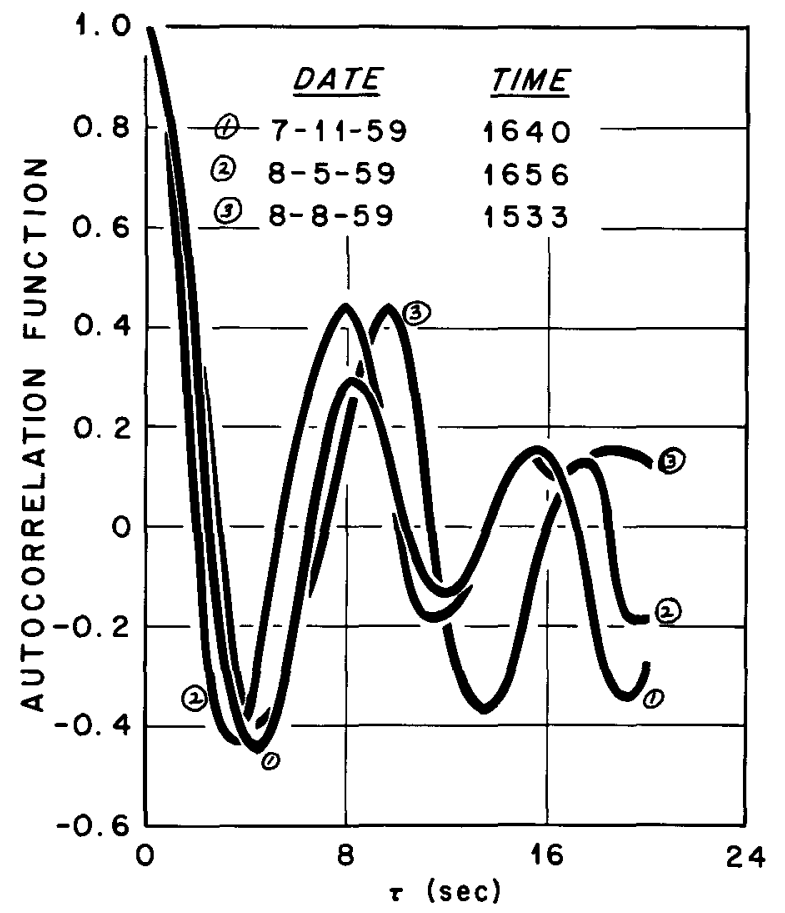

Slide 8. Autocorrelation Function Obtained from HRT Under Different Operating Conditions 


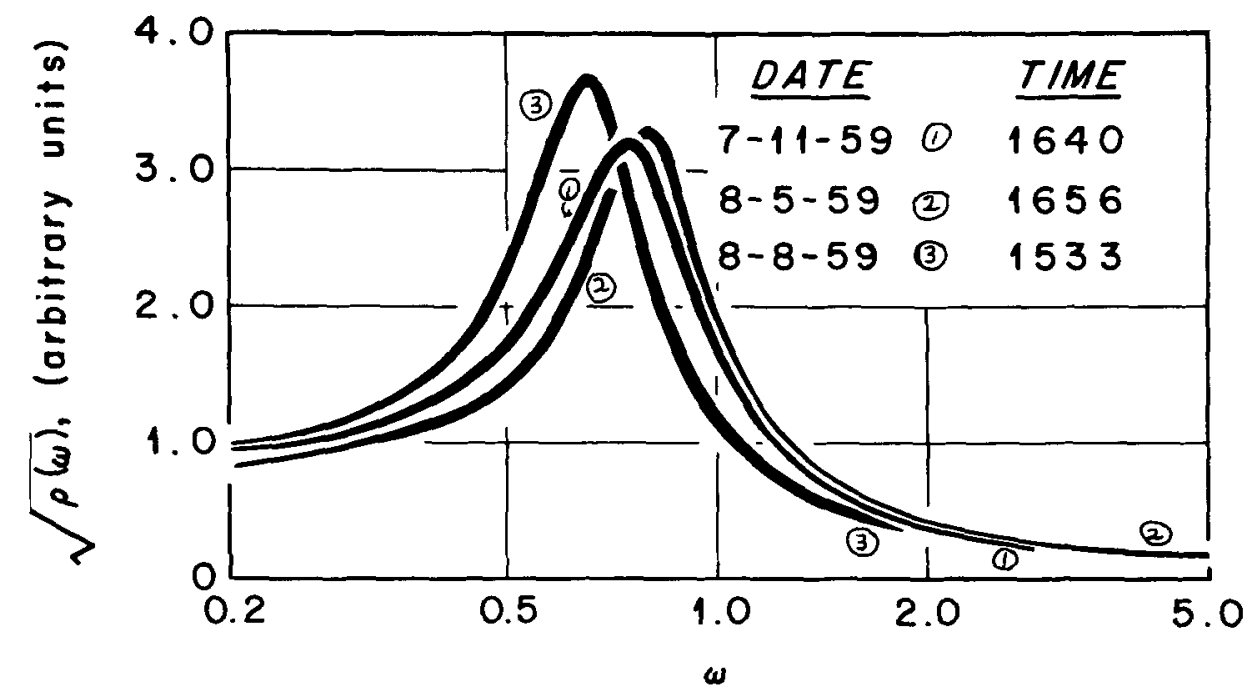

FOURIER AMPLITUDE

Slide 9. Shape of HRT Transfer Function as a Function of Frequency

characteristic peak--it's rather sharp; the " $Q$ " value is normally above two in these stable conditions. Slide 10 shows the autocorrelation function when there had been a fuel loss from the solution. As shown, the shapes of the autocorrelation functions are quite different than those in Slide 8. The results given in Slide 10 correspond to rapid-loss

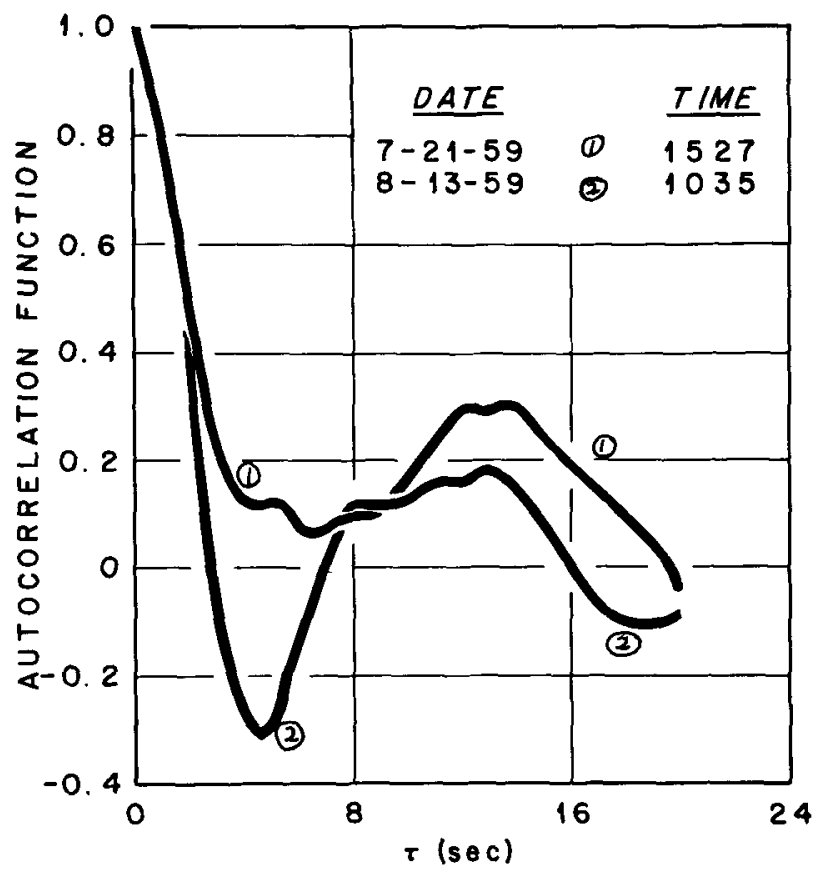

AUTOCORRELATION FUNCTION (DISTORTED)

Slide 10. Autocorrelation Function Obtained from HRT Under Some Fuel Loss Conditions 
conditions; also, the different curves shown in Slides 8 and 10 correspond to different reactor powers, different reactor pressures, different reactor temperatures, so that the transfer function would not necessarily be the same even under stable conditions.

SIide 11 shows the shape of the transfer function for a stable and unstable fuel condition. The curve labeled (2) corresponds to an unstable fuel condition, and the curve labeled (1) corresponds to a relatively stable fuel condition. The change in curve shape with decreasing fuel stablitity corresponds to a decrease in the " $Q$ " and peak of the resonance.

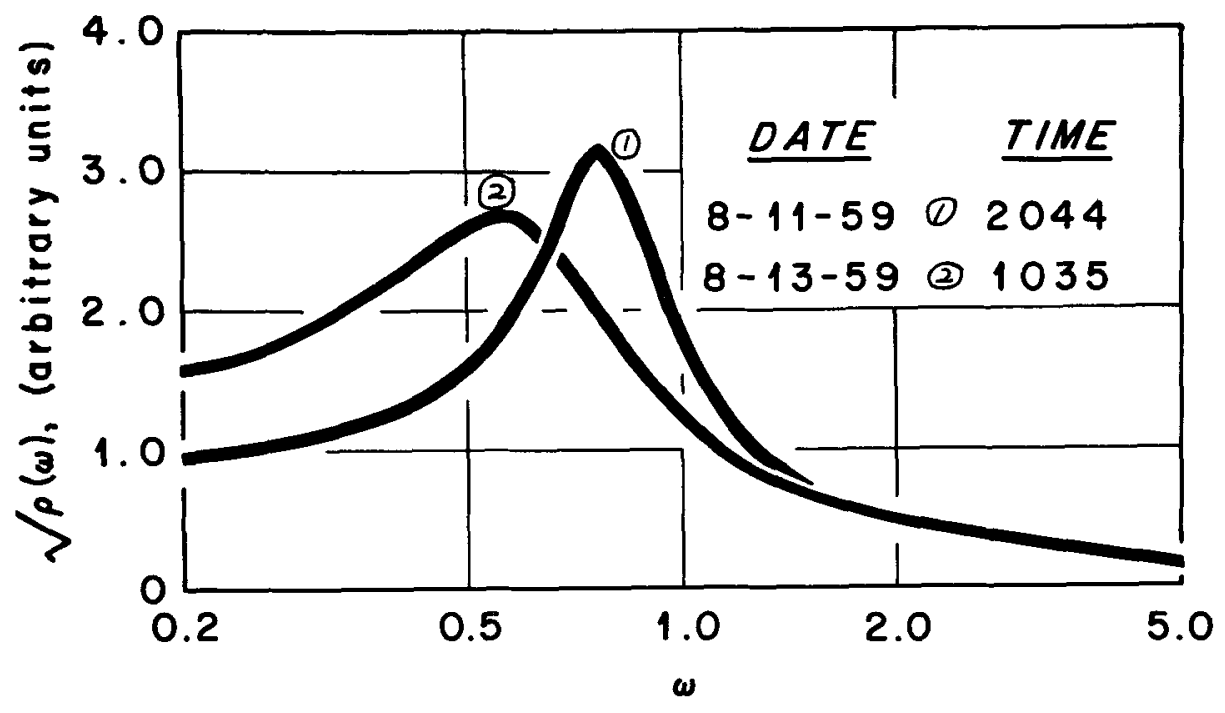

\section{CHANGE IN FOURIER AMPLITUDE DUE TO FUEL INSTABILITY}

\section{Slide 11. Shape of Transfer Function for Two Fuel Conditions}

Some analytical work based on the equations of motion was also done. The equations utilized in obtaining the shape of the analytical transfer function are given in Slide 12. The first equation is the power equation. I think that most of you will recognize the various terms. These are linearized equations, of course. The second equation corresponds to the delayed neutrons. The third equation is the energy equation. The fourth is the hydrodynamic equation which considers the core connected to a pressurizer. The last equation is the continuity equation. This set of equations was analyzed to give the shape of the transfer function. Some results are shown in Slide 13, which gives the transfer function for different reactor power levels, and for a specified fluid compressibility. Curve (1) corresponds to zero core power, while curve (5) corresponds to a reactor power of about $5 \mathrm{Mw}$ (thermal). As 


$$
\begin{aligned}
& \dot{n}=D\left(k_{i n}+\frac{d k}{d \rho} \rho\right)-\frac{\beta}{l} n+\sum_{i}^{6} \lambda_{i} C_{i} \\
& \dot{C}_{i}=E_{i}\left(k_{i n}+\frac{d k}{d \rho} \rho\right)+\frac{B_{i}}{l} n-\lambda_{i} C_{i} \\
& \dot{T}=\frac{1}{S} n-a T \\
& \dot{u}=F\left(\left|\frac{d \rho}{d T}\right| T+\rho\right)-r u \\
& \dot{\rho}=-J u
\end{aligned}
$$

Slide 12. Equations Used in Analysis of HRT

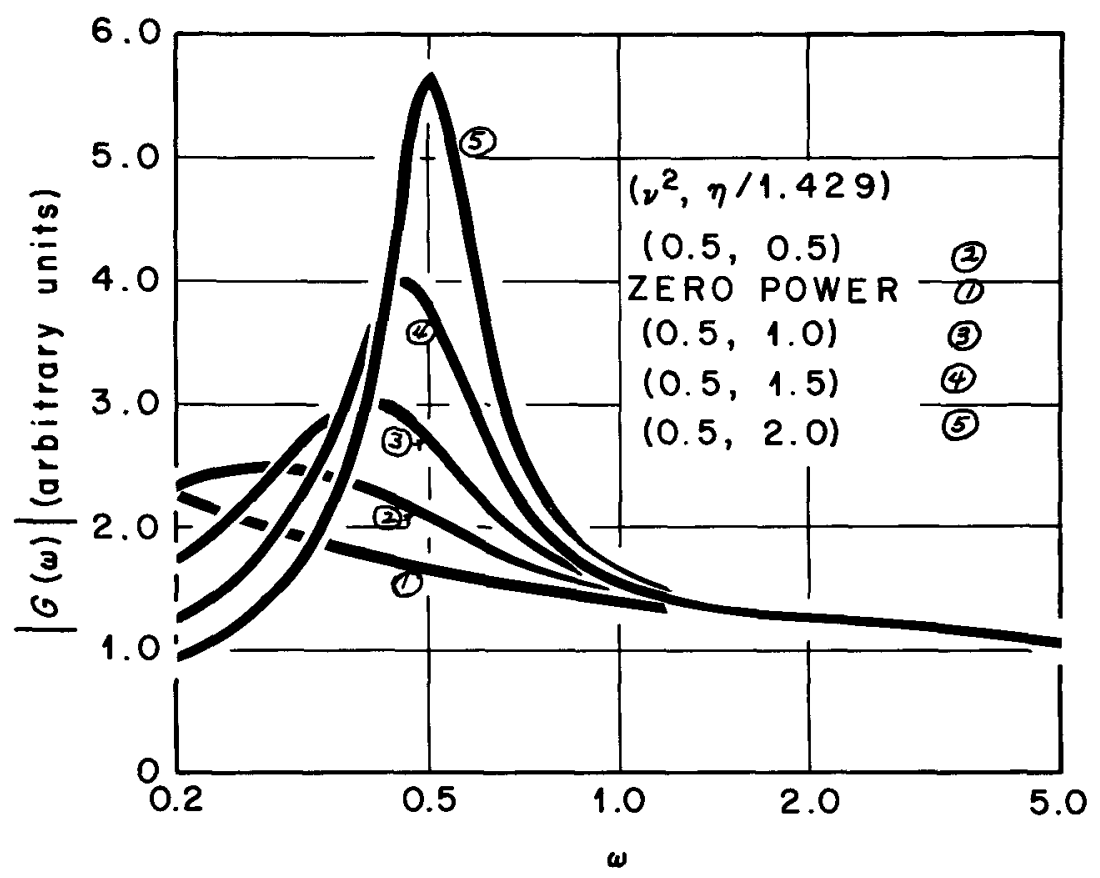

Slide 13. Calculated Transfer Function of HRT as a Function of Power Level 
shown, the resonant frequency increased with increasing power. Decreasing the fluid compressibility decreased the resonant frequency. Hydraulic frequencies ranging from 0.5 to $1.5 \mathrm{sec}^{-1}$ covered the measured frequencies of resonance obtained by noise analysis; however, such hydraulic frequencies are much lower than the value of about $60 \mathrm{sec}^{-1}$, corresponding to the presence of no gas in the core. This indicates that there was gas present in the reactor.

Also, the results in Slide 1 show a decrease in the resonant frequency with decreasing fuel stability; such a decrease can be associated with an increase in the compressibility of the fluid (decrease in hydraulic frequency), and may be due to uranium deposition on the core wall and an increase in gas and vapor within the core.

The results obtained indicate that a change in the shape of the transfer function may give some indication of a change in operating conditions. Noise analysis permits one to obtain the shape of the transfer function rather readily, and so this information can be obtained during reactor operation. A change in shape may not be understood, but it may still indicate undesirable changes in reactor operation. I would like to ask the panel for their comments concerning whether it would be worthwhile for power reactor people to automatically display the shape of the transfer function on a scope or on some kind of recorder; a change in the shape might be an indication of a "getting-into-trouble" situation which may not be measured readily in other ways.

I have essentially reported the work of Jitsuya Hirota, given in ORNL-CF-60-1-107. Jitsuya was at Oak Ridge National Laboratory for about two years, but has recently returned to Japan. He did the analysis with a desk calculator, and so quite a bit of labor went into this work. At the present time, Jim Kolb is extending the work*. We do not plan to analyze the past results obtained from the reactor, because we want to spend most of our efforts in analyzing reactor behavior under present conditions. The core holes have now been patched, and the reactor should be back in operation within a few weeks.

*

Note entered by Kasten in review: Subsequent work shows that the approximation of the curves in Slide 8 by a damped cosine is inadequate for long periods of time. Machine calculations indicate that the autocorrelation function did not damp out, and that the noise generation was not random with time. 
Lipinski: I'd like to start out by asking a question of Mr. Gyftopoulos. In the presentation on the random binary pulse, this was an assumed step which is not physically realizable. What are the restrictions if you have to assume a ramp input which is what really exists when you perform your experiment?

Gyftopoulos: First, let me say that the signal that I described on the board is realizable because it is only a question of turning something on or off electrically. The restriction here is that the rise or decay time be a fraction of the duration of the pulse. In fact, even when the rise or decay times are appreciable the derived formalism is applicable, the only difference being that the autocorrelation function of the input signal is slightly distorted.

Second, let me emphasize that the control rod does not necessarily follow, faithfully, the input signal. This essentially implies that the control rod introduces an extra filter in the overall system function. Therefore, one measures the transfer function from input command to power output.

Iipinski: Well, this is not the transfer function of interst. You want reactivity input to power output. This is the transfer function of interest, not the electrical command signal.

Gyftopoulos: No, because no matter how you do the measurement, the transfer function from control rod to reactor is always involved.

Lipinski: Yes, but in your assumption of the impulse, it is necessary that this be an impulse. If it's a ramp, then you have approximations involved.

\section{Gyftopoulos: Definitely so.}

Lipinski: Okay. Are there any questions from the audience? Joe Thie?

Thie: I have two comments. One pertains to the remark of Fred Storrer on a dangerous situation where you have a period which turns into a faster period. This has actually been done on Borax II and is reported in--I think it's ANL-6135. It came about by doing a Spert-type experiment where you eject a eontrol rod and in the Borax II core you have a positive void coefficient in the center so the period tumed into another period of about twice--one-half, rather, the value. Untimately, the excursion turned over because of voids having a measurable effect. The other comment is on the signal-to-noise ratio of Wasserman. On Borax IV, Argonne did a transfer function measurement with a much more unfavorable signal-to-noise ratio. The control rod had roughly the same signal that the reactor itself had; that is, the transfer function was being measured while the reactor was unstable, and it was possible actually to attain beats between the control rod's frequency and the reactor's own natural frequency. Needless to say, this experiment was rather complex and did not lead to a simple answer.

Lipinski: What was the All number, Joe? 
Thie: Of the Borax-II excursion? ANL-6135, "An Experimental Investigation of Boiling Reactor Safety".

Lipinski: Mr. Ergen?

Ergen: I also want to ask Mr. Storrer if he twists the lion's tail by going up on this particular exponential, and then he gets a sort of stable region where the periods will not decrease and--now let's take a period where it would increase, and the question is, can one show somehow that if you twist the lion's tail by putting not an exponential but from some other curve, that you will get approximately the same region of stability?

Storrer: Actually, I showed you there the results only for an exponential, and leaving this exponential and going to another one. But you can do this for any shape transient.

Ergen: Is there any simple or complicated correlation between the regions which you get?

Storrer: Well, I get the same expression. When you have an arbitrary power rise and you know the reactivity variation required to achieve this power rise, which is arbitrary, and then you define the transfer function the same way I did over there, you'll find the same thing whatever the shape is. It is only dependent on Reff.

Ergen: Could you say again how you find $R_{e f f}$ ?

Storrer: $R_{\text {eff }}$ is the actual neutronic reactivity. It is the inserted reactivity from which you add or subtract the feedback. So, it is the real reactivity in the physical sense of the word.

Lipinski: Mr. Chernick.

Chernick: I again address my question to Dr. Storrer. Isn't it true that you're using the linearized version of stability and you're looking at the real roots? Now, unfortunately, they run quite a bit higher--I don't know how much because you haven't actually looked at the case of pure oscillations. The only thing you can trust in the linear theory is the curve you get for pure oscillation. When you get any distance away from that, you may be in trouble. And now, since the real curve is some distance away from the curve of pure oscillation, again, I don't think you can trust a Iinear theory there.

Storrer: Well, it is certainly true that it is not satisfactory to look only at the real positive roots. You should look at the complex roots with positive real parts.

\section{Iipinski: Any other questions?}

Wasserman: I'd like to ask one of Joe Thie. You commented about the fact that one way to get the transfer function data was the transformation of time domain, step response information, for example, where the 
power didn't vary over more than a decade and I was wondering whether any really good transfer functions had been extracted from that kind of an experiment that you know of.

Thie: That depends upon how you define "good". Usually, the results are in proportion to the amount of work you do. There have been comparisons of the step transfer functions with those which were obtained with great effort by rod oscillation on EBWR, and we found that the agreement was within a couple of decibels, typically around the resonant peak. However, if you do a step twice, you don't get the same answer so I think for a quick and dirty test the step is all right. For precision, however, I think you have to do many steps and average them, or otherwise oscillate the reactor.

Lipinski: Mr. Storrer.

Storrer: I'd like to comment on this. The same transfer function that I have defined can also be used when you have a step. That is, if you want to calculate the power behavior after a step in reactivity, one usually uses the transfer function $Q(s)$ minus $P_{e x}{ }^{*}$, so if you want to find the power change after a step--or let's see, $P(s)$, where $P(s)$ is the Iaplace transform of the relative power change--you write this where you put on top here the transform of your reactivity step, which is $\mathrm{R} / \mathrm{S}$. Here also it is better, at least at the beginning, to use a transfer function where you have minus $R$ where the step appears also in the denominator. And you can show, in particular, that this is absolutely exact in the absence of feedback. When you have no feedback this is zero, and if you solve this, you get the exact response to a step change. You do not, if you do not put $R$ here. You have in the denominator the inhour relation; $Q$ is the inverse of the zeropower transfer function, so $\mathrm{Q}(\mathrm{s})$ equal to $\mathrm{R}$ is the inhour relation, so I get exactly the right roots by this method. So, I think it's better to use this as a transfer function when you have a step, rather than omitting the $\mathrm{R}$.

Gyftopoulos: Well, you wouldn't be able to use the other without the reactivity in the denominator because it is not applicable. It so happens here that when the reactivity is a step you have no timedependence, or at least your equations are not non-linear. Now, there is another point that I want to make. You said you find the same expression regardless of what you assume for your power variation? I question this, because it seems to be that what you've done here is to introduce this period, $P$, which has shifted the abscissa of convergence of the Laplace transform by $P$, so that you can handle by the Laplace transform the exponential that you assumed. Had you assumed that exponential right, the expression wouldn't be the same. Introduction of $P$ there, is effectively changing the abscissa of convergence or, in other words, the origin of the S-plane.

* Editors Note: The equation written by Mr. Storrer and his review of this discussion session were not available at the time of publication. 
Storrer: Well, the transfer function may be different, but I think they both would be the same. Not the transfer function--the initial conditions--I mean if you have a different power variation, the initial conditions would be different, but they disappear exponentially--those initial conditions are damped, both the delayed neutron effects and the feedback effects decreasing with time. Now I'd like to go back to this: when you said you cannot use this reactivity in this . . . . .

Gyftopoulos: I did not say you cannot use it; I'm just saying that you couldn't use the other one, for the step response, without putting $R$ in the denominator.

Storrer: You cannot use it?

Gyftopoulos: No.

Storrer: It has been done.

Gyftopoulos: Many things have been done wrong you know.

Storrer: Dr. Bethe has done it this way and it gives pretty good results. But for the initial power variation you get better results with $R$ in the denominator. For long times you get better results without $R$ in the denominator.

Gyftopoulos: I agree with you that you should have it in the denominator. There's no question about it.

Storrer: But you do not get the right final value, though, if you use this. For the final value of the power you should use it without $R$. I think there is a difference here; you should always have the actual reactivity in the denominator and at the end of your transients when the effective reactivity becomes zero, you have better results by not considering this $\mathrm{R}$ in the denominator.

Lipinski: While you have the light there I should like to call everybody's attention to a new ANL report that will be out shortly, covering further theoretical analysis of boiling reactors. This will be numbered ANL-622l, and the author will be Akcasu. It's a further extension of the early work that was done by Beckjord. The title of it is "Theoretical Feedback Analysis in Boiling Water Reactors". Any other questions? Beckjord?

Beckjord: Can I make a comment instead of asking a question?

Lipinski: Sure.

Beckjord: Well, two years ago, looking ahead to the Dresden startup, there was a problem of how do you determine stability? There are several practical problems involved. The main one was a disturbance, and should we make a special control rod that would cycle at high enough frequencies. We decided at the time that we wouldn't because this was very expensive and, instead, the course of action was to use the control rod which was 
already there which cannot be controlled linearly. The rod drive operates between fixed notches about 8 in. apart. We oscillated this rod in trapezoidal wave forms at low frequencies to measure the low frequency transfer function. For the intermediate and high frequency range we used a combination of noise analyses with filters--using electronic filters and integrators, and this type of thing. And also, for analysis of the reactor noise, we used some methods developed by Wes Harker which utilized a digital code or two to correlate the data and take the Fourier transforms of the correlations. And finally, well, two final things: one was a question of spatial stability. We fortunately had ion chambers placed throughout the core which enabled us to compare the spatial response to rod oscillation and also to correlate the noise effects from one part of the core to the other. And the last question was of large signal responses which was done by means of changing pressure and changing load and observing the effects on neutron flux. So, really, we combined all of these methods in order to arrive at a conclusion about the reactor stability.

Lipinski: I have a question on your trapezoidal input. Was this reduced to finite samples by hand and readings converted to digits in order to perform your computations?

Beckjord: Well, of course, as far as the analysis by the computer which Wes has been--Wes Harker has been working on, the type of wave form is immaterial as long as you know what it is. Now, in actual fact, we didn't know exactly what the wave form was because we don't have a measurement of rod position at all points. We know when it reaches the end of the notch, so the problem here is that you lose your high frequency information, so you can't tell for sure from the rod oscillation tests what the high-frequency behavior is. For low-frequency behavior, as far as measuring the phase shift and that sort of thing, we just used Fourier analysis. You can determine what the phase shift of the fundamental is relative to the reference square wave which you are putting into the reactor, because the trapezoidal flux response shifts and the wave of the--the flux wave moves backwards or forward, depending on what the phase shift is, and by a Fourier analysis you determine what the shift is.

Lipinski: What range of frequencies did this work cover? From the low end to the high end?

Beckjord: The lowest frequency of oscillation was one cycle a minute because it was apparent that the flux had reached its final value at that time except for possible Xenon effects. The maximum speed of operation of the rod was 3.7 sec per cycle, a period of 3.7 seconds. For higher frequency information--we relied on noise measurements for the data at higher frequencies.

\section{Lipinski: Any other comments or questions?}

Thompson: Mr. Kasten of ORNL.-may I direct my question to him--stated that the HRT was working under fuel-loss conditions. I gather this was a period during which the fuel had plated out on the sides of the 
reactor, and I also gather, that in this condition that there were effects on the transfer function. I wonder if he had gone any further into the mechanism of this; in other words, was this due to the change in both the distributions through the core, or just what sort of effect does he expect this is due to?

Kasten: Presumably, the fuel had been "Iost" someplace within the system; whether it had plated on the wall or in the heat exchanger, it's hard to say. Also, we don't know whether the shape of the transfer function had continually changed because of a change in the probability distribution of possible reactivity changes. The reactivity input noise might have changed if the noise was partially due to the fuel movement. It's very difficult to know what the transfer function shape would mean in that particular case. I think a change in shape, however, can be used as a signal.

Thompson: You mean that this might be due to a kinetic transfer and removal of the fuel from a given point, or is it due to the heat developed from this fuel located and distributed in odd places?

Kasten: I think it would be due to changes in fluel position.

Thompson: Is the time of interest short enough that there can be movement of fuel on surfaces and so on, so that these effects show up as a change in transfer function--are these changes fast enough to show up this way?

Kasten: These changes we observed took place over a relatively long period of time. It's not something that happens all at once--the frequencies of interest are about 10 cycles per minute.

Thompson: But that's still fairly fast to lose an appreciable trace amount of fuel on the surfaces and off the surfaces, isn't it?

Kasten: It depends on how it moves. If it's associated with fuel movement in the fluid stream, it certainly could take place in that period of time. I can't answer your question.

Lipinski: Mr. Ergen.

Ergen: Didn't you indicate that the transfer function showed a change in the gas content, and point out that, if there is fuel concentrated at some point inside the reactor, this could mean that you get boiling and this shows in your transfer function.

Kasten: That's true. The fluid compressibility appeared to increase with decreased fuel stability. The resonant peak decreased as we went to an unstable fuel situation. However, everything isn't explained, and we hope to do much more work in this direction when we operate the reactor again. We hope we don't see the above changes.

Lipinski: Paul, how about fuel-phase changes, is this a possibility? 
Kasten: It was assumed that the above behavior was associated with fuel deposition, but the surface was hot enough to cause a phase change.

Lipinski: Mr. Chernick.

Chernick: Well, I was going to ask if the power went to a higher level, whether it would indicate that the fuel was actually depositing or operating at a higher reactivity?

Kasten: We do get different shapes at different power levels. As we went to a non-stable situation, the power disturbances were greater; but just what this meant in terms of what happened to the fuel, we really don't know.

Chernick: I was just asking if the average level went up, which would indicate that you've got more fuel; some fixed, some circulatory.

Kasten: As we went to an unstable situation, the nuclear temperature always dropped, which means that the fuel went to a region of less importance; this region of lesser importance might be either the core wall, or the extermal system.

\section{Lipinski: Any other questions?}

Henry: I can't understand what you mean by an "unstable situation". How do you know it's an unstable situation?

Kasten: The word "unstable" here does not have the meaning used in other presentations at this conference. Here we are talking about fuel instability, a chemical instability, where the fuel solution physically "Ioses" uranium. This is determined by chemical analyses. But these analyses are relatively slow and are performed on a routine basis-you take a sample one day and let it cool; the next day or two days later a chemical analysis is obtained.

Henry: Depending on the analysis, you then say "on this day I was in an unstable fuel situation".

Kasten: Yes. To get back to the question, "is transfer function shape really a good criteria to help determine when things are going wrong", use of this information may be very useful because we could get it relatively rapidly.

Lipinski: Any other questions or comments? Thie?

Thie: I'd like to ask Eric Beckjord something. Does spatial correlation of chambers--does this lead to any interesting development, such as phase shifts that were significant and frequency dependence?

Beckjord: No, because within the range of frequency which we studied-which was basically what you could see on the maximum chart speed of a Brush recorder, there is no phase shift across the core. There's no evidence that we can find of spatial variations in flux. 
Lipinski: Is this true of your noise as well?

Beckjord: It's true of the noise as well. You find axial variations of noise in the upper chambers, probably due to "boiling noise", but the basic variations in power in the core, correlate from bottom to top and from side to side. These results will be reported at the ANS meeting in December.

Lipinski: When you say "Brush recorder"--that has a 100 cycle capability? Beckjord: Yes. You can't see a phase shift across the core.

Lipinski: Interesting. Mr. Thompson?

Thompson: Does the entire core always behave as a single unit?

Beckjord: Yes.

Thompson: What about the case where you took the reactor almost critical on one side and took it on up to critical on the other side, and the difference in the .......

Beckjord: The stability measurements to which I refer were made from about $60 \mathrm{Mw}$ thermal on up. No power-stability type tests were made near reactor criticality.

Lipinski: You have differences there in the time scale between the two sides as much as two cycles.

Snyder: I think the important thing is that concept, which I'd like to call the neutron transmitting property of a multiplying medium. If you have a high buckling, then you get poor transmission; i.e., you have high attenuation. In a pretty well flattened reactor, for example, a power reactor operating at power, you have very good long-distance transmission, because $k_{\infty}$ is very nearly one. The characteristic length for neutron transmission is the migration length divided by the square root of $k_{\infty}-1$. This is a big number in a flattened core and, for this reason, the core is more closely tied together than is the case when the core is either highly subcritical or highly supercritical.

Thompson: This is true, but you also, of course, have problems with the stability at startup and at times when you are not at power. You have, therefore, the problem of getting it to power.

Beckjord: Well, we also made one measurement with, as far as we could tell, very little boiling in the core, and the results were of the same character there; that if you made a change in the rod position you could see it throughout the core--that is, throughout the chambers--and the variation was the same percent.

Thompson: Did you ever find the case where when you withdrew a control rod and you found that your flux decreased in any of your chambers? 
Beckjord: No.

Henry: That can't be done unless there is negative power feedback through an external loop. You can nowhere reduce power by the withdrawal of a control rod. You can tilt the power, but only up; it cannot decrease anywhere until there is external feedback. I was convinced of this at our meeting two years ago when I tried to present a curve that showed a dip associated with a tilt, and Dan St. John balked.

Lipinski: Mr. McCullough?

McCullough: May I ask--your statement was that if the core is flattened-if the flux is flattened in the core, then the neutron transmission is very good; and, therefore, what occurs in one part is very quickly reflected in another. Is the converse equally true that if you should put a valley in the flux in the center of the core then the transmission is not good--the deeper the valley, the less you know about the transmission?

Snyder: I think this is true. In other words, you begin to have two reactors instead of one.

McCullough: Well, the point that I'm getting at is ( $I$ haven't imagined how one could get such a perturbation in the flux) if such a perturbation should occur, then if you're depending on a signal on one side to tell you what is happening on the other, and this signal becomes more and more unreliable.

Lipinski: Or, at least, insensitive.

McCullough: All right.

\section{Lipinski: Mr. Wasserman?}

Wasserman: I just have a quickie of Eric. How small a phase shift do you think you could have seen in that experiment?

Beckjord: Well, let's see, the Brush recorder--now you do see--I should perhaps amend one of the statements--axially, you see a phase shift, a small phase shift, which is due to the flow time through the reactor because a change in the bottom is carried up the core, so you do see a small shift there. Now, the smallest change that we have seen would be governed by the frequency response of the Brush and of the chart speed and, roughly, I would say the Brush has probably $30^{\circ}$ or $40^{\circ}$ phase shift at somewhere around 50 or 60 cycles and, also, the maximum chart speed, I believe, is somewhere around $10 \mathrm{~cm}$ a second and you can read roughly to a millimeter. So, this tells you it would be . . . (figuring on blackboard) we can write it out later--about $10^{\circ}$ of angle at the smallest cyclic period of $3.7 \mathrm{sec}$, and about $2^{\circ}$ for the longest period of $60 \mathrm{sec}$, which was spread over $40 \mathrm{~cm}$ of chart.

Lipinski: I have a general question to pose for the audience to see-who knows the answer, what time do they stop serving food? We can continue until we exhaust all discussion, but it would be hazardous if we couldn't get fed after we got through. Does anyone know what the hours are in the cafeteria?

Unidentified: I believe they are open continuously.

Lipinski: They don't have any specific lunch hour? Any further comments? Mr. Silver? 
Silver: Yes, I have a brief comment for the purpose of which I'd like to show Slide 1 , which as I said, I didn't show before. I mentioned at the beginning of my talk, that once you knew $\ell / \beta$, you could use it to measure unknown reactivities, and I just want to give you an example of that because I think it may have application where fairly large reactivity effects are concerned that you can't conveniently measure by positive methods, $\$ 3, \$ 4$, $\$ 5$ reactivity, say, but they're very conveniently measured in the negative domain by the pulsed method. This is a graph of--just over the range in this case of $\$ 1.4$ because that's all that was accessible to our inhour technique, although we attribute the change to the control rod measured by both the usual inhour method and by our pulsed-neutron technique. You will note that in the range over which we can check the agreement it is very satisfactory, the triangles represent the pulsed-neutron measurement. We have made other measurements extending to 7, 8, 10 dollar range of reactivity worth with multiple control rods and, in general, the answers are plausible although we don't have any good way of checking them against other experiments. We have seen rod shadowing and shadowing effects and things of this sort, just about as you would expect.

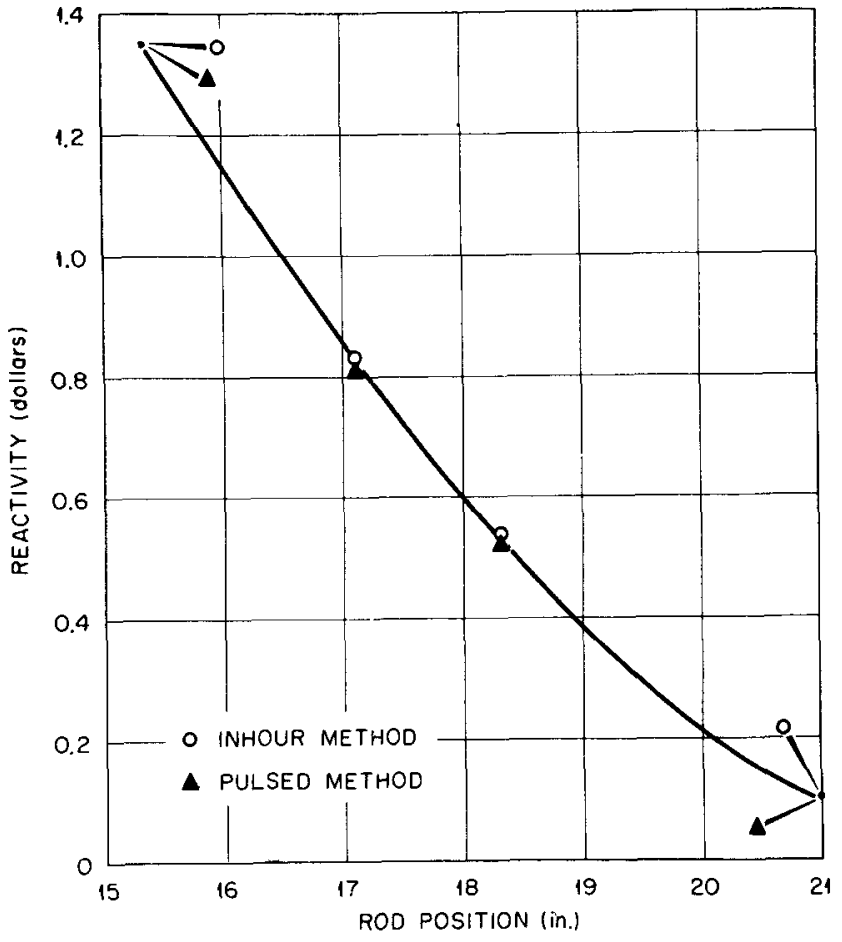

Slide 1. Reactivity of a Central Control Rod in Aluminum Pool-Type Reactor, Measured by Inhour Method, and Pulsed-Neutron Method. 
Lipinski: $\mathrm{Mr}$. Thompson.

Thompson: I think Dr. Kasten asked a question of the experts which was not answered; namely, is there any advantage in displaying the reactivity curve on the scope in a power reactor and the transfer function, in order to show the oncoming of something unexpected. Isn't this right, Paul, you asked that question.

Kasten: I wanted to see if there were any comments whether displaying the shape might prove to give useful information. I think this would be one way of finding if things are changing in a reactor and finding it very rapidly.

Lipinski: Let me see if I understand the question. Knowing what the neutron variation $1 s$, you want to compute backwards as to what the $\Delta k$ input had to be?

Kasten: No, just display the shape of the transfer function on a scope or even some kind of other instrument. By seeing this shape change with time, practice may enable one to have knowledge of what a change means.

Iipinski: I might comment on this. The work we are doing at Argonne Is in the direction where we can take the noise, and perform a computation. We won't display fully the transfer function but we will give an indication of how close the system is to being unstable, based on the linear extrapolations.

Grace: I should think that in an experimental power reactor, such as EBWR, that might be a useful tool, because of the many different changes that are made in the testing procedures. But so far as operations for power or propulsion are concerned, we feel we have to analyze thoroughly beforehand all possible modes of operation to make sure that the operator can't get into a range of instability. We would, of course, never depend on an operator watching the scope to determine whether he's getting into an unstable range. All stability analyses are done over the full range of combinations of operating conditions possible, and then the operator must stay within those bounds. But I think it probably would be of considerable use when one is changing power levels significantly in experimental power reactors, trying to anticipate how close he is getting to instability.

Lipinski: I'd like to add a further comment to that. In the case of EBWR where we're striving for maximum power production, the question arises as to whether the stability is the same over the life of the core. There seems to be some indication that when we start the reactor we will have a given set of stability conditions, but as that reactor ages it can become more unstable.

Grace: That is certainly true in these other power reactors I'm talking about. When I say we must analyze it for all possible operating conditions, I mean at several stages throughout the lifetime of the plant. 
Iipinski: Well, the ability to make a stability calculation strictly from analysis is certainly progress. Mr. Kasten?

Kasten: It seems to me that one cannot assume that all circumstances that could possibly arise in a reactor will have been looked at. If one displays the shape (although under normal conditions no changes would occur) a change in shape could be an indication for the operator to take safety action.

Shyder: Well, I think--didn't Mr. Storrer really give an example where this display would have been of no use?

Storrer: If you should display the transfer function during one of those transients you would display exactIy the transfer function that I have mentioned, and you could observe the change of shape of the transfer function because you are in the midale of a transient.

Snyder: But, in this case, the power is more sensitive to what's going on than the transfer function is.

Wasserman: May I make a comment?

Llpinsk1: Yes.

Wasserman: It seems to me that a display of that sort would be most useful in a situation where you were going to have time to do something, but where you could not so quickly recognize that something was wrong by observing other thermodynamic variables.

Lipinsk1: Well, I visualize another instrument that would be effective the same as a period meter; that could be coupled directly into the reactor system--not requiring any thought upon the part of an operator. Mr. Beckjord?

Beckjord: Well, I think you ought to distinguish between a presentation that would show noise spectrum which is--to do this requires rather little equipment, a band-pass filter and some sort of a sweeping device, maybe an integrating circuit, and, on the other hand, a tmue transfer function representation which requires a lot of expensive and complicated equipment, and I think you have less reliability with a transfer function determination. Also, while I think of it the turbine people have a, I think they called it a Framm vibration indicator. All it is is a whole series of little cantilever beams, each one tuned to a different frequency, and when the turbine begins to vibrate the indicator at that frequency starts to show a large vibration.

Iipinski: Right, your point is well-taken. You're not interested in the entire spectrum, only the portion of the spectrum that's going to give you difficulty. Any comments? Mr. Kasten?

Kasten: We have to remember that the shape of the transfer function does not tell us whether we are in an unstable situation. In fact, it doesn't really apply unless we're in a stable situation. And so, what 
we are really trying to find from this is whether the reactor is tending to go in a particular direction which we don't understand. It's not that we're having an accident or anything like that, it's just that something is happening and we don't really know that something has happened--under normal circumstances.

Lipinski: Yes, but this isn't happening over four decades. It's only within a region.

Kasten: That's true.

Iipinski: So that to talk about the entire transfer function from very low frequencies to very high frequencies is not the point of interest.

Kasten: No, it's just that a certain portion of it might be of interest to be shown as a function of time.

Lipinski: Perhaps at our next meeting I'Il have more definite information on this in terms of noise and transfer function.

Snyder: Is there anybody that has a feeling about the relative sensitivity of looking at a transfer function and looking at the sort of thing Joe Thie was talking about, where you begin to see better and better defined wave trains? What's the relative sensitivity of these two schemes for detecting the onset of instability?

Lipinski: Well, where you have a noise input--instead of inserting the artificial step test, the noise surge is the input. Then you analyze the output response as a result of this input.

Snyder: Now, Joe was saying you don't analyze the noise, you just look at it, and .....

\section{Lipinski: Oh, yes, on one of those exponential rising forms.}

Thie: I think to answer your question is to do all of these things just to be sure you're covering the field. We really don't have a good feel for the thing that you can do that will take the place of all others. Maybe in a while we will, but for the time being, do as much as you can.

Lipinski: Right now we have to coordinate all the various methods before we san say anything definite about which is the best. Everything is in its infancy right now. Mr. Gyftopoulos?

Gyftopoulos: I would like to make the comment that there is no simple relationship between a particular instant of time and a particular frequency value. All frequency components contribute to the definition of one value in the time domain and vice versa. The only exceptions to this rule are initial and final times and large and small frequencies, respectively. Then, as far as the previous conment about frequencies is concerned, there is no need to display the transfer function as such. It is just as expedient to work with the impulse response in the time domain. There are criteria of stability and transient response in 
the time domain which are equivalent to the ones used in the frequency domain. Now, even if you don't want to rely on the operator for watching the impulse response, you can build what usually in controls is called an adaptive control system, and by interpreting the criteria for impulse responses or whatever you are using, have the control system readjust so that the system comes back to the desired frequency or impulse response. Of course, in order to do this you have to have information about the impulse response; so it is useful sometimes to have that kind of information available during operation.

Lipinski: You require a known input?

Gyftopoulos: Yes.

Iipinski: You will have to generate it artificially.

Gyftopoulos: We1l, you never buy something for nothing.

Lipinski: It seems the best method is the one that takes advantage of the existing system as it stands without operating on it artificially-simply analyzing the information present.

Gyftopoulos: That's true. Then one has to comment that we'll have to get better information about the statistics.

Iipinski: That is correct. This is my understanding. Mr. Boland?

Boland: I would like to ask a question here. Has anyone ever been around an operating reactor that was starting to change conditions and you didn't know it just by watching a linear power trace? I've never seen one; maybe there is one somewhere. I think in your case, when you started having fuel troubles, you started seeing spikes on your power recordings, didn't you? Somebody reported that the fuel would come out of and go back into solution and you would see big spikes on your power-level recordings. In boiling reactors you have essentially no noise at low power and the noise increases at higher powers.

Iipinski: Can you quantitatively state, based on the power you're at, as to what higher power this would occur at, in terms of numbers. If you're at $20 \mathrm{Mw}$, can you predict that the flux would exponentially rise to $30 \mathrm{MW}$ prior to getting there?

Boland: No, I'm not proposing that this is the way you extrapolate behavior, but we're talking about monitoring what is happening in the existing reactor where you have an established power and you go up there and operate. It doesn't take much of an operator to see when things start to change after you've run it at power for a day or two, or even less than that.

Iipinski: Yes, but the question is, can you have some othex technique that will possibly illustrate this before the flux starts behaving violently? 
Boland: Well, I don't expect the case of violent change to show up, and I think any change that will show up in any of these systems will probably show up in the amplitude of the noise as you have proposed with your RMS noise detector. Of course, if you can build an instrument and couple it in to the scram system, then you don't rely on the operator. This is fine if this is necessary, but I really wonder how much you would buy with a dollar you put in one of these constant monitoring systems on a power reactor.

Lipinski: Well, if it's that close the reactor is marginal and is overdesigned. Mr. Kasten?

Kasten: Certain indications at the HRT point out that our changes took place over a period of time. When the reactor went from a so-called stable to an unstable fuel situation, we might only notice a change in power fluctuations from 2 to $3 \%$, and this is very difficult to associate with an actual event in the reactor. You can't say this was due to fuel instability, because there might be something else which caused this change. Now, if this took place over a long period of time and eventually led to higher peaks, maybe $5 \%$, it would be noticed. But if we could get this information, "has something happened" before we got the larger peaks, this would be useful. And I would think one could conceivably have the same circumstance in other reactors.

Lipinski: Questions or comments? Yes, Mr. Storrer?

Storrer: Do you think I'd have some time to-rabout five minutes--to say something on the influence of decay heat on the reactor kinetics?

Lipinski: Well, it's about 12 minutes to one. I think we ought to terminate at one $o^{\prime}$ clock.

Storrer: Oh, well, five minutes?

Lipinski: Let's see. Could we have it presented in another session, appropriately, possibly? Mr. deBoisblanc?

deBoisblanc: Isn't the real key point raised in this last question, how rapidly, as a function of power, the transition is made in the locus of the poles of the transfer function--how quickly this transition is made as a function of power, shifting the pole over to the $\mathrm{f}$-omega axis. Now if you have a situation in which the power alters some physical variables, say as you increase power you have a factor of two increase in power from the situation where you have essentially a flat transfer function to the situation where you have a sharply rising positive function of frequency--it is not necessary to go to the point where you have an infinite bump on the transfer function before you would be considered to be in trouble in this business, but there is-I believe there were data presented showing this transition as a function of power level, and I think that this is the real key point. If you can show that the system, in anticipating this sort of thing, (the approach of an oscillatory mode) changes perhaps even a factor of two in power before the acceptable and the unacceptable condition is 
reached, then the comment this gentleman made is valid. You would have many ways of looking at this. For example, the noise spectrum, if you did no harmonic analysis on it at all, just a visual analysis of the noise observed, you would observe these trains of pulses tending to have the--to select out of this spectrum of random noise the frequency corresponding to the locus, not of the actual frequency of oscillation or where the bump is, but where the positive slope reaches a certain value. And at that point you would see a structure there and this, of course, would have an envelope shape. And as you go to a sharper and sharper transfer function the envelope must necessarily select out the single frequency that corresponds to the peak. And this is so obvious when you're looking at a scope, or it's so obvious when you select it with a filter, and so obvious when you do a correlation function analysis or you do a transfer Iunction; it's obvious in any way, provided only that the transition is not a discontinuous thing in power. If, for example, you made a fuel element--I believe Stu Forbes has brought this point up--you made a fuel element which suddenly at a certain power can expand in this direction and that direction and then meet, right at one point, the next little increment of power and you have a fuel element which essentially can produce an oscillatory condition. Below this you have no indication of it, but it's an unstable and undesirable condition. In that case, no analysis, would be of any value, so the real key point is in the analysis of the onset of the instability as a physical mechanism, whether it has to do with bubble size, void volume, or pressure vs void formation rate, or any other variables of that nature. If you can look at the physical variables and could decide how much margin you have, then you can decide whether you can use one or the other of these techniques.

Lipinski: I have only one comment on this from the Borax data. We saw these wave-trains and, likewise, on increasing power you would get to the multidecade exponential chugging. I'd like to ask Joe Thie a question on that. Were you ever able to relate the power increase from the chugging state to just the pure noise steady power case? In numbers?

Thie: Well, not quantitatively. I think on the Borax set of experiments the theory was rather naive, so you only have qualitative indications, I'm afraid.

Iipinski: Well, there we didn't have the transfer functions, so we had no evaluation of the system. But the thing you're faced with, in making these observations you describe, is: How far away are you from the point where your power's going to increase a factor of 10 or 100, and your fuel temperatures, likewise?

deBoisblanc: You mention this chugging. Is there any necessary connection between the chugging phenomena and the transfer function? In my opinion, the chugging phenomenon is a representation of essentially a reactor going from one reactor to another reactor. And now there is an extremely nonlinear situation in which all of this frequency analysis is hardly valid.

Lipinski: That is correct, but I am of the opinion that the chugging phenomena will follow the linear instability indication. 
deBoisblanc: Stu? You better.... .

Forbes: No comment.

Lipinski: Right now it's a "no-man's land" as to where chugging is with respect to the nonlinear prediction.

Langmuir: By "chugging" do you mean the Spert oscillations? Is that chugging? Or is that something else?

Lipinski: No, Borax-type oscillations where you're running along at a I MW average power with about a 10\% noise signal, and the reactor sits there and runs for ten minutes, and all of a sudden it has a mind of its own and you've got your power going up to $57 \mathrm{Mw}$ on a peak, and down to $10 \mathrm{kw}$, and then up to $60 \mathrm{MW}$......

Langmuir: But with the amplitude increasing in an envelope . . . .

Lipinski: No. A straight noise envelope. In fact, the description that Thie gave didn't appear on that particular record. It was a random noise; it didn't have the exponential envelope, yet the reactor went into the condition of a limit cycle, essentially, but it varied over the order of three decades after having sat there for several minutes.

Langmuir: You mean the first deviation from the noise equals three decades higher than the average power?

Lipinski: No, this was a gradual process where each successive peak was higher than the preceding one.

Langmuir: Well, then, we would say this was subject to linear analysis and predictable by transfer function methods. This is the conclusion of our out-of-pile measurements.

Lipinski: The nonlinear chugging?

Langmuir: The divergent Spert oscillations--the critical power at which they take off appears to be predictable by a combination of out-of-pile measured power-void transfer functions and neutron kinetics.

Lipinski: Any other comment?

Grace: I think that the reactivity instability experiment I described earlier further confirms that point of view. In fact, ours was actually in-pile.

Lipinski: Mr. Beckjord?

Beckjord: I was just thinking that if you operate a reactor with $10 \%$ noise you can pretty well say that you're sitting right on the fence. That's awfully high.

Lipinski: As I recall, on the EBWR it was $50 \mathrm{MW}$. 
Beckjord: It was also on the fence, wasn't it?

Lipinski: No, we said we could get another $12 \mathrm{Mw}$. Well, if there are no further questions, I guess we'Il conclude this session. There are a few announcements to be made first.

Bright: Please don't anyone panic--I don't have any slides. The next session will be at 7:30 this evening, so this gives you the afternoon off to get your notes together, read reports, whatever. In case you'd like to do something else, however, the weather, I think, is good enough that if you keep moving you shouldn't be in any great trouble. One thing--the ski lift is not operating; we're sorry, it doesn't operate today, but there is bowling, bicycling, horses, whatever you would like to do. The other bit of information is the Pirates won today. 
$$
\text { (5) }
$$
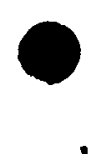

.

.

.<smiles>[I-]</smiles> 
SESSION IV

REACTOR INSTABILITY AND THE THERMAL-HYDRAULIC PROBLEM

Chairman: T. M. Snyder (GE-APD)

Review Paper: N. Zuber (R-W)

Panelists: R. P. Anderson (ANL)

E. S. Beckjord (GE-APD)

J. Chernick (BNL)

J. A. Fleck (UCRL-I)

J. N. Grace (W-Bettis)

D. B. Langmuir $(R-W)$

V. E. Schrock (UC)

THERMAL HYDRODYNAMIC INSTABILITY

Review Paper*
by
N. Zuber $(R-W)$

\section{Introduction}

In this paper, I shall discuss thermal-hydrodynamic instability. Only if we obtain a better understanding of basic two-phase flow instability will we be able to understand fully the stability of a water-cooled reactor with boiling. In addition to summarizing the considerable progress made in this country, I shall review recent advances which have been recorded in the foreign literature and which may not be generally known, as yet, here. I hope that from my efforts and from those of the panelists to follow, an integrated picture of the entire problem of thermal-hydrodynamic instability will emerge.

The problem of two-phase flow with boiling can really be divided into three separate problems: The problem of the voids, the thermal problem, and the hydraulics problem. These three problems are intimately interrelated. Their solution requires specification of both the system and the operating conditions involved. In specifying the

*Ed. Note: Transcription of the introductory remarks of Dr. Snyder and portions of this paper were incomplete due to a malfunction in the sound system. The paper presented here was compiled by A. A. Wasserman from the transcription and notes taken during the session. Dr. Zuber's review of the paper was not available at the time of publication. 
system one must include: (a) the geometry of the system, (b) the thermodynamic properties of the fuel, (c) the nature of the surface of the fuel in contact with the coolant, and (d) the thermodynamic properties of the coolant. In specifying the operating conditions, one must include: (a) the static pressure, (b) the inlet temperature and velocity of the coolant, and (c) the mean power level of reactor operation.

The interrelation of these three parts of the two-phase-flow problem may be indicated as follows: The hydrodynamics of the coolant in the heated region is determined by: (a) the system geometry, (b) the static pressure, (c) the inlet coolant conditions, (d) the nature of the fuel-coolant surface, (e) the thermodynamic properties of the coolant, and $(f)$ the heat flux into the coolant from the fuel. The heat flux into the coolant is determined by: (a) the power level of reactor, (b) the geometry and properties of the fuel, (c) the system pressure, and (d) the effective heat-transfer coefficient from fuel to coolant. The effective heat-transfer coefficient is determined by: (a) the nature of the surface of the fuel, (b) the thermodynamic properties of the coolant, (c) the inlet conditions of the coolant, (d) the system pressure, and (e) the effective void fraction in the coolant. The effective void fraction is determined by: (a) the system pressure, (b) the nature of the fuel-coolant interface, (c) the fuel surface temperature, and $(d)$ the vapor removal rate, fluid flow pattern, and pressure distribution, all three of which are manifestations of the hydrodynamics of the fluid, with which we began.

I shall start this review by considering the problem of the voids, which includes the problems of nucleation, bubble growth, and vapor removal. With present theoretical and experimental knowledge, the se mechanisms of bubble dynamics are now well understood for steady-state boiling systems. In discussing bubble dynamics, I shall include the important role of the characteristics of the heat-transfer surface on both nucleation and on the rate of heat transfer to the liquid. From the se relationships one can predict the void distribution on a heated plate. The problem of vapor removal also involves the slip velocity ratio which I consider later.

Next, as part of the thermal problem (along with the effect of surface characteristics), I consider the effect of pressure variation on the boiling heat-transfer coefficient. This is especially important in boiling-water reactors. I shall show that increasing pressure facilitates nucleation (tending to increase the number of nucleating centers) and decreases bubble growth rates (tending to make the boiling process smoother). The net effect is an increase in the nucleate boiling heat flux.

As a start on the hydraulics problem, I then turn to a discussion of the general one-dimensional, two-phase transient flow equations, followed by remarks on the effect of the two-dimensionality of twophase flow patterms and how the se may be taken into account. This leads to a discussion of the determination of the slip velocity ratio and of the vapor removal rate, including results by the Russians 
which now permit confident prediction of the slip velocjty ratio in stable two-phase flow systems. This is followed by presentation of a simple expression for the two-phase pressure drop which has been analytically derived and experimentally verified.

In the final portion of the talk, the effect of both nucleation properties and two-dimensional flow patterns on flow stability is discussed. Included here is a discussion of "chugging" as a phenomenon which occurs in the transition from the stable bubble-flow regime to the unstable slug-flow regime, and which is associated with poorly nucleating surfaces. The stabilizing effect of pressure increase is explained. An ultimate criterion for two-phase flow stability is presented which empirically relates the two-phase pressure drop, the nonboiling-length pressure drop, the total pressure drop, and the absolute pressure.

\section{Bubble Nucleation and Growth}

The problem of predicting the void fraction from the factors which determine it, is conveniently divided into three phases which may be studied separately: (a) bubble nucleation, (b) bubble growth, and (c) vapor removal. We consider here, briefly, the first two of these phases.

For a bubble to begin to grow from a vapor pocket in a heated surface, the effective radius of the vapor pocket must exceed a critical radius $R_{c}$ given (from a force balance) by

$$
p_{V}-p_{L}=\frac{2 \sigma}{R_{C}}
$$

where $\sigma$ is the surface tension of the liquid, $p_{v}$ is the vapor pressure of the liquid at the wall temperature $\mathrm{T}_{\mathrm{W}}$, and $\mathrm{p}_{\mathrm{L}}$ is the system pressure in the liquid. Or, conversely, for a surface of a given roughness characterized by $R_{c}$, the vapor pressure must exceed the static pressure by an amount specified by Equation (I) in order for nucleation to occur.

An alternative way of expressing this relation (using the ClausiusClapeyron relation between vapor pressure and saturation temperature) is

$$
T_{W}-T_{\text {sat }}=\frac{2 \sigma T\left(\rho^{\prime}-\rho^{\prime \prime}\right)}{R_{C} L \rho^{\prime} \rho^{\prime \prime}}
$$

where $T$ is the liquid temperature, $\rho^{\prime}$ is the liquid density, $p^{\prime \prime}$ is the vapor density, $L$ is the latent heat of vaporization, and $T_{\text {sat }}$ is the saturation temperature at the system pressure. According to Equation (2), the larger the difference between the wall temperature and the saturation temperature (i.e., the larger the superheat), the smaller the size of the pocket in the metal surface from which nucleation can 
occur and the greater the number of active nucleating sites for a given surface. Conversely, the smoother the surface, the larger the superheat required for nucleation.

From Equation (2), it is also evident that nucleation at a given surface can be increased either by increasing the wall temperature or by decreasing the saturation temperature as a result of reducing the system pressure. Because the latent heat of vaporization and the vapor density increase with pressure, the change in saturation temperature with pressure is smaller at high pressure than at low pressure. Since, also, the surface tension decreases with pressure, the temperature superheat above saturation required to give a fixed degree of nucleation at a given surface is reduced as the pressure is increased. In other words, nucleation is easier at high pressure than at low pressure.

We turn from the problem of nucleation at a heated surface to the problem of bubble growth rate. If one assumes that the heat transferred from the surface near a growing bubble goes to maintain the evaporation associated with bubble growth, one can derive the following equation for the radius of the bubble as a function of time

$$
R(t)=F(\theta) \frac{K}{L \rho^{\prime \prime}} \int_{0}^{t}\left(\frac{\partial T}{\partial y}\right)_{y=0} d \xi
$$

where $(\partial \mathrm{T} / \partial \mathrm{y})_{\mathrm{y}=0}$ is the temperature gradient in the liquid at the solidliquid interface, $K$ is the heat conductivity of the liquid, and $F(\theta)$ is a function of the contact angle $\theta$ which is constant for a given solidliquid pair. $F(\theta)$ is approximately unity for all systems.

For a slab-geometry transient with exponential heating (as in a reactor wi.th plate-type fuel elements), the temperature gradient in Equation (3) is obtained from the solution of the one-dimensional heat conduction equation. This yields

$$
\left(\frac{\partial T}{\partial y}\right)_{y=0}=\frac{T_{W}-T_{s a t}}{\sqrt{\pi a \xi}}
$$

Combining (3) and (4), one obtains for the bubble radius as a function of time

$$
R(t)=\frac{2 K\left(T_{W}-T_{S a t}\right)}{L \rho^{\prime \prime}} \sqrt{\frac{t}{\pi a}}
$$

where $a$ is the heat diffusivity of the liquid. Thus, the bubble radius increases asymptotically as the square root of the time from initial nucleation and is proportional to the amount of superheat. As the pressure 
increases, the bubble growth rate decreases because $L$, $\rho^{\prime \prime}$, and $T_{\text {sat }}$ increase. The result is that high pressure boiling will generally be "smoother" than low-pressure boiling.

\section{Prediction of amount of Vapor on the Surface}

To predict the amount of vapor on a heated surface, we must have information on the bubble population $\mathrm{n}$. Lienard, a doctoral thesis student of Professor Schrock, has derived the following relevant equation

$$
\frac{v}{A}=\int_{0}^{t} \frac{n(\xi)}{A} \frac{d v_{b}(t-\xi)}{d(t-\xi)} d \xi
$$

where V/A is the volume of vapor per unit area of the surface, and $v_{b}$ is the volume of a bubble. The rate of change of $v_{b}$ with time can be predicted by an equation of the form of (5).

Thus, if the bubble population $n$ can be determined, the amount of vapor on the surface can be determined. As yet there is no analytical expression for $n$. Lienard has proposed that such an expression be obtained from steady-state boiling for. which there is a relation between heat flux $\mathrm{g} / \mathrm{A}$ and bubble population of the form

$$
q / A=B(n / A)^{\gamma}
$$

where $\gamma$ varies between $1 / 3$ and unity. (Professor Schrock will discuss further the use of (7) and (6) to determine the amount of vapor on the surface.)

Experiments on water at MIT and other experiments on methanol have verified equation (5) very well, so that to a first approximation, at least, we can predict the rates of bubble growth on a surface both for steady-state and transient conditions.

Data of Berenson of MIT on nucleate pool boiling in methanol and other data from Purdue show that changes in surface finish produce marked changes in the curve of heat flux vs superheat. The smoother the surface (i.e., the smaller the radius of the nulleating centers), the higher the superheat required to trigger boiling and to obtain a given heat flux in nucleate boiling. But, as remarked earlier, low superheats are associated with low bubble growth rates while high superheats lead to "explosive" bubble growth rates. This can play an important role in the stability of a boiling loop.

If one plots the heat transfer rate $q$ vs the number of nucleating centers per unit area n/A (as done by Meyer of Purdue), one finds that a smooth surface, which requires a larger superheat, also requires a 
smaller density of nucleating centers than a rough surface to get a given q. The reason is because the heat transfer in boiling is enhanced by agitation of the water layer adjacent to the surface by the bubbles. If the agitation produced by a single bubble is large, it is not necessary to have many bubbles to get large heat transfer rates. This is the condition for smooth surfaces with large superheat and large consequent bubble growth rates. For rough surfaces with low superheats, bubble growth rates are low so that the substantial agitation of the water layer required to achieve high heat transfer rates demands a high density of nucleating centers.

From the experimental results, one can write the following powerlaw formula for the dependence of the heat flux on the superheat at constant surface roughness

$$
\mathrm{q} / \mathrm{A}=\mathrm{B}_{\mathrm{I}}\left(\mathrm{T}_{\mathrm{W}}-\mathrm{T}_{\text {sat }}\right)^{\mathrm{m}}
$$

where $3<\mathrm{m}<24$. The heat transfer coefficient $\mathrm{h}$, defined as the ratio of the heat flux to the superheat, becomes

$$
\mathrm{h}=\mathrm{B}_{1}\left(\mathrm{~T}_{\mathrm{W}}-\mathrm{T}_{\mathrm{sat}}\right)^{\mathrm{m}-1}
$$

Alternately, from the experimental results mentioned, instead of expressing the heat flux in terms of the superheat, we can express it in terms of a power-law dependence on the bubble population $n$, at constant surface roughness, as in Equation (7).

We note from ( $8 \mathrm{a}$ ) that $\mathrm{h}$ can be increased either by increasing the wall temperature $\mathrm{T}_{\mathrm{W}}$ or by decreasing the saturation temperature $\mathrm{T}_{\text {sat }}$ by a decrease in pressure. For a given $\mathrm{T}_{\mathrm{W}}$, a sufficient increase in pressure can decrease $h$ to zero. Hence, it is plain that a boiling system with pressure fluctuations will have a variable heat transfer coefficient coupled with the hydrodynamics of a system, and one might expect that this effect can lead to instability. We shall discuss this further later on.

From experimental results we can formulate an expression for the nucleate boiling heat flux as a function of both the bubble population and the superheat; e.g.,

$$
\begin{aligned}
q / A & =\beta \frac{K\left(T_{w}-T_{s a t}\right)}{D_{b}}\left[\frac{\left(T_{w}-T_{s a t}\right) C \rho \sqrt{\pi a}}{L \rho^{\prime \prime}}\right]^{1 / 2}\left(\frac{n}{A}\right)^{I / 4}\left(\frac{D_{b}}{\mu}\right)^{I / 2} P_{r}{ }^{1 / 3} \\
& =B\left(T_{w}-T_{\text {sat }}\right)^{3 / 2}(n / A)
\end{aligned}
$$


From an equation like (9) based on experimental studies, we can determine the bubble population by measuring the heat flux, wall temperature, and pressure. If this method of Professor Shrock's is successful in predicting the bubble population from steady boiling, then we can apply the results also to the transient problem. It is not clear whether or not applying steady-state results to the transient problem will lead to valid results, but this question should be answerable from the experiments of Professor Shrock and those of Art Morse at Ramo-Wooldridge. If the answer is yes, then we can determine $\mathrm{n} / \mathrm{A}$ from a steady-state equation like (9), insert the result under the integral in (6), and deduce the amount of vapor on the surface. (For example, experimentally, if $\mathrm{m}$ is $3, \gamma$ is $3 / 4$; if $\mathrm{m}$ is $8, \gamma$ is $1 / 3$. )

\section{Recommended Heat Transfer Correlation}

On another point, the question often arises as to the best correlation to use to predict the nucleate boiling heat transfer coefficient. A current recommended nucleate boiling correlation formula for the heat transfer coefficient $h_{B}$ (developed at Oak Ridge and similar to one derived by Kutadeladze in Russia) is

$$
\mathrm{Nu}=0.001 \gamma^{0.85} \operatorname{Re}^{0.7} \operatorname{Pr}^{0.4}(\mathrm{Db} / \mathrm{D})^{1 / 3}
$$

where

$$
\begin{aligned}
\mathrm{Nu} & =\mathrm{h}_{\mathrm{B}} \mathrm{D}_{\mathrm{b}} / \mathrm{K} \\
\boldsymbol{X} & \left.=\mathrm{P} / \sigma \mathrm{g}\left(\rho^{\prime}-\rho^{\prime \prime}\right)\right]^{I / 2} \\
\operatorname{Re} & =(\mathrm{q} / \mathrm{A})\left(\mathrm{D}_{\mathrm{b}} \rho^{\prime}\right) / \mathrm{L} \rho^{\prime \prime} \mu \\
\mathrm{Pr} & =\mathrm{C} \mu / \mathrm{K} \\
\mathrm{D}_{\mathrm{b}}= & {\left[\sigma / \mathrm{g}\left(\rho^{\prime}-\rho^{\prime \prime}\right)\right]^{I / 2} } \\
\mathrm{D} & =\text { four times the coolant flow area divided by the wetted } \\
\mathrm{P} & =\text { serimeter } \\
\mathrm{g} & =\text { gravitational acceleration } \\
\mu & =\text { viscosity of liquid } \\
\mathrm{C} & =\text { specific heat of liquid }
\end{aligned}
$$




\section{Hydrodynamics Equations}

The most general equations (Lagrangian) describing the hydrodynamics of a two-phase reactor coolant in one-dimensional flow are the following:

a. Mass Balance in Coolant:

$$
\begin{aligned}
\frac{\partial}{\partial t} & {\left[(1-\alpha) \rho^{\prime}+\alpha \rho^{\prime \prime}\right]+\frac{\partial}{\partial x}\left[(1-\rho) \rho^{\prime} u^{\prime}+\alpha p^{\prime \prime} u^{\prime \prime}\right]=0, \quad \text { (II) } } \\
& \text { b. . Momentum Balance in Coolant: } \\
-\frac{\partial p}{\partial x}= & (1-\alpha) \rho^{\prime}\left[\frac{\partial u^{\prime}}{\partial t}+u^{\prime} \frac{\partial u^{\prime}}{\partial x}\right]+\alpha \rho^{\prime \prime}\left[\frac{\partial u^{\prime \prime}}{\partial t}+u^{\prime \prime} \frac{\partial u^{\prime \prime}}{\partial x}\right]+ \\
& +g\left[(1-\alpha) \rho^{\prime}+\alpha \rho^{\prime \prime}\right]+\left(\frac{\partial p}{\partial x}\right)_{T P F}+\left(u^{\prime \prime}-u^{\prime}\right)\left[\frac{\partial}{\partial t}\left(\alpha \rho^{\prime \prime}\right)+\frac{\partial}{\partial x_{X}}\left(\alpha \rho^{\prime \prime} u^{\prime \prime}\right)\right.
\end{aligned}
$$

c. Energy Balance in Coolant:

$$
\frac{4 h_{B}\left(T_{w}-T_{S}\right)}{D}=\frac{\partial}{\partial t}\left[(1-\alpha) \rho^{\prime} i^{\prime}+\alpha \rho^{\prime \prime} i^{\prime \prime}\right]+\frac{\partial}{\partial x}\left[(I-\alpha) \rho^{\prime} i^{\prime} u^{\prime}+\alpha \rho^{\prime \prime} I^{\prime \prime} u^{\prime \prime}\right],
$$

d. Energy Balance in Lumped Solid Fuel Region:

$$
H_{S} \frac{d T_{W}}{d t}=Q-h_{B}\left(T_{W}-T_{S}\right) D \pi
$$

e. Relation Between Heat Transfer Coefficient and Superheat in Boiling:

$$
h_{B}=B\left(T_{W}-T_{S}\right)^{m-1}
$$


where a single prime refers to the liquid phase and a double prime refers to the vapor phase, and where

$$
\begin{aligned}
\alpha & =\text { void fraction at } x, t \\
u & =\text { velocity at } x, t \\
p & =\text { density at } x, t \\
P & =\text { pressure at } x, t \\
h_{B} & =\text { nucleate boiling heat transfer coefficient at } x, t \\
T_{W} & =\text { fuel surface temperature at } x, t \\
T_{S} & =\text { saturation temperature at } x, t \\
D / 4 & =\text { flow area divided by wetted perimeter } \\
i & =\text { enthalpy per unit mass at } x, t \\
H_{S} & =\text { heat capacity of fuel region per unit length in } x \text {-direction } \\
Q & =\text { heat generation in fuel per unit length in } x \text {-direction } \\
B, m & =\text { constants for given fuel surface, coolant liquid, and system }
\end{aligned}
$$

The derivation of these equations is given, for example, in a book on Supersonic Hydrodynamics by Shapiro, Volume I.

In equation (II) the first group of terms is the transient part, the second group is the convective part. In equation (12), the lefthand side is the total pressure drop; the first group of terms on the right is the rate of momentum change in the liquid; the second group is the same in the vapor; the third group is the hydrostatic term; $(\partial P / \partial x)_{T P F}$ is the frictional pressure drop in two-phase flow; and the last group of terms is associated with the vaporization process. If the transient terms are set equal to zero, one obtains the equation of Martinelli derived in 1946. If one integrates equation (12) between two limits $x_{1}$ and $x_{2}$, one obtains the integral form of the equation, derived by Dr. Fleck in 1958. In equation (13), the first group of terms on the right is again the transient term, the second is the convective term.

Suppose one increases the pressure so $\mathrm{T}_{\mathrm{W}}-\mathrm{T}_{\text {sat }}=0$ in (15). Then if one proceeds to heat the fuel at a constant rate, we see from (14) that the temperature of the solid increases. Then by the time a bubble is triggered, there is so much superheat that the bubble grows explosively. This increases the transient vaporization term in equation (12) and can lead to instability in the hydrodynamics as described by equations (11), (12), and (13). 
All models which have been considered in this country thus far are derivable from the above equations by making various additional restrictive assumptions. Dr. Fleck is the only one I know who has integrated these equations. He did this by first making four simplifying assumptions. These were: (I) the boiling point is translated as the power is generated, (2) the slip ratio is constant, (3) the heat transfer coefficient in boiling is constant, and (4) there are no pressure effects.

Concerning the development of analytical models for the hydrodynamics of boiling reactors, if one plans to use a computer and a linearized model it makes more sense to start by linearizing equations (11)-(13) rather than some simplified form of them. Equations (11)-(13) are the most general set of equations for one-dimensional hydrodynamics. By starting with them one knows explicitly the assumptions introduced by the various simplifications which are subsequently made. One can then analyze the linearized problem on a computer and evaluate the non-linear terms which have been neglected. (The non-linearities arise primarily from the convective terms in the equations.)

To solve equations (11) through (15), we must know the heat transfer coefficient $h_{B}$ and the two-phase pressure drops. For $h_{B}$, we can use the value obtained for steady boiling. Some new correlations for two-phase pressure drop have been proposed recently and we shall discuss these later; alternately, we can use the Martinelli equation.

We must ask the basic question as to whether it is valid to describe a two-phase flow system by means of one-space-dimension equations. For example, in single-phase flow, we treat separately the laminar and the turbulent flow regimes because the flow patterns or velocity profiles are different. We analyze the heat transfer in each flow regime separately. To an even greater extent we might, therefore, expect differences in flow patterns to add another degree of freedom to two-phase-flow problems, possibly requiring a multi-dimensional description for accurate predictions. In two-phase systems, three flow regimes are possible; we can have bubble flow, slug flow, or annular flow. A one-dimensional description may very well be inadequate to represent the se significantly different flow patterns. We must do more work in comparing predictions of the one-dimensional model with experimental two-phase-flow results.

It might prove fruitful first to use a two-dimensional steady-state analysis to obtain information on the flow patterns and flow characteristics in a given system. The results could then be used in the onedimensional hydrodynamics equations (11)-(15) to solve the transient problem. It does not appear to be feasible to solve the transient problem directly for a two-dimensional model.

6. The Two-Dimensional Problem and the Derivation of the SlipVelocity Ratio

From a two-dimensional analysis, one can derive the slipvelocity ratio. Once the slip-velocity ratio is known, one can solve the problem of vapor removal from a system. This problem was analyzed by Bankoff at Northwestern University and by Griffith at MIT. The basis for the analysis is the following. 
A bubble located near the wall of a channel with upflow of liquid exists in a shear flow regime having a velocity profile which increases toward the center of the channel. As a result, the bubble has a Bernoulli Effect acting on it. Also, because of the velocity gradient associated with the shear flow, there is a circulation within the bubble which causes a Magnus Effect. These two effects tend to draw the bubble toward the center of the channel with the result that the vapor is concentrated in a central "core". The concentration of the vapor in the center of the channel distorts the velocity flow pattern because of density difference. As a result, the velocity profile becomes quite peaked in the center, rather as in ordinary laminar flow. Also, the vapor bubbles are transported much faster than the mean velocity of the liquid, the ratio of vapor and liquid velocities being the slipvelocity ratio. Actually, there is no real slip. The vapor bubbles can have a velocity relative to the liquid of only about $24 \mathrm{~cm} / \mathrm{sec}$. But, because they are located in a high-velocity region, they are transported much faster and this introduces the slip velocity.

For a channel with downflow of liquid, the Bernoulli Effect still pulls a bubble away from the wall of the channel, but because the circulation in the bubble is now in the opposite sense from before, the net effect is to concentrate the bubbles in an annular region rather than in the center of the channel. As a result, the velocity distribution is more complex, being "positively" peaked at the center of the channel and "negatively" peaked in the annular region containing the vapor, because the buoyant force due to the density difference now tends to oppose the main direction of flow of the liquid. Such flow patterns and density distributions have been observed at Argonne, at MIT, and at the Ramo-Wooldridge Laboratory.

The above discussions indicate how the two-dimensional problem can be analyzed to obtain the density and velocity distributions. These can then be applied to a one-dimensional model to derive the slip-velocity ratio as follows. The static void fraction $\alpha$ is defined by

$$
\alpha \equiv A^{\prime \prime} /\left(A^{\prime \prime}+A^{\prime}\right)
$$

where $A^{\prime \prime}$ is the part of the cross-section filled with vapor and $A^{\prime}$ is the part filled with liquid at a given distance up the channel. The superficial or reduced vapor velocity is defined by

$$
u_{0}^{\prime \prime} \equiv \frac{V^{\prime \prime}}{A} \equiv \frac{G^{\prime \prime} / \rho^{\prime \prime}}{A}=\infty u^{\prime \prime}
$$


where

$$
\begin{aligned}
& V^{\prime \prime}=\text { the local volumetric vapor flow rate } \\
& A^{\prime}=\text { the local channel cross section }=\left(A^{\prime}+A^{\prime \prime}\right) \\
& G^{\prime \prime}=\text { the local vapor mass flow rate } \\
& \rho^{\prime \prime}=\text { the local vapor density } \\
& u^{\prime \prime}=\text { the actual local vapor velocity }
\end{aligned}
$$

The superficial or reduced liquid velocity is similarly given by

$$
u_{0}^{2} \equiv \frac{V_{0}^{\prime}}{A} \equiv \frac{G^{3} / p^{2}}{A}=(1-\alpha) u^{2}
$$

where the various quantities involved have analogous definitions to the above, except that they refer to the liquid rather than the vapor phase.

We define the dynamic void fraction $\beta$ as the fractional volumetric flow rate of vapor. It is given by

$$
\beta \equiv \frac{V^{\prime \prime}}{V^{\prime \prime}+V^{\prime}}=\frac{u^{\prime \prime}{ }_{0}}{u_{0}^{\prime \prime}+u_{0}^{\prime}}=\frac{\rho^{\prime} x}{\rho^{\prime \prime}+x\left(\rho^{\prime}-\rho^{\prime \prime}\right)}
$$

where $X=$ the local vapor quality (or fractional mass of vapor). The essential difference between $\beta$ and $\alpha$ is that $\beta$ takes account of the effect of the concentration of the vapor in a particular region of the channel where the liquid velocity may be quite different from the mean-liquid velocity. The static void fraction $\alpha$ can be measured by $\mathrm{X}$-ray or $\boldsymbol{\gamma}$-ray transmission measurements; the dynamic void fraction $\beta$ can be calculated from equation 19.

The Russians have done extensive experiments to investigate the twodimensional character of the flow as described by the density distribution equation

$$
\left(\alpha / \alpha_{m}\right)=1-(y / R)^{1 / n}
$$

and the velocity distribution equation

$$
\left(u^{\prime} / u_{m}^{\prime}\right)=1-(y / R)^{1 / m}
$$


where $\mathrm{y}$ is the transverse distance from the center of the channel, $\mathrm{R}$ is the channel radius, $\alpha_{m}$ is the static void fraction and $u{ }^{\prime} m$ is the liquid velocity, both at the axis of the channel, and $\mathrm{n}$ and $\mathrm{m}$ are constants. Bankoff at Northwestern has analytically derived the flow parameter $\mathrm{C}$ defined by

$$
\mathrm{C} \equiv \alpha / \beta
$$

He found that $\mathrm{C}$ depends only on the exponents of the density and velocity distributions $\mathrm{n}$ and $\mathrm{m}$. The flow parameter $\mathrm{C}$ expresses the interaction of the velocity and density fields in a flow system. Bankoff also derived the following equation for the slip-velocity ratio $\left(u^{\prime \prime} / u^{\prime}\right)$

$$
\left(u^{\prime \prime} / u^{\prime}\right)=(I-\alpha) /(c-\alpha)
$$

We note that the slip-velocity ratio does not depend on the quality $x$. We used to think that the slip velocity ratio was given by

$$
\left(u^{\prime \prime} / u^{\prime}\right)=\left(\frac{x}{1-x}\right)\left(\frac{1-\alpha}{\alpha}\right)\left(\frac{p^{\prime}}{p^{\prime \prime}}\right)
$$

which results directly from combining equations (19), (18), and (17). But Bankoff's analysis leading to (23) shows that the quality is not actually involved.

We note also from (23), that if the slip-velocity ratio is unity, then $\mathrm{C}$ is unity and we have a uniform distribution of the vapor all the way across the channel.

We next examine the slip-velocity ratio which would be deduced from a one-dimensional model. In 1959, Levy showed that by using the Martinelli equation one could derive the following equation for the slip-velocity ratio

$$
\left(u^{\prime \prime} / u^{\prime}\right)=\left(20 p^{\prime} / p^{\prime \prime}\right)^{1 / 2}
$$

If, as an example, we take a value of $\alpha=0.1$ for bubble flow, and $\rho^{\prime} / \rho^{\prime \prime}=1500$ for water at atmospheric pressure, then we obtain $\left(u^{\prime \prime} / u^{\prime}\right) \approx 17$. This indicates that the vapor is traveling 17 times faster than the liquid. If, then, the liquid were traveling at a velocity of $2 \mathrm{ft} / \mathrm{sec}$, then the vapor would be traveling effectively at $34 \mathrm{ft} / \mathrm{sec}$.

One then asks what kind of interface can be stable under these dynamic conditions. We argue as follows: The stagnation pressure on the bubble is given by $\rho\left(u^{\prime \prime}-u^{\prime}\right)^{2} / 2$. This pressure tends to deform the bubble and is counteracted only by the negative surface tension pressure $2 \sigma / R$, 
where $R$ is the bubble radius. Then for equilibrium one must have

$$
2 \sigma / R \approx \rho^{\prime}\left(u^{\prime \prime}-u^{\prime}\right)^{2} / 2
$$

If we insert the value for vapor velocity derived in the example above into (26), we find that for stability of the bubble-liquid interface, the bubble radius $R$ must be of the order of $10^{-4} \mathrm{~cm}$. We know that this is not correct because in fact we observe much larger bubbles. We conclude that the separated flow model (i.e., a purely one-dimensional approach) cannot be used for the correct analysis of the bubble flow regime. We must use a two-dimensional approach such as Bankoff's.

One additional point of importance. From the above two-dimensional line of analysis one can derive an expression for the frictional pressure drop for two-phase flow $\triangle \mathrm{P}_{\mathrm{TPF}}$ in the form

$$
\Delta P_{T P F}=f \frac{l}{D} \frac{\rho^{\prime} u_{i}^{2}}{2}\left(I+A \frac{u_{0}^{\prime \prime}}{u_{1}}\right)
$$

where $f$ is the Fanning friction factor, $u_{i}$ is the inlet liquid velocity, $u^{\prime \prime}$ is the superficial vapor velocity as before, and $A$ is a constant which depends only upon the pressure. Equation (27) proves to be excellent in correlating experimental data over a pressure range from atmospheric nearly to the critical point. It is a very simple relation, being linear in quality, and is an excellent simplification for use in the analysis of two-phase flow problems.

Data obtained by the Russians verifies the prediction of Bankoff's equation that a plot of $\alpha$ vs $\beta$ is linear over a large range of $\alpha$. (When $\alpha$ reaches about 0.6 , there is a departure from linearity between $\alpha$ and $\beta$, probably due to a change in flow regimes.) Hence, we can use the above analysis to predict the slip-velocity ratio.

There is another point to note; namely, as the pressure increases, the slip-velocity ratio decreases. For pressures over, say, 90 atmospheres, the slip-velocity ratio may be taken equal to unity for all practical purposes.

Schwartz has developed a relatively simple expression for the correlation of two-phase pressure-drop data which is of the same form as equation (27), with the constant A being a function of pressure. The data correlated was taken in a 25-mm diameter pipe, both at 12 atmospheres and at 181 atmospheres. The coefficient A varies from unity to zero as the pressure is increased and since the specific nature of the variation is determined from experimental results, equation (27) can be used to predict two-phase pressure drops with confidence.

The parameter $C$ (equation (22)) should vary between 0.7 and 1.0 , according to Bankoff's prediction. From experimental results it is 
found to vary from 0.78 to about 1.0, in excellent agreement with this prediction.

These results have also been verified for mercury two-phase flow systems.

Data was taken by Fleischer at Ramo-Wooldridge on the void distribution in the transverse direction as a function of axial distance in a simulated spert I channel. For a channel having good nucleating centers due to the soldered construction, the flow was very stable at a power input of 500 watts. Near the inlet, the void distribution was highest near the soldered corners of the channel where nucleation was good and lowest in the center of the channel. In moving farther up the channel, the density distribution gradually changed from being concave upward to convex upward; that is, the voids tended to concentrate more in the center of the channel. For another channel having smooth surfaces and welded construction, nucleation was poor. The voids were concentrated in the center, even at the inlet to the channel, and there was essentially no change in the flow pattern in moving up the channel. In this case the flow was unstable; there was "chugging" at a power input of 500 watts.

Clearly, to establish whether or not flow will be stable in a heated channel, it is not enough to specify the heat input and the inlet water temperature and velocity (i.e., it is not enough to specify the external circuit); one must also consider the internal surface characteristics of the heated channel as well. Also, from the experimental results it is clear that to determine the average void fraction, one must consider the transverse distribution as well as the longitudinal distribution, because the transverse distribution can vary, depending upon the surface characteristics.

We consider next why one channel had stable flow and the other unstable flow. The answer involves both the nature of the flow patterns and the pressure propagation in the two-phase flow mixture. A thesis done under Professor Griffith at MIT shows that the change from the bubble flow regime to the slug flow regime represents a propagation phenomenon. If there is an interaction among bubbles formed at the bottom of a channel, it takes some time for the bubules to form a slug; hence, there is a propagation phenomenon associated with the change in flow regimes. For a short channel one does not observe a large slug. For a relatively long channel, however, there is time to go from the bubble flow regime to the slug flow regime and large slugs are observed. The bubble flow regime would be stable; the slug flow regime would be unstable because it would chug. Therefore, in the twophase flow analysis we must consider this propagation phenomenon. The MII experiments demonstrate this.

Therefore, for two-phase flow we must have a flow map such as that devised by Professor Griffith (Slide 1), in which the "dynamic void coefficient $\beta$ is plotted against the Froude Number $\left(u_{0}^{\prime}+u_{0}\right)^{2} / g D_{p}$ where $D_{p}$ is the pipe diameter. 


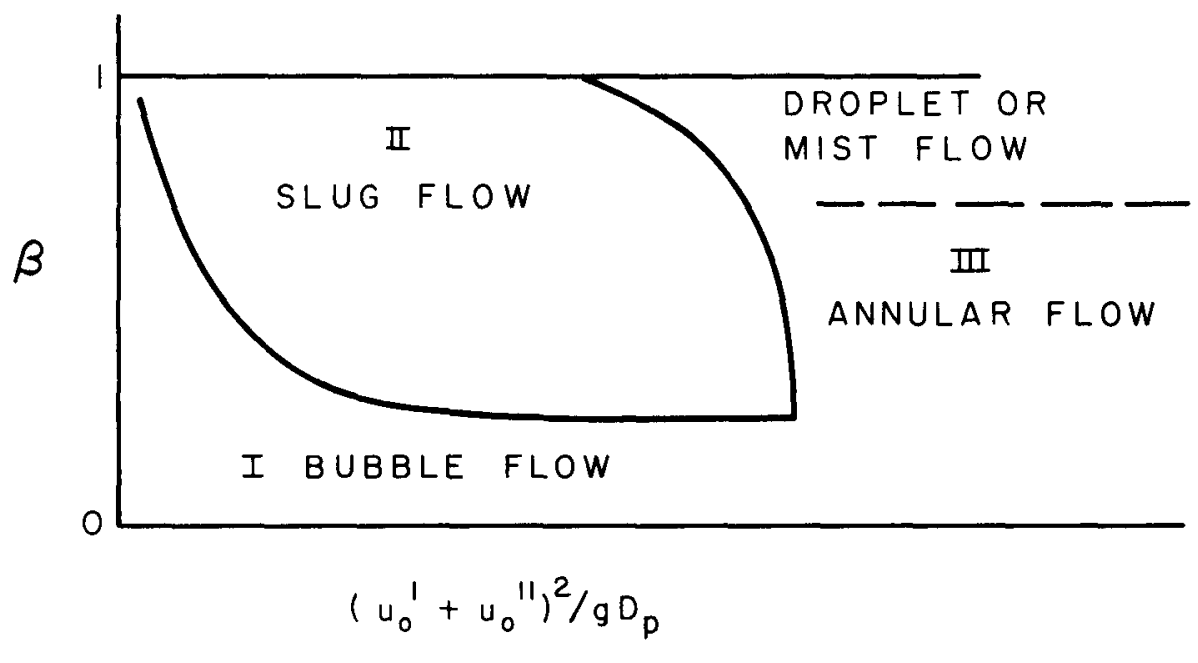

SIide 1. Flow Map

In domain I, the bubble flow regime, the system is stable and there is no flow oscillation. In domain II, the slug flow regime, the system is unstable and there is chugging. In domain III, the annular flow regime, the system is again stable. Above the dotted line in region three is the mist or droplet flow regime.

The effect of pressure on this flow map is as follows: From experimental results we know that as the pressure is increased, the domains of bubble flow regime and the annular flow regime increase while the domain of the slug flow regime decreases. Hence the system becomes more stable at high pressures, as observed in almost all experiments. At very high pressures the slug flow regime decreases to zero.

How, then, do we apply these results to explaining the different behavior of the two channels having different nucleating surfaces in the Ramo-Wooldridge experiments? We have seen that nucleation depends on the size of the surface cavities. If, as for a rough surface, the cavities are large, there is no need for large superheat to trigger nucleation (see equation 2). Also, if the superheat is low, we have seen that bubble growth is slow and the boiling process is very stable. For smooth surfaces with small nucleating cavities, on the other hand, once nucleation is triggered by the large superheats required, the bubble growth is explosively fast. As a result tilere is a pressure wave transmitted through the two-phase flow region. This pressure wave does two things. First, because of the increase in saturation temperature with pressure, it causes condensation at the nucleation centers in 
advance and behind the center from which the pressure wave is propagated. This means it will also change the heat transfer coefficient as indicated in equation (28)

$$
h=B\left\{T_{W}-\left[T_{S}(0)+\frac{T\left(\rho^{\prime}-\rho^{\prime \prime}\right)}{L \rho^{\prime \prime} \rho^{\prime}} \delta P\right]\right\}^{m-1}
$$

(which is the same as equation (8a), except that the effect of the pressure on the saturation temperature is shown explicitly). The increasing pressure decreases the heat transfer coefficient and can even lead to a zero value for it. If the heat transfer coefficient is reduced to zero, the temperature of the heated surface must increase.

The second effect of the pressure increase is to reduce the bubble growth rate due to the decrease in superheat as indicated in equation (29)

$$
\dot{R}=-\frac{K}{L \rho^{\prime \prime} \sqrt{\pi a t}}\left\{T_{W}-\left[T_{S}(0)+\frac{T\left(\rho^{\prime}-\rho^{\prime \prime}\right)}{L \rho^{\prime \prime} \rho^{\prime}} \delta P\right]\right\}
$$

(which is the derivative of (5) with respect to time). For a sufficiently large pressure wave, the bubble growth rate is reduced to zero.

If the pressure is then suddenly decreased by the rarification which follows the initial pressure wave, there is a new explosive vapor growth at the new sites. Hence, we see that the process described here can lead to a dynamic chugging in the system.

Now this effect clearly is important only at low pressures because explosive bubble growth rates are possible only at low pressures. At high pressures, growth rates are slower so the tendency to dynamic chugging is damped.

Unstable oscillations can occur if the pressure wave due to explosive bubble growth is so large as to reduce the heat transfer coefficient to zero very quickly, causing an increase in plate temperature. When the pressure wave has passed, then the increased plate temperature means that the superheat is greater than it was before. This leads to still more violent bubble growth rates and larger pressure waves, and so on. This mechanism can lead to divergent oscillations in a pure boiling, low pressure, out-of-pile system due to the feedback between pressure and superheat.

How can such divergent oscillations in a low-pressure system be suppressed? One way is to apply damping by orificing the flow. The Russians have proposed the following pressure criterion: If $\triangle P_{O}$ is the pressure drop across the orifice, $\triangle \mathrm{P}_{\mathrm{NB}}$ is the pressure drop in the non-boiling region, and $\Delta \mathrm{P}_{\mathrm{B}}$ is the two-phase pressure drop in the 
boiling region, then for the system to be stable we must have that

$$
\Delta P_{0}+\Delta P_{N B} / \Delta P_{B}>a
$$

where " $a$ " is a quantity which the Russians have determined by experiment. The quantity " $a$ " is found to decrease with pressure. For stability, a high pressure drop is clearly essential. As the pressure increases, however, the required pressure drop for stability decreases.

We see from equation (30) how important it is to be able to predict the two-phase pressure drop $\Delta P_{B}$, because we could then, by means of (30), predict whether or not a given system would be stable or unstable.

We next review some additional pertinent Russian experimental data. A plot they have obtained of the heat flux $q / A$ divided by the latent heat of vaporization $L$ times the vapor density $\rho^{\prime \prime}$ times the initial liquid velocity $u_{i}$ (a dimensionless ratio) vs the ratio of the non-boiling length to the total length of the pipe (also a dimensionless ratio) shows two things. First, if constant superheat is maintained then the ratio of nonboiling length to total length remains constant, and if also the inlet velocity is held constant but the heat flux is increased, then the plot indicates that one can reach a region in which large oscillations are obtained. The same result is obtained if the heat flux is maintained constant, but the inlet velocity is reduced. The plot shows also that instability can also be obtained simply by varying the length of the nonboiling region.

In another Russian plot having as the ordinate the ratio of the period of the oscillations to the total transit time in the system, and the abscissa the Reynolds number of the system, shows that the ratio varies with the Reynolds number at a given pressure, going from a value less than unity at low Reynolds numbers to a value greater than unity at larger Reynolds numbers. The curve is shifted as the pressure is changed.

In another Russian plot, one can examine the effect of variations in the dynamic void coefficient $\beta$ on the pressure fluctuations in a two-phase system. The pressure variations are seen to change markedly in going from the bubble flow regime to the slug flow regime; both the amplitude and the period of the pressure fluctuations change. It is clear that by measuring the pressure fluctuations one can get useful information on the nature of the flow and hence on the tendency toward flow instability.

In summary we can state the following:

1. We are presently in a position to accurately predict the course of void growth.

2. Within a year, the experiments being carried on now at Berkeley and at Ramo-Wooldridge should permit us to say whether or not 
we can use steady-boiling measurements to obtain bubble populations and use these in prediction of transient boiling behavior.

3. It is essential to be able to predict the two-phase pressure drop since stability depends intimately upon it, and criteria can be established for predicting instability if only the twophase pressure drop is known.

4. I believe that the course of boiling transients can be predicted from the general one-dimensional equations. However, this remains to be proved by detailed comparisons with expeximents. (This has not yet been done except for the work of Dr. Fleck.) Clearly, it will be important to bring to bear knowledge of the two-dimensional flow patterns, but I believe that when this is done we will find that the one-dimensional formulation of the dynamic problem will give predictions in good agreement with experiment.

What further work must be done? Because reactors are so complicated, it makes the most sense to pursue the answers to the remaining questions on hydrodynamic instability in experiments conducted out-of-pile. We need good measurements of four quantities:

1. The void coefficient.

2. Pressure drop measurements. These are needed both to determine the two-phase pressure drops and to be able to predict the dynamic effect of the pressure waves propagated in a two-phase flow mixture. This is one of the most important areas for further work because I believe that the different flow regimes which occur in boiling are connected with a kind of shock-wave phenomenon in two-phase flow mixtures. (The reason is because the velocity of sound in boiling water is only one meter per second.) I believe that the flow patterns are related to this propagation phenomenon in a two-phase flow mixture.

3. Temperature measurements in the axial direction along the channel with accuracies at least of $0.5^{\circ} \mathrm{C}$. This is important because nucleation is very sensitive to the plate temperature.

4. Flow-pattern correlation in two-phase flow.

If we can get good measurements of these four quantities in the next two years, I would confidently predict that we could say at that time that we had solved the problem of hydrodynamic instability in boiling systems completely.

Blizard: Have any experiments been done using different "controlled" surfaces to study the effect of the surface characteristics? 
Zuber: Some experiments of this sort have been done at Ramo-Wooldridge, as I mentioned. These will be discussed by Wright and Langmuir. In a reactor, of course, this question becomes somewhat academic because of the existence of sharp corners in the construction of the fuel elements and because of the effect of radiation on nucleation bubbles.

Fleck: Is bubble nucleation affected by radiation? How do we know?

Zuber: A comparison of experiments done with pure water and with a uranyl sulfate solution showed that whereas a water system permitted the development of hundreds of degrees superheat, the uranyl sulfate system kept the superheat down to only a few degrees.

Thie: The British have done some work on the radiation effect on nucleation. They find that the effect is greatest at low pressures.

Zuber: On another subject, I might mention that Karplus has made extensive studies of the velocity of sound in two-phase water systems.

Beck: Three questions occur to me: First, what happens if the channels are very, very narrow so that the slip-velocity effects are not so effective? Second, what happens if the geometry of a channel changes as one goes along in the direction of flow? Third, in a vertical channel in which the void fraction changes with vertical distance, how does the flow vary with the vertical distance?

Zuber: In answer to the first question I would point out that shear effects give higher slip velocities in narrower channels. At very high velocities one goes into the annular flow regime. One cannot carry the analysis I have discussed in my talk to the annular flow regime. This must be analyzed separately. 
SESSION IV

PANEL PRESENTATIONS

Snyder: The first panelist will be Virgil Schrock from the University of California.

\author{
Transient Boiling Experiments \\ by \\ V. E. Schrock (UC)
}

Schrock: Thank you, Thoma. I'd like to describe, in some slightly greater detail, the experiments which we are doing at Berkeley. I'm doing this work in collaboration with Professor H. A. Johnson. The work is essentially an out-of-pile, bench-scale, transient bolling experiment which is an extension of the Rosenthal and Miller work reported in ORNL-2294.* The extension is to include the measurement of the steam volume developed as a function of time and to make these measurements over a range of pressures as high as those of interest in reactors, and with flow imposed over the surface as well as with stagnant conditions. The equipment which we are using is substantially the same as that used by Rosenthal and Miller; in fact, we have the Oak Ridge transient power generator which consists of a set of a hundred thyratrons that act as switches and successively, on a predetermined schedule, add a contribution to the current which builds up the power exponentially.

This admittedly adds power in steps; the steps are usually quite small, however. There may be some question regarding how well this approximates a true exponential function. Slide I presents a particular run, which is typical of the data which we get, showing calculated power based on measured current and voltage as a function of time on semi-log paper. You can see that the approximation of the exponential is really quite good.

Now, to accomplish this work we first had to design a high-pressure system; we had to make some decisions about how we would measure the volume developed. The decision that we made was to make measurements photographically, getting three simultaneous views of the boiling taking place on the thin ribbon, and in addition to this, to make measurements using X-ray absorption similar to those that are made by the people at Ramo-Wooldridge. So a good deal of the effort went into developing this high-pressure system which is just now completed and ready to go. The information that I have to offer at this point, is a summarization of some preliminary atmospheric pressure work which we did for several reasons. First, it is, of course, of interest to have atmospheric

*N. W. Rosenthal and R. L. Miller, "An Experimental Study of Transient Boiling", ORNL-2294 (1957). 


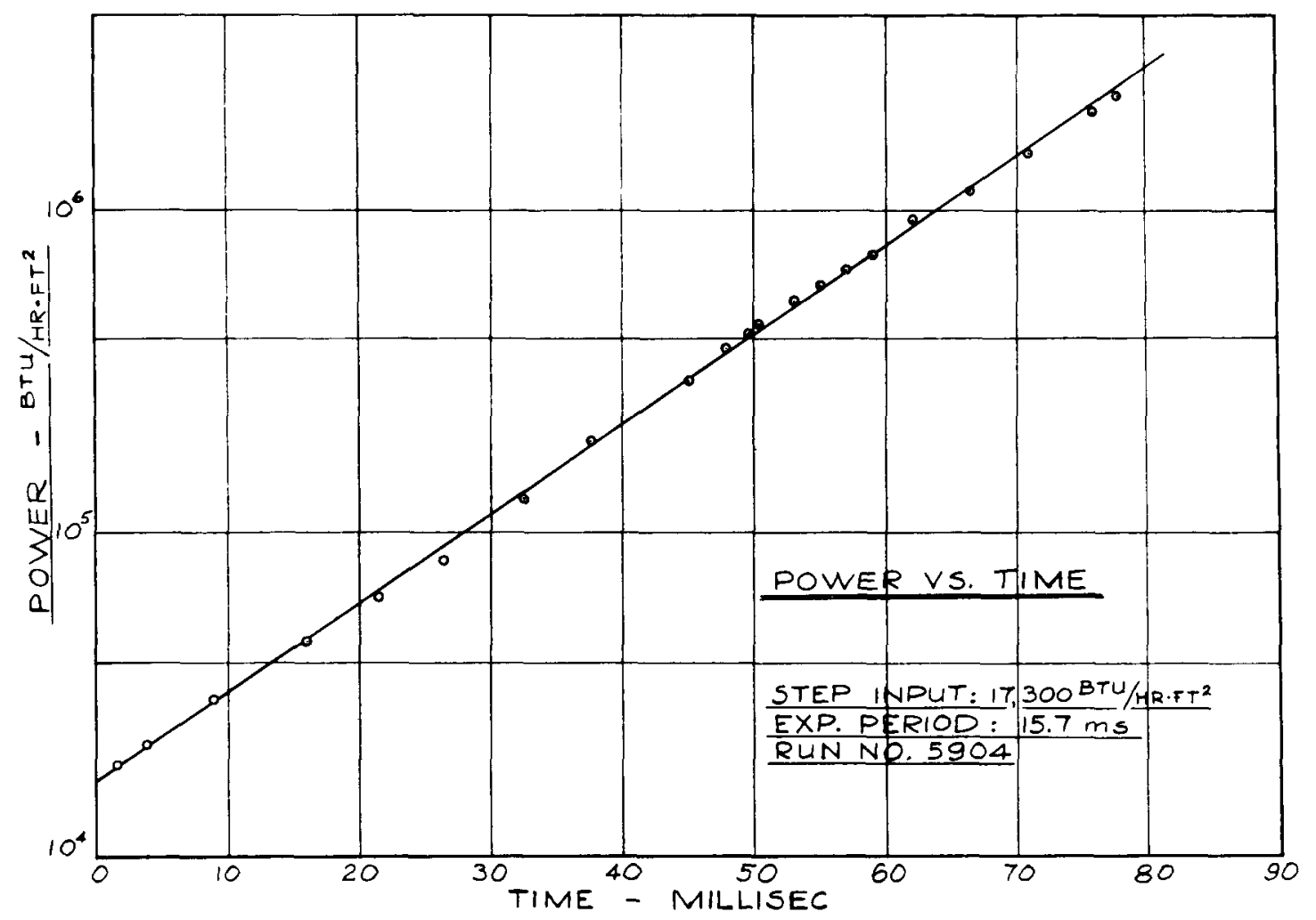

Slide 1. Power Calculated from Current and Voltage Measurements as a Function of Time

pressure data; secondly, we had to learn how to make the measurements; we had to learn how to get satisfactory photographs, and we used these tests to develop our experimental techniques. I brought a few slides which will illustrate rather simply the nature of the results which we get. These measurements have covered a range of exponential periods from about $5 \mathrm{msec}$ to as high as $100 \mathrm{msec}$, most of them taken at 5, 15, 25 and $50 \mathrm{msec}$. The initial water temperature or the degree of subcooling was varied. Three standard values of water temperature used were $100^{\circ} \mathrm{F}, 170^{\circ} \mathrm{F}$ and $210^{\circ} \mathrm{F}$, which gives just enough subcooling so that we don't have an excess of vapor in the system before we begin the test. Now, in Slide 2, we see the sort of thing which was reported by Rosenthal and Miller, and I'd like to describe rather briefly how we interpret what happens here. As the heating takes place we have, first, a regime in which the mechanism is one of pure conduction--this has already been brought out earlier in the meeting. During this period of time we can calculate the temperature rise, and you see in this slide the comparison of experimental determination of the temperature as a function of time with the conduction solution. After the surface temperature passes the saturation temperature, superheat begins to develop in the liquid so that nucleation then becomes possible. The rate of heat addition, of course, continues increasing exponentially. The heat flux tends to outrun the usual steady-state condition, and we go to superheats which 


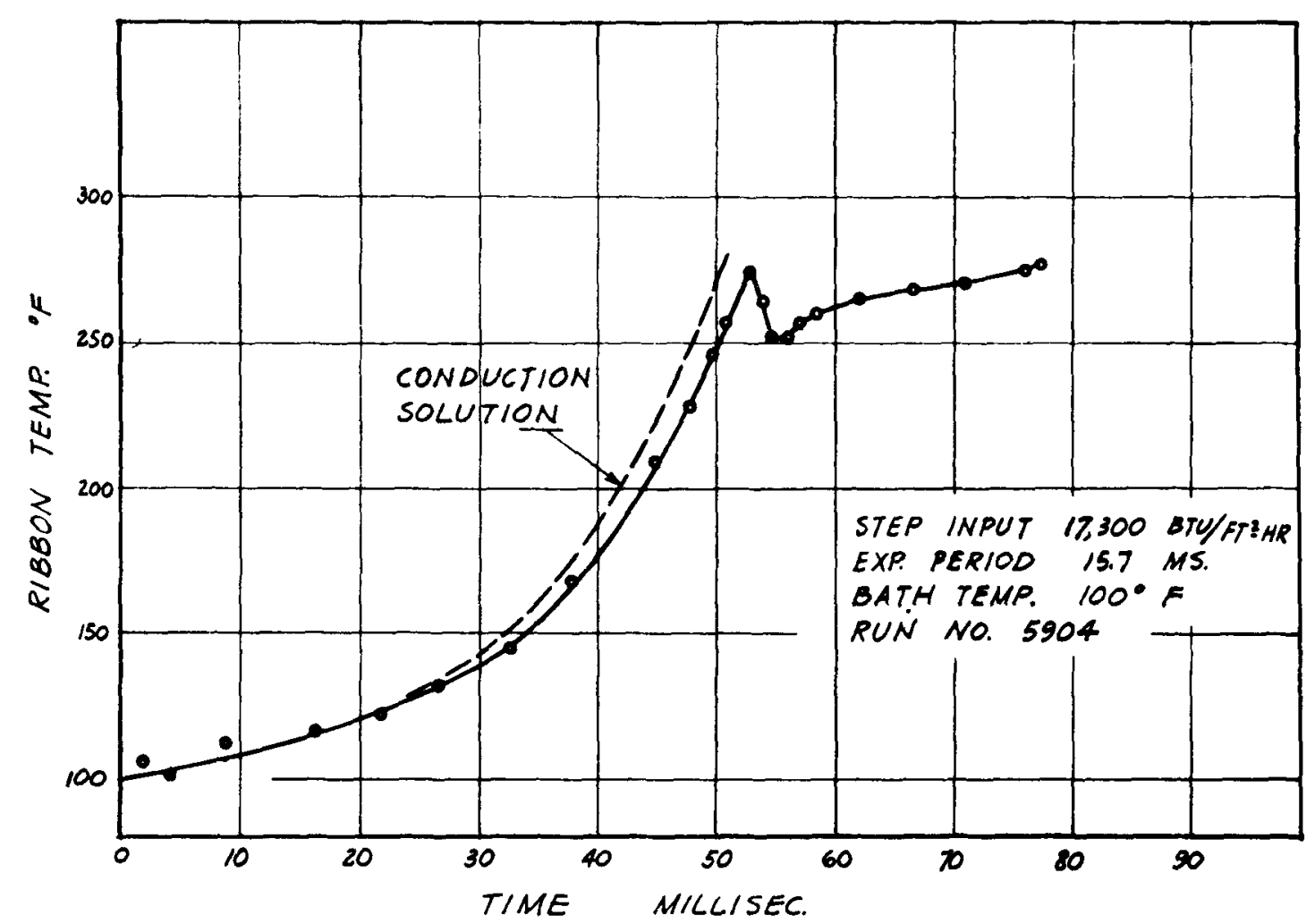

Slide 2. Ribbon Temperature as a Function of Time for a $15.7 \mathrm{msec}$ Period Test

would exist normally in steady state at the inception of nucleate boiling. So, we get this sort of "overshoot". The overshoot is partially attributed to the low thermal capacity of our system and there is a very good possibility--some people feel strongly--that this kind of overshoot will not exist if we have a larger thermal capacity, as in the plate-type, MTR fuel element. During this period, somewhere near the peak temperature, nucleation begins (actually, our data indicate that it takes place very, very close to the peak temperature) and that the first generation of bubbles, because they are growing in an environment of superheat which is very much greater than the usual superheat in steady-state boiling, grow to a very much larger size. They grow very rapidly because of the high superheat, and they tend to cause a cooling effect which is so large that it outruns the increase in heat flux, even; and for this low thermal capacity the ribbon experiences this decrease in temperature. This is then followed by a steady rise in temperature, but now the heat transfer rate is improved so greatly that we no longer have an exponential rise in temperature--in fact, the temperature remains relatively steady. In this particular run we've terminated the transient before the ribbon burned out, but in later runs-and I'll show you some movies where we 
have actually burned them out--the data would plot, generally, like this, and then when burnout takes place, the temperature rises almost vertically. Slide 3 shows an effect which is also reported by Rosenthal and Miller. They define a temperature overshoot in terms of the saturation temperature of the liquid--the difference between the temperature at the peak of the overshoot and the saturation temperature was defined as the overshoot temperature, and an attempt was made to correlate this in terms of the exponential period. As you can see, the general tendency is for the overshoot temperature to be reduced with increases in exponential period. Rosenthal and Miller reported data going to zero and, of course, as we know, this could not be possible because it is always necessary to have some degree of superheat in the liquid to promote nucleation. Slide 4 shows the result of the photographic measurements of the steam void. This is done by studying the individual frames of Fastax film, scaling the bubbles, estimating the best equivalent shape for them, and calculating the volume--a very tedious process, of course. And this is the sort of result that we get at the three temperatures used. Close to saturation we have a monotonic increase in the volume. For $172^{\circ}$, or $40^{\circ}$ of subcooling, we get a marked overshoot (again, this may not occur if we have a higher thermal capacity). There is not only an overshoot in the temperature, but there is an overshoot in volume as well. This is associated with the fact that this first

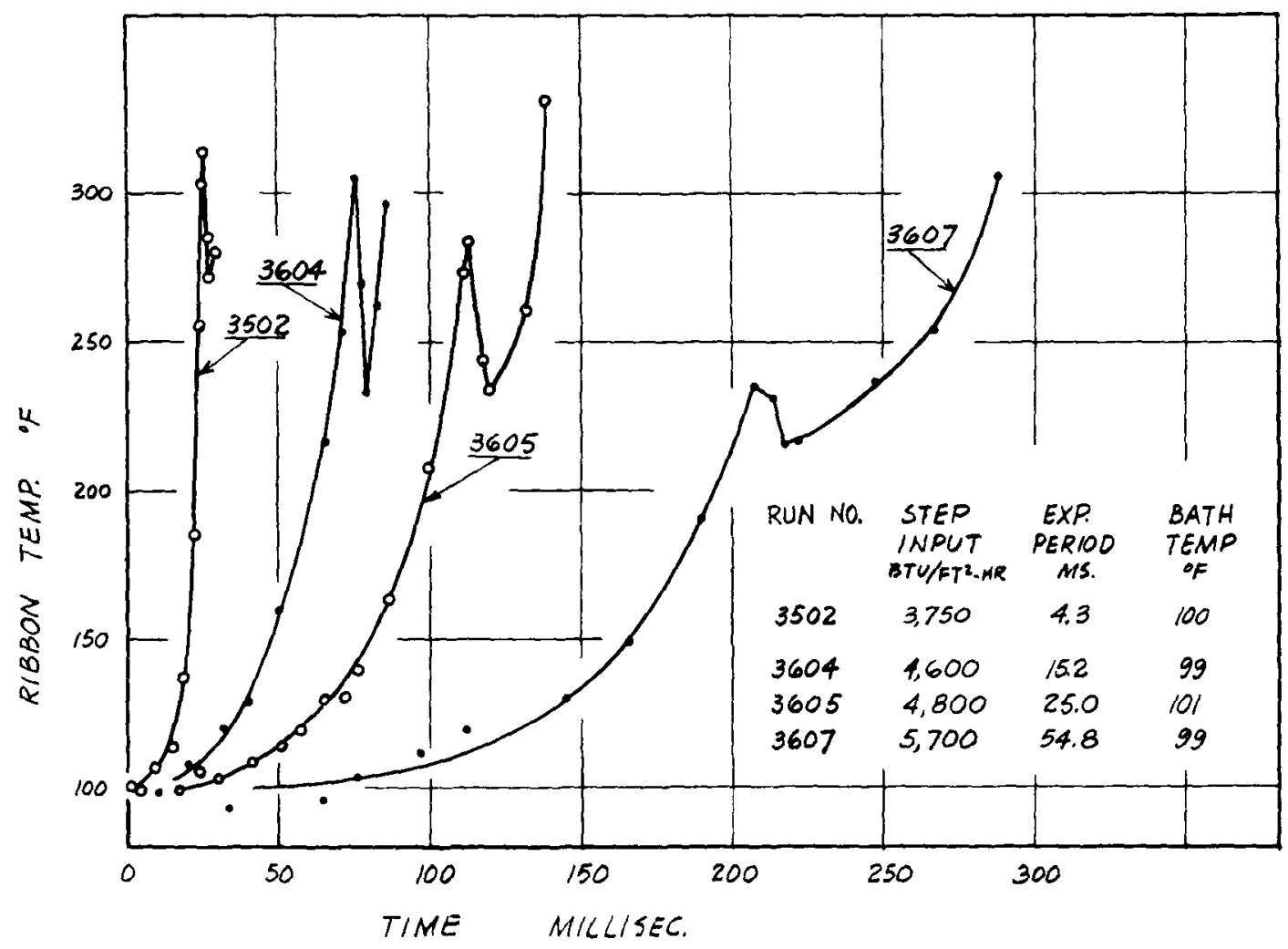

Slide 3. Ribbon Temperature as a Function of Time with Period a Parameter 


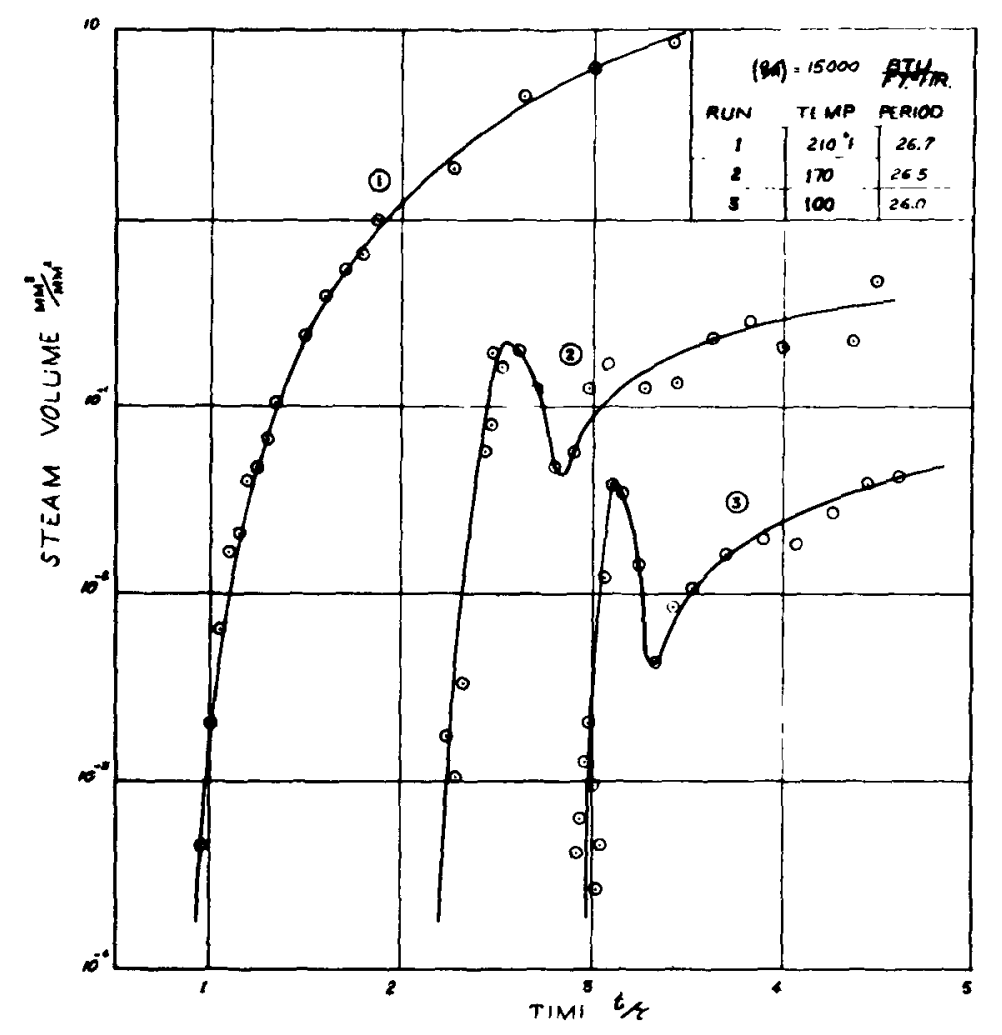

Slide 4. Steam Volume Calculated from Photographic Measurements as a Function of Time

generation of bubbles grows to a larger size than subsequent bubbles, and can be seen quite clearly in some of the movies which I'Il show in a moment. When the water temperature is subcooled by some $112^{\circ} \mathrm{F}$, we see that we have almost an order of magnitude change in the volume after it reaches a peak value. In some of these muns we get a first burst of large bubbles, followed by their collapse and a completely clean surface results. Then the development of what looks like an entirely different sort of bolling occurs with very fine bubbles and very large numbers of bubbles. And it takes some time before the volume develops again to the same level that it had attained at the peak of the overshoot.

Let's take a moment now to discuss what you're going to see in the movies. We have mirrors arranged to show the ribbon in three simultaneous views. The ribbon is orientated horizontally and the top surface is seen in a mirror above the ribbon. The edge view of the ribbon appears as a fine, shiny line in the movies; it is difficult to see until it gets bubbles on it. And then you see another view below which is the underside of the ribbon in another mirror. (These three views are indicated in the first picture in slide 5). 
The conditions for the three runs that I selected are as follows (one at each of the temperatures): the first one is at $100^{\circ} \mathrm{F}$, and has a period of about $15 \mathrm{msec}$; the second one is at $170^{\circ} \mathrm{F}$ and has a period of $25 \mathrm{msec}$; and the last one is at $210^{\circ} \mathrm{F}$ and has a period, again, of $15 \mathrm{msec}$. Now, this gives you a little indication of the effect of period as well as the effect of the water temperature on the amount of steam which develops on the surface. Along with each of these you might be interested in knowing the amount of vapor which develops on the surface for these particular tests: at the peak of the overshoot, where there is an overshoot (of course, in the last case there is none), and at the burnout condition. In the $100^{\circ} \mathrm{F}$ case (Slides 5, $6,7,8)$ we get a volume per unit area at the overshoot peak of 0.0085 centimeters, and at the burnout condition we have 0.012 centimeters. The $170^{\circ}$ mun (Slides $9,10,11$ ) has 0.02 , and 0.03 , and the $210^{\circ}$ run (Slides 12, 13, 14) has a value of 2. The last case, of course, is not too meaningful if you consider a Spert channel which is a good deal thinner than 2 centimeters. It lacks significance also, because of the fact that the ribbon is only 1/10 of an inch wide and the steam developed completely surrounds the ribbon, and we're looking at vapor that surrounds it near the burnout condition. I think that that's really all I want to say about the movies-perhaps I can add comments as we go along.*

The reel was assembled in just the wrong way, because we should look at the slow one first, and the fast one last.

In Slide 5 the edge view, upper surface, and the lower view are indicated. In this sequence you see the fine bubbles that developed and finally it burned out.

* A set of still pictures (Slides 5 through 14) are presented for the runs shown in motion pictures at the Conference. The period, intitial water temperature, $T_{O}$, and the initial heat flux, qo are indicated for each run. 


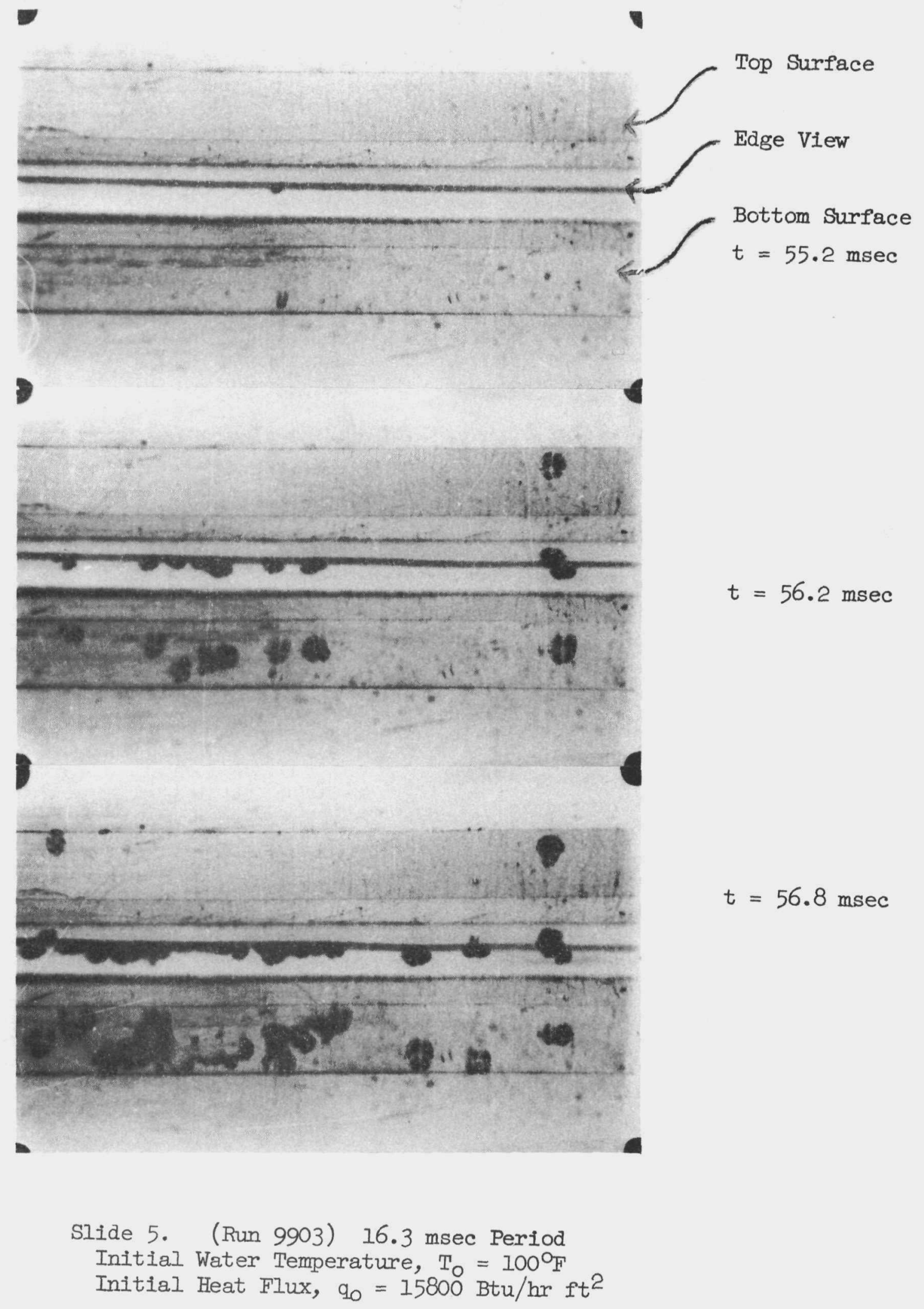




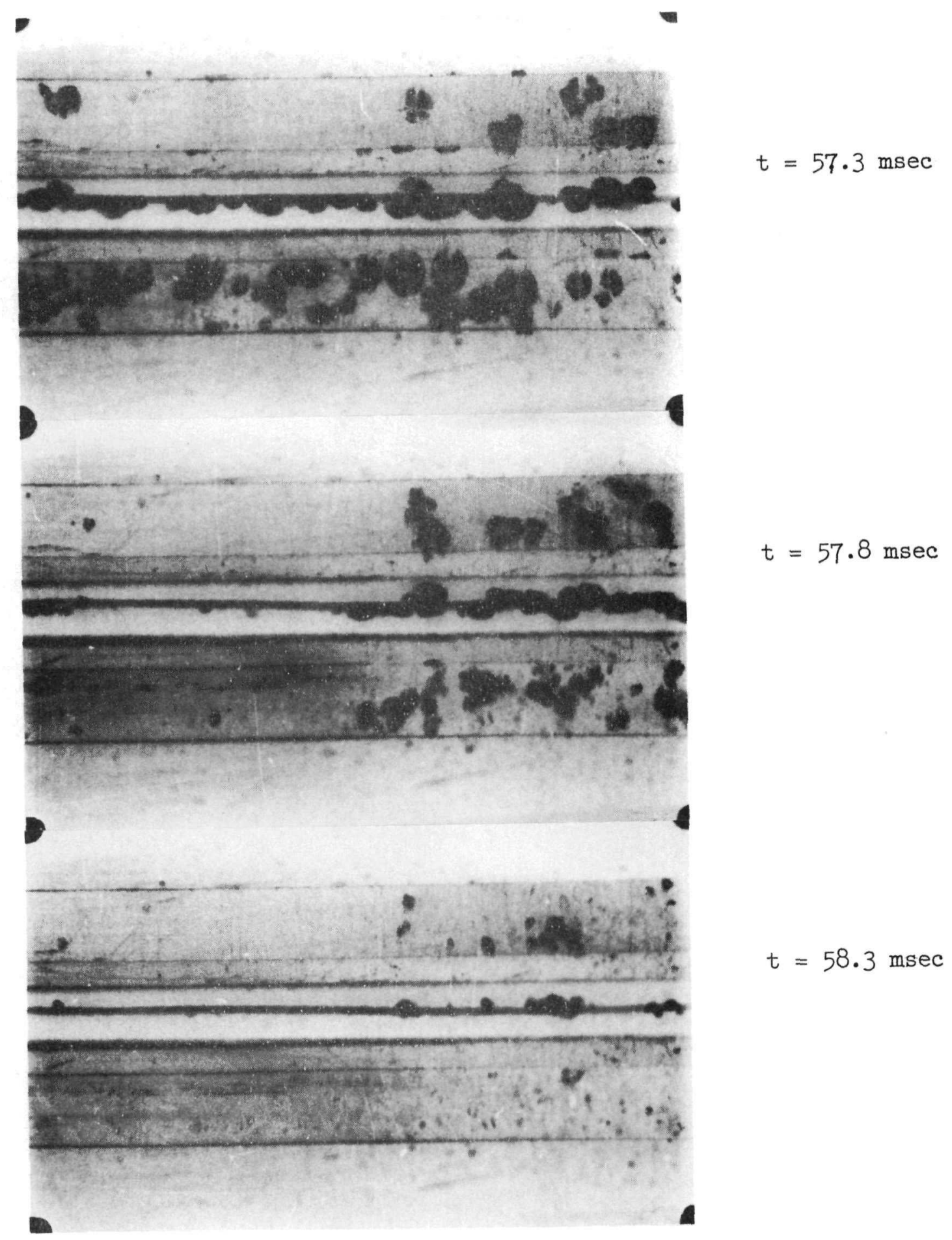

Slide 6. Run 9903 (Continued) 


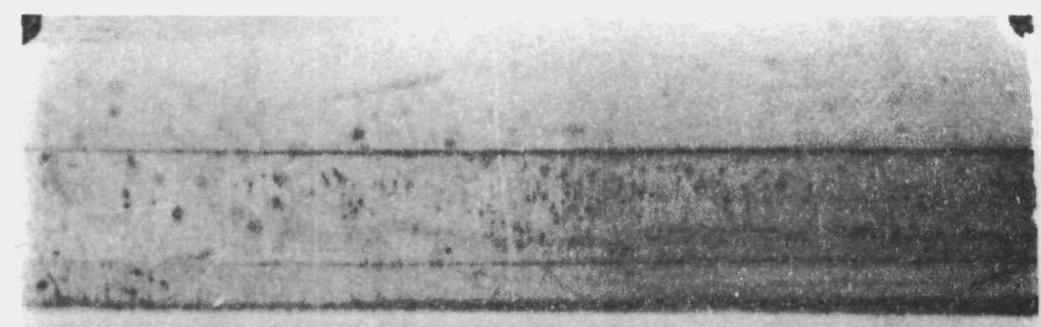

$$
t=58.8 \mathrm{msec}
$$
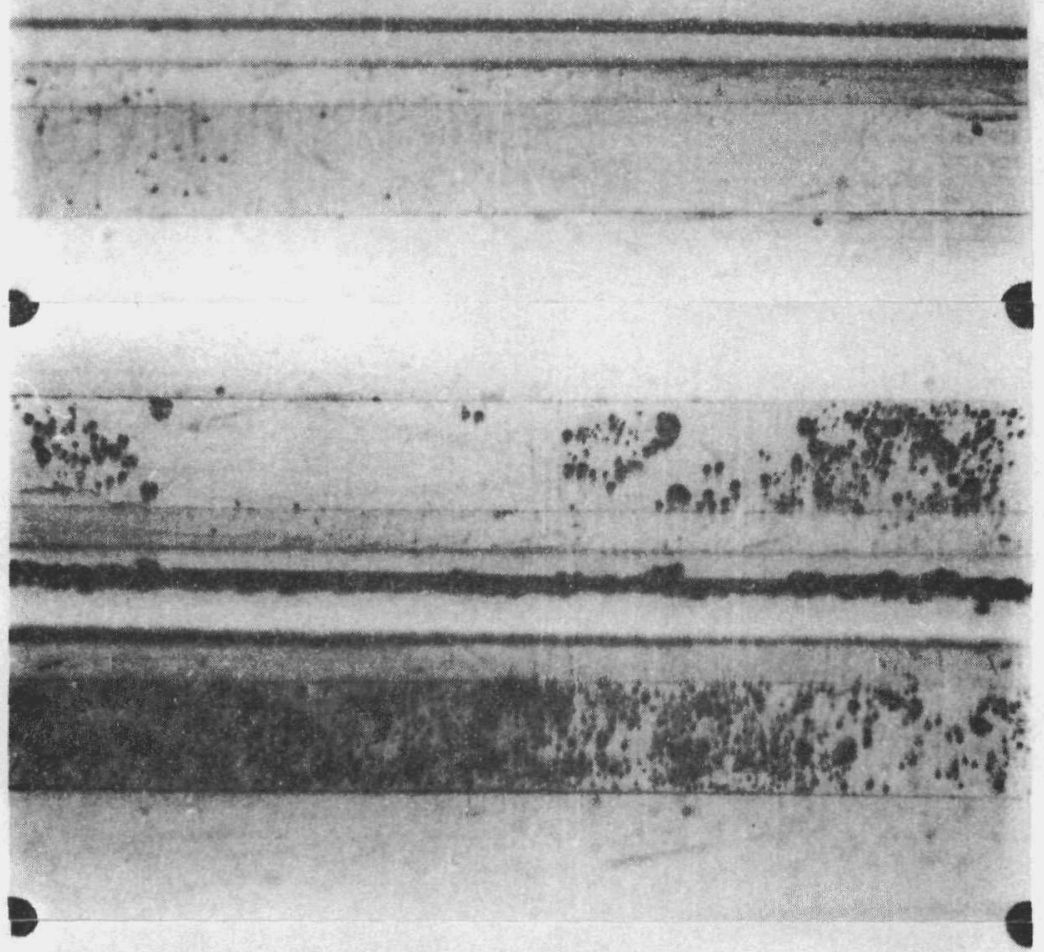

$$
t=67.9 \mathrm{msec}
$$

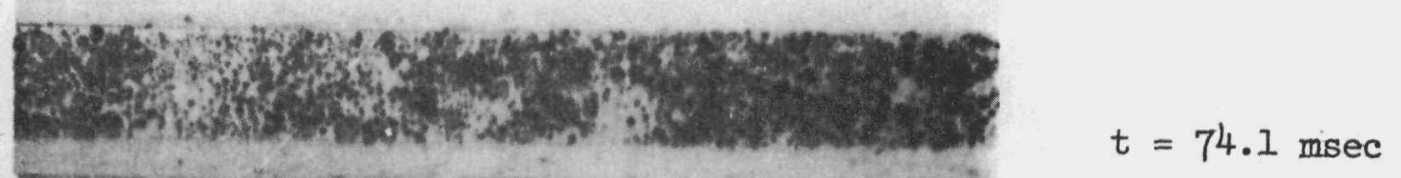

Slide 7. Run 9903 (Continued) 


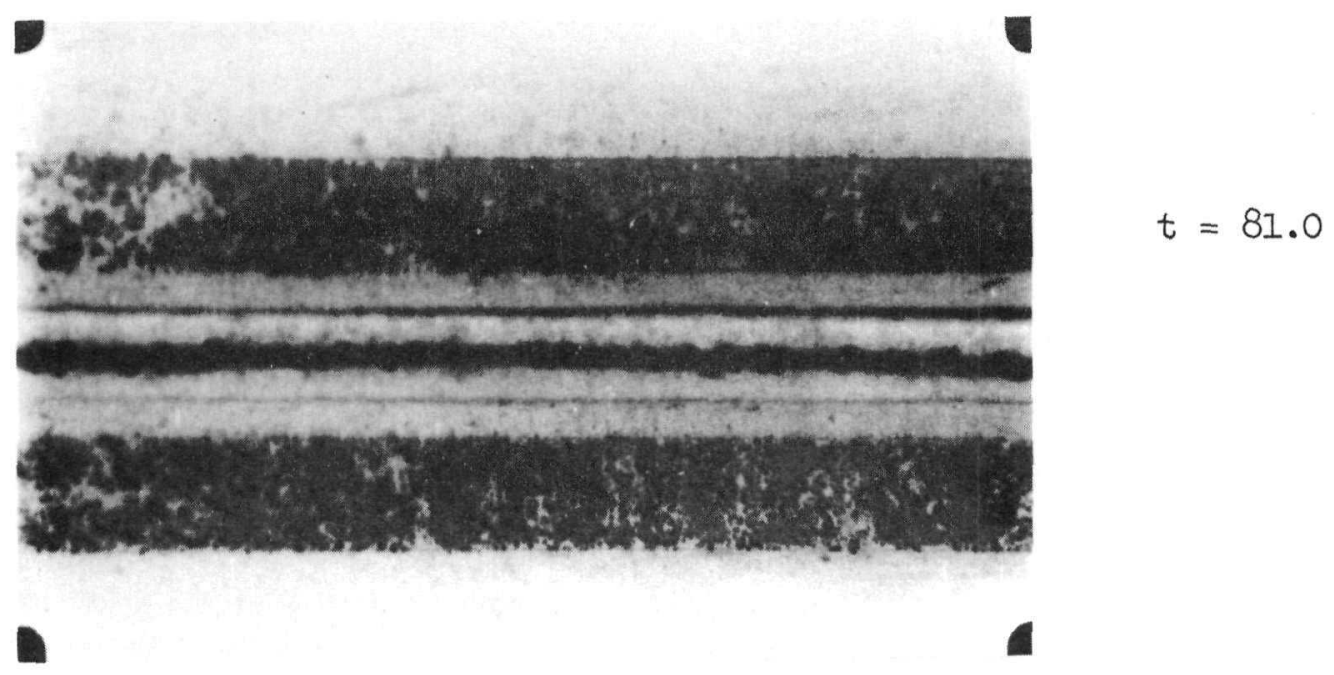

Slide 8. Run 9903 (Concluded)

I should have mentioned that the ribbons in this particular set of photographs were one-mil-thick platinum. We've done tests with thicker platinum ribbons; we've also done tests with aluminum and with a 50-50 iron-nickel alloy. The results don't seem to be significantly different as far as we've been able to tell so far.

Unidentified: What is the time scale we are watching?

Schrock: This is magnified in time about 250 to 300 times. There are about four frames per millisecond, and if we're showing this at 16 frames per second I think that comes out to be close to 300 .

In this case, Slides 9, 10, 11 you can see film boiling which developed first--at the point where it burned out--right at the edge of the field. We're not sure what the small bubbles remaining are-possibly a little disassociation of the water vapor caused by hot platinum at the time it burned out. 


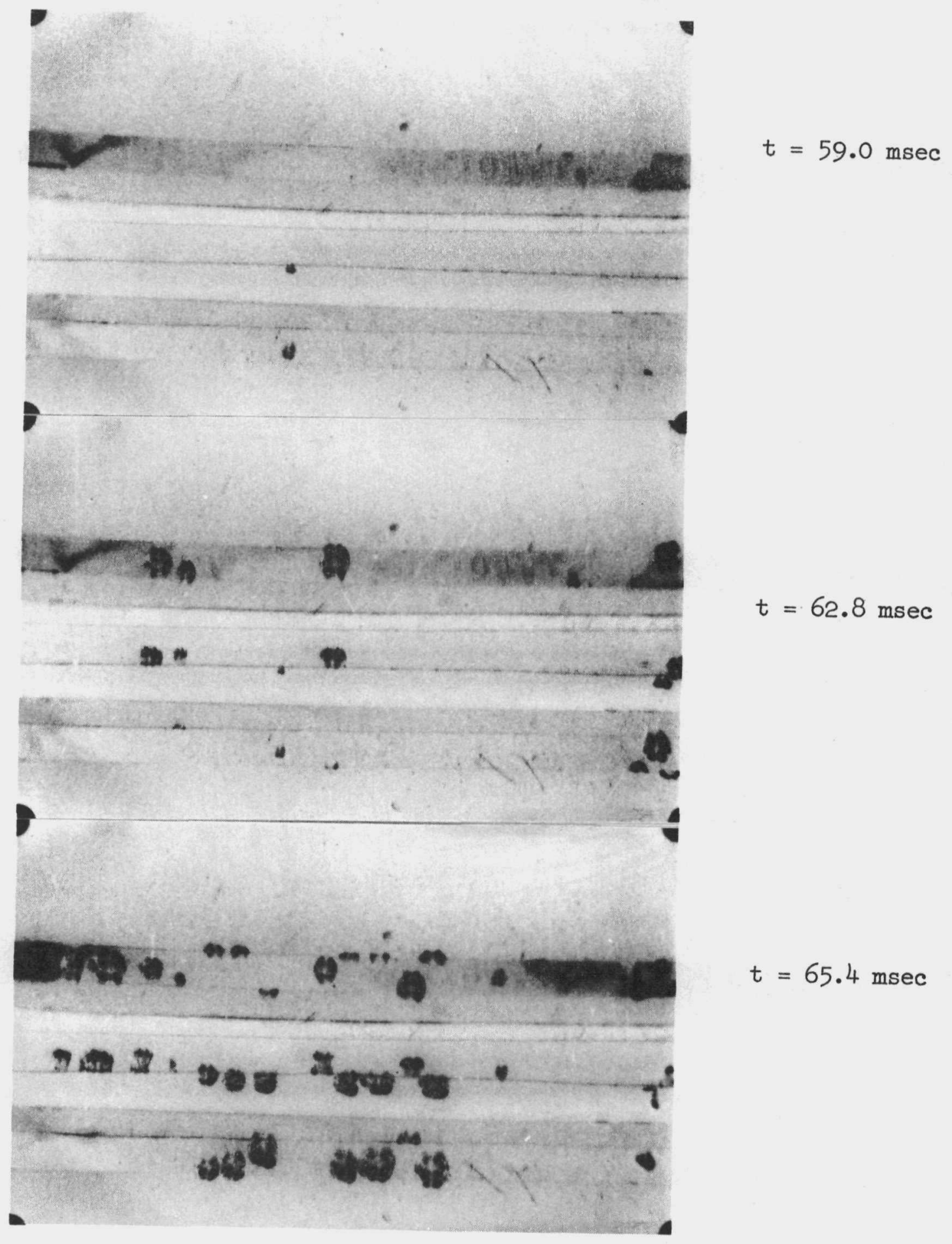

Slide 9. (Run 8705) $26.4 \mathrm{msec}$ Period

$\mathrm{T}_{\mathrm{O}}=170^{\circ} \mathrm{F} \quad \mathrm{q}_{0}=17,000 \mathrm{Btu} / \mathrm{hr} \mathrm{ft}^{2}$ 


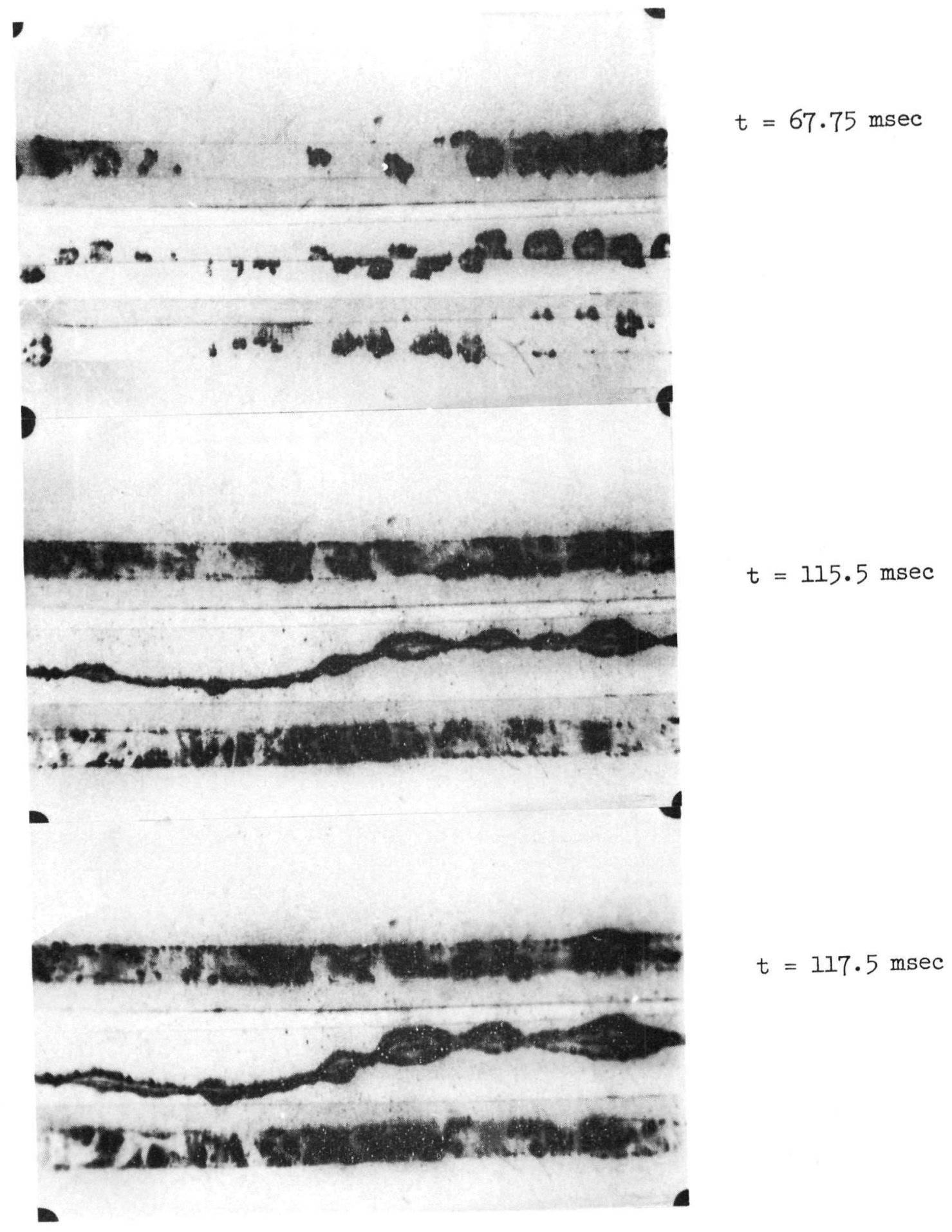

Slide 10. Run 8705 (Continued) 


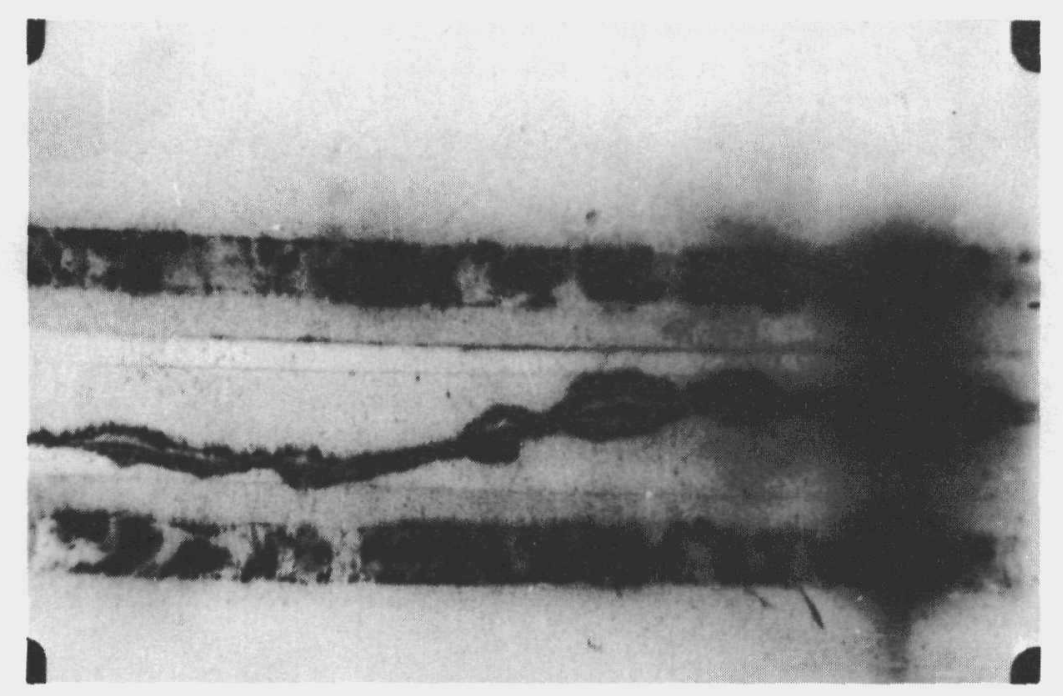

$$
t=118.7 \mathrm{msec}
$$

Slide 11. Run 8705 (Concluded)

Water is reasonably well degassed in these tests, although it's difficult to maintain well degassed water which is exposed to the atmosphere. We deaerate by boiling the water for a period of time prior to the run. Then in the cooling process for subcooled runs at $100^{\circ} \mathrm{F}$, while it stands exposed to the atmosphere, it takes on about half the amount of air that it had before the degassing process.

The last run, Slides 12, 13, 14, is close to saturation and so you don't see any significant collapsing of bubbles. The bubbles that form continue to grow and you quickly have the whole region covered with vapor. 


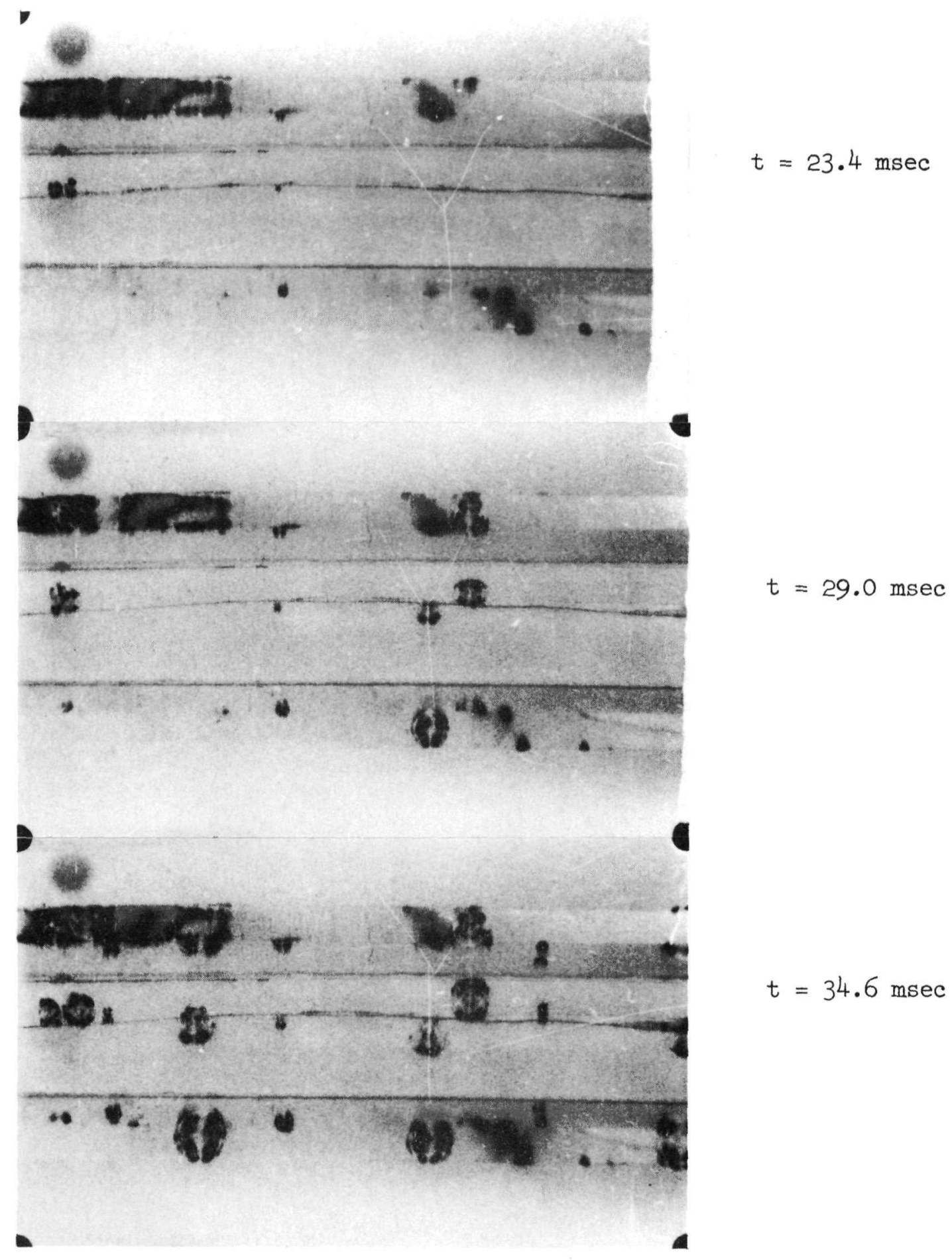

Slide 12. (Run 6609) $15 \mathrm{msec}$ Period

$$
\mathrm{T}_{0}=210^{\circ} \mathrm{F} \quad q_{0}=15,000 \mathrm{Btu} / \mathrm{hr} \mathrm{ft}^{2}
$$




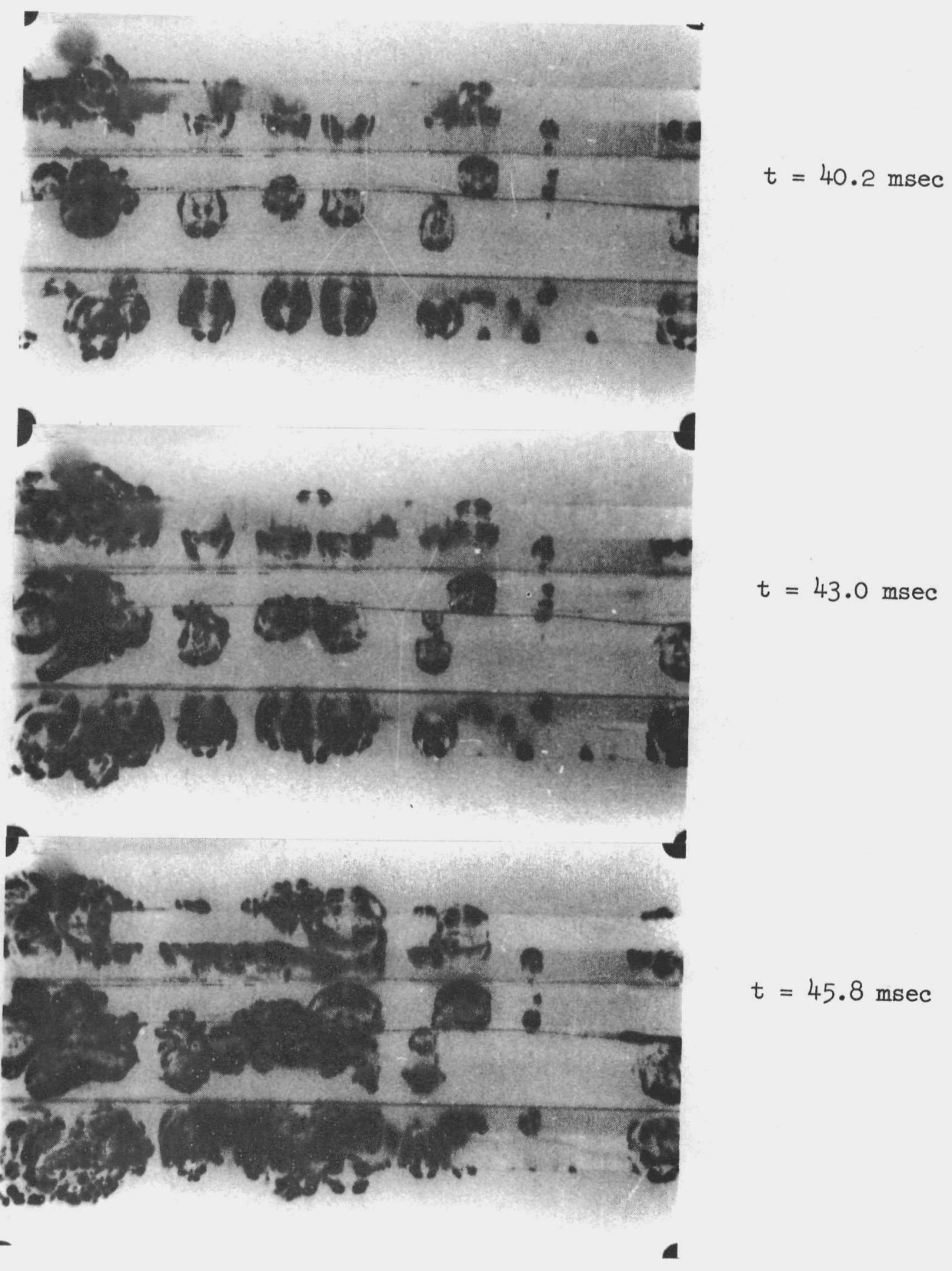

Slide 13. Run 6609 (Continued) 


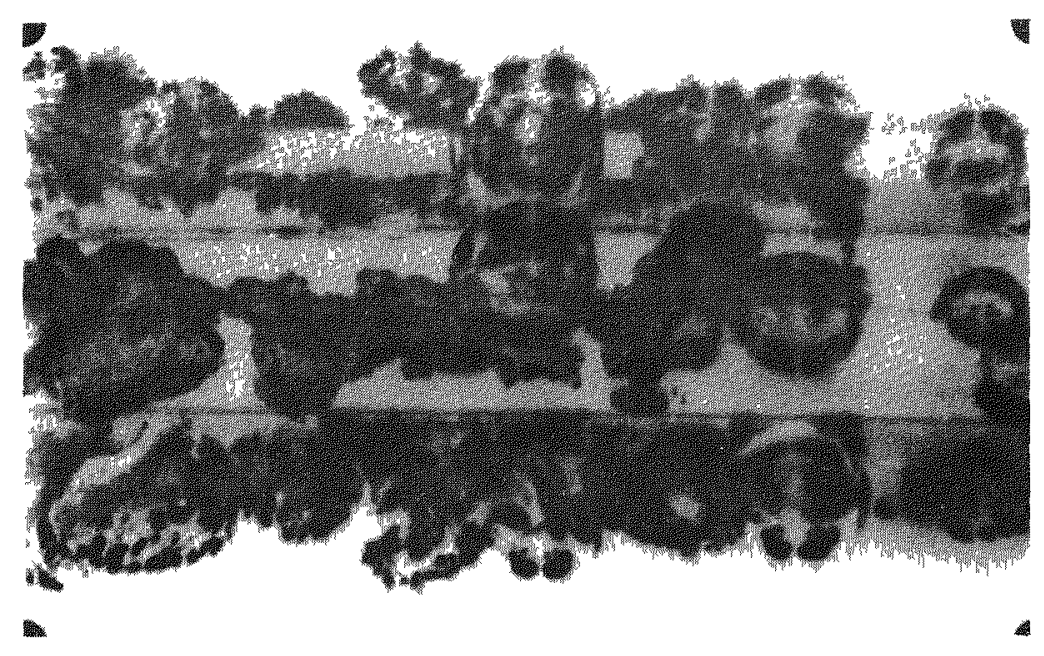

$t=48.6 \mathrm{msec}$

Slide 14. Run 6609 (Continued)

I think you can appreciate our desire to make this measurement using $X$-ray absorption when you see the number of bubbles that have to be counted. At that, the statistical aspect of the process is quite prominent. If we repeat runs as closely as possible, we find that the boiling process does not always begin at the same time. We find that the amount of vapor which develops is somewhat different, even for the same ribbon where we've made one test with the ribbon and not burned it out and then repeated the test within an hour or two.

Novak (Zuber) mentioned some of the analytical effort of our group. One analysis of void volume growth has been developed which gives reasonable results for the near saturated case. Bubbles are assumed to grow according to the Forster-Zuber asymptotic solution. Steady-state nucleation site density data of Westwater is assumed to apply for the instantaneous heat flux and only one bubble is assumed to grow at each activated site. Relations obtained from these assumptions were substituted into the general integral (which Zuber showed) to account for simultaneous growth and site activation. The upper limit of the integral was the variable time measured from the appearance of the first bubble. Integration gave the time dependence of the vold volume. The analysis does not consider collapse of bubbles (as occurs in subcooled boling) and is, therefore, restricted to the saturated case. For short periods, on the order of 5 to $10 \mathrm{msec}$, the theory and experiment are within a factor of two and this deteriorates to a factor of four for a period of $80 \mathrm{msec}$.

Now, the real key problems, I think, to focus attention on in this particular case, are the rates at which the vapor is generated and the time at which the nucleation first takes place. We have had a limited amount of success, also, in predicting when the first bubble appears. Rosenthal and Miller's work, of course, attempted to give us some information as to where the peak of the overshoot would occur and if we assume that this time coincides with apperance of the first bubble, this might be one way of getting at it. We find that our data scatter very badly, however, when we use this approach, and we can't get much more than a trend out of it. There is a tendency for the overshoot to decrease as 
the exponential period is increased, but the experimental data scatter very badly. We've tried to do it on a more fundamental basis using, first of all, the 1dea that perhaps nucleation takes place when we have developed a superheated layer which is equal in thickness to the critical bubble size corresponding to the instantaneous value of the wall temperature (superheat). If, at any particular instant, a superheated layer has developed of sufficient thickness so that its average superheat corresponds to the value of the critical bubble size, in Novak's notation, $R_{c}$--we would then say that now the nucleation process can take place. We tried this and found that the actual beginning of boiling takes place very much later. The next thing which we tried was to investigate the energy content of the layer which has the thickness $R_{c}$ as a function of time. Since the critical bubble size diminishes as the temperature--superheat temperature, increases, but the specific enthalpy of the fluid contained within that region is increasing with time, we find that we have a maximum in the total enthalpy contained. If we plot the total enthalpy as a function of time it first increases and then decreases--it has a rather flat peak, however. Now if we argue then that, for some reason which may not be too clear physically, the probability of nucleation occurring is greatest at this maximum, then we would have some indication of when the first bubble appears. We have since refined this analysis, or made it a little bit more elegant, by putting it in terms of the thermodynamic potential and the availability function which gives it, perhaps, a little bit sounder physical base. The end result is identical, whether we are considering the maximum total enthalpy contained or whether we're considering a stability criterion based on the thermodynamic potential combined with the avallability function. The experimental data have been checked for saturated boiling--or closely saturated boiling, and we find that we can predict the time of the first bubble within about $\pm 10 \mathrm{msec}$, on the basis of this idea. We hope that we can refine the analysis and get better predictions, but I'm afraid that at this point the best that I can say is that our prediction on the basis of this theory would be no better then the prediction on the basis of the temperature overshoot with the assumption that nucleation begins at the temperature overshoot. Novak?

Zuber: I think Dr. Schrock has here the solution to the problem in the following way.

We can estimate the rate of evaporation by using the energy considerations of Boshjakovic and Jakob (see AECU-4439, Sect. II). Following Fritz and Ende (Phys. Zeitsch V. 37, p. 391, 1936) the problem is formulated by considering the one-dimensional transient heat conduction problem

$$
\begin{aligned}
a \frac{\partial^{2} T}{\partial x^{2}} & =\frac{\partial T}{\partial t} \\
t=0: T(x, 0) & =\left(T_{W}-T_{L}\right) f\left(\frac{x}{d}\right) \\
t>0 \quad T(0, t) & =T_{s}
\end{aligned}
$$


where

$$
\begin{aligned}
T_{W}= & \begin{array}{l}
\text { temperature of the solid wall at the time the bubble } \\
\text { starts to grow }
\end{array} \\
T_{L}= & \text { temperature of the bulk liquid } \\
T_{S}= & \text { saturation temperature } \\
a= & \text { thermal diffusivity } \\
\left(T_{W}-T_{L}\right) f\left(\frac{x}{d}\right)= & \begin{array}{l}
\text { temperature distribution in the liquid at the time } \\
\text { the bubble starts growing, 1.e., when evaporation } \\
\text { starts. }
\end{array}
\end{aligned}
$$

For exponential heating:

$$
f\left(\frac{x}{d}\right)=e^{-x / \sqrt{a \tau}}
$$

where $\tau$ is the period.

The rate of evaporation, $R$, is obtained from the energy balance:

$$
I \boldsymbol{\rho}_{\mathrm{V}} \dot{\mathrm{R}}=\left.\mathrm{K} \frac{\partial \mathrm{T}}{\partial \mathbf{x}}\right|_{\mathrm{x}=0}
$$

where

$$
\begin{aligned}
L & =\text { heat of vaporization } \\
\rho_{\mathrm{V}} & =\text { the density of the vapor } \\
\mathrm{K} & =\text { thermal conductivity of the liquid. }
\end{aligned}
$$

Solving (1) for the case of exponential heating, the rate of evaporation is given by

$$
I \rho_{V} \dot{R}=K\left[\frac{T_{W}-T_{S}}{\sqrt{\pi a t}}-\frac{T_{W}-T_{L}}{\sqrt{a \tau}} e^{t / \tau}\left(1-\operatorname{erf} \sqrt{\frac{t}{L}}\right)\right]
$$

when $t \ll \tau$ this simplifies to

$$
L \rho_{V} \dot{R}=K \frac{T_{W}-T_{S}}{\sqrt{\pi a t}}\left[1-\frac{T_{W}-T_{L}}{T_{W}-T_{S}} \sqrt{\frac{\pi t}{\tau}}\right]
$$


It is seen that we can estimate the rate of vapor formation if the temperature of the wall, $T_{W}$, is known at the instant the first bubbles nucleate, $1 . e .$, at the inciplent bolling conditions. At what wall temperature the nucleation will start depends on the liquid temperature, the dimensions of the surface cavity (surface pit), contact angle and the amount of entrapped gas or vapor (see discussion in Sect. I-I and I-2, AECU-4439). For a uniformly superheated liquid the problem is solved. However, as yet, there is no satisfactory theory of nucleation in a non-uniform temperature field as is the case in bolling, either transient or steady.

Several criteria for nucleation in non-uniform temperature fields have been proposed. Virgil told us a possible nucleation criterion may be the condition that the thickness of the superheated liquid film adjacent to the surface be equal to the critical bubble size corresponding to the instantaneous value of the wall temperature. A few years ago, a nucleation criterion for transient boiling was advanced by Faneuff, McLean and Scherrer (J. Appl. Phys., V. 29, p. 80, 1958). They proposed that a bubble will nucleate when the saturation isotherm has advanced a distance $R_{O}$ from the heating surface. This distance $R_{O}$ was equal to $2 / 3$ of the critical bubble radius $R_{c}=2 \sigma / \Delta P$. I found that, in steady nucleate boiling, the criterion appears to be a requirement that the saturation isotherm has advanced a distance equal to the diameter of the nucleating pit on the heating surface (N. Zuber, AECU4439, Sect. II-5). Although this condition was deduced from data for steady bolling, I belleve that it may be also valid in transitional bolling. I say this because in steady bolling (steady bubble emission) the growth and departure of bubbles induce locally transient thermal conditions.

It can be seen that the preceding criteria all imply the same condit1on: the saturation isotherm must advance a given distance from the heating plate before a bubble can nucleate. If the characteristic distance is taken as the radius, $r_{c}$, of the nucleating pit on the plate, then, in exponential heating, the temperature of the plate $\mathbb{T}_{W}$ at which the bubble will nucleate is

$$
T_{W}-T_{S}=\left(T_{S}-T_{L}\right)\left(e^{r_{c} / \sqrt{a \tau}}-I\right)+\frac{2 \sigma T\left(\rho_{I}-\rho_{V}\right)}{L \rho_{V} \rho_{L}} \frac{I}{r_{c}} e^{r_{c} / \sqrt{a \tau}}
$$

where

$$
\begin{aligned}
\sigma & =\text { surface tension } \\
\rho_{I} & =\text { density of the liquid }
\end{aligned}
$$

What are the implications? There are several and rather interesting.

(1) Consider a given pit size and a given pressure (constant thermodynamic properties). Then for constant temperature of the liquid, 
$\mathrm{T}_{\mathrm{L}}$, the wall temperature, $\mathrm{T}_{\mathrm{W}}$, will decrease with increasing period, $\tau$.

(2) For the same pit and at the same pressure, keeping the period $\tau$ constant, but reducing the temperature of the liquid Th (i.e., increasing the "subcooling", $\mathbb{T}_{B}-T_{L}$ ), results in an increase of the wall temperature.

(3) It can be seen from Equations (4) and (5) that the higher is the liquid superheat temperature difference, $T_{W}-T_{S}$, the faster is the rate of vapor formation and the larger is the amount of vapor formed.

(4) In transient bolling it appears even possible (theoretically at least) to control the wall temperature $\mathrm{T}_{\mathrm{W}}$ by varying the nucleating characteristics of the surface.

(5) For a given size of nucleating pits and for a given Tf and heating rate, we could estimate then both $\mathbb{T}_{W}$ and the "delay time" required for vapor formation.

(6) For a given $\mathfrak{T}_{\mathrm{W}}$ and operating pressure and liquid we could estimate then the rate of vapor formation.

(7) From the preceding information we could estimate the maximum possible amount of vapor formed. This can be done in a rather simple manner. If the criterion for nucleation is the requirement that the liquid at a distance, say equal to $r_{c}$, i.e., equal to the radius of the surface pit, reaches the temperature given by Gibb's relation, then the temperature of the wall can be estimated from Equation (5). The Iiquid adjacent to the heating surface is superheated and it is therefore in a thermodynamically unstable state. Assuming, for simplicity, a linear temperature profile in the liquid adjacent to the heating surface, the average enthalpy of the superheated liquid film is equal to $\left(T_{W}-T_{S}\right) c \rho_{L} r_{c} A / 2$ where $A$ is the area of the heating surface. If this energy (which is in an unstable state) is used entirely for the process of vaporization, then the maximum amount of vapor that can be formed is obtained from the energy balance

$$
A D L \rho_{V}=\left(T_{W}-T_{S}\right) \subset \rho_{L} r_{C} A / 2
$$

Solving for $D$ we get the maximum possible vapor thickness at the wall.

Other models and energy balances can be postulated but the key to the problem still remains the estimate of the wall temperature. This was the reason for my statement that if a criterion for nucleation were established the solution of the initial transients would follow soon after. I am just looking for experimental data to show it. 
Schrock: Well, I'm glad Novak is so optimistic. He set a rather optimistic goal for us of having adequate predictions of volume within a year, and I hope we can meet this, but I must say that I'm a little more pessimistic than that.

To summarize again, the key questions, I think, which we must attack are the rate at which the volume grows once it does begin and the determination of the inception of boiling. The determination of the inception of boiling is quite critical because the volume grows so rapidly once it begins that the termination of the transient, if it depends to a large extent on formation of steam void, is going to be accomplished in a very short period of time, and so we must have good accuracy in the prediction of the first bubble--good accuracy in the determination of the beginning of the boiling. Thank you. 
SESSION IV

PANEL PRESENTATIONS

Snyder: The next panelist will be Eric Beckjord from General Electric.

Instability Problems

by

E. S. Beckjord (GE-APD)

Beckjord: The stability of boiling water power reactors at normal operating pressures involves, in my opinion, three questions. The first is the question of the stability of the nuclear-thermal feedback in the absence of hydraulic oscillations. The second question has to do with systems which would be stable from the point of view of nuclear feedback but which could have hydraulic instability. The third question has to do with the interactions of the two phenomena. I want' to talk this evening exclusively about a hydraulic instability which is of interest at higher pressures and at steam qualities below 10 percent. It is also an instability which so far as we have been able to tell experimentally is the dominant case for power reactor interest. Now, first of all, we have done some experiments in electrically heated natural circulation loops at $1,000 \mathrm{psi}$ and have made several observations from these experiments which were conducted over a period of the last several years. The first observation is that the period of instability-and when I say instability, I mean a pulsation in flow--the period of instability is always a bit longer than the mean transit time of fluid across the vertical two-phase portion of the test loop. The second observation is that this pulsation occurs under a wide range of conditions, a wide range in regard to loop subcooling, and in regard to quality. A third observation is that these oscillations can begin with no apparent external disturbance of the loop. The oscillations might begin after a time of steady operation, grow to a limiting amplitude, and then die away. You might observe this process three or four times and then the next time the oscillation won't stop, but will increase and the test section will burn out.

I want to describe briefly the attempts to get at the kinematics of this flow pulsation. Several models were considered. The first, already mentioned by Novak Zuber, is the curve of equilibrium pressure drop vs flow shown in Slide l. Now the pressure drop curve with a negative slope region is by itself an insufficient condition for undiminished flow oscilletions. Force or energy considerations show that a disturbance at $B$, which is a dynamically unstable point, will cause the system to accelerate to $\mathrm{A}$ or $\mathrm{C}$, which are both stable points.

Let us consider a second pressure drop vs flow curve which can result in oscillations, shown in Slide 2. Without finding a physical cause of such a curve, we can see what the result might be. Suppose the system is accelerating from $A$ to $B$. When the flow reaches $B$, the pressure loss increases to $\mathrm{C}$ and the system decelerates to D. Flow 


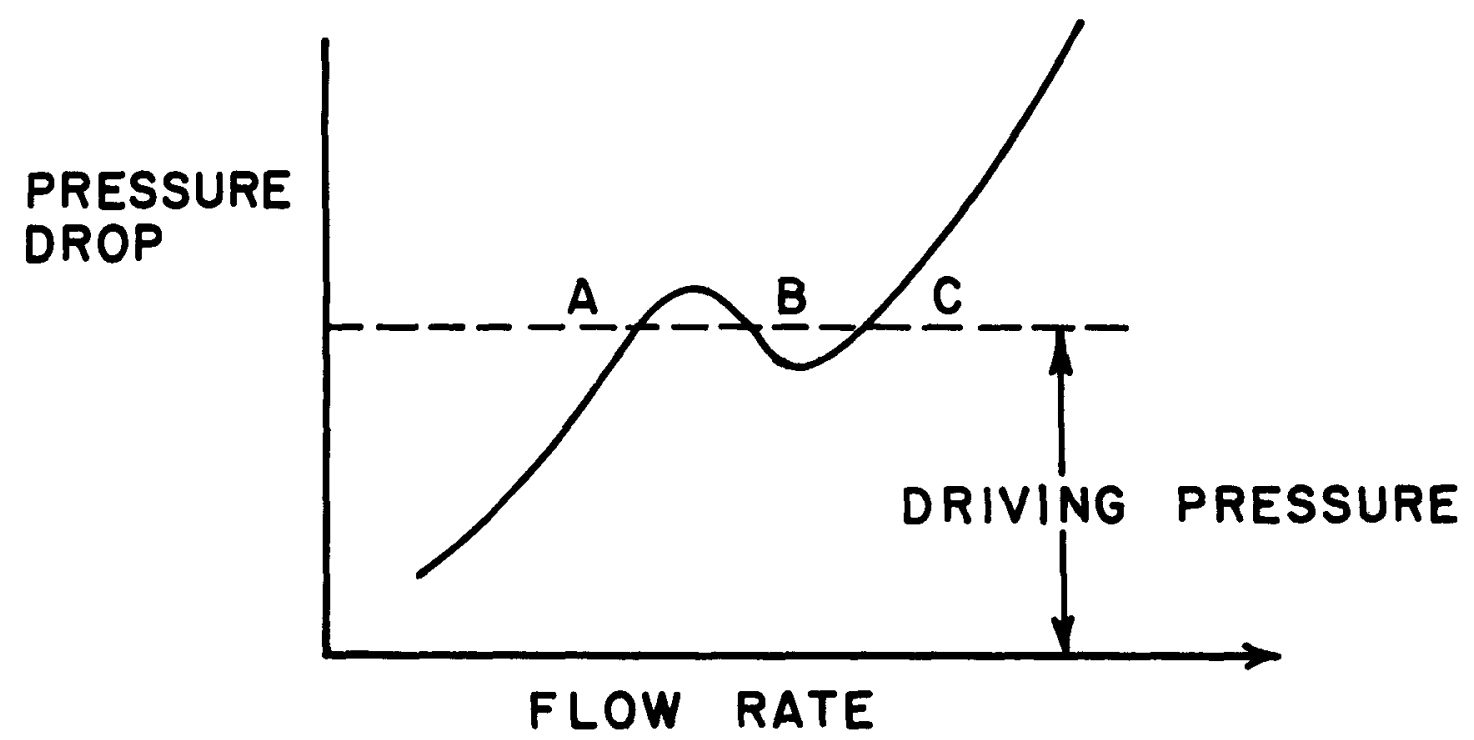

Slide 1. Unstable but not Oscillatory Case

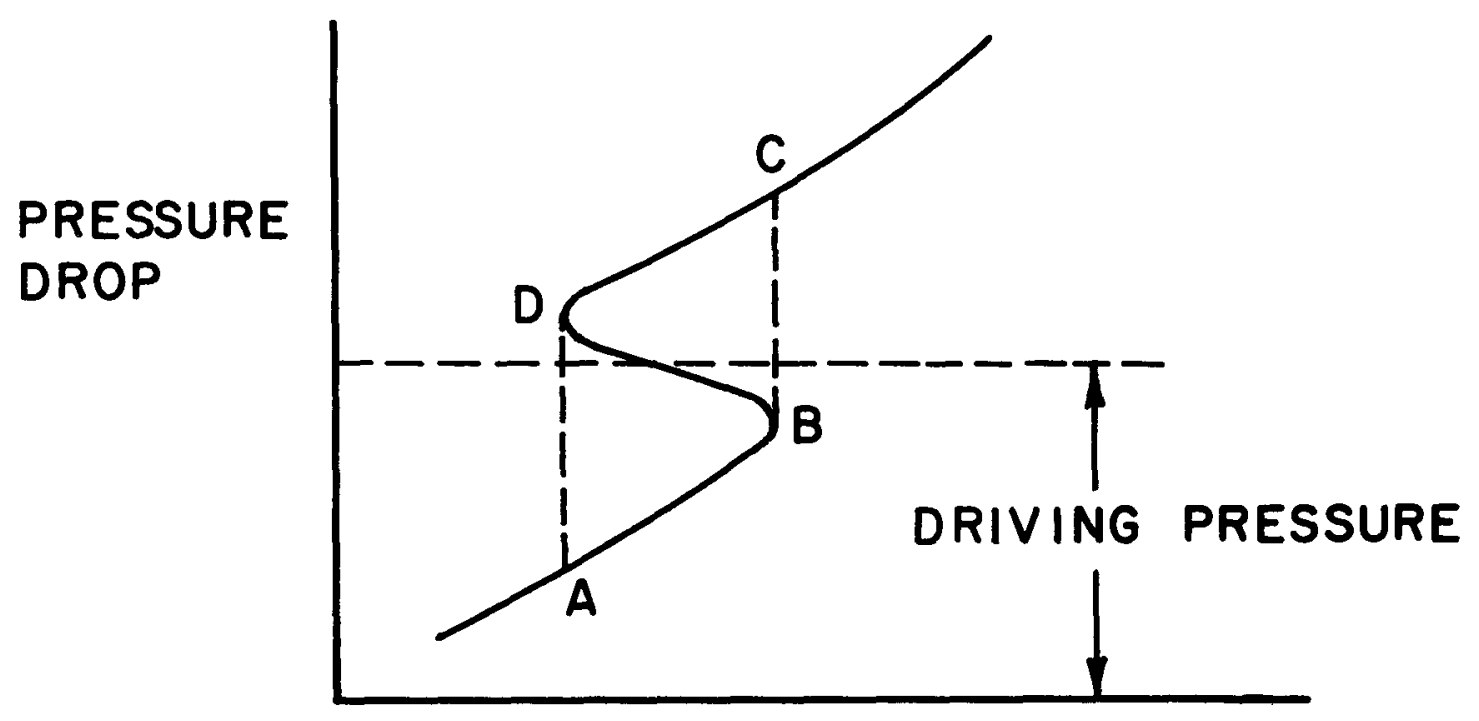

FLOW RATE

Slide 2. Oscillatory Case 
cannot change instantaneously, and the pressure loss drops to $\mathrm{A}$, and the cycle repeats itself. The system constitutes a hydraulic relaxation oscillator. When this model was applied to our experiments, it was found that the predicted period of oscillation was one-tenth of the observed period. The model was therefore discarded.

A more promising model for describing the two-phase flow oscillations is based upon the static head of the two-phase fluid and the transit time of fluid across the vertical two-phase section. If the fraction of water in the vertical section should increase, the static pressure drop will increase in a period of time governed by the transit of fluid across the section. A simple equation which includes inertia, friction loss, static head and driving head shows that such a system can oscillate at periods between 1 and 2 times the transit time. This period corresponds to the experimental observations we made. The work described above was reported in an ASME paper by S. Levy and myself: "Hydraulic Instability in a Natural Circulation Loop with Net Steam Generation at 1000 psia", (paper No. 60-HT-27).

Now, I want to pass on to the question of the two-phase flow mechanics to get at the cause of pulsation. Novak Zuber has shown the equations of two-phase flow. W. H. Harker of GE integrated similar equations numerically a year and a half ago. Typical results are shown in slide 3. The circled points are the data taken by Howard Cook at Argonne (Boiling Density in Vertical Rectangular Multi-Channel Sections with Natural Circulation, ANL-5621). The dotted lines are calculated a priori using

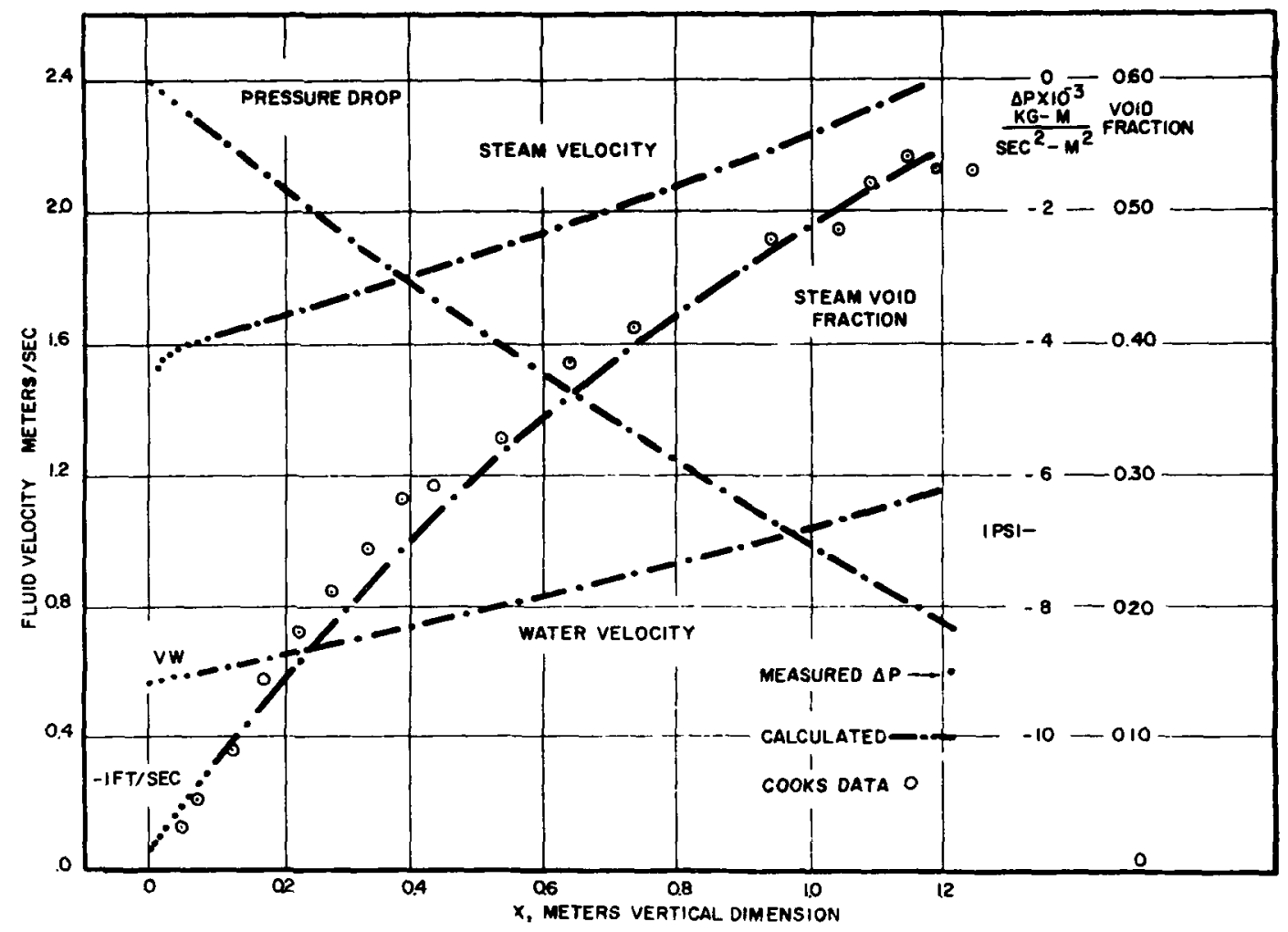

Slide 3. Comparison of Measured and Experimental Void Fraction of Cook's Run 445 
the boundary conditions of inlet velocity to the section, heat input, and pressure. The experimental data of steam void fraction and pressure drop and the calculated points correspond well. Checks were made with other data of Cook at 600 psia, and with Marchaterre's data at lower pressure. The correspondence is good until about 100 psia.

The calculations described are for the steady-state. In order to obtain transient solutions, many simplifications are necessary, such as restriction to one dimension of space. We find, nevertheless, that the simplified model which was developed for transients predicts the stability of the test loops.

The experiments are typically run by establishing steady conditions of heat input, flow rate, subcooling and pressure in a natural circulation boiling loop. The degree of flow stability to disturbances can be controlled by a variable restriction in the single-phase downcomer section. Stability is tested by changing the heat input rate and observing flow response. Comparison with the model is made by means of locating the roots of the model characteristic equation, and also with the aid of an analogue computer. For example, if you increase the heat rate to the loop, there is an impulsive action caused by increased steam generation which temporarliy accelerates water above the heating point, and decelerates water below it. The analogue model predicts this behavior and its degree.

The model indicates ways of stabilizing two-phase flow loops: one way is by downcomer restriction. Another is by inlet subcooling, which I want to discuss, because subcooling turns out to be an important consideration. Consider the equation:

$$
\text { steaming heat rate }=\text { input heat rate }-\underset{\text { mass flow rate } \mathrm{x} \text { inlet }}{\text { subcooling }}
$$

We observe that steaming rate decreases with increasing flow rate for a fixed subcooling. Now suppose that a loop is operating in a steady state, and that the flow rate is caused to increase. The steaming rate decreases and after a transit time of the two-phase section the pipe has more water in it than before. The driving head is therefore decreased, and the loop decelerates. Now the steaming rate rises, and the pipe fills with steam. Driving head increases and the loop accelerates. Under the right conditions, the system will oscillate.

The stability of two-phase flow systems is affected by many parameters of geometry, of hydrodynamics, and of thermodynamics. Inlet subcooling is particularly important among these.

This concludes what I have to say about two-phase flow instabilities. Thank you. 
Snyder: I'll ask for the next panelist who will be Dick Anderson from Argonne.

Experimental Hydraulic Programs at ANL

by

R. P. Anderson (ANL)

Anderson: I would like to very briefly outline some of the experimental work being done at Argonne now in the field of hydrodynamics. Due to a lack of an adequate method for predicting where a system will go unstable in reactor design, we periodically are forced to measure the performance of a mockup in a heated test loop of a given reactor design. A couple. of recent experiments like this, one on EBWR, $100 \mathrm{MW}$, and one on Borax V boiling regions have led to some interesting results. In the EBWR, 100-MW operation, we were curious what the effect of the skewed flux distribution would be on the total power that could be extracted from a given element, so we ran two tests, one with a four foot section, linear power input, another with a four foot section, linear power input in the first two feet, no power input in the last two feet, with the proper riser, etc., on it. We found we could get the same total power input into both of these before we burned out, or, this is a factor of two in the local burnout heat flux, and we attributed this to the fact that burnout followed very closely after the system went unstable and this was drastically affected if not completely caused by the fact that the system was unstable. In a similar manner we ran tests on Borax $V$, which is the boiling core, boiling outside of a rod-square array. We attempted to mock this up by boiling inside parallel round tubes with the same equivalent diameter as the flow areas between the rods, and again found an effect, both on the flux distribution and diameter which is different than the effect that you would expect just on a burnout and is attributable to the fact that the burnout was caused by the stability of the system. We weren't surprised that the power could be increased when it was put into the bottom half but we were rather surprised that you could get the same total power out.

I would like to mention a couple of thesis topics that are being worked on at Argonne. One is being worked on by an Illinois Institute of Technology student. He is doing the autocorrelation noise analysis on the void and flow traces from an instrumented hydrodynamic loop. If there are any questions on this, I will refer you to Joe Thie, who was his advisor until quite recently. Helge Christensen is measuring the power-to-void transfer function in a heated test loop at pressures up to $1200 \mathrm{psi}$, similar to the atmospheric work being done at Ramo-Wooldridge. Any questions about this I would refer to Professor Gyftopoulos who is the advisor for this gentleman. Finally, I would like to mention an experimental program measuring unstable behavior in a loop containing quite a bit of fast-response instmumentation. The purpose of this is to measure the influence of pressure test section and riser geonetry, 
water level above the riser and, to some extent, inlet temperature on the inception power; that is, the power at the inception of instability, the oscillation amplitude and the oscillation period. As of yet, none of these programs has produced too many results.

I would like to make one point. Novak (Zuber) said that in the paper by $\mathrm{Dr}$. Lottes and myself, we attributed an unstable behavior to a triple valued pressure drop flow curve. Now this wasn't quite correct. What we said was, that Ledenegg's model based on this mechanism predicted all the correct trends and to a fair degree predicted the right quantities. We also presented a figure, shown in Slide 1, which showed that this wasn't the mechanism that caused our loop to go unstable. We had pressure drop flow. This was the natural circulation point. We measured, at the same subcooling and different subcooling in forced dirculation, the pressure drop vs flow curve and did not get this triple valued type thing. We concluded that this wasn't the mechanism but the fact remains that the model based on the mechanism did predict most of the trends. No further conclusions were mentioned or drawn.

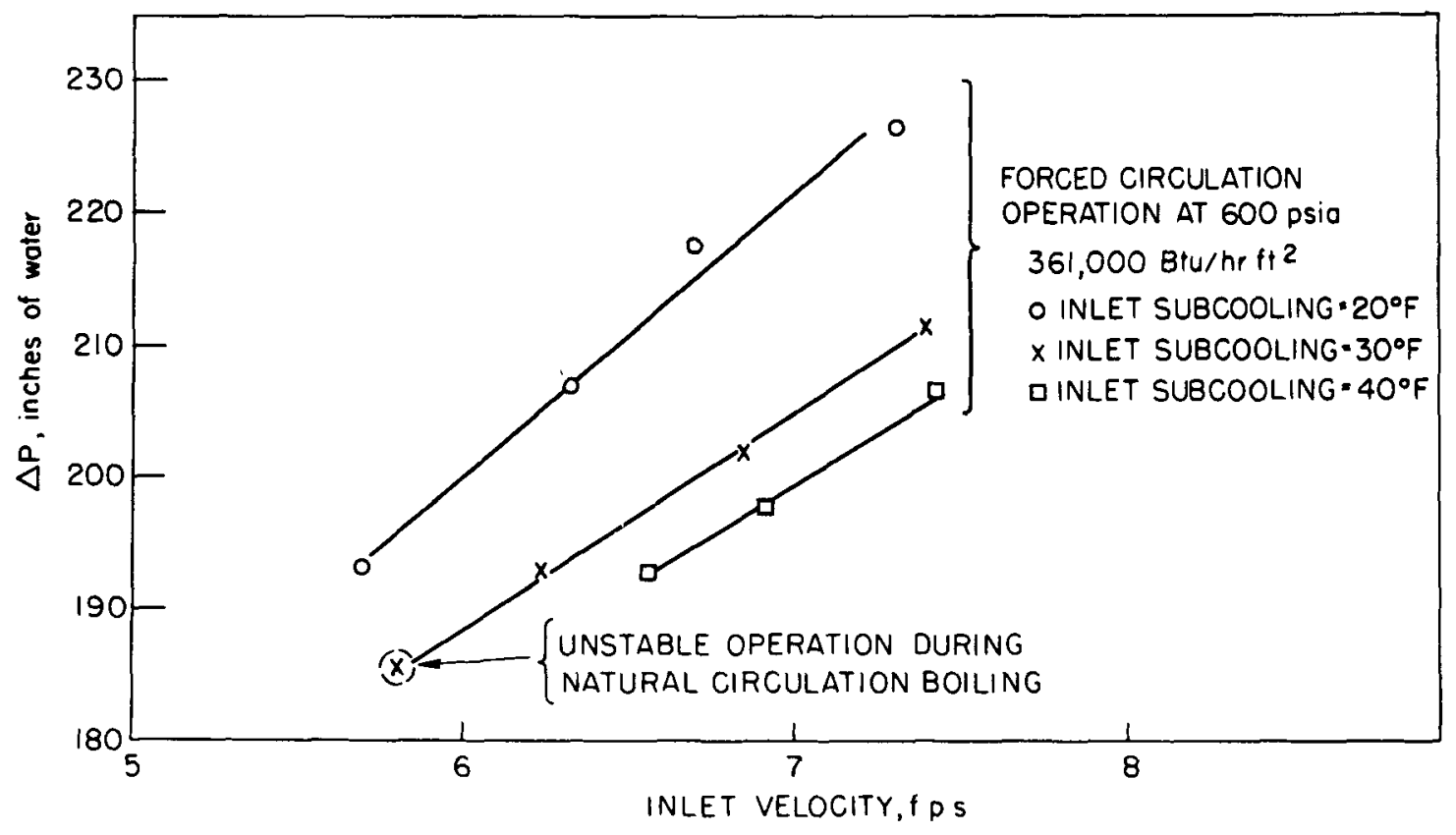

Slide 1. Pressure Drop as a Function of Inlet Velocity for a l-in. ID $\times$ 48-in. Vertical Pipe with a l-in. ID

by lo-ft High Chimney 
SESSTON IV

PANEL PRESENTATIONS

Snyder: The next panelist is Nelson Grace of Westinghouse.

\author{
Presentation of Bettis Program* \\ by \\ J. N. Grace (W-Bettis)
}

Grace: We are touching this evening on a very broad subject, especially when we include reactivity feedback simultaneously with hydrodynamic effects. Let me first outline briefly the scope of our program at Bettis on these combined problems, and then indicate the status of the development of the work. I think I can do this in less than ten minutes, as requested by the Chairman; if I don't, cut me off.

We are concerned, as you know, at Bettis in developing methods of analysis for prediction of the onset of instability to enable us to design cores which we can guarantee to be safe before the core is manufactured. We are currently spending some time on the reactivity instability problem (which I described this morning), the hydrodynamic instability problem, and the combination of the two. There are situations where either mechanism may cause instability independent of the other, and there are other situations where interaction exists. We are interested in both stability and transient analysis of the se two phenomena. Stability in the absolute sense is required in core design since we would not allow the power level to hunt continuously in the steady state. However, it is conceivable that one might allow momentary ringing during some transients; but a design criterion for this has not yet been established. We are developing both analog and digital means of stability and transient analysis of these problems. And we are supplementing the analytical work with both in-pile and out-of-pile experiments.

This morning I described a method of analysis for prediction of reactivity feedback instability exclusive of any hydrodynamic effect and an experiment that was designed for testing this method of analysis. Therefore, it is appropriate that we switch our attention now to hydrodynamic instability.

The type of hydrodynamic instability that we are primarily concerned with is different from the types described by the previous speakers. I think that if we are to communicate well on this subject, and understand each other, we must be careful each time we talk about hydrodynamic instability, to define very carefully what mechanism, what mode of instability, we are talking about.

*This paper was issued as WAPD-OP-11-Part II. 
In our particular problem, we are concerned with hydrodynamic oscillations in a reactor coolant channel having a constant applied pressure drop, determined by the flow through the rest of the channels, and constant heat flux. In some cases we may get into a feedback effect where there is a fluctuation in the applied $\triangle P$. But, for the most part, we're concerned with a constant applied pressure drop driving a hot channel which goes into oscillation. The inclusion of the continuity equation with fluid expansion--that is, allowing the mass velocity to vary along the channel length--is an essential part of this particular mode of oscillation. Now, this oscillation, unlike the one described by Eric Beckjord, does not depend on the external loop. He was talking about a loop oscillation characteristic of a natural circulation system. ours is simply a hot channel that has a constant pressure drop applied to it. Reactivity feedback instability was described as more of an overall core problem; that is, the average core channel must be boiling in order to get sufficient reactivity feedback to cause this oscillation. However, hydrodynamic instability usually involves one hot channel which might start chugging while the rest of the core is operating satisfactorily.

The first attempt to try to explain these hydrodynamic instabilities at Bett1s was done by Earl Quandt. 'Te was working in the thermal and hydraulics laboratory and participated in the running of the experiments that brought out these instabilities. He developed an analytical model which essentially involved the derivation of a transfer function relating inlet flow response to variations in the heat flux. Now, he probably could have chosen some other transfer function to derive, since he was mainly interested in getting the characteristic equation of the system, which is a common denominator to several of the transfer functions involved. The characteristic equation turned out to be a quadratic. The stability criterion for quadratics is quite simple; that is, all coefficients must have the same sign.

In Quandt's model the reduction of the constant term in the quadratic to zero corresponds to a type of instability that has been mentioned several times already this evening; that is, where the partial derivative with respect to flow levels off and becomes zero. In our experiments there was none of this type of instability observed; in fact, this curve was always monotonic in these particular experiments. We would not really call this an oscillatory condition, but a condition of excursion where it is possible to move over from one part of the curve to the other and probably stay there.

The second coefficient, in other words, the damping term in the quadratic, was examined to see what would make it zero or negative. This coefficient is rather complicated. It is very difficult to see intuitively what is going on in the terms that make up this coefficient, and $I$ am not able to postulate a simple physical argument for what is causing this instability. But Quandt's model did predict very well the hydrodynamic oscillation experiments done in our thermal and hydraulics laboratory, using the sign of the damping term as the criterion. The trends in the experimental data were predicted extremely well, although quantitative comparisons in some cases were not too good. This, however, was our first attempt. It was encouraging, but clearly the model needed 
some improvement--in particular, more detail in its representation. This model was a stability criterion model, as distinguished from a transient model.

Since then, John Meyer at Bettis has done extensive work on trying different representations of the hydrodynamic system, this boiling channel, to determine the best method for representing hydrodynamic instability. The model that he has now selected he calls MIM (Momentum Integral Model), because it involves integration of the momentum equation, such that he essentially applies $f=$ ma to the total channel, so far as the momentum is concerned. The flow distribution within the channel varies with time during the transient, but the momentum equation is applied to the overall channel rather than being distributed within. The continuity and energy equations are distributed, as is the equation of state, of course. The flux distribution with position is assumed known, but is held constant in time. The MIM model for prediction of channel hydrodynamic instability is currently being programmed for digital solution.

I have done little more here than mention the existence of these methods of analysis of channel instabilities. However, I can refer you to a paper by quandt, which was presented at the Fourth National Heat Conference in Buffalo. His paper was sponsored by AIChE and is identified as preprint No. 27. It will soon be published by $A I C h E(1)$. John Meyer is going to present the gevelopment of his MIM model at the next ANS meeting in San Francisco(2). Other contributors to channel instability analysis are Eliot Duncombe and Ron Rose; however, they have not published any available reports that I can refer you to.

I had better skip along now and mention the analog model under development and then sit down. The analog model is designed to perform transient analysis including both reactivity and hydrodynamic effects simultaneously. The different parts of the problem of simulation of the total system have been pretty well worked out such that we are now ready to compose the complete simulator. This work is being done by John Reihing and Neil Curlee.

I have some slides showing the results of the checkout of one particular aspect of the simulator model which I want to show you.

The curves in Slide 1 show the result of a loss-of-flow transient, where the inlet flow vs time was specified as an input function. What we see here is a ringing, or an oscillation, in the reactor power and in the outlet enthalpy, as a function of time, caused by reactivity feedback. Now, this particular test of the analog model was to explore the effect of not assuming a constant mass velocity vs position, but including the distributed continuity equation, and allowing the mass to bunch up and expand. It shows here that when the conservation of mass or the continuity equation is included correctly, the magnitude of the reactivity oscillation is reduced. This is an interesting consequence, but we cannot claim any advantage from this yet, until we set up the complete simulator and include the variation of inlet flow rate which can occur. What we are seeing here is truly a reactivity oscillation, and we are showing a hydrodynamic interaction. These two sets of curves 


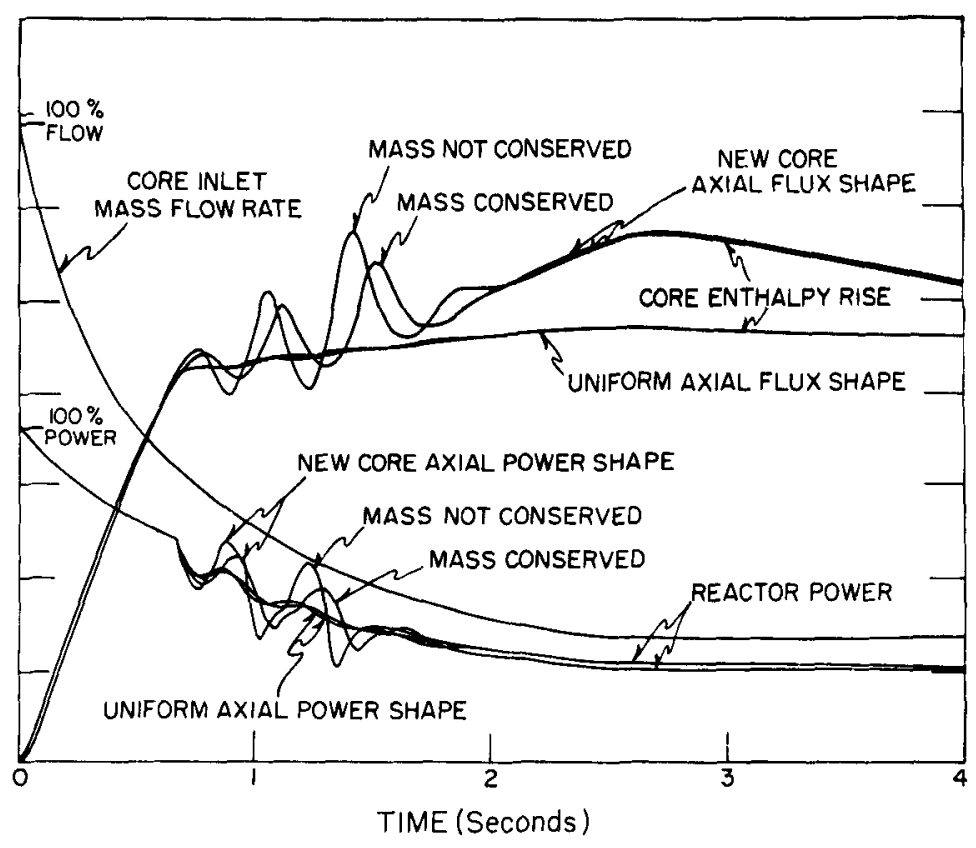

Slide 1. Bolling Reactor Channel Analog Simulation of Response to a Loss-of-Coolant Flow. Axial Flux Shape Effects and Conservation of Mass Effects.

show the difference between a uniform power distribution and a power distribution which is skewed toward the bottom of the core. As I mentioned, a very important part of the reactivity feedback oscillation is the phase-lag resulting from the transport delay of the coolant from the lower region of the core to the upper region of the core. This makes the instability worse when the power is shifted toward the bottom of the core. When the flux is flattened, you might say some of the time delay is short-circuited, and this reduces the severity of the oscillation; In fact, in this case it essentially eliminated it.

Slide 2 shows the effect of including slip (a fixed value independent of position and time) on this same kind of transient. Here it appears that as we go from the fog-flow model, or a slip value of one, to larger values of slip, the amplitude of the reactivity feedback oscillation is reduced.

I have presented these curves only as examples of some of the tests that have been done on different aspects of the simulation problem. We are ready now, as I say, to put together the whole package in an attempt to simulate all reactivity feedback and hydrodynamic equations simultaneously, for transient analysis. The analog model will include actually two channels: one, the nominal channel, which may play the more important role in reactivity feedback, and another, a hot channel, which may show up hydrodynamic instabilities before the other channel oscillates. There are other points I wanted to make, but I have already run overtime. Thank you. 


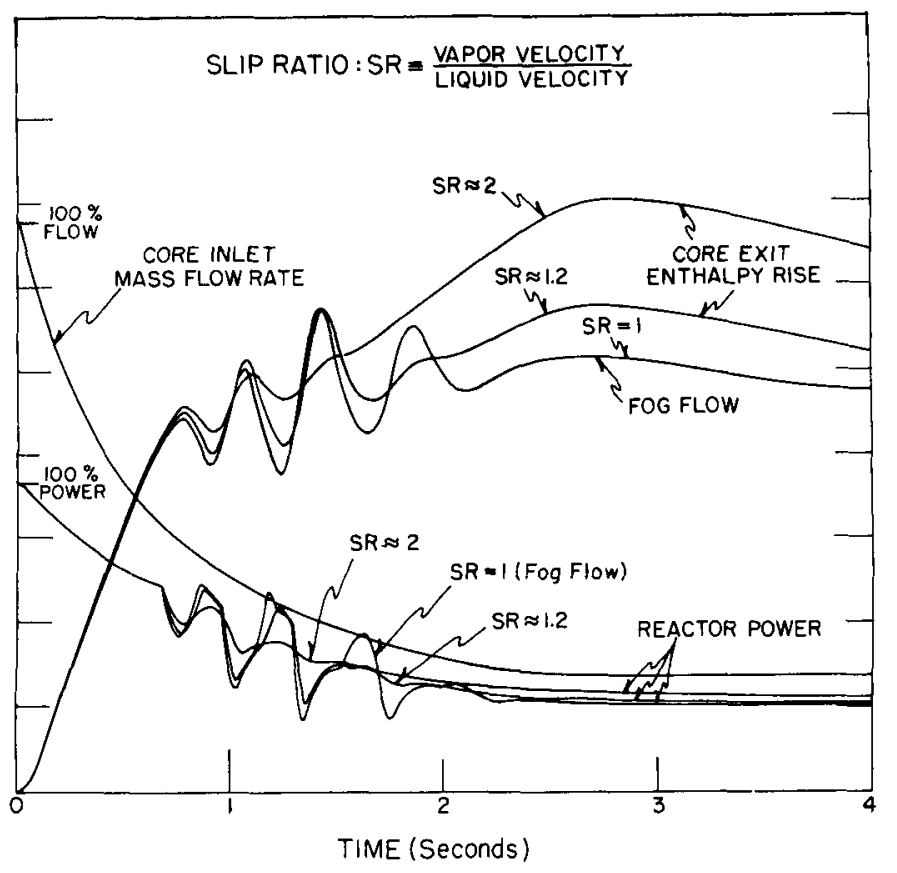

Slide 2. Boiling Reactor Channel Analog Simulation of Response to a Loss-of-Coolant Flow.

Slip Flow Effects. 


\section{REF'ERENCES}

1. E. R. Quandt, "Analysis and Measurements of Flow Oscillations", Chemical Engineering Progress, Monograph and Symposium Series No. 32-S, Heat Transfer, Buffalo, pp. 111-126 (August 14-17, 1960).

2. J. E. Meyer, "Hydrodynamic Models for the Treatment of Reactor Thermal Transients", Trans. Am. Nuclear Soc., 3, p. 521 (December 1960). 


\title{
SESSION IV
}

\section{PANEL PRESENTATIONS}

Snyder: The next panelist will be Joe Fleck from Livermore.

\author{
A Model for Hydraulic Instability \\ by \\ J. A. Fleck (UCRL-I)
}

Fleck: Zuber, in his paper, has already mentioned a number of special "nonmechanical" mechanisms which might lead to hydraulic instability. The point of view which I have taken is that hydraulic loop instability, at least in some cases, could well be the unstable behavior of a mechanical system acting under friction and buoyant forces. What one wishes to do under this assumption is to derive a system of differential equations describing the dynamic behavior of the system and then to examine the solutions to these differential equations. The conditions for unstable solutions would then presumably shed light on the nature of the instability mechanisms. One could, for example, test Ledinegg's hypothesis that a condition in which more than one flow rate corresponds to a given pressure drop causes hydraulic instability. One would simply substitute this condition into the dynamic equations and solve them. If the solution were unstable, one would then have proved the Ledinegg hypothesis. Without such an analysis Ledinegg's hypothesis would be on shaky ground. Hydraulic instability has also been attributed to another condition; namely, when the system is operating on the downward side of the flow rate vs power curve. Solution of the dynamic equations, however, has shown that a natural circulation system can operate quite stably under such a condition. Therefore, such a condition should not be a sufficient condition for hydraulic instability.

It should be mentioned here that whenever one attempts to make a calculation of the hydraulic behavior of a multi-channel reactor or to mockup the reactor by an out-of-plle loop experiment one generally approximates the multi-channel system by a single channel system. It has yet to be demonstrated how suitable this approximation is. The following questions might, for example, be raised. What is the appropriate power to be used in the channel; the central channel power, an average power, and if so what average? Also, is the single channel system hydraulically equivalent to the multichannel system, particularly if different channels operate at different power levels and have different vold fractions and flow rates? One might also wonder how one determines the conditions for hydraulic instability for a parallel-channel system from the conditions for hydraulic instability in a single-channel system. Does the parallel-channel system go unstable when unstable conditions occur in the central channel, or does some entirely different condition govern unstable behavior in the parallel-channel system? In my opinion, this whole question of the relation between the natural circulation flow in parallel and single channels deserves both theoretical and experimental investigation in the future.

The work of Lottes $(1)$ and his co-workers at Argonne National Laboratory has shown that the steady-state behavior of an out-of-pile hydraulic loop can be described rather well by means of a simple one-dimensional hydrodynamic model. The model makes use of mass and energy conservation together 
with the principle that the sum of the pressure drops around the convection loop equals zero. A simple model of two-phase friction pressure arop is also used; namely, the increased friction in two-phase flow is assumed due to the reduction of flow area by steam.

One would like to be able to generalize this simple one-dimensional treatment to dynamic conditions. This generalization is in principle fairly simple. One adds to the energy and mass balance equation terms involving the time rates of change, in the boiling volume, of the energy and mass, respectively. It remains then to generalize the pressure drop theorem. This one does by using an analogy with $D^{\prime}$ Alembert's principle in mechanics. This principle states that any dynamic system may be regarded as a system

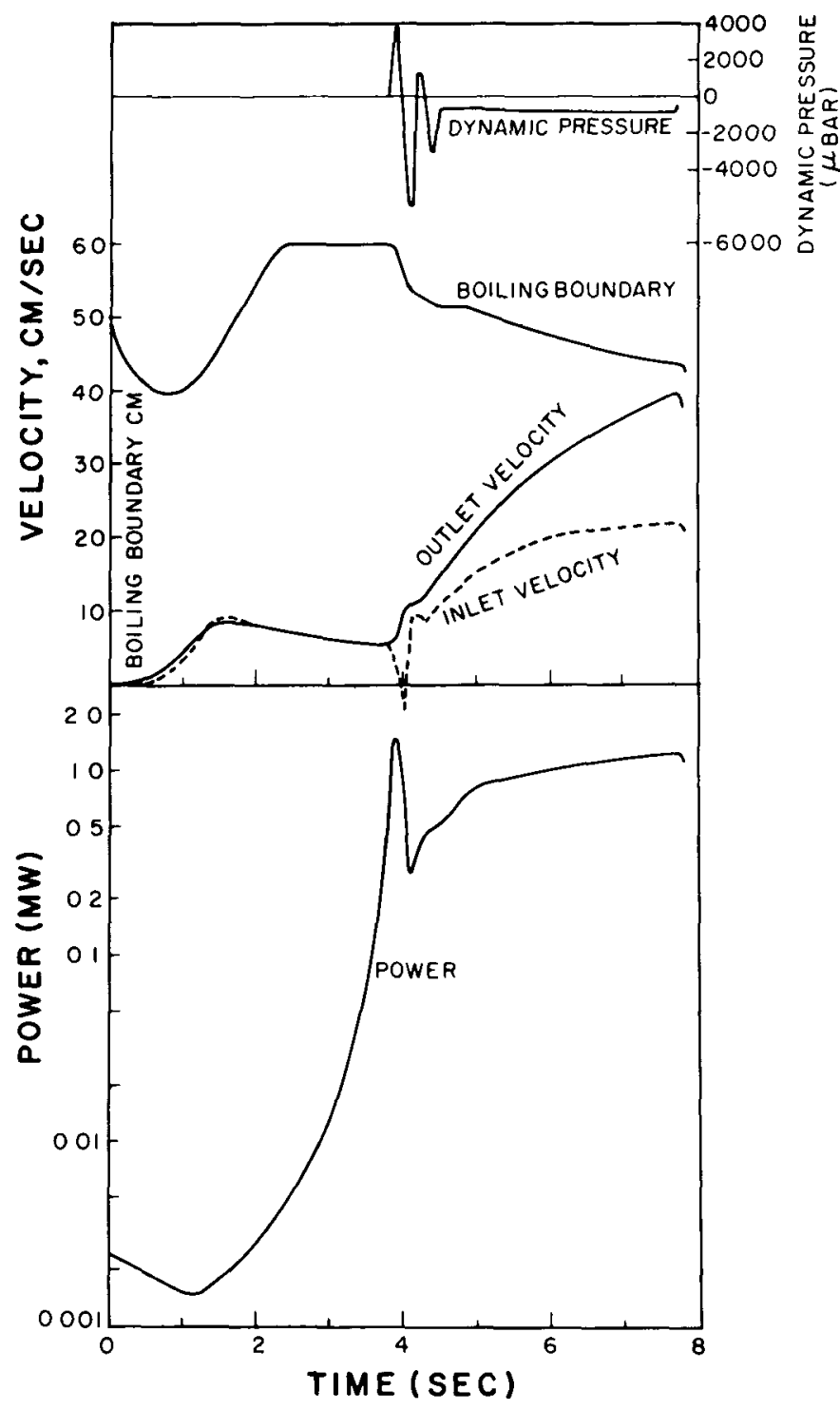

Slide 1. Time Behavior of Variables from Hydrodynamic Model Using Spert I Parameters in static equilibrium provided that to the true forces on the system one adds a system of fictitious forces equal to the negative of the time derivatives of the various momenta in the system. In the case of a natural circulation loop the analogy is between pressure $d r o p$ and force. The theorem that the sum of the pressure drops around the loop equals zero holds for dynamic as well as steady-state conditions provided that one includes a set of fictitious pressure drops (D'Alembert pressure drops) which are proportional to minus the time derivative of fluid momentum in various parts of the convection loop. The preceding analysis can be used to derive a system of ordinary differential equations involving inlet velocity, outlet velocity, outlet void fraction, and boiling length(2). The equations can be integrated on a digital computer to obtain the flow behavior of the boiling reactor system. Recently the method has been extended to take into account the effect of pressure on such steam properties as saturation temperature and density $(3)$.

\section{A typical calculation is} indicated in Slide 1 . The parameters refer as nearly as could be guessed to the Spert I 
reactor. Indicated are the behavior of the power level and inlet and outlet water velocities. The power burst is accompanied by the ejection of water from the top of the core and the simultaneous ejection of fluid from the bottom of the core due to momentum conservation. The pressure changes affecting steam formation result both from the acceleration of fluid and from the changing position of the boiling boundary.

Calculations with this model have indicated both feedback and hydraulic forms of instability. The hydraulic form of instability is a rather curious one. To occur, a special model of steam slip is required; namely, the steam slip ratio is assumed large and either constant or slowly varying with inlet water velocity. Then, if for a sufficiently high head of water above the core the void fraction exceeds some critical level, the flow becomes unstable and reverses completely. The significance of these conditions for instability is that with a high relatively constant slip ratio small changes in circulation velocity can cause large changes in the rate of steam removal, for example, would be translated into top reflector motion. The momentum imparted to this reflector would have to be removed from the convection circuit causing a slowdown of circulation velocity.

It should be emphasized that this unstable behavior is a mathemat 1cal consequence of the model employed but need not occur if other models of steam slip are employed. To me the most interesting fact about the Spert I and Borax I instabilities was that the power threshold depended on water height. The abmupt and rapid divergence of the oscillations which developed when this threshold was reached is also curious and suggests some sort of nonlinear phenomenon. Of course, the effect of water head on stability might also be explained by changes in subcooling and consequently changes in boiling length arising from changes in water head. Calculations made on the $\mathrm{D}_{2} \mathrm{O}$ boiling reactor at Halden, Norway, indicated decreased overall stability (in terms of damping rate) for increased subcooling at a given total void fraction. Unfortunately, the question of the effect of subcooling on hydraulic stability has not yet been investigated very thoroughly using this model.

In conclusion, while the present model does provide a method for calculating boiling reactor dynamic behavior and gives some insight into the dynamics of natural convection flow, yielding possible mechanisms of instability, it does not as yet enable pinning down the precise nature of boiling reactor instability. Hopefully, however, models such as this one can be an aid in understanding the hydraulic and reactor data which is becoming increasingly more plentiful.

Snyder: Thank you, Joe. 


\section{REFHRENCES}

1. P. A. Lottes and W. S. Flinn, Nuc. Sci. and Eng., I, p. 461 (1956).

2. J. A. Fleck, Jr., "The Dynamic Behavior of Boiling Water Reactors," J. Nucl. Energy, Part 4: Reactor Science, pp. 114-130 (1960).

3. J.A. Fleck, Jr., "The Influence of Pressure on Boiling Water Reactor Behavior at Atmospheric Pressure," Nuc. Sci. and Eng., 2, p. 271 (1961). 
SESSION TV

PANEL PRESENTATIONS

Snyder: The next panelist will be Jack Chernick from Brookhaven.

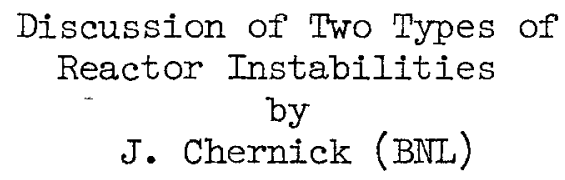

Chernick: I want to assure you that I'm having as much trouble keeping alert as the audience, so I'll try to be brief. I want to discuss in a simple way two rather important types of reactor instability.

The first of these is a high-frequency oscillation that is due to delay in the negative temperature coefficient of the moderator. The importance of this effect was given its impetus by Bethe's work on the analysis of the EBR-I fast reactor incident. It was originally conceived by Kinchin but neither he nor Bethe thought up a reasonable mechanism for the transfer function of the EBR-I and for the analysis of the incident. In particular, they could not really find a negative temperature coefficient--though they suggested a possibility--which was delayed long enough, something of the order of 10 seconds; and it is very difficult to envision a delay of that length. On the other hand, it is immediately evident that the problem of a delayed temperature coefficient applies to all of the heterogeneous systems that we're interested in. There are at least three barriers to heating up the moderator of the reactor: heat has to diffuse through the fuel to the surface; you have a heat transfer delay across the surface of the fuel element; and, finally, you have to have bulk heating of the moderator to get the full temperature effect. Now it's true that there is always some prompt temperature effect; in fact, Dr. McCullough raised this question. Several percent of the heat of fission is generally dissipated in the moderator in a prompt manner by gamma-ray heating and by slowing down of neutrons. In addition, there is sometimes a negative temperature coefficient in the fuel itself and, of course, the prime example of this is the Doppler effect in the natural uranium or slightly enriched uranium systems that are so frequently used.

The other problem that I want to talk about is completely different. These are the long osclilations due to xenon which have been particularly annoying in the high flux reactors of the production or power type. We've been giving some consideration to this problem recently, particularly to finding out what it takes to excite space-independent oscillations. Now, the thing you'd like to do is to get rid of oselllations in both types. In the case of xenon you'd like as high a temperature coefficient as possible and, in this way, you can suppress the spatial xenon oscillations as well as fundamental mode xenon oscillations. On the other hand, if you don ${ }^{2} t$ have a large negative temperature coefficient, you have less of a problem with delayed temperature effects. So the question is, can you design a reactor which will be safe against both types of oscillations--highfrequency oscillations and the low-frequency xenon oscillations? I 
would like to discuss the achievement of these oscillations. The first one $I^{\prime} I I$ mention is due to the delay in the temperature effect. This was discussed by Dr. Lellouche at the last ANS meeting in Chicago( $(\mathrm{I})$. I should say something about the model that was used. The three types of delay terms that I mentioned were put into the equations. The reactor was of the PWR nonboiling type. The objective was to determine at what power level or temperature decrement the reactor would become unstable. The particular case involved a $0.3 \mathrm{sec}$ transient time of the coolant through the reactor and a total of $0.1 \mathrm{sec}$ for the delay times. At about $4 \%$ excess reactivity the oscillations were damped, at about 4-1/2\% we saw growing oscillations. The oscillations can be gotten by making any small perturbation from equilibrium; in this case, the inlet temperature was dropped $10^{\circ}$; it doesn't matter except that the oscillations take a little longer to build up if there were a tenth of a degree drop in temperature. The particular level of power (or excess reactivity) is unstable. our objective is to determine the effect of the delay times and coolant transient times in the initiation of these oscillations and this work is still going on. The particular oscillation had a $0.2 \mathrm{sec}$ period, and it looks like it was tied to the transient time of the coolant. It was slightly offset because the power distribution is roughly sinusoidal under these circumstances.

Now the second problem that I want to talk about is fundamental mode xenon oscillations. There $\frac{i}{2}$ an article in the last issue of Nuclear Science and Engineering (2) in which the problem has been solved for a reactor with a zero temperature coefficient--one that is controlled by xenon alone. The slides that I want to show now include the effect of temperature. Because of the fact that these oscillations are so slow, we can use a very simple model for the equations. In fact, since xenon burnup, in the worst case, gives a time constant of at least the order of minutes, you can neglect the details of the delayed neutron emitter equations--they play no role in the determination of the conditions for instability. You can neglect the exact mechanisms for all other power induced effects and simply lump them into one prompt coefficient for the purpose of determining the stability curve. These equations boil down to a single equation for the flux. We can define an effective neutron generation time $\tau_{e}$ which is averaged over the prompt neutrons and all the delayed neutrons, and if you remember the inhour equation-this is simply the sum of the delayed neutron yields times their mean lifetimes. Then we write the flux equation in the form

$$
\tau_{e} \phi^{\prime}=\left(\delta_{0}-c X-\gamma\right) \phi
$$

where $\varnothing$ is the flux, so the excess reactivity, $\mathrm{X}$ the xenon concentration and $\gamma$ the flux coefficient. One sees immediately that the flux is bounded when $\gamma>\delta / \gamma$. Thus, unbounded oscillations or divergent solutions cannot occur.

I think now we're ready for Slide 1 . One finds a stability curve that is S-shaped. The left side of the slide corresponds to a negative temperature or negative flux coefficient. The right side corresponds to 


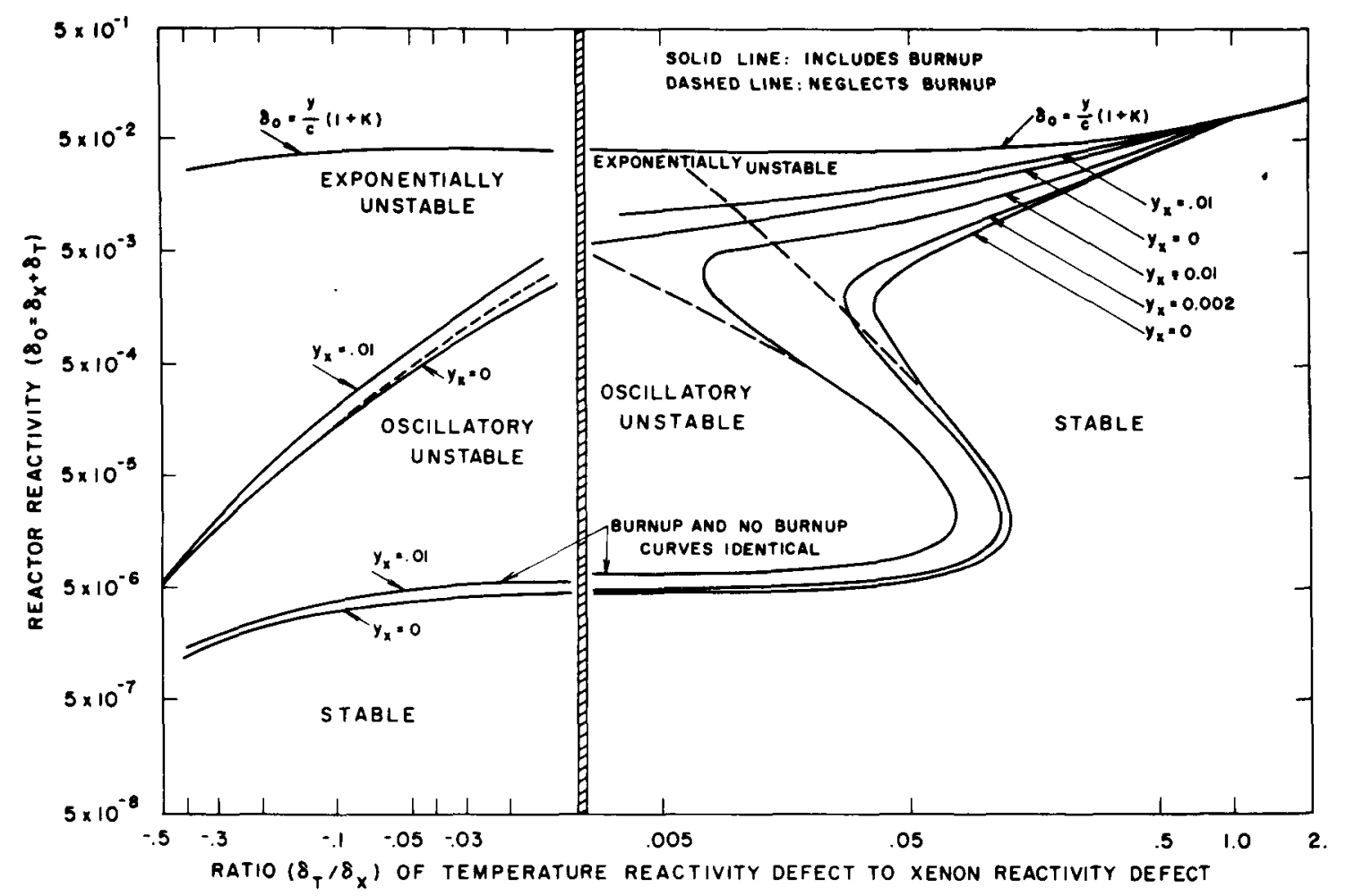

Slide 1. Regions of Iinear Stability against Xenon and Temperature

a positive temperature coefficient. Now, what we have plotted here is the Initial reactivity $\delta_{0}=\delta_{x}+\delta_{t}$, which must be overcome by both xenon and temperature, $\delta_{x}$ is the xenon defect, $\delta_{t}$ is the temperature defect, against a ratio which is convenient for reactor designers; namely, the ratio of the temperature reactivity defect to the xenon defect. One finds that if one is operating at very low flux or very low reactivity, the initial reactor period is so slow even compared to xenon decay that one is in a stable region, but then as one moves up in reactivity, one enters a region which, in linear approximation, is oscillatory unstable. Now, as you go up higher the temperature effect becomes more important, the reactor responds faster to the temperature effect, and in the absence of burnup (indicated by the dotted Iine) above some reactivity, it would again be absolutely stable. So, the first bend is due to the importance of temperature at higher fluxes; the second bend in the curve, towards instability, is due to xenon burnup. This continues until one reaches a point where the temperature defect is so large that the reactor is absolutely stable. We've drawn the line which corresponds to infinite flux, to indicate the high-flux region. The effect of the prompt xenon yield, $y_{x}$, tends to make things somewhat more stable as indicated by the fact that these curves bulge out. One finds even for positive coefficients that there is a very small region of stability. However, it is obviously not very large and it wouldn't take much of a change, say, in coolant temperature, to move up into the oscillatory region, or into the exponentially unstable 
region. The nonlinear solutions of the equations, which we've investigated, because one can have only limited trust in the linear equations, indicates some differences from the linear theory. The first difference, obviously, is that there are no such things as oscillatory or exponential instability for a reactor with a negative flux coefficient. The solutions can depend on initial conditions. For example, for a reactor with a positive flux coefficlent, the initial flux and hence the initial reactivity could be so high that it could not be balanced even by saturated xenon and the reactor power would then diverge. In general, we find that the linear theory is a poor guide for a reactor with a positive flux coefficient and is not completely reliable for a reactor with a negative flux coefficient.

I'd like to next discuss Slide 2, which shows the regions of linear stability in terms of flux against the flux coefficient. The equilibrium flux values are the solid lines--there are actually two solutions in the region of positive flux coefficient--one is always unstable, and except for the very narrow region, both solutions are unstable. The next point of interest is that a tangent line can be drawn such that for the flux coefficients to the right of this line, the reactor is stable. The reactivity effect can be found by mutiplying the flux coefficient by the flux level. Now the most interesting region--and the one $I$ want to talk about now--is from $10^{13}$ neutrons $/ \mathrm{cm}^{2}$ sec up. At $10^{13}$ flux, it turns out when you multiply the flux coefficient times the flux, that one needs about 1\% in temperature defect to be stable, but when you

move up from 1013 to $10^{14}$ neutrons $/ \mathrm{cm}^{2} \mathrm{sec}$, one quickly

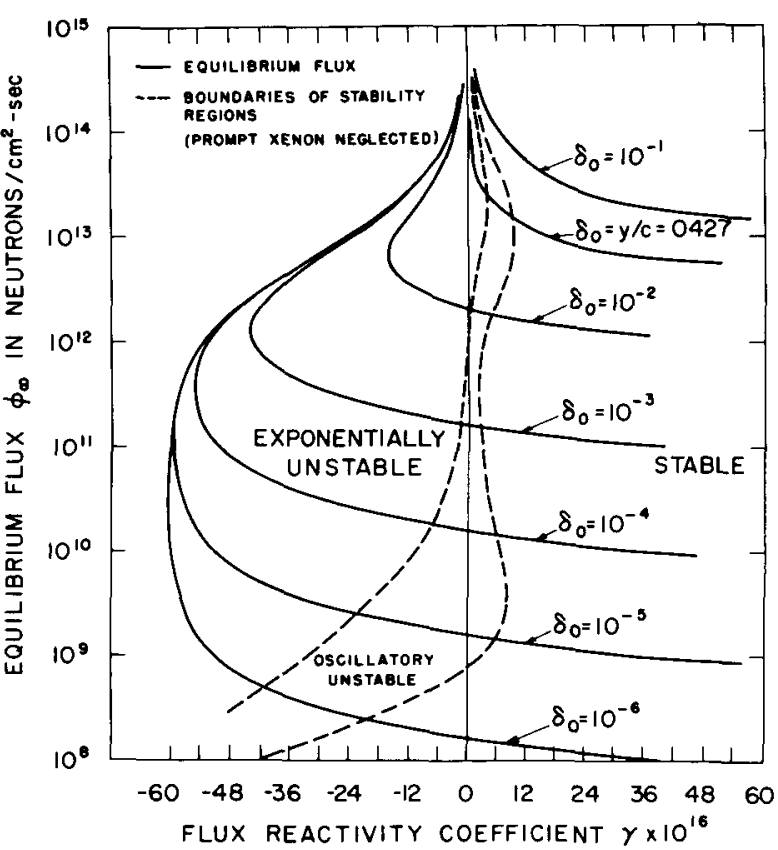

Slide 2. Regions of Linear Stability Against Xenon and Temperature goes toward the limit for saturated xenon and you need a $4 \%$ temperature defect to be absolutely stable against fundamental xenon oscillation. These results are typlcal for $3 \%$-enriched fuel. If the fuel were natural uranium these numbers would drop-you'd need about $3 \%$ excess $k$ to be stable at any flux level. There is one other interesting point, and that is if one is in stable operation in this region with a temperature defect of a couple of percent, and one goes down to lower power without reducing the coolant flow rate, one can go into an unstable oscillation. Of course, one can keep the fuel temperatures up and remain stable by reducing the coolant flow rate. This relationship between xenon stability and coolant velocity 
may be of interest from the engineering viewpoint. It is interesting, also, to notice that you can get into unstable regions at relatively low fluxes with efficiently cooled reactors which run at very high flow rates but at low power levels. There is a practical problem, however, because the temperature effect becomes quite small, and one needs a system that isn't too sensitive to changes in coolant $f l o w$, and $s x$ on, to get an experiment like this to work. So far, no one has recorded such space-independent xenon oscillations. It would take a long, careful experiment to observe them. The best reactor for the purpose would be some high-power reactor. There is one other point, and that is if you design a reactor to be stable against space independent xenon oscillation it may be expected that it will generally be stable against harmonics and therefore against spatial oscillations as well. Dick Cohen is looking at this problem, and may have something to say about it later. Thank you.

\section{REFHERENCES}

1. G. S. Lellouche, "Nonlinear Space-Dependent Kinetics", Trans. Am. Nuclear Soc., 3, p. 180 (June 1960).

2. J. Chernick, "The Dynamics of a Xenon-Controlled Reactor", Nucl. Sci. and Eng., 8, pp. 233-243 (1960). 
Snyder: Our last panelist tonight will be Dave Langmuir from RamoWooldridge.

\author{
Presentation of Ramo-Wooldridge Program \\ by \\ D. B. Langmuir $(\mathrm{R}-\mathrm{W})$
}

Langmuir: I shall try to summarize some results which illustrate points which have been mentioned by a number of previous speakers, and also to point out some unfinished lines of work and outstanding questions.

With regard to the interaction of hydraulic instabilities and neutron coupling, we are forced to conclude from our detailed studies of a single out-of-pile spert channel that both these subjects must be taken into account in interpreting Spert oscillations. Our measured power-to-void transfer function shows no sign of a resonance peak and shows no way, by itself, of accounting for instabilities or oscillations. Nor does the zero-power transfer function by itself. If you combine them, however, the combined system shows a resonance peak, and it predicts oscillations of the frequency observed by Spert, one cycle per second. The power density is also very close to that exhibited by spert. If we take this channel and raise it to higher steady power, it will indeed oscillate all by itself and exhibit hydraulic instabilities; but at a power density a factor of two higher than that at which spert oscillates and at a frequency also a factor of two higher. We conclude, therefore, that this mode of natural hydraulic instability does not conform to the Spert oscillations but that at lower powers, comblned with a zero-power transfer function, the combination of the hydraulic system and the neutron coupling system seems to explain the Spert channel well. We have no choice but to draw this conclusion from our measurements as far as they go. This applies only to the channel that has lots of nucleation centers, and is therefore relatively very reproducible and stable. It is not a piece of an actual spert channel. We are guessing that the spert channel also has a prolific supply of nuclei and that we therefore probably match the conditions fairly well.

The next subject is that of purely hydraulic instabilities. There are three main things which have been mentioned before, to touch on, and one new item. First, every channel we have tried will oscillate all by itself with constant power input. Second, these channels show widely different oscillatory characteristics depending on how they are made. We have run some tests to try to control the nucleation density in the surfaces. It seemed that sandblasting surely would be a wonderful way of putting lots of nuclei into the surface; but it makes almost no difference; it is very ineffective. By all odds, the best way of introducing lots of nuclei is to have some solder and some soldering flux on the surface. But, a more convincing and a much cleaner way 
is to make the channel by simply riveting two flat sheets to some metal spacers. Advance-metal spacers have a high enough electrical resistance to have only a small heating effect, since the current goes mostly through the flat aluminum sheets which simulate the Spert fuel elements. This leaves some nice cracks on either side which are very reproducible, reliable, and beautiful nucleation centers. Presumably such cracks are there in reactor fuel elements too, which consist of plates put into slots. We can't vouch for the exact similitude here, but it is suggestive of what may be present in the Spert reactors.

The third point on hydraulic instability is the "3-D effect", if you like. Dr. Zuber presented curves showing that the void varies not only vertically up and down the axis of the channel, but also laterally across the l-in. width of the channel. We thus can now claim to have measured the void in three dimensions. It is averaged pretty well through the thickness of this channel by the X-ray absorption observations; we have scanned the other two dimensions of the channel, and also have measured it in time by measuring the transfer functions. It appears that the hydraulic instability is very much correlated with the spatial distribution of void in the channel. What we make of this remains to be seen; hopefully, in the coming year.

There is one more point that has not been mentioned before on hydraulic instability. One of the unstable channels has been examined for hydraulic instability in a series of experiments in which as many things as feasible were varied; namely, the power, the subcooling, and the head. The frequency of the natural hydraulic oscillation was observed. The results correlate in a way which is so neat as to be extremely suggestive. Over the entire range of parameters the results all merge themselves well, if you correlate the frequency of this oscillation with the nonboiling length in the channel. The frequency varies as the reciprocal of the non-boiling length with a surprising degree of accuracy.

Next, I will discuss the status of the interpretations of the transfer function data. At the outset the status seems very poor indeed. We cannot calculate even the most simple thing; namely, the static characteristics of the channel. Take a simulated Spert channel at atmospheric pressure, put it at a given power, what will be the vertical distribution of voids? We do not know, for reasons I have just stated--it all depends on funny nucleation phenomena, upon whether you have soldered it or whether you have welded it. On that we admit total defeat so far. This is a structure-sensitive property which we do not yet understand.

From here on, though, the status of interpretation is interesting and encouraging. Suppose we measure the static characteristics of a channel and accept it as a basic characteristic, a constant of the channel. Then with some simple assumptions, the interpretation seems to be able to go quite a long way. The approach is based upon the observations that, as you change the power, the whole void distribution curve shifts longitudinally along the channel. With higher power the void starts lower down in the channel, but increases with height in a 
manner which is independent of power and which can be taken as a kind of a master distribution. Particularly for small oscillations of power, the evidence is very convincing that the effect of a change of power is simply a vertical shift of this void distribution. This concept has been used by applying the method of Storrer, and making some other reasonable and plausible assumptions. These are too detailed to discuss at the moment, but discussion on them is invited. The power-void transfer function which has been thus calculated agrees with the experimental results with very encouraging accuracy. I will not show the slides, but will state the conclusion reached by Mr. Zivi and Mr. Wright: if the thermal time constant of the fuel element is less than the residence time of the fluid in the channel, then you can have a large phase shift without a large attenuation of the power-void transfer function. The combination of appreciable amplitude with large phase shift leads to instability and a key condition seems to be that the residence time of the water is long compared with thermal time constants; in other words, transportation effects dominate.

The extension to the void phase of the approach which storrer has applied to transportation effects in the fluid phase is the principa? philosophy underlying these calculations, and it seems to be encouraging and productive.

The most important single additional factor to consider would appear to us to be the effect of flow on void; so the measurement of a flow-tovoid transfer function directly is one of our immediate objectives. This has been mentioned, implicitly or otherwise, by a number of previous speakers. Several have also mentioned the fact that when liquid bursts into void in the middle of the channel, water is expelled both from the top and the bottom. This, again, is part of the mechanism of interpretation of our results and very essentially so, it would seem.

Finally, two big remaining questions seem to me to be the following-and now I am presenting personal views and not necessarily those of people in my own company who know more about this than $I$. Joe Thie mentioned one big question: How far can the transfer function approach be pushed? Can you take care of all hydraulic instabilities by transfer function methods? Probably not when you have a thing like superheated water being triggered, and you do not know when it is going to trigger. This is not something with which you will go very far using the Nyquist criterion. But I would feel encouraged by the probability that most of the real channels we deal with consist of a large number of statistical ensembles of triggers and that they will average out pretty well so as to show essentially smooth, continuous characteristics. I strongly suspect that studies of hydraulic instabilities will piece themselves together and lead to a rational interpretation in terms of transfer functions measured or soon to be measured, such as the power-to-void transfer function, the flow-to-void transfer function, the pressure-tovoid transfer function. The combined phase shifts and amplitudes of these in a closed loop may lead to correct interpretation of hydraulic instabilities. It may become possible to take measurements at powers below the unstable point and predict the power and frequency at which purely hydraulic instabilities will occur. This is a controversial 
question and a personal opinion, but I think I see it in the wind.

A second burning question is, how can we reduce nucleation and triggering-phenomena to a predictable basis? What can we do to see this nonlinearity around the corner where we have not been yet? On this, I do not have any panacea to suggest. It is a question raised here by many speakers and is one of the great topics for the coming year. 
SESSION IV

GENERAL DISCUSSION

Snyder: Well, that concludes our presentation, and we've heard a wide variety of subjects discussed here tonight starting with, among other things, the effects of nucleation on stability--will a reactor avoid this problem by having lots of radiolytic gas in the reactor? Then we've heard about the problems of predicting several different types of two-phase flow, and then we've heard about a number of mechanisms for instability. I think we still have a lot of questions you people want to ask; on the other hand, the howr is getting late, so I think that $I^{\prime} I I$ ask you to restrlct your questions to those that you think will have a pretty good audience interest. Fred?

Storrer: I'd like to ask Dr. Chernick about the first slides you showed. Chernick: Yes.

Storrer: I still don't understand the ordinate.

Chernick: The ordinate was the integrated power over a channel and the increase in temperature, or the extt temperature rather, less the inlet temperature.

Storrer: Yes, but then when you explained the different curves you mentioned a $\Delta k$ of $4 \%$ and for .....

Chernick: Ah, yes, because the reactivity decrement is the product of the temperature rise and the temperature coefficient. The temperature rise depends on the power level you're operating at and this product of coefficient times temperature is the temperature decrement. Therefore, this is the reactivity compensated by temperature that was something over $4 \%$. I don't think it was quite exactly pinned down.

Storrer: Yes.

Snyder: Scott?

Sleeper: I'd like to raise some questions and make a couple of comments about the effect of radiation on nucleation phenomena.

I think that Dr. Zuber has already indicated, on the basis of experiments that he did a number of years ago, that the presence of radiation remarkably affects the degree of superheat that one can maintain in a bubble or in a superheated system; in effect, it becomes then a bubblechamber. We've been discussing here situations where one talks about out-of-pile experiments without the presence of the effects of radiation. One might ask can one separate those effects and study only the effects of radiation and not those due to superheat.

We have done some experiments in the Triga reactor which inadvertently 
give us some qualitative information on this general subject. Under both steady-state and transient conditions we are able to produce a considerable number of radiation centers in the coolant water without having any high degree of superheat in the system. We have shown that at, say, levels of the order of $100 \mathrm{kw}$, and certainly at $200 \mathrm{kw}$, without the presence of boiling in the system at all, one can observe steadystate generation of radiolytic gas bubbles.

When one does subcooled transient experiments and initiates large reactivity insertions of from $\$ 1$ to $\$ 3$ insertion, we can observe the presence of a large amount of radiolytic gas formed during these bursts. We do not have any detalled studies of when these gas bubbles are formed, but we do have good reason to expect that they do not affect the shutdown characteristics of the reactor because of the large prompt negative temperature coefficient. It is interesting that we generate something on the order of one to two liters of gas in the Triga reactor during these large bursts, so that there is some indication here of essentially a supersaturated system where your knock-on protons are producing a lot of hydrogen and oxygen, and we can actually measure the generation and the ratio of hydrogen-oxygen to nitrogen generated from the dissolved gas in the system.

Now, I think that there are much cleaner experiments of this nature that have been done by Spert people: Capsule experiments, where they put capsules of deaerated distilled water in the center of the reactor during these transients and watch to find out when the bubbles are formed. And, in general, I belleve that they found that the bubbles are formed much too late to account for the observed shutdown characteristics. On the other hand, I believe they have done some other experiments in which they study the spert conditions by putting fuel plates in the water and observe the results. If I'm not mistaken, one observes in that condition that when the fuel reaches saturation temperature one suddenly observes bubble growth, so that one does not observe any great or substantial degree of superheat in the system as a result of the presence of nucleation centers in the water, independent of any mechanical nucleation centers on the surface.

Snyder: So, you say that nucleation is not a problem in a reactor?

Sleeper: That's what I'm saying, essentially.

Snyder: Yes?

Wright: That would be nice, and I would think, not having a reactor, that there is a nice simple experiment somebody could do who does have a reactor. Take a simple atmospheric pressure boiling loop that 's unstable because of poor mucleation, and see if the radiation in the core stabilizes it.

\section{Snyder: Dick?}

Cohen: I want to add some remarks to Jack Chernick's and perhaps we could have this one slide on the xenon stability. 
Snyder: Would you like to come up here?

Cohen: . . . I've looked at the problem considering xenon in the presence of reactor buckling and temperature coefficient. Below a flux level

of $10^{15}$ the effect of neutron lifetime is negligible, so that the buckling effects and the Plux coefficient are equivalent, and I find the following condition: that the "temperature" defect--(or the flux coefficient times the flux level) for stability, must be greater (i.e., more negative) than $b$, where the critical value, $k$ is given by:

$$
b=\frac{y}{v}\left[\frac{\sigma \phi}{\lambda_{1}+\lambda_{2}+\sigma \phi}\left(\frac{\sigma \phi}{\lambda_{2}+\sigma \phi}-x\right)\right]-\frac{M^{2} \Delta B^{2}}{k_{\infty}}
$$

In this equation the symbols have the following meanings:

$$
\begin{aligned}
& y=\text { total xenon yleld } \\
& x=\text { fraction of total xenon which is directly produced ( } 0.05 \text { for } \\
& \text { U-235) }
\end{aligned}
$$

Slide I shows the stable and unstable regions; for a high feedback

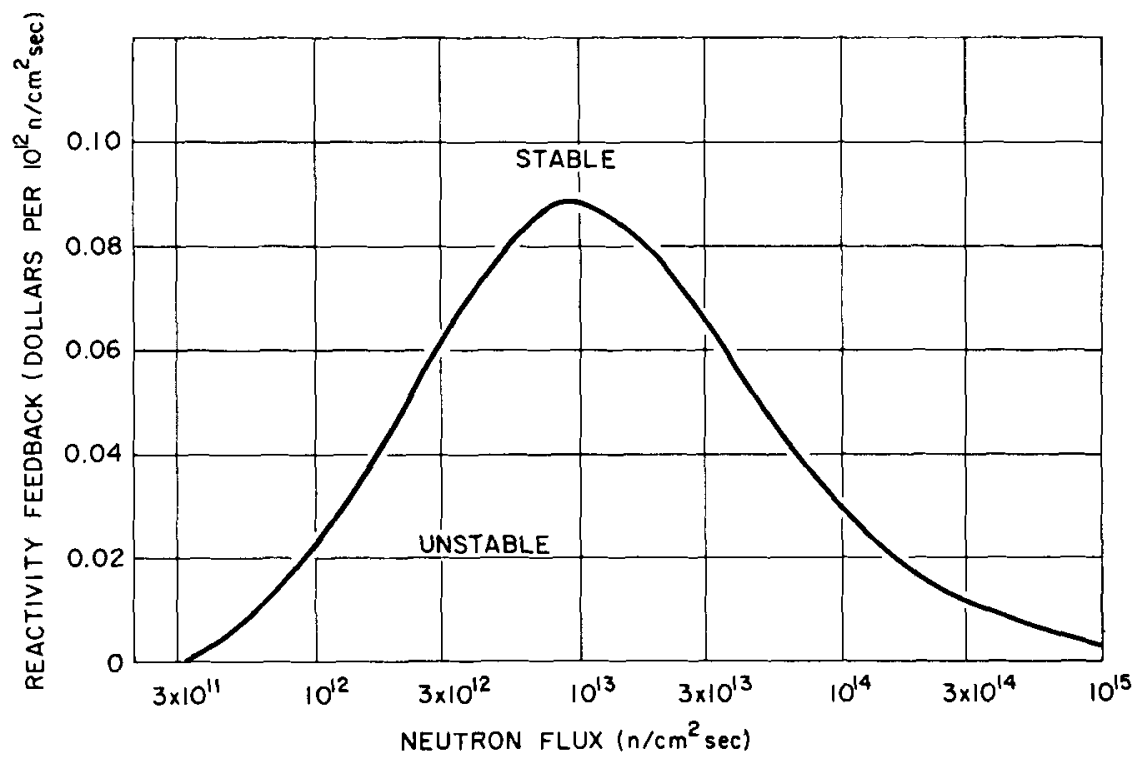

Slide 1. Feedback Requirements for Xenon Stab1lity 
effect we have stability, for a lower feedback effect we have instability. The curve has a maximum and drops off above a flux of $1013 \mathrm{n} / \mathrm{cm}^{2}-\mathrm{sec}$ because as the flux increases we only need a smaller coefficient per unit flux. For example, at a flux of $10^{13}$ this means that about $90 \%$ in temperature defect will stabilize against xenon; at 1014 about a $\$ 3$ effect is required to stabilize against xenon. There is one point $I^{\prime} d$ like to check with Jack on, and that is, we find that the zero point is a reflection of the prompt xenon production, and if that were zero, there would be no stable region without a temperature coefficient to stabilize the reactor. Now, I believe your curve showed a stable region.

Chernick: Yes.

Cohen: At low flux.....

Chernick: Yes, but at extremely low flux--about several times 108, well, somewhere below that, even without prompt xenon, there is a stable level. Physically we interpret this as meaning that the reactor is stable when the reactivity is so small and the periods are so long, that even the decay in xenon is prompt; at least that is the way we interpret that setup. And so this is approximately within our stable region--it isn't a very large region. It gets bigger, of course.

Cohen: Yes.

\section{Snyder: Rogers?}

McCullough: I'd like to ask Mr. Fleck if I understood him correctly when he said that a high-water head and a high slip-velocity ratio is conducive to the nonlinear instability, and I'm reminded that swimming pools, as compared to Spert, have water heads of the order of $18 \mathrm{ft} \mathrm{vs}$ 2 or $3 \mathrm{ft}-4 \mathrm{ft}$, and I should like to inquire that if we have a swinming pool reactor and if the plates become hot enough so that we begin to get steam bubbles, and if this is one of these high-power swimming pools with a downdraft, this is very high slip-velocity ratio. Let's extend this now to a tank-type reactor with a down flow which is not supposed to be boiling, and suppose we pressurize it to avoid boiling, but the plates get hot enough so that we begin to get steam bubbles. Again, we have a high head and we have a high slip-velocity ratio. Does this infer that these are subject to instability?

Fleck: If this type of instability can be believed, these systems that you're talking about might be susceptible; depending on how much steam is able to form.

McCullough: The point that I'm getting at is that when the steam begins to form on the plates that's the time we are beginning to get into trouble.

Fleck: Well, at the beginning you have very little reactivity held in steam.

McCullough: That's right. 
Fleck: Therefore, one would expect instability if it occurs to be hydraulic.

Ergen: Now wait a minute. You're postulating something that has forcedcirculation.

McCullough: Well, I was using the forced circulation to get a high slipvelocity ratio.

Fleck: The analysis here assumes natural circulation and high slip ratios.

McCullough: Yes--but I don't quite see what difference--as long as the relative velocities--I don't think that the system knows whether something is cooling it or not.

Ergen: Oh, yes, because the mechanism involved lets you explain that the head, being Ilquid, draws momentum out of the circulation. If you have a problem that overrides this mechanism then it would not . . . .

Fleck: That's right. Forced circulation tends to keep flow velocity constant and therefore minimize this momentum exchange effect.

MeCullough: That still leaves the swimming pool case, though.

Snyder: You mean the open pool.

McCullough: Yes. All I'm suggesting is $I^{\prime} d$ llke to see this looked at a little more thoroughly to be sure that that answer is not applicable.

Snyder: Did I see a hand back there?

Wright: I don't have the numbers with me, but I remember looking at the high-head spert I instability tests. They were quite consistent with the simple effect of the increase in the subcooling. For the same reactivity insertion you need nearly the same void compensated reactivity at steady power, and this will occur at a higher power level. The flow will change little in this range. The zero-power transfer function gain is directly proportional to the power level, so the instability would be somewhat more severe at the higher head with the resulting higher steady-state power.

Snyder: Well, I think, if there are no more questions, we'd better call this meeting to a close and thank our speaker and our panelists. 


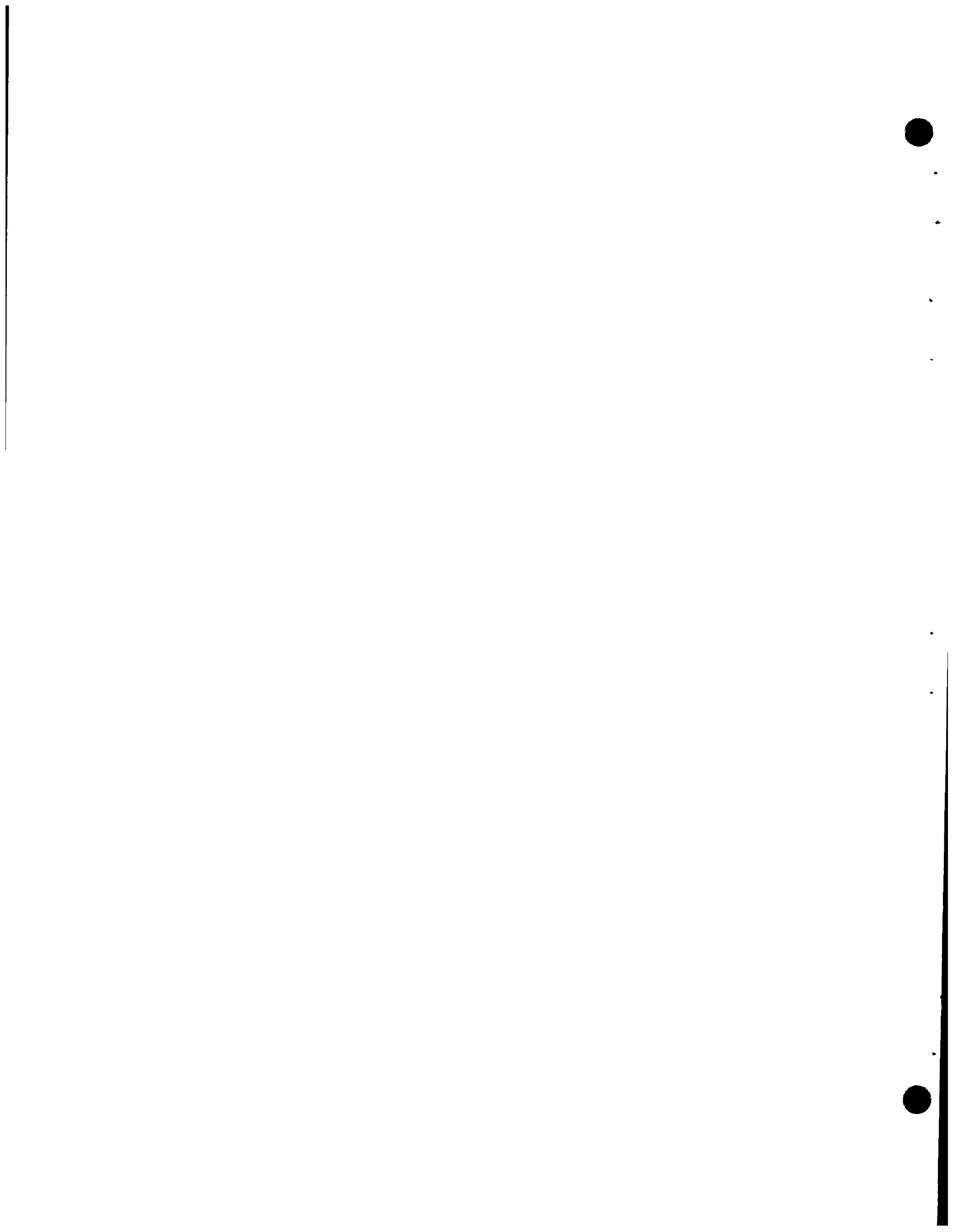


SESSION V

ANALYTICAL METHODS IN REACTOR KINETICS

\author{
Chairman: J. Chernick (BNL) \\ Review Paper: A. F. Henry (W-Bettis) \\ Panelists: E. R. Cohen (AI) \\ W. K. Ergen (ORNL) \\ S. G. Forbes $(R-W)$ \\ H. H. HummeI (ANL) \\ S. O. Johnson (W-Bettis) \\ W. R. Stratton (LASL)
}




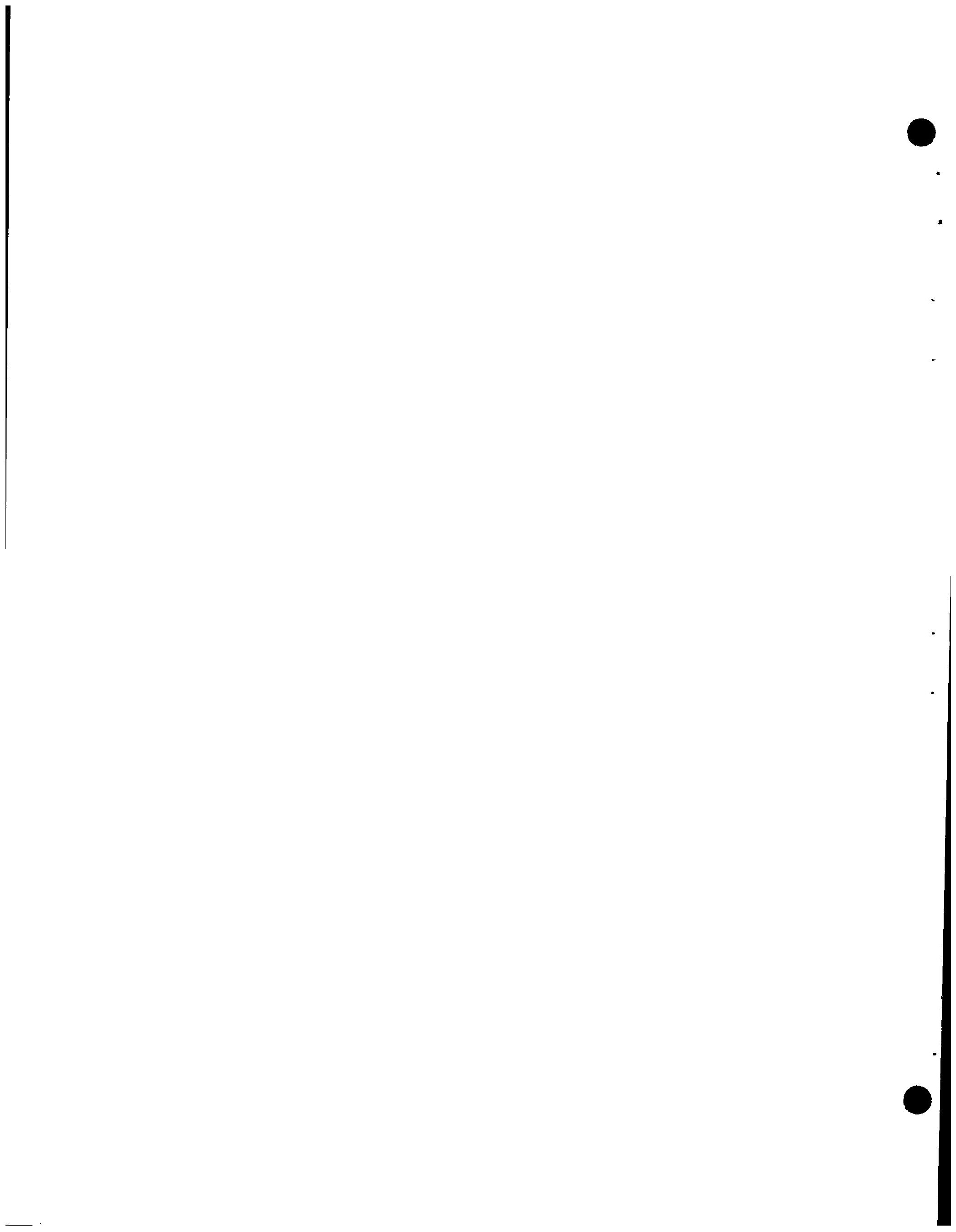




\section{ANAIYYTICAI METHODS IN REACTOR KINETICS * \\ Review Paper \\ by \\ A. F. Henry (W-Bettis)}

\section{INTRODUCTION}

When Glenn Bright first called me to describe this meeting, I took his request for a review paper rather literally. I went back and looked over my old journals and read a lot of articles I'd never read before-and should have, and reviewed some that I had read and forgotten. Having done it this way, I should like to try to describe from a historical viewpoint my notion of the present status of the various theoretical approaches to reactor kinetics problems. I've split the review into four subject categories: the statistical part, the part having to do with the conventional kinetics equations, that having to do with space-time problems, and finally that having to do with space, overall reactor plant description.

\section{STATISTICAI PROBLEMS}

The statistical problem is one which I expect first got examined in connection with the bomb. A lot of pertinent work on the subject is probably buried in secret documents. It has come up again in the study of autocorrelation functions, and in some of the Godiva experiments at Los Alamos.

A theoretical foundation for attacking the statistical problem has been laid through studies of Brownian Motion, and stellar radiation. The review papers of Chandrasekhar $(1)$ and of Wang and Uhlenbeck $(2)$ are fundamental to the subject. The quantity which is of interest in a statistical consideration is not the neutron density but one of a group of probabilities. First of all one defines the "state" of a system as the numerical value, or values, of the observables in which one is interested. These can be the positions and momenta of all the neutrons in the system and all the delayed precursors at any particular time. Alternately the state can be simply the position of a single neutron. One then considers the system as changing from one state to another. Thus one speaks of the state $A$, at a time, $t_{I}$, which goes to state $B$ at time $t_{2}$. Frequently this process is a continuous one. However, it makes it easier to talk about if we consider that the history of a system is a series of discrete states.

The things that one wants to compute about the system are probabilities. First of all, there is the probability that a system will be in state $A$ at time $t_{1}$. Next comes what is called the "joint probability" that it will be in state $A$ at $t_{1}$, state $B$ at $t_{2}$, state $C$ at $t_{3}$, etc. Finally, and most important, there is the "conditional probability:" Given the fact that the system is in state $A$ at time $t_{1}$, the probability that it will be in state $B$ at time $\bar{t}_{2}$. This "conditional probability," is the quantity which in a

*This paper was issued as WAPD-OP-10. 
sense takes the place of the neutron density in the Boltzmann equation. Thus the equation fundamental to the statistical problem involves conditional probabilities. It is called the Smoluchowski equation and states that the conditional probability of going from state $\mathrm{A}$ at time $\mathrm{t}_{1}$ to state $\mathrm{C}$ at time $t_{3}$ is equal to an integral of the conditional probability of going from A to B multiplied by the probability of going from B to C, integrated over all possible states B during times at which these states can occur. It is thus an integral equation involving the conditional probabilities and is sort of like the transport equation, only ten times worse; it has yet to be solved for any practical reactor situations. (I should remark that the Smoluchowski equation is based on the assumption that the particular state, say $B$, of a system at time $t_{2}$, depends only on the prior condition; that is, on the state $A$ at time $t_{1}$, and not on any past states of a system. Systems where this situation obtains are said to obey a Markov process.)

Under the assumption that during the interval $\left(t_{3}-t_{1}\right.$ ) there is very little change in the quantities which specify the state, one can expand the probability of going from $A$ to $B$ in a Taylor series about the coordinates of state $A$, and, keeping only the first two terms, can transform the integral equation into a differential equation. This procedure is analogous to what is done in the age theory or the Greuling-Goertzel approximation; one takes a kernel and expands it retaining only one or two moments. In the statistical case the manipulation leads to the Fokker-Planck equation, a partial differential equation still very difficult to solve, but somewhat simpler than the initial integral equation.

Well, such is the formal system for treatment of the statistical problem. The solution consists in finding the conditional probability. One can in principle start with a basic probability, determined from some physical model, and build up more complex probabilities by successive applications of the Smoluchowski equation. For example, if one is interested in the probability that a neutron, initially in state $A$, will be in state $B$ in exactly two collisions, physical models can be used to specify the probabilities of going in one collision from $A$ to any state, $A^{\prime}$, (excluding $B$ ) and from $A^{\prime}$ to $B$. Integration over all states $A^{\prime}$ gives the desired result. Clearly an extension to very many more collisions will lead to impossibly difficult complexities. As a result only very simple physical models, for which the number of intermediate states, $A^{\prime}$, is very small are at all tractable, This has limited studies to stationary, homogeneous, nonmultiplying systems $(3)$ or to one group, homogeneous, infinite medium multiplying systems $(4,5,6)$.

Two areas of kinetics were mentioned in which statistical considerations become important. I shan't review the work on autocorrelation functions because it has been covered in prior sessions. ${ }^{*}$ In dealing with the problem of starting up without an artificial source, there have been two lines of attack taken. Both assume a one-group, space-independent model. However, one approach makes adgitional assumptions concerning delayed neutron behavior (Gordon Hansen's work $(5)$, and just recently Henry Hurwitz's $(6)$ ). These assumptions permit actual computation of the probability distribution. Thus this attack has one very definite advantage; it gives an answer-one gets a probability distribution. It has the limitation that it is based on certain assumptions which are difficult to evaluate.

*Also see references $7,8,9$ and 10 . 
The other approach (4), which is the one Don Harris has taken, is to treat the delayed neutrons exactly. Because of the resultant mathematical headaches, Don has so far been able to get only moments of the probability distribution. In fact, to find even the first two moments is extremely difficult. So, one treatment of delayed neutron effects is theoretically perfect, but requires knowledge of a distribution which must be determined (approximately) by other means; the other one gives a distribution, but is based on assumptions the validity of which is difficult to assess. Clearly, what has to be done is to attack the problem from both ends and to meet somewhere in the middle so that we thoroughly understand what is going on. The work so far represents a big step in that direction.

\section{CONVENTIONAL KMNETICS EQUATIONS}

\section{A. Formalism}

Well, so much for the statistical considerations. If one considers the average behavior of the system, one obtains a deterministic equation for the neutron flux; namely, the transport equation. And from that, one can derive the kinetics equations. It would have been a remarkable record had we been able to go through this whole meeting without once writing down the kinetics equations, and I would hesitate to break this record except that Paul Kasten had them on a slide last night. So it's already a one-hitter, and I'll go ahead and write them down again. Like everybody else, I'll use my own particular notation.

$$
\begin{aligned}
& \frac{d T}{d t}=\frac{\rho-\bar{\beta}}{\Lambda} T+\sum_{i} C_{i} \lambda_{i}+Q \\
& \frac{d C_{i}}{d t}=\frac{\bar{\beta}_{i}}{\Lambda} T-C_{i} \lambda_{i}
\end{aligned}
$$

I should like to review the evolution of these equations.

So far as I can determine, the form was originally derived from physical considerations. The first paper I was able to find on it was Nordheim's $(11,12)$ about 1946. He credits Wigner, Fermi, Wheeler, Christy, and Schwinger with development of the fundamental ideas. The quantities, $\Lambda$ and $\bar{\beta}$, were physical in nature. It was Wigner $(13)$ who first suggested that in order to find the reactivity, $\rho$, one could use an adjoint flux. He did this for two groups, and being interested only in getting $\rho$ (not the inhour formula), didn't consider delayed neutrons. Almost simultaneously, Nordheim(14) (who credits Wigner with the suggestion to use the adjoint) derived the inhour formula for two-group multi-region caseg. This was generalized by Brooks first to a multigroup diffusion theory model (15) and then to the transport equation description(16). Again, these efforts resulted in a more accurate inhour formula.

Henry Hurwitz made a very major contribution(17), in my judgment, by giving a physical significance or definition to the adjoint flux. He called it the iterated fission probability. This notion is based on the observation that if, in a neutron-free critical assembly, one introduces a (statistically large) group of neutrons of a particular variety (i.e., position and velocity) ultimately a steady-state condition involving release of a definite number of neutrons per generation will be reached. 
This "number per generation" is the adjoint. (Normalization depends on the number of neutrons in the introduced sample and is hence arbitrary.) Thus to find the adjoint for neutrons of any velocity at any point in a critical reactor, one simply takes a fixed sample of neutrons, gives them the velocity of interest, introduces them at the point of interest, waits until an asymptotic situation is reached, and counts the number of neutrons per generation. This number is the desired adjoint. From this definition Hurwitz derived the Nordheim perturbation formula.

Ussachoff in a 1955 Geneva Conference paper(18) defined the adjoint or importance in a way which, until wednesday night, I thought was identical with Henry Hurwitz's. Fred Storrer convinced me that it is slightly different. Ussachoff took the same sample of neutrons, but rather than talking about the number of neutrons per generation asymptotically, talked about the power level or neutrons per second asymptotically. For a given initial sample, the two numbers are different but directly proportional.

Ussachoff, from his definition of importance, then derived the stationary transport equation, for the adjoint flux, and from this went on to derive the kinetics equations (1)--not just the inhour formula, the actual kinetics equations themselves. I remember this very well because while everybody was in Geneva having a good time, I did the same thing and greeted Sid Krasik when he returned with the information that I had achieved this wonderful derivation. Sid pulled out Ussachoff's paper and gave it to me; we published my work as a Bettis report (19) with the appropriate scoop-acknowledging footnote.**

Both derivations assumed separability; that is, that one could separate the total behavior of the neutron flux into a spatial part $\Psi(\underline{r}, \underline{\Omega}, u)$ multiplied by a time function, $T(t)$. The rise and fall of this fixed shape then specified the kinetic behavior of the system. It was, I guess, a year or so later that we found one didn't have to make that assumption (2l); that is, that one could actually talk in terms of a nonseparable shape function $\psi(\underline{r}, \Omega u, t)$ along with the time function $T(t)$ of Eq. (1). The shape function must be constrained such that the adjoint weighted integral

$$
\int \psi_{O}^{*}(\underline{r}, \underline{\Omega}, u) \frac{1}{v(u)} \psi(\underline{r}, \underline{\Omega}, u, t)
$$

is constant in time. However it can wiggle and slosh around inside this construction. Thus, no assumption of separability is needed formally to get Eqs. (1). Of course, the whole process is merely a recasting of the time dependent Boltzmann equation into another form. It is convenient to have it in this form for certain further approximations, but one has not solved the nonseparable time dependent problem by doing this, since one must still resort to approximations to find the nonseparable shape function. Still, as a formalism, it is a helpful thing to have since use of the adjoint tends to minimize errors if $\rho, \bar{\beta}$ and $\Lambda$ are computed using some approximation to $\psi(\underline{r}, \underline{\Omega}, u, t)$. In cases which are separable, the procedure is rigorous--for example, when the flux is on an asymptotic

** Fischer at GEANP had a similar experience (20). 
period. Gross and Marable ${ }^{(22)}$ in their paper actually use the shape function associated with the asymptotic period. As a result their formalism is rigorous for the case considered.

The next development of the formalism is really a generalization of the meaning of adjoint. It was proposed by Dave Selengut in a very excellent paper which has only appeared, so far as I know, as part of a Hanford progress report (23). His intent was not really to extend the formalism of reactor kinetics so much as to provide a systematic method for developing approximation methods. But in so doing he introduced a physical definition of an adjoint for a subcritical system. The definition is an operational one and is in terms of the total reaction rate for any arbitrary process the observer wishes to choose. Thus, for example, if one wishes to find, in terms of the total fission rate, the adjoint for neutrons of energy, $E$, traveling in direction, $\Omega$, at point, $\underline{r}$, one introduces a constant point source ( $N$ neutrons per second) of neutrons of this variety ( $E$ and $\Omega$ ) at $r$ and notes (after steady-state is reached) the total fission rate throughout the system. This rate is the adjoint. To find the corresponding adjoint for some other point $\left.\overline{(E}^{\prime}, \Omega{ }^{\prime}, \mathrm{r}^{\prime}\right)$, one introduces neutrons (N per second) of this variety and again measures the total fission rate. Again this rate is the adjoint. Thus the adjoint flux (with respect to total fission rate) can be found throughout the entire system. If the observer wishes to define in terms of some other reaction rate (for example, the counting rate in one or a series of detectors located somewhere in or about the reactor), one merely notes the asymptotic reading of these counters as the $N$ neutrons per second from the source are changed in energy, direction and location. As the system approaches criticality, the relative readings of all reaction rates approach one another, so that except for a normalization constant this definition of the adjoint goes over into the UssachoffHurwitz definition for critical systems.

The adjoipt concept has been generalized further, in recent papers by Lewins. (2h,25), to the time-dependent situation. As I view it, Lewins uses Selengut's procedure but sticks in a single test neutron whose adjoint he wishes to measure at a particular time, $t$, and then notes the counter reading at a time, $t_{f},\left(t_{f}>t\right)$. This reading is the adjoint, which now depends on $t_{f}$, as well as on the position, energy and direction of the test neutron and on the location and nature of the reaction with respect to which the adjoint is being defined. In this definition the reactor can be critical, subcritical, or supercritical. The adjoint which one gets is a time-dependent function, and is indeed adjoint to the time-dependent Boltzmann equation.

Well, I don't think we can get more general than this, unless we pursue generality for its own sake. One caution occurs to me concerning this last procedure: One cannot get the kinetics equations ( 1 ) using a time-dependent adjoint, without some approximation. For a rigorous derivation, one must use a stationary adjoint appropriate to the (possibly fictitious) critical reactor with respect to which changes in the system are being compared.*

* In 1955 at Bettis, Don Harris, by concocting a Lagrangian density, derived time-dependent adjoint equations identical with Lewins's. Since no practical use for them could be seen, the work was published internally. 
So much for the history of the adjoint flux.

As the more general formalisms have come in, definitions of the quantities, $\bar{\beta}, \Lambda$, and $P$ have become more and more mathematical. One cannot in general define them physically and then derive mathematical expressions for them. The reason I am so sure of this is that I can change the value of them at will simply by a slight renormalization. Even in the most fundamental experiments all that has physical significance is a ratio of quantities, beta to lambda, and so on. Along with the increase in abstraction evaporated the argument, (which I remember could become very heated some time ago) as to whether reactivity was $8 \mathrm{k} / \mathrm{k}$, or whether it was $\delta \mathrm{k}$, or whether one put a $\mathrm{k}$ eff in the delayed neutron equations. In this connection, Corben (26) by a matrix transformation showed that one form can be translated to another by a redefinition of quantities. Others $(27,28)$ have started with the time dependent) $k_{\text {eff }}$ in the delayed equations and have then neglected it as a small effect--usually a perfectly satisfactory procedure. One illuminating point on this question was supplied by Lewins in a paper, a year or so ago (29), in which he pointed out that if one considers $\rho$ as a change in multiplication divided by the production rate of neutrons one gets equations of the form (1). If one divides by the destruction rate, (sum of the absorption and leakage) one gets the set with the $\mathrm{k}_{\mathrm{eff}}$ in the delayed equations. I recommend the form (I) simply because it's easier to deal with a set in which all external perturbations (for most practical problems) affect only one coefficient $(\rho)$.

B. Determination of Parameters in the Kinetics Equations

I should like to mention a word about the determination of the quantity,

$$
\frac{\rho-\bar{\beta}}{\Lambda} \equiv \alpha
$$

in the kinetics equations. Determinations of some of the other quantities are discussed in References 19, 21, 26, 30, 31, 32, 33, 34, and 35. For nonmultiplying systems pulsed-neutron experiments measure this quantity (36-40). (For some fomulations of the kinetics equations, $p, \bar{\beta}$, and $\Lambda$ are not defined for nonmultiplying systems. But $\rho / \Lambda(=\alpha)$ and $\bar{\beta} / \Lambda \quad(=0)$ are.) I'll write now the only other equation I'm going to; namely, that for the decay constant, $\alpha$, in a nonmultiplying pulsed-neutron experiment. This constant is written as a power series in the buckling of the medium.

$$
\alpha=v \Sigma_{a}+D_{O} B^{2}-\mathrm{CB}^{4} .
$$

Here $v \Sigma_{a}$ represents a spectrum weighted average of the neutron speed and the absorption cross section of the medium; $D_{0}$ is an average diffusion constant (units of $\mathrm{cm}^{2} / \mathrm{sec}$ ) and $\mathrm{C}$ is the diffusion cooling coefficient. In experiments with pulsed sources one measures the asymptotic decay of the neutron population ( $\sim e^{-\alpha t}$ ) for various sizes of the system (values of $B^{2}$ ) and thereby tests a theoretical model by seeing whether it will compute the coefficients of the various powers of $B^{2}$ in $E q$. (2). There is one major difficulty: The buckling of the system really depends on the extrapolation distance, which in turn depends on the geometry and on $\alpha(41)$. So, all that one can do, rigorously, in exploring a pulsed- 
neutron measurement is to see whether a consistent theoretical model of the system works. One must first use this model in order to determine the $\mathrm{B}^{2}$ for a given geometrical size and then infer "experimental values" of the coefficients, to be tested against further predictions of the model. Of the various theoretical inyestigations of pulsed source experiments in nonmultiplying media $(42,43,44)$, the most complete, in my opinion, is Mark Nelkin's (44). By expanding all pertinent functions in powers of $\mathrm{B}^{2}$, he shows clearly the contributions of spectrum and transport effects to each coefficient. In particular he shows that one must go at least to a P-3 approximation to the transport equation to obtain a meaningful computation of the diffusion cooling coefficient. A diffusion theory treatment can yield the correct numerical value of $\mathrm{C}$ only though a cancellation of errors.

In multiplying mediums the analogous experiments to determine $(\rho-\bar{\beta} / \Lambda)$ are the Rossi-AIpha experiments $(30,45)$ and the pulse experiments with reactor systems $(46-50)$. One has a stationary problem from the point of view of the calculations, except that the eigenvalue is no longer the keff of the system but the decay constant, $\alpha$, which can be computed by essentially the same standard reactor techniques used to determine $\mathrm{k}_{\text {eff }}$. One can use the formalism of perturbation theory in order to estimate $\alpha$. This is the approach that Simmons at KAPL has taken in his analysis (51)* others $(52,53)$ prefer merely to compute $\alpha$ directly as an eigenvalue. If one has mathematical tools for computing that particular eigenvalue, I think this is a preferable procedure; if not, (i.e., if available mathematical procedures are applicable only to finding $k_{\text {eff }}$ of a system) then I think the kinetics formalism is appropriate; it will usually yield quite accurate results.

\section{Analytical Solutions of Reactor Kinetics Equations}

Finding an exact analytical solution of the kinetics equations (I) (with $\bar{\beta}$ and $\Lambda$ constant in time) seems to have intrigued a number of the more mathematically inclined, and a moderate amount of work has been done on the problem, none of which, as far as I know, has provided any suprises to the man with the analog computing machine. Sylvan Wallach ( 54 ) is the first person I know of who treated the linear ramp rate of insertion of reactivity. (Everybody can do the step change.) Unfortunately I have never really been able to follow his paper; the solution is in terms of a contour integral--it goes out and it comes back, and I don't really know how to evaluate it. Some clarification was supplied by Garabedian, Varga and Bilodeau (55) who applied what looks to me to be the same method to a particular case. Ernest Wilkins $(56)$ then extended the attack somewhat further. Finally, Smets (57), in a Geneva conference paper, presented a rather general study of the problem, showing for what particular functional behavior of $\rho$ (step, linear, inverse linear, or exponential) one could get an analytic solution to the equations.

* The term "perturbation theory" may be misleading. Simmons's analysis involves no approximation, since he uses the flux shape associated with the asymptotically decaying mode. Use of some more approximate flux shape would yield an expression more properly termed "perturbation". 
Approximate solutions of $\mathrm{Eq}$. (1), valid under various conditions, have been suggested by several authors $(28,58,59)$. These are, I think, of considerable interest to the person doing a safeguard report, since they are in the form of simple formulas which yield results remarkably close to the right answer for certain limiting cases--even with power feedback included.

Another aspect to the analytical attack on the kinetics equations-and one which I think is of tremendous significance--concerns the stability characteristics of the solution, particularly the nonlinear stability. (Linear stability has been well covered in the last two days.) The question to be answered is whether a reactor with a given set of feedback characteristics will automatically seek an equilibrium power level after any arbitrary power perturbation. So far as I know, the first one to consider the problem was
Jack Chermick in his study of the stability of the Brookhaven reactor (60). With the reactor temperature assumed to decay according to Newton's law of cooling, he established by a mathematical argument the sufficient conditions for stability. Stability criterion applicable to some other systems of cooling were considered in a paper of Ergen and Weinberg (6I). They used a mechanical analog. That is, they established the similarity of the kinetics equations* plus feedback equations to those for an oscillating mechanical system and then, by deriving the total energy (or more accurately, the Lagrangian) of the system, and applying the stability theorems of classical mechanics, established the sufficient conditions for stability. The procedure was made more formal in another paper by Bill Ergen $(64)$ in which he (and coauthors Lipkin and Nohel) applied the Liapunov theorems of stability and thereby provided a somewhat firmer mathematical basis for the conditions sufficient to guarantee stability. Welton $(65,66)$ extended the work to include a feedback kernel which had a time delay in it; the resultant instability criterion has become pretty much a standard.

Further investigations of the stability problem are described in References 27 and 67 to 72 . The last of these, namely, the paper of Gyftopoulos and Devooght $(72)$ contains a generalization which I think is quite important. In effect, all of the other studies assume that $\Lambda$ and $\bar{\beta}$ are time-independent, i.e., that they are constant during the transient. Now this is not rigorously true unless the flux shape is constant in time. (Its amplitude can go up and down in accord with Eqs. (1); but the shape must be constant for $\Lambda$ and $\bar{\beta}$ to be constant.) It seems to me that in a great many nonstable situations the shape is changing and therefore that one should worry about the adequacy of a stability criterion which does not account for this fact. Gyftopoulos and Devooght do account for the possible feedback effects of flux tilt--at least to the first order. They do this by concentrating, not on the amplitude function, T, of Eq.(1), but rather on an integral over-all direction, energy and space of the neutron density plus the delayed neutron emitter concentrations (weighted by appropriate adjoints). Since this integral, which the authors designate as $P(t)$--it's a sort of generalized power--is over the whole core, flux

* Delayed neutron effects were neglected. Ergen (62) later showed they always increased instability. See also Reference 63. 
tilting effects are automatically included, and a reactor will be stable if

$\lim _{t \rightarrow \infty} P(t)=C$

where $\mathrm{C}$ is a constant. Unfortunately, to get a tractable equation for $P(t)$ one must expand a certain time dependent operator which arises from manipulation of the reactor equations with feedback effects included. Since only the first two terms in this expansion are retained, the resulting stability criterion is not completely general. Nevertheless, it makes me feel a little more comfortable about possible effects of tilting on stability. Even though higher terms in the operator expansion are neglected, all parts of treatment appear to be in more consistent balance.

This brings me to the final two points I want to make on the subject of nonlinear instability criteria. The first is that they are only sufficient (and not necessary) conditions for stability. Perfectly stable systems exist for which these sufficient conditions are not met. Secondly, being based on approximations which simplify the mathematical description of the feedback mechanisms, there are systems for which they are not valid. Thus, the sufficient conditions are not necessarily sufficient. I wonder seriously, for example, whether they are applicable to the xenon-tilt instability $(73,74) ;{ }^{*}$ there the flux shape may well be changing too much for the approximations on which the criterion is based to be valid. I worry further about a boiling reactor during a transient in which all the water is frothing out of the core and falling back in again. For this, the flux shape changes so significantly that I have no confidence the criterion is applicable. I have no suggestions as to how to improve matters. It's miraculous to me how people have gotten this far. But I think it should be kept in mind that, so far, only a sufficiency criterion has been established and that the assumptions upon which it is based are open to question in certain pertinent cases.

\section{NONSEPARABLE PROBIFMS}

Let me now turn to the nonseparable space-time-energy problems. There have been four general lines of attack on such problems. As a general rule, the most suitable approach is determined by the nature of the problem.

\section{A. Modal Analysis}

Any complete set of functions obeying the boundary conditions of a particular reactor assembly can be used to describe the space-time behavior of the neutron and delayed emitter population in an assembly. An initial condition for the assembly can be described by appropriate selection of the coefficients in a sum of such functions, and the subsequent behavior is then specified by the time behavior of these coefficients as determined by the time-dependent equations for the assembly.

One particularly convenient set of functions is that determined by the reactor equations themselves. These modes, since they are defined by non-self-adjoint equations, are not necessarily complete. But there

* The discovery and initial analysis of the xenon-tilt phenomenon was made by $E$. W. Hones and R. R. Haefner. I know of no reference in open literature to this important work. 
are an infinite number of them, and physicists generally think that's enough. As a practical matter, the fundamental mode of such a set can be found by any standard criticality computation procedure. However unless there is a good deal of symmetry, finding higher order modes requires special computational procedures.

An early application of modal analysis was made by Weinberg and Schweinler in their paper on the pile oscillator technique $(75)$. Ward used modes in his analysis of the xenop tilt problem 73 L-as do Morhizuki and Takeda $(76)$ and Margolis and Kaplan $(77)$. Dick Cohen discusses kinetics in terms of modes in his Second Geneva Conference paper(78) and they have been used by Kaplan and Margolis (79) to show the significant influence of delayed neutrons in flux tilt problems.

Any parameter appearing as a linear coefficient in the descriptive equations of a given reactor model can be used as eigenvalue in a modal expansion. The two most common choices are $1 / k_{e f f}$, the coefficient of the fission source term, and $\omega$, the time constant replacing the operator $\partial / \partial t$ in the time dependent equations. For descriptions in terms of functions not defined by the reactor equations (for example, functions defined by $\left.\Delta^{2} \phi=B^{2} \phi\right)$ the buckling is the eigenvalue most frequently used. Garabedian and Leffert $(80)$ make use of such an expansion. These functions have the advantage of being simple and complete; however a series of them must be used to represent even the steady-state flux shape in any core other than a bare one of simple geometry.

B. Nodal Analysis

A nodal treatment, rather than expanding in a series of functions, splits the reactor into sections and ascribes to each section a separate kinetic behavior determined partly by its own characteristics and partly by a coupling, through neutron leakage to neighboring sections. Probably the best known analysis by this method is Bob Avery's treatment of the half-fast reactor $(81,82)$. With one part of the reactor fast and the other thermal, nodal analysis was particularly pertinent for thig problem. An application to the Argonaut reactor was made by $\operatorname{Baldwin}(83)$.

One can also use nodes in applying the formalism of Eq. (1) to nonseparable cases $(84,85,86)$. In this application one use a nodal method to calculate the shape function. For example if the flux shape at any instant is assumed to depend only on the average temperatures in various regions of the core* a series of auxiliary static problems can be run, a priori, to establish this relationship. Thus $\rho$, (which depends on the local temperature through cross section changes and flux shape change), $\bar{\beta}$ and $\Lambda$ (which depend on local temperatures primarily through flux shape changes) can be expressed as a function (usually a power series fit) of the average temperatures throughout the core. Thus these average temperatures determine instantaneous values of $\rho, \bar{\beta}$, and $\Lambda$ which, through Eq. (l), determine the amplitude, $T(t)$, which, through coupled heat transfer and fluid flow equations (in combination with the shape function) determines the average temperatures, and the circle is complete.

* Generally an invalid assumption $(79,86)$. 
Whether one should take a modal or a nodal approach to a given problem of course depends to a large extent on the nature of the problem. My personal preference is for a nodal approach. It seems to me this method fits more naturally into situations involving nonlinear feedback, and I suspect it is more practical for three-dimensional problems. Others disagree, though.

\section{The Transform Approach}

The finding of detailed space-time-energy solutions to reactor transients by Laplace transform methods is restricted to systems in which there is either no feedback or in which feedback effects are linearized. Because of this restriction and because of the difficulties of inverting the transforms in practical problems, most studies have been concerned with the transients associated with a pulse of neutrons. Voorhis $(87)$ and Deverall(88) have considered one energy group space-time situations,* while Krieger and Zweifel $(52)$ and Koppel $(89)$ have been more concerned with the energy-time behavior. Extension of any of these methods to complicated geometrical configurations is very difficult.

\section{Finite Difference Techniques}

Now, the final way that one goes about treating the energy-spacetime problem is the "bludgeon" method: multigroup, space-time-differencing. One simply takes the multigroup equations (and associated delayed neutron equations), writes all space and time derivatives in finite difference form and solves the resultant algebraic equations.

Except no computer built is big. enough to do this. Even a few group, one dimensional procedure can strain all but the most plush computing budgets. One reason for this is that the prompt neutron lifetime in, say, a thermal power reactor is $\sim 1 \mathrm{O}^{-4} \mathrm{sec}$, and the delayed neutrons have a characteristic time the order of $10 \mathrm{sec}$, or so. Thus, if the details of prompt effects are important, extremely small time steps must be used. Yet, if any delayed effects are important the problem must be run for 10 seconds or so. Thus the number of time steps becomes very large. If the reactor is nearly critical, an additional severe problem arises in that the convergence of the spatial calculation at each time step is very slow. This is because such a calculation is in effect a computation of the flux shape due to a source in a very slightly subcritical reactor. Inhomogeneous problems of this kind are very slowly convergent. For many cases, one can neglect the prompt neutron behavior, (assume that

$$
\left.\frac{I}{v} \frac{\partial \phi}{\partial t}=0\right) \text {. }
$$

This simplification (90) speeds the computation if it can be used. A procedure called "source purification" was investigated by Ely Gelbard a year or so ago. This method speeds convergence of the spatial calculation at any time step by removing from the initial source the fundamental mode component, the shape and time dependence ( $e^{\omega t}$; of which can readily

* Voorhis also outlines an extension to two-energy groups. 
be found from standard criticality procedures. With no fundamental mode component present, convergence of the spatial iterations is much faster; the behavior of this mode can then be added back in to get the final

answer.

\section{THE EFFECT OF THERMAL FEEDBACK}

The ultimate objective of reactor kinetics is to describe the overall time behavior of a reactor plant including the feedback effects due to such things as power excursion and changes in coolant flow or power demand. The empirical approaches to this problem have been covered in previous sessions. Frank Schroeder's paper, notably, described the various "correlational" approaches to accounting for the behavior of feedback mechanisms during fast power transients.* So let me just cover the attempts which have been more--I hesitate to use the word, "basic"--but, at least, which employ fudge constants somewhat more fundamental than those which can only be obtained by actually running the system. I have in mind film coefficients, heat capacities, viscosities, and things of this sort which I regard as being somewhat like cross sections in the transport equation. One does not fret because the transport equation is not adequate to compute the inelastic scattering cross section of iron; it is assumed to be given, and I think, in treating these more general problems of interaction of a sys tem, we shall have to assume, as given, certain of the hydrodynamic and thermal parameters. I'm not sure we know as yet what the most appropriate ones are. Continued work on heat transfer and fluid flow phenomena will, hopefully, lead to an understanding of when it is most expeditious to replace a detailed model, involving a physical mechanism, by a single coefficient. The detailed model will then be used to fill in the gaps between experimental measurements of the coefficient. Then the coefficient, and not the detailed model, will be used in an overall mathematical description of the system.

The need to describe the system in a total way, as accurately as possible, and to predict, a priori, its behavior has motivated the kinetics program at Bettis from the start. The reason for this is, of course, that the kinetic behavior largely determines the thermal and hence physics design of a power reactor. One can compute initial criticality without too much trouble. The problem comes after several thousand hours of operation when much of the fuel has been depleted out of the reactor system. Where is the hot spot? How hot is it? Will it melt down? Unless we can answer these questions we must overdesign the core to insure reliability.

Most of the very extensive Bettis work on overall plant descriptions involves details of naval reactor design and is hence classified.** Some

* See also References 91 to 97. **In the unclassified literature, Monty Schultz's book (98) describes many of the techniques applied. Also there are some unclassified reports by Nelson Grace, Stan Johnson, and their associates (99-104). Recent efforts to code for digital solution some of the reactor transient problems are described in References 105 to 109. 
significant papers from other laboratories have appeared $(110-117)$ on various aspects of the subject. To my mind, however, they do not reflect the great amount of detailed work which has actually been done in this area.

I am encouraged by two impressions of the situation as I view it today. One is that the majority of people in the field seem now to believe that an overall kinetic description of a reactor plant is possible. There were, I think, a number of people, some years ago, who felt it was not possible and that one would always have to depend on empirical measurements of the plant itself--a procedure which is more of a correlation than a prediction.

My second impression--and I find it most encouraging--is that there are groups actually working on the individual parts of the description. I think last night's session provided an excellent example of such an effort, this particular one being in the field of heat transfer. There is similar effort being put into studying other phases of the problem. It seems to me that when we thoroughly understand aIl the pieces which are being studied, we'll be in a position to put some of them together-certainly not in all of their generality, any more than in the case of reactor physics we put the transport equation into our design computation codes. But we will get precise mathematical descriptions against which to check definitive isolated experiments, and then simple mathematical models will be devised which are adequate (and economical) approximations to the more exact procedures. A composite description of the whole plant can then be solved with a finite amount of computing machine time. As I say, I think that the present status is very encouraging. Still, we have a long way to go. 


\section{REFFERENCES}

1. S. Chandrasekhar, "Stochastic Problems in Physics and Astronomy," Rev. Mod. Phys., 15, p. 1 (1943).

2. M. C. Wang and G. E. Unlenbeck, "On the Theory of the Brownian Motion," Rev. Mod. Phys., 17, p. 323 (1945).

3. C. N. Klahr, "Calculation of Neutron Distribution by the Methods of Stochastic Process," Nucl. Sci and Eng., 3, 269 (1958).

4. D. R. Harris, "Stochastic Fluctuations in a Power Reactor," WAPD-TM-190 (November 1958).

5. G. E. Hansen, "Assembly of Fissionable Material in the Presence of a Weak Neutron Source," Nucl. Sci. and Eng., 8, p. 709 (1960).

6. H. Hurwitz, Jr., "Reactor Startup with Statistical Fluctuations," G. E. Research Lab Report No. 60-R2-(2405E) (ApriI 1960).

7. M. N. Moore, "The Determination of Reactor Transfer Functions from Measurements at Steady Operation," Nucl. Sci. and Eng., 3, 387 (1958).

8. C. Velez, "Autocorrelation Functions of Counting Rate in Nuclear Reactors," Nucl. Sci. and Eng., 6, p. 414 (1959).

9. M. N. Moore, "The Power Noise Transfer Function of a Reactor," Nucl. Sci. and Eng., 6, p. 448 (1959).

10. C. E. Cohn, "A Simplified Theory of Pile Noise," Nucl. Sci. and Eng., I, p. 472 (1960).

11. L. W. Nordheim, "Pile Kinetics", MDDC-35 (1946).

12. L. W. Nordheim, "Pile Kinetics" (Abstract) Phys. Rev., 70, p. 115 A (1946).

13. E. P. Wigner, CP-3048 (1945), (out of print).

14. L. W. Nordheim, "Weight Factors and Period Relationship for Composite Piles," CP-2824 (1945).

15. H. Brooks, "Perturbation Methods in Multigroup Calculations," KAPL-71 (1948).

16. H. Brooks, "Perturbation Theory for Boltzmann Equation," KAPL-304 (1950).

17. H. Hurwitz, Jr., "Note on the Theory of Danger Coefficients," KAPL-98, (1948)

18. L. N. Ussachoff, "Equation for the Importance of Neutrons, Reactor Kinetics and the Theory of Perturbation, "Ist U.N. Int. Conf. on the Peaceful Uses of Atomic Energy, Geneva, A/CONF. P/656 (1955). 
19. A. F. Henry, "Computation of Parameters Appearing in the Reactor Kinetics Equations," WAPD-142 (December 1955).

20. P. G. Fischer, "Reactor Kinetic Theory", APEX-443 (1958).

21. A. F. Henry, "Application of Reactor Kinetics to the Analysis of Experiments," Nucl. Sci. and Eng., 3, p. 52 (1958).

22. E. E. Gross and J. H. Marable, "Static and Dynamic Multiplication Factors and Their Relation to the Inhour Formula," Nucl. Sci. and Eng., 1, p. 281 (1960).

23. D. S. Selengut, "Variational Analysis of Multi-dimensional Systems," HW-59126 (1959).

24. J. Lewins, "The Time-Dependent Importance of Neutrons and Precursors," Nucl. Sci. and Eng., I, p. 268 (1960).

25. J. Lewins, "Variational Representations in Reactor Physics Derived from a Physical Principle," Nucl. Sci. and Eng., 8, p. 95 (1960).

26. H. C. Corben, "The Computation of Excess Reactivity from Power Traces," Nucl. Sci. and Eng., 5, p. 127 (1959).

27. H. A. Sandmeier, "Nonlinear Treatment of Large Perturbation in Power Reactor Stability," Nucl. Sci. and Eng., 6, p. 85 (1959).

28. A. Z. Akcasu, "General Solution of the Reactor Kinetic Equations Without Feedback," Nucl. Sci. and Eng., 3, p. 456 (1958).

29. J. Lewins, "The Use of the Generation Time in Reactor Kinetics," Nucl. Sci. and Eng., 7, p. 122 (1960).

30. G. E. Haynam and M. F. Crouch, "Monte Carlo Calculation of Slowing Down Time Distribution for Neutrons in $\mathrm{H}_{2}$, " Nucl. Sci. and Eng., 2, p. 626 (1957).

31. S. Kaplan and A. F. Henry, "An Experiment to Measure Effective Delayed Neutron Fractions," WAPD-TM-209 (February 1960).

32. F. deHoffmann, The Science and Engineering of Nuclear Power, Vol. 2, Ch. 9, pp. 103-119, Addison-Wesley Press, Inc., (1949).

33. T. F. Ruane, D. J. Anthony, P. Buck, D. A. Gavin, and H. B. Stewart, "A Measurement of the Effective Delayed Neutron Fraction by the Substitution Method," Trans. Am. Nuclear Soc., I, (2), p. 142 (1958).

34. G. R. Keepin, "Period Reactivity Relations Determined Directly from Prompt Burst Neutron Decay Data," Nucl. Sci. and Eng., 5, p. 132 (1959).

35. R. E. Skinner and E. R. Cohen, "Reduced Delayed Neutron Group Representations," Nucl. Sci. and Eng., 2, p. 291 (1959). 
36. Proceedings of Second Symposium on Applications of Pulsed Neutron Source Techniques, UCRL-5665 (December 1958).

37. G. F. Von Dardel, "Study of the Interaction of Neutrons with Moderating Materials," Phys. Rev., 24, p. 1272 (1954).

38. G. F. Von Dardel and N. G. Sjostrand, "Diffusion Parameters of Thermal Neutrons in Water," Phys. Rev., 96, p. 1245 (1954).

39. K. H. Beckurts, "Measurements with a Pulsed Neutron Source," Nucl. Sci. and Eng., 2, p. 516 (1957).

40. E. C. Campbell and P. H. Stetson, Physics Division Semi-Annual Progress Report for Peri od ending March 10, 1956, ORLL-2076 (1956).

41. E. M. Gelbard, J. A. Davis and J. J. Pearson, "Space-Fnergy Separability in the Thermal Neutron Group," Trans. Am. Nuclear Soc., 2, (2), p. 95 (1959).

42. N. G. Sjostrand, "On the Theory Underlying Diffusion Measurements with Pulsed Neutron Sources," Arkiv f Fys., 15, p. 147 (1959).

43. W. Hafele and I. Dresner, "On the Theory of the Diffusion Cooling Effect in Homogeneous and Heterogeneous Assemblies, "Nucl. Sci. and Eng., I, p. 304 (1960).

44. M. S. Nelkin, "The Decay of a Thermalized Neutron Pulse," Nucl. Sci. and Eng., 7, p. 210 (1960).

45. J. D. Orndoff, "Prompt Neutron Periods of Metal Critical Assemblies," Nucl. Sci. and Eng., 2, p. 450 (1957).

46. B. E. Simmons and J. S. King, "A Pulsed Neutron Technique for Reactivity Determination," Nucl. Sci. and Eng., 3, p. 595 (1958).

47. J. Bengston, et al, "Determination of Prompt Neutron Decay Constants of Multiplying Systems", UCRL-5159 (1958).

48. A. J. Kirschbaum. Proceedings of the French-American Conference on Graphite Reactors, November 12-15, 1957. BNL-489, 275-290 (1957).

49. H. L. Reynolds, Critical Measurements and Calculations for EnrichedUranium Graphite-Moderated Systems, UCRL-5175 (1958). Also A/Conf. $15 / \mathrm{P} / 2408$ (1958).

50. F. A. Kloverstrom and T. T. Komoto," Pulsed Neutron Source Studies of Graphite-Enriched Uranium Systems," UCRL-5477 (1959).

51. B. E. Simmons, "The Dynamic Reactivity Interpretation of Pulsed Neutron Measurements," Nucl. Sci. and Eng., 5, 254 (1959).

52. T. J. Krieger and P. F. Zweifel, "Theory of Pulsed Neutron Experiments in Multiplying Media," Nucl. Sci. and Eng., 5, p. 21 (1959). 
53. S. C. Fultz, "The Time Dependent Thermal Neutron Flux from a Pulsed Subcritical Assembly," Nucl. Sci. and Eng., 6, p. 313 (1959).

54. S. Wallach, "Solutions of the Pile Kinetic Equations when the Reactivity is a Linear Function of the Time," WAPD-13 (November 1950).

55. H. Garabedian, R. Varga, and G. Bilodeau, "Reactor Response to a Reactivity Change during Xenon Transient," Nucl. Sci. and Eng., 3, p. 548 (1958).

56. J. E. Wilkins, Jr., "The Behavior of a Reactor at Prompt Critical when the Reactivity is a Linear Function of Time," Nucl. Sci. and Eng., 2, p. 207 (1957).

57. H. B. Smets, "Reactor Dynamics at Low Power," 2nd U.N. Int. Conf. on the Peaceful Uses of Atomic Energy, Geneva, A/Conf. P/107 (1958).

58. H. Hurwitz, Jr., "Derivation and Integration of the Pile Kinetic Equations," Nucleonics, 5, p. 61 (1949).

59. H. Furwitz, Jr., "Approximate Analysis of Reactor Start-up Incidents," Nucl. Sci. and Eng., 6, p.11 (1959).

60. J. Chernick, "The Dependence of Reactor Kinetics on Temperature," BNL-173 (1951).

61. W. K. Ergen and A. M. Weinberg, "Some Aspects of Nonlinear Reactor Dynamics," Physica, 20, p. 413 (1954).

62. W. K. Ergen, "Kinetics of the Circulating-Fuel Nuclear Reactor", J. Appl. Phys., 25, 702 (1954).

63. E. P. Gyftopoulos and J. Devooght, "Effect of Delayed Neutrons on Nonlinear Reactor Stability," Nucl. Sci. and Eng., 8, p. 244 (1960).

64. W. K. Ergen, H. J. Lipkin, and J. A. Nohel, "Applications of Liapunov's Second Method in React or Dynamics," Jour. Math. and Phys., 36, p.36 (1957).

65. T. A. Welton, "A Stability Criterion for Reactor Systems, " ORNL-1894 (1955).

66. T. A. Welton, "Kinetics of Stationary Reactor Systems," Ist U.N. Int. Conf. on the Peaceful Uses of Atomic Energy, Geneva, A/Conf. P/610 (1955).

67. H. B. Smets, "Notes on the Inherent Stability of Muclear Reactors," and U.N. Int. Conf. on the Peaceful Uses of Atomic Energy, Geneva, A/Conf. P/107 (1958).

68. G. W. Stuart and W. H. Harker, "Non-Linear Reactor Stability," VAI. 41 (1957). Also Trans. Am. Nuclear Soc., 1, (1), p. 111 (1958).

69. H. B. Smets, "On Welton's Stability Criterion for Nuclear Reactors," Jour. Appl. Phys., 30, 1623 (1959).

70. H. B. Smets and E. P. Gyftopoulos, "The Application of Topological Methods to the Kinetics of Homogeneous Reactors," Nucl. Sci. and Eng., 6, p. 341 (1959).

71. A. Z. Akcasu and A. Dalfes, "A Study of Nonlinear Reactor Dynamics," Nucl. Sci. and Eng., 8, p. 89 (1960). 
72. E. P. Gyftopoulos and J. Devooght, "Boundedness and Stability in Nonlinear Reactor Dynamics," Nucl. Sci. and Eng., I, p. 533 (1960).

73. A. G. Ward, "The Problem of Flux Instability in Large Power Reactors," CRRP-657 (July 1956).

74. A. F. Henry and J. D. Germann, "Oscillations in the Power Distribution Within a Reactor," Nucl. Sci. and Eng., 2, p. 469 (1957).

75. A. M. Weinberg and H. C. Schweinler, "Theory of Oscillating Absorber in a Chain Reactor", Phys. Rev., 74, 851 (1948).

76. K. Mochizuki and A. Takeda, "An Analysis of Neutron Flux Spatial Oscillation Due to Xenon Buildup in a Large Power Reactor Core," Nucl. Sci. and Eng., 7, p. 336 (1960).

77. S. G. Margolis and S. Kaplan, "Nonlinear Effects on Spatial Power Distribution Transients and Oscillations with Xenon Reactivity Feedback," Trans. Am. Nuclear Soc., 3, (1) p. 183 (1960).

78. R. Cohen, "Some Topics in Reactor Kinetics," and U.N. Int. Conf. On the Peaceful Uses of Atomic Energy, Geneva, A/Conf. P/629 (1958).

79. S. Kaplan and S. G. Margolis, "Delayed Neutron Effects during Flux Tilt Transients," (Letter to Editor) Nucl. Sci. and Eng., I, p. 276 (1960).

80. H. L. Garabedian and C. B. Leffert, "A Time-Dependent Analysis of Spatial Flux Distributions," Nucl. Sci. and Eng., 6, p. 26 (1959).

81. R. Avery, "Kinetics of Coupled Assemblies," Trans. Am. Nuclear Soc., 1, (1), p. 165 (1958).

82. R. Avery, "Theory of Coupled Reactors," Ist U.N. Int. Conf. on the Peaceful Uses of Atomic Energy, reneva, A/Conf. P/1858 (1955).

83. G. C. Baldwin, "Kinetics of a Reactor Composed of Two Loosely Coupled Cores," Nucl. Sci. and Eng., 6, p. 320 (1959).

84. A. F. Henry and N. J. Curlee, "Verification of a Method for Treating Neutron Space-Time Problems," Mucl. Sci. and Eng., 4, p. 727 (1958).

85. N. J. Curlee, "Nonseparable Space-Time Transients Resulting from Changes in Inlet Coolant Temperatures, " Nucl. Sci. and Eng., 6, 1, (1959).

86. A. F. Henry and R. S. Willey, "Determination of Reactor Transients When Space and Time are not Separable," Trans. Am. Nuclear Soc., ㄹ, (2) p. 63 (1959).

87. A. D. Voorhis, "Kinetics of a Plane Neutron Burst in an Infinite Homogeneous Medium," WAPD-145 (February 1956).

88. L. I. Deverall, "Solution of the Time-Dependent Thermal Neutron Diffusion Equations," (Letter to Editor), Nucl. Sci. and Eng., 4, p. 495 (1958).

89. J. Koppel, "Time-Dependent Neutron Spectra," Nucl. Sci. and Eng., 8 , p. 157 (1960).

90. H. D. Brown, "A General Treatment of Flux Transients," Nucl. Sci. and Eng., 2., p. 687 (1957). 
91. L. W. Nordheim, "Pile Kinetics," MDDC-35 (1946).

92. J. C. Allred and D. S. Carter, "Kinetics of Homogeneous Power Reactors of the LAPRE Thpe," Nucl. Sci. and Eng., 3, p. 482 (1958).

93. R. E. Skinner and D. L. Hetrick, "The Transfer Function of a Water Boiler," Nucl. Sci. and Eng., 3, p. 573 (1958).

94. R. S. Stone, et al., "Transient Behavior of TRIGA, a Zirconium-Hydride, Water-Moderated Reactor," Nucl. Sci. and Eng., 6, p. 255 (1959).

95. W. A. Horning, "A Theory of Free Oscillations in the Spert Reactor," Third Annual Meeting of the American Nuclear Society, Paper 2-6 (1957). H. C. Corben, "Theory of Small Oscillations of the Spert Reactor," Third Annual Meeting of the American Nuclear Society, Paper 2-7 (1957).

96. L. I. Deverall and G. W. Griffing, "Energy Model for the Initial Behavior of Spert I Transients Initiated by a Step Addition of Reactivity," Second Winter Meeting of the American Nuclear Society, Paper 15-3 (1957).

97. ن. S. Drunenfeld and D. P. Gamble, "Analytic Solutions to KEWB Kinetics Equations for Short Period Transients," Trans. Am. Nuclear Soc., ㄹ, (2), p. 62 (1959).

98. M. A. Schultz, Control of- Nuclear Reactors and Power Plants, McGrawHill Book Co., Inc., New York, (1955).

99. J. N. Grace, "Synthesis of Control Systems for Nuclear Power Plants," Institute of Radio Engineers Convention Record (March 1954).

100. J. N. Grace, M. A. Schultz, T. E. Fairey, "Inherent Reactor Stability," Proceedings 1955 Conference on Nuclear Engineering, UCLA, California Book Co., Ltd., Berkeley, California (1955).

101. W. H. Hamilton, J. N. Grace, O. R. Meyer, N. E. Wilson, "Power and Temperature Control of Pressurized Water Cooled Reactors," WAPD-T259 (1955).

102. J. N. Grace and S. O. Johnson, "Analog Computation in Nuclear Engineering," Nucleonics, 15, No. 5, p. 72 (1957).

103. S. O. Johnson, N. J. Curlee, and J. V. Reihing, "Simulation of Hot Channel Boiling in Water Cooled Reactor," IRE Transactions on Nuclear Science, NS-5, No. 1, 1-9 (1958).

104. R. P. Rose and S. O. Johnson, "A Method of Evaluating Heat Transfer Simulation in Pressurized Water Reactor," Bettis Technical Review, WAPD-BT-8, pp. 137-147 (June 1958).

105. J. E. Meyer and W. D. Peterson, "ART-04 - A Modification of the ART Program for the Treatment of Reactor Thermal Transients on the IBM-704," WAPD-TM-202 (May 1960). 
106. J. E. Meyer and W. D. Long, "A Channel Integral Model for the Treatment of Transient Fluid Flow," Bettis Technical Review, WAPD-BT-23, pp. 1 - 16 (1961).

107. R. I. Miller and R. S. Pyle, "TITE - A Digital Program for the Prediction of Two-Dimensional Two-Phase Hydrodynamics," WAPD-TM-240 (to be issued).

108. J. B. Callaghan and J. S. Williams, Jr., "F0020 - An IBM-704 Thermal Transient Analysis Code," WAPD-TM-145 (January 1959).

109. J. E. Meyer, "Hydrodynamic Models for the Treatment of Reactor Thermal Transients," Trans. Am. Nuclear Soc., 3, 521 (1960).

110. M. W. Fleck, "Transient Pressures in Nuclear Reactors," Nucl. Sci. and Eng., 2, p. 694 (1957).

111. R. B. Nicholson, "A High Frequency Reactor Instability Mechanism," Nucl. Sci. and Eng., 3, p. 620 (1958).

112. E. P. Gyftopoulos and H. B. Smets, "Transfer Functions of Distributed Parameters in Nuclear Reactor Systems," Nucl. Sci. and Eng., 5, p. 405 (1959).

113. R. Siegel and H. Hurwitz, Jr., "The Effect of Positive Temperature Coefficients on Reactor Stability and Reactor Transfer Functions," KAPL-1138 (1955).

114. C. Caillet and M. Deat, "Possibilities and Limitations of Analog Calculations for Dynamic Study of Nuclear Power Stations," and U.N. Int. Conf. on the Peaceful Uses of Atomic Energy, Geneva, A/Conf. P/1196 (1958).

115. J. A. Thie, "Theoretical Reactor Statics and Kinetics of Boiling Reactors," 2nd U.N. Int. Conf. on the Peaceful Uses of Atomic Energy, Geneva, A/Conf. P/638 (1958).

116. M. E. Remley, et al., "Experimental Studies on Kinetic Behavior of Water Boiler Type Reactors," and U.N. Int. Conf. on the Peaceful Uses of Atomic Energy, Geneva, A/Conf. P/1079 (1958).

117. J. A. Fleck, Jr., and J. Husely, "A Math. Model for Calculating Boiling Reactor Transients," and U.N. Int. Conf. on the Peaceful Uses of Atomic Energy, Geneva, A/Conf. P/581 (1958). 
SESSION $V$

PANEL PRESENTATIONS

Chernick: The rest of the papers will take up some aspects of the analytical methods but will range pretty far afield. The first person I would like to call upon is Bill stratton of Los Alamos, who has spent a great deal of time in reviewing what we usually euphemistically call nuclear incidents at various sites in the country and in the 10 minutes or so that Bill expects to spend, obviously he can't review the large number of incidents but I think he will probably pick some of the relevant samples.

\section{Review of Some Prompt Critical Accidents by \\ W. R. Stratton (LASI)}

Stratton: As Jack (Chernick) said, a few months ago I finished a study of the prompt critical accidents that have occurred in this country. There have been some 18 of these. I won't try to cover all of these this morning, but I'll try to hit a few that are typical of each class. One can group

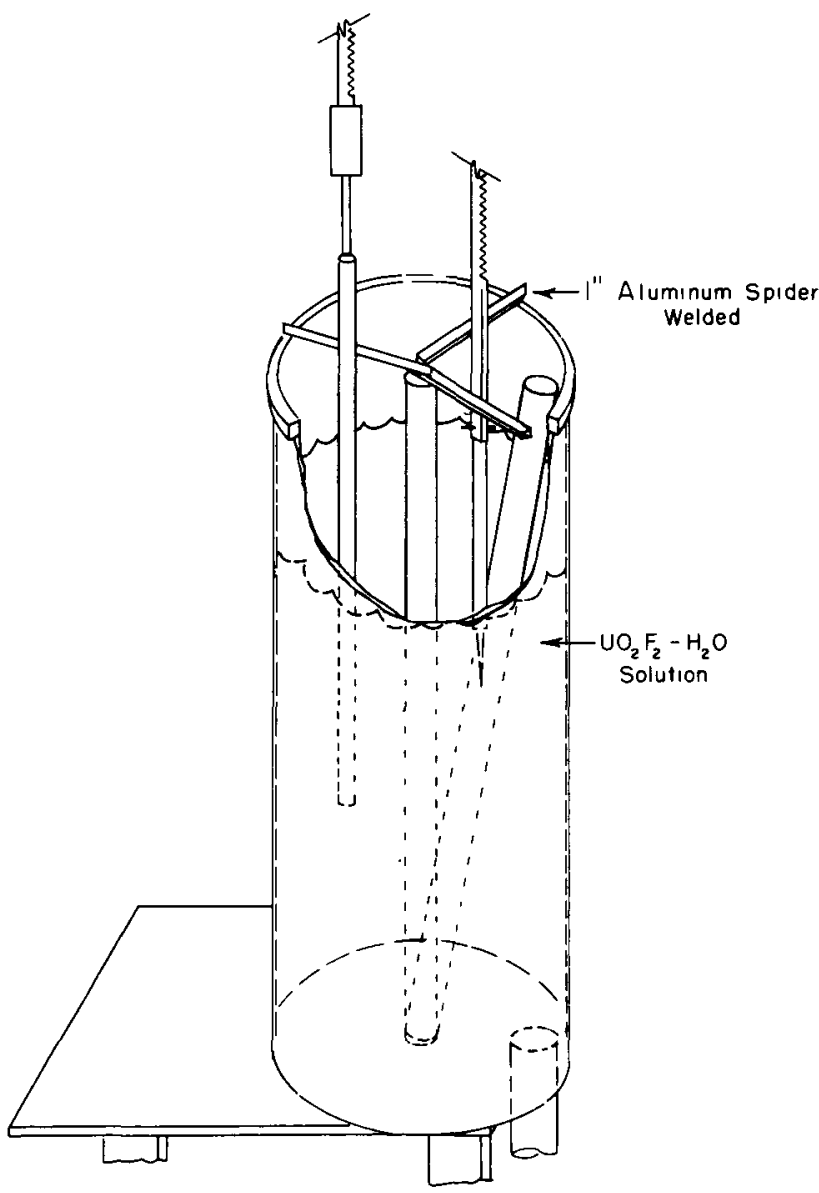

Slide 1. Schematic of the ORNL Uranium Solution Indicating the Normal and Detached Positions of the Central Cadmium-Coated Cylinder the incidents into solution systems, metal systems, heterogeneous systems and such like. Almost all of them seem to differ and we have usually an inadequate amount of information to work with when we try to figure out what happened. Accidents are traditionally underinstrumented so we don't have all the data such as exist for the Godiva, Triga, Spert and Kewb experiments. Ideally, I think one would like to look at these afterwards and be able to say, "What has the reactivity done as a function of time? What caused it to do this? What's the power history? What's the energy release? What are the quenching action or actions?" We very seldom have anywhere near the amount of information one would like to have. So without delaying, I'll hit a few fairly typical ones.

The first of these I would like to mention is the Oak Ridge National Laboratory incident in May of 1954. A schematic of the equipment is shown in Slide 1 . They were studying solutions of uranium in annular cylinders in which the central rod was cadmiumclad. The initial situation was 
with the rod in the vertical position. They were just about at critical when the mechanism at the top became detached and the central rod fell to one side. Since it was cadmium coated, it was essentially the same as removing a poison rod from the system and the reactivity started to rise at about the rate of $3-1 / 3$ dollars per second. When it was all over, the fission yield was about 1017. There was no damage and no real problem except why it happened. The reconstmaction of this inc1dent was one of the best I know of. Dixon Callihan and his group followed this one through and found the worth of the cadmium-coated cylinder as it tilted as a function of time. And by using a simple energy model along with the bubble model that the Kewb people suggest, one can come out with what looks to be a plausible power and reactivity history. The initial spike must have been half the total yield, about $5 \times 10^{16}$ fissions with the peak power around $8 \times 10^{18}$ fissions/sec and a minimum period of about 2 msec. The rest of the yield occurred after the initial spike when the central cylinder was sill falling, going to the outside cylinder. Scram occurred immediately, of course, and solution started to drain and this finally turned it off.

The second incident that I would like to mention is the $Y-12$ plant accident of 1958 in their (ORNL) Chemical Processing Plant. These plants are complicated things, but I think the pertinent point in this case is that it was being remodeled in places and an inventory was in process. Unknown to the operator a quantity of uranyl nitrate was transferred from the region that had been remodeled and was being used, to the region where he was leak testing various pipes. The pipes he was testing were "ever-safe", but when he finished what he thought was leak testing, and started to drain the solution into a 50-gallon drum, it was no longer ever-safe, and became critical. The official yield was $1.3 \times 10^{18}$ fissions. There were about $18 \mathrm{~kg}$ of uranium and about 55 liters of water, I believe, and in this case all that we know is that there was an initial spike of unknown magnitude, and it happened at an unknown time. As there was no source it was a zero-power startup. It could easily have been over prompt critical when the first spike occurred, and I think it most likely was; one can guess that the spike yield must have been of the order of $10^{16}$ fissions, because there was not much in the way of splashing or damage. The excursion was seen by a boron-lined ionization chamber some $1400 \mathrm{ft}$ away, the record of which suggests a sharp initial spike of some unknown magnitude followed by power oscillations of apparently much lower magnitude, and finally boiling. In this case, one really cannot say much quantitatively about what happened.

The next one I will describe is the rather grim case of the Harry Daghlian incident of Los Alamos, on August 21, 1945. He was handstacking 4.4-kilogram tungsten carbide bricks around a $6.2-\mathrm{kg}$ plutonium core which was in the form of two hemispheres coated with 5 mils of nickel. The final brick was held in his left hand over the assembly when he noticed by some monitoring counters that the system was already critical, or very near critical. He started to remove the brick and it slipped out of his hands and fell onto the center of the assembly. This made the system prompt critical, a burst occurred, and he proceeded to disassemble it by hand. In this case the total yield was about 1016 fissions and one can guess several reasonable paths for the power history. It appears to me that the falling brick must have been 
a step addition of reactivity to slightly over prompt critical. By analogy to Jezebel, the Los Alamos bare plutonium reactor, one can guess a negative temperature coefficient of reactivity for the system and by using the fact that the nickel canning did not rupture (the excursion was not in the inertial region), the reactivity can be limited to about 10 cents over prompt critical. This looks not unreasonable and we come out with a spike yield of $2 \times 10^{15}$ fissions. The majority of the fissions must have occurred in the region of the plateau.

The next incident is the final Godiva accident. The experiment underway was to irradiate a large mass of graphite and polyethylene stacked near Godiva. Sometime just before the final burst was initiated by shooting the burst rod in, we think that this material somehow slumped nearer Godiva and added an extra 15\%, so that the burst rod, instead of inserting a dollar and 4 or 5 cents starting from delayed. critical, inserted this much starting from about $15 \%$ over delayed critical. The yield in this case was $1.2 \times 10^{17}$ fissions and we think it was about 2$] \notin$ over prompt critical. It damaged Godiva pretty badly, as can be seen in Slide 2 and Slide 3. It's obvious that the center was close to the melting point; the material near the center actually started to move out. The burst rod was stretched and almost ruptured, and the plates were warped and very badly oxidized. As you know, we didn't even try to fix it. This case was well into the inertial region and I think it is indicative of the danger of operating such a metal system. It is teetering on the edge of destruction a good bit of the time.

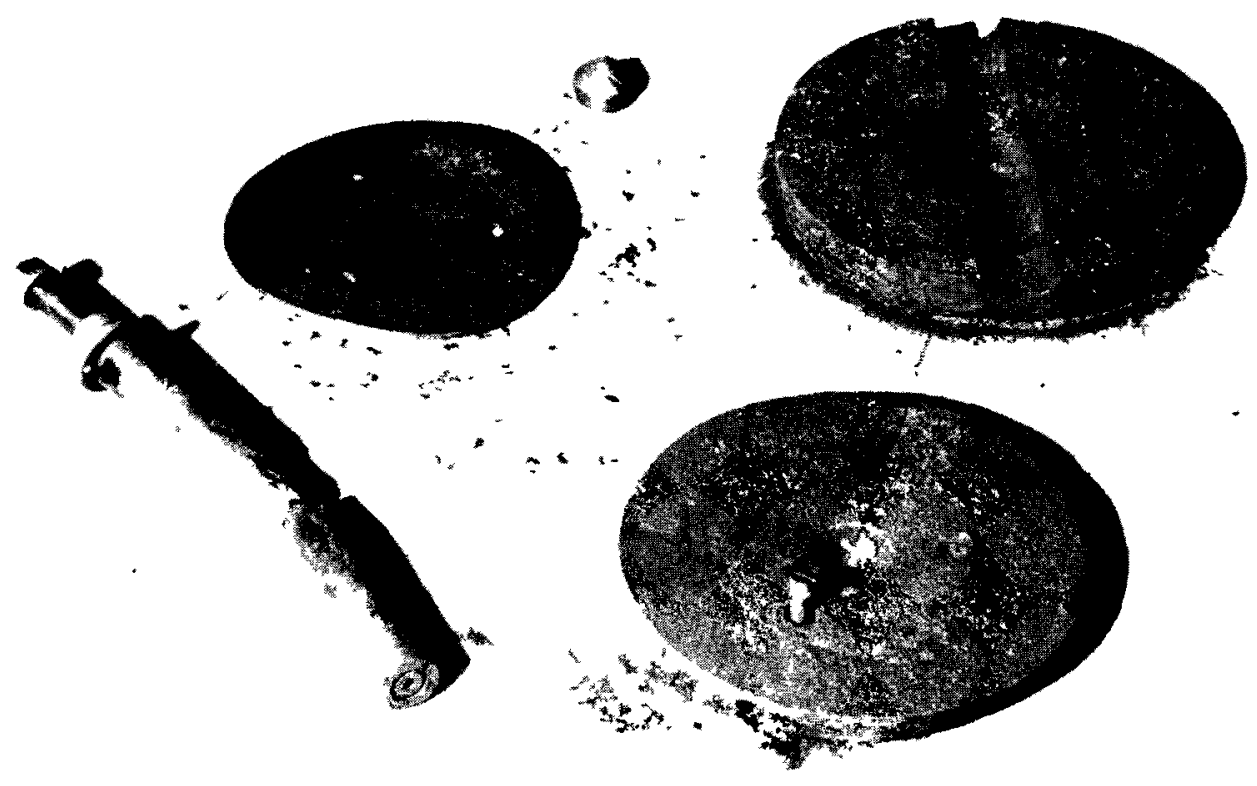

Slide 2. Photograph of the burst rod and several sections of Godiva-I showing the oxidation and warpage which accompanied the second accidental excursion 


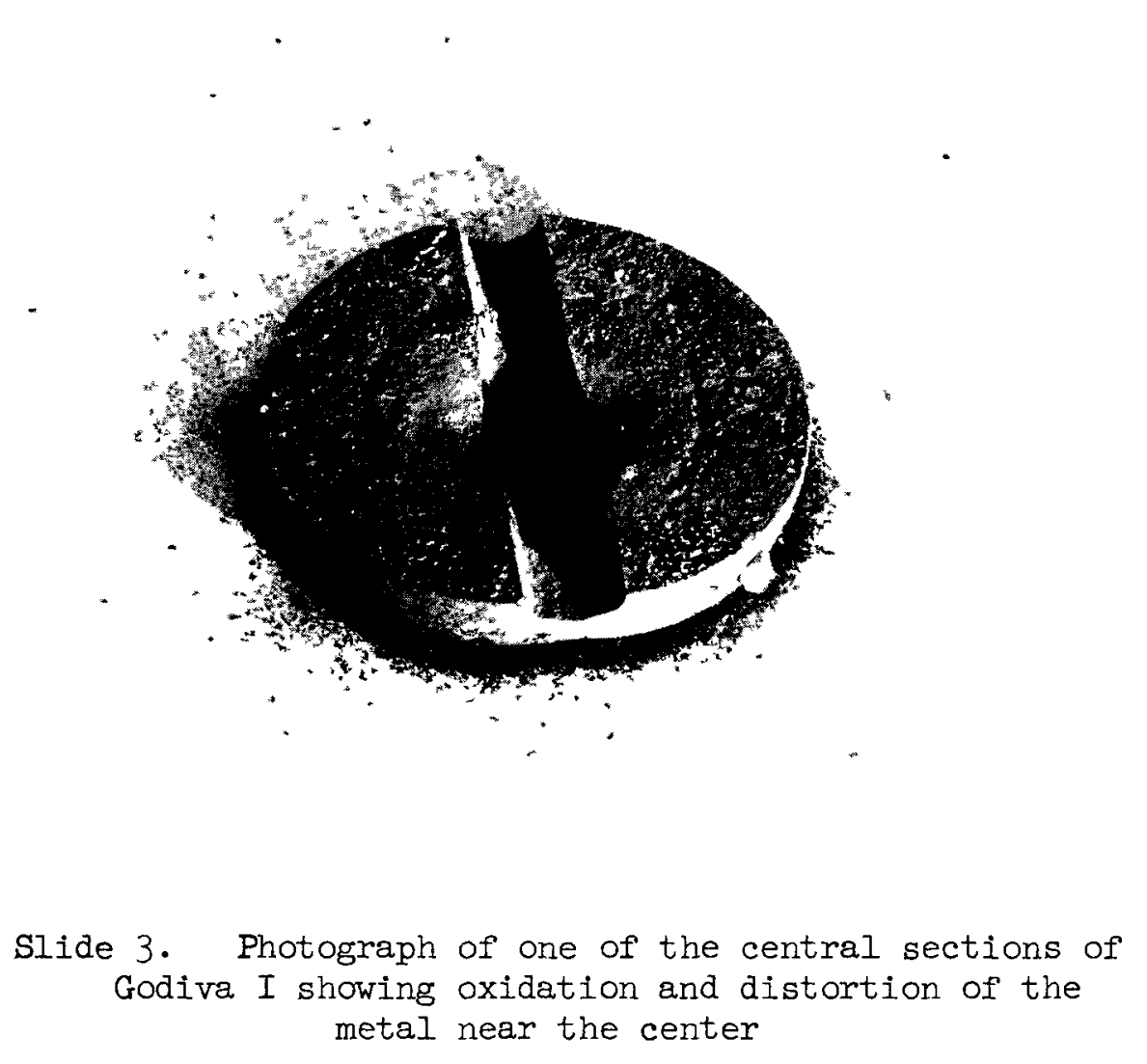

Slide 4 is a schematic of the Los Alamos Aquarium machine. The experiment under way at the time was to find the critical separation distance of two large masses of enriched uranium metal in water. The lefthand piece was a solid cylinder of $24 \mathrm{~kg}$ with $10 \mathrm{mils}$ of cadmium on the outer surface and the right-hand piece was a hollow cylinder of $38 \mathrm{~kg}$ with cadmium on the inner surface. The system was made for immersion experiments of a single piece in water and when it was decided to do this separation experiment, a traveling support was added. The scrams consisted of a lift for the left-hand piece, a cadmium screen to separate the two metal masses and a water drain. The scrams had never been used with active material prior to this experiment and when it was completed, at a multiplication of 65 , the system was scrammed as a final flourish. Simultaneously, the water started draining, the cadmium screen dropped, and the solid cylinder on the left started to lift. An excursion was observed as jamming of counters and evidence on television of a vapor cloud above the water. Later reconstruction showed that the center of reactivity of the left-hand piece was below that of the stationary piece and the rapid lift through the water created Bernoulli forces which swung the cylinders closer together. The prompt burst was certainly 


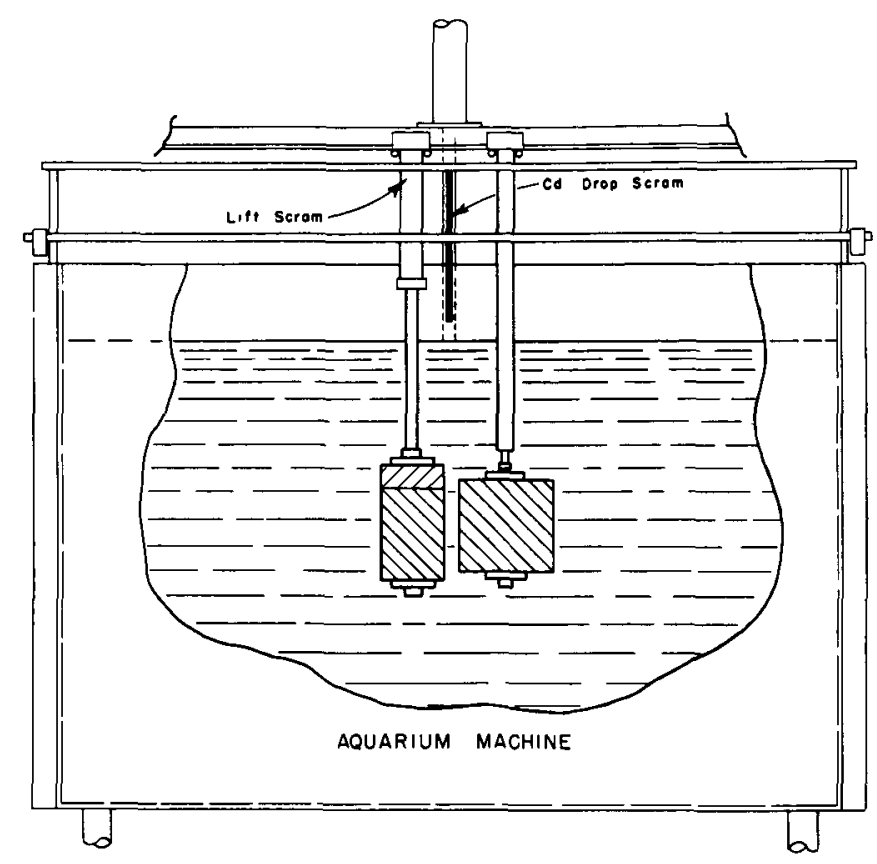

Slide 4. Schematic of the LASL Aquarium AssembIy machine which was employed for measurements of critical

quenched by boiling of the water on the surface of the metal, but an exact description is nearly impossible. I merely tried to find out what spike yield ( $\sim 6 \times 1015$ fissions) would raise the surface of the metal above the boiling point of water, and let it go at that. The total yield for this incident was $10^{17}$ fissions.

The final system I would like to mention is the Los Alamos Honeycomb, a photo of which can be seen in Slide 5. This large-assembly machine is used for early studies of rather complex systems. The usual stacking consists of uranium foils ( 2 to 5 mils thick) sandwiched between layers of moderating and reflecting material. The particular stacking in July of 1956, when we had an excursion of $3.2 \times 10^{16}$ fissions in this machine, was with these uranium foils sandwiched between graphite slabs. The total mass was about $50 \mathrm{~kg}$ of oralloy and I think the reflector was mostly beryllium. A change had been made from the previous critical, larger than had been thought, and the closure rate of $0.2 \mathrm{in.} / \mathrm{sec}(\sim \mathrm{s} / \mathrm{sec})$ created a prompt critical geometry before the moving cart reached the stationary half. The system was saved by the scram system ejecting beryllium control rods and reversing the motion of the cart. In this particular case, it was a very close thing, because we can see no quenching mechanism for an assembly of this type, aside from the vaporization of the foils. A slightly faster insertion rate or slower rod response could have put us in this yield region, which would not result in an explosion, but would have most seriously contaminated the Kiva. 


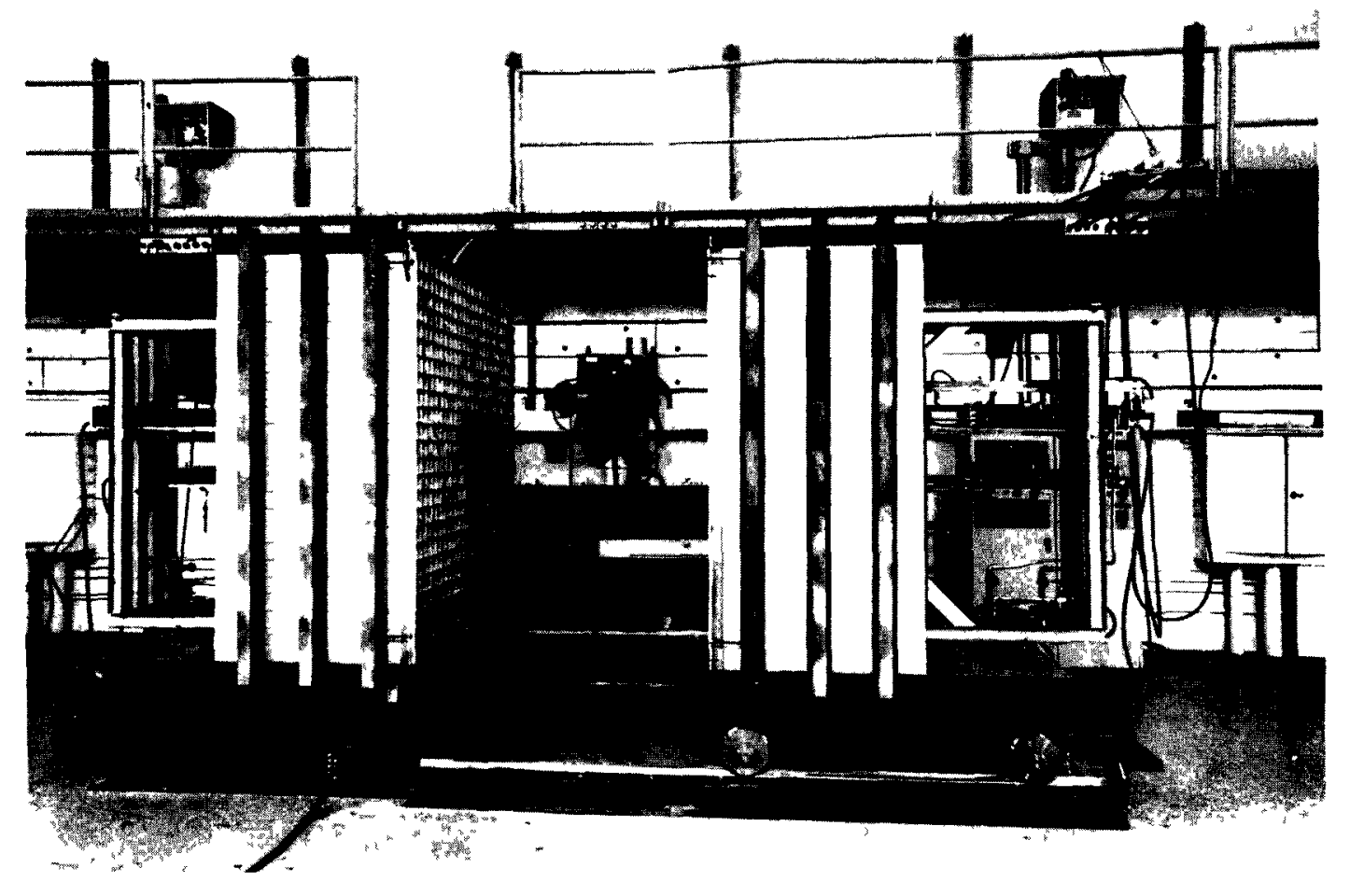

Slide 5. Photograph of the IASI Honeycomb assembly machine. The movable section (right) is in the withdrawn position and the aluminum matrix is only partially loaded.

These are just a few examples that I have run into, and after going around on these a few times, I have come to the conclusion that we have quite a bit more to learn before we fully understand accidental power excursions.

Chernick: Thank you, Bill. There is just one question I would like to ask you. People are always trying to put upper limits on these excursions and come up with magic numbers, and so on. I wonder what your feeling is about the range of total fissions which might be possible in accidents?

Stratton: I don't think there is any real upper limit. For a slow insertion of reactivity it has been suggested a number of times--stu Forbes suggested it to me--that phase changes constitute a limit, where water starts to boil, or metal starts to melt, or to vaporize. The fission density corresponding to such a change would be a rough upper limit for the special case of slow insertion rates. If you want to dump enough reactivity in, it's pretty hard to find an upper limit. And then we get into cases like the Y-12 incident last fall, where the system just sits. It goes on until enough water has boiled off. One can get into some very large numbers here. 
Chernick: I would now like to call on the next panelist, Stu Forbes from Ramo-Wooldridge, to talk about the dependence of burst behavior on the specific shutdown law.

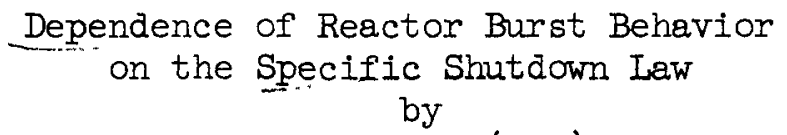

S. G. Forbes (R-W)

Forbes: Now, one of the things that the people in the HEB and ACRS always ask about, and certainly would like to have, is some sort of equation for the figure of merit of a reactor as far as safety is concerned. Presumably, this would have all the possible design parameters in it and you put them in and turn the crank and out you get a score or grade for this reactor which you decide is suitable or unsuitable. This, of course, is quite unrealistic and probably will never be done, but we thought it would be interesting to try it for the very few reactor parameters which are conceivably at the disposal of the reactor designer and see what might be accomplished. One of the things we have noticed in the various models, both our own and other people's, is that very often the dependence of, say, things like the maximum energy release, peak power, and what not, comes out quite different in its functional form depending upon what the model looks like. And this led to some work, which is just getting written up in report form $(1)$, which I will say a few words about.

Actually, a number of the models were worked on and treated in this report. I'm only going to talk about one, and the generalization of this one, to show for two different simplifications of this generalized model-how much they differ in their behavior. Slide 1 is an electrical analog of the simple conduction heat transfer, lumped-parameter system. We have

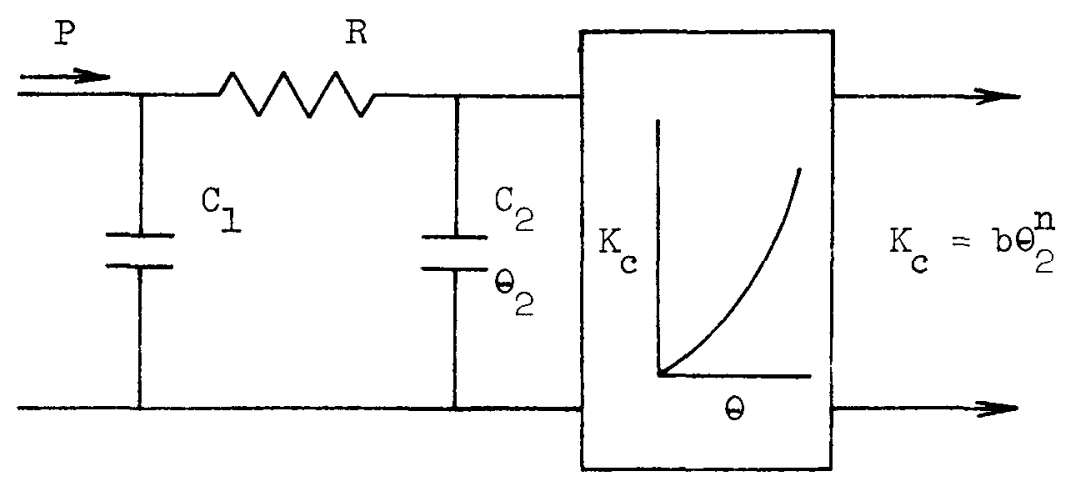

Slide 1. Electrical Analog of a Reactor System 
the power input, $P$, from fission; we have the heat capacity of the fuel plate, marked $\mathrm{C}_{I}$, the thermal resistance, $\mathrm{R}$, at the plate-water interface; and heat capacity $\mathrm{C}_{2}$ of the water channel, again Iumped; the moderator temperature, which we will call $\theta_{2}$; followed by some sort of nonlinear function generator, represented by the box, in which the reactivity compensation is some nonlinear function of the temperature. Out of this we get a $\mathrm{k}$ compensated. The simplest nonlinear function that you can handle analytically is just a power law; $\mathrm{k}_{\mathrm{c}}$ is some constant times the temperature rise to the $n^{\text {th }}$ power, where $n$ is now the disposable parameter and actually changes the model. For example, there might be, say, a square law. The expansion of water is approximately that kind of law below about $250^{\circ} \mathrm{C}$. Then the prompt approximation to the reactor kinetics equations can take this kind of form

$$
\begin{aligned}
& \frac{\dot{P}}{\mathrm{P}}=\alpha_{0}-b \psi^{n} \\
& \psi=\int-H_{-}-\int P a t^{\mu}
\end{aligned}
$$

and I'm already generalizing from this model. The usual thing, the period, the inttial step, and some function $\psi^{\text {n }}$ where $\psi$, for the case I showed, would be the double integral of the power, but we might conceivably have a series of such integrators. (This, of course, is only appropriate for the high frequency limit of an R-C clrcuit of this type. The solution for the low frequency limit is simplified a great deal). There might be many such integrators in the feedback loop. So $\mu$ now becomes a disposable parameter along with $n$ and this becomes a general model from which the simple energy model, the long-delay model and many others can be derived just by selection of $\mu$ and $n$. This can, believe it or not, be solved in the sense that we can get an expression for the peak power and, in this case, the energy release, $E_{M}$, at the time of peak power, in terms of initial step, $\alpha_{0}$, the shutdown coefficient, and $\mu$ and $n$ which

$$
\mathrm{E}_{\mathrm{M}}=\alpha_{0}^{u-1}\left(\frac{\alpha_{0}}{b}\right)^{1 / n} \cdot \mathrm{c}
$$

are the disposable parameters in our model, times a constant. This constant, $C$, is a particular value of an unnamed function which is the solution to a dimensionless nonlinear, differential equation of order $\mu$; but nevertheless, all the parameters and constants are determinable; you get a recursion formula so you can crank it out on the machine and find that constant. It's a number, just from very general considerations, between $I$ and 2 at the time of maximum power. This energy expression gives us what we need for the step case. The analogy between ramps and steps has been carried out for the simple energy model (I believe sombody mentioned Smets' work this morning), and other people have done it 
too. It was possible to derive the same general expression under certain conditions,

$$
\alpha_{\mathrm{m}}=\sqrt{\frac{2 \dot{k}}{2} \ln \frac{\mathrm{P}_{\mathrm{M}}}{\mathrm{P}_{\mathrm{O}}}}=\alpha_{\mathrm{O}}
$$

an approximate relation between the maximum alpha, minimum reciprocal period, the ramp rate $\vec{k}$ over the lifetime, times an implicit function here, of the peak power over the initial power (the natural log of this). Under certain conditions, as long as this ( $10 \mathrm{~g}$ ) term is relatively large, then, this is a very good representation. Under those same conditions we can equate $\alpha_{0}$ to $\alpha_{\mathrm{m}}$ and use this expression to establish the equivalence, just as we did in the simple model, for this more general model. Now, let us suppose that we are going to take as a figure of merit for a reactor, either the maximum permissible step, or the maximum permissible ramp which will just lead to, say for example, fuel plate melting; then the energy release has to be kept under that which will just produce melting of the core and we can immediately say how the functional dependence of these figures of merit--what the functional dependence will beon the things we will call the design parameters: the peak energy that it will tolerate, the void coefficient, and the prompt neutron lifetime. Let's take two cases here, using two different figures of merit, and see what the functional dependence is.

\begin{tabular}{|c|c|c|}
\hline & $\Delta \mathrm{k}_{\mathrm{M}}$ & $\dot{\mathrm{k}}_{\mathrm{M}}$ \\
\hline $\mathrm{n}=\mu=1$ & $\mathrm{E}_{\mathrm{M}}^{1} \mathrm{C}_{\mathrm{V}}{ }^{1} \ell^{0}$ & $\mathrm{E}_{\mathrm{M}}{ }^{2} \mathrm{C}_{\mathrm{V}}{ }^{2} \ell^{-1}$ \\
\hline $\mathrm{n}=\mu=2$ & $\mathrm{E}_{\mathrm{M}}^{2 / 3} \mathrm{C}_{\mathrm{n}}^{1 / 3} \ell^{2 / 3}$ & $\mathrm{E}_{\mathrm{M}}^{4 / 3} \mathrm{C}_{\mathrm{V}}^{2 / 3} \ell^{1 / 3}$ \\
\hline
\end{tabular}

The first row of the table is the simple energy model in which both $\mathrm{n}$ and $\mu$ are one (this is the same old thing we've all seen) the permissible step, $\Delta \mathrm{k}_{\mathrm{m}}$, goes as the first power of the permissible energy, goes as the first power of the void coefficient, the zeroth power of the lifetime so it is independent of the prompt neutron lifetime for the step-case. In the ramp case we see that the permissible ramp rate lingoes as the energy squared and inversely as the prompt neutron lifetime. Let's look at the next case, the second row in the table, which is really the one I had in the network in which both $\mathrm{n}$ and $\mu$ are two--a square law nonlinearity and integration. For the step-case we find $E$ to the $2 / 3$ power, so if you make a change in say, the melting point, we gain not too fast; $1 / 3$ power of the void coefficient (it isn't going to do much good to change this); the $2 / 3$ power of the prompt neutron lifetime. Here we find in contrast to the simple model that it does make a difference, even in the step case, what the lifetime is. Finally, the ramp case for this simple nonlinear model (and you can work out lots of others), 4/3 power of the energy, $2 / 3$ power of the void coefficient, and the $1 / 3$ power of the lifetime. And I would like to point out a couple of things; suppose that you have a reactor and it looks like it is not too good from a step or a ramp 
toleration standpoint. You say, "Well now I'll raise the melting point of the fuel, that ought to help me. I'll use stainless steel instead of aluminum". If you have this simple model that's right. You will gain as the square of the melting point roughly, if the heat capacity is about the same. In this nonlinear model, it isn't going to help you nearly so much. The same is true with the void coefficient, you gain a lot on this linear model; you gain not very much in this (nonlinear) case; as a matter of fact, this general weakened dependence from nonlinear models on void coefficient, which Schroeder mentioned, has been demonstrated in the spert experiments. Most interestingly here, we have the inverse lifetime effect and here we have a positive exponent. Now, in the generalized model, this has limits of minus one and plus one for the limits of $n$ and $\mu$ large or small. So, we find that not only are we not sure (if we don't know the coupling equation) which of these things to try to vary but, in this case, we don't even know which way to go. The short lifetime reactor in a prompt shutdown, a linear shutdown, has a distinct advantage in a ramp over a long lifetime reactor. On the other hand, if you have these nonlinearities, it's the other way around. So we find, and this is the point I really want to make, that in trying to set down even these very elementary figures of merit, and on some very simple types of models, we find a very wide variation in the functional dependence on the parameters. So a reactor designer will, at the very least, have to have a general notion of what kind of coupling he has, before he has any idea of which of the parameters he should try to adjust and even to know whether he should increase or decrease these particular design parameters.

\section{REFERENCE}

1. S. G. Forbes, "The Dependence of Reactor Behavior on the SelfShutdown Mode", IDO-16635, Apri1 25, 1961. 
SESSION $V$

PANEL PRESENTATIONS

Chernick: The next panelist is Dr. Ergen of the Oak Ridge National Laboratory who is going to speak about the developments in reactor safety at the Oak Ridge National Laboratory. Dr. Ergen.

Developments in Reactor Safety at ORIN by W. K. Ergen (ORNL)

Ergen: In deference to the title of this session which concerns itself with reactor-kinetic computation, I just want to mention that ORNL has embarked on the problem of computing Doppler effects in a fuel element which has a nonhomogeneous temperature distribution inside its volume (I). This, of course, is of interest in reactor kinetics because if you, say, add a delta function in power to the reactor and then see what happens thereafter, the heat will flow out of the fuel element, the temperature distribution in the fuel element will change and the effect will probably be different from what you've guessed if you just followed the change in the average temperature.

A second point I want to mention since something was said in the introduction to this meeting about the dissemination of information. The Oak Ridge School of Reactor Technology is offering two courses, one for reactor operators and one specifically on reactor safety, but I must state that the transition from the original reactor-technology course to these specific courses is somewhat gradual, partly because of the philosophy that a good reactor-safety man should understand the reactor theory, and partly because of the fact that it is not very easy to produce a well organized safety course (even if you know the subject) in a step-function fashion. Year after year the courses become more safety oriented, changing more or less continuously from the original curriculum of the reactor school. Also, in line with this dissemination of information there are a number of journals being edited at the laboratory and I believe $\mathrm{Mr}$. Blizard wants to say something about this this afternoon.

What I mainly wanted to mention was the three reactors which ORNL is proposing to build in the foreseeable future. I want to mention, what constitutes, at least in my personal opinion, the main safety feature by which each of those reactors is somewhat different from those reactors which receive, justifiably, the main attention these days: namely, the boiling-water reactors, pressurized-water reactors, and so on. The first one I want to mention is the High-Flux Isotope Reactor (HFIR) which is being built, as the name implies, to produce isotopes and, in particular, transplutonium elements. There is a very high premium of increasing the flux for this particular purpose and the elements which we hope to produce have half-lives of the order of years down to about 60 days, so it is the average flux over a period of this length which counts and, consequently, it would be very sad if the reactor would be unreliable and would have a great fraction of downtimes because this would decrease the average. 
However, with this limitation of minimizing the downtime, it is the deliberate intent to drive the known technology of essentially MTR-type reactors (light-water-moderated, plate-type reactors) as far as one can go. One of the things that is being done, obviously, is using a flux trap (which is a water volume which contains the target material) and surrounding it by two concentric, cylindrical fuel elements (to get away from corners which would occur with a rectangular shape). Each of the fuel elements, Slide 1, has involute plates which have the property that the gap is constant throughout the radial direction--it looks somewhat like a wet book. The distance between the plates is somewhat smaller than they are in the MTR-ETR; originally, it was a 40-mil plate and a 40-mil gap. This has been relaxed by $10 \mathrm{mils}$. They also use such tricks as shaping the fuel loading inside each of these plates. It is really an effort to drive the technology as far as this can be done. The power density, and also the maximum of the heat flux out of the element, is not much greater than it is in the ETR but, the point is, here this is going to be over the whole volume whereas in the EIR the flux I was referring to is at the hot spot which occurs only in a fraction of one of the fuel elements. The main safety problem in this reactor, I think, is that by reason of this flux trapping the removing of water from the trap causes a positive reactivity change so that the overall temperature coefficient, or void coefficient, would be essentially zero or only slightly negative. The other problem is that the distribution of fuel and water is not optimum to get the maximum reactivity; in other words, if there is a change in distribution due to an accident it could add reactivity. One is, of course concerned about the -shaped fuel loading giving rise to

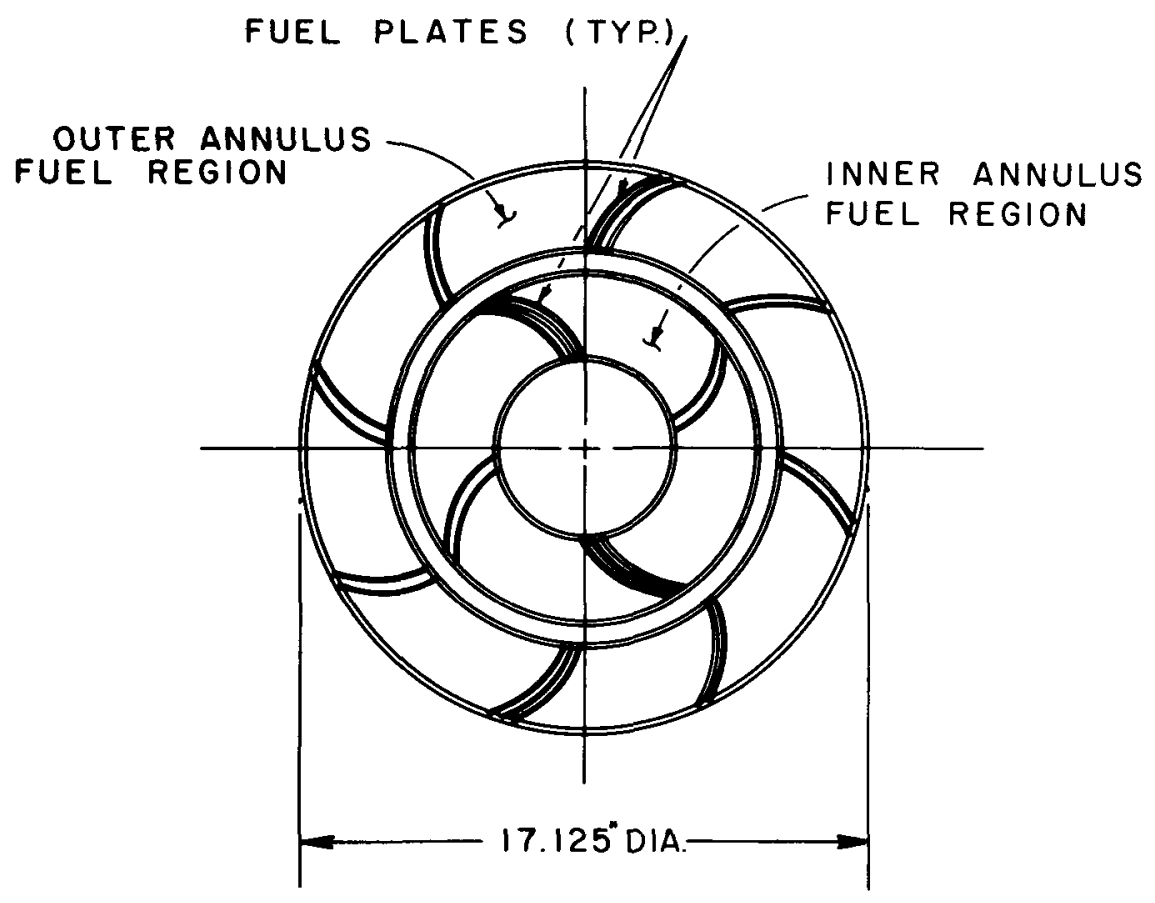

Slide 1. HFIR Fuel Assembly - Horizontal Section 
unknown stress distribution and stability. This is very, very carefully looked at, just for this reason. These are, I think, the salient features in this reactor.

The second reactor $I$ want to mention is a Godiva-type reactor which should allow a little more power to be extracted because the gamma phase of uranium is stabilized by addition of 10 per cent molybdenum. It will give only one pulse per half hour, the pulse length is $38 \mathrm{msec}$, the maximum power is $63,000 \mathrm{Mw}$. In the stationary state, the reactor will run at $1 \mathrm{kw}$, or for a short time at a maximum of $10 \mathrm{kw}$ if air cooling is applied. The maximum accident in this reactor is considered to be the following: if some animal to be irradiated comes close to the reactor with some hydrogeneous part of his body and he stands there just right then when the burst rod is withdrawn, this would give a greater burst, of course, than was anticipated, analogous to the accident Mr. Stratton described. This would, in this reactor, give 1019 neutrons (the final safety report has not been written, but the main theme the report is probably going to use is this). The reactor has such a small heat removal capacity that it would completely disintegrate before it produces any appreciable amount of radioactive fission fragments. It is going to be remotely operated in a remote location. The accident, postulated above will give only something like 100 curies of iodine, for instance.

Finally, the last reactor I want to mention is the molten-salt reactor, MSR, which is a conservatively designed descendant of the somewhat fantastic aircraft reactor. Its main purpose is to demonstrate, and to convince the world, that reactors of this type can be operated safely. It is a liquid-fuel reactor, such as the aqueous homogeneous reactor, except that it uses molten fluorides--lithium beryllium fluoride with zirconium and uranium fluorides in it--as the working fluid. It has the nice property that the xenon comes out during operation and consequently one doesn't have to make an allowance for xenon poisoning. It cannot have a loss-ofcoolant accident because the coolant is the fuel itself and would drain out. Due to the fact that it is homogeneous--in the same sense that Triga is-it cannot have a cold-water accident. Also, it cannot possibly have a rod-withdrawal accident because it doesn't have any control rods, at least according to the present plans; however, of course, this is fictitious because you have to bring it to criticality and keep it there by feeding it pills of concentrated uranium and, if the pill-vending machine would go crazy, you would have the equivalent of a rod-withdrawal accident but you just have to design around this. From the reactor dynamics viewpoint, a point of interest is that an accident that one can visualize with this reactor can raise the operating temperature (which is normally around $1200^{\circ} \mathrm{F}$ ) to about $1350^{\circ} \mathrm{F}$; this is the worst accident anybody could conceive. This is $1350^{\circ} \mathrm{F}$ as compared with 1500 or 1600 at which the ARE, its predecessor, has operated and as compared to $2000^{\circ} \mathrm{F}$ which would be the temperature at which real damage would occur. The low temperature, $1200^{\circ}$, comes from the necessity of running a long time at low corrosion rates and the small rise comes from the low power density of this reactor.

The salient feature I would be concerned about in this reactor is that this particular model is graphite-moderated and though the power density is very low, if compared to its fantastic predecessor, it is quite 
high for a graphite-moderated reactor, considerably greater than for the gas-cooled reactor, for instance, and the deformation of the graphite could occur. If you just plug up one of the channels it would't hurt anything, you couldn't tell the difference, and the thing you really want to worry about is how do you tell the difference if enough channels plug up to cause you trouble.

Well, this is all I wanted to say in review of our reactor-safety developments.

\section{REFERENCE}

1. L. Dresner, "Some Remarks on the Effect of a Nonuniform Temperature Distribution on the Temperature Dependence of Resonance Absorption," Nuc. Sci and Eng., 11, No.1, 39 (1961). 


\section{PANEL PRESENTATIONS}

Chernick: Right now we will call on the next panelist, Stan Johnson of Bettis, to talk on analytical models for burst transients with special consideration of a resulting pressure effect.

\section{Analytical Models for Burst Transients* \\ by}

S. 0. Johnson (W-Bettis)

Johnson: We at Bettis, like everyone else, must give quite a bit of consideration to the safety problems when we make a new facility or when we make changes in an existing facility. Part of this safety analysis is, of course, the response of the reactor to the maximum credible accident; and we must for each of these reactors, by some hook or crook, calculate what the response of the reactor will be. There are several models for doing this, many of them quite simple, quite approximate. The difficulty with this type of analysis is that you must make a number of simplifying assumptions, each of which must be in a conservative direction. This can pile conservatism on top of conservatism and pretty soon, by use of some of these models, you can predict that Spert should blow up on a 100-msec period. Obviously this can lead to severe restrictions on the operation of the facilities and the type of the experiment which can be done. To attempt to get around this problem of excessive conservatism, we have taken the different path of using a relatively complicated model--one which can be subjected to machine computations and in principle, at least, can be made as detailed as we wish. One such model was presented at an ANS meeting in Washington about a year ago--I guess it was the winter meeting(1). I would like, just to get us on the same grounds, to talk for a moment about it. It's quite straightforward. It is the conduction model which schroeder mentioned. It has one departure from the usual. Prior to bolling it is essentially a three-region conduction model, see Slide 1 , having a meat, a clad, and a water region governed by the

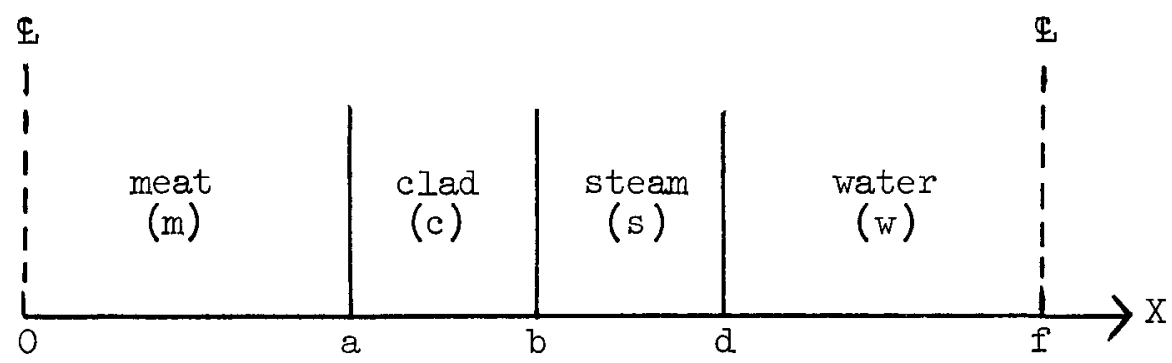

Slide 1. Regions for Conduction Mode1

*This paper was issued as WAPD-OP-11. 
normal heat conduction equation. This can be solved on the computer in as much detail as you wish, within the limitation of the size of the computer, and so for that simple system in which there is no boiling you should be able to get a rather detailed estimate of the behavior. You can put in as many reactivity coefficients as you wish--for instance, the expansion of the fuel, Doppler effect, heating of the water, and so forth--and feed these back continuously, so that it is a continuous feedback model. As in all models the diffieulty arises when you try to consider boiling, and I think the discussions yesterday indicated why; there is a lot of progress in the field of boiling, but we still don't know an awful lot about it. In this model we took an easy way out, one which was relatively easily solved. We assumed that when the plate got to saturation temperature or some arbitrary temperature above saturation we immediately went into film boiling without any nucleate boiling. The departure, then, from the usual model in which you assume a certain fraction of the total heat leaving the plate goes into the formation of voids is indicated in the following equation:

$$
h+g \frac{d}{d t} \int_{b}^{d} \rho_{s} d x=-\left.k_{s} \frac{\partial t_{s}}{\partial x}\right|_{b}+\left.k_{w} \frac{\partial t_{w}}{\partial x}\right|_{d}
$$

We assume that when boiling begins the surface of the water at the steam-water interface remains at the saturation temperature. Having done this we can calculate the heat flux into the water--having the gradient in the clad you can calculate the heat flux into the steam. This particular model then neglects heat capacity in superheat of the steam, so the difference of those two heat fluxes must go into the generation of steam. This is the approximation presently in the model. We hope to improve upon it considerably as more information becomes available.

Now, of course, any analytical model is only as good as it predicts experiment. We have the Borax and Spert experiments to compare it with, and we attempt to do this continuously as data become available. Slide 2 shows not a comparison, because we do not yet have the data. There has been, I think, considerable worry about the effects of pressure, and this is part of the impetus for Spert III studies. We have put into this model coefficients for the fuel plate expansion which ejects water, coolant expansion from direct and conduction heating, and steam void generation, and we examined the behavior of the peak power and the fraction of the total reactivity compensated at peak power as a function of the initial system pressure. We start at atmospheric--the power scale here is arbitrary and would match approximately the Spert III results if we assume that the major fraction of the energy is released in about 20 per cent of the core volume. There is some normalization required. We indicate, then, that at atmospheric most of the shutdown is from the steam void generation, amounting to about 60 per cent. We 


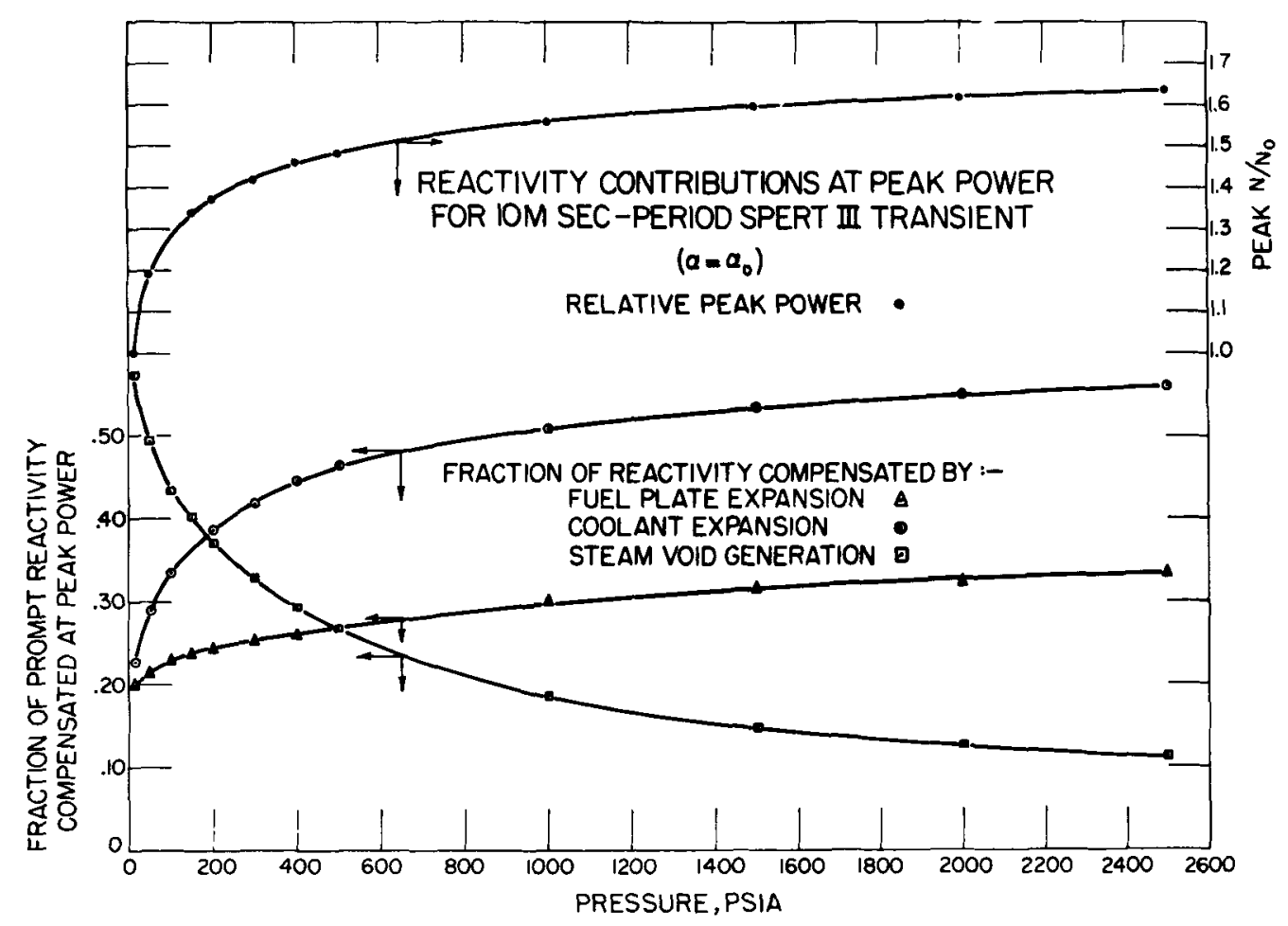

Slide 2. Calculated Transient Reactivity Contributions for $\alpha=\alpha_{0}$

get about equal contributions from fuel plate expansion and coolant expansion. As we increase the pressure, as you would expect, the fraction that is compensated by steam void generation goes down and the fraction attributed to the other two effects increases. We note that even pressurizing up to 2500 pounds with this particular model gives you an increase of only 60 per cent in the peak power, and that most of that increase comes within the first 400 pounds of pressurization. I should mention that we have an alpha (effectlve coefficient of expansion of fuel) equal alpha zero where alpha zero is the linear coefficient of expansion of the fuel plate. We were using the model at the time to investigate the importance of these various effects-how important it was to calculate how much the fuel plate would expand. This assumes that it expands only in one direction. Slide 3 is the same sort of thing using an effective coefficient of expansion of the fuel of 1.8 times the linear coefficient. This assumes that you neglect the axial expansion--we have not completely calculated it yet--but it takes two directions in the radial or in the horizontal plane, one of which is restricted because of the configuration of the fuel, and the 1.8 then comes from the Poisson ratio. We note that with that change of approximately a factor of 2 we get somewhat different effects-boiling is suppressed at 2000 pounds. Unity on the power scale corresponds to approximately 0.85 on the previous slide. In this case we 


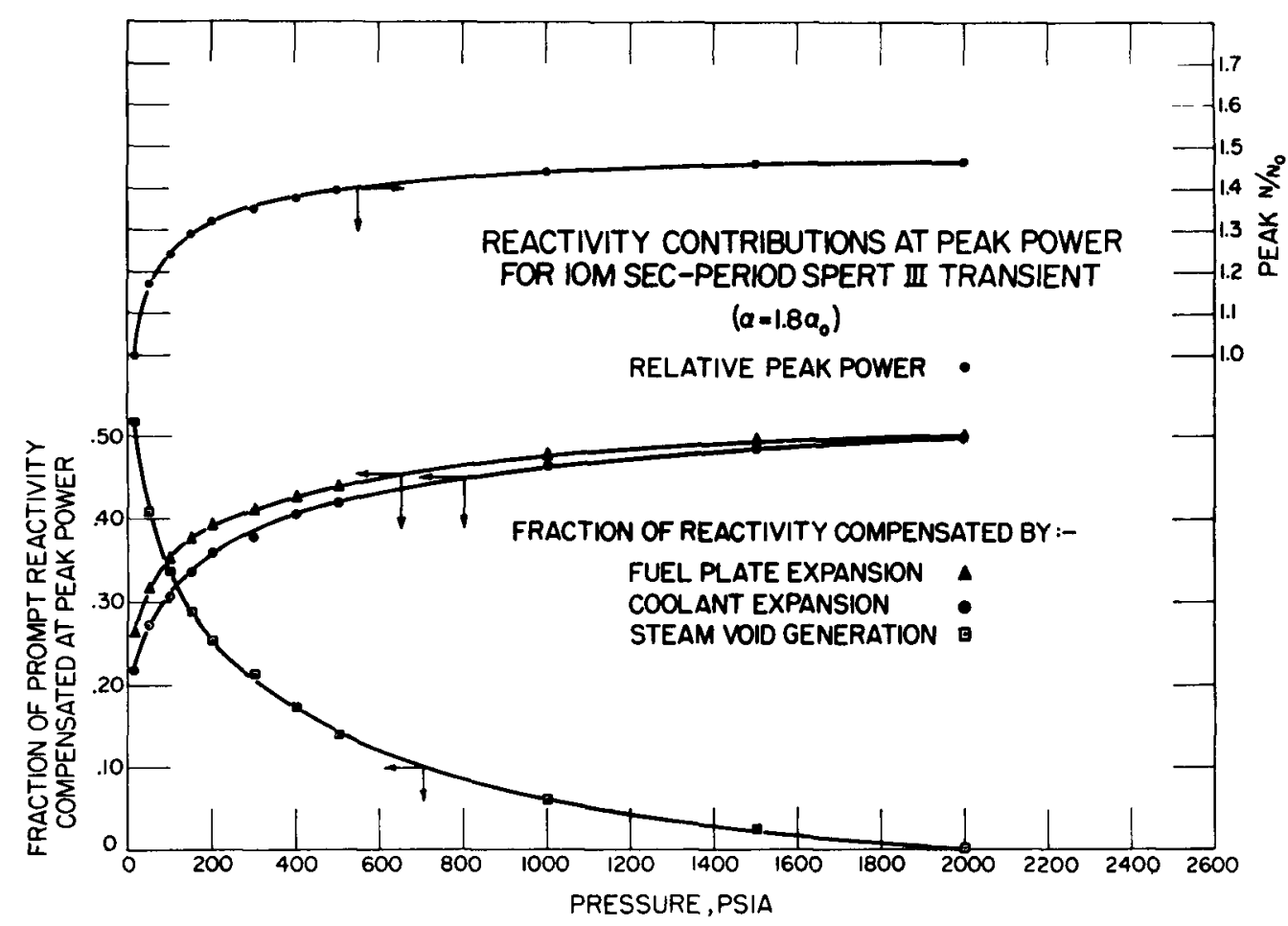

Slide 3. Calculated Transient Reactivity Contributions for $\alpha=1.8 \alpha_{0}$

we get something like a 45 per cent increase in the peak power with pressurization. By word of mouth from Frank Schroeder this seems to give the proper trend. I don't think we have solved the problem of correctly predicting absolute magnitudes. We certainly hope to do much better with this model in the future, and try to get some of the conservatism out of safety calculations so that we are less restricted in our safeguards considerations. We are using this model, then, to investigate the importance of the various shutdown mechanisms. We have not yet considered Doppler coefficients--these are U-235 cores and there are questions as to the magnitude and even to the sign of the Doppler coefficient in U-235. We are looking somewhat into the possibility of microbubbles and radiolytic gas generation. These will take some time. They can be easily incorporated into the model, and their effect can be investigated, at least in a qualitative way.

Chernick: Thank you. Do I understand you to say that you have got a complete steam blanket around the fuel in this model?

Johnson: In this model we have a complete steam blanket over that part of the fuel which is above saturation or some arbitrary temperature above saturation, and that which is still subcooled is considered to be in contact with water. 
Chernick: I just wonder what the plate temperature would go to--have you looked at that?

Johnson: Well, these are calculated for some conduction models. On some tests of this model we did against spert I, our surface plate temperature was somewhat below what their thermocouple indication was. Just inside the plate, or $1 / 6$ of the way through the clad, I believe, i.t was above; so we seem to straddle, then, their thermocouple indication.

Wright: Did you say that you included inertial pressure effects on the model?

Johnson: We have done this. For the transients which we have been studying, the inertial pressure does not seem to be important in the range of' $10 \mathrm{msec}$. It becomes important, I believe, starting at about $5 \mathrm{msec}$, depending on the core configuration. We do have that provision in the model.

\section{REFERENCE}

1. W. A. Vogels and S. O. Johnson, "Simulation of Prompt-Critical Excursions in Water-Moderated Reactors," Trans. Am. Nuclear Soc., ㄹ, (2) p. 58 (1959). 
SESSION V

PANEL PRESENTATIONS

Chernick: I would like now to call on Dick Cohen who is going to tell us something about Ixtraction of Reactivity from Power Traces.

\author{
Discussion of Extraction of Reactivity from Power Traces \\ and Equilibrium Bubble Size Analysis \\ by \\ E. R. Cohen (AI)
}

Cohen: Actually the title that Jack quoted is a little bit too general. All I am really going to do is discuss the procedures that we have been using at $A I$ in the extraction of the reactivity from the Kewb transient power traces. We've had quite a bit of difficulty with this problem, and therefore it is probably worthwhile, at least to point out some of the pitfalls in hopes that other people will be able to avoid them.

The power trace in a Kewb transient is recorded on a strip chart using linear ion chambers which are not able to cover individually the full range of the power excursion. We have to either change scale, or equally, jump from one detector to another to trace out the power excursion over its full range. Therefore, on the strip charts, the output consists of a trace that starts up on an initial rise, runs off scale, and then a second one picks up, and then a third detector channel may get the peak. We may then have several sections coming down on the other side. The problem is to take all of these five or six sections of trace, normalize them, put them together into a single power trace from which we can proceed to further steps. Slide 1 is a direct photograph of the Kewb transient strip chart record. As usual in these charts time increases toward the left. The transient represented had a $3.18 \mathrm{msec}$ initial period and reached a peak power of $218 \mathrm{Mw}$. The high frequency sine wave trace is a $2.5 \mathrm{kc}$ time standard. The saturation of two channels is clearly evident and one of the remaining minor problems in the analysis is to be sure that the various segments which we analyze are terminated before the saturation effect has distorted the curve. If this is not done an extraneous reactivity variation appears in the output. The reproduction is slightly overexposed and the actual strip chart trace is somewhat sharper, the lines being approximately half as broad as indicated in the photograph. The traces are read on an automatic reader and converted to a digital form. We have, after quite a bit of work getting all the bugs out, a digital code which takes the last few points of the first trace and matches them against the first few points of the second trace to determine a normalization factor which rescales these to a common base. There is, of course, the problem of throwing away the noise in the lower end of trace $B$ and the saturation effects in the upper end of trace $A$, but we have conditions built into the code which evaluate this. After the data have all been renormalized, we found that it is then necessary to make at least one sweep through the essentially raw normalized data to smooth it, in order that we don't get extraneous noise in our further analysis. What we are now doing is fitting the logarithm of the power to a cubic polynomial with a running ll-point smoothing function. This smoothing operation 


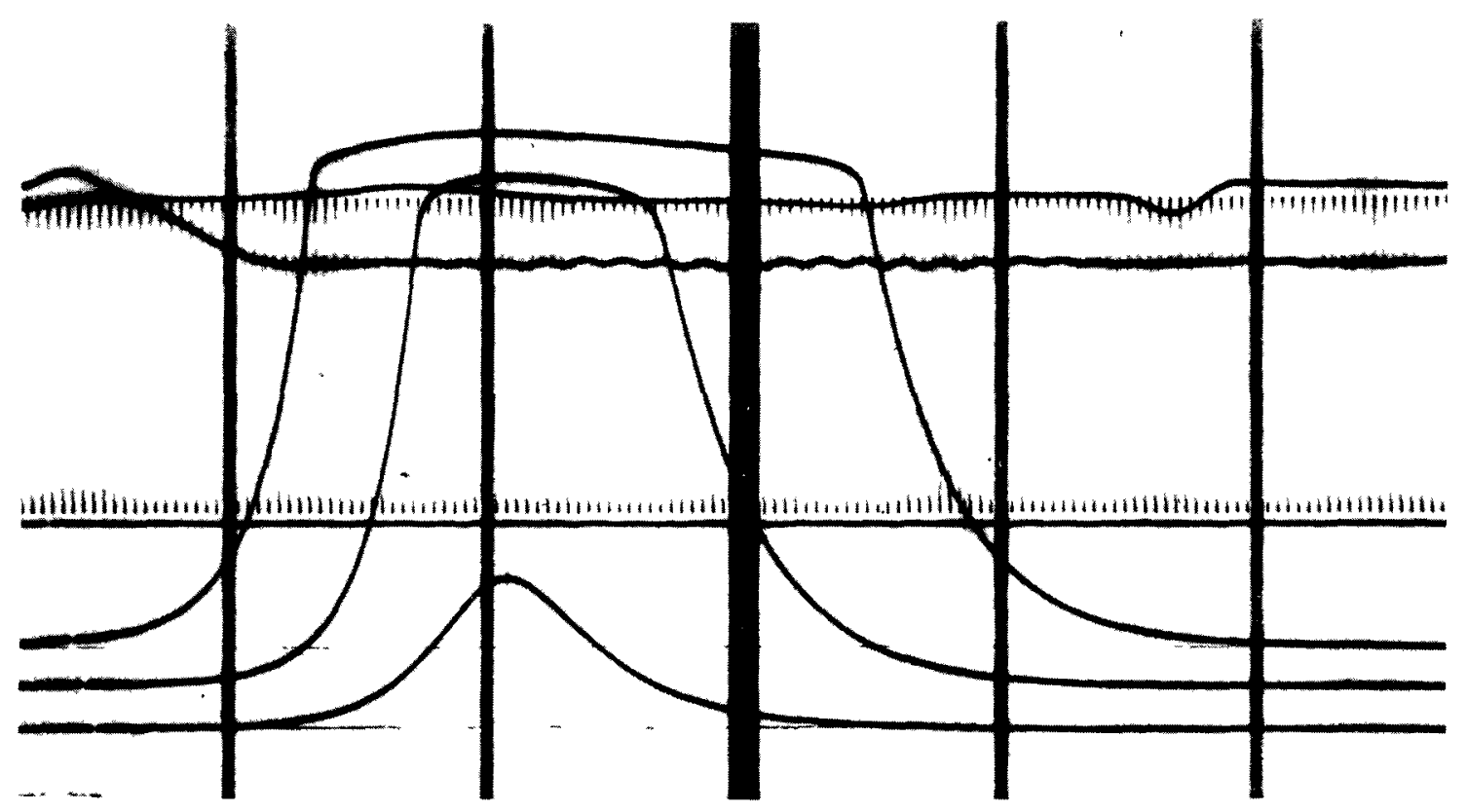

\section{Slide 1. Kewb Transient Strip Chart Record}

fits the cubic by least-squares (five points to each side of the point you are interested in) from which we evaluate not only the logarithm of the power but also a quadratic fit to the instantaneous inverse period-that is, the derivative of the logarithm of the power. Then, with this instantaneous effective inverse period, we take the kinetics equations (and since Al Henry has already written them down this morning, I don't have to repeat them) and solve for the reactivity which means, of course, that we have to perform an integration over the delayed neutron components in order to be able to subtract out that component and get the reactivity itself. This is a fairly involved program--as I say, I think it finally works--and now that we finally have the code going, we have been able to analyze a hundred power traces that have been accumulated over the last few years in about a half hour or so of (IBM 709) machine time.

The other point which I wanted to make, completely separate from this, is a discussion that perhaps should have been thrown in last night but which, because of the lateness of the hour, I postponed, taking advantage of my position today. This is to describe some work that Jim Bick has done at AI on the equilibrium bubble size when a vapor bubble forms on a heated plate. Slide 2 represents isothermal contours for the bubble on a heated plate whose surface is held at constant temperature. $T^{\prime}$ is the dimensionless temperature expressed in terms of the difference between the plate temperature and the bulk fluid temperature. The concentration of isotherms at the "cornex" produces a logarithmic singularity in the heat flow and makes an equilibrium solution impossible. 


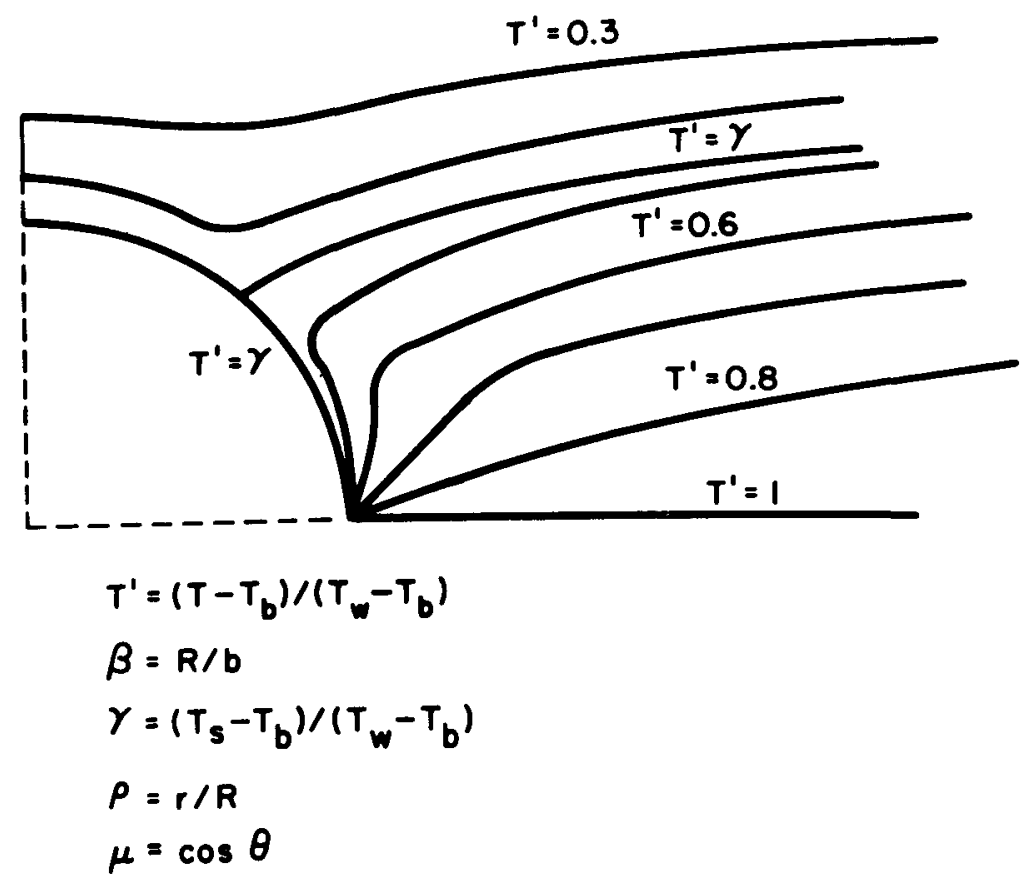

Slide 2. Isothermal Contours for Bubble on Heated Plate

The problem is one of having a hemispherical bubble sitting on a plate, the wall temperature, $\mathrm{T}_{\mathrm{W}}$, being greater than the saturation temperature, $\mathrm{T}_{S}$; and we assume that at large distances from the bubbles, there is a temperature gradient characterized by a parameter $b$, so that $T$ is equal to $\mathrm{T}_{\mathrm{wall}}-\mathrm{bx}$ and we assume that the bubble wall is at temperature $\mathrm{T}_{\mathrm{S}}$. We then ask what the temperature isotherms are, and what the equilibrium bubble radius is. Well, as this problem is stated, unfortunately, it has no solution; because, if we look at the corner where the bubble wall meets the plate, we have two different isotherms coming together and all the isotherms between $T_{W}$ and $T_{S}$ come into a singularity at this point. Just at the corner there is an infinite temperature gradient; the net result being that there is a logarithmic infinity in the heat flow into the bubble, so that there is no equilibrium radius. It took us a little bit of worrying before we actually realized this. We were finding that the equilibrium radius depended upon our approximations-on the order of the Legendre polynomial expansion, and then we realized what the actual cause was. The solution to this difficulty was to specify the wall temperature only at infinity, and to consider the conductivity of the base-plate as well as the heat flow in the water. We now have a three-region problem: the water, the plate, and the bubble. There is a problem about what the temperature boundary condition is on the metalvapor interface, but this is bounded by considering either that this section of the wall is at temperature $\mathrm{T}_{S}$, or by considering that there is no heat flow across that section of the wall $(\partial \mathrm{T} / \partial \mathrm{x}=0)$.

The problems of circulation of the vapor in the bubble we've essentially ignored, because if there is circulation, then there 
probably also is not a hemispherical shape to the bubble contour, and therefore our entire problem is violated. So, with the conditions as stated, we can expand $T(r, \theta)$ in Legendre functions

$$
T(r, \theta)=\Sigma A_{n} r^{n-1} P_{n}(\cos \theta)
$$

and extract the equilibrium radius in terms of a ratio of two infinite determinants, but we find, in fact, that by considering fifth order, sixth order, tenth order, fifteenth order, the ratio converges quite rapidly and we then can get the equilibrium bubble radius. This, of course, in itself, is not important. It points out, however, that there are some difficulties; for example, in Griffin's work in which he considered the original boundary conditions that I quoted he apparently finds an equilibrium bubble radius. The problem that he was solving, however, was really the initial transient problem, so that the fact that the equilibrium was false is not really of too much importance to him. It does, however, indicate that you've got to be a little careful in trying to extrapolate some of his results. The equilibrium that he found, I'm sure, was the result of his finite mesh size; and had he used a different mesh size, we would have found that the equilibrium radius would probably have gone as something like the logarithm of the spatial mesh that he was using.

Having extracted the equilibrium radius, we are now ready to work on the transient problem of the growing bubble and the temperature distributions on the wall and in the liquid. And we are, if not optimistic about being able to get a solution, not being pessimistic about it, and I think we will be able to push the problem further.

Chernick: Thank you. 


\title{
SESSION $V$
}

\author{
PANEL PRESENTATIONS
}

Chernick: We finally have someone to talk about fast reactors from Argonne; we've been dominated by thermal reactor people. Harry Hummel will speak about fast reactor kinetics.

Fast Reactor Kinetics

by

H. H. Hummel

Hummel: Well, in fast reactors one is primarily concerned with thermal expansions and related small mechanical motions of the system which are more important in fast reactors because they're smaller than thermal reactors. Because of this, the behavior of the fast reactors tends to be fairly specifically tied to the particular idiosyncrasies of design, so that it is somewhat difficult to extrapolate abnormal behavior from one fast reactor to another, unfortunately. So far we haven't had the interesting boiling phenomena that have taken up so much of the attention of this meeting, although there has been some mention of boiling fast reactors in the future, particularly for space applications, and these certainly promise to lead to some very interesting dynamic investigations, but this is not yet.

What I want to do today is, first, make a few remarks about the status of agreement between experiment and theory for the EBR-I experiments, and then go on to say a little bit about calculations for EBR-II. Regarding EBR-I, Mark II, the one that melted down--it had the resonance behavior--I was interested to hear from Dick Smith that recent investigations at EBR-I have given rise to ideas that seem to point the way to explaining those long time lags of about 10 seconds in the negative coefficient-that seem to be necessary to produce the resonance discussed by Bethe in APDA-117--and this has long been a puzzlement to people that looked over this question. It would certainly be nice to feel that this, at least, was solved and possibly if someone is interested in this, Dick will be willing to go into this a little further and discuss what ideas they have about this. I'll now pass on to the Mark III core, which is the ribbed one which eliminated the bowing question which caused part of the difficulty in Mark II. Of course, there's been a long series of experiments concerned with this reactor, and a detailed study of them has been published in ANL-6124 by Carter, Sparks and Jessier. Now, the data showed us marked nonlinearity of the feedback with power--it would be more proper to talk of the describing function, which was what was actually measured, and Carter, et al., were able to explain this by postulating a model in which thermal expansion was restrained by frictional contact among the fuel elements, which led to a sticking and slipping phenomenon. Carter's first investigation was concerned with a purely unrestrained thermal expansion, and it was found that the amplitude of the feedback observed was two to three times less than you'd get with this model, and the phase lag was also two or three times greater. Now, John Long at Idaho used a more accurate temperature integration method 
than Carter's; Carter's work was done on an analog where you divided the rods up into three radial regions and a number of axial regions. Long's work was done with an IBM-704 code, using the temperature integration method described by Fred storrer in APDA-132. Well, in EBR-I, Mark III, they were able to measure temperatures during the oscillations and Long found very good agreement between the calculated and observed temperatures, but he was not able to compute the feedback satisfactorily, which agrees with Carter's experience. Now, Fred Storrer, at the May reactor stapility meeting at Argonne, presented an analysis of fast reactor kinetics $(1)$ in which he studies what happens if you extrapolate to a zero frequencyyou extrapolate oscillator measurements to zero frequencies, and then tie things in with the steady-state power coefficients. At that time he concluded that the time constants observed for the temperature and for the feedback were close enough that he didn't seem to feel that the model of Carter was really necessary in order to explain the time lag. Now, this seems a rather puzzling contradiction which can probably be cleared up with a little more analytical investigation. Well, it's clear that while the model of Carter has not received universal acceptance, anything that's going to work must explain the nonlinearity that's involved, and there's no way you can get this out of unrestrained thermal expansion, I don't believe, so that something more complicated is going to be necessary.

Following the ribbed Mark III experiments some of the ribs were stripped off and further investigations were carried forth, and I don't believe there have been any attempts at detailed analytical investigations of the results. It was found that the feedback amplitude was reduced, apparently by bowing, which, of course, was intended; but there were additional complicating phenomena apparently associated, according to Dick Smith, with swelling, bending and distortion of the fuel rods.

Passing now to EBR-II, it's our current belief that the feedback will be due almost entirely to unrestrained thermal expansion so that if we do a good job of measuring the reactivity coefficients and use $\mathrm{Mr}$. Storrer's temperature integration method, we ought to be in pretty good shape. A series of reactivity measurements on ZPR-III has been completed and will now be put into the code that Long has devised with some--(we've made some) trivial extensions of this to make it more useful for application to EBR-II. We haven't really used these new data yet to obtain a closed loop transfer function. There have been some preliminary investigations with guesses at the reactivity coefficients which indicate that the reactor should be completely stable. It's not possible to get big enough phase lags with the unrestrained thermal expansion to bring one into the region of instability. EBR-II is actually specifically designed in such a way that the upper supporting structure doesn't contribute to the kinetics of the system; the subassemblies themselves are completely supported at the bottom so that this is expected to eliminate any long time lags which would tend to bring one into the region of instability. Now, there are a couple of possible complications; one would be subassembly bowing. This has been examined in the past. It's mentioned in EBR-II hazards report. It's a very difficult thing to estimate. It's not believed that it's going to be a very big effect at all, because considerable effort has been made to keep the subassemblies rigidly supported next to each other to eliminate this phenomenon. 
Of course, there have to be certain clearances in order to get the subassemblies in and out of the reactor, and certainly some small bowing effects can occur. Whether they are going to be enough to be observable is something else. Joe Carter, I think, is planning some additional investigation of this question.

Another possibility that might complicate life is the alpha to gamma transition in uranium which occurs at $550^{\circ} \mathrm{C}--$ Walt Loewenstein put the finger on this question--this is within the operating temperature range of the fuel. Now, this transition occurs in uranium instantaneously, but in fissium--that is, with the recycled fission products that are going to be present in EBR-II, it is believed that this transition is rather sluggish and it may be too slow to be observed in oscillator measurements, but should be present in the steady-state power coefficients. So, this could complicate the application of Fred Storrer's idea of extrapolating from finite frequencies to zero frequencies in order to tie things in with the steady-state power coefficient. Well, we'll be making additional investigations of this point. We expect to do most of the purely linear work for EBR-II on the IBM-704, using the Storrer integration technique. We can do a real good job on the thermal feedback this way and what I would hope to do then is to fit the resulting feedback as a function of omega--possibly feed this into the analog where we would then handle any nonlinear effects that might seem to be applicable, and possibly make a Fourier analysis of these to feed into the thermal portion, so that the thermal feedback would become Fourier series in omega. Of course, the analog setup at present is simply a straight finite difference calculation where there are three radial regions in the pin and eight axial regions. This is not completely satisfactory because in the limit of low frequency I don't believe it would give a very good steady-state temperature distribution in the pin. I guess that's all I have to say.

\section{REFERENCE}

1. F. Storrer, "Analysis of the Power Feedback Relations in Fast Reactors," Proceedings of the Conference on Transfer Function Measurement and Reactor Stability Analysis, ANL-6205, p. 251 (May 1960). 


\section{SESSION V}

\section{GENERAL DISCUSSION}

Chernick: I think we have plenty of time for questions. This is the last paper. Dick?

Smith: I would like to comment on the influence of the Mark II shield plate. This plate, 4 in. in thickness, has a series of broach holes through which the fuel rods penetrate. When a hot burst of NaK hits this plate, the first thing that happens is the plate dishes. As the plate dishes, the axes of the fuel rods are tilted away from the normal, thereby modifying the effective size of the core. Such motions have associated time constants of an order of magnitude consistent with experimental facts.

I would also like to comment on the origin of nonlinearities in the power coefficient. It looks as if nonlinearities are the results of a power-dependent system of clearances. As you bring a reactor from room temperature to operating temperature, the clearances between fuel rods increase, mainly because the stainless steel components expand more than the fuel. As the clearances between fuel rods increase, the radial coupling decreases and the power coefficient is reduced.

If, however, you put power across the reactor, the outer structure remains at constant temperature. The core temperature increases relative to that of the structure so that eventually fuel expansion catches up with the structure and the rods finally become tight; i.e., radially coupled.

There are actually three regions in the power coefficient curve; at low power there is a loss of radial coupling, at a higher level of power the fuel expansion starts to catch up with the structure. Here, there is some radial coupling and the power coefficient increases. Finally, at high levels of power all the clearances are closed between fuel rods, between fuel rods and hexes, and between hexes. Here, there is a denial of radial expansion freedom and the power coefficient decreases. The result of all this is a power coefficient curve characterized by a low slope, a sharp slope and then a slightly decreased slope. I think that this is probably a more logical explanation of the nonlinearities. It is, of course, practically impossible to prove these points. All one can do is to cite favorable pieces of evidence.

\section{Chernick: Harry?}

Hummel: Dick, would you try to explain a little more about how these temperature changes act, you say the structure gets heated more than the fuel.

Smith: If you heated the system electrically and brought it from room temperature to, say $100^{\circ} \mathrm{C}$, the stainless steel would expand more than the uranium fuel. The rods actually become somewhat looser. 


\section{Chernick: Rogers?}

McCullough: I'd like to ask you when you expand these things and lose your clearances, have you looked into how much force you have put on these things which are now in contact and do you exceed the elastic limit?

Smith: This problem hasn't been looked at, but I think someone should. One thing that complicates any detailed treatment is the condition of the rods. There's a lot of evidence showing that the rods are affected mechanically; they're warped and swelled. We even found bends of as much as $1 / 2$ in. in some of them. Such effects, of course, modify the clearance and, consequently, the coupling.

\section{Chernick: Fred?}

Storrer: About these $10 \mathrm{sec}$ delays in the negative coefficient in the EBR-I. It's certainly interesting to hear what Dick Smith said; that it takes a few seconds for this top plate to expand. There is a second form for this which is worth mentioning. That is, if it takes one-half a second for the coolant to go from the core to the upper plate, it takes ten times as long for the temperature--at low frequency, anyway. A temperature signal travels slower than the coolant at low frequencies by a large factor, and this factor is the ratio of the total heat capacity to that of the coolant plus that of the fuel, or the fuel expansion to the heat capacity of the fuel. So that should give a half-second times ten is $5 \mathrm{sec}$. If you've got an extra $5 \mathrm{sec}$ delay within your plate, that gives you the ten seconds. If it was purely heat conduction delay within the plates it should be of a conductive type which would be attenuated. This power coefficient with a very large delay had not very much amplitude in attenuation which seems to indicate that a large fraction of it is due to transport.

Chernick: But, roughly speaking, you're back to the old Kinchin-Bethe model. I was wondering what you thought of the Acroyd explanation, which involved the blanket and hotter fluid entering through the edges of the core and the cooler fluid through the center. I think, hydrodynamically, there certainly could be questions raised about this model.

Smith: I think we've unearthed enough evidence that we just don't accept this point of view any longer. We tried real hard to look for strong blanket feedback affects in Mark III. We've even modified the core to promote additional heat transfer across the flow separator and we can see no evidence whatsoever of any strong effect. We also placed thermocouples in certain inner blanket channels in an attempt to detect heat transfer across the separator. At precisely the spot where you'd expect the maximum effect we find none at all. Any oscillations we have found in the temperature of outlet blanket coolant can be explained on the basis of heat generated in the blanket. In brief, we just don't believe that there are any significant blanket feedback effects.

Chernick: Briefly, then, you think there's enough mixing in the coolant to prevent this explanation. 
Smith: Right. We couldn't find any evidence of temperature oscillations larger than $\pm 0.5^{\circ} \mathrm{C}$ in the mixed coolant leaving the blanket.

Chernick: Yes?

deBoisblanc: I'd like to ask Dick how suddenly the change in slope occurred. I would suspect that since the sodium coolant volume is rather small, that the center part of the structure would expand first and then there would be a sudden transition of slope of the power coefficient. Is this the way the effect actually . . . . .

Smith: Are you referring to the discontinuities in the power coefficient curve?

deBoisblanc: Yes. Changes in the slopes of the power coefficient vs power.

Smith: From 0 - $200 \mathrm{kw}$ the power coefficient is practically zero; from 200 - $600 \mathrm{~kW}$ it is very strong. The strong power coefficient in this region most likely is the result of axial and radial expansion and coolant expulsion. From $600-1200 \mathrm{kw}$ the slope of the power coefficient is intermediate in magnitude.

deBoisblanc: This is one of the examples of the use for transfer functions to predict the conditions at a higher power level. If this is a phenomenon--that is, a discontinuous phenomenon--then there is no meaning to the extrapolation. of the transfer function--from low power to high power. This is the one thing--I think a most significant one--is the use of that type of extrapolation to clearly eliminate any possible discontinuous power coefficients. For if the peak of the transfer function grows smoothly with power, there can be no sudden appearance of a large positive power coefficient.

Smith: Even though you might make large errors in predicting the negative feedback, you're still a long way from cancellation of the feedback with the inverse zero-power gain. If you make an error by a factor of two, you will still be a long way from making an error of a factor of two in your stability prediction. It would still be safe. You can tolerate large errors here.

deBoisblanc: Well, that's really a good point because a lot of people talk about oscillations as if they are bad in reactors. Some of them are bad and some of them are not bad, but the philosophic question is whether you could predict from a low-power transfer-function measurement something that happened after one of these transitions. It is clear you cannot, simply because the system does not contain in its describing equation that coefficient--that new coefficient.

Smith: Well, you just can't place any reliability on very wide extrapolations. We have to be very careful about this.

deBoisblanc: The definition of wide has to do with our sharpest transitions. 
Smith: Yes.

\section{Chernick: Bob?}

Wright: I would like to mention very briefly an addition which we have recently made to our model of the power void transfer functions which we've measured in our simulated spert I-A channel. I didn't get a chance last night. This has been to add to a description of essentially the transportation effects in the channel, which we gave at the Argonne Transfer Function Conference and the June ANS meeting, the effect of flow fluctuations or flow modulation in the boiling region of the channel. This produces a large quantitative change in the irequency region of interest for the instabilities in the Spert I-A reactor. I have a couple of slides.

Chernick: Why don't you come up, Bob?

Wright: Okay.

Chernick: Thank you.

Wright: The model of power void transfer functions we've considered is shown in Slide 1 in which we have power modulation going in and what we

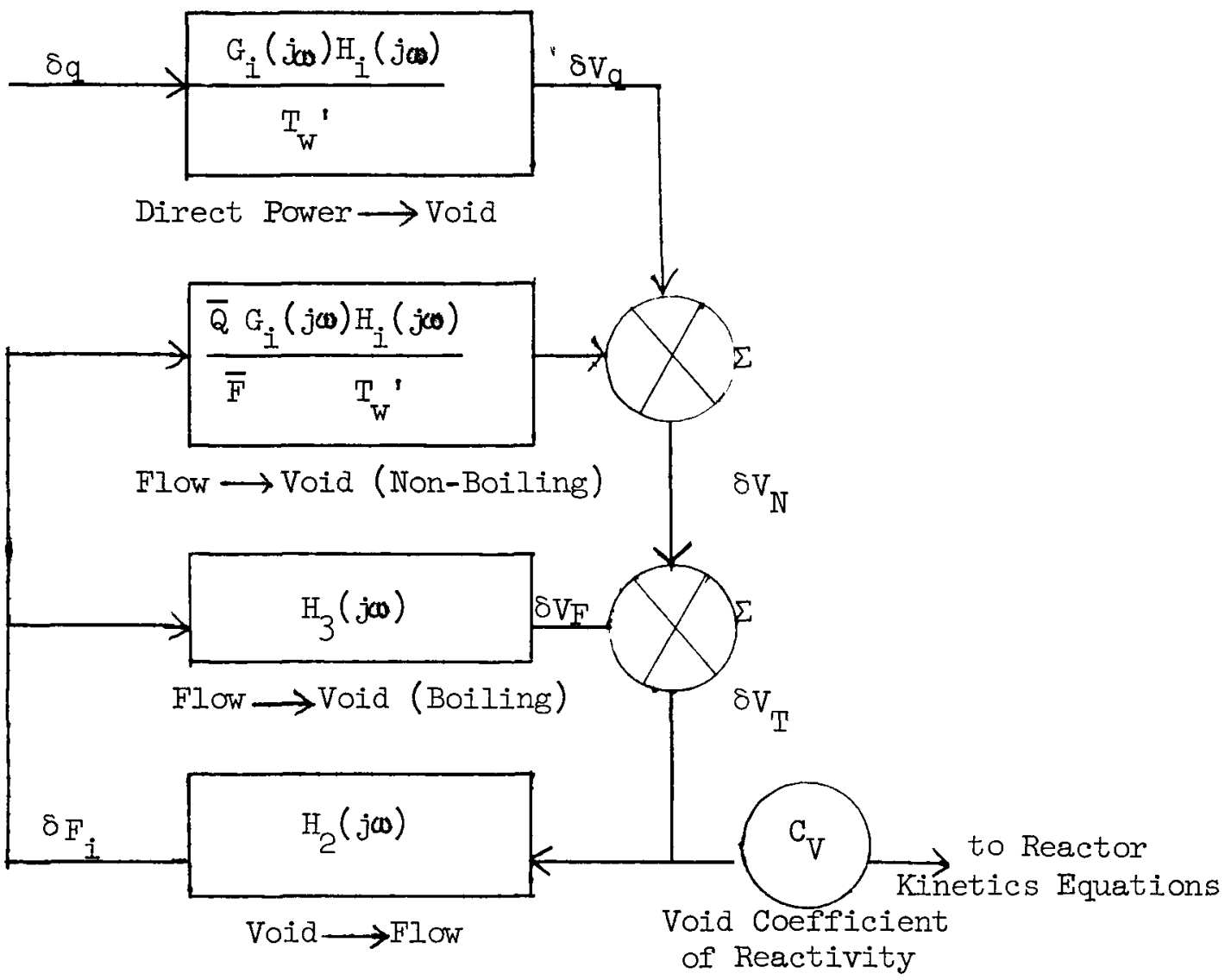

Slide 1. Block Diagram of Power-Void-Flow System of Transfer Function Model 
call "direct void" modulation resulting. This results from a storrer analysis of the subcooled length, integrating up the channel the temperature modulation, to get the motion of the bulk saturation point, translating the steady-state void fraction curve vertically in response to this motion and adding a time-lag due to the water transportation time in the boiling region. This was what we presented before. In addition, now, we are considering the effect of the flow modulation which results from the vold response. We've shown that the measured flow modulation is consistent with this void response and peaks in the region of one cycle a second. It falls when the void response gets out of phase with itself in the boiling region. Previously, we had considered the flow effect in the non-boiling length of the channel which you can incorporate directly into the Storrer analysis, equivalent to a power modulation, and we had qualitative agreement with the measured power-void transfer function. However, in the region which is important for stability we had $90^{\circ}$ too much phase shift and we were too low by a factor of two in the amplitude. Sam Zivi has just recently added the effect of flow in the boiling region as essentially the geometrical displacement of voids by this inlet flow modulation. There are no adjustable parameters in these calculations. Slide 2 shows the first of the new results. These are experimentally measured void fraction phase shifts vs power modulation frequency in this natural circulation channel. The lower two curves were the calculated phase shifts from the simple model first described, and the upper curve gives the phase when this flow modulation effect is added to the calculation. I think the waviness is, at least partially, due to errors in the

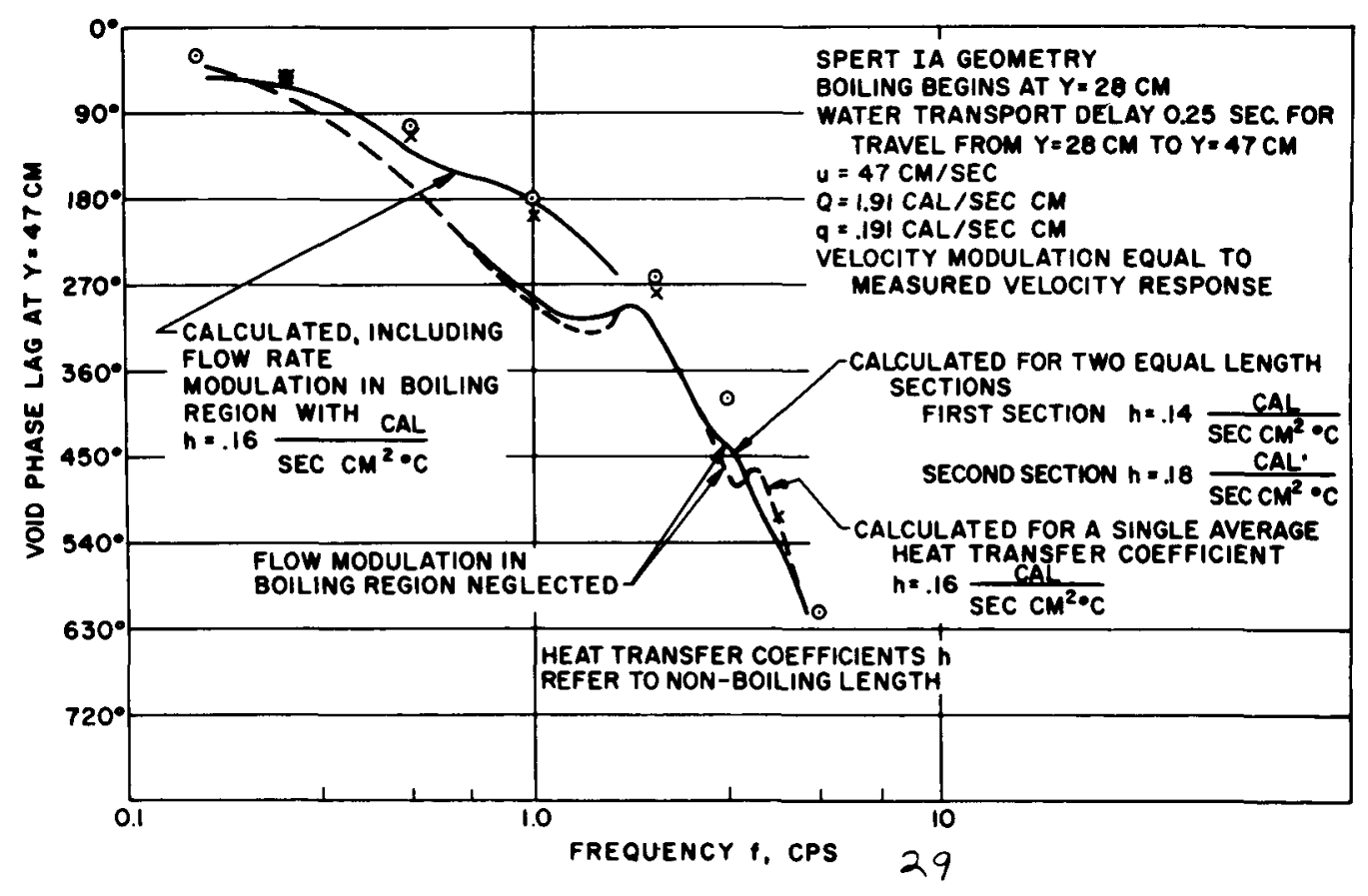

Slide 2. Calculated and Measured Phase-Lag of Power-Void Transfer Function, 500 watts Mean Power 
graphical analysis that we did. This addition to the model gets rid of $90^{\circ}$ too much phase shift at one cycle per second in the region of interest for Spert I-A instability. Flow response to power modulation goes away in our channel, under these operating conditions, in the region above two cycles per second, and also our measurement--our instrumentation--gets had so the curve is not continued. Slide 3 shows how the amplitude is heiped by this addition to the model of the flow response in the boiling region. Incidentally, in line with the previous comments on the fast reactors, this power-void transfer function goes directly to the steady-state void fraction characteristic in the zero-frequency limit of scale. You can see that we've picked up a very helpful factor of two in the void response amplitude; in fact, in the region where you're getting flow response which is the region of interest for Spert I-A instability, the effects of the flow response in the boiling region have become dominant. Except in this frequency region the transportation effects are dominant.

I'll mention one thing more with respect to Slide 1 . It's of interest to us that the flow-void feedback loop shown seems to dominate the power-void transfer function in the frequency region of interest for Spert I-A instability; that is, if we're making any sense with our model. And the feedback loop, of course, can go unstable all by itself. This may be one mode of purely hydrodynamic oscillation and it's one which we're investigating. In some pressure modulation experiments we've seen some suspiciously resonant-looking void and flow responses. We've only made one set of measurements, however. I think last night

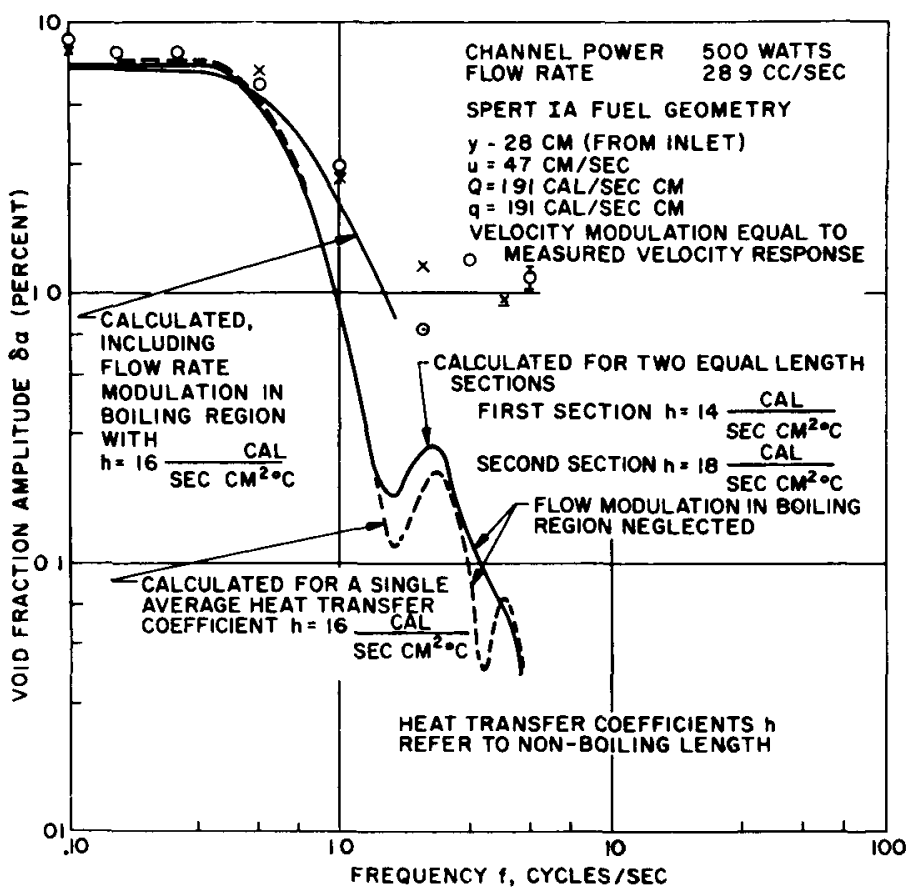

Slide 3. Calculated and Measured Amplitude of Power-Void Transfer Function, 500 watts Mean Power 
Dave Langmuir mentioned that in looking at the frequency of oscillation, purely hydrodynamic oscillation, when the non-boiling length was changed in various ways, we found essentially a constant phase-shift condition across the non-boiling length. That is, the frequency of the hydrodynamic oscillations was found experimentally to be about inversely proportional to the non-boiling length of the channel. Now, the non-boiling flow-void box shown is the only one of the three mechanisms in this loop which is sensitive to the non-boiling length, the flow-void response in the boiling length and the void-flow response have phase shifts which are essentially insensitive to changes in non-boiling length, so that, if you were exciting this loop for purely hydrodynamic instability, you would have this constant phase shift condition which we have observed. Thank you.

Chernick: Yes, Fred.

Storrer: I'd like to make a few comments in connection with what Harry (Humel) mentioned, that the phase lag on EBR-I seems to be larger than what the temperature would predict. Also, on EBR-I Mark II, we had this problem of trying to explain that large phase lag. So, we thought of a mechanism that would give--could give--this additional phase lag and the results are negative. When we write the transfer function, there's one thing we're really neglecting. All the power is not fission power. Some happen later on due to the decay heat of the fission products. So, you really should add another transfer function as a factor which is a transfer function giving the total power in the heat, including the fission power and the decay heat over the fission power only. So, we calculated the transfer function which is total power over fission power which is equal to one plus the decay power over fission power. We took a curve showing the decay power response to a burst of fissions. We took a Laplace transform of this and for zero frequency we get $7 \%$, and then we have a phase lag of the fission power--the decay power behind the fission power which goes to $90^{\circ}$ at infinite frequencies. But what we are rea.ly interested in is not this but is in one plus this, so we nave to add one on the real axis. Our results showed the gain and phase of the transfer function of total power over fission power and the negative conclusion is that the maximum phase lag is at about one radian per second and about 0.8 degrees, so this certainly cannot give an additional phase lag. The positive result is that it shows also the gain, so if you want to analyze feedback very accurately, and I'm not talking here about boiling-water reactors, but about fast reactors or PWR, you should take this into account. You have a change in gain of $7 \%$; I mean, when you increase the frequency even to one radian per second almost all of the decay heat can't follow, so you have a reduction of $7 \%$ in the gain. But the phase lag is 0.8 degrees so that's way within the accuracy.

Chernick: Thank you. Any other questions? Yes? I have been a little remiss in this but I would like the questioners to give their names, because there's a possibility that they might want to know who made what statements later on.

Forbes: You just defeated your own purpose. (Iaughter) I have a question for Stan Johnson. Where did he go? Oh. And also a comment which I'm sure he knows. Did you take into account the nonlinear expansion of water in this model? 
Johnson: In what I've shown here, we didn't. We can easily do this with a simple modification.

Forbes: I'm sure Frank would be delighted if you would do so, because this is one of the stickiest problems in trying to interpret the highpressure mus in which boiling was suppressed and make sure that you still have agreement on water expansion and plate expansion. I'm not quite sure that I agree; in fact, I'm sure I don't, with not using the bulk expansion coefficient for the plates. You neglected axial expansion and use 1.8 which I understand took into account Poisson's ratio, but actually what happens--the plates are clamped on the sides by fairly rigid members and they buckle and they really can expand three dimensionally and push water out of the core without changing the core periphery diameter. It's an opinion, I would be glad to argue with you sometime.

Johnson: I didn't mean to imply that 1.8 was the right number.

Forbes: I insist that 3 is the right number. (General laughter).

Johnson: I will argue with you on that because you do not have free expansion in the axial direction . . . . .

Forbes: It's virtually ......

Johnson: If it's free, then Poisson's ratio......

Forbes: Yes. If it's a clamped and rigid beam, but it isn't. It just buckles itself and it doesn't get any axial loading. Now, there is some complication--the fact that the sides are braced, so--. Well, it's a fine discussion point for some other time.

Johnson: Well, I think you bring up a very interesting point, particularly at high pressure it appears that plate expansion is important. It also appears to be rather important to know rather precisely just how the plate behaves when it gets hot.

Forbes: That's right. It's not a trivial contribution. One other thing, and this is the one I'm sure you know. It's been brought up in this meeting and in our own work too. But, this assumption of film boiling has never been observed experimentally; in fact, quite the contrary, every bubble picture you have ever seen of a transient first has the small bubbles, maybe later, as Professor Schrock showed us, you may get films, but not at the initial phase. At least, we've never seen them yet.

Chernick: Thank you, Stu. Al?

Henry: I have a question for Bill Ergen. What are the initials HFIR? Another question--how do you control it? What I'm really after is: Will the control scheme change this power distribution that you have so carefully tailored to be uniform? Similarly, since you are going to load the plates in a nonuniform manner, will depletion disturb this uniformity? 
Ergen: Well, it doesn't. The depletion is, of course, considerable and it will change the flux pattern inside the core to some extent. However, this has been taken into account--and what one essentially does is, one shapes this for some form of an average condition and it's not too bad at the beginning and not too bad at the end. The controls are between this ring which I showed you and the reflector and they're gray--gray rods and black rods which come in there and this distortion also has to be taken into account and matched against the distribution because essentially the cycle of the thing is completely set. There is no intention, at least, to run at high power, low power, and so on. Epler would be the one to give details on these controls.

Chernick: Yes. While you're on the floor here, Bill, there were a couple of questions which occurred to me in your explanation of the safety of the molten salt reactor because we had to go through a number of these problems ourselves on the liquid metal fuel reactor. The first is, how do you feel about the possibility of penetration of graphite by fuel which could be a safety hazard, and this depends on what you've found out about penetration of graphite by molten salts. The second question had to do-you mentioned plugging channels. In general, the problem there was not so much what this would do in reactivity but how hot the fuel would get if it were blocked and had to just sit around there. If you had a couple of plugged channels, what the temperature distributions would be in the fuel and surrounding graphite.

Ergen: Well, the answer to both of those questions is that for this particular reactor the power density is quite low as these reactors go. It turns out to be, I think, $40 \mathrm{w} / \mathrm{cc}$ of the reactor. Anyway, it is 67 cubic feet in the whole reactor and the maximum power is going to run 10, or for the benefit of $\mathrm{Dr}$. McCullough, maybe $9.9 \mathrm{Mw}$. (General laughter) Well, anyway, the power density is very low. Consequently, my reference to the plugged channel was really to exactly what you said. This will change the temperature but if you plug one channel then the adjacent channels will take all the heat away with a very few degrees temperature rise. You won't be able to tell the difference. However, if you plug very many channels, you ultimately must get into trouble, and my comment was that one really has to design a way of finding out what happens, because you can't tell the difference in one channel but sometimes you would be able to tell the difference. Also, in answer to your first question, there are various techniques of decreasing the penetrability of the graphite. In the first place, you use fairly high density graphite, but that is not so good because of the physical properties. One idea is to soak the graphite with fluorides to begin wi.th, and this penetration of the graphite is something that is being experimentally investigated. It is not very critical in this reactor because of the low-power density. Even if you generate a certain amount of heat in the center, it can very easily flow out of these 2-in. graphite blocks, the moderator blocks, into the coolant channel.

Chernick: Yes, thank you. I feel maybe I can put in a plug for experiments at this stage, because here is obviously an example where these problems can be licked in a low-power reactor and then when you go to a 
thousand megawatts and things are really crucial, at least you will have the fundamental information.

Ergen: Yes. And experiments are being done; however, at least my personal opinion is, that if you go to a high-power reactor, you won't need a graphite moderator because the graphite moderator is in there to reduce the size of this reactor to roughly $5 \mathrm{ft}$ in diameter. A much larger size would pertain if you would allow only the moderation of the fluoride itself. But the fluoride, in contrast to the bismuth, is a good enough moderator so you can build a homogeneous fluoride reactor, if you know what to do with the power you get out of the machine.

Chernick: It's not exactly thermal.

Ergen: It will not be exactly thermal, but it is approximately so. The age, I think, is about three times the age of graphite.

Chernick: Thank you. Yes?

Smith: I have a question for stratton. Is the simulation you showed thermal or fast?

Stratton: I'm sorry.

Smith: Is the simulation in the Los Alamos machine thermal or fast?

Stratton: The critical assembly?

Smith: Yes.

Stratton: That was pretty thermal.

Chernick: Well, I don't see any--well--okay.

Wasserman: This is not a question but just a fill-in of an inadvertent omission in my talk yesterday. Just for the record . . . . . .

Chernick: Please announce your name.

Wasserman: Art Wasserman of Spert. I just wanted to give due credit to Dick Wagner of Spert, who assisted in the describing function calculations that I mentioned yesterday.

Chernick: All right. Well, I think the questions are petering out but before we close the meeting I have two announcements. First, that the three o'clock checkout time does not apply to conference attendees; they may check out at any time today. The second announcement is for people who are catching the 1I:15 flight out of Twin Falls tonight; there will be a special bus leaving the Challenger Inn at 8:15 p.m. for those who are making the 11:15 flight out of Twin Falls. Thank you. 
SESSION VI

GENERAL REVIEW

\author{
Chairman: W. E. Nyer (PPCo) \\ Panelists: C. K. Beck (AEC) \\ E. P. Blizard (ORNL) \\ I. D. P. King (LASL) \\ C. R. McCullough (Mon) \\ T. J. Thompson (NIT)
}

Nyer: This last session was to provide us with an opportunity to have a review of all of the things that we have discussed during the meeting and all of the things that might have come up in various conversations outside the meeting. For this purpose we have listed several of the well-known and senior people in the field, Mr. King, Mr. Blizard and Mr. Thompson. In addition, because of the fine opening that we had to our discussions in the first session, I thought it was appropriate to ask Mr. Beck and Mr. McCullough to comment again. Because Cliff is leaving, or will have to leave early to catch a plane, I'll call on him first. Cliff.

Summarizing Remarks

by

C. K. Beck (AEC)

Beck: As has been said so many times, it's easy to prepare an hour's talk but very difficult to prepare one of five minutes. I will a.ttempt to record here in a five-minute statement, however, some of my impressions of this meeting. As a non-expert in these specialized areas I have tried to understand what has been said in these sessions which would influence and illuminate the evaluations and judgments we have to make in our own safety surveillance program.

First, my strongest impression is that there is in progress substantially more work, more extensive studies particularly in the areas of theoretical understanding of stability, factors affecting stability, possibilities of predicting instability and the inter-relationships of hydraulic and thermal parameters on nuclear behavior, than I had realized. I am impressed with the level of sophistication in these studies now being made and the superior quality of scientific talent being brought to bear on the problem.

Second, it is my impression that there is not any particularly obvious lack of communication among the various groups working on these problems 
at the present time. The line of questions which have been asked and the frequent cross-references by various speakers to work, even recent work, of other groups, and the general complementarity and similarity of patterns of approach described by the various speakers, is evidence that the level of communication, though I am sure it is not ideal, is apparently not too unsatisfactory. Whether this is so beyond the relatively narrow domain of the expert workers in this field is not clear and cannot be determined from this conference.

Third, it is my impression that the broad basis of explanation, interpretation and understanding of reactor kinetic behavior, including the main categories of parameters, and characteristics which influence stability, is on a sound and generally agreed-on basis. The main stream of present efforts and the focus of attention is on the tedious, time-consuming, complex and confused, but exceedingly necessary, in fact essential, business of assigning or sorting out the minor and major parameters, assigning practical values, defining of practical relationships and identifying the boundaries and limitations within which behavior can be confidently predicted. In most of these principal areas of kinetics, the present status seems to be at the height of the sorting, organizing and filling-in-ofdetails period, out of which boundaries, limitations, areas of validity, and working rules will emerge; but little of the latter has been accomplished to the present time.

Fourth, in my opening comments offered at the beginning of this conference, I suggest that there are some house-cleaning tasks now overdue in collecting, organizing, and writing-up with interpretations some of the work already done in these areas of kinetics. I had in mind a broad coverage, in one comprehensive treatment, of the systematic description of experimental work, matched and integrated with the best corresponding analysis and interpretations now available. I still believe there is a need for this in some areas but I believe it would be premature in most of these areas at the present time. For example, I believe it would be profitable at this time for a comprehensive coverage of the experience and theoretical work which has been carried out on transient behavior in Spert, Borax, and related activities. On the other hand, from the impression I have gained at this meeting, I believe that such an attempt would be at least a year, and possibly two years, premature in the general field of reactor instabilities and advanced prediction of instabilities and in the boiling vapor formation and the thermal and hydraulic complexities relating thereto. This is not to say that frequent and full reporting of detailed studies, and investigations of component parts of these fields should not be encouraged. This is precisely what should be encouraged, in supplementation of frequent meetings such as the one we are now holding as a means of informal and oral exchange of views and ideas. It simply appears to me that major efforts to generate comprehensive, organized descriptions of these fields would not be as worthwhile now as they will be a little later when some of the numerous gaps in knowledge have been filled in.

Fifth, it is my impression that our general direction of research effort now in progress is the right one. There needs to be more of it in the same direction. Primarily this is toward more extensive and 
diverse types of experiments on transient behavior in reactors of different types and characteristics. A wide variety of large scale, inpile and laboratory scale, out-of-pile work on hydraulic, thermal and nuclear parameters involved in instability and in the detailed understanding of the boiling process in reactors.

Sixth, finally, in view of the many gaps in knowledge in these vital areas in reactor design and operation and having sat through this conference, I am much more aware than I was formerly of the uncertainties and complexities in so many of these areas; I believe that there can be only one approach that we in the regulatory end can take. It is the one we have been taking and is articulated most precisely in the words of Mr. Henry earlier today when he said of the magnitude and location of the hot spots of a partially burned out core, "Where we cannot predict it we must overdesign to avoid it". Since this is the case in so many areas, this is the approach that must be taken and we can only recede from that insistence on overdesign when knowledge becomes clarified and confidence can be gained of how much the margins really are. It becomes clear, therefore, how important it is both to the reactor designers and to the reactor regulators to hasten the full understanding in these vital areas of reactor behavior.

I would add finally, and I have no hesitation in saying, that this has been the most interesting and profitable technical discussion it has been my pleasure to attend for a long time. I think that the hosts and planners of the conference are due our full thanks for their efforts which have made this so. 
SESSION VI

PANEL PRESENTATIONS

Nyer: Thank you, both for the comments and the compliments. Now perhaps we can hear from Perc King. Perc, would you mind.

\author{
Summarizing Remarks \\ by \\ L. D. P. King (LASI)
}

King: I think I am the only member of this panel that isn't connected in some way with reactor safeguards, so perhaps my point of view in making these remarks is slightly different. I would first like to thank the sponsors of this program for inviting me to these sessions. It was under the condition that I would not be considered an expert that I agreed to attend and now, in trying to summarize some of the results of this meeting, I find myself in the dubious position of trying to talk to experts on a subject they know much better. I would first like to say that I think these meetings have been highly successful. The informality, small size, volume of information and results have shown that this approach is very good. I think the arrangement of giving a review paper and then status reports by experts is one of the best methods that I have ever run into at a meeting. It is also evident that we are making substantial progress in this field.

I would like to say a few things about the possible future needs that seem to have come out of discussions and observations. It looks as though perhaps we need better dissemination of the "exact state of the art" reports for non-experts. It has already been mentioned that one method is to clean house, get rid of some of the old information, and put us up-todate. It would also be helpful if we could get quicker dissemination of data and especially report writing. I think the type of meeting which we have had here, plus ANS meetings, are probably the quickest way of trying to disseminate data of immediate interest. It would be nice if one could somehow have summary status reports of wider distribution than are presently available. An important point that was brought up is the great care that must be exercised by the expert in pointing out the usefulness and applicability of rules of thumb. Unless it is very clearly stated how these rules apply, the non-expert is very apt to use them incorrectly.

Now a few remarks about encouraging signs of progress and better understanding. It would appear that some of the simpler systems are now almost completely understood. One of the most promising things that I have heard in this meeting is the beginning of the use of numerous differential experiments to supplement the excellent integral work in such facilities as Spert and Kewb. We have two types of these, in-pile and out-of-pile experiments. Some examples of the in-pile experiments that are being considered or have just been completed were the use of a small additional cylinder on the Kewb experiment to obtain better pressure determinations, the indications of nucleation centers observed in Triga 
bursts, and the Treat experiments on fuel element behavior during power bursts. We have another very important group which are out-of-pile tests, these, I think, were beautifully demonstrated by the results of RamoWooldridge in the nucleation of bubbles, Argonne's electrical heating tests of fuel elements and the University of California's rate of bubble formation. I think these supplementary tests are highly important and necessary for a complete understanding of some of the kinetic problems.

There were a few surprises for me, I must say. The first was the starting of reactors without a source. I think if you are of the old school when reactors were first operated, this philosophy gives you a little bit of a shudder. I think this idea should only be considered in reactors of well-known design or those with essentially duplicate cores, where one really knows how such a reactor behaves. If one has built one reactor very similar to another, one probably has sufficient feeling and understanding that this philosophy might be used. It is possible to imagine some sort of a mechanical device putting in reactivity steps with known delays so that you can almost guarantee the safety of such a startup in a rather well-known type of reactor. The other surprise was that fast reactors with built-in prompt positive temperature coefficients are under serious consideration. This would always appear to be unsafe.

Next, there are a few discouraging facts of life. 1) It has been clearly brought out how small, very minor, changes in reactor design can produce a radically different stability behavior in a reactor. Changes in $U^{238}$ concentration can effect the Doppler effect. Very small changes in surface conditions, geometry, pressure, or channel flow rates can radically effect bubble nucleation and therefore reactor stability. Changes in fuel cladding alone may make it possible to get a more critical geometry by fuel expulsion through holes in the cladding. This was shown by aluminum versus stainless-steel cladding in one of the Argonne $\mathrm{UO}_{2}$ experiments and in the inability to duplicate the precise conditions of the EBR-I runaway where one really attempted to make an identical system. 2) I think it has been shown that mies of thumb, or extrapolation from results from one reactor to another, do not necessarily hold unless we have the complete understanding of the kinetic dynamic behavior of the reactor systems involved. 3) The appearance of non-understood shutdown mechanisms for very fast reactor periods as have recently appeared in Kewb and Godiva experiments and are tentatively explained by assuming additional neutron delay periods. The appearance of unexpected, overlooked mechanisms have led to most reactor incidents and surprises, such as the importance of. long delayed negative temperature coefficients as occurred in the EBR-I incident, must still be possible. 4) There is still a lot of empirical fitting and the arbitrary use of adjustable constants to explain reactor response. 5) Complete kinetic prediction of most reactors still appears to be some time off.

We had some discussion on responsibility in case of accidents. I think it was the unanimous decision of the group here that the ultimate responsibility really must lie with the designer and operator. I think a word of caution is needed here in how far the AEC attempts to apply detailed regulatory processes and rules because I believe they run the danger of throwing the full load of responsibility in their direction. 
Only when we really know the detailed kinetic behavior of the reactor in question can one apply detailed regulations.

Finally, let me summarize a few recommendations for the future. 1) I think one of the most important things which should be done is for designers to apply much more effort towards designing inherently safe reactors, even if some present types have to be dropped. 2) An increase in differential types of experiments in which we have recently made so much progress is desirable. 3) We should continue the present good work and close exchange of information between experts as illustrated by this meeting. 4) We need more experiments in dynamic problems where other variables besides reactivity are used such as pressure, flow, temperature, etc. 5) Work should be carried out on very short reactor periods in order to assist in the complete understanding of the kinetic behavior even if this may require the sacrifice of a few reactors. Such tests also would contribute to solid-state physics. 6) More work is needed on the information of shock waves in rapid transitions. This, after all, is probably one of the principal factors which would result in the rupture of the container. 7) More thought should be put on how unusual dynamic properties of fission processes can usefully be employed. 8) Total energy release is the important factor in most reactor incidents, yet most of the effort has only gone into the primary pulse, with very little looking at possible secondary factors. A more detailed understanding of the effect on stability of secondary and tertiary neutrons, gamma rays and even beta rays and soft gammas is needed. 9) Some theoretical physicists should not get caught with too much mathematical formulation and play, and lose sight of the physical quantities really desired.

I would like to close with a couple of questions, I believe have been unanswered. One of these is whether safety tests of some sort should be made on power reactors before startup and, if so, when and how many? Should power reactors be designed and run at constant load in order to minimize the hazards of the startup and shutdown regions? Could a continuous transfer function analysis in the reactor contribute to the safety of its operation by display or scram action? Finally, I would like to congratulate this group on this very excellent, high quality meeting. 


\section{SESSION VI}

\section{PANEL PRESENTATIONS}

Nyer: Now one of the points that has kept recurring many times has had to do with the matter of disseminating information, and I think we have someone from whom we can perhaps get some special comments on this; Blizard?

Surmarizing Remarks

by

E. P. Blizard (ORNL)

Blizard: Thank you, Warren. And, Perc, thank you very much for giving most of what I had to say; it's very kind of you and should save time in the long run. Going through a short list of things I had to say, I will pick out one or two that Perc hasn't already said. I would like to say that I think a particularly good aspect of this meeting has been that there have not been parallel sessions because, I think if there had been, very likely you would have had those whose experiments are more nearly of an engineering type in one room, and those of more physics type in the other room and the purpose of the meeting, it seems to me, would have been very nearly defeated. It seems to me, that it has been an exceptionally fine arrangement when you make the physicists sit and listen to the engineering papers and vice versa. I didn't get a chance to ask a question the other day, is it legal for me to ask it now, Warren?

\section{Nyer: Certainly:}

Blizard: It's for Mr. Zuber. I wanted to know whether any thought had been given in this business of nucleate boiling in the presence of radiation, whether, for example, aluminum fuel elements for which there might be considerably more $(n, \alpha)$ reaction than with, say, stainless steel, has ever been observed, in particular, to be better from the point of view of providing nuclei for boiling. The effects of the alphas would give you considerably higher concentration of ionization, I would expect. I don't know whether you want to answer this or not.

Zuber: Well, I would say that, in my opinion, any nucleation due to the alphas won't be such an important thing, because of the way the channel is made. This is what Dr. Langmuir has also talked of, aside from the radiation, the thing which is going to be present all the time, this is the poor stability due to the change of the flow pattern when we go from bubble flow to slug flow, so that, whether we have radiation or whether we don't have it, we shall always have this instability. May I again say that the essential point is for the future research to mark this flow regime, so if we know that for these operating conditions, for this system, for this geometry, and for this pressure, the operation in this flow regime shall be stable, this will be independent of the radiation; and this is the essential program for the future. I think the problem of nucleation is probably a minor effect, especially at high pressures. 
Blizard: Thank you sir, and thank you for letting me get in my question. I note that the program was listed as Reactor Kinetics, it really should be listed in its full-blown reality of Reactor System Kinetics, and I think this is an appropriate expansion of the field.

In the nuclear energy business, there are two things which will determine, some time from now, whether we have been successful or not. One is whether we have been able to beat the cost of fossil fuel power production, and the other will be whether we have been able to do this safely or not and of the two, I wouldn't choose which is more important, but safety seems to be an exceptionally important thing and it seems to me that the business of studying the systems as a whole and studying them in detail is going to yield a great deal in the matter of making reactors and reactor systems safe. Perc King did mention already this business of testing power reactors conceivably to destruction, perhaps not through destruction but perhaps up to destruction, and we may very well be forced to demonstrate in the case of new power reactors that we really do believe that they are safe and that we know how far one can go in reactivity steps, for example, or ramps. And one might challenge us by saying that, if we don't dare to test these reactors out in Idaho, what business have we installing them in some large city. What we must be careful about, though, is the criterion that we use in choosing how far we go in these things. I think that it is important to be sure that we are not merely running these tests to see if the predictions of behavior were correct, just in a general way you can do these perhaps in other, cheaper, systems. We don't want to be put in a position, not of testing these new power reactors but merely testing Frank Schroeder. I think it is important to beware of this.

Perc has said everything else that I had to say, with the exception of the publications, and I might say that there are two publications which are edited at Oak Ridge, which have to do with the business of this meeting. One of these is published under the aegis of the AEC. The editor is Bill Cottrell. This is the Nriclear Safety Review and the modus operandi of this publication is that a number of related recent papers are given to somebody knowledgeable in the field, and he is asked to prepare a review of them which is published as one of the chapters or papers in an individual issue. This comes out four times a year. I hope those of you who have found it, have found it useful and interesting. If you have not, it is incumbent on you to write to Bill Cottrell and tell him why not. And I might say also that, if there are occasions when you think that you might contribute a chapter to this publication, I am sure that Cottrell would be interested to hear from you about it. As regards Nuclear Science and Engineering, the journal of the American Nuclear Society, we have had a change of editor within the past year and some change of editorial policy; I guess this is natural. I plan to continue to publish, from time to time, review articles, realizing that such articles can serve a useful purpose in educating people who are not necessarily directly in the field that is covered. Furthermore, they can kind of document the work and make sure they get all the references in a single available place for those who are in the field. I would like to suggest that you, any of you, who feel you are sufficiently familiar with a part of the reactor system kinetics business, consider 
submitting a paper, a review paper, of this sort. I would like to suggest it would be a good idea to correspond with me first to make sure I don't get four on the same subject; whenever you are dealing with non-original pieces you have to be very careful about this, of course. As always, we are very anxious to receive original pieces and have received a number of them and published them. You will be interested to know that there is a set of 16 papers from Los Alamos on Fast Reactor Criticals which should appear in the December issue and there is also a series of papers coming out now, one at a time, on Reactor Operating Experience and these may also be of interest to you as, in essence, data in the large on the reactor operating business. I echo the sentiments of the previous speakers regarding the high caliber of this meeting.

Nyer: Thank you.

Chernick: Warren, may we address the panelist?

Nyer: Yes, by all means. I assumed that everybody was a little tired so I didn't ask.

Chernick: I don't like to turn a recommendation back at the person that is doing the recommending, but how much of the present meeting do you think would be appropriate for somebody like Warren to send the programs to Bill Cottrell and perhaps make an arrangement with him, conceivably, whereby the chairman, or some other knowledgeable person, write up the session. Perhaps the reviewer might submit his paper, it seems to me that this would be entirely appropriate, and I think that you should approach it.

Ergen: I think that one very good way of handling just this, as well as maybe similar other situations, would be if cottrell could be given the rough drafts of the papers which were given, obviously for the purpose of making a review of the things which he would have done in the usual way. It is not necessary that all information be in good English or something like this, and it would considerably advance the day when this would be available, and also would tip off potential readers of the final edited paper that this thing exists. Along the same line, it just occurred to me, while Blizard was talking regarding the Safety Review, that there is the situation now that, most of the time, Cottrell gets his journals when he finds them $\therefore$ n the ORNL library, when everybody else has seen them too, and the advantage is only that they are reviewed and abstracted. But, if anybody writes a paper pertinent to reactor safety, I think it would be very much appreciated if he would send a pre-print at the same time he sends it to the journal, to Cottrell, with the stipulation that, of course, Cottrell shouldn't print it before it appears as a paper, but he could then print it very soon thereafter.

Nyer: Well, I assure everybody that I want to get out of the frying pan on this reporting business. I feel some obligation to do what we can to try to report on this meeting, if everyone wants it that way, and we also have to have an obligation to the people who did speak to make sure that we don't say things that they don't want us to say. We will try to meet most of these, and I think that this is a good suggestion, that 
we think more of getting this published in one of these journals than having it come out as a TID, because there is always an objection to some of the AEC documents that they do not carry quite the same weight as an article that appears in NSE. I think that is a very good suggestion.* Thank you.

$---\cdots-\cdots---\cdots-\cdots$

*Editor's Note: A review of the meeting was prepared by an attendee, M. F. Valerino, for Power Reactor Technology 4, 2, pp. 22-30 (March 1961). 
SESSION VI

PANEL PRESENTATIONS

Nyer: I would like to call on Professor Thompson who, I think, has some more critical comments to make.

\author{
Sumnarizing Remarks \\ by \\ T. J. Thompson (MIT)
}

Thompson: This has been a rather hard conference on the people that are here from MIT. I still have yet to get my luggage. It has never come in. This is the same shirt I arrived in. My fellow colleague from MIT, Professor Gyftopoulos, is presently next door in the hospital. He is somewhat better today, but I must say this has been a hard session on us. I missed the first day, so I don't feel I can report on it. I would like to talk about the parts of this conference which I did listen to.

I want to compliment Al Henry and Joe Thie on the rather remarkable summary papers they have presented here. I think that these papers said about everything that anyone could say on reactor kinetics and on transfer functions from the theoretical and practical viewpoints. I want to ask, though, a few questions about what we have done, what we are doing, and where we are going. In the practical sense, what we are really doing is looking at some of the most important problems of reactor safety. I will try to deal principally with the implications of reactor kinetics on these problems in my discussion this afternoon. I believe that in considering reactor safety and kinetics only in one place do we get into problems which verge on reactor economics and that is in the burnout problem. Every reactor designer and operator wants to run his reactor at the highest power at which he can run it safely, and this means that he runs it as close to the point where he will get fuel burnout as he can, safely. Depending upon the reactor and upon the management and upon the operators and upon AEC controls and many other things, this margin is small or large. It is only in the problem of burnout that we get into any other than the safety problems. In the burnout problem I must say there are economic aspects, the rest of these considerations are almost pure safety.

Basically, a transfer function is a tool. It is not a fundamental physics concept; it has nothing to do with Newton's laws or the actual movement of neutrons through materials. It is a tool. If you judge it from the safety viewpoint, it is only on the basis of whether or not this tool is useful that the ideas and theories of transfer functions can be said to be useful. The first thing in using a transfer function is to see whether or not the reactor has possible operating conditions where it demonstrates instability, let's say, and after one determines that there may be such conditions one has to make a second judgment as 
to whether these instabilities are a serious threat to the safety of the reactor. As an example, at MIT recently we had a student who did a study on the transfer function of the automatic control system of the Yankee reactor. To do this, he took the original specifications on the system and put the right numbers in the black boxes of the schematic block diagram, supposedly, and came out with an answer that the safety system was such, and the automatic control system was such, that it would oscillate in power with a period of about 15 or 20 minutes and perhaps with an amplitude such that the temperature changes in the reactor would be say $3-$ or $4{ }^{\circ} \mathrm{F}$. In the absolute sense, there was an instability, but I can't bring myself to believe that this was a problem that seriously affected the safety of the reactor. I think that the Xenon situation, which has been discussed here at length, is an example of this sort. I think, in general, that the Xenon situation is not basically a safety problem but is basically an operating problem. If you have reasonable instrumentation in the core--for example a good operating chamber in each quadrant of the core and one at the top and one at the bottom--then likely you can detect any Xenon effects which will be large enough to be a serious worry and by proper operation of the control rods you can tend to counteract this. You probably cannot eliminate it, but you can counteract it in such a way that it is likely not to be a safety problem or a serious operating problem. This is a problem which we can argue about, but I've never seen an occasion in which it looked to me as though it were really a serious safety problem, assuming one has reasonable core instrumentation. This instrumentation, I think, can still be outside the core and be reasonable.

It seems to me that the biggest worry with transfer functions is that they don't always work. This was brought out this morning by Mr. deBoisblanc. I was going to say the same thing myself. Basically, the fundamental worry is that, if you have a discontinuous change in the core or in the reactor system somewhere, the old transfer function goes completely out the window, and you have to start again with some sort of a new transfer function. Say, that you have a change in phase involved or a change in the metallurgy of the fuel, it may lead you into a situation in which you have a completely different transfer function. Somehow, one must not only answer the question of what the transfer function is of the existing system, but ask also what other transfer function might possibly occur if such and such happens. As a part of your design, you must go one step further and answer three questions instead of two. First of all, do we have any instabilities? Second, how serious are they? Third, what other possibilities exist for transfer functions that could happen with this reactor? Then you must go through the analysis and then somehow make a judgment as to whether or not these make a difficulty in the reactor design.

It seems to me that the analogy between aircraft and nuclear reactors is very close, indeed. Both aircraft and reactors require designers. There is an equivalent to the Hazards Evaluation Branch and to the ACRS in the Federal Aviation Authority. Both require inspection and licensed operators. There is an operator, the airlines, and there is the interested public, those who are underneath the airplanes as they fly overhead and those who fly in the airplanes. I believe that what actually happened 
in the case of the Electra is that, basically, the designers understood what they were doing up to a certain point--they had the right transfer functions. But at some point there suddenly occurred a vibration which was not in their original calculations--there was a discontinuity in the transfer function. This vibration was a function of the speed of the aircraft, evidently, and this vibration was a discontinuous function. It started suddenly, and destroyed the aircraft in a manner not predicted by the calculations at all. This is basically the same situation that we face with the use of the transfer function or any other of our safety calculational tools. What are the unknowns that we face? The other day a group of us were discussing this problem, and somebody said, "Every day you drive down strange roads and you don't get into troubles." That is true, you do drive down strange roads and you don't get into trouble, but you see road signs as you go along and, hopefully, you don't come to a place where there is suddenly a cliff. There are exceptions to even this. If you go through Ohio you will see occasionally, places where it says "Danger - Mine Fires". If you don't know the history of it, it means nothing, but basically the mines in ohio are burning out underground and, as they do, suddenly great chunks of earth drop down into the caves created by the fires and leave huge holes in the road. If you are unlucky enough to come along when one of these had just caved in, you're in trouble. And so, in the same way, even roads have their problems on occasion.

As a safety tool, then, what you really need is, first, an understanding of the transfer function representation of the components of every part of your system. Now this is something that Dr. Henry mentioned this morning. A good deal of progress is being made in understanding what goes in the "black boxes" in the transfer function, and when you know exactly what to put in every black box, and when you know you've got all of the "black boxes", you have the right transfer function; but, until you do, there is a good deal of uncertainty. Once you know what goes in the black boxes you have to have the ability to combine these into an overall transfer function which will predict instabilities. And, hopefully, the prediction can be extrapolated beyond experience. It is a little bit like predicting by $\mathrm{I} / \mathrm{M}$ curves where a reactor will go critical. In the same way you ought to be able to predict where an instability will occur as was pointed out by Joe Thie. The work at Argonne, on the reactors there, has been rather remarkable in that it does predict these things quite well. And, as long as you can assume they have the right transfer function, it ought to work very well. What we have to be careful of is that there is no place where there is a discontinuous vibration in our aircraft, or a hole in the road, if you will. These are the serious cases that one has to worry about.

Another point that I would like to re-emphasize (it's been said before) is that the transfer function method predicts only a threshold in instability. If the instability itself then leads to a change in the reactor system, then you must worry about what this means in terms of the subsequent behavior of the reactor. In other words, there are, indeed, a great many reactor systems about which you can only gain information by real transient tests. I believe that this statement is the heart of the answer to the question which has been posed here in 
regard to power reactor tests. No reasonable commercial power reactor group, I think, will resist doing some nice, calm, quiet, oscillator tests, except for the economic resistance which, I think, you will find on the part of everybody. But I think every commercial power operator will resist to the last ditch a transient which will in any sense endanger his system. If you really want to solve the transient problem, what you must do, and $I$ am appealing to you now as experts, is to conjure up some way to do transient tests which you will guarantee will not get the system into trouble. That's a difficult problem--it may even be impossible--but that's really what you must do. By the time you get 50 or 100 million dollars invested in a single reactor vessel and a single core and plant, you're not going to risk it on some test which may, or may not, give you additional information and which, in any case, the owner will believe will never occur in his reactor in normal operations anyway. The only way that I think such a test could ever be done is by having the AEC require it, and $I$ would resist that to the last ditch also. I just don't think the major reactor transient studies should be done on cores in commercial power plants.

There is one other philosophical point here and this goes back to Dr. Kasten and his question asked about the reactor at Oak Ridge, the homogeneous reactor. The question was, "Should you display the transfer function on a little board in the control room?" And the answer, it seems to me, is "Maybe". The only reason for displaying such a signal in the control room is if it is the best way and the fastest way to gain information about a transient, or about an unusual condition in the reactor. As a matter of fact, I think the information on the problem in regard to the fuel in the homogeneous reactor was not gained from the transfer function, but was gained rather from the fact that somewhere they were losing some fuel, and this was the hint that something was wrong. Therefore, in this case it looks like the transfer function display wasn't really doing much good. But with the previous knowledge they had gained, if they should want to put one on the next homogeneous reactor with the hope that they would first learn from the transfer function that something was going wrong, then I would say, by all means put it in. But don't require that everybody do it because it is not at all clear that the transfer function that you see on that scope is the right transfer function. It's not at all clear you will get information in time, and it may even lead you to an unsafe condition because you will look at the rest of your instrumentation and you will say, "I have a little problem," but you look at your transfer function system and you say, "That says no problem", and you will be tempted to believe your transfer function system. I am afraid that more often than not the transfer function system display will lead you to a false sense of security. I'd rather see an alert operator with a little bit of a worried frown all the time, than one who believes completely in any single instrument which, after all, is using a rather remotely derived system which is complex and which may actually be displaying, say, a range of frequencies in which there may be no possibilities of a transient, but perhaps the range is not broad enough to cover the dangerous frequencies.

If you want to look at the ideal situation, it would be nice if each one of us could predict during the initial design of his reactor exactly 
what instabilities will occur and hopefully design them all out--design out the xenon instabilities, design out the spatial instabilities, and so on as you design the reactor. This will probably never be achieved and probably is completely impossible, but at least one should continue to try for it. At this time, the best we can do is to develop the transfer functions of the components, do the best we can on design, and then probably plan a series of tests during startup. Some reactor groups are already considering what sort of oscillators they should put in. This was brought out by $G E$ in discussing the Dresden reactor. This is fine and I think everybody should plan ahead what these transfer function tests will be and have proper instrumentation designed into and installed in the reactor while other design and constmuction work is going on. Of course, after having done the test, and I still think that you must maintain a certain degree of skepticism, it is really this feeling of healthy skepticism that I would like to leave with you.

I feel quite strongly that we are at the moment on the threshold of a somewhat new era. At this moment, the second and third large core water reactors are on the line or going on the line. I do not feel, in any sense, that we understand large core kinetics and I think we need a good deal of development in the field of large core kinetics. It is certainly possible to have effectively at least two sub-reactors in the same large core and maybe three or four, if you don't do things just right. I believe I am correct in saying that in the shippingport reactor, because the control rods were left perhaps a little long in the same places, there were basically four different sub-reactors in the system. It would be nice to know a little bit more about the space-time representation of one of these large cores when it is split up into parts and it is easy to split it into parts by, let's say, improper control rod programming, or other things that may occur during the life of the reactor. One of the things that makes the new power reactors going on line of real interest is that they, for the first time, have some real in-core instrumentation. In particular, I would like to compliment the Dresden group on the efficiency in the work on their in-core instrumentation. My compliment goes back further than that because we ought to go back and compliment Savannah River. They are the ones that developed the in-core chambers in the first place and first used chambers in high-flux regions for long periods of time and got good results. These have been translated from aluminum into stainless steel and are now in Dresden and are giving good results, as nearly as I have been able to see from the preIiminary data. I think it is quite remarkable and, for the first time, gives one a chance to study one of these large cores as it runs, and as it undergoes its control rod programs, and in certain of its transient effects. This information will have important economic, as well as safety, implications. I think that the General Electric Company, if I may say so, and the Dresden people have a real obligation here not only to produce the results themselves, but to publish some of the things that they are finding out so that the reactor community can find out what they are learning--in particular, to publish the raw data and the results that they are getting from as many measurements as they can and as fast as they can. This will accelerate our understanding of these problems. As other reactors come on the line, they should follow the same procedure. 
One of the things that was discovered on the Dresden reactor is very interesting, if I have been informed correctly, and I'd like to describe it. On one of these large power reactors, so that it will last long enough to give a reasonable core life, you must carry a large amount of excess reactivity, which is held down by control rods in one way or another. This is true for any of them. First you put a chamber $A$ on one side of this reactor and a chamber $B$ on the other side. The region near A is made to go critical. I believe in Dresden you can do that by withdrawing four control rods completely and then pull the fifth one up in the center and the region goes critical. This reactor within a reactor is not a very big reactor, it's a long thin cylinder. After the region near A goes critical, then it is carefully adjusted so that $k_{\text {eff }}$ there is 0.99. Then the other side near region $B$ is made critical. If you consider chamber $B$, and withdraw the rod near $B$, the flux will rise on a period. Then let's say that you push the rods in until the power levels off, and then shut the region near B down by scramming those rods, and the flux will go back down. If you look at what has happened in chamber A during this time, you find a considerable delay before the flux rises, levels off, and falls, with considerable delays and different apparent periods I am told. The difference in time between these two may be as much as 2 to 5 minutes. Now these are cold tests, to the best of my knowledge, and are not typical of the operating core. I believe it was stated the other day that then the core behaves very much as one unit. Now I don't think this is unique in Dresden, I think it is common in all of them. One might ask the question, what would. happen if we had a reactor like this with one side in which we are withdrawing rods and the other side almost critical so that $k_{\text {eff }}$ is 0.99 all over the reactor. We then have a minor cold-water accident and a slug of cold water comes into one area. If you combine this with an improper location of chambers or other possibilities, it might be that you wouldn't even see a transient which was occurring on one side of the reactor until it was quite well advanced on the other side. Whether this can really happen, I don't know, but it seems to me that a lot of these problems of loosely coupled reactors which are large and which can be split up into minor sub-reactors, either by control rod programming or by other means, are worthy of a good deal more investigation than has been given to date. If I may say so, it would seem to me that this is a good deal more important than trying to determine how to start a reactor up with very low-level sources. I think it is also probably just as challenging. This is really quite a hard problem. If I am incorrect on any of these Dresden facts, I would like to be corrected. I think I had it roughly right. These are orders of magnitude and I am just roughing out the problem. I am sure the same sort of thing is true in Yankee and I am sure that it's going to be true in ConEd. I don't know about Shippingport; I don't know whether they have done tests like this. They have done some flux tilts where they purposely jammed the rods in on one side and started xenon tilting to see the flux pattern move. This is a somewhat different test. Some of these things, I think, are worth looking at and considering very seriously, and now is the time to do it. There are reactors on line, or going on line, which have some of this data available and also I think the spert program is going to be in position shortly and is already beginning to fill in some of these gaps in the larger core, water reactors. 
I should point out, also, control rod problems are a real bugaboo. The programming of one of these reactors is, I think, a real problem; it is not all reactor kinetics, but is partly that. of all the accidents that you can have in a reactor, I think the cold-water accident is by far the worst. I believe I have a fair amount of agreement from others on this. I see Stu Forbes shaking his head vertically, and I think Warren Nyer is shaking his up here. We have discussed this before and I think the three of us are in agreement that it wouldn't be a bad idea if we forgot some of the large negative temperature coefficient that we put in some of the big reactors now and try to make the temperature coefficient perhaps positive at room temperature and only negative at operating temperatures, if this can be adjusted closely. It is already adjusted automatically in the swimming pools; they have a positive temperature coefficient at low room temperatures and negative at elevated temperatures. I would caution here, though, that economics can take a part in setting the metal-water ratio, and it may be that the economics are quite a bit worse than the core, I suggest. This requires further study, but I do think that, if anything, one ought to tend to lower the negative temperature coefficient rather than to raise it. I believe that this is probably in a safer direction.

In summary of the large-core kinetics, I think a lot of additional work needs to be done. I think that all of Al Henry's remarks this morning on space-time variations and sequencing are pertinent and I think a good deal more work could profitably be put into this field.

Another region in which a great many problems exist is in the startup of a reactor. Transfer functions, I think, don't give very good information. Joe Thie pointed this out the other morning, and it seems to me that there are a number of problems there that deserve looking into. Hydraulic instability is one. I was particularly impressed with the Ramo-Wooldridge work mostly because it shows, as Dr. King has already pointed out, that these supplementary tests, in addition to the integral tests, are meaningful and worthwhile. Normally, I tend to resist these, but I think I was converted a little bit. The problem that always worries me is that, unless you have the neutron and gamma radiation and all other reactor environment effects in with it, you are not looking at the whole picture. In particular, I'Il cite, for instance, the behavior of uranium oxide. Normally, it is essentially insoluble in water. But, I am assured by some of my metallurgist friends that, if you put it in the middle of the reactor for a while under high flux conditions, it is soluble in water and you can leach out quite a bit of it into the solution. So that things change under radiation and, in particular, if you take a transient it isn't so clear that the radiation or other reactor conditions don't have some effect. This was pointed out by Everitt Blizard, alpha particles shooting out into the coolant stream and perturbations of this sort. It carries me back about four years to when Art Wasserman and I tried to find some of these effects in a very crude apparatus at MIT and failed completely. I still believe they exist but outside of spert little is being done to study them.

I would like to mention one point that was not brought out here. A group in Milan, Italy, under Professor Silvestri, at the CISE laboratory 
are doing some very nice work in the field of burnout. He is using twophase flow equipment, and I think it would be worthwhile if somebody interested in this field would check with him. Yes?

Zuber: This work concerned the thermal burnout problem, and I was informed that this was not actually the topic for this meeting, so I did not include it in the discussion of hydraulic problems. If I can take two minutes I would like to say, that the problem that Professor Silvestri is studying, is very interesting. It's a two-phase flow problem for the burnout. It is one model which can occur at high quality; however, there is another model for burnout in the flow regime which can again occur due to pressure oscillations. I think this is a model which can occur at very low quality. The problems to investigate now are two. One has to look at a high quality problem or at the low quality which can occur in subcooled boiling. A differential is involved, one is tied to the pressure oscillation, in the two-phase flow. These two, I think, possibly could give an answer to the burnout problem in two-phase flow forced convection.

Thompson: Thank you. One of the things I want to say about this work, which I have just recently seen, is that he gets an effect which I would like to mention as possibly being of value as well as interesting. This effect occurs just before the burnout condition. He observes an increased heat transfer, essentially a drop in temperature (he is measuring the temperature on the wall), just before burnout occurs. It struck me that it might be possible, if this is real, to make suitable instrumentation so that, if you could place such an instrument at a hot spot in the core and you have instrumentation which will resist the high radiation level, you could predict or have a little bit of advance warning--it's not much-that burnout was about to occur in your hottest element and do something about it. I am not suggesting that this is necessarily a solution, but at least it's worth thinking about because, after all, burnout is one of our most difficult problems. This was mentioned to Professor Silvestri and I think he is thinking about it too.

In the case that Bill Ergen mentioned this morning, a universal fuel element, I would like to mention one other if I may. The universal fuel element that Bill is working on reminds me a little of the one that Carl Muehlhause has suggested. Carl, as most of you know, is a former Brookhaven scientist who is now at the Bureau of Standards. He has suggested an element, mainly for heavy-water reactors, in which one has a series of concentric rings. By appropriately adjusting thermal absorbers, such as boron, and uranium content, one gradually increases the thermal neutron absorption of the rings as they go toward the center so that, if it is adjusted just right, one ought to be able to make an element in which $k_{\text {eff }}$ is exactly equal to 1 . Hence, in the core one would really be unable to detect whether the fuel element is there or not. The innermost tube would be essentially a black thermal absorber and by so doing, a high fast flux would exist in the center of the element together with a low thermal flux. Thus, fast neutron radiation damage can be studied while, at the same time, the thermal flux is low and the reactor, reactivity-wise, never knows what is in the center of the fuel at all. This is an idealized element, but it's something Carl Muehlhause is working on and we are going to do some experiments for him up at MIT in the 
very near future. So far as test reactors are concerned, I think this may make some interesting sense.

May I offer a comment on Bill Stratton's talk on criticality accidents this morning. In looking over a good many of these, I think it becomes apparent that three or four things usually happen. There is usually something wrong with instrumentation--almost always there is an instrument error on any criticality accident or there is a lack of instrumentation as Bill said this morning. Second, there is usually something wrong with the design, there ${ }^{2} \mathrm{~s}$ a faulty design feature somewhere in the system. This design flaw may be subdivided into two types: built-in inherent safety problems, such as positive reactivity coefficients, and faulty mechanical or nuclear design. Third, there is usually an operator error. Normally, the operator error is made by a supervisor or management. You usually find these combined in varying degrees. If you study all of the criticality accidents that have occurred, it appears that the worst of all these usually occurs when one brings two large chunks of an assembly together. Of course, this is because the multiplication, the $1 / M$ curve, remains flat for long periods and then just suddenly drops immediately to critical. One can find, time and again, situations in which large masses are brought together and these are the most dangerous ones--the ones which you would hopefully like to avoid. It may even be possible to bring a large reactor together if you, say, have the core somehow isolated, one part from another, by control rods and. suddenly raise these control rods. Those control rods, by the way, are worth a great deal of reactivity.

In commenting on one other point that was made earlier, one can make a very strong case for building reactors out in the desert somewhere and testing the reactor kinetics, testing the reactor behavior in the desert over long periods of time, rather than do the in-pile loops and studies on materials and loops and so on, because it normally does not cost much more to build a reactor and try the whole thing out than it does to do all the partial experiments. This is, in no sense, said in depreciation of the things that have already been said on such systems.

One final comment: it seems to me that, as a previous speaker this afternoon has said, we need more information dissemination, I would like to see more summaries of the type that have been given by Al Henry and Joe Thie and so on. But, in addition, summaries of full fields, in what I would like to call monographs, which would narrow down the problems in regard to the safeguard discussions that have to go on everywhere. If one had, for instance, a monograph which stated a basic philosophy of reactor kinetics, I don't care whether it is the best one or not, but stated one which was reasonably accepted by everybody (there might be some disagreements) but there is something at least to start from, which one can argue. Then, when one goes into the Atomic Energy Commission and to the HEB one could say, "We have designed this according to this monograph, or this set of information, with the following exceptions", and immediately the area of discussion is narrowed down to a relatively small part of the system. Now, if one disagrees with something in this monograph you can come in and say, "Look, I just don't believe this at all and I think this is a better way", one can present his case and it 
would then be easy to shift the system so that a new set of criteria could gradually be developed. In this way I think the understanding and the agreement between the designers, the safeguard people, the operators, and so on, would be substantially improved and, at the same time, a good deal more information would be made available to everyone. In particular, for insiance Al Fenry in his survey this morning mentioned a paper which has appeared only in a Hanford progress report. Now who in the world would read all the Hanford progress reports in order to find this paper? And somebody needs to dig some of these things out and put them where everyone can get at them. Some of these are classified, some people would never see these classified documents. If you have what you feel is a really fundamental document, write it up, if you can, in an unclassified version. Somehow get it out where people can read it. In your own words, summarize your work so that it makes good sense and so that you can state conclusions which will be useful to others who have to come after you. In particular, I think I would have liked to have seen a few more conclusions on some of the papers here, such as, "I think that my method is good in such and such a regiori or it has the following problems", and so on. I know it's early and I realize that in many cases you can $t$ do this, but anything that you can do in the direction of helping to formulate general policy and helping to formulate mutual understanding with the people who come after you, is all to the good. Many times recently, I have seen people making errors and repeating mistakes that a good many people knew about eight or ten years ago. The new men have just never heard about it, it's in an unpublished Oak Ridge report or something of this sort. Anything anyone of you can do in the way of helping to supply better fundamental information is certainly worthwile.

Chernick: May I ask a question? Professor Thompson, you have raised one very important, and I am afraid controversial question, and this is that of core instrumentation in a high-pressure system. Now, you did mention Dresden, as an example. This is a rather unique subject because it is such a long column that your seals could be put into place where there is a relatively weak radiation field. I am not an engineer, but I have to argue with them, and I think you will have to convince the top of Argonne Engineers that this is possible in most power reactors, that generalely tend to be more compact, the shells tend to be a lot more compact, and industrial designers, I don't think I have to mention any one group in particular. . . .

Thompson: I think the answer goes something like this. In the big power reactors and almost any reactor, in which there is associated some temperature and pressure, one of the chief worries is, "What happens to the pressure vessel". Nobody honestly knows the answer to that to this day. Since they do now know the answer, they try to put the pressure vessel in a relatively low flux. Iet's take the Dresden or Yankee vessels. If you make a seal, let's say in Yankee, it's way up in the top lid. From this seal there are a serles of little tubes that come down into the core and fan out, and go into the various fuel element positions, and these are inserted after the fuel is put into place and it is possible then to slide instrument wires, or chambers, down into the core. A similar system exists also for Dresden. The answer to your question is 
this, that since people don't know about how much pressure vessels will stand, they're going to try to keep the pressure vessels in a low-flux region, by thermal shields, etc., and when they do that they also, at the same time, make it possible to put in seals which can hold instrumentation. It does not come for free. It costs a lot of money, but I think I am correct in saying that if Dresden, and I am speaking now I think for the Commonwealth people, that if they were to do it again they would repeat exactly what they have done. They are quite happy with what they are doing and they probably will be able to operate a core so as to justify, economically, the insertion of this instrumentation in the core. It is certainly too early to say that about Yankee but certainly you can say it for Dresden, I think.

Chernick: Well, I think particularly if you could get some instrumentation to measure fuel temperatures or, at least, temperatures in individual cooling channels, this would be even more important than the question of xenon oscillations that you raised. But there is one other point in that region, can you get away without using plastic materials which damage a lot more easily than steel?

Thompson: You mean can you make chambers without plastic materials?

Chernick: Part of the cover is plastic and I know that the EBWR-I had a plastic seal that was removed every once in awhile because it did damage very easily.

Thompson: No, I think you will find that the things that are put in these large power reactors do not contain plastics--the tubes are welded into place (there is atmospheric pressure inside so you can slide anything in the tubes you want) and standard type seals are made involving nothing except steel and stainless steel 0-rings, etc. You can make it without any plastic parts. You can also thank the Savannah River Project who make chambers which will resist these high fluxes and high temperatures. I think you are in business. The necessary techniques exist today and can be used. I think there is no question about it. Even if these chambers in Dresden were gradually to go out, they have enough life now so that they have indicated they are really quite good.

Nyer: I think I would like to comment on a couple of points brought up during the last discussion. I think I would emphasize the point, Tomy, that safety is economic, to a large measure. I think there is a little bit of a distinction in the point that you were making.

Thompson: I think that you can even argue that you can take all the reactor accidents to date, and safely say that if they had been built a little safer they would have been better off economically. You can say this without any question. It pays to shut down whenever you're in doubt and see what the trouble is, rather than to try to mu a little more, and see if you can't make a little more power and endanger the system.

Nyer: Well, I also meant that, in order to insure safety, we have to overdesign, which results in an increased cost. One other point I would disagree with you on is that the addition of any particuldr instrument, whether it be to display a transfer function or whatever it displays, 
necessarily adds to the hazard by giving people a false sense of security. You can look at any combination of instmuments we have in there already as having this property, and the way you try to avoid this, as an operator, is to say that whenever there is a disagreement, we shut the thing down. Now occasionally people won't do that, but this is the way you try to avoid that problem.

Just a little additional bit of information, spert IV was designed so that we can test these large cores. There are two other comments I feel I would like to make if you won't object to me taking the soap box for a while. For some time, we have been talking about this viewpoint toward positive coefficients, and I think that maybe the way we should summarize the comment at the end of this conference is, there is a little bit more of a trend for people to look at the possibility of shaping the void coefficient to attain a degree of safety, I don't think there is any need to elaborate on that, but at least this is somewhat of a change in viewpoint and it is in the direction we were talking about here. Al, do you have a comment?

Henry: On that I'd like to make a comment, and I think maybe this is the opportune time. It is possible for some of the small reactors (and I don't know about the large ones, I've never tried to design one) to create a reactor which has a zero temperature defect, by virtue of the fact that the reflector water has a positive component to the temperature coefficient; nevertheless, this reactor, if it runs away in an interior fashion, will have a highly negative prompt coefficient, as we call it, so that it would seem possible to make the flow come in, in such a way as to take advantage of the best of all possible worlds, a combination of such effects. At least this is the attempt in some of the futuristic designs in small reactors.

Nyer: A zero adiabatic coefficient and a very strong negative prompt coefficient .... .

Henry: There may be other ways of doing it, so that a loss of flow accident-well, once you get loose with this idea, you can fill up blackboards with possible schemes. But I think it should be added to the list.

Thompson: You can't do it by reflectors in large reactors, though, because the reflector has such a small effect that it is essentially . . .

Henry: There are large reactor designs which are essentially controlled, at least for which proposals have been made, which are controlled in such a way that you might do this. The Combustion design for the Advanced Power, which had the boxes of poison for control would be an example, there is lots of water which might have one effect or the other.

Nyer: Well, I do think that what has happened is that the designers now are looking upon the temperature coefficient, or the void coefficient, as a design parameter that can be shaped to gain certain ends. I think it might be worthwhile to elaborate on a comment that stu Forbes made earlier, and that is that all of the models that we have looked at, at 
Spert, end up with the parameters of safety that are involved as being: the energy that can be allowed to be released, the reactivity coefficient, and the lifetime. Depending on which particular form of the coupling equation is used, these enter in different degrees. The thing I want to add to this is, that if we look at each of these separately, the energy which can be permitted, or tolerated, in a release doesn't vary over a factor of ten for all known substances, just because of the fact that you can't change the melting point very much when you go from one substance to another, at least not much over this factor of ten. When we look at the lifetime, however, it can vary over many orders of magnitude, but this is not with quite the same freedom; that is, the designer doesn't have the full freedom of using all of the lifetime latitude that exists, because for any given class of reactor, he's always somewhat restricted and maybe he can't vary that more than a factor of ten, either. On the other hand, the void coefficient can vary by many orders of magnitude, so that the argument that I think one could make on this is that the place where we have the big chance of making the greatest gains with respect to safety is in the void coefficient itself, rather than in fooling with changing the materials of construction.

Perhaps we should get on with the last formal speaker. The reason I want to get on is that some of the people may have to leave and we can continue the informal discussion afterwards. 
SESSION VI

PANEL PRESENTATIONS

Nyer: I would like to call on Dr. McCullough.

\author{
Surmarizing Remarks \\ by \\ C. R. McCullough (Mon)
}

McCullough: Mr. Chairman, Gentlemen. There is not much I can add. I think the thing has been pretty well covered, but I would like to take this opportunity to enumerate some of the things. In the first place, I want to say that $I$ am very pleased and happy that $I$ was invited to this conference. I have gotten a great deal out of it. Other people have said, and I want to second what they have said, that this has been, from my point of view, a very excellent conference. I think I see evidence of very good work in both the theoretical region and experimental region and also in the region of theory and experiment on the components. Now, as I said to begin with, I think this is exceedingly important; and I am very glad to see this integration. I am so enthusiastic that I almost moved to say everything is perfect; and, of course, I don't believe that things are ever perfect. I think that the people that sponsor this work, whether it's Stan Szawlewicz or whether it is the Naval Reactors Branch or who it is should be very proud of the work that is going on. I think you people that are doing the work should be proud; but don't get too proud, don't forget, there's a lot more to be done.

The second point I wanted to make, just a couple of things in the fast reactors. I think there has been a real improvement in the last several years in the study of the fast reactor systems and I think we have really done a pretty good job of understanding the theory, and we have gone a long way toward the experimental verification of some of these factors. I think we are quite a long ways from the end yet. Now this problem of bowing of fuel elements and clearances of fuel elements obviously is a kinetics problem, but I can't help but be impressed, as $I$ sit and listen to this, with the fact that there is a very large and very difficult mechanical engineering problem that somebody should attack. Further discussion of this I do not think is pertinent at this conference. The third point I want to mention has been mentioned several times, the startup without source, and I will pass that on by saying I am completely confused, I want to go back home and sort out my ideas. The fourth point I want to mention is that I want to commend the very interesting and. valuable work that has been done by Treat and Spert. This is an example of work that I think has done a great deal to point up many of our ideas and the theoretical work that we have done. The Treat work is just beginning and I think we will get a great deal of information about the behavior of fuel elements as the work continues. What I learned about it at this meeting has been very illuminating, and I am just anxious to see a whole lot more. 
Now with regard to this nucleation problem, which has considerable theoretical interest, I am still uneasy about the state of the art. I hate to leave it and say if you put some soldering flux and a little bit of--speck of solder--or leave some cracks in the fuel elements, that everything is lovely. Now maybe we shouldn't be running fuel elements to near enough their melting point so that this is a problem, but in power reactors the economic pressure is to move that temperature up as far as you dare. If we know that this automatic inherent shutdown mechanism will take over at some distance below the melting point we might be in much better shape. And, if the superheat can keep on going until past the melting point, why then we are in very bad trouble.

There is another point which has been mentioned repeatedly: obviously, we would much prefer to have an inherently safe reactor. I am afraid that the world isn't that perfect yet, so we do have to rely on gadgets, some kind of control mechanisms. I think we need to. Epler made some points about this which I think need to be thought about much further. Of course, people tell us that they can't get their reactors on fast transients; this was the core of the remark that I made the other day when I questioned, "How do you go about getting a two-millisecond period", and I got my ears pinned back. Well, so I say that the question is really open, that we are not sure yet that we can't get very short periods, particularly with the coldwater accident and we must now put the proper balance between reliable fast shutdown mechanisms as well as the inherent ones.

The sixth point that I would like to cover is the problem of those of us who are engaged in giving advice about and studying the safety of various reactor systems. I am sure you recognize that we do not completely review any system. That would be impossible. The person that knows the most about the system is the man, the group of men, that designed it. Now all we can do is to go over a few of the details and try to make sure that the designer really had an adequate understanding of what he was doing and had made sufficient tests. You recognize that commercial pressure and economic pressures tend to short-circuit some of the studies and analytical examinations and experiments that you might like to make. Now it is very gratifying, therefore, to come to a conference like this and see the amount of effort that is really going into understanding why you put certain design features in and, although we are not going to rely on that completely, it gives us a real feeling of satisfaction that this kind of work is going on. Now Stu Forbes said that we are groping for a figure of merit of safety for reactors, and we are. I also want to assure you that the longer that I have been working in this field the more I've come to realize that it is not a simple number--it is not a simple concept--we have to examine a variety of factors and a variety of concepts. At the present time there are only a very few areas where I think it possible to write down a rule of thumb or a figure of merit and, when we do this, we must always put four kinds of danger signals around it saying it's workable under certain conditions. Now the essential problem I think we should never lose sight of and that is, as far as transients are concerned, we want to be assured that there is no part of the reactor 
which can release fission products. That's the thing we are really talking about. The reactor owner and the reactor designer may have a secondary objective; mainly, that under no conditions of transient can the reactor be damaged so that he can't run it. But I really think that's another way of saying that he mustn't release fission products. Now the only thing that I can think of which is really important, although I can think of some secondary effects, is such a high temperature that something melts. Now if you talk about one little part of the reactor and begin to examine what can happen to that little part, always keeping in mind what your real objective is, and then deal with how the reactor behavior or other parts of the system relate with this part, or how this part looks over in another location, that then the examination of transient and space dependency, and what-not, has meaning. But I think we want to keep in our minds that the release of the fission products from any part of the core is the thing we are trying to prevent. And secondary to that, almost identical, is that you mustn't let any part of the cladding or the fuel melt. I should comment, however, the center of certain oxide elements probably do melt and, at the moment, we are not particularly sure that's bad.

I want to endorse the comment that $\mathrm{Dr}$. Beck made, that we do need more critical evaluation of the data that we have, and putting it in my own words and endorsing what other people have said, we do have a mass of data. It is very difficult for those of us who are not working directly in the field to know how to put this data into its proper context, its proper weight of importance. How we go about getting this critical review, I am not sure. Those of you working in the field can help a lot by taking time to review your work, and I don't think it's necessarily a useless labor because if you sit back and review your work you may see some areas that you have overlooked, some areas that you haven't properly appreciated, and perhaps it will suggest where you could do further work. As a matter of fact, my feeling is that the mass of information in this field, in the Atomic Energy Field, is so great that there is a crying need to sit down and examine it; and I have, just for simplicity, separated the kind of judgments that you'd try to make of the reviews that you should make of this data. One, there is certain data which is adequate for the purposes of using certain simple limited rules of thumb. Secondly, there is a field where if you'd only do a few more experiments, and you see clearly how you can do these experiments, that you'd be able to (if the experiments turned out well) set up certain limited rules of thumb. There is another category where you just don't know enough about the business to ever hope to be able to set up criteria or any of these simple rules of thumb. In these areas (except in the places where you think it's interesting and useful to do experimental work) from a standpoint of criteria the only thing we can wait for is experience and in the meanwhile just use judgment.

Now, one final point. I don't know to what extent you people realize the increasing need for not only translating the language of the expert into language which is understood by the technical person but not expert in the field. This has been mentioned before and this is a very important need. In addition to that, we must 
increasingly educate the layman and this is exceedingly difficult to do. I have made a few attempts at it and I find it very frustrating and difficult. Now I don't know who does this job, but certainly to the extent that you can express your ideas and your conclusions in an intelligible manner to the technical person, then that makes it that much more easy for someone to translate it to the layman and is, believe me, becoming very important. Thank you.

Nyer: In just a minute or two I'd like to call for a coffee break and then we'll get into any discussion period that may be left but before we do that, while everybody is still here, I'd like to mention that I think that the people who spoke in the first session and this last one probably had the most difficult job of all. I personally feel that this was handled admirably. In addition to that general comment, I would like to thank everybody who participated in the meeting because I believe we had a successful meeting. The comments I have heard have certainly been in that vein. This would not have been achieved without the efforts of all those people who attended, prepared papers and took part in the discussion. 
-
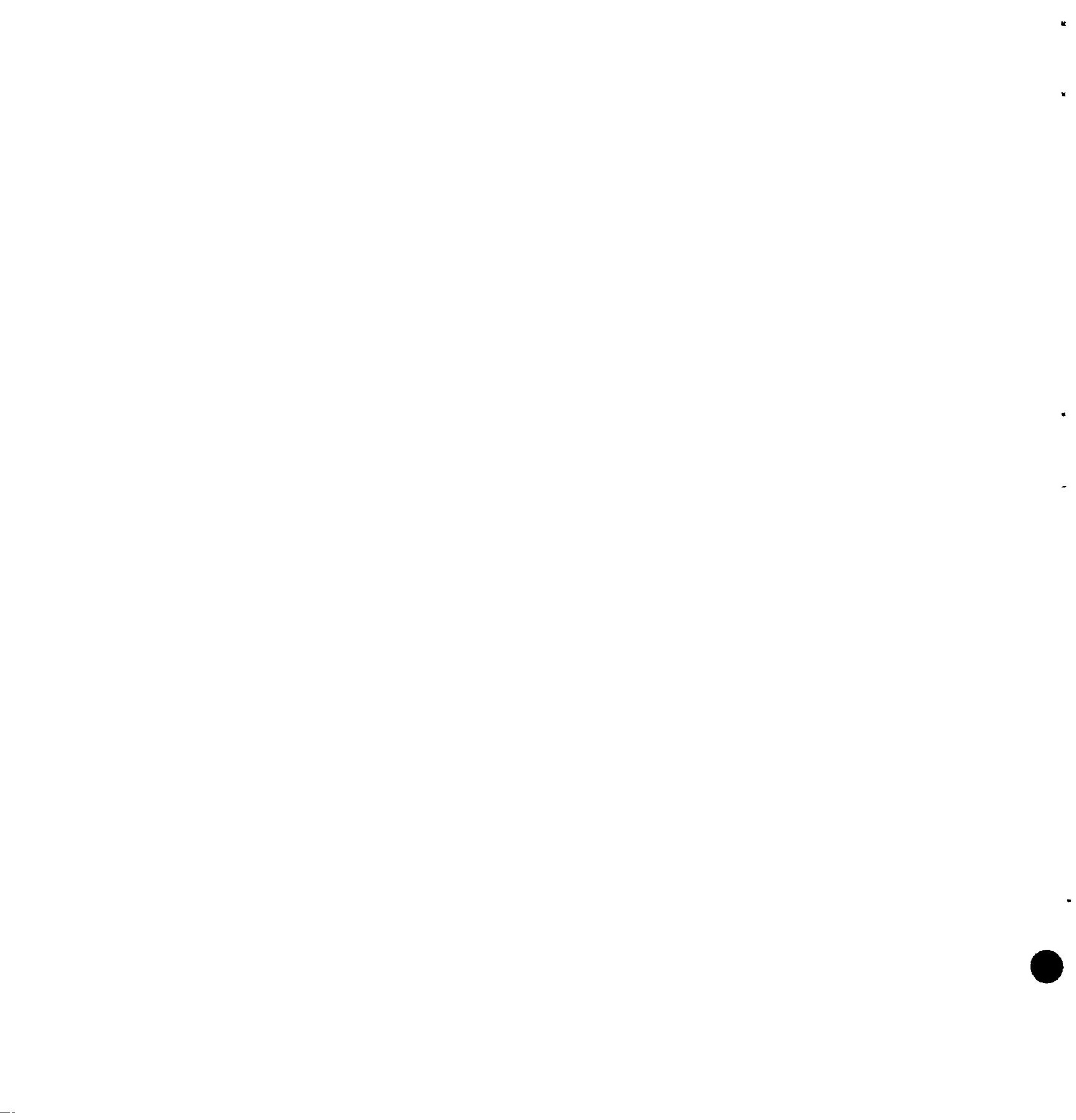

-

$-$

. 
Nyer: We've had three days of rather long meetings. Everybody is probably pretty well tired, and we may have said all that we possibly can, but as long as anybody else is willing to stay here and argue awhile, I'm willing to do the same. So I'd like to call for any questions or comments from the floor. Stu, do you want to make a speech?

Forbes: You bet. Since I limited myself to the prescribed ten minutes this morning, I feel cheated.

Nyer: Have at 1 .

Forbes: Commenting along the line that Tommy started here, I'd Iike to take one more thump on the drum for testing reactors, as built, during commissioning, and make a parallel analogy. You design a new aircraft, you build a few prototypes-you literally fly the wings off--often destructively, at least on occasion. But, you don't quit there; when you start the production line, the plane comes off the production line-it's tested, and it's not ground tested, it's flight tested--and it's flight tested under conditions more severe than those you expect them to operate under, and only then do they go into passenger service, and most of the time the plane is in pretty good shape. Now, as a passenger on this airplane, if the flight test of the one $I^{3} \mathrm{~m}$ in is neglected--I object. And I would think that, as a resident of a place close to a reactor, I would have the same feeling about flight testing of reactors.

Nyer: May I add a comment before I call on any other objection. I think we have not entirely stated what we mean by this, Stu. One thing--we're not suggesting that you should take every reactor and see what you can do to make the sides bulge. But there are a number of tests that can be carried out and they are transient in nature--they are excursion tests-and we believe they can be done with complete safety. Now, if the people who own a particular reactor say, "We could carry out any tests you want, but we want to do it in complete safety", then I think these tests should still be done, but with the understanding that they may not be able to be carried far enough to yield much useful information. And I think the point that we just haven't stressed enough is that we feel that you can do these tests safely if you want to do them safely. How far you want to carry tests of this type is a second question. Tommy?

Thompson: I think there's no real disagreement at all on this, Stu, and Warren both. As far as $I^{\prime} \mathrm{m}$ concerned, every reactor does go through a flight testing during startup; and your problem, as I see it, the only controversy might be on the transient parts of such experiments and what I said, I think, was that if you can figure out ways to do this so that you can convince an owner that he's not likely to have the thing blow up in his face, then by all means--let's do it. But we have to figure out first-and I think that you have to do this by using Spert as your testing of the original models of these things--to show that 
sush tests can be done with safety in the core and, once you've done that, then you can convince individual owners that there's no problem and then you can do transient tests on each one as it starts up.

Nyer: I hope that by next year we will have a type form of the kind of tests that can be performed.

Thompson: That's what you're here for.

Nyer: Walt?

Lipinski: I'd like to inject a comment with regard to the nucleate bolling phenomenon. These tests are performed in a laboratory under ideal conditions. If as a result of these studies an optimum surface were to be designed and installed in the reactor, the purchaser of the plant might be surprised, in shooting for his megawatt-days per ton, to find the dynamics of the plant have changed considerably due to the fact that material has been deposited on the surfaces. A particular example to cite would be the 5-mil crud covering on the EBWR plates. The part that hurts is that it occurs at the point of maximum boiling. These studies should be carried through to include the case of the corroded plates, and see what the resultant nucleate boiling is on old plates.

Nyer: Mr. Storrer?

Storrer: With regard to the flight testing of reactors, if you made those wild transient runs in the reactor itself, it's like flight testing the reactor with the passengers in it. If you're testing, for instance, any reactor near a big city. . . . .

Nyer: Please answer that, Stu.

Forbes: Have you got more to say before I start to... . (laughter).

Storrer: This was not the main subject I wanted to discuss, this was just the ..... (laughter).

Forbes: The fact of the matter is, you don't have the passengers in it because you don't have the fission product inventory, for one thing. And, second, you use the phrase "wild transient test"--I disagree. Transient tests are not wild, but quite ordinary things and, once you've seen a few of them, you realize this; in fact, I'll cite an example, in BSR-II; the core was at spert and I dropped in near the end of their experiments and asked "What is doing today?" They say, "Oh, nothing interesting at all, just an old slow 20-msec transient". And then he caught himself-and he said, "Good heavens, if I go back to Oak Ridge and I say this, they ${ }^{\prime \prime l}$ think I've lost my mind". . But these are not power-dive tests of our passenger plane, and don't resemble them at all; I think the first thing I can cite is, first, people who have stood and watched a few of these things seem to get very blase about it. In fact, there's danger even there. The other fact is that we ran--I don't know--a thousand or twelve hundred of these things, and deliberately skirted the area of very destructive excursions, and not just stopping where 
we knew we were safe, we pushed well on past this and have yet to step over the brink, and if people do this a thousand times--or whatever we have done it--certainly we want to be a little more conservative, I don't see that there's any real big problem. And this is the difference in viewpoint. They're not power-dive tests, they're not wild excursions, they're quite ordinary tests.

Nyer: Yes, along with Stu, I think those of us who have been close to these tests have a feeling that the results are much more predictable than is believed by people who have not been close to them.

Storrer: I agree with you that this absence of fission products is really a very important point of consideration. Now I'd like to talk a little bit about the prompt positive coefficient in fast reactors.

Nyer: Would you like to come up here and use the microphone?

Storrer: ..... My voice isn't loud enough?

Nyer: Why don't you use one of these on the side--I think the people in back might be having a little difficulty in hearing you.

Storrer: I don't think I explained it very well, yesterday, when I aaid, "prompt positive coefficients". We always postulate that the next steadystate coefficient is negative.

Nyer: That doesn't satisfy me. Anybody else?

Storrer: No--well-I would like to amplify a little bit on this. First, obviously, suppose that our steady-state coefficient is negative. Now let's assume that our Doppler coefficient is positive, this is certainly a prompt positive coefficient. One certain way to avoid any trouble is to have a coefficient which is just as fast and is negative and larger, that's the obvious way, of course; and fuel expansion is really just about as fast as Doppler, except in an atomic bomb. I mean--for our reactors in normal operation the fuel expansion does not play any role in our stability studies.

Unidentified: You mean, then, that you don ${ }^{7} t$ object to having one contribution to the prompt coefficient which is positive but the overall has to be negative?

Storrer: The steady-state one or the overall prompt?

Unidentified: The overall prompt.

Storrer: Well, that's my number two point. You can also have a prompt positive coefficient and a delayed negative coefficient, but the ratio of their time constants and their magnitude will be such that even if you go up at a very short period, as short as you want, that the net feedback is always negative. And you can show this very well by a simple example. If you have a sum of two coefficients and I write $x_{t}$, which is the coefficient, this would be the coefficient when you 
have an exponential with inverse period $P$. Let's say we have two coefficients of first order. This is the positive one, and then we have a negative one. We have, of course, that a is smaller than $b$, because in steady-state $x_{0}$ would be $a-b$, $a$ must be smaller than $b$ to have a net negative coefficient. We say prompt positive which means that $\tau_{\mathrm{a}}$ is smaller than $\tau_{b}$, but is this acceptable. Well let's look at $x_{p}$ when $p$ becomes very large, and let $p$ even go into infinity--then this goes to--the positive coefficient goes as $a / \tau_{a}$ and the negative coefficient as $\mathrm{b} / \tau_{\mathrm{b}}$. And, if this is smaller than this, then you never have a positive net contribution, whatever your period is. And so this tells us that a does not have to be smaller than $b$, only that $a / b$ must be smaller than this ratio $\tau_{\mathrm{a}} / \tau_{\mathrm{b}}$ which is smaller than one.

Editors Note: No editing of this discussion of prompt coefficients has been effected. Mr. Storrer's review of this transcription was not available at time of publication.

Cohen: You don't have a prompt positive coefficient.

Storrer: Now we must define what we call prompt. This is what you would have if you had a prompt Doppler, and a delayed coolant effect.

Nyer: The coolant is negative--that's the point.

Cohen: But even prompt, the net coefficient is negative.

Storrer: That is right.

Cohen: There's a contribution that's .....

Storrer: That is right.

Cohen: In other words, it's your positive that's deteriorating.

Storrer: Well, that's now a matter of definition; $k$, which is the feedback responsible for balance, is always negative--that's what you're talking about. But here--I call this a prompt positive coefficient. Maybe my definition is odd, but in reactors when you have coefficients acting in parallel, I always used to call it the prompt positive coefficient.

Cohen: Don't you think a case like this is due to inherent coefficients?

Nyer: Well, I just--one point--we have two questions; first, Jack, would you go ahead.

Chernick: Yes--I'II be very brief. We've been warned about being too theoretical. I would think if I were on the Safeguard Comittee, the first question of interest would be whether there are any accidents when you could go prompt critical. And, I think this is the most important question that should be asked when you're talking about prompt coefficients. If you can never get anywhere near there--then it doesn't matter very much and you can expect the negative delayed 
effects to take care of the situation. We are all impressed by Los Alamos experiments where their safe bursts are always counted in cents above prompt critical and I think this is really the crucial fix.

Nyer: I think I'd like to ask your question in another way, Jack, and this might answer the question for Dick, too. If you have a reactivity perturbation, does the period tend to shorten or does it tend to lengthen? And this avoids the semantic difficulty we're having so far--maybe you'd answer it that way. If you have a reactivity perturbation, does the period tend to lengthen or shorten?

Storrer: Yes, that's exactly it (laughter)--we--that's the question-does it lengthen or shorten. When you talk about prompt critical, if you always have a negative coefficient, as you have here, a net negative, even for any period, then if you move your control rods slow enough you will never get into prompt criticality; even if you insert 5 dollars the feedback will always catch up with you. The prompt critical is the only danger--is the main danger of fast reactors. You would run into trouble if you had a positive coefficient--I mean if this ever became positive--in this sense. But that does not mean that some component cannot be prompt as we have here--or positive.

Kouts: I think a single temperature has been discussed here. The temperature coefficient comes from several sources, and it's possible that under some conditions, this negative coefficient just isn't here or it is not strong enough, so your condition for stability is violated. If it comes from heat transfer to fluid, for instance, then you're certainly not safe for no-flow conditions. For instance, if you have a positive Doppler coefficient you would always try to design so that the fuel expansion coefficient, which is just as fast in normal operation, would be negative and larger.

Storrer: Let's say we calculated the Doppler to be positive and $90 \%$ in magnitude of the fuel expansion. Well, it is not a magic number; if we miscalculate a little bit and the Doppler is 1.1 times the negative expansion, it is not a catastrophe, this is what I wanted to point out, just the sort of things like that.

McCullough: Perhaps another way of saying this then is that, although you may have a positive component to your temperature coefficient, you insist that your temperature coefficient is always negative and, in addition, that the components of it cannot be separated.

Storrer: That's right. And, as Dr. Kouts points out, of course we have to look not only at the full-flow conditions without all kinds of other conditions, but using the same criterion.

Nyer: Yes, Fric.

Beckjord: I want to make--as a user--an occasional user of transfer functions I am impelled to say something. And, this is a controversial subject and I think that the opposite views are expressed on the one hand by a sayjing that the students used to have at a well-known electrical 
engineering school, when they said that, "The transfer function is the One Way, and the Head of the Department is Its Prophet", not to mention any names. The opposite view is expressed by a physicist from the same institution who refers derisively to all transfer function users as $j-$ omega artists. I find myself in complete and whole-hearted agreement with Professor Thompson's very well expressed thoughts on the subject. And, in particular, I wanted to say just for a minute what I think transfer functions as a tool are good for. And, first of all, I think it's useful for looking at a problem that you initially don't know anything about--it's good for the process of linearization, trying to gain an understanding, initially. In some instances it's been demonstrated that you can extend the transfer function and the linear method to nonlinear problems, but these are a pretty limited class of nonlinear problems. You can go on into the area of design, extending transfer functions with the use of analog computers to very useful studies of large and complicated systems, provided you know enough about the component parts of the system, and I think that's pretty obvious. I think in regard to--as a practical matter-in regard to predicting reactor stability, I think that again the transfer function is useful for small extrapolations in going to higher power, providing you drive slowly enough so that you don't run off Professor Thompson's cliff or, at least, you go slowly enough so that maybe you can get some indication that you're coming close to it. Well, it's definitely limited, and it's limited by the cliff problem, but there's another limitation which is essentially this: the transfer function was designed to handle electrical network problems. And as soon as you get into transport problems and partial differential equations, and that kind of thing, the limitations begin to show up fast and, in particular, in studying this hydraulic instability problem, I think, myself, many of the governing or perhaps the most important governing factors here are the delays, the transport delays. And, when you come to study a delay problem with a transfer function, you find that if you have a principal delay in a system, no matter what you do to the other parameters of the system, the response is pretty much governed by this delay, and it's obvious in the function $\mathrm{e}^{\tau \mathrm{s}}$, and the phase of that function drops off very sharply--with frequency--and, to use the example of the two-phase flow loop again, if you go and make transfer function measurements, for example, on the loops that we've been working on, no matter what particular condition of instability you're studying--because there are many conditions--you find that the transfer function is going to be pretty much the same over this whole wide range of conditions. Therefore, what does the transfer function tell you about this system, and I say, if you don't know what the governing parameters are of this system, the transfer function doesn't tell you very much, and so that--well, I think in some cases--transfer function measurements--oscillator measurements--are useful, I think that if it's a question of spending a lot of money and getting the equipment together to do these tests; if it were my decision, I would prefer to put the money into better instrumentation rather than expensive oscillatory devices. Oh--and the only--one other thought, going back to what transfer functions are good for, they appear to be about the best way that I know of, to measure the feedback-negative feedback coefficients in boiling-water reactors, and maybe in other types, but certainly in case of water-moderated reactors. 
Nyer: Nelson?

Grace: Nelson Grace of Bettis. I think I agree with most of what Professor Thompson says and what Eric says. I think we have to clarify a point, though, that there is a difference between the use of transfer functions for the prediction of stability and the understanding of the important mechanisms on the one hand and, on the other hand, the measurement of transfer functions and trying to extrapolate them into areas of the unknown. When it comes to trying to predict something, or analyze something thoroughly on the basis of fundamental principles, one derives a transfer function. It is quite clear in every instance, when you've established a criterion, a stability criterion, what parameters are important and what parameters have to be changed in order to increase the stability or reduce the instability. It is when we get into the measurement of transfer functions--that isn't based necessarily on fundamental principles or basic equations--that we get into an area of really risky business, I think. And I think that the transfer function application to reactor stability has been extremely useful to us in the last ten years at Bettis. We have never used it exclusively to predict stability or safe operation; we have always supplemented it with analog computing studies and digital computer studies where the nonlinearities are included. So I think that I do agree with Professor Thompon that it is dangerous to measure transfer functions and then try to extrapolate them too far without attempting to understand the fundamental principles, since you haven't necessarily anticipated what you're going to get into in these other regions. Professor Thompson used a favorite example of mine--the Electra airplane--suggesting that there was a kind of instability that apparently had not entered into previous designs for airplanes. I think this is a good example which helps justify the investigation of different modes of instability even though they haven't given us any difficulty in the past. If there is a possible mode of oscillation, let's examine it and try to understand it by transfer function techniques supplemented by experiments and nonlinear transient analysis, but let's not measure something in one region and then assume that it applies over too large a range.

Nyer: Bob?

Wright: I think that in transfer function measurements, say measuring frequency response characteristics of a noisy black box, it's a whale of a lot easier to measure, to get a decent measurement, on an a-c characteristic than on a d-c characteristic. I think that you can gain information on the system this way and you can make better measurements sometimes; and there's a second question. $I^{\prime} m$ not sure that I understood all that Eric Beckjord was talking about, but you can certainly measure frequency characteristics which do not amount to solutions of simple differential equations and which cannot be represented by simple time constants. I think there has maybe been a tendency to represent unknown black boxes by throwing in one or two time constants when they have little to do with the real situation. But we have been pleasantly surprised, I think, with the way that you could get frequency response measurements on unknown systems, on the part of the system which involves the reactivity feedback in the boiling reactor in our 
case, and can gain understanding of it by these frequency response neasurements.

Nyer: Let me see--Jim?

Boland: I'd like to say a couple of words that come to mind, listening to these discussions in regard to transfer function limitations. I think it's basic, if you don't know what the devil the physical system is, it doesn't make any difference what your mechanism is for investigating it, you're not going to get any place. A transfer function, a transient analysis or anything else is no good. The tone has been "don't extrapolate transfer function data". One shouldn't extrapolate transient data either. They're all one and the same thing and you have to know the physical system or extrapolations can get you in trouble. Something else that comes to mind along this line is that the transient analysis work reported here, at least in so far as I have followed $1 t$, has been concerned with transients starting at low power levels. It does not seem to me that we have paid enough attention to transients at power when we're operating our power reactor 20\%, 10\%, 50\% below the burnout heat flux. It seems to me that considerably more consideration should be given to this type of transient analysis.

\section{Nyer: Any other comments? Joe?}

Thie: I'd like to make a point, giving a little bit more thought to using transfer functions to approach Professor Thompson's cliff situation. Admittedly, you can have conditions where a change in some constant occurs at a certain power point and your dynamic analysis is completely different and you go from one set of differential equations to another. So you can run transfer functions all you want at $30 \mathrm{MW}$ but at $60 \mathrm{MW}$ they don't tell you a thing. But you might have a way of approaching $60 \mathrm{Mw}$ by running transfer functions; for example, just continually oscillate your reactor as you raise your power. This is just like dipping your toe in the water and pulling it out when your bathtub is warming up. You're sort of feeling your way and--I'm not saying that this will cover or protect you against every possible cliff. It's possible that there may be certain types of instability that we could get some information on. For example, if you put in a sinusoidal reactivity and you observe as your output in power a sine wave with a peak clipped off, then you suspect as you go up in power another megawatt or so, there's a discontinuity that's going to occur. So I think we should give more thought to experimental methods and not just increase the power without any transfer functions or any experiments at all. One has to have some alternative to the transfer function if he's going to abandon it.

Nyer: Beckjord?

Beckjord: Perhaps I didn't make myself clear--I'll try and make myself clearer. I was thinking of the work that you'd done at Ramo-Wooldridge on the channel and I don't see any objection to using the method--the transfer function method--for all it's worth. What I'm saying is this-the basic phenomenon which you're studying is the hydraulic problem-it seems to me-because the reactor part is known, that is the 
bare kinetics and, to a large extent, the nuclear feedback is known. But I think in getting at the--as has been said again and again--getting at the basic physical facts of what the hydraulic instability is, I don ${ }^{\prime} t$ think the transfer function or the oscillation method is going to explain, or by itself alone, is going to explain the phenomenon.

Nyer: Billm-I thought you had a comment--didn't you?

Ergen: Well, Warren, I had a minor comment. I think I know what Paul Kasten was trying to say in here about the transfer function being displayed in his particular case of a homogeneous reactor. In this respect he is saying that if he had a transfer function he would have noticed that there was something wrong before the chemical analysis was made which they said they could do. I think this is a very special case and I think his question was--were there any other cases like this--and the question I think, in this particular case, does make sense.

Nyer: I think we're getting rather particularized on this--so let's not $\widehat{d w e l l}$ too much on it anymore, but I'll start on this end. Tommy?

Thompson: In answer to a large part of Jim Boland's comments, I think these are very pertinent and I'd just like to see them applied to the large cores. If anyone can get into this problem, I think you should take a slug of cold water and put it in the center of an operating core and let's see what happens. Let's try it once--I mean not experimentally, but theoretically. A comment to Joe Thie, I would say that I was not proposing to tear down the system, but let's look at the realities of it and, if possible, let's get some sort of little feelers out in front of this hypothetical car so that as it approaches the edge of the cliff we'll know before we reach there. Maybe your situation is exactly the place to put in some sort of a transfer function display system such as was pointed out by Bill Ergen.

Henry: I'd like a little further discussion on the accuracy and scope of our calculational ability. It's the large reactors which you seem very wary about, and I don't think the situation is as bad as the impression I get from your remarks. We've even been able to describe some pretty complicated nonseparable space-time transients.

Thompson: I don't think it's bad--I just think there's not as much known as there should be.

Henry: I can't disagree with that.

Nyer: We're all against sin. Bob?

Wright: Very briefly, Eric, in these various modes of hydrodynamic instability, and I think we all agree there are various ways it can go; one of the big questions is, are these linear or are they a nonlinear sort of thing like a flip-flop. And one of the easiest ways to tell is to do some transfer function measurements, and see if you can excite them, and you can maybe separate out the ones that go this way and those that go the other way. 
Nyer: Any more comments? Walt?

Lipinski: I have only one comment. Unless somebody can propose some better experimental techniques, I don't know of any other method of finding out about a dynamic black box. You can have static meters hanging all over the place, but they don't tell you anything. Your step tests or transfer functions are equivalent--we're finding out more about noise--but, until we know what the source of the noise is and what its characteristic is, we won't be able to tell any more about the system.

Nyer: I'd like to go one better on that, but maybe Stu is going to do it for us.

Forbes: No--If you're going to reinforce what he's saying--go ahead.

Nyer: Go ahead. Be my guest:

Forbes: Well, there is one respect in which impulse testing and transfer function or harmonic testing are not equivalent. I'm sure you know this, but it's absolutely impossible to do a transfer function measurement in the Spert I reactor at $2000 \mathrm{Mw}$; however, you can get it up there for a few milliseconds and see what's happening. We ran into this--I'm sure Art Wasserman was going to talk to you about trying to do a transfer function in Spert I--it was not designed for heat removal and it was a heck of a job--so you can't really tell whether you can correlate this, even in low power, and you can't operate it at high enough power to see the peak.

Lipinski: Well, you've got to qualify your transfer function measurement. These are basically nonlinear processes and all you're doing is linearizing them about an operating point to extract information. Now if you want to assign too much significance to your measurements, then you're naturally going to have errors. If it's a nonlinear process you must limit yourself to small signal analysis and you have to have all your nonlinear parameters steady. In your case of Spert I, your nonlinear parameters are not steady; consequently, you are not measuring a true transfer function at the point.

Nyer: Art Wasserman would like to make a brief comment.

Wasserman: The whole point here is the distinction between concerns over power operation and concerns with safety and accidents. Period.

Nyer: That was too brief.

Wasserman: Well, another sentence. I think that Walt's concerns have been the power operation and the degree of the stability of power operation. Stu's concerns have been with the safety of this kind of a reactor under accidental conditions. Under accidental conditions you do achieve levels of power for short periods of time which throw the reactor system into a situation where its characteristics may be rather different than it is at operating power levels; therefore, even measurements, if we had 
been able to do them at Spert I, at $20 \mathrm{Mw}-$-or what have you--will still be unlikely to be extrapolable to predict or explain the course of a reactor transient which reaches a peak power of $2000 \mathrm{Mw}$.

Nyer: Bill?

Ergen: I have one comment on a different point--a sort of phobia--. . .

Nyer: I think it is.

Ergen: My concern was that these experiments on Spert and Borax usually refer to the first peak, and not very much is done about the rest. I always rationalize this in the sense that the inherent characteristics of the reactor are supposed to shut the reactor down on the first transient; then, the control mechanism is supposed to work and this is why you are justified in confining yourselves essentially to the first transient. Am I right in this?

Nyer: Yes--I think I must get some more comments. But, I believe that the point is that if you get a large amount of reactivity into the system, somehow you have to take care of this ultimately. The thing that the inherent safety--as we've been looking at it--does for you is to buy you a lot of time to do something in, because in the very short period region--the concern has always been--you can't make any mechanical system that will react fast enough.

Stu, you had your hand up.

Forbes: One thing we did observe on the runouts on the subcooled tests, and we did run some of them way out, and that was that nothing else happened. The thing perks along at $5 \mathrm{Mw}$ and you get the fuel hot and it makes it hard to instrument so we quit it. But that's not always true in boiling transients because the threshold for instability--(about 1.5 per cent) that we've found in that system is also about the biggest transient that you can put in step-wise, and it's possible to get a step in and then have the thing go into oscillations which are very nearly--or even as bad--or maybe even worse than they were in the first burst and then you're in trouble.

\section{Nyer: Any more comments? Eric?}

Beckjord: I'd like to just comment on the effect of rapid flow through the core as a fact in this matter because, if rapid flow occurs, then any self-shutdown mechanism which you might have which stops the first pulse is not going to handle a second one very quickly.

Ergen: Well, if it were possible to have a type of fuel element which continues feeding heat at the same rate as the flow removes it, then you get sort of a stationary situation.

Nyer: Fred? 
Storrer: I realize that I forgot to mention the point three which is really the most important one. Suppose $x_{t}$ does become positive for a very large peak. For instance, if we have a positive Doppler and a negative expansion coefficient which is larger, when $P$ is very large or the period gets down to a microsecond or something like this, when the inertial forces prevent expansion, $x_{t}$ will be positive. But can we operate such a reactor--that's, I think, a question that should be answered--but of course we will never operate a reactor with that short a period. But the problem is as follows: Let's suppose that you operate a reactor with values of $P$, with reciprocal period, much shorter than the critical $\mathrm{P}$ for which $\mathrm{x}_{t}$ becomes positive. Can the reactor ever, by itself, choose this shorter period. And I think this is a theoretical problem which should be answered and this is what I was trying to do, in what I showed yesterday. An example is EBR-I. For EBR-I, Mark-II, this "P" corresponded to a period of 60 seconds. When the period occurred, in 60 seconds $x_{t}$ was positive. Now the question is, could you ever excite this short period without programming it? of course, we would not want a reactor where $x_{t}$ became positive at 60 second periods, but if you have a positive Doppler this may happen at the microsecond period--I don't know. So this is the theoretical problem that I think should be answered, and this is really a net--a prompt positive coefficient. Thank you.

Nyer: Herb?

Kouts: We were looking at the asymptotic situation here. I should think there would be situations where you could also get into trouble under conditions of prompt jump if you're working that close to operating limits.

Nyer: I think the questions are petering out a little bit and unless someone feels that he has something extremely important to discuss--I guess Walt does.

Lipinski: I think I'll have to recall a previous statement that Stu took exception to. It's the magnitude of the step we disagree on. My size of step is a small linear incremental step, not a large nonlinear perturbation.

Nyer: Unless somebody else has something to come to our attention we can adjourn the meeting--and my thanks to all of you again. 


\section{APPENDIX I \\ 1960 Idaho Conference on Reactor Kinetics ACKNOWLEDGEMENTIS}

In addition to the conference attendees the editors acknowledge the direct assistance of the following people:

G. F. Brockett for recording the proceedings;

Mrs. Charlotte Brunelli, Mrs. Jean Cramer, Mrs. Tera Lee Crumley, and Mrs. Agnes Noice for transcribing the proceedings and preparing review drafts;

Lance Taylor for editorial assistance;

Numerous members of the Spert staff for proofing copy; and

Mrs. Donna George for transcribing the proceedings, preparing review drafts and for make-up and typing of the final copy. 
-

5

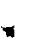

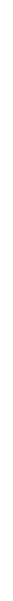




\section{APPENDIX II}

1960 Idaho Conference on Reactor Kinetics

LIST OF ATTENDEES

Anderson, R. P. (ANL-A)

Beck, C. K. (AEC-G)

Beckjord, E. S. (GE-APD)

Bentzen, F. L. (PPCo)

Blizard, E. P. (ORNL)

Boland, J. F. (ANL-I)

Bright, G. O. (PPCo) (I)

Brittan, R. O. (ANL-A)

Brockett, G. F. (PPCO)

Chernick, J. (BNL)

Cohen, E. R. (AI)

deBoisblanc, D. R. (PPCo)

Doan, R. I. (PPCO)

Epler, E. P. (ORNL)

Ergen, W. K. (ORNL)

Fischer, P. G. (GE-ANP)

Fleck, J. A. (UCRI-I)

Forbes, S. G. (R-W)

Grace, J. N. (W-Bettis)

Gyftopoulos, E. P. (MIT)

Haire, J. C. (PPCo)

Head, M. A. (GE-APED)

Henry, A. F. (W-Bettis)

Hummel, H. H. (ANL-A)

Jarrett, A. A. (AI)

Johnson, S. O. (W-Bettis)

Kasten, P. R. (ORNL)

King, L. D. P. (ORNL)

Koutz, H. J. C. (BNL)

Kunze, J. F. (GE-ANP)

Langmuir, D. B. (R-W)

Lipinski, W. C. (ANL-A)

Litteneker, P. E. (W-IRR )
Marsden, R. S. (PPCo)

McCullough, C. R. (Mon) (3)

Nyer, W. E. (PPCo)

Porter, R. A. (AEC-I) (4)

Remley, M. E. (AI)

Romie, F. (ATL)

Rothleder, B. M. (W-NRF)

Ruiz, A. L. (GE-H)

Schrock, V. E. (UC)

Schroeder, F. (PPCo)

Silver, E. R. (ORNL)

Sleeper, H. P. (GA)

Smith, R. R. (ANL-I)

Snyder, T. M. (GE-APD)

Solbrig, A. W. (ANNL-I)

Spano. A. H. (PPCo)

Stahl, R. H. (GA)

Stitt, R. K. (AI)

St John, D. S. (duP-SRL)

Stone, R. S. (GA)

Storrer, F. (APDA)

Stratton, W. R. (IASL)

Swickard, E. O. (LASL)

Szawlewicz, S. A. (AEC-G)

Thie, J. A. (McL-R) (6)

Thompson, T. J. (MIT)

Valerino, M. F. (GNEC)

Wade, J. W. (duP-SRL)

Walker, V. A. (AEC-I)

Wasserman, A. A. (PPCO)

Williams, J. S. (W-NRF)

Wimett, T. F. (IASI)

Wood, R. E. (GE-ANP)

Wright, R. W. (R-W)

Zuber, N. $(\mathrm{R}-\mathrm{W})(7)$

(1) Now with University of Oklahoma

(2) Now with Phillips Petroleum Company

(3) Now with Nuclear Utilities Services, Inc.

(4) Now with Martin Company

(5) Now with W-Bettis

(6) Now with J. A. Thie, Consultant

(7) Now with GE-Research Lab. 
•

;

\section{.}




\section{APPENDIX III}

1960 Idaho Conference on Reactor Kinetics

ORGANIZATION AFFIIIATION ABBREVIATIONS

\begin{tabular}{|c|c|}
\hline$A E C-G$ & Atomic Energy Commission - Germantown, Maryland \\
\hline$A E C-I$ & Atomic Energy Commission - Idaho \\
\hline ANL-A & Argonne National Laboratory - Argonne, Illinois \\
\hline$A N L-I$ & Argonne National Iaboratory - NRTS, Idaho \\
\hline ATI & Advanced Technology Laboratory \\
\hline APDA & Atomic Power Development Associates \\
\hline $\mathrm{AI}$ & Atomics International \\
\hline BNL & Brookhaven National Laboratory \\
\hline duP-SRI & duPont-Savannah River Laboraties \\
\hline GA & General Atomic \\
\hline GE-ANP & General Electric Company - Aircraft Nuclear Propulsion Dept. \\
\hline GE-APD & General Electric Company - Atomic Power Dept. \\
\hline GE-APED & General Electric Company - Atomic Power Equipment Division \\
\hline $\mathrm{GE}-\mathrm{H}$ & General Electric Company - Hanford \\
\hline GNEC & General Nuclear Engineering Corporation \\
\hline IASI & Ios Alamos Scientific Laboratory \\
\hline McL-R & MeLain-Rodgers Associates \\
\hline MIT & Massachusetts Institute of Technology \\
\hline Mon & Monsanto Chemical Company \\
\hline ORIL & Oak Ridge National Laboratory \\
\hline PPCo & Phillips Petroleum Company \\
\hline$R-W$ & Ramo-Wooldridge Corporation \\
\hline UCRL-I & University of California Radiation Laboratory-Livermore \\
\hline UC & University of California \\
\hline W-Bettis & Westinghouse - Bettis \\
\hline $\mathrm{W}-\mathrm{NRF}$ & Westinghouse - Naval Reactors Facility \\
\hline
\end{tabular}

